\title{
Explizite Prozesskoordination von Entscheidungsfindungsgruppen
}

\author{
Dissertation \\ zur Erlangung des Doktorgrades \\ der Mathematisch-Naturwissenschaftlichen Fakultäten \\ der Georg-August-Universität zu Göttingen
}

\author{
vorgelegt von \\ Michaela Kolbe \\ aus Halle/Saale
}

Göttingen 2006 
D7

Referentin: Prof. Dr. Margarete Boos Korreferentin: DP Dr. Micha Strack Tag der mündlichen Prüfung: 18.01.2007 


\section{Danksagung}

An dieser Stelle möchte ich allen danken, die mir im Verlauf dieser Arbeit auf unterschiedliche Weise geholfen haben.

Mein herzlicher Dank gilt meiner Doktormutter Margarete Boos - Danke für Dein Vertrauen in meine Arbeit, Deine stete Ansprechbarkeit und fachliche Unterstützung sowie für die zahlreichen Diskussionen über Koordination. Bei Micha Strack bedanke ich mich nicht nur für die Übernahme des Korreferats, - Danke vor allem für Deine methodische Unterstützung und die manchmal herausfordernden Diskussionen über Sinn und Zweck diverser statistischer Kenngrößen.

Mein innigster Dank gilt Andrea Gurtner. Merci vielmal für Deine Unterstützung, für Bern und für Deine unerschütterliche Zuversicht im Hinblick auf das Fertigwerden dieser Arbeit. Ich danke auch Franziska Tschan für die Möglichkeit des Forschungsaufenthalts in Neuchâtel.

Luisa Becker und Alexandra Stein haben als Diplomandinnen wesentlich zu dieser Arbeit beigetragen. Euch gilt mein großer Dank für Euer Engagement und Eure beruhigende Verlässlichkeit.

Große Hilfe haben Eva Biermeyer, Jan Häusser, Jule Hinkel, Melanie Jäger und Cornelia Schnur als studentische und wissenschaftliche Hilfskräfte geleistet. Ohne Eure Unterstützung beim Kodieren, ..., Versuchsleiten hätte ich das nicht geschafft! Bastian Funken, ich danke Dir für Deine unbeirrbare Förderung der Funktionsfähigkeit meines Notebooks.

Für weitere fachliche Unterstützung danke ich Adrian Bangerter, Yvonne Bogenstätter, Martin Goerges, Andreas Mojzisch, Andrea Müller, Christof Nägele, Margarita Neff-Heinrich, Barbara Schauenburg und Christina Sommer. Jule Hinkel und Carsten Marx - ich danke Euch für Euer pionierartiges Korrekturlesen. Oliver Rack - ich danke Dir für die ISF und für Das wird schon. Lisette Morris und York Hagmayer - danke besonders für Eure exklusive kulinarische Unterstützung. Martina Hake - ich danke Dir für die Hotline und die emotionale Unterstützung. Tristan Preuk - ich danke Dir für die literarischen, musikalischen und vor allem fotografischen Anregungen.

Ein wesentlicher Dank gilt meinen Eltern, Barbara und Udo Kolbe. Danke für Eure vielseitige Unterstützung und Euer Vertrauen in meine Arbeit.

Darüber hinaus möchte an dieser Stelle allen Interviewpartnern/-innen für ihre Zeit und ihre Gesprächsbereitschaft herzlich danken.

Teile der vorliegenden Arbeit wurden finanziell gefördert vom Deutschen Akademischen Austauschdienst (DAAD), der Universitätsstiftung Göttingen sowie dem Universitätsbund Göttingen e.V., ich bedanke mich herzlich für die Unterstützung. 


\begin{abstract}
This dissertation focuses on coordination of decision-making groups. Following basic concepts of group coordination and empirical results of decision-making, it is assumed that group decision processes are very complex and involve high coordination demands. Based on the model of coordination mode (Wittenbaum, Vaughan \& Stasser, 1998), the importance of explicit coordination during the group interaction process for decision quality is analyzed.
\end{abstract}

In Study 1 and 2, subjective coordination theories of experts and non-experts on group coordination and facilitation are investigated, showing that well-known coordination mechanisms ranging from simple to complex are performed with different intentions. Concerning the efficiency of coordination mechanisms, Study 3 experimentally analyzes the influence of explicit coordination on group decision quality. A trained group facilitator who was only superficially informed about the decision task and did not know the correct solution performed two explicit coordination mechanisms, asking for information and repeating information. Results indicate that asking for information does neither improve group information exchange nor decision quality. However, if the group facilitator regularly repeats already mentioned information, information exchange and decision quality improve. It is discussed that next to analyzing coordination intentions and evaluating the efficiency of coordination mechanisms, detailed investigating of coordination during the interaction process is essential for making predictions regarding both situational and end-result effectiveness of specific coordination mechanisms. For this level of investigating, a method that allows for micro-analytic assessment of coordination during group discussions is developed. Additionally, as there is no existing methodology for generating micro coding units, a technique for coding unit segmentation is developed using grammar-based rules. Using these new methods, a design is developed that allows for a systematic comparison of coordination of successful and unsuccessful decision-making groups. 


\section{Zusammenfassung}

Im Fokus der vorliegenden Arbeit steht die Koordination von Entscheidungsprozessen in Gruppen. Vor dem Hintergrund einschlägiger theoretischer Konzepte der Gruppenkoordination und empirischer Befunde zur Entscheidungsfindung wird davon ausgegangen, dass Gruppenentscheidungsprozesse sehr komplex und mit hohen Koordinationsanforderungen an die Gruppenmitglieder verbunden sind. Ausgehend von dem Modell der Koordinationsmodi (Wittenbaum et al., 1998) wird die Bedeutung expliziter Koordination während des Interaktionsprozesses für das Entscheiden von Gruppen untersucht.

In Studie 1 und 2 werden subjektive Koordinationstheorien von Experten/-innen und Nicht-Experten/-innen erhoben. Die Ergebnisse zeigen, dass eine Vielzahl einfacher und komplexer Mechanismen zur Koordination von Gruppen bekannt sind, die mit unterschiedlichen Intentionen eingesetzt werden. Um der Frage nachzugehen, zu welchen Konsequenzen der Einsatz dieser Mechanismen führt, wird in Studie 3 der Einfluss expliziter Prozesskoordination auf die Entscheidungsgüte experimentell überprüft. Die Koordinationsmechanismen Informationen erfragen und Informationen wiederholen werden von einem/r inhaltlich neutralen, geschulten Moderator/-in ausgeführt. Es zeigt sich, dass das Erfragen von Informationen weder den Informationsaustausch noch die Entscheidung verbessert. Durch das Wiederholen von Informationen kann hingegen eine Optimierung des Informationsaustauschs und dadurch eine bessere Gruppenentscheidung erreicht werden.

Es wird diskutiert, dass neben der Erhebung der subjektiven Perspektive auf die Koordination und der Überprüfung der Wirksamkeit einzelner Koordinationsmechanismen die Untersuchung von Koordination im Interaktionsprozess wesentlich ist, um Aussagen über die situative Effektivität einzelner Koordinationsmechanismen zu treffen. Dazu wird eine Methode entwickelt, die eine mikroanalytische Erfassung der Koordination von Gruppendiskussionen erlaubt. Ausgehend von dem bisher uneinheitlichen Vorgehen in der Aufbereitung von 
transkribierten Gruppendiskussionen, und den fehlenden Methoden zur Definition von Kodiereinheiten, wird zusätzlich ein Verfahren entwickelt, das eine regelgeleitete und reliable Bildung von Kodiereinheiten ermöglicht. Auf Grundlage der entwickelten Verfahren wird abschließend ein Design entwickelt, welches einen systematischen Vergleich der Koordinationsmechanismen erfolgreicher und nicht erfolgreicher Entscheidungsfindungsgruppen erlaubt. 


\section{Inhaltsverzeichnis}

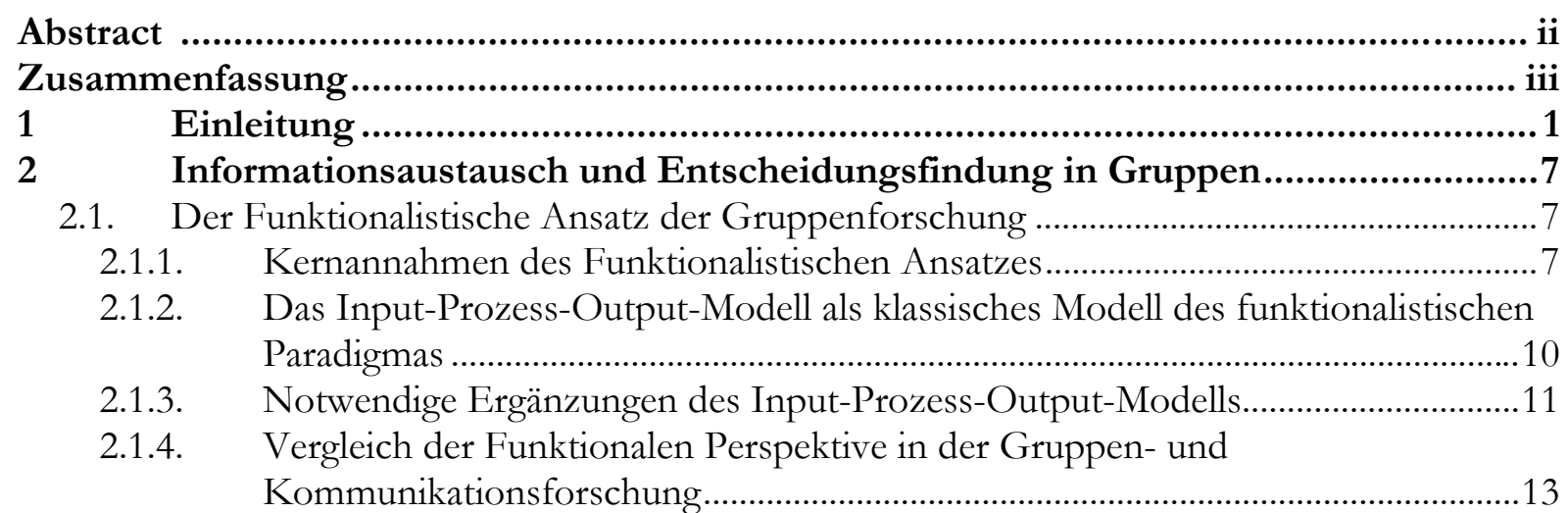

2.2. Eine funktionale Theorie zur Entscheidungsfindung in Gruppen ...................................14

2.3. Empirische Befunde zum Informationsaustausch und zur Entscheidungsfindung in

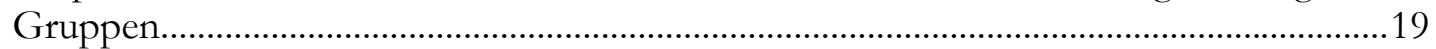

2.4. Fazit zur Entscheidungsfindung und zum Informationsaustausch .................................20

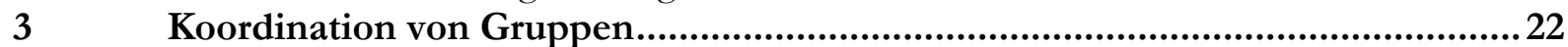

3.1. Koordinationsbedarf bei Gruppenentscheidungsaufgaben ..............................................22

3.1.1. „Verluste“ und „Gewinne“ während des Gruppenprozesses .....................................24

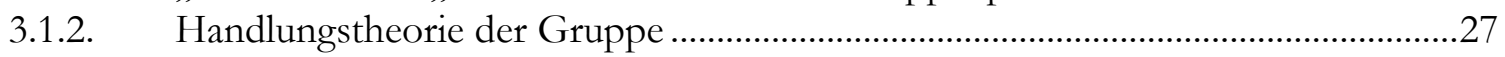

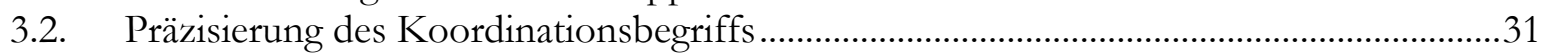

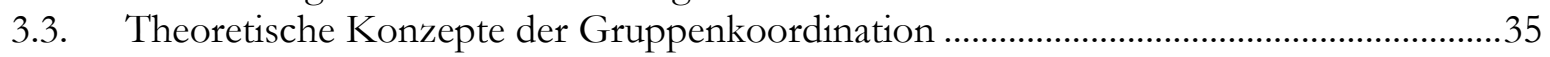

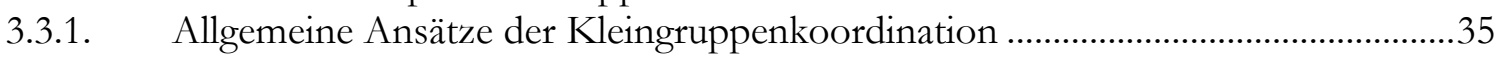

3.3.2. Implizite und explizite Koordinationsmechanismen - Das Modell von Espinosa, Kraut und Lerch (2004) .................................................................................................. 38

3.3.3. Das Modell der Koordinationsmodi von Wittenbaum, Vaughan und Stasser

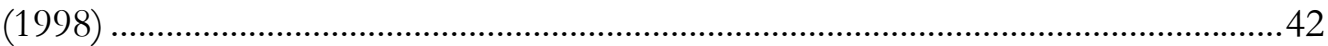

3.4. Explizite Prozesskoordination durch Kommunikation........................................................51

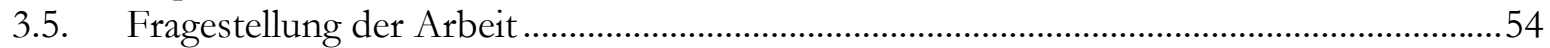

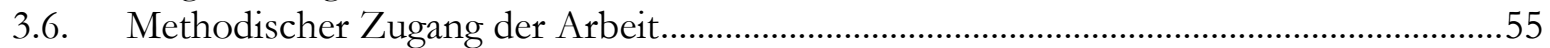

4 Subjektive Theorien expliziter Koordination - Koordination aus Perspektive der

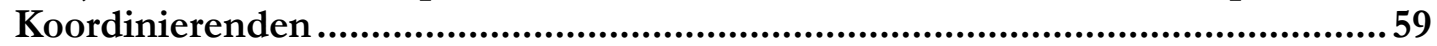

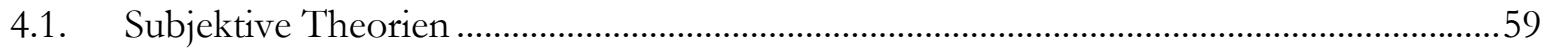

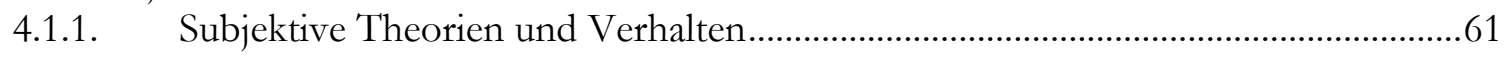

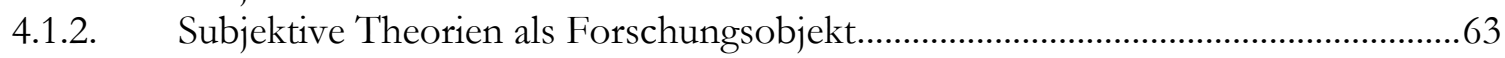

4.1.3. Subjektive Theorien expliziter Koordination...............................................................64

4.2. Studie 1: Subjektive Theorien der Koordination: Die naive Perspektive der

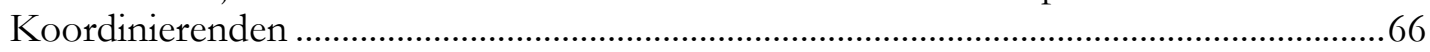

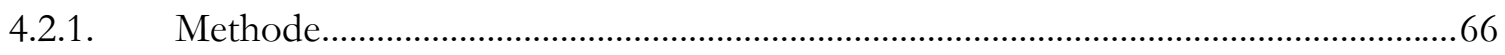

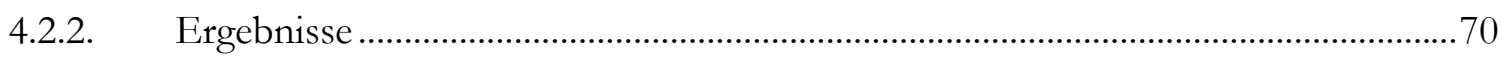

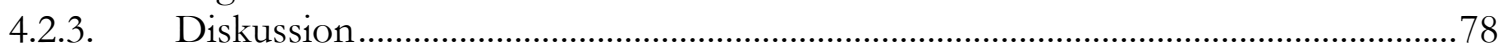

4.3. Studie 2: Subjektive Theorien der Koordination: Die erfahrene Perspektive der

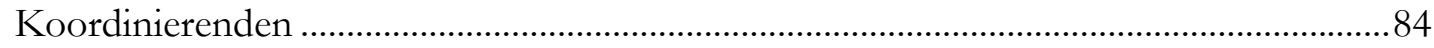

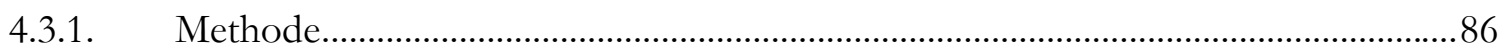

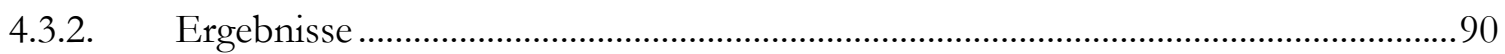

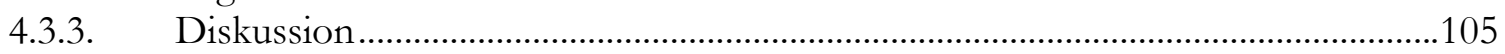

4.4. Studie 1 und Studie 2 - Implikationen der Studien zur Erfassung subjektiver

Koordinationstheorien .................................................................................................111

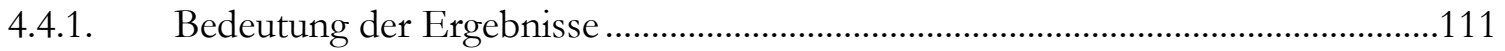

4.4.2. Implikationen für das weitere Vorgehen ..................................................................115

$5 \quad$ Studie 3: Der Einfluss von Koordinationsmechanismen auf die

Entscheidungsfindung - eine experimentelle Überprüfung ...............................116 


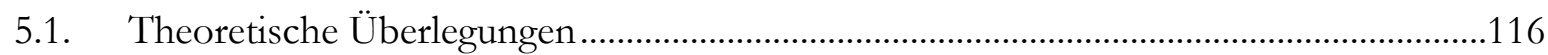

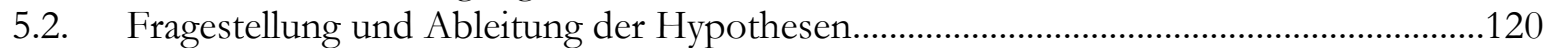

5.3. Methode

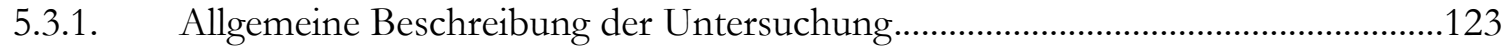

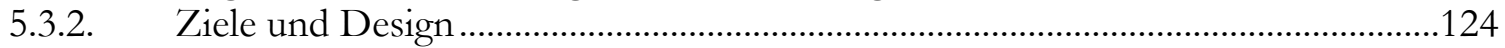

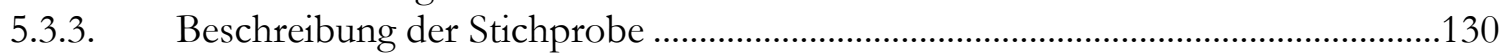

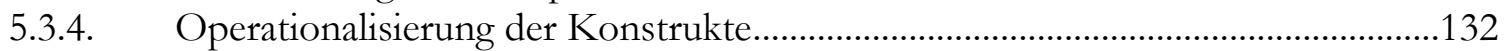

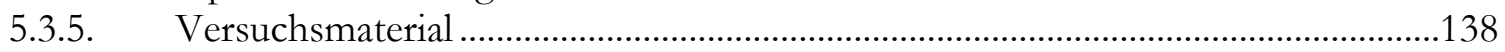

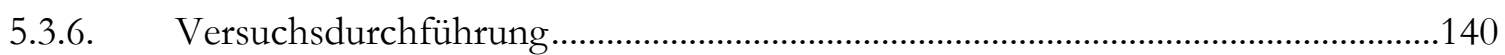

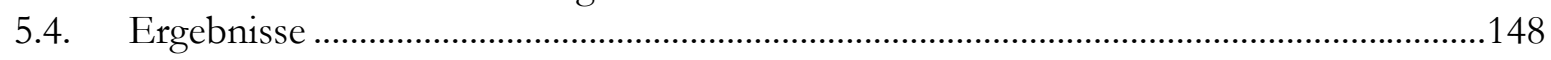

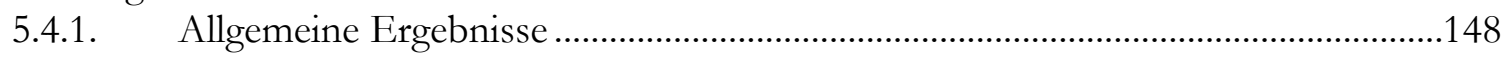

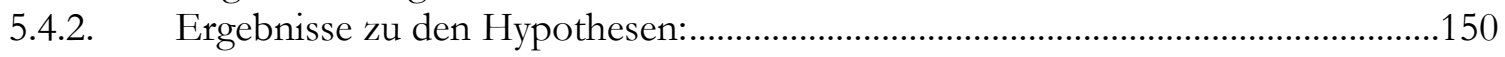

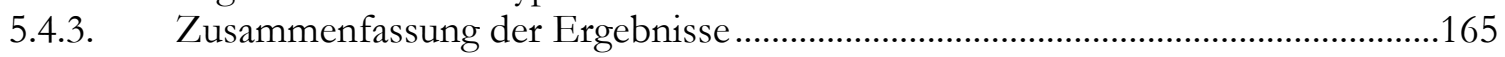

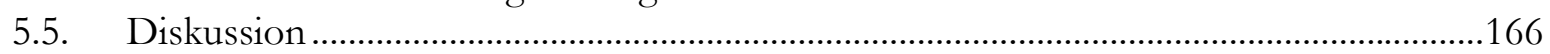

5.5.1. Interpretation und Diskussion der erhaltenen Ergebnisse .....................................166

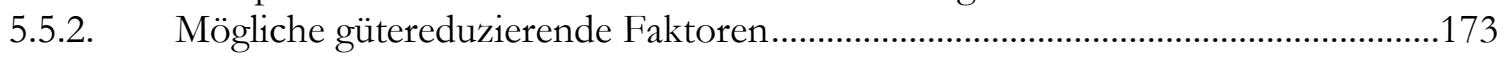

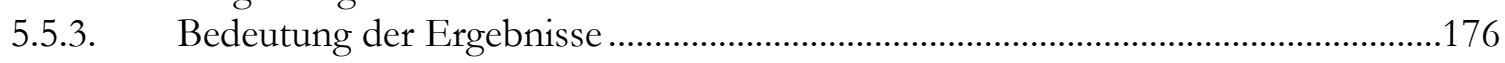

$6 \quad$ Methodenentwicklung 1: Ein Kodiersystem zur mikroanalytischen Erfassung von Koordination in Gruppendiskussionen ...............................................181

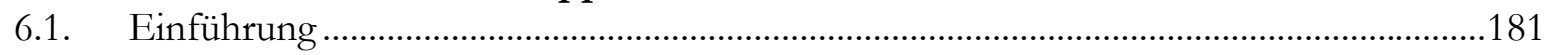

6.1.1. Theoretischer Hintergrund: Mikroanalytische Verhaltensbeobachtung in der

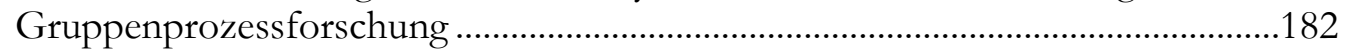

6.1.2. Koordinationsmechanismen als Gegenstand des Kodierverfahrens.......................185

6.1.3. Ziel des Kategoriensystems ………….................................................................187

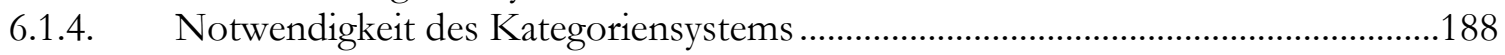

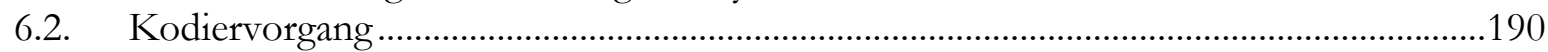

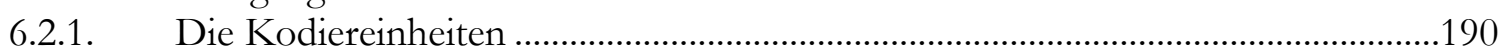

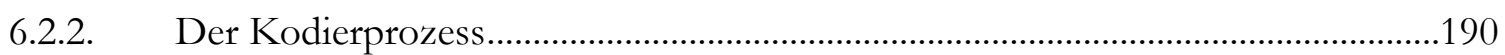

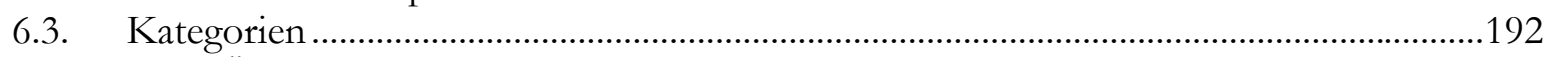

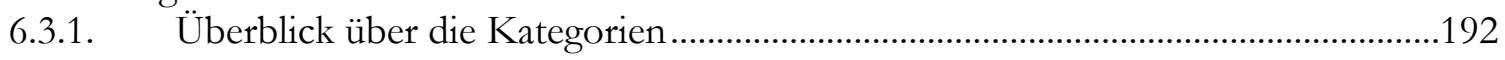

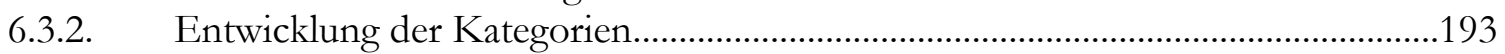

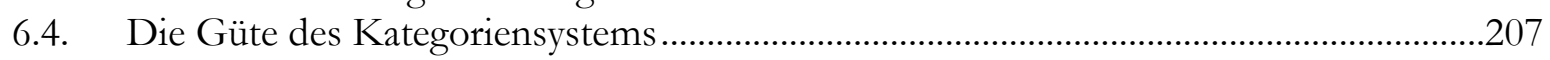

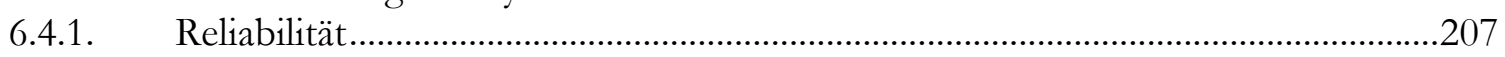

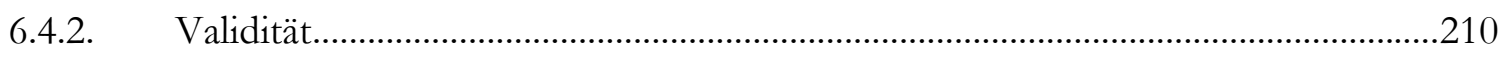

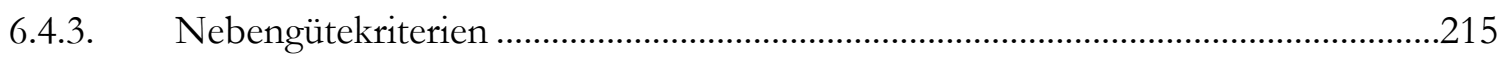

$7 \quad$ Methodenentwicklung 2: Eine Methode zur Bildung von Kodiereinheiten in

Transkripten verbaler Interaktionsprozesse ................................................... 217

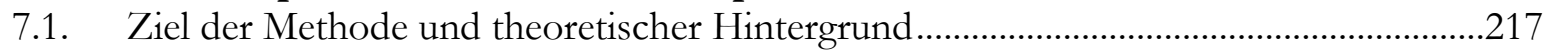

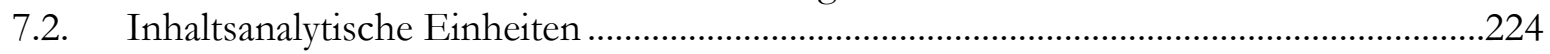

7.3. Formale Gestaltung der Zergliederung einzelner Redebeiträge in Kodiereinheiten: ....226

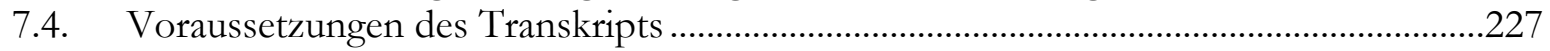

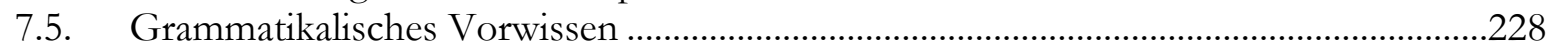

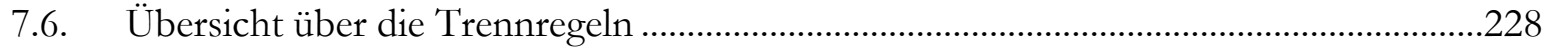

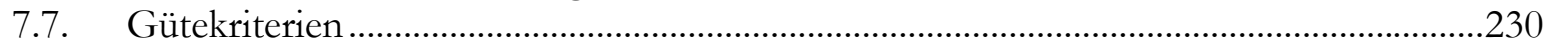

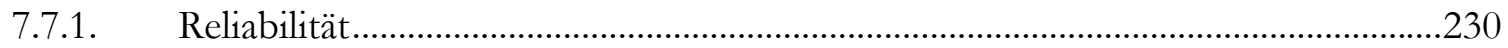

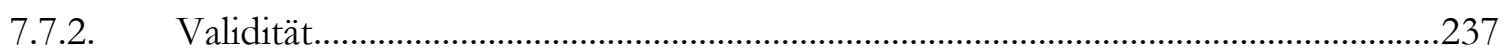

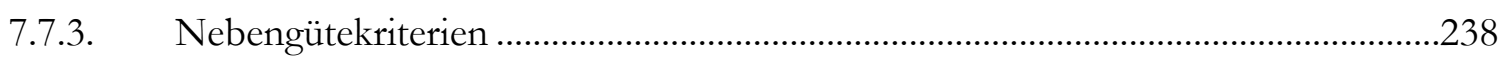

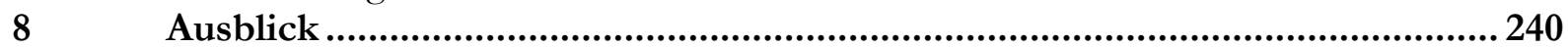

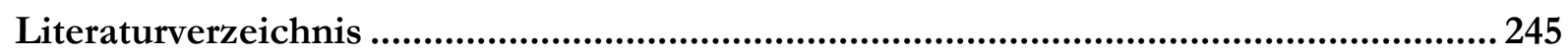

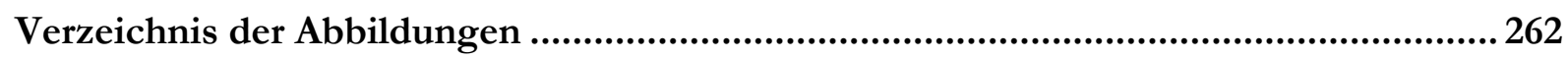

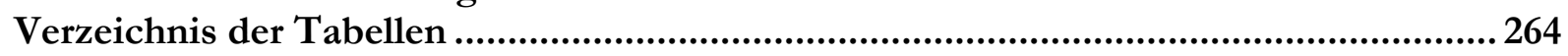

Anhang 


\section{$1 \quad$ Einleitung}

Die Psychologie der Gruppe ist seit einem Jahrhundert Gegenstand psychologischer Forschung (vgl. Boos, 1997). Dabei hat sich gezeigt, dass die Gruppe nicht nur ein Aggregat ihrer Mitglieder ist, sondern eine eigene Dynamik entfaltet und in ihrer Gesamtheit untersuchenswert ist. Arbeitsgruppen sind Kleingruppen interdependenter Individuen, die in abgestimmter Weise an unterschiedlichen Aufgaben arbeiten, um spezifische und gemeinsame Ziele zu erreichen (Brannick \& Prince, 1997) und sich ihre Verantwortung für organisationale Ergebnisse teilen (Sundstrom, 1990).

In Organisationen werden wichtige und komplexe Entscheidungen oft Gruppen übertragen. Dabei wird angenommen, dass die Gruppenmitglieder über unterschiedliche Expertisen verfügen und der Gruppe dadurch einen breiten Zugang zu entscheidungsrelevanten Ressourcen ermöglichen (Larson, Foster-Fishman \& Franz, 1998b). Beispielsweise muss ein Managementteam eine gemeinsame Entscheidung über die strategische Unternehmensplanung mit der Garantie einer langfristigen Gewinnmaximierung treffen, wobei die Mitglieder des Managements individuell durchaus unterschiedliche Ziele haben können. Personalauswablkommissionen mit Experten/-innen unterschiedlicher Fachabteilungen müssen entscheiden, welche/r Bewerber/-in den ausgeschriebenen Arbeitsplatz am besten ausfüllen wird. OP-Crews ${ }^{1}$ müssen unter Zeitdruck entscheiden, wie in unvorhergesehenen Notfällen in der Behandlung des/der Patienten/-in weiter verfahren wird, wobei insbesondere die Abstimmung zwischen Chirurgen/-innen und Anästhesisten/-innen fehlerlos funktionieren muss: Die Chirurgen/-innen sind auf die verlässliche Rückmeldung aktueller Patientendaten durch die Anästhesisten/-innen angewiesen, nur gemeinsam können sie eine korrekte Versorgung gewährleisten.

\footnotetext{
${ }^{1}$ Arbeitsgruppen im Operationssaal (OP) zeichnen sich durch eine Crew-Struktur aus, da die Gruppenmitglieder nicht dauerhaft in gleicher Zusammensetzung arbeiten, sondern vielmehr aus einem Pool verfügbarer Fachkräfte ausgewählt werden (vgl. auch Arrow, McGrath \& Berdahl, 2000).
} 
Diese Beispiele zeigen den so genannten Synergieeffekt. Er besagt, dass die Gruppe als Ganzes mehr leisten kann als die Summe der individuellen Leistungen ergäbe (West, 2004; Wilson, 2005) und ist der Grund für den strategischen Einsatz von Arbeitsgruppen (Scherm, 1998). Das Synergiepotential der Gruppe besteht bei Entscheidungsaufgaben in der Unterschiedlichkeit der Expertisen und Problemsichten der Gruppenmitglieder. Die Synergie wird möglich über den Austausch dieser unterschiedlichen Problemsichten und Erfahrungen und das gemeinsame Abwägen verschiedener, insbesondere negativer, Folgen von Handlungs- und Entscheidungsalternativen. Im Gegensatz zur reinen Ideenfindungsaufgabe erfordern Problemlöse- und Entscheidungsaufgaben von der Gruppe den effektiven Umgang mit Interdependenz, die in Organisationen durch unterschiedliche, aber sich gegenseitig beeinflussende, Verantwortungsbereiche der Gruppenmitglieder entsteht. Die Entscheidungsfindung erfordert zusätzlich eine Diskussion und Übereinkunft über die der Entscheidung zugrunde liegenden Bewertungsmaßstäbe (Boos \& Sassenberg, 2001). Durch diese hohen Aufgabenanforderungen erreicht der Gruppenentscheidungsprozess eine Komplexität, die von den Gruppenmitgliedern nicht immer optimal bewältigt wird (Kerr \& Tindale, 2004; Mojzisch \& Schulz-Hardt, 2006; Stasser \& Titus, 1985). Diese Komplexität der Entscheidungsaufgabe erfordert, dass die Gruppenmitglieder ihre individuellen Handlungen koordinieren (Boos \& Sassenberg, 2001; Dickinson \& McIntyre, 1997; Scharpf, 1988; Tschan \& Semmer, 2001). Koordination stellt eine grundlegende Anforderung an Gruppenarbeit dar (Brauner, 2001) und ist in Gruppen hauptsächlich über Kommunikation möglich (Brauner, 2001). Die Art dieser durch Kommunikation ermöglichten Koordination und ihrer Wirkungen ist Gegenstand der vorliegenden Arbeit.

Grundlage der Arbeit ist das Modell der Koordinations-Modi von Wittenbaum, Vaughan und Stasser (1998). Es betrachtet Koordination auf zwei Dimensionen: Zeitpunkt und Explizitheit der Koordination. Der Fokus dieser Arbeit liegt auf expliziter Koordination während des Interaktionsprozesses, die im Folgenden als explizite Prozesskoordination bezeichnet wird. 
Das Ziel der vorliegenden Arbeit besteht in der Beschreibung expliziter Prozesskoordination von Arbeitsgruppen und in der Analyse ihrer Wirkung auf die Entscheidungsfindung. Thematisch lässt sich die Arbeit einordnen in die sozialpsychologische Forschung zur Entscheidungsfindung, Koordination und Interaktion in Gruppen (Becker-Beck, 1997; Boos, 1996b; Boos, Morguet, Meier \& Fisch, 1990; Boos \& Sassenberg, 2001; Ellis \& Fisher, 1994; Gurtner, 2003; Gurtner, Tschan, Semmer \& Nägele, in press; Hackman \& Morris, 1975; Hirokawa \& Salazar, 1999; Kerr \& Tindale, 2004; Larson, Christensen, Franz \& Abbott, 1998a; Larson et al., 1998b; Sexton, 2004; Sexton et al., 2004; Stasser \& Titus, 1987; Tschan, 2000; Tschan, Semmer, Nägele \& Gurtner, 2000; Wittenbaum et al., 1998).

Nach der Darstellung des relevanten theoretischen und empirischen Hintergrundes soll explizite Prozesskoordination in Studie 1 und 2 aus der Perspektive der koordinierenden Personen bzw. auf der Bedeutungsebene betrachtet werden: Explizite Koordination wird von Gruppenleitern/-innen oder -mitgliedern intentional eingesetzt. Die individuellen Intentionen und Heuristiken des Einsatzes expliziter Koordinationsmechanismen werden im Rahmen subjektiver Theorien in der praktischen Gruppenkoordination unerfahrener Personen (Studie 1) und erfahrener Personen (Studie 2) erhoben. Dadurch soll, neben Erkenntnissen über die individuelle Bedeutung expliziter Koordination, eine differenzierte Phänomenbeschreibung im Rahmen der Grounded Theory ${ }^{2}$ (Glaser \& Strauss, 2005) ermöglicht werden.

Nach der Betrachtung der subjektiven Perspektive soll auf der Handlungsebene die Umsetzung der Koordination in konkrete Handlungen untersucht werden. Im Fokus steht die Effektivität expliziter Koordinationshandlungen: Führen Handlungen expliziter Koordination während des Gruppenentscheidungsprozesses zu besseren Entscheidungen? Dazu werden in Studie 3 zwei ausgewählte explizite Koordinationsmechanismen in einer Hidden-Profile-

\footnotetext{
2 Unter Grounded Theory ist keine Theorie, sondern eine Konzeptualisierung von Theoriebildung zu verstehen (Howitt \& Cramer, 2005). Sie beinhaltet Richtlinien zur Erfassung, Kodierung und Analyse von Daten zur Generierung von Theorien (Glaser \& Strauss, 2005) und legt einen flexiblen Gebrauch von Daten und schwerpunktmäßig komperative Analysen nahe (Glaser \& Strauss, 2005).
} 
Gruppenentscheidungsaufgabe bezüglich ihrer Wirkungen auf die Gruppenentscheidung untersucht.

Die Ergebnisse der Studien 1 bis 3 werden ausführlich diskutiert, wobei die Notwendigkeit des systematischen Vergleichs des Einsatzes von Koordinationsmechanismen im Entscheidungsprozess von erfolgreichen versus erfolglosen Entscheidungsgruppen verdeutlicht wird.

Zur handlungsanalytischen Untersuchung wurden daher zwei Methoden zur Erfassung expliziter Prozesskoordination entwickelt: Das Kodiersystem zur mikroanalytischen Erfassung von Koordination in Gruppendiskussionen (Methodenentwicklung 1) dient der detaillierten Beschreibung von Koordinationshandlungen der Gruppenmitglieder und ermöglicht deren sequentielle Betrachtung. Es wurde sowohl deduktiv (theoriegeleitet und ähnliche Verfahren integrierend) als auch induktiv (datennah) entwickelt. Die Methode zur Einteilung von Kodiereinheiten in Transkripten verbaler Interaktionsprozesse (Methodenentwicklung 2) widmet sich dem methodischen Problem bisher nahezu fehlender Verfahren zur systematischen Extraktion von Kodiereinheiten aus den Redebeiträgen der Gruppendiskussionen. Es werden ein Design zur Anwendung beider Verfahren im Rahmen eine Prozessanalyse zum systematischen Vergleich erfolgreicher und erfolgloser Entscheidungsgruppen ${ }^{3}$ sowie Empfehlungen für weiterführende Forschungen entwickelt.

Tabelle 1 fasst den skizzierten Forschungsplan zusammen.

\footnotetext{
${ }^{3}$ Die Umsetzung des vorgestellten Designs ist nicht mehr Gegenstand der vorliegenden Arbeit.
} 
Tabelle 1: Übersicht über den Forschungsplan der vorliegenden Arbeit

\begin{tabular}{|c|c|c|c|}
\hline & $\begin{array}{l}\text { Theoretische } \\
\text { Anforderungen }\end{array}$ & Methodische Anforderungen & Erwartete Resultate \\
\hline $\begin{array}{l}\text { 1. Phänomen- } \\
\text { beschreibung }\end{array}$ & keine & $\begin{array}{l}\text { Qualitativ-quantitative Analyse von } \\
\text { Gruppenkoordination } \\
\text { (Studie 1, Studie 2) }\end{array}$ & $\begin{array}{l}\text { Phänomenologische } \\
\text { Beschreibung von } \\
\text { Gruppenkoordination aus der } \\
\text { Perspektive der } \\
\text { Koordinierenden } \\
\text { (Bedeutungsebene) }\end{array}$ \\
\hline $\begin{array}{l}\text { 2. Analyse } \\
\text { kausaler } \\
\text { Beziehungen }\end{array}$ & $\begin{array}{l}\text { Hypothesen } \\
\text { über den Erfolg } \\
\text { von } \\
\text { Koordinations- } \\
\text { mechanismen }\end{array}$ & $\begin{array}{l}\text { Experiment zur Untersuchung des } \\
\text { Einflusses von } \\
\text { Koordinationsmechanismen auf die } \\
\text { Entscheidungsfindung } \\
\text { (Studie 3) }\end{array}$ & $\begin{array}{l}\text { Koordinationsmechanismen, } \\
\text { die als Interventionsmaßnahme } \\
\text { die } \\
\text { Gruppenentscheidungsfindung } \\
\text { verbessern } \\
\text { (Handlungsebene) }\end{array}$ \\
\hline $\begin{array}{l}\text { 3. (Entwicklung } \\
\text { von } \\
\text { Verfahren } \\
\text { zur) Prozess- } \\
\text { analyse }\end{array}$ & $\begin{array}{l}\text { Formales } \\
\text { Arbeitsmodell }\end{array}$ & $\begin{array}{l}\text { Entwicklung von } \\
\text { Verhaltensbeobachtungsinstrumenten } \\
\text { zur Erfassung von Koordination } \\
\text { (Methodenentwicklung 1, } \\
\text { Methodenentwicklung 2) }\end{array}$ & $\begin{array}{l}\text { Aussagen über konkrete } \\
\text { Koordinationsverhaltens- } \\
\text { abfolgen und -muster, die } \\
\text { erfolgreiche Gruppen von nicht } \\
\text { erfolgreichen unterscheiden } \\
\text { (Handlungsebene) }\end{array}$ \\
\hline
\end{tabular}

Die genannten Ziele legen der vorliegenden Arbeit forschungsmethodisch sowohl einen quantitativen als auch einen qualitativen Ansatz zugrunde (vgl. Kapitel 3.6). Die Untersuchung von Gruppenkoordination im Rahmen sozialpsychologischer Kleingruppenforschung entbehrt bisher einer grundlegenden Theorie der Koordination. Es existieren Taxonomien einzelner Koordinationsformen (vgl. Wittenbaum et al., 1998) und vereinzelte empirische Befunde zur situativen Erscheinung und Effektivität dieser Koordinationsformen (vgl. Espinosa, Lerch \& Kraut, 2004; Grote, Zala-Mezö \& Grommes, 2003). Jedoch wurde bisher weder eine formale Theorie zu Inhalt, Bedingungen und Wirkungsweise noch eine normative Theorie zum idealtypischen Ablauf von Koordination postuliert, was allerdings durch die sehr unterschiedlichen Koordinationsanforderungen der jeweiligen Gruppenaufgabe (motorische Aufgabe, Ideen- oder Entscheidungsfindung) bedingt sein mag. Die vorliegende Arbeit verfolgt 
nicht den Anspruch, eine Theorie der Koordination aufzustellen. Vielmehr soll sie einen Beitrag dazu leisten, bisherige theoretische Ansätze (Wittenbaum et al., 1998) zu präzisieren und den Gegenstand expliziter Prozesskoordination differenziert zu betrachten. Um dieses Ziel zu erreichen, ist einerseits die Verwendung qualitativer Methoden erforderlich (Interviews und Verhaltensbeobachtung), um neben einer quantitativ-experimentellen Überprüfung Koordination aus verschiedenen Perspektiven betrachten zu können: Subjektive Sichten koordinierender Personen (Studie 1 und Studie 2), Effektivität von Koordinationsmechanismen (Studie 3) und Verbaltensbeobacbtung durch Dritte (Methodenentwicklung 1 \& 2).

Vor der Darstellung der durchgeführten Studien werden grundlegende theoretische Konzepte zum Informationsaustausch und zur Entscheidungsfindung in Gruppen (Kapitel 2) sowie zur Koordination von Gruppen (Kapitel 3) erläutert. 


\section{Informationsaustausch und Entscheidungsfindung in Gruppen}

Die vorliegende Arbeit geht von zwei Annahmen aus:

1. Damit Gruppen Entscheidungen treffen können, müssen die Gruppenmitglieder ihre individuellen Informationen austauschen.

2. Der Informationsaustausch bei Entscheidungsaufgaben ist ein komplexer Prozess und bedarf der Koordination.

Die Forschung zur Koordination sowie zum Informationsaustausch und zur Entscheidungsfindung in Gruppen basiert auf dem funktionalistischen Paradigma der Gruppenforschung (Wittenbaum et al., 2004b). Dieses Paradigma wird zunächst vorgestellt (2.1). Anschließend werden eine funktionale Theorie sowie empirische Befunde zur Entscheidungsfindung in Gruppen (2.2 bzw. 2.3) erläutert.

\subsection{Der Funktionalistische Ansatz der Gruppenforschung}

\subsubsection{Kernannahmen des Funktionalistischen Ansatzes}

Gruppen sind komplexe soziale Systeme (Arrow et al., 2000; Cranach, Ochsenbein \& Valach, 1986; Gurtner, 2003), weshalb zu ihrer Untersuchung eine Reihe unterschiedlicher Betrachtungsebenen existieren: beispielsweise die Perspektive der Sozialen Identität (Hogg, Abrams, Otten \& Hinkle, 2004), die Symbolisch-Interpretative Perspektive (Frey, 2004), die zeitliche Perspektive (Arrow, Poole, Henry, Wheelan \& Moreland, 2004), die psychodynamische Perspektive (McLeod \& Kettner-Polley, 2004), die Netzwerk-Perspektive (Katz, Lazer, Arrow \& Contractor, 2004) und die Funktionale Perspektive (Wittenbaum et al., 2004b) ${ }^{4}$.

Für die vorliegende Arbeit eignet sich die funktionale Perspektive der Gruppenforschung, da sie ein normatives Vorgehen zur Beschreibung und Vorhersage von Gruppenleistung bietet

\footnotetext{
${ }^{4}$ Eine erläuternde Übersicht der Perspektiven ist in Hollingshead und Poole (2004) und in Poole, Hollingshead, McGrath, Moreland und Rohrbaugh (2004) zu finden, ein differenzierter Überblick über die Forschungsrichtungen in Arrow, McGrath und Berdahl (2000).
} 
(Wittenbaum et al., 2004b). Ihr Betrachtungsfokus liegt auf so genannten Inputfaktoren, welche die

Gruppenleistung, vermittelt über den Interaktionsprozess der Gruppenmitglieder, beeinflussen:

"Success is not only a function of team members' talents and available resources but also the processes team members use to interact with each other to accomplish the work." (Marks, Mathieu \& Zaccaro, 2001, S. 356, Herv. im Original)

Durch die Analyse des Interaktionsprozesses soll erklärt werden, warum einige Gruppen erfolgreich sind, andere hingegen nicht (Wittenbaum et al., 2004b). Drei Kernannahmen bilden den Schwerpunkt des funktionalistischen Ansatzes:

1. Gruppen sind zielorientiert.

2. Die Gruppenleistung variiert und kann evaluiert werden.

3. Vermittelt über den Interaktionsprozess beeinflussen interne (z.B. Gruppengröße, Persönlichkeit der Gruppenmitglieder) und externe Faktoren (z.B. Charakteristika der übergeordneten Organisation, Aufgabe) die Gruppenleistung.

Diese Annahmen bieten die Anwendung der funktionalistischen Perspektive zur Untersuchung des Einflusses expliziter Koordinationsmechanismen auf die Gruppenleistung an, da Koordinationsmechanismen den Interaktionsprozess koordinieren sollen und ihre Wirksamkeit daher im Rahmen des Input-Prozess-Output-Wirkungsgefüges untersucht werden muss. Abbildung 1 zeigt für die vorliegende Arbeit die Relation der Konzepte Entscheidungsfindung, Informationsaustausch und Koordination im Rahmen der funktionalistischen Perspektive: Das Entscheiden ist der Gruppenaufgabentyp und stellt damit eine Input-Variable dar. Während des Entscheidungsprozesses müssen die Gruppenmitglieder ihre vorhandenen Informationen austauschen, daher bildet der Informationsaustausch den Prozess. Aufgrund der Komplexität der Entscheidungsaufgabe muss der Informationsaustausch koordiniert werden, die explizite Prozesskoordination stellt daher eine Prozessvariable dar. 
Input

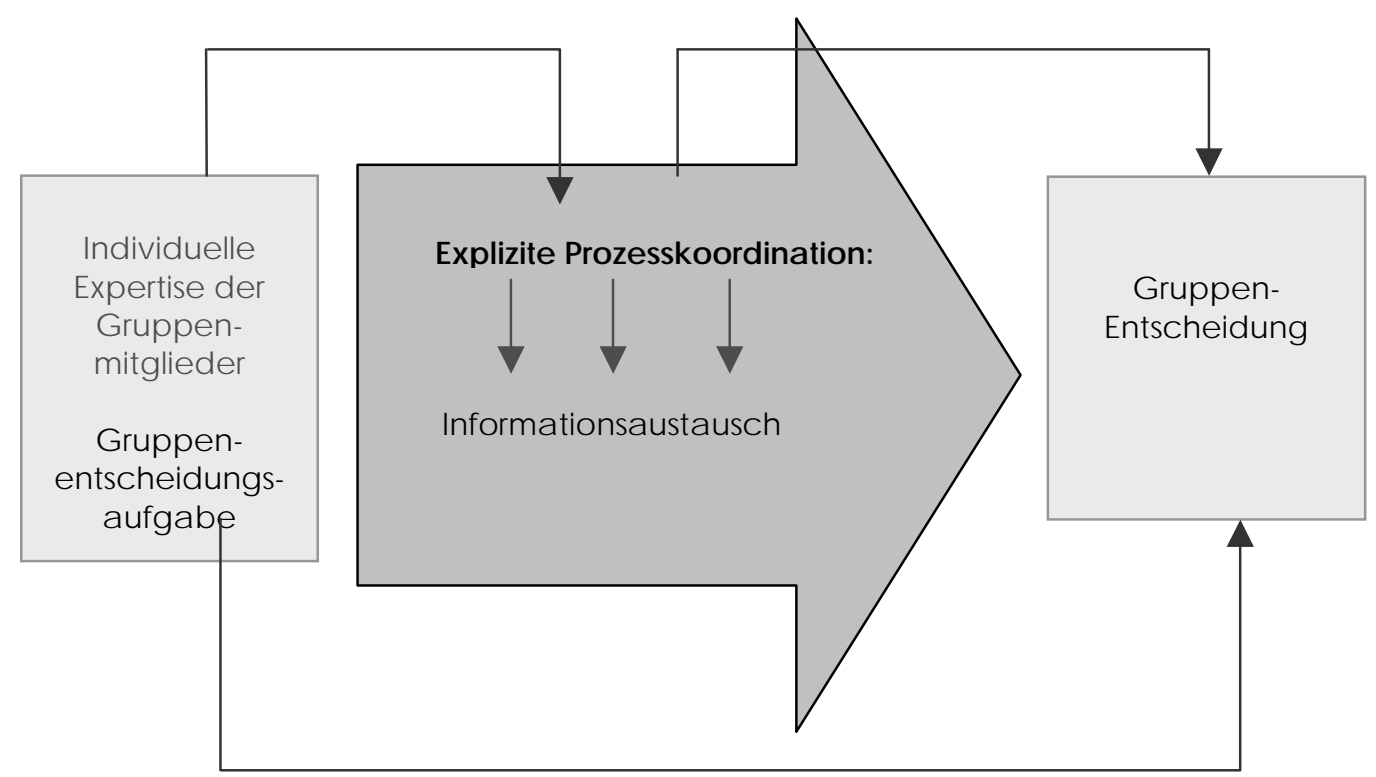

Abbildung 1: Einordnung der Konzepte Entscheidungsfindung, Informationsaustausch und explizite Prozesskoordination in das funktionale Paradigma

Der Vorteil des funktionalistischen Ansatzes besteht darin, dass er die Aufstellung testbarer Hypothesen erlaubt (Wittenbaum et al., 2004b). Nachteilig kann der implizite Fokus auf lediglich aufgabenorientierte Ziele wirken. Fraglich bleibt auch, ob der Ansatz eine Betrachtung der Gruppe als komplexes, adaptives und dynamisches System erlaubt (Wittenbaum et al., 2004b). Als Forschungsparadigma hat er in den letzten Jahren hauptsächlich Untersuchungen zu kausalen Wirkungsbeziehungen zwischen Input- bzw. Prozessvariablen und Outputvariablen angeregt (Wittenbaum et al., 2004b). Diese Studien ermöglichen wichtige Einblicke in einzelne Mechanismen der Gruppenleistung, dennoch wird mittlerweile der Ruf nach einer stärker bedingungs- und interventionsorientierten, statt einer ursachenorientierten, Gruppenforschung laut (Hackman, 2006), interessanterweise vom Autor des einschlägigen Input-Prozess-OutputModells (IPO, Hackman \& Morris, 1975), welches im folgenden Abschnitt kurz erläutert wird. 


\subsubsection{Das Input-Prozess-Output-Modell als klassisches Modell des funktionalistischen Paradigmas}

Das Input-Prozess-Output (IPO)-Modell betont die Analyse von Inputvariablen und ihren durch den Interaktionsprozess vermittelten Einfluss auf den Output, um Antworten auf die Frage zu finden, warum manche Arbeitsgruppen effektiver sind als andere. Die Inputfaktoren werden auf drei Ebenen betrachtet: Ebene des Gruppenmitgliedes, Ebene der Gruppe, Ebene des Kontextes der Gruppe. Der Grundgedanke des Modells besteht darin, dass die Inputfaktoren keinen direkten Einfluss auf das Gruppenergebnis ausüben, sondern über den Interaktionsprozess der Gruppenmitglieder mediiert werden (siehe Abbildung 2).

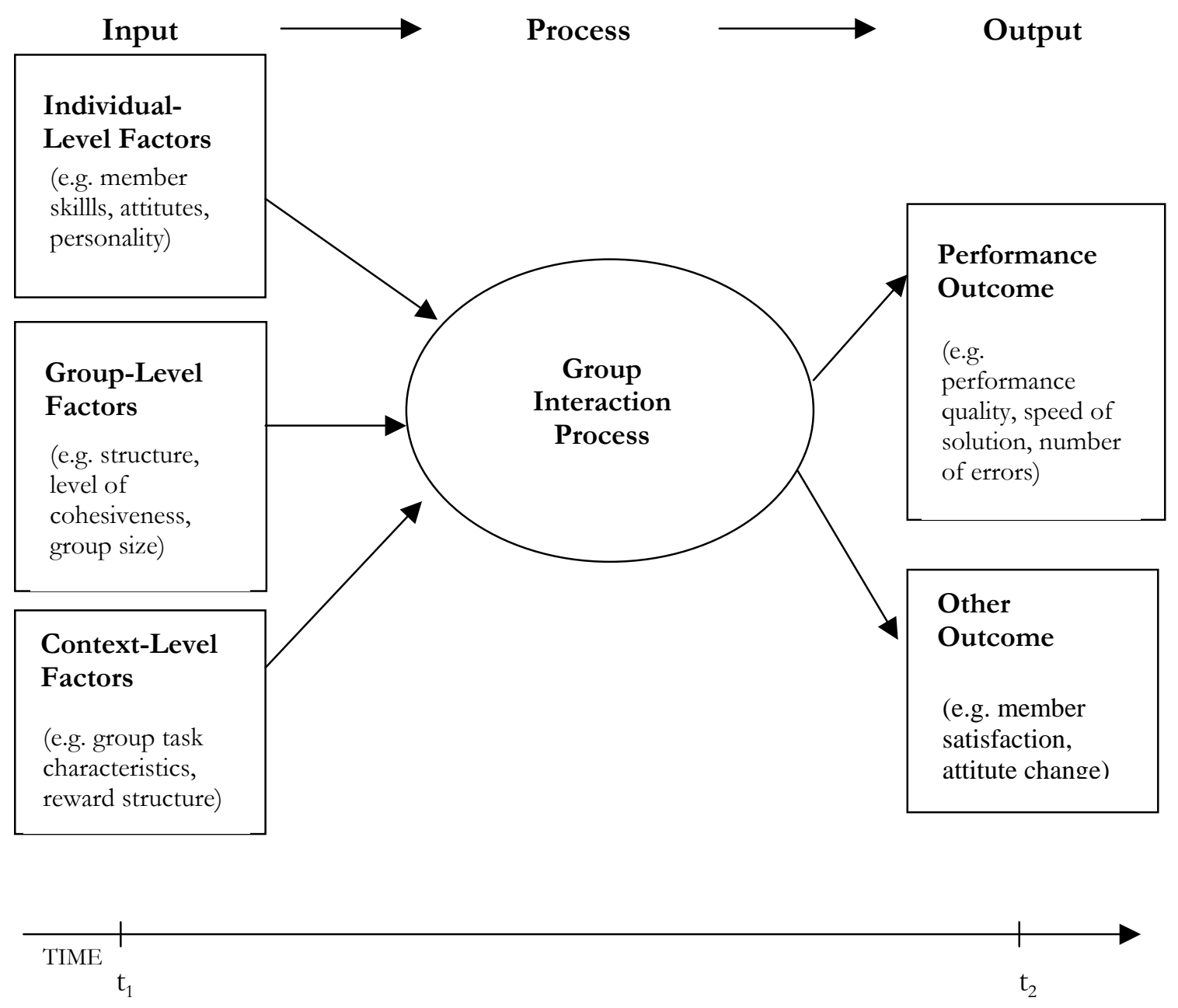

Abbildung 2: Traditionelles Paradigma zur Analyse der Gruppeninteraktion als Mediator von Gruppenergebnissen. Nach Hackman und Morris (1975) sowie McGrath (1970) 
Der Interaktionsprozess entspricht allem beobachtbaren Verhalten zwischen zwei (künstlichen) Zeitpunkten ( $t_{1}$ und $t_{2}$ ) und kann beispielsweise nur einige Sekunden oder aber ein Jahr lang sein (Hackman \& Morris, 1975). Zwar weisen Hackman und Morris (1975) darauf hin, dass die Kausalität (Input $\rightarrow$ Prozess $\rightarrow$ Output) auch zirkulär sein kann, indem beispielsweise so genannte Summary Variables (Kompetenzen der Gruppenmitglieder, Art und Nutzung von Strategie der Aufgabenbewältigung und das Niveau und die Koordination der Bemühungen der Gruppenmitglieder) auf den Interaktionsprozess zurückwirken können, allerdings gäbe es häufig einen klaren Endpunkt der Gruppeninteraktion, so dass der Prozess unzyklisch betrachtet werden könne (Hackman \& Morris, 1975). Diese lineare Modellierung des Einflusses der Inputfaktoren ist Gegenstand der Kritik am IPO-Modell, auf die im Folgenden näher eingegangen wird.

\subsubsection{Notwendige Ergänzungen des Input-Prozess-Output-Modells}

Die wesentlichen Kritikpunkte an der auf dem IPO-Modell basierenden Forschung betreffen die Vernachlässigung der zeitlichen Perspektive im Verlauf des Gruppenarbeitsprozesses und die einseitige Untersuchung von Leistung als Output-Kriterium.

Die Rolle der Zeit

Die einseitig gerichtete Untersuchung des Einflusses der über den Interaktionsprozess wirkenden Inputfaktoren und die fehlende Berücksichtigung des Feedbacks durch den aktuellen Stand der Aufgabenbewältigung sind mehrfach kritisiert worden. So schlagen Ilgen, Hollenbeck, Johnson und Jundt (2005) vor, statt IPO besser vom Input Mediator Output Input -IMOI- zu sprechen. Durch die Substitution des $P$ durch das $M$ wird die Bedeutung der mediierenden Wirkung des Interaktionsprozesses klarer, das Hinzufügen des $I$ verweist auf zyklische Wirkung des Feedbacks (Ilgen et al., 2005). 
Der Ansatz von Marks, Mathieu und Zaccaro (2001) greift den bei Hackman und Morris (1975) fehlenden expliziten Verweis auf die zyklische Natur jeder Input-Prozess-Output-Episode auf. Sie konstatieren, dass zwar Modelle zum zyklischen Charakter des Gruppenarbeitsprozesses entwickelt wurden (Gersick, 1988), diese allerdings wenig in andere Modelle zur Gruppenarbeit integriert wurden. Marks und Kollegen betonen, dass „team performance trajectories most commonly consist of several I-P-O-type cycles that run sequentielly and simultaneously“ (Marks et al., 2001, S. 359). Die temporären Zyklen zielgerichteter Aktivität nennen sie Episoden (Marks et al., 2001). Der gesamte Arbeitsprozess einer Gruppe besteht aus vielen Episoden bzw. funktionalen IPO-Einheiten. Auch Boos (1996b) konstatiert, dass der Problemlöseprozess iterativ und zyklisch sei, wonach bestimmte funktionale Handlungen mehrfach durchgeführt würden.

Neben der Betonung des temporalen Rhythmus des Gruppenarbeitsprozesses spezifizieren Marks und Kollegen den Gruppenprozess selbst. In ihrer Taxonomie der Teamprozesse unterscheiden sie drei Prozessdimensionen: Übergangsprozesse, Handlungsprozesse und interpersonale Prozesse (Marks et al., 2001), wobei sie ihren Betrachtungsfokus auf die ersten beiden Prozesse legen. Während der Handlungsprozesse führt die Gruppe Handlungen aus, die direkt der Zielerreichung dienen. Hingegen dienen die Übergangsprozesse der Planung und Evaluation der Zielerreichung. Die Überlegung, dass unterschiedliche Gruppenprozesse zu bestimmten Zeitpunkten der Gruppenarbeit besonders wichtig sind, wurde auch von Hackman und Wageman (2005) in ihrer Theorie des TeamCoaching aufgegriffen. Sie postulieren, dass zu Beginn der Gruppenarbeit interpersonale Interventionen besonders effektiv sind. Strategieinterventionen seien ,,around the midpoints of the team's work“" angemessen (Hackman \& Wageman, 2005, S. 276), am Ende der Gruppenarbeit seien schließlich edukative Maßnahmen sinnvoll. Auch wenn eine Konkretisierung dieser Coaching-Maßnahmen wünschenswert ist, so leistet Hackman und Wageman's Ansatz einen wichtigen Beitrag zur Integration empirischer Befunde zur zeitlichen und situativen 
Angemessenheit von Interventionen in den Gruppenprozess und der praktischen Beratung von Gruppen im organisationalen Alltag (z.B. Teamentwicklungsmaßnahmen).

\section{Die Spezifizierung der Output-Variablen}

Wie bereits in der Kritik des funktionalen Paradigmas erwähnt, sind neben der impliziten linearen Wirkungsrichtung der Inputfaktoren die einseitige Konzentration in der Forschung auf Leistungs- und Erfolgsaspekte und die Vernachlässigung anderer, nicht unmittelbar auf die Leistung bezogenen Ergebnisse, kritisiert worden. Dazu gehören beispielsweise die Zufriedenheit der Gruppenmitglieder, das Gruppenklima, das Fortbestehen der Gruppe etc. (Wittenbaum et al., 2004b). McGrath (1991, S. 154) geht davon aus, dass alle Gruppen im Sinne dreier wichtiger Funktionen agieren: das Erfüllen der Projektziele (production), die Gruppenerhaltung (well-being) und die Unterstützung einzelner Gruppenmitglieder (member support).

\subsubsection{Vergleich der Funktionalen Perspektive in der Gruppen- und Kommunikationsforschung}

Hirokawa und Salazar (1999) unterscheiden drei theoretische Perspektiven, die sich mit dem Zusammenhang zwischen der aufgabenbezogenen Kommunikation in der Gruppe und ihrer Entscheidungsfindung beschäftigen:

Mediational Perspective. Der Interaktionsprozess wird als Mediator betrachtet, durch welchen die eigentlichen Determinanten der Gruppenleistung ihren Einfluss ausüben können.

Functional Perspective. Der Interaktionsprozess wird als ein soziales Tool betrachtet, welches die Gruppenmitglieder als Voraussetzung für ihr Entscheidungsfindungsverhalten verwenden.

Constitutive Perspective. Gruppenentscheidungen werden als soziale Produkte betrachtet, wobei die Kommunikation soziale Konvergenz entstehen lässt.

Der Mediational Perspective ordnen Hirokawa und Salazar (1999) das IPO-Modell von Hackman und Morris (1975) und darauf aufbauende Arbeiten zu. Diese Zuordnung erscheint 
sinnvoll, da Hackman und Morris (1975) den Interaktionsprozess als Mediator betrachten. Lediglich die Terminologie der theoretischen Perspektiven wird nun verwirrend, da das IPOModell in das Funktionale Paradigma der Gruppenforschung eingeordnet wird (Wittenbaum et al., 2004b), im Rahmen der Kommunikationsforschung zur Gruppenkommunikation und -entscheidungsfindung allerdings der Mediational Perspective, dort aber auch eine (andere) Functional Perspective postuliert wird. Diese Functional Perspective der Kommunikationsforschung unterscheidet sich von der Mediational Perspective bzw. der funktionalen Perspektive der Gruppenforschung insofern, als dass der Interaktionsprozess nicht nur als Mediator betrachtet wird, sondern eine aktivere Rolle einnimmt. Die Kommunikation wird als Mittel betrachtet, dessen sich die Gruppenmitglieder aktiv zur Aufgabenerfüllung bedienen. Die Kernaussage dieser Perspektive besteht darin, dass die Gruppe gemäß der zu erledigenden Aufgabe relevante, d.h. funktionale Inhalte kommunizieren muss, z.B. müssen vor dem Fällen der Gruppenentscheidung die Entscheidungsalternativen abgewogen und über ihre Vor- und Nachteile diskutiert werden (Hirokawa, 1985). Wittenbaums (2004b) Zuordnung der Functional Perspective der Kommunikationsforschung bzw. der Funktionalen Theorie der Gruppenentscheidungsfindung (Hirokawa, 1985; Orlitzky \& Hirokawa, 2001) zum Funktionalen Paradigma der Gruppenforschung scheint gerechtfertigt, da sie, mit Fokus auf dem Interaktionsprozess, Annahmen zum Zusammenhang zwischen der Kommunikation der Gruppenmitglieder und der Entscheidungsleistung der Gruppe trifft.

Die Funktionale Theorie der Gruppenentscheidungsfindung (Hirokawa, 1985) wird im folgenden Abschnitt dargestellt.

\subsection{Eine funktionale Theorie zur Entscheidungsfindung in Gruppen}

In der Existenz unterschiedlicher Problemsichten besteht das Synergiepotential einer Gruppe. Wie kann dieses Potential genutzt werden? Wie können die unterschiedlichen Problemsichten und Expertisen integriert werden? Dazu müssen zunächst die individuell 
unterschiedlichen Informationen durch Kommunikation ausgetauscht werden. Die funktionale Theorie der Gruppenentscheidungsfindung (Gouran \& Hirokawa, 1996; Hirokawa, 1983, 1985, 1990; Orlitzky \& Hirokawa, 2001) beinhaltet Aussagen zum Zusammenhang zwischen dem Gruppeninteraktionsverhalten und der Entscheidungsfindung. Das effektive Erfüllen der komplexen und unstrukturierten Entscheidungsaufgabe erfordert, dass die Interaktion der Gruppenmitglieder vier kritische Funktionen erfüllen muss:

1. Das bestehende Problem muss analysiert werden. Die Gruppe muss ein gründliches und genaues Verständnis der Natur, des Ausmaßes, der Ernsthaftigkeit und der Gründe des Problems entwickeln.

2. Es müssen Bewertungskriterien aufgestellt werden, die beschreiben, wie das Entscheidungsergebnis aussehen soll.

3. Entsprechend den Bewertungskriterien müssen Lösungsalternativen generiert werden, die sowohl realistisch als auch akzeptabel sind.

4. Die generierten Lösungsalternativen müssen sorgfältig bezüglich ihrer möglichen positiven und negativen Konsequenzen bewertet werden.

Das Erfüllen dieser kritischen Kommunikationsfunktionen beeinflusst die Entscheidungsfindung positiv. Besonders wesentlich für die effektive Gruppenentscheidungsfindung ist neben der Problemanalyse die Bewertung eventueller negativer Konsequenzen einzelner Entscheidungsalternativen (Hirokawa, 1985; Orlitzky \& Hirokawa, 2001).

Die bisherige Forschung konnte zeigen, dass die Kommunikationsfunktionen weniger im Sinne eines normativen Ablaufmodells nacheinander in der gleichen Reihenfolge auftreten müssen. Ausschlaggebend für die Qualität der Gruppenentscheidung ist lediglich die Erfüllung der funktionalen Anforderungen, weniger die Einhaltung bestimmter Diskussionsprozeduren (Hirokawa, 1985). Es scheint nicht den einzig richtigen Diskussionsablauf im Sinne einer gleichförmigen Sequenz bestimmter Kommunikationsphasen zu geben, der Gruppen mit effektiver Entscheidungsfindung von solchen mit ineffektiver unterscheidet (Hirokawa, 1983). Eine sequentiell lineare Abfolge funktional unterschiedlicher Phasen mag in Gruppen als 
implizite Norm des Vorgehens vorhanden sein, ist empirisch hingegen nicht zu finden und entspricht einer zu starken Vereinfachung des Gruppenprozesses (Poole, 1983 zit. n. Boos, 1996b). Boos (1996a) konnte in Gruppendiskussionen im Rahmen einer Problemlösungsaufgabe ebenfalls keine funktional voneinander abgrenzbaren Phasen finden. Allerdings konnten dennoch Unterschiede im zeitlichen Verlauf zwischen erfolgreichen und nicht erfolgreichen Gruppen festgestellt werden: Erstere analysieren zunächst das Problem, bevor sie Entscheidungsalternativen sammeln, erfolglose Gruppen hingegen beginnen sofort mit der Lösungssuche, ohne das Problem genau analysiert zu haben (Hirokawa, 1983). Tschan (1995) kam ausgehend von der Handlungstheorie in einer Untersuchung von Gruppenprozessen bei einer Konstruktionsaufgabe zu ähnlichen Ergebnisse: Gruppen, deren Kommunikation in einem so genannten idealen Zyklus ablief (beginnend mit Orientierung und Planung, endend mit Evaluation) zeigten bessere Leistung als solche, deren Kommunikation nicht dem idealen Zyklus entsprach.

Darüber hinaus muss auch überlegt werden, inwieweit tatsächlich allein die Orientierung an funktionalen Anforderungen das Entscheidungsverhalten von Gruppen erklären kann und nicht noch weitere Faktoren, z.B. die Dynamik der Gruppe, als verhaltensbestimmende Faktoren hinzugezogen werden sollten (Boos, 1996b).

Die Annahmen der funktionalen Theorie der Entscheidungsfindung konnten empirisch bisher teilweise unterstützt werden (Gouran \& Hirokawa, 1996). In Bezug auf soziale Einflüsse, die die Gruppe möglicherweise daran hindern, die kritischen Funktionen umzusetzen, diskutieren und revidieren Gouran und Hirokawa (1996) Funktionen, deren Erfüllung durch die Gruppe eine gute Entscheidung ermöglicht. Sie postulieren, dass gute Gruppenentscheidungen dann möglich werden, wenn die Gruppenmitglieder

1. ihr Interesse an der bestmöglichen Gruppenentscheidung betonen,

2. relevante Entscheidungsfindungsressourcen identifizieren, 
3. eventuell auftretende Hindernisse erkennen,

4. einzuhaltende Verfahrensweisen spezifizieren,

5. Grundregeln der Interaktion festlegen,

6. versuchen, die wesentlichen Aufgabenerfordernisse zu erfüllen, indem sie

a. ein korrektes Problemverständnis zeigen,

b. die Minimalkriterien bestimmen, denen jede akzeptable Alternative gerecht werden muss,

c. relevante und realistische Alternativen generieren,

d. die Alternativen sorgfältig bezüglich der Minimalkriterien überprüfen,

e. die Alternative auswählen, die am wahrscheinlichsten die angestrebten Merkmale enthalten wird,

7. angemessene Interventionen einsetzen, um kognitive, affiliative und egozentrische Einschränkungen, welche die Erfüllung der wesentlichen Aufgabenerfordernisse beeinträchtigen könnten, zu überwinden und

8. den Entscheidungsfindungsprozess kritisch prüfen und gegebenenfalls die Entscheidung als getroffen ansehen (Gouran \& Hirokawa, 1996).

Durch die Erweiterung der Bedingungen, die die Gruppenmitglieder zur Entscheidungsfindung beachten müssen, verdeutlichen Gouran und Hirokawa (1996), dass neben den reinen performanzbezogenen Prozesskriterien (a-e) der Gruppenentscheidungsfindungsprozess auch soziale und kognitive Anforderungen an die Gruppe stellt. Darüber hinaus benennen sie nun den in der ursprünglichen Theorie fehlenden Schritt des Treffens der Entscheidung, auch wenn dieser im Vergleich zu anderen Entscheidungsmodellen (z.B. Ellis \& Fisher, 1994) immer noch relativ wenig spezifiziert wird. Der Vorteil der funktionalen Theorie der Entscheidungsfindung liegt jedoch in der Postulierung kritischer Funktionen, die während der Gruppenentscheidungsfindung erfüllt werden müssen. Die Effektivität des Erfüllens dieser Funktionen ist teilweise empirisch sehr gut bestätigt (Orlitzky \& Hirokawa, 2001) und bietet Entscheidungsfindungsgruppen daher eine Prozessunterstützung, die insbesondere durch die Führungskraft oder den/die Diskussionsleiter/-in beachtet werden können (Wilson, 2005). Darüber hinaus ermöglicht die 
funktionale Theorie die theoriegeleitete Beobachtung des Gruppenprozesses (Hirokawa, 1982), deren Ergebnisse zur Rückmeldung an die Gruppe im Rahmen von Interventionen und zu Forschungszwecken genutzt werden können. Sie verdeutlicht die Komplexität und den damit verbundenen Koordinationsbedarf von Gruppenentscheidungen, auf den in Kapitel 3.1 genauer eingegangen wird.

Festzuhalten bleibt, dass für eine gute Gruppenentscheidung die kritische Bewertung vorgeschlagener Lösungsmöglichkeiten und Entscheidungsalternativen durch alle Gruppenmitglieder wesentlich ist (Gouran \& Hirokawa, 1996; Orlitzky \& Hirokawa, 2001). Nur so kann das Wissen aller Gruppenmitglieder ausgeschöpft werden und der empirisch vielfach bestätigten Tendenz von Gruppen, sehr risikofreudige Entscheidungen (Wittenbaum et al., 2004b) zu treffen, entgegengewirkt werden. Inwieweit die Gruppenmitglieder ihre individuellen Informationen tatsächlich austauschen, wird im nächsten Abschnitt dargestellt. 


\subsection{Empirische Befunde zum Informationsaustausch und zur Entscheidungsfindung in Gruppen}

Im vorangegangenen Kapitel wurde gezeigt, dass die Gruppenentscheidungsfindung eine komplexe Aufgabe ist, für deren effektive Lösung die Gruppenmitglieder ihre unterschiedlichen Problemsichten und individuellen Informationen austauschen müssen. Gleichzeitig ist das Suchen, Speichern, Verarbeiten und Benutzen von Informationen eine der wesentlichsten Funktionen verschiedenster Gruppenarten (Arrow et al., 2000). Gruppendiskussionen dienen dazu, unterschiedliche Problemsichten, Lösungsvorschläge und Informationen auszutauschen und das gemeinsame Handeln und Entscheiden abzustimmen (Boos, 1996b). Allerdings konnte in einer Vielzahl von Studien gezeigt werden, dass Gruppen die unterschiedlichen Informationen ihrer Mitglieder und damit ihr Synergiepotential nur unzureichend ausnutzen.

Zwei Arten von Qualitätsgewinn durch Gruppendiskussionen werden unterschieden: individueller Wissenszuwachs und Verbesserung der Gruppenentscheidung (Kerschreiter, Mojzisch, Schulz-Hardt, Brodbeck \& Frey, 2003). Leider neigen Gruppenmitglieder dazu, Informationen auszutauschen, die bereits allen bekannt sind (geteilte Information). Hingegen wird wesentlich weniger über Informationen gesprochen, die nur ein oder wenige Gruppenmitglied/er besitzt/-en (ungeteilte Information) und die daher neu für die anderen Gruppenmitglieder wären. Durch diesen in der Forschung vielfach bestätigten Nennungsvorteil der geteilten Information wird der individuelle Wissenszuwachs der Gruppenmitglieder verhindert und eine gute Gruppenentscheidung weniger wahrscheinlich. Der Nennungsvorteil geteilter Informationen konnte in Untersuchungen, denen ein Hidden-Profile-Paradigma zugrunde liegt, vielfach gezeigt werden (Larson et al., 1998b; Larson, Foster-Fishman \& Keys, 1994; Stasser \& Titus, 1985, 2006). Das Hidden-Profile-Paradigma wird als Untersuchungsparadigma für den Informationsaustausch und die Entscheidungsfindung in Gruppen genutzt, indem die Informationsverteilung der Gruppenmitglieder gezielt manipuliert und der 
Informationsaustausch über den Diskussionsprozess hinweg verfolgt werden kann (SchulzHardt, Brodbeck, Mojzisch, Kerschreiter \& Frey, in press; Stasser \& Titus, 1985). In den meisten Fällen wird die Informationsverteilung so manipuliert, dass die individuellen Informationen die falsche Entscheidung implizieren, die korrekte Entscheidung hingegen ausschließlich durch den Austausch ungeteilter Informationen erreicht werden kann (vgl. Hidden-Profile in Kapitel 5.3.5). Als Gründe für die in diesen Untersuchungen suboptimale Gruppenentscheidung und den Nennungsvorteil geteilter Informationen werden auf Gruppenebene beispielsweise stochastische Nennungsvorteile im Collective Information Sampling (CIS) Modell (Stasser \& Titus, 1985, 2006), kommunikationsbedingte gegenseitige positive Verstärkung (Mutual Enhancement, Wittenbaum, Hubbell \& Zuckerman, 1999) oder die Verfolgung anfänglicher Entscheidungspräferenžen (Gigone \& Hastie, 1993; Winquist \& Larson Jr., 1998) diskutiert. Auf individueller Ebene wurden beispielsweise die präferen₹konsistente Informationsverarbeitung (Greitemeyer \& Schulz-Hardt, 2003; Greitemeyer, Schulz-Hardt \& Frey, 2003) oder der Einfluss individueller Ziele der Gruppenmitglieder (Schauenburg, 2004; Wittenbaum, Hollingshead \& Botero, 2004a) untersucht. Eine systematische Darstellung der aktuellen Befundlage zum Informationsaustausch in Entscheidungsaufgaben ist bei Mojzisch, Kerschreiter, Schulz-Hardt und Frey (2004) sowie bei Kerschreiter und Kollegen (2003) zu finden.

\subsection{Fazit zur Entscheidungsfindung und zum Informationsaustausch}

Bisher wurden Modelle und empirische Befunde zur Entscheidungsfindung und dem damit verbundenen Informationsaustausch in Gruppen dargestellt. Bezugnehmend auf die eingangs vorgestellte Veranschaulichung der wesentlichen Konzepte der vorliegenden Arbeit (Abbildung 1) wurden die Merkmale der Inputvariable Entscheidungsaufgabe und des Prozesses des Informationsaustauschs diskutiert. Insgesamt hat die Forschung zur Effektivität von Gruppenentscheidungen gezeigt, dass Gruppen keineswegs immer die Ressourcen ihrer Mitglieder so ausnutzen, dass ihr unterschiedliches Wissen und ihre unterschiedliche Expertise 
tatsächlich für das Finden der Gruppenentscheidung benutzt werden. Im Gegenteil, häufig treffen Gruppen Entscheidungen, die entweder den individuellen oder initial genannten Präferenzen der Mitglieder entsprechen. Vielmehr nutzt die Gruppe ihr Synergiepotential zu Entscheidungsfindung nicht aus, da die Gruppenmitglieder wesentliche Informationen nur unzureichend diskutieren (Scherm, 1998). Fehlerhafte Gruppenentscheidungen lassen sich in alltäglichen, organisationalen und politischen Bereichen finden (Ellis \& Fisher, 1994; Thompson, 2004) und können insbesondere in Arbeitssituationen mit hohem Risiko (z.B. in der Luftfahrt oder in der Medizin) fatale Folgen haben (Campion, 2003; Hyey \& Wickens, 1993; Thiele \& Manser, 2001). Im folgenden Kapitel wird dargestellt, inwieweit die Entscheidungsfindung in Gruppen ein sehr komplexer Prozess ist, der hohe Koordinationsanforderungen an die Gruppe stellt und inwieweit die Koordination als Prozessvariable dazu beitragen kann, dass Synergiepotential der Gruppe zur Entscheidungsfindung zu nutzen. 


\section{$3 \quad$ Koordination von Gruppen}

Vor einer Präzisierung des Koordinationsbegriffes (3.2) wird zunächst auf den Koordinationsbedarf eingegangen, der speziell von Entscheidungsaufgaben ausgeht (3.1). Anschließend werden theoretische Konzepte der Koordination dargestellt (3.3) und auf den Zusammenhang von Koordination und Kommunikation eingegangen (3.4). Zum Abschluss des Kapitels werden die Fragen präzisiert, denen in den folgenden Studien nachgegangen wird (3.5).

\subsection{Koordinationsbedarf bei Gruppenentscheidungsaufgaben}

Gruppenaufgaben vom Typus des Entscheidens sind nach der Einordnung in das Circumplexmodell der Aufgabentypen von McGrath (1984) eher konzeptionell und konflikthaft und erfordern von der Gruppe Aktivitäten, die McGrath als „choose“ bezeichnet: Die Aufgabenstruktur ist nur schwer beschreibbar und es gibt keine Lösung, die als richtig entdeckt werden kann (Boos \& Sassenberg, 2001).

Hirokawa (1990) unterscheidet drei wesentliche Charakteristika der Aufgabe: Struktur, Informationserfordernisse und Evaluationsanforderungen. Die Aufgabenstruktur wird bestimmt aus der Klarheit der Ziele, der Wege und Schritte der Zielerreichung und Barrieren auf Weg zur Zielerreichung. Einfache Aufgaben zeichnen sich durch klare Ziele und Wege der Zielerreichung, geringe notwendige Schritte und wenig Barrieren aus. Bei komplexen Aufgaben hingegen sind das Ziel und die Wege der Zielerreichung unklar, viele Schritte notwendig und viele Barrieren vorhanden (Hirokawa, 1990). Die Informationserfordernisse werden durch die Informationsverteilung und die Notwendigkeit zur Informationsverarbeitung bestimmt (Hirokawa, 1990). Auch Daft und Lengel (1984) gehen in der Media Richness Theorie davon aus, dass sich Aufgaben in den Anforderungen an den Informationsreichtum der Kommunikation unterscheiden (Daft \& Lengel, 1984). Dennis uns Valacich (1999) entwickelten diese Annahmen in der Media Synchronicity Theorie weiter und unterscheiden - ähnlich Hirokawa (1990) - zwei generische Kommunikationsprozesse: Informationsübermittlungsprozesse (conveyance) und konvergente 
Kommunikationsprozesse (convergence). Informationsübermittlungsprozesse ermöglichen die Versorgung aller Gruppenmitglieder mit möglichst vielen Informationen, wohingegen konvergente Kommunikationsprozesse der Strukturierung, Verdichtung der Informationen und der Entwicklung eines gemeinsamen Verständnisses über das Problem und seiner Lösungsmöglichkeiten dienen (Dennis \& Valacich, 1999; Schwabe, 2001b).

Neben der Aufgabenstruktur und den Informationsanforderungen betrachtet Hirokawa (1990) die Evaluationsanforderungen als Aufgabencharakteristikum. Diese werden bestimmt durch die Anzahl der Lösungsmöglichkeiten, die Zielklarheit und durch das Ausmaß, indem die Lösung als korrekt überprüft werden kann.

Betrachtet man die Gruppenentscheidungsaufgabe auf den drei geschilderten Merkmalen, so wird deutlich, warum der Entscheidungsprozess koordiniert werden muss:

1. Die Entscheidungsaufgabe ist komplex, weil das Ziel und die Wege der Zielerreichung am Aufgabenbeginn unklar sind, ihre Festlegung ist selbst Gegenstand der Aufgabe.

2. Die Informationsanforderungen sind hoch, denn die Informationen sind ungleich über die Gruppenmitglieder verteilt. Eine optimale Lösung kann nur durch Wissensaustausch gefunden werden kann - dieser Wissensaustausch muss koordiniert werden, da sonst Informationen entweder gar nicht erst genannt werden (Stasser \& Titus, 1985) oder während des Diskussionsprozesses verloren gehen, weil sie nicht wieder aufgegriffen, wiederholt oder zusammengefasst werden (Prozessverlust, siehe folgender Abschnitt 3.1.1). Allerdings reicht der Austausch von Informationen zur Entscheidungsfindung nicht aus. Gruppen, die mit einer Entscheidungsaufgabe betraut werden, bestehen meist aus unterschiedlichen Experten/-innen. Nur die Heterogenität der Gruppenmitglieder kann das Vorhandensein einer breiten Auswahl von Entscheidungsfindungsressourcen gewährleisten. Die Gruppenmitglieder besitzen allerdings aufgrund ihrer unterschiedlichen Expertise auch unterschiedliche Problemsichten und Bewertungskriterien, die sich beispielsweise in unterschiedlichen moralischen oder ethischen Vorstellungen oder in unterschiedlichen Zielen zeigen.

3. Die Evaluationsanforderungen sind hoch, da prinzipiell viele Entscheidungen möglich sind, deren Korrektheit nicht unmittelbar festgestellt werden kann.

Aufgrund der beschriebenen Merkmale gelten für Entscheidungsaufgaben besonders hohe Koordinationsanforderungen. Individuell unterschiedliche Meinungen, Präferenzen und Bewertungskriterien müssen ausgetauscht werden (Boos \& Sassenberg, 2001), wobei die 
Bedeutung von Informationen für die Entscheidung zunächst unklar sein kann und erst diskutiert werden muss (Ambiguität der Information) (vgl. auch Poole \& Hirokawa, 1996). Die aufgrund der Diskussion getroffene gemeinsame Entscheidung sollte idealer Weise von allen Gruppenmitglieder getragen werden können.

Die Bedeutung von Koordination für die Gruppenleistung und die Konzeptionalisierung des Koordinationsprozesses werden im Folgenden anhand klassischer Modelle der Gruppenproduktivität und des Gruppenhandelns dargestellt.

\subsection{1. „Verluste“ und „Gewinne“ während des Gruppenprozesses}

In seinem klassischen Werk zu Gruppenprozessen und -produktivität postulierte Steiner (1972), dass sich die tatsächliche Gruppenleistung aus der potentiellen Gruppenleistung, abzüglich der Prozessverluste, ergibt. Die maximal mögliche Gruppenproduktivität wird durch die Aufgabenanforderungen und die individuellen Ressourcen bestimmt (Steiner, 1972). Den Prozess hingegen betrachtet Steiner eher als eine Quelle möglicher Fehler, die beispielsweise durch „different views concerning the proper pattern of collective action" (Steiner, 1972, S. 8), Unstimmigkeiten oder schlecht koordiniertes Verhalten die Gruppenleistung negativ beeinflussen. Steiners Postulat hatte zu Folge, dass der Schwerpunkt der Gruppenproduktivitätsforschung sich hauptsächlich mit Prozessverlusten, kaum aber mit Prozessgewinnen beschäftigte. Sein Modell wird daher auch häufig als pessimistisches Gruppenleistungsmodell (Tschan, 2000) bezeichnet. Konzeptuell haben Stroebe und Frey (1982) die Prozessverluste in Motivationsverluste und Koordinationsverluste unterschieden, wobei wiederum erstere den Großteil der Forschung bildeten und Phänomene wie Social Loafing, Trittbrettfahren, Ringelmanneffekt untersucht wurden (z.B. Huguet, Charbonnier \& Monteil, 1999; Karau, 1993; Smith, 2001). Die Gewinne (z.B. zeitsparende implizite Koordination aufgrund geteilter mentaler Modelle, vgl. Kapitel 3.3.3) und Verluste (z.B. doppelte Arbeit aufgrund fehlender Absprachen), 
die durch die Koordination der Gruppenmitglieder während ihres Interaktionsprozesses entstehen, waren weit weniger Gegenstand der Forschung.

„The process by which groups coordinate the completion of single - let alone multiple projects has received relatively little attention in the social psychology literature" (Arrow et al., 2000, S. 102).

Auch Kerr und Tindale (2004) diskutieren in ihrem aktuellen Review zu Gruppenleistung und Entscheidungsfindung die Befunde $\mathrm{zu}$ Motivationsverlusten und -gewinnen, Koordinationsverluste- oder gewinne führen sie hingegen nicht an. Darüber hinaus ist unklar, inwieweit Motivation und Koordination konzeptuell streng getrennt betrachtet werden sollten. So konnten Larson und Schaumann (1993) zeigen, dass das von den Gruppenmitgliedern wahrgenommene Koordinationsvermögen der Gruppe den Einfluss der motivierenden Wirkung von Gruppenzielen auf die individuelle Arbeitsmotivation moderiert: Bestehen sowohl hoher Koordinationsbedarf als auch viele Koordinationsmöglichkeiten, so wirken schwierige Ziele motivationserhöhend, leichte Ziele motivationsschwächend. Hat die Gruppe aber trotz hoher Koordinationsanforderung keine Möglichkeit, diese $\mathrm{zu}$ bewältigen, so zeigen sich keine Unterschiede in der Wirkung von schwierigen im Vergleich zu leichten Zielen (Larson \& Schaumann, 1993). Dieser Befund impliziert, dass erstens Motivations- und Koordinationsprozesse nicht getrennt voneinander ablaufen. Zweitens betont er die Koordinationsnotwendigkeit von Gruppenentscheidungsaufgaben.

Ausgehend von der Annahme, dass der Gruppeninteraktionsprozess unweigerlich mit Prozessverlusten einhergeht, hat sich die Forschung damit beschäftigt, inwieweit Gruppen bessere Leistung zeigen, wenn die Interaktion ihrer Mitglieder reduziert wird. So konnte gezeigt werden, dass nominelle Gruppen in Ideenfindungsaufgaben interagierenden Gruppen überlegen sind (Diehl \& Stroebe, 1987). Ebenso wurden Moderationstechniken entwickelt, durch die der Interaktionsprozess der Gruppenmitglieder systematisch kontrolliert wird (Witte, 2001). Allerdings können diese Ansätze nicht auf Gruppenentscheidungsfindung übertragen werden, da 
die Gruppenmitglieder interagieren müssen, um die für die Entscheidung kritischen Funktionen erfüllen zu können. Interaktion ist daher für das gemeinsame Entscheiden notwendig, interagierende Gruppen sind nominellen Gruppen in der Entscheidungsfindung weitaus überlegen (Burleson, 1984). Die Interaktion während des Entscheidungsfindungsprozesses muss koordiniert werden.

Festzuhalten bleibt, dass die Koordination des Gruppenprozesses neben der Motivation der Gruppenmitglieder die wesentliche Voraussetzung für die Effektivität von Gruppen (Boos \& Sassenberg, 2001; Steiner, 1972) und insbesondere bei Entscheidungsfindungsaufgaben unerlässlich ist. Fehlende oder schlechte Koordination führt zu schlechten Gruppenentscheidungen und kann als klassischer Prozessverlust betrachtet werden. Zwei wesentliche Koordinationsverluste können dabei unterschieden werden: die fehlende Abstimmung zielgerichteter Entscheidungsfindungsabsichten und mangelnde Nutzung vorhandener Ressourcen (Scherm, 1998). In Anlehnung an Steiner (1972) postulieren Wittenbaum und Kollegen/-innen (1998), dass Koordinationsverluste durch unangemessene Ressourcenaufteilung entstehen, beispielsweise unnötige Doppelanfertigung einiger Teilaufgaben, so dass andere unerledigt bleiben, oder Fehler in der zeitlichen Aufteilung der Arbeit. Auch die fehlende Gewichtung der Arbeit der anderen Gruppenmitglieder und falsche Annahmen über die Arbeit der anderen kann zu Koordinationsverlusten führen (Steiner, 1972). Daher muss der Frage nachgegangen werden, wie der Gruppenentscheidungsprozess im Hinblick auf eine effektive Gruppenentscheidung und zufriedene Gruppenmitglieder koordiniert werden sollte.

Zur Beantwortung dieser Frage liefert Steiners Modell (1972) wenig Ansatzpunkte. Vielmehr lohnt sich eine konkrete Betrachtung des Interaktionsprozesses (Hackman \& Morris, 1975) - wobei der Komplexität des Entscheidungsfindungsprozesses Rechnung getragen werden muss. Eine Theorie, welche die Gruppe als komplexes System betrachtet, deren Handlungen auf verschiedenen sowohl sequentiellen als auch hierarchischen Ebenen betrachtet werden, ist die 
Handlungstheorie der Gruppe (Cranach, 1996; Cranach et al., 1986; Tschan, 2000), die im Folgenden erläutert wird.

\subsubsection{Handlungstheorie der Gruppe}

Die Handlungstheorie der Gruppe postuliert, dass Gruppen aktiv auf ein bestimmtes Ziel hin streben und dieses durch gerichtetes Verbalten zu erreichen beabsichtigen (Cranach et al., 1986). Die Gruppe wird als handelnde Einheit verstanden.

Der Grundgedanke der Handlungstheorie der Gruppe besteht in der hierarchischsequentiellen Organisation des Gruppenhandelns. Die Gruppenaufgabe kann als ein Ziel formuliert werden, welches in Teilziele zerlegt werden kann. Die bierarchische Struktur der Ziele und Teilziele postuliert, dass es ein übergeordnetes, bewusstseinpflichtiges Ziel bzw. Purpose gibt (z.B. Entscheidung treffen), welches auf verschiedenen Ebenen in ebenfalls bewusstseinspflichtige Subziele (Planning) zergliedert werden kann (z.B. Problemanalyse, Entscheidungsalternativen generieren usw.). Die Subziele können wiederum in kleinste Teilhandlungen (Performance) zergliedert werden, deren Erfüllung meist automatisiert ist und die daher nicht mehr bewusstseinspflichtig repräsentiert sein müssen. Die sequentielle Struktur der Handlung bezieht sich auf die zeitliche Ordnung, wonach ein Teilziel nach dem anderen abgearbeitet wird (z.B. erst Problemanalyse, anschließend Entscheidungsalternativen generieren). Eine Darstellung wesentlicher Annahmen der Handlungstheorie der Gruppe inklusive Erweiterungen und anschaulichen Beispielen ist in Tschan (2000) zu finden. Eine Integration handlungs- und systemtheoretischer Ansätze wurde von McGrath und Tschan (2004) vorgenommen werden.

Ähnlich der Unterscheidung in Motivations- und Koordinationsprozesse (Steiner, 1972; Stroebe \& Frey, 1982) wird in der Handlungstheorie der Gruppe zwischen zwei für das zielgerichtete Gruppenhandeln wesentlichen Prozessen unterschieden: Steuerung und Energetisierung (Cranach, 1996; Cranach et al., 1986). Durch Steuerung wird Gruppenhandeln 
zielgerichtet, Energetisierung beeinflusst die Energie, die die Gruppe zum Handeln bewegt (Cranach, 1996). Im Folgenden wird dennoch der Koordinationsbegriff beibehalten, da er einerseits konkreter zu beschreiben und zu erfassen und damit deskriptiver ist, andererseits der Steuerungsbegriff eine inhärente Manipulationsintention enthält und damit eher in den Bereich der Gruppenführung fällt. Beiden Begriffen ist gemein, dass sie die Zielgerichtetheit der jeweiligen Prozesse betonen, sowohl Steuerung als auch Koordination geschehen nicht um ihrer selbst Willen, sondern dienen ausschließlich dem Erreichen eines Ziels. Goerges (2005) betrachtet Steuerung und Koordination aus systemtheoretischer Perspektive und postuliert, dass sich beide Begriffe funktional ergänzen. Steuerung bestimme den Sinn und die Richtung von Handlungen. Koordination hingegen beziehe sich nur auf den Moment, der eine Verknüpfung von Handlungen während des Prozesses erfordert (Goerges, 2005). In Bezug auf die Handlungstheorie kann damit überlegt werden, dass durch Koordination der Übergang zwischen einzelnen Handlungsphasen realisiert wird. Diese Präzisierung des Koordinationsbegriffs wird in Kapitel 3.2 wieder aufgegriffen..

Wesentlich für die Betrachtung von Koordinationsprozessen ist, dass die handlungstheoretische Struktur der Aufgabe die Koordinationsanforderungen an den Handlungsprozess bestimmt. Da die Entscheidungsaufgabe sowohl hierarchisch als auch sequentiell komplex ist, ist ihr Koordinationsanspruch entsprechend hoch.

In der funktionalen Theorie zur Entscheidungsfindung in Gruppen (Hirokawa, 1985) wird die Bedeutung einer profunden Problemanalyse als kritische Funktion zur effektiven Entscheidungsfindung betont. Ähnlich wird auch in der handlungstheoretischen Perspektive davon ausgegangen, dass die Repräsentation der Aufgabe durch die Gruppe der Ausgangspunkt für koordiniertes Handeln und gute Gruppenleistung ist (Tschan, 2000).

Damit weist Tschan auf die durch die zu bewältigende Gruppenaufgabe vorgegebenen Koordinationsanforderungen an den Gruppenprozess hin. Ähnliche Überlegungen stammen von 
Grote und Kollegen/-innen (Grote et al., 2003; Grote, Zala-Mezö \& Grommes, 2004): Die Arbeitsbelastung während der Aufgabenerfüllung bzw. der Workload der Aufgabe determiniert die notwenige Koordination während des Arbeitsprozesses.

Abbildung 3 zeigt exemplarisch die hierarchisch-sequentielle Struktur einer Gruppenentscheidungsaufgabe. Auf der hierarchisch höchsten Ebene ist das Ziel der Gruppenentscheidung repräsentiert, welches sich durch die Gruppenaufgabe ergibt. Als Teilziele fungieren die der funktionalen Theorie zur Entscheidungsfindung in Gruppen (Hirokawa, 1985; Orlitzky \& Hirokawa, 2001) entnommenen kritischen Funktionen, die eine Gruppe während der Entscheidungsfindung bzw. des Informationsaustauschprozesses erfüllen muss (vgl. auch Abbildung 1).

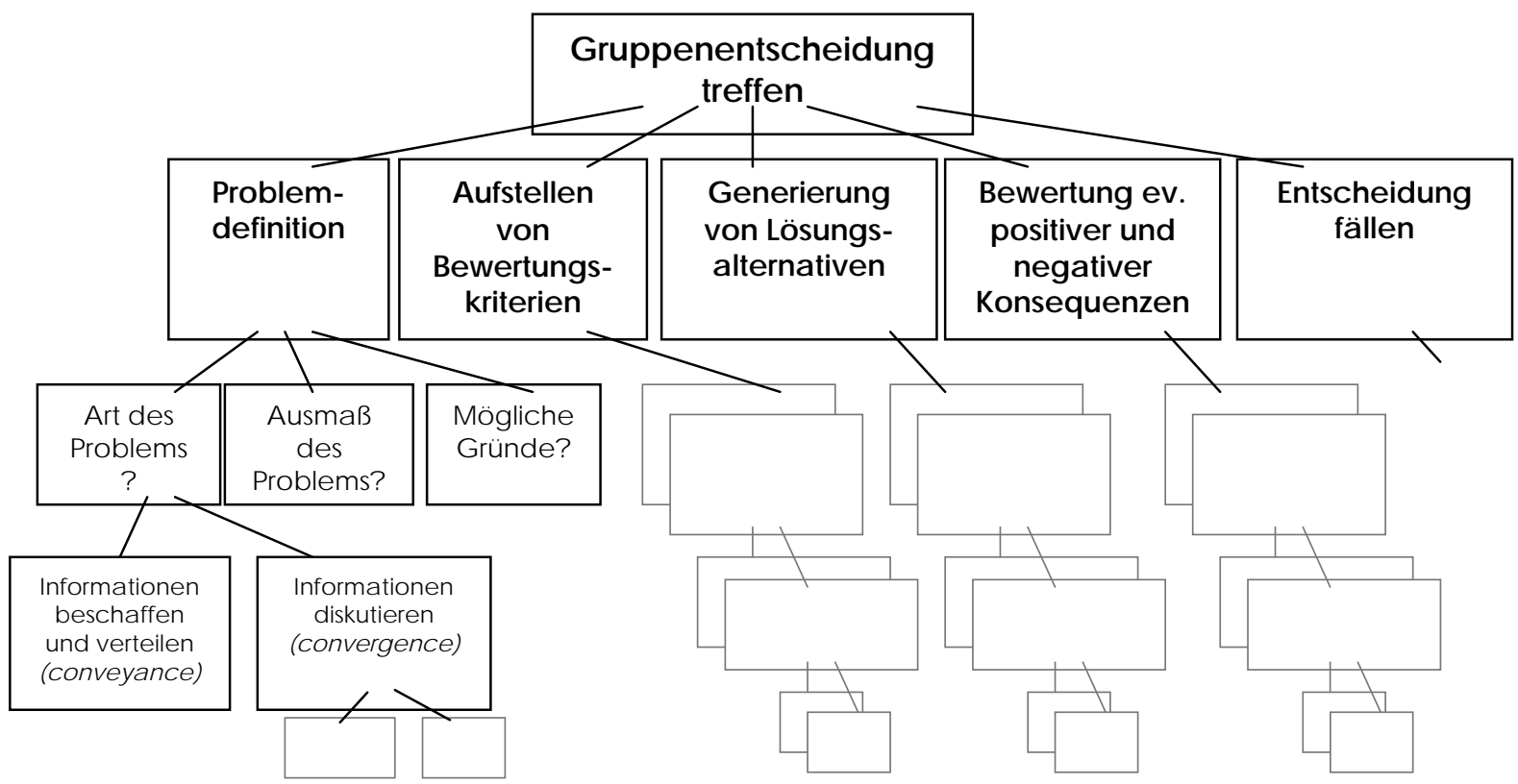

Abbildung 3: Exemplarische Darstellung der hierarchisch-sequentiellen Struktur der Gruppenentscheidungsaufgabe

Der sequentielle Charakter der Handlungsstruktur impliziert, dass es Funktionen gibt, die vor anderen Funktionen erfüllt sein müssen (z.B. Generierung von Lösungsalternativen vor der Bewertung). Diese idealtypische sequentielle Struktur ist zwar intuitiv plausibel, dennoch gibt es berechtigte Kritik an der Annahme einer idealtypischen Phasenabfolge, auf die bereits im Abschnitt 2.2 eingegangen wurde. 
Die Effektivität so genannter idealer Kommunikationsaylklen, die sowohl hierarchisch als auch sequentiell korrekt im Sinne der Aufgabenstruktur sind (vgl. Tschan, 2000, S. 155), konnte bei einer Konstruktionsaufgabe festgestellt werden (Tschan, 1995, 2000). Inwieweit die idealen Kommunikationszyklen auch auf Entscheidungsaufgaben übertragen werden können, ist unklar und auch nicht Gegenstand der vorliegenden Arbeit. Die bisherige Empirie deutet nicht auf die Existenz solcher Zyklen hin (Boos, 1996a; Poole, 1983 zit. n. Boos, 1996b; Hirokawa, 1983). Möglich ist, dass ideale Kommunikationszyklen sich zwar nicht über den gesamten Entscheidungsprozess hinweg finden lassen (Makroebene ${ }^{5}$ ), jedoch im Rahmen der iterativen Struktur als ideale Teilzyklen existieren (Mikroebene $)^{6}$.

Durch die in Abbildung 3 vereinfachte Darstellung der Struktur der Gruppenentscheidungsaufgabe soll der hohe Koordinationsbedarf und die damit verbundenen Kommunikationserfordernisse verdeutlicht werden. Alle kritischen Funktionen auf der Teil-ZielEbene benötigen zu ihrer Erfüllung auf der Performanz- bzw. Handlungsebene Kommunikation. Nur durch Kommunikation ist der Austausch von Informationen und deren Bedeutungen und damit die Gruppenentscheidung möglich (vgl. Abbildung 1). Kommunikation erfüllt während des Entscheidungsfindungsprozesses zwei wesentliche Funktionen: Erstens ist es das Arbeitsmittel, durch welches die Gruppendiskussion im Sinne des Austauschs von Informationen und Bedeutungen überhaupt möglich ist. Zweitens ist Kommunikation auch ein Koordinationsmittel, wodurch der Ablauf des komplexen Entscheidungsfindungsprozesses strukturiert und reguliert werden kann.

Zusammenfassend postuliert Tschan (2000), dass „Gruppen besser leisten, wenn sie folgende Merkmale aufweisen: Sie entwickeln ein gemeinsames Abbild oder ,Mentales Modell' der zu bearbeitenden Aufgabe, sie nutzen die in der Gruppe vorhandenen Ressourcen optimal,

\footnotetext{
${ }^{5}$ Unter Makroebene wird hier die Betrachtung des gesamten Entscheidungsfindungsprozesses verstanden.

${ }^{6}$ Auf der Mikroebene wird der Entscheidungsprozess in feinerer Auflösung betrachtet, z.B. auf Ebene einzelner individueller Äußerungen innerhalb der Phase der Problemanalyse.
} 
und sie strukturieren und koordinieren ihren Prozess gemäß den Anforderungen der Aufgabe“ (Tschan, 2000, S. 10).

Für handelnde Gruppen, also auch für Entscheidungsfindungsgruppen, gilt, dass sie die handlungsbegleitende Kommunikation und die Handlungsausführung parallel koordinieren müssen. Neben der Koordination der gemeinsamen Arbeit muss die handlungsleitende Informationsverarbeitung adäquat (hierarchisch und sequentiell) zu den Aufgabenanforderungen stattfinden (Tschan, 2000).

Gurtner (2003) konnte zeigen, dass die Leistung von Teams in einer Luftraumüberwachungsaufgabe umso höher ist, je besser die individuellen Beiträge der Gruppenmitglieder koordiniert sind. Koordiniertes Handeln musste in dieser komplexen Aufgabe durch effiziente Kommunikation zwischen den Gruppenmitgliedern hergestellt werden.

Bei Entscheidungsaufgaben steht nicht die Koordination von Handlungen (Clark, 1991) im Vordergrund, wesentlicher sind die Koordination von Wissen und Bedentungen, wodurch die Kommunikation das einzige Koordinationsmittel darstellt. Im Folgenden werden nach einer Begriffspräzisierung theoretische Konzepte vorgestellt, welche die Möglichkeiten der Koordination aufzeigen.

\subsection{Präzisierung des Koordinationsbegriffs}

Gruppen können nur effektiv handeln, wenn sich ihre Gruppenmitglieder koordinieren. Durch Koordination soll nicht nur Doppelarbeit vermieden und die handlungstheoretisch sinnvolle Erfüllung zentraler Funktionen ermöglicht, sondern auch Flexibilität gewährleistet werden (vgl. Ellwein, 1990). Doch was genau bedeutet Gruppenkoordination? Intuitiv ist der Begriff Koordination zunächst verständlich (Malone \& Crowston, 1994), doch so vielfältig die Forschung zu Koordination von Gruppen und Organisationen auch ist, Koordination wird häufig nicht (Bornstein, Gneezy \& Nagel, 2002; Brauner, 2001; Ellwein, 1990; Foushee, 1982; 
Fussell et al., 1998; Godart et al., 2001; Hyey \& Wickens, 1993; Kanki, Lozito \& Foushee, 1989; Larson \& Schaumann, 1993; Leedom \& Simon, 1995) oder ungenau (Clark, 1991) definiert. Malone und Crowston (1994) diskutieren, dass die Verschiedenheit bestehender Definitionen die Schwierigkeit der begrifflichen Präzisierung von Koordination widerspiegelt.

Tabelle 2 zeigt einschlägige Definitionen von Koordination, die sich auf die Koordination von Kleingruppen beziehen. Definitionen, die sich auf die Koordination von Organisationen beziehen, werden hier nicht aufgeführt (bis auf Van de Ven, Delbecq \& Koenig, 1976).

Beim Betrachten der Koordinationsdefinitionen fällt auf, dass zwischen Koordination als Prozess (Arrow et al., 2000; Boos \& Sassenberg, 2001; Brannick \& Prince, 1997; Grote et al., 2003; Malone \& Crowston, 1994; Marks et al., 2001; Tschan, 1995; Van de Ven et al., 1976; Wittenbaum et al., 1998) und Koordination als Zustand bzw. Ergebnis (Dickinson \& McIntyre, 1997) unterschieden wird. Andere Autoren/-innen geben explizit beide Betrachtungsweisen an (Berteotti \& Seibold, 1994; Espinosa et al., 2004). Koordination als Prozess beschreibt den Weg der Erreichung eines koordinierten Zustandes.

Tabelle 2: Definitionen von Koordination und ihre Merkmale

\begin{tabular}{|c|c|c|c|}
\hline $\begin{array}{l}\text { Autoren/- } \\
\text { innen }\end{array}$ & Definition & $\begin{array}{l}\text { Koordination } \\
\text { als Prozess } \\
\text { oder } \\
\text { Ergebnis }\end{array}$ & $\begin{array}{l}\text { Definitions- } \\
\text { merkmale }\end{array}$ \\
\hline $\begin{array}{l}\text { (Arrow et al., } \\
2000, \text { S. 104) }\end{array}$ & $\begin{array}{l}\text { Coordination is the structured patterning of within-group } \\
\text { activities by which groups strive to achieve their goal. }\end{array}$ & Prozess & Gemeinsames Ziel \\
\hline \multirow{2}{*}{$\begin{array}{l}\text { (Boos \& } \\
\text { Sassenberg, } \\
\text { 2001, S. 199) }\end{array}$} & \multirow{2}{*}{$\begin{array}{l}\text { Koordination }[\ldots] \text { als ein sozialer Prozess der } \\
\text { aufgabenbedingt notwendigen Informationsverarbeitung } \\
\text { in einer Gruppe }[\ldots] \text { Koordination von Bedeutungen und } \\
\text { Wissen. }\end{array}$} & \multirow[t]{2}{*}{ Prozess } & $\begin{array}{l}\text { Aufgaben- } \\
\text { abhängigkeit }\end{array}$ \\
\hline & & & $\begin{array}{l}\text { Koordination von } \\
\text { Bedeutungen und } \\
\text { Wissen }\end{array}$ \\
\hline \multirow[t]{2}{*}{$\begin{array}{l}\text { (Berteotti \& } \\
\text { Seibold, 1994, } \\
\text { S. 108) }\end{array}$} & $\begin{array}{l}\text { Coordination }[. . .] \text { is the extent to which the work activities } \\
\text { of members cohere logically, temporally, and effectively. }\end{array}$ & Ergebnis & $\begin{array}{l}\text { zeitliche \& } \\
\text { logische } \\
\text { Effektivität }\end{array}$ \\
\hline & $\begin{array}{l}{[\ldots] \text { coordination is evidenced by how well group members }} \\
\text { as a whole perform in accord with their roles in the system }\end{array}$ & Prozess & $\begin{array}{l}\text { Verhalten im } \\
\text { Einklang mit Rolle } \\
\text { und System }\end{array}$ \\
\hline
\end{tabular}




\section{Autoren/- Definition innen}

\section{Koordination Definitions- als Prozess merkmale \\ oder \\ Ergebnis}

Prince, 1997, that one or more of the team members makes so that the

S. 4) goal is reached.

Übergeordnetes Ziel

(Dickinson \& Coordination reflects the execution of team activities such Ergebnis Gemeinsames Ziel

McIntyre, 1997, S. 22) that members respond as a function of the behavior of others. Successful coordination implies the effective operation of other components of teamwork (e.g., communication, monitoring, and backup). In this way, the actions of individual members are merged to produce synchronizes team performance.

(Espinosa et $\quad \ldots$ coordination as the effective management of al., 2004, S. dependencies among subtasks, resources (e.g., equipment, $109 / 110)$ tools), and people...

The process of coordinating can be defined as the activities Prozess carried out by team members when managing dependencies.

Coordination as an outcome (i.e., coordination success) can be defined as the extent to which dependencies have been effectively managed.

(Grote et al., Coordination defined as tuning interdependent work 2003, S. 127) processes to promote concerted action towards a superordinate goal

Prozess

Umgang mit Interdependenz

Synchronisation

Umgang mit Interdependenz

Effektivität

Ergebnis Umgang mit Interdependenz Übergeordnetes Ziel

(Malone \& Coordination is managing dependencies among activities.

Prozess

Umgang mit Interdependenz 1994, S. 90)

(Marks et al., 2001, S. 363)

Orchestrating the sequence and timing of interdependent actions.

(Tschan, 1995, Good coordination can be described as adopting a S. 371) common goal, building a mutual representation of the task, and structuring the interaction process in such a way that group members optimally combine their efforts.

(Van de Ven et Coordination means integrating or linking together al., 1976, S. different parts of an organization to accomplish a 322) collective set of task.

(Wittenbaum The way in which group members synchronize their et al., 1998, S. actions in order to complete successfully the group task. 177)
Zeit und

Reihenfolge

Umgang mit Interdependenz

Prozess Gemeinsames Ziel

Prozessstrukturierung

Effektivität

Prozess Integration

Gemeinsames Ziel

Prozess Synchronisation

Gemeinsames Ziel 
Die Autoren/-innen legen darüber hinaus unterschiedliche Schwerpunkte in ihrer Definition. Sehr häufig wird betont, dass Koordination der Erreichung eines gemeinsamen oder übergeordneten Ziels dient (Arrow et al., 2000; Brannick \& Prince, 1997; Dickinson \& McIntyre, 1997; Grote et al., 2003; Tschan, 1995; Van de Ven et al., 1976; Wittenbaum et al., 1998) und dieses nach Möglichkeit effektiv erreicht werden soll (Berteotti \& Seibold, 1994; Espinosa et al., 2004; Tschan, 1995). Einige Autoren/-innen sehen nicht nur in der Erreichung eines gemeinsames Ziels den Zweck der Koordination, sondern gehen davon aus, dass Koordination hauptsächlich der Handhabung von Interdependenz̨en (Espinosa et al., 2004; Grote et al., 2003; Malone \& Crowston, 1994; Marks et al., 2001) oder der logischen und zeitlichen Abstimmung von Teilhandlungen dient (Berteotti \& Seibold, 1994; Brannick \& Prince, 1997), die auch als Synchronisation bezeichnet werden kann (Dickinson \& McIntyre, 1997; Wittenbaum et al., 1998). Spezifischere Definitionen beziehen die Strukturierung des Interaktionsprozesses (Tschan, 1995) bzw. die Koordinationsinhalte Wissen und Bedeutungen ein (Boos \& Sassenberg, 2001). Lediglich in der Definition von Boos und Sassenberg (2001) wird auf die Aufgabenabhängigkeit der Koordination hingewiesen.

Nahezu allen hier aufgeführten Definitionen von Koordination ist gemein, dass sie Koordination als ein Mittel zum Umgang mit Komplexität aus handlungstheoretischer Perspektive betrachten.

Diese Definitionen integrierend wird Koordination in der vorliegenden Arbeit als der aufgabenabhängige Umgang mit Interdependenz zur effektiven Erreichung eines gemeinsamen Ziels im Sinne einer Abstimmung von Teilhandlungen und einer hierarchisch und sequentiell logischen Verarbeitung von Informationen betrachtet. Der Interdependenz der Aufgabe kommt dabei eine besondere Rolle zu: Erfordert die Aufgabe keine interdependenten Handlungen, so ist Koordination nicht notwendig. 
Der Begriff Koordinationsmechanismus beschreibt Verhaltensweisen, durch welche die Koordination während des Interaktionsprozesses umgesetzt wird. In Anlehnung an ihre Definition von Koordination betrachten Espinosa et al. (2004) Koordinationsmechanismus als etwas, das Teams im Umgang mit Interdependenzen hilft:

"A coordination mechanism is one that helps teams manage dependencies" (Espinosa et al., 2004, S. 111).

Im folgenden Kapitel wird dargelegt, wie Koordinationsmechanismen zur Koordination von Kleingruppen eingesetzt werden können.

\subsection{Theoretische Konzepte der Gruppenkoordination}

Im Folgenden werden theoretische Konzeptionen der Kleingruppenkoordination vorgestellt. Zunächst werden allgemeine Ansätze der Kleingruppenkoordination vorgestellt, der Schwerpunkt liegt jedoch auf der Darstellung zweier Modelle der Kleingruppenkoordination: das Modell der Koordinationsmechanismen von Espinosa, Kraut und Lerch (2004) sowie das Modell der Koordinationsmodi von Wittenbaum, Vaughan und Stasser (1998). Da sich die vorliegende Arbeit mit der Koordination von Entscheidungsfindungsprozessen in Kleingruppen beschäftigt, werden nur die theoretischen Konzeptionen vorgestellt, die sich tatsächlich mit Koordination von Intragruppenprozessen auseinandersetzen. Auf Ansätze zur Koordination in Organisationen (z.B. Bechky, 2003; Weick, 1995; Wilke, 2001) wird nicht eingegangen.

\subsubsection{Allgemeine Ansätze der Kleingruppenkoordination}

Arrow, McGrath und Berdahl (2000) betrachten Gruppen als komplexe Systeme und gehen davon aus, dass die Bewältigung von Gruppenaufgaben in unterschiedlichem Ausmaß drei verschiedener Arten von Koordination bedarf: Der Koordination von Zielen und Interessen der Mitglieder, der Koordination von Handlungen (Synchronisation) und der Koordination von Bedeutungen. Letztere Koordinationsanforderung gilt insbesondere für Aufgaben, in denen 
Informationen verarbeitet und Probleme gelöst werden müssen (Arrow et al., 2000, S. 102 ff.) und ist daher auch für Entscheidungsaufgaben relevant. Die Koordination von Bedeutungen beinhaltet das Austauschen und Teilen von Informationen und das Etablieren von Bedeutungen dieser Informationen für die Gruppe. Sie erfordert, dass bestimmt wird, welche Informationen die Gruppe benötigt, woher diese Informationen bezogen werden können, ob die Bezugsquellen vertrauenswürdig sind sowie das Festlegen der Bedeutung der gewonnenen Information für die Gruppenaufgabe. Darüber hinaus muss entschieden werden, wer die Informationsbeschaffung und den Informationsaustausch überwacht. Außerdem müssen Bewertungs- und Integrationsregeln zum Umgang mit den Informationen bestimmt werden (Arrow et al., 2000).

Malone und Crowsten (1994) betrachten Koordination fächerübergreifend, indem sie Koordinationsprozesse annehmen, die in unterschiedlichen Disziplinen (z.B. Computerwissenschaften, Organisationstheorie) in ähnlicher Weise auftreten. Sie unterscheiden vier Koordinationsprozesse: 1.) den Umgang mit geteilten Ressourcen, 2.) die Handhabung von Produzent-Konsument-Beziehungen, 3.) den Umgang mit simultanen Bedingungen sowie 4.) den Umgang mit Aufgaben und deren Teilaufgaben. Im Hinblick auf Gruppenentscheidungsfindung diskutieren Malone und Crowsten (1994) allerdings nicht die Bedeutung von Koordination für den Entscheidungsfindungsprozess, sie betrachten vielmehr umgekehrt die Rolle der Gruppenentscheidungen während der Koordination, indem beispielsweise während des Umgangs mit geteilten Ressourcen entschieden werden muss, welches Gruppenmitglied zu welchem Zeitpunkt über die Ressource verfügen kann.

Schwabe betrachtet Koordination in Anlehnung an Malone und Crowston (1994) als die Handhabung von Abhängigkeiten zwischen einzelnen Aktivitäten (Schwabe, 2001a). Koordinationswerkženge dienen dazu, diese Handhabung von Abhängigkeiten zu ermöglichen und zu erleichtern (Schwabe, 2001a). Unklar ist, ob Schwabe Koordinationswerkzeuge mit Koordinationsmechanismen gleichsetzt, derer er fünf unterscheidet: 
1. Terminkoordination (z. B. über elektronische Gruppenterminkalender),

2. zentrale Koordination von strukturiert ablaufenden Aktivitäten durch Vorgangssteuerung (nach vorher festgelegten Regeln, z.B. Dienstweg),

3. zentrale Koordination von unstrukturiert ablaufenden Aktivitäten durch Projektmanagementwerkzeuge,

4. dezentrale Koordination durch gemeinsames Material (z.B. To-Do-Listen mit offenen Aktivitäten, elektronisches Tagebuch),

5. Koordination in computerunterstützten Sitzungen.

Deutlich wird zum einen, dass die aufgeführten Koordinationsmechanismen sich auf computervermittelte Zusammenarbeit beziehen. Zum anderen unterscheidet Schwabe (2001a) zwischen zentraler und dezentraler Koordination. Diese Unterscheidung führt er allerdings nicht explizit aus, eine theoretische Konzeption von Koordination und von Merkmalen, nach denen die genannten Koordinationsmechanismen unterschieden werden, fehlt. Daher eignet sich Schwabes Betrachtungsweise der Koordination kaum zur Untersuchung der Koordination von Gruppenentscheidungsfindungsprozessen.

Van de Ven und Kollegen (1976) unterscheiden drei Koordinationsmodi, die in Abhängigkeit der mit der Aufgabe verbundenen Unsicherheit und Interdependenz sowie der Gruppengröße einzusetzen sind: Die unpersönliche Koordination koordiniert durch vorgegebene Pläne, Zeitpläne, formalisierte Regeln oder standardisierte Informationen. Persönliche und Gruppenkoordination koordinieren durch persönliches oder gemeinsames Feedback im Sinne eines gegenseitigen Anpassens an neue Informationen. Persönliche Koordination findet dabei häufig durch vertikale und horizontale Kommunikation statt, Gruppenkoordination in regelmäßigen und unregelmäßigen Gruppensitzungen (Van de Ven et al., 1976). Gruppenkoordination wird als Koordinationsmodus betrachtet, innerhalb dessen Van de Ven et al. (1976) nicht weiter ausdifferenzieren, wie die Gruppensitzungen selbst koordiniert werden. Aufgrund der fehlenden Differenziertheit im Hinblick auf intragruppale Entscheidungsprozesse eignet sich der Ansatz von Van de Ven et al. (1976) eher für die Betrachtung organisationaler Koordination, weniger für die Analyse der Koordination innerhalb von Kleingruppen. Er wird dennoch in der 
Untersuchung natürlicher Gruppen verwendet (Berteotti \& Seibold, 1994) und findet sich auch im Modell der Koordinationsmodi von Wittenbaum et al. (1998) wieder, welches im Folgenden gemeinsam mit dem Modell zur Unterscheidung impliziter und expliziter Koordination vorgestellt wird.

\subsubsection{Implizite und explizite Koordinationsmechanismen - Das Modell von Espinosa, Kraut und Lerch (2004)}

Espinosa, Lerch und Kraut (2004) definieren Koordination als Management von Dependenzen. Sie unterscheiden Koordination als Ziel vom Koordinationsprozess an sich (vgl. Tabelle 2): Zur Erreichung des Ziels der Koordination, d.h. zur Bewältigung gegenseitiger Abhängigkeiten, nutzen die Gruppenmitglieder Koordinationsmechanismen. Diese Mechanismen werden danach unterschieden, inwieweit ihr Einsatz expliæit oder impliæit ist. Zu den expliziten Mechanismen werden die Organisation der Aufgabe durch Programme (Zeitpläne, festgelegte Abläufe) und Kommunikation gezählt. Auf eine ähnliche Systematik wurde bereits in früheren Arbeiten hingewiesen (Fussell et al., 1998). Diese Mechanismen werden als explizit bezeichnet, weil sie mit Koordinationsabsicht ausgeführt werden:

“..., explicit coordination mechanisms can be defined as those mechanisms explicitly employed by a team to help manage task dependencies" (Espinosa et al., 2004, S. 111).

Diese Definition der Explizitheit erscheint allerdings zirkulär und erlaubt außer der Intentionalität der Koordination keine weitere Unterscheidung von impliziter Koordination. Implizite Koordinationsmechanismen entstehen durch Gruppenkognition, die auf geteilten mentalen Modellen ${ }^{7}$ basiert (Espinosa et al., 2004). Sie werden nicht absichtlich zur Koordination verwendet.

\footnotetext{
${ }^{7}$ Als geteilte mentale Modelle werden Wissensstrukturen der Gruppenmitglieder bezeichnet, die sie befähigen, richtige Erklärungen und Erwartungen der Aufgabe zu bilden (Cannon-Bowers, Salas \& Converse ,1993, zit. n. Gurtner et al., in press). Geteilte mentale Modelle beziehen sich nicht nur auf die Aufgabe, sondern auch auf die Gruppenmitglieder, die vorhandene Technologie, die Umgebung sowie auf Metakognition und Interaktion (Tschan \& Semmer, 2001).
} 
"..., we define implicit coordination mechanisms as those that are available to team members from shared cognition that enable them to explain and anticipate task states and member actions, thus helping them manage task dependencies." (Espinosa et al., 2004, S.112).

Explizite und implizite Koordinationsmechanismen sind nicht unabhängig voneinander, so bestimmt beispielsweise das Niveau der Teamkognition bzw. der Grad des geteilten Gruppenwissens den Bedarf expliziter Absprachen (Espinosa et al., 2004). Explizite Koordinationsmechanismen bedürfen mehr Zeit und Anstrengung (z.B. formale Besprechungen; Erstellen von Plänen) und werden, insbesondere unter Zeitdruck, eher durch implizite Mechanismen substituiert (Espinosa et al., 2004). Dieser Zusammenhang wird im Modell (vgl. Abbildung 4) durch die Doppelpfeile zwischen den expliziten und impliziten Mechanismen repräsentiert.

Espinosa und Kollegen ziehen demnach den bewussten Einsatz eines Koordinationsmechanismus als Kriterium zur Bestimmung des Grades der Explizitheit heran. Weitere Merkmale beider Koordinationsmechanismen erläutern die Autoren nicht. Trotz dieser konzeptuellen Schwäche von Explizitheit ist das aufgestellte Rahmenmodell der Teamkoordination und Teamleistung (vgl. Abbildung 4) im Hinblick auf Koordination eine notwendige Erweiterung des klassischen Input-Prozess-Output-Modells (vgl. Abbildung 2).

In dem Rahmenmodell wird Koordination einerseits als Prozess (durch explizite und implizite Koordinationsmechanismen) und andererseits als Ergebnis (z.B. Strategie) betrachtet. Sowohl die Koordinationsmechanismen als auch die Koordination als Ergebnis mediieren die Wirkung der Inputfaktoren auf die Gruppenleistung, wobei zunächst die Koordinationsmechanismen die Beziehung zwischen den Inputfaktoren und der Koordination als Ergebnis vermitteln. Allerdings stellt Koordination zwar eine notwendige, aber keine hinreichende Bedingung für gute Gruppenleistung dar, andere Voraussetzungen müssen in Abhängigkeit der Aufgabe ebenfalls erfüllt werden (Espinosa et al., 2004). 


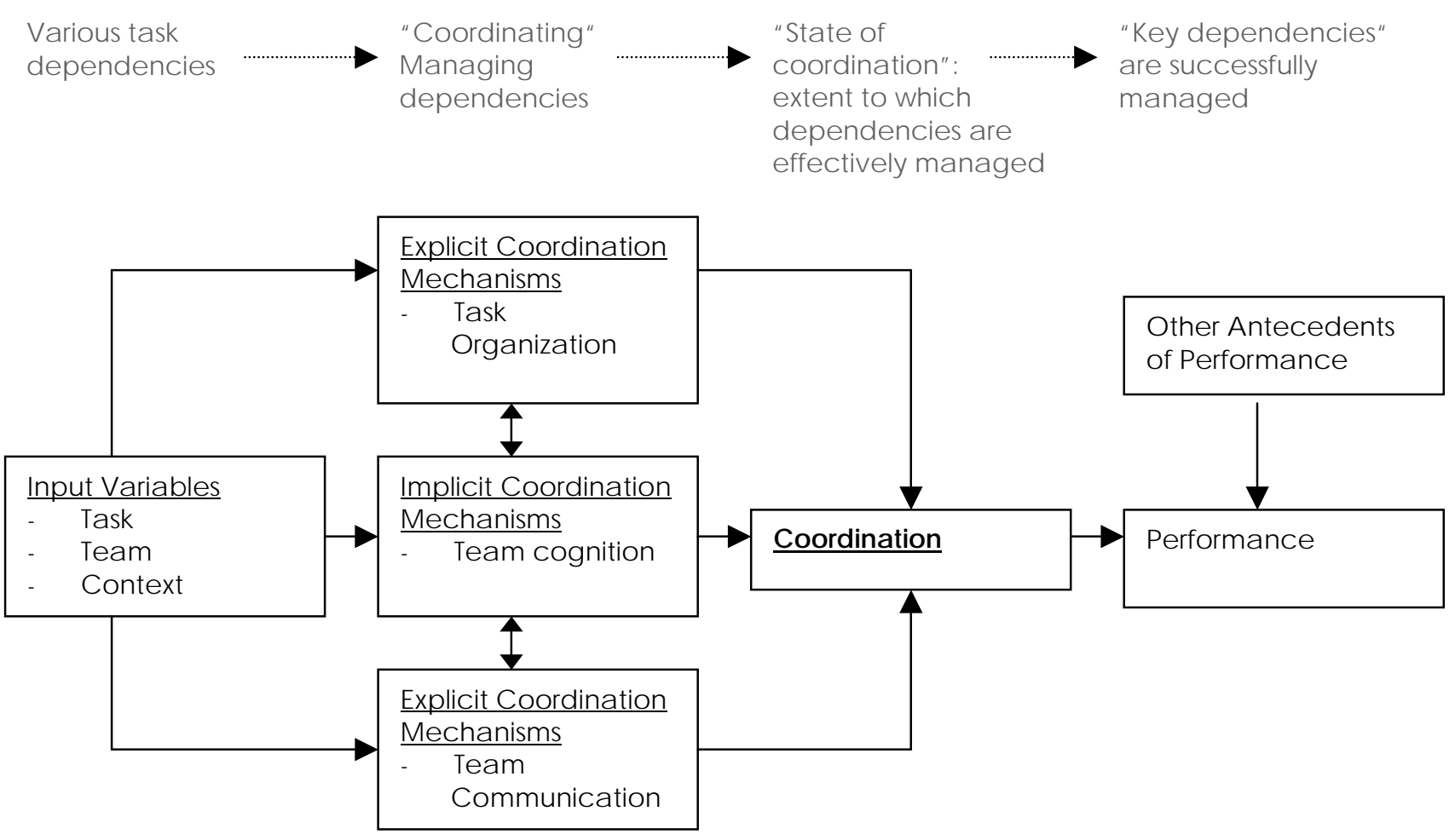

Abbildung 4: Integriertes Rabmenmodell der Teamkoordination und Leistung (Espinosa et al., 2004, S. 110 \&118)

Darüber hinaus weist das Modell auf die situative Effektivität der Koordinationsmechanismen hin: Wesentlich für die Angemessenheit der Mechanismen sind die Koordinationsanforderungen der Gruppenaufgabe. So besteht beispielsweise bei mangelnder Komplexität und fehlender Interdependenz der Teilaufgaben kein Koordinationsbedarf. Allerdings werden im Modell keine Angaben zur funktionalen oder normativen Beziehung einzelner Aufgabenanforderungen und bestimmter Koordinationsmechanismen postuliert.

Darüber hinaus sind beide Koordinationsmechanismen sind nicht in gleicher Wiese geeignet, um die mit unterschiedlichen Aufgabenerfordernissen wechselnden Koordinationsanforderungen erfolgreich zu handhaben (Espinosa et al., 2004).

“..., a team will employ a mix of coordination mechanisms that it deems most suitable to manage task dependencies in a given context. [...] task, team, and context variables influence the number, complexity, and types of dependencies that are present in the task 
and, consequently, which coordination mechanisms are used by team members to manage these dependencies" (Espinosa et al., 2004, S. 114).

Im Hinblick auf explizite Koordination scheint die Betrachtung von Kommunikation als Koordinationsmechanismus sehr oberflächlich, da keine Aussagen darüber getroffen werden, welches Gruppenmitglied wann wie kommunizieren muss, um den Gruppenprozess explizit zu koordinieren. Espinosa und Kollegen postulieren lediglich, dass Kommunikation insbesondere bei komplexen intellektuellen Aufgaben mit unklaren Interdependenzen und in frühen Phasen der Zusammenarbeit notwendig ist (Espinosa et al., 2004). Neben der undifferenzierten Betrachtung von Kommunikation als explizitem Koordinationsmechanismus ist der Einsatz nonund paraverbaler Kommunikation auch als implizitem Koordinationsmechanismus denkbar, indem sich die Gruppenmitglieder beispielsweise per Blickkontakt verständigen oder im Verlauf ihrer Zusammenarbeit lernen, minimale Botschaften zu senden und zu verstehen.

Die konzeptuelle Unterscheidung von impliziter und expliziter Koordination wird von Grote und Kollegen (2003) kritisiert. Sie konstatieren, dass es zwar einige interessante Forschungen zu diesem Thema gäbe, diese jedoch keine geeigneten Indikatoren liefere, beide Koordinationsmechanismen konkret voneinander $\mathrm{zu}$ trennen. Neben der fehlenden Funktionalität bemängeln sie die unklaren Definitionen und somit auch Operationalisierungen von Implizitheit und Explizitheit. Wird beispielsweise in einer Gruppe deutlich und direkt miteinander kommuniziert, dann wird dies oft als Indikator für explizite Koordination verwendet, mag aber für implizite Koordination auch teilweise zutreffen, wenn dabei z.B. ungefragt Informationen an andere Personen weitergegeben werden (Grote et al., 2003). Grote und Kollegen/-innen (2003) schlagen keine Alternative für eine andere bzw. eine erweiterte Definitionen expliziter und impliziter Koordination vor, sondern postulieren, dass die Arbeitsbeanspruchungen der Aufgabe die Koordinationsanforderungen innerhalb des Teams bedingen. Das Einbeziehen von Arbeitsanforderungen in ein Koordinationsmodell erscheint sinnvoll, da Koordination zielgerichtet und aufgabenabhängig ist: Je nach Aufgabe und Kontext 
ist ein anderer Koordinationsmechanismus effektiv. Ein entsprechendes Modell zum Zusammenhang von Arbeitsanforderungen bzw. Arbeitsbeanspruchung und Koordination schlagen Grote et al. jedoch nicht vor. Vor dem Hintergrund bisheriger Forschung scheint es nicht angemessen, die konzeptionelle Differenzierung zwischen expliziter und impliziter Koordination aufgrund noch nicht präzisierter Definitionen und erfolgreicher Operationalisierungen zu verwerfen. Insbesondere die Forschung zur Interaktion in risikoreichen Arbeitsumgebungen konnte zeigen, dass je nach situativen Anforderungen entweder implizite oder explizite Koordination effizient waren (Sexton, 2004), so dass sich die Konzeptionalisierung impliziter versus expliziter Koordination bereits bewährt hat, auch wenn ihre Operationalisierung nicht einfach ist (Boos, Kolbe \& Strack, 2006).

Insgesamt kann davon ausgegangen werden, dass Kommunikation sowohl zur expliziten als auch zur impliziten Gruppenkoordination eingesetzt werden kann. Zur Untersuchung der Funktionalität von Kommunikation im Hinblick auf Koordination muss die Dimension der Explizitheit präzisiert sowie überlegt werden, inwieweit Explizitheit als einziges Unterscheidungskriterium der Koordinationsmechanismen ausreichen ist. Dazu wird im Folgenden die Taxonomie der Koordinationsmechanismen von Wittenbaum, Vaughan und Stasser (1998) herangezogen.

\subsubsection{Das Modell der Koordinationsmodi von Wittenbaum, Vaughan und Stasser (1998)}

Wittenbaum, Vaughan und Stasser (1998) betrachten Koordination als Synchronisation der Handlungen der Gruppenmitglieder, um die Gruppenaufgabe erfolgreich lösen zu können.

"In other words, group coordination involves who among the members does what, as well when, where, and how they complete their designated tasks" (Wittenbaum et al., 1998, S. 177).

Wittenbaum und Kollegen/-innen (1998) postulieren, dass Gruppenkoordination auf mindestens zwei Dimensionen variieren kann und ziehen neben der Dimension Expliæitheit zusätzlich die 
Dimension des Zeitpunktes der Koordination heran. Die Gruppenmitglieder können ihre Zusammenarbeit vor dem eigentlichen Arbeitsbeginn oder während des Arbeitsprozesses koordinieren. Die Koordination kann einerseits implizit bzw. stillschweigend sein und auf unausgesprochenen Erwartungen und Absichten basieren. Anderseits kann explizit koordiniert werden - durch verbale Abstimmungen oder zuvor beschlossene Pläne, aus welchen klar hervorgeht, welches Gruppenmitglied welche Aufgabe zu welchem Zeitpunkt zu erledigen. Obwohl sowohl die Explizitheit als auch der Koordinationszeitpunkt kontinuierlich sind, können sie zur vereinfachten Darstellung dichotomisiert werden, so dass sich vier Koordinationsmodi ableiten lassen (vgl. Abbildung 5): Vor der Zusammenarbeit kann durch Preplans und Tacit PreCoordination koordiniert werden, während des Arbeitsprozesses durch In-Process-Planning und durch In-Process-Tacit Coordination (Wittenbaum et al., 1998). Diese vier Koordinationsmodi werden im Folgenden näher betrachtet.

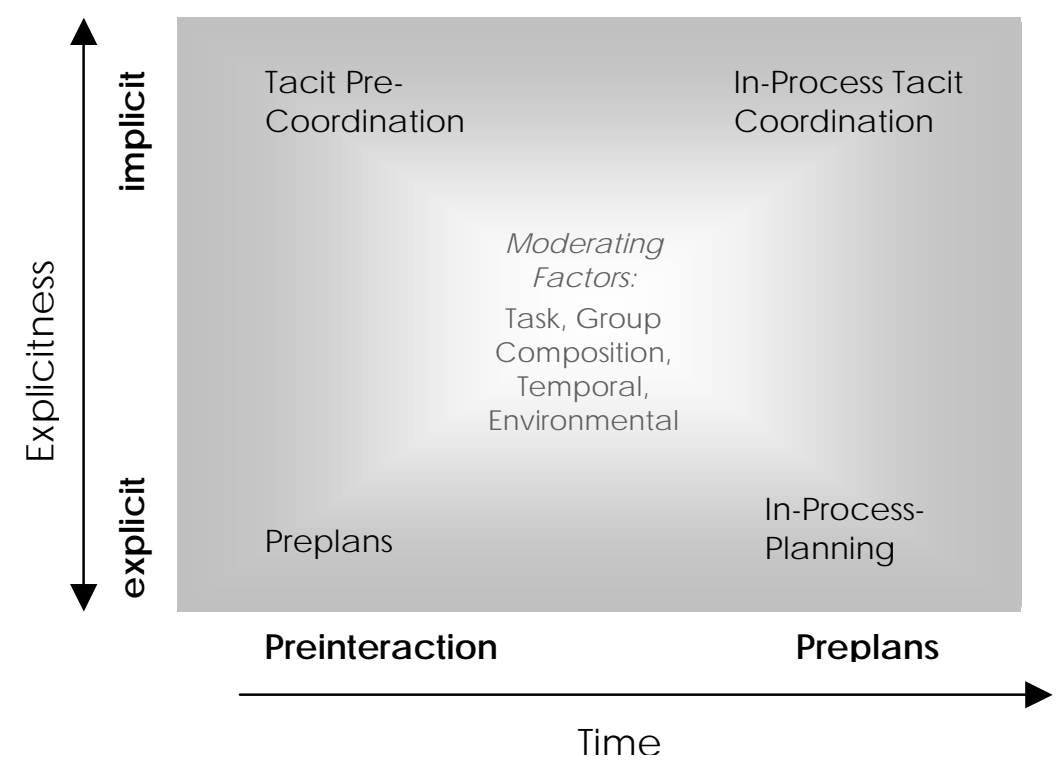

Abbildung 5: Modell der Koordinationsmodi in Task-Performing-Gruppen (Wittenbaum et al., 1998, S. 178)

Preplans (vor der Interaktion; expliqit) umfassen alle Arten von Rollen- und Tätigkeitsbeschreibungen, organisationalen Regeln oder Zeitplänen, die vor dem Beginn der 
Zusammenarbeit der Gruppe in schriftlicher oder auch mündlicher Form an die Gruppenmitglieder herangetragen werden (Wittenbaum et al., 1998). Ein Beispiel für Preplans stellen Standard Operating Procedures (SPOs) dar, in denen das Vorgehen in risikoreichen Situationen schrittweise erläutert ist.

Während des In-Process-Plannings (während der Interaktion, explizit) werden ebenfalls die Arbeitsrollen einzelner Gruppenmitglieder definiert. Allerdings findet diese Form der Koordination durch die Kommunikation von Strategieplänen während der Aufgabenbewältigung statt. Das In-Process-Planning zeigt sich in der Diskussion der Fähigkeiten und Erfahrungen der Gruppenmitglieder, Zuordnung von Teilaufgaben zu einzelnen Gruppenmitgliedern, dem Erfragen der Tätigkeiten der anderen oder in der Festlegung, wie und wann eine Aufgabe fertiggestellt werden soll (Wittenbaum et al., 1998).

Der Mechanismus der Tacit Pre-Coordination (vor der Interaktion, implizit) beschreibt einen Modus der Koordination, in dem die Gruppenmitglieder aufgrund ihrer bereits vor dem Arbeitsprozess getroffenen Hypothesen bezüglich der Aufgabe, dem Aufgabenkontext und vor allem bezüglich der anderen Gruppenmitglieder handeln. Die Gruppenmitglieder treffen zunächst eigene Annahmen über die Aufgabenanforderungen und die wahrscheinlichen Beiträge der anderen Gruppenmitglieder und passen ihr eigenes Handeln daran an. Damit orientieren sich alle Gruppenmitglieder stillschweigend an ihren gegenseitigen Erwartungen, um ihre Handlungen untereinander abzustimmen (Wittenbaum, Stasser \& Merry, 1996; Wittenbaum et al., 1998). Dabei ist der Aufbau und Zugriff auf geteilte mentale Modelle der Gruppenmitglieder, der Aufgabe und der Zusammenarbeit wesentlich (Mathieu, Goodwin, Heffner, Salas \& CannonBowers, 2000). Sie können das Erkennen von Aufgabenkonstellationen und ihren Handlungsanforderungen erleichtern und durch das Anknüpfen an bestehende Erfahrungen die Bildung geeigneter Erwartungen über die zukünftigen Handlungsweisen der anderen Gruppenmitglieder ermöglichen (Gurtner, 2003). Denkbar ist auch, dass im Hinblick auf die eigenen Erwartungsbilder auch auf frühere Erfahrungen und mentale Modelle zurückgegriffen 
wird, die im Umgang mit Aufgaben und Arbeitskollegen bereits gewonnen wurden. Die In-Process Tacit Coordination (während der Interaktion, implizit) findet statt, wenn die Gruppenmitglieder basierend auf ihren Beobachtungen ihre Arbeitsstrategien während des Arbeitsprozesses stillschweigend gegenseitig anpassen. In Anlehnung an Hackman und Morris (1975) diskutieren Wittenbaum und Kollegen/-innen (1998), dass die In-Process Tacit Coordination von den Gruppenmitgliedern als Ersatz für eine explizite Diskussion des Vorgehens während der Aufgabenbearbeitung (In-Process-Planning) gewählt wird, da es in Gruppen Normen gäbe, das Vorgehen nicht explizit zu besprechen (Wittenbaum et al., 1998). Beide Modi impliziter Koordination bergen sowohl Vor- als auch Nachteile in sich. Einerseits ist die implizite Koordination weniger zeitaufwendig und erlaubt den Gruppen, mehr Zeit für die eigentliche Aufgabenerledigung zu verwenden. Zusätzlich ermöglicht sie die sozialverträgliche Zuweisung von Expertise und Nicht-Expertise, indem beispielsweise eine komplizierte Teilaufgabe automatisch dem/der vermuteten Experten/-in, nicht aber der Person, die die Aufgabe vermutlich nicht bewältigen kann, übertragen wird, ohne explizit über die vorhandene Expertise sprechen zu müssen. Andererseits birgt implizite Koordination das Potential für Koordinationsfehler. Erstens basieren die Hypothesen und Erwartungen bezüglich des Verhaltens anderer Gruppenmitglieder auf minimalen Hinweisreizen, wodurch fehlerhafte Schlussfolgerungen, z.B. fälschliche Zuweisung von Expertise, möglich sind. Zweitens können diese fehlerhaften Annahmen im Rahmen selbsterfüllender Prophezeiungen sozial verstärkt werden. Drittens ist ein implizites Rollengefüge besonders anfällig gegenüber Fluktuation der Gruppenmitglieder, da aufgrund mangelnden expliziten Rollenwissens neue Gruppenmitglieder schwer eingearbeitet werden können (Wittenbaum et al., 1998).

Die Annahmen über den Zusammenhang zwischen impliziter Koordination und geteilten mentalen Modellen sind Gegenstand der Kritik. Cannon-Bowers und Salas (2001) sind der Ansicht, dass die Annahme, die Bildung geteilter mentaler Modelle sei notwendig, um die Koordination in der Gruppe und das Endergebnis zu verbessern, zu einfach wäre, um mit ihrer 
Hilfe ein komplexes Phänomen wie das der Gruppenkoordination zu erklären. Ihrer Meinung nach besteht weiterer Forschungsbedarf zur Klärung der Frage, welches Wissen in der Gruppe geteilt werden muss und in welchen Situationen bestimmte Koordinationsmechanismen effektiv sind.

Wann wird welcher Koordinationsmodus eingesetzt?

Wittenbaum und Kollegen/-innen (1998) postulieren so genannte moderierende Faktoren, die bestimmen, unter welchen Bedingungen welcher Koordinationsmodus geeignet ist. Zu den moderierenden Faktoren gehören neben der Gruppenaufgabe die Gruppenzusammensetzung, die zur Aufgabenerledigung vorhandene Zeit und Faktoren der Umgebung. Auf die Implikationen der Ausprägung der moderierenden Faktoren wird im Folgenden näher eingegangen.

\section{Gruppenaufgabe}

In Anlehnung an Van de Ven et al. (1976) postulieren Wittenbaum und Kollegen/-innen, dass sowohl die Interdependenz und die Unsicherheit der Aufgabe den Einsatz der Koordinationsmodi bestimmen.

Interdependen, beschreibt das Ausmaß, in dem ein Gruppenmitglied seine Aufgabe nur in Abhängigkeit der Aufgabenerfüllung eines anderen Gruppenmitgliedes erledigen kann. Je interdependenter die Gruppenaufgabe ist, desto höher ist ihr Bedarf an expliziter Koordination.

\footnotetext{
"Tasks that create high dependence among members add to the complexity of synchronization and may render tacit coordination strategies ineffective. Therefore, the more coordination required of members, the more they should use explicit strategies, particularly during interaction" (Wittenbaum et al., 1998, S. 189).
}

Demnach führt explizite Koordination nur dann zu einer besseren Gruppenleistung, wenn die Aufgabe durch ihre Dependenz der Koordination bedarf (Hackman, Brousseau \& Weiss, 1976). Gruppenentscheidungsaufgaben gelten als komplex und interdependent (vgl. 
Kapitel 3.1), daher ist ihr Koordinationsbedarf hoch und explizite Koordination scheint die angemessene Koordinationsform zu sein.

Die Aufgabenunsicherbeit bezieht sich auf die Schwierigkeit und Variabilität der Aufgabe (Van de Ven et al., 1976). Mit zunehmender Aufgabenunsicherheit tendieren Gruppen zu InProcess-Planning (Wittenbaum et al., 1998). Da Gruppenentscheidungsaufgaben keine einzig richtige Lösung versprechen, sondern die Konsequenzen aller Entscheidungsalternativen von der Gruppe verantwortlich abgewogen (Hirokawa, 1985) und die entscheidungsrelevanten Ressourcen und das Wissen um ihre Bedeutung im Entscheidungsprozess integriert werden müssen, können Gruppenentscheidungsaufgaben als unsichere Aufgabe im Sinne obiger Definition betrachtet und von einem hohen Bedarf expliziter Koordination ausgegangen werden.

"Thus, the more variability and complexity involved in the task, the greater the coordination demand, and the more likely that in-process planning will be needed to achieve successful group performance" (Wittenbaum et al., 1998, S. 191).

\section{Gruppenzusammensetzung}

Bezüglich der Gruppenzusammensetzung beeinflussen neben der Größe der Gruppe auch Neuzusammensetzung als auch die Diversität der Gruppenmitglieder die Koordinationsanforderungen. Koordinationsprobleme treten häufig in großen Gruppen auf, verbunden mit der Tendenz zu impliziter Koordination, da explizite Koordination aufgrund eingeschränkter Kommunikationsmöglichkeiten kaum realisierbar ist (Wittenbaum et al., 1998). Auch die Neuฉusammensetæung der Gruppe, beispielsweise wenn ältere Gruppenmitglieder die Gruppe verlassen und neue hinzukommen, kann Koordinationsprobleme verursachen und kurzfristig explizite Koordination erfordern. In Gruppen mit hoher Diversität können die Gruppenmitglieder aufgrund vorhandener Stereotype leichter Hypothesen über das Verhalten der anderen bilden und sich dadurch möglicherweise leichter implizit koordinieren (Wittenbaum et al., 1998). 


\section{$\underline{\text { Zeit }}$}

Je nach der für die Aufgabe zur Verfügung stehenden Zeit und des Auftretens sogenannter zeitlicher Meilensteine ändert sich der Koordinationsbedarf. Zeitdruck führt häufig dazu, dass Gruppen sofort mit der Aufgabenbearbeitung beginnen, ohne sich vorher explizit abzustimmen, was zu Leistungseinbußen führt. In Anlehnung an Gersick (1988; 1989) diskutieren Wittenbaum et al. die Bedeutung zeitlicher Meilensteine für die Koordination. Gersick (1988) konnte zeigen, dass sich Gruppen nach der Hälfte der ihnen zur Verfügung stehenden Arbeitszeit neu orientieren, ihre bisherigen Leistungen evaluieren und gegebenenfalls ihre Arbeitsweise ändern. Eine derartige Krise könnte dazu führen, dass die bisherige implizite Koordination nun zeitweise explizit werden muss, um die weiteren Arbeitprozesse planen zu können (Wittenbaum et al., 1998).

\section{Umgebung}

Abschließend diskutieren Wittenbaum und Kollegen/-innen (1998) die Rolle von umgebenden Faktoren wie Feedback, Zielsetzung, Interventionen und Arbeitskontext.

Positives Feedback bezüglich der Gruppenleistung kann implizite Koordination aufrechterhalten; negatives Feedback hingegen kann Koordinationsroutinen unterbrechen und explizite Koordination erforderlich machen (Wittenbaum et al., 1998). Denkbar ist allerdings auch, dass Gruppen selbst bei negativem Feedback ihre Interaktionsmuster beibehalten und ihr Verhalten im Sinne eines eskalierenden Commitments nicht ändern (Wittenbaum et al., 1998).

Herausfordernde Gruppenziele führen zu vermehrtem In-Process-Planning. Dabei moderiert das von den Gruppenmitgliedern wahrgenommene Koordinationsvermögen der Gruppe den Einfluss der motivierenden Wirkung von Gruppenzielen auf die individuelle Arbeitsmotivation (Larson \& Schaumann, 1993). 
Auch die Art externer Interventionen in den Gruppenprozess, z.B. durch Training, können verschiedene Einflüsse auf den Koordinationsmodus haben. Beispielsweise kann ein Gruppentraining den Aufbau geteilter mentaler Modelle und dadurch die Entwicklung impliziter Koordinationsmechanismen fördern (Wittenbaum et al., 1998).

Schließlich diskutieren Wittenbaum und Kollegen/-innen, inwieweit der Kontext, in dem die Gruppe arbeitet, zu unterschiedlichem Einsatz der Koordinationsmodi führt. Sie beziehen sich dabei auf den Unterschied zwischen Laborgruppen und natürlichen Gruppen und postulieren, dass Laborgruppen vermutlich implizite Koordinationsmodi, natürliche Gruppen hingegen explizite Modi bevorzugen (Wittenbaum et al., 1998). Diese Annahme erscheint nicht plausibel, da die Gruppenmitglieder erst im Verlauf ihrer Zusammenarbeit gegenseitiges Vertrauen und geteilte mentale Modelle aufbauen, welche die Koordination mit impliziten Mechanismen fördern.

Bezüglich der geschilderten Wirkungsweise der moderierenden Faktoren auf den Einsatz und die Effektivität der Koordinationsmodi weisen Wittenbaum und Kollegen/-innen darauf hin, dass ein Großteil der postulierten Zusammenhänge bisher empirisch noch nicht untersucht ist. Forschungsbedarf besteht insbesondere bezüglich der Fragen, ob und inwieweit die Koordinationsmodi sequentiell oder parallel eingesetzt werden, mit welchen sozialen und leistungsbezogenen Konsequenzen die Koordinationsmodi verbunden sind und wie der Prozess der impliziten Koordination funktioniert (Wittenbaum et al., 1998).

Neben der fehlenden empirischen Überprüfung der postulierten Annahmen scheint die Bezeichnung des Ansatzes von Wittenbaum und Kollegen/-innen als Modell sehr weitgegriffen, da systematische Annahmen zur Voraussetzungen und Konsequenzen der Koordinationsmechanismen nicht spezifiziert werden. Die moderierenden Faktoren werden nicht in einen systematischen Zusammenhang mit den Koordinationsmechanismen gestellt. Vor dem Hintergrund der fehlenden empirischen Überprüfung erlaubt der Ansatz daher noch keine 
normativen Aussagen zur situativen Effektivität und sollte daher eher als Taxonomie von vier idealtypischen Koordinationsmechanismen betrachtet werden.

Welche Implikationen birgt der Ansatz, von Wittenbaum und Kollegen/-innen für die Koordination von Entscheidungsfindungsgruppen?

Gruppenentscheidungsfindung ist eine komplexe Aufgabe, bei der die Gruppenmitglieder interdependent arbeiten müssen. Sie erfordert die insbesondere Koordination des Austauschs individuellen Wissens und dessen Bedeutung. Sie ist eine unsichere Aufgabe, da sie keine einzig richtige Lösung impliziert und die Korrektheit der getroffenen Entscheidung nicht unmittelbar evaluiert werden kann. Daher wurde bereits konstatiert, dass Gruppenentscheidungsaufgaben hohe Koordinationsanforderungen an die Gruppe stellen (vgl. Kapitel 3.1). In Anlehnung an Wittenbaum et al. (1998) kann nun davon ausgegangen werden, dass Entscheidungsgruppen ihren Entscheidungsfindungsprozess explizit durch In-ProcessPlanning koordinieren sollen.

"However, when groups work on a highly uncertain or variable task, or experience member recomposition, the need for in-process planning may increase, making preplans less effective“ (Wittenbaum et al., 1998, S. 200)

Daher steht das In-Process-Planning in Entscheidungsfindungsgruppen im Vordergrund der vorliegenden Arbeit. Den Ausführungen von Wittenbaum et al. (1998) kann nicht entnommen werden, wie der Koordinationsmechanismus des In-Process-Planning bei Gruppenentscheidungen funktioniert. Aus Gründen der Einfachheit wird dieser Koordinationsmechanismus im Folgenden als explizite Prozesskoordination bezeichnet. In der vorliegenden Arbeit wird den Fragen nachgegangen, wie explizite Prozesskoordination in Gruppenentscheidungsprozessen abläuft und mit welchen sozialen und leistungsbezogenen Konsequenzen dieser Koordinationsmechanismus verbunden ist.

Auch wenn die explizite Prozesskoordination während der Entscheidungsfindung von Gruppen noch nicht systematisch untersucht wurde, so kann vor dem Hintergrund bisheriger 
Befunde davon ausgegangen werden, dass a) die Güte der Gruppenentscheidung wesentlich vom Interaktionsprozess der Gruppenmitglieder abhängt, da die Gruppenaufgabe sowohl strukturell komplex als auch mit hohen Informations- und Bewertungsanforderungen verbunden ist (Hirokawa, 1990), und dass b) Kommunikation ein wesentliches Mittel expliziter Prozesskoordination des Gruppenentscheidungsfindungsprozesses ist (Espinosa et al., 2004; Wittenbaum et al., 1998). Darauf wird im Folgenden näher eingegangen.

\subsection{Explizite Prozesskoordination durch Kommunikation}

Boos (1996b) betrachtet die Gruppe als ein funktionales Beziehungsgefüge, dass „durch Kommunikation zusammengehalten wird“ (S. 22). Kommunikation gilt als Hauptkomponente des Teamprozesses (Dickinson \& McIntyre, 1997) und dient als verbindender Mechanismus anderer Teamarbeitkomponenten. Dabei dient sie dem gegenseitigen Verstehen bzw. dem Erreichen eines gemeinsamen Verständnisses einer Situation oder eines Problems (Grounding, Clark, 2004). Kommunikation ermöglicht die Entstehung von konversationaler Kohärenz im Sinne gegenseitiger Bezugnahme und der Entwicklung gemeinsamer Themen.Sie beinhaltet sowohl den Informationsaustausch zwischen zwei oder mehreren Teammitgliedern, die angemessene Bereitstellung von Informationen durch ein einzelnes Teammitglied (Dickinson \& McIntyre, 1997) und die weitere Verarbeitung von Informationen in der Gruppe (Cranach et al., 1986). Im Hinblick auf die Entscheidungsfindung betonen auch Poole und Hirokawa die Instrumentalität der Kommunikation:

"As a primary tool of social action, communication mediates the effects of knowledge, preferences, task characteristics, and scores of other influences on decision-making" (Poole \& Hirokawa, 1996, S. 7).

Die explizite Koordination des Austauschs von Wissen und dessen Bedeutung erfolgt durch Kommunikation. Espinosa und Kollegen betrachten Kommunikation selbst gar als 
expliziten Koordinationsmechanismus. Auch Wittenbaum et al. betonen die Rolle der Kommunikation für explizite Koordination:

"If the coordination demands for a group are high (e.g. high task uncertainty or interdependence, difficult goal), then making in-process ore preplanning difficult, by narrowing or eliminating communication channels, will invariably hurt group productivity"(Wittenbaum et al., 1998, S. 201).

Kommunikation ist für die Koordination der Gruppe instrumentell. Dabei moderieren die Koordinationsanforderungen der Aufgabe den Einfluss der Kommunikation auf die Gruppenleistung: Je komplexer die Aufgabe ist, desto höher ist der Koordinationsbedarf und desto mehr beeinflusst eine gelungene Kommunikation die Gruppenleistung (Boos \& Sassenberg, 2001). Demzufolge ist der Koordinationsbedarf der Gruppe abhängig von der Aufgabe, der Gruppenzusammensetzung und dem Kommunikationsmedium (Boos \& Sassenberg, 2001). Die Gruppenentscheidungsfindung verlangt von den Gruppenmitgliedern, ihre individuellen Problemsichten und ihr Wissen koordiniert auszutauschen. Diese Koordination des Wissensaustauschs ist über Kommunikation möglich (Brauner, 2001). Je mehr die Kommunikation in der Gruppe den Aufgabenanforderungen entspricht, desto mehr wirkt sie sich positiv auf die Gruppenleistung aus (Tschan, 1995).

Empirisch ist der Zusammenhang zwischen Koordination, Kommunikation und Gruppenleistung insbesondere im Bereich der Luftfahrt untersucht worden. Es konnte gezeigt werden, dass sich gute von schlechten Crews in ihrer Kommunikation unterscheiden (Kanki \& Foushee, 1989). Auch in der Medizin wird angesichts der Vielzahl von Behandlungsfehlen die Rolle der Koordination und Kommunikation untersucht. Mittlerweile sind Kommunikationsdefizite als eine Ursache von Behandlungsfehlern empirisch bestätigt. Beispielsweise müssen sich müssen sich Chirurgen/-innen und Anästhesisten/-innen während einer Operation ständig über den Ablauf, Komplikationen und die Vitalparameter des/der Patienten/-in austauschen. Werden die relevanten Informationen nicht rechtzeitig und vollständig ausgetauscht, kann auf Änderungen nicht adäquat reagiert werden, und die Sicherheit 
des/der Patienten/-in wird gefährdet (Thiele \& Manser, 2001). Auch Fehler- oder Unverträglichkeitsmeldungen werden teilweise nicht weitergeleitet (Campion, 2003). Hyey und Wickens (1993) zeigten, dass neben einem ineffektiven Umgang mit Ablenkungen und Konflikten das fehlende Infragestellen von Entscheidungen zu den Hauptfehlerquellen in der Arbeit von Crews gehört

Sexton und Helmreich (1999) zeigten in Simulationsstudien, dass es nicht nur darauf ankommt, worüber sondern wie gesprochen wird. Beispielsweise war schon allein der häufige Gebrauch des Wortes „wir“ mit einer besseren Crewleistung verbunden (Sexton \& Helmreich, 1999), was als möglicher Ausdruck von Vertrautheit diskutiert wird. Der gezielte Einsatz von Kommunikation ist besonders in solchen Situationen wichtig, in denen plötzlich Stress und Unsicherheit etc. auftreten (Sexton, 2004): Tauchen Probleme auf, sollte viel darüber kommuniziert werden, das eigene Vorgehen in der Problemlösung sollte mit einfachen Sätzen verbal begleitet werden und eine Führungsrolle sollte übernommen werden (möglichst ausschließlich). In derartigen Situationen ist die Kommunikation von Handlungsabsichten und Erwartungen bezüglich möglicherweise eintreffender Ereignisse ein bestimmender Faktor für eine gute Gruppenleistung (Dietrich, 2004). Wesentlich ist dabei auch, dass Kommunikation in der Gruppe das Gefühl der Gruppenmitglieder, kognitiv überlastet zu sein, verhindern kann (Fussell et al., 1998).

Gruppenentscheidungsfindung findet nicht immer in risikoreichen Arbeitsumgebungen wie im Cockpit oder im OP statt. Dennoch sind Gruppenentscheidungsaufgaben unsichere und interdependente Aufgaben, weshalb sie der Koordination - vorzugsweise expliziter Prozesskoordination durch verbale Kommunikation (Wittenbaum et al., 1998)- bedürfen. Vor dem Hintergrund der gegenwärtigen Befundlage kann noch nicht gesagt werden, wie Gruppenmitglieder zur explizite Prozesskoordination des Informationsaustauschs und der Entscheidungsfindung kommunizieren müssen. Die explizite Prozesskoordination muss sicherstellen, dass die für die Entscheidungsfindung kritischen Funktionen (Gouran \& Hirokawa, 
1996; Hirokawa, 1985; Orlitzky \& Hirokawa, 2001) erfüllt werden, der Austausch ungeteilter Informationen (Mojzisch et al., 2004; Stasser \& Titus, 2006) erhöht wird, die Gruppenmitglieder dabei effizient vorgehen (Tschan, 2000; Wittenbaum et al., 1998) und anschließend mit der Entscheidungsfindung zufrieden sind (Poole \& Hirokawa, 1996). Inwieweit die explizite Prozesskoordination von Entscheidungsfindungsgruppen im Rahmen der vorliegenden Arbeit untersucht werden soll, wird im folgenden Abschnitt erläutert.

\subsection{Fragestellung der Arbeit}

"...facilitating the successful coordination of group members may be a key ingredient to improving group performance” (Wittenbaum et al., 1998, S. 199).

Im Fokus der vorliegenden Arbeit steht die explizite Prozesskoordination. Diese Form der Koordination ist aus Wittenbaums et al. (1998) Taxonomie der Koordinationsmodi abzuleiten und beinhaltet Koordinationsmechanismen, die explizit und während des Interaktionsprozesses eingesetzt werden und dazu dienen, Gruppen bei interdependenten und unsicheren Aufgaben zu koordinieren. Gruppenentscheidungsaufgaben sind sowohl interdependent als auch unsicher und bedürfen daher expliziter Prozesskoordination. In Anlehnung an die Handlungstheorie der Gruppe (McGrath \& Tschan, 2004; Tschan, 2000) soll explizite Prozesskoordination auf verschiedenen Ebenen des Entscheidungsfindungsprozesses untersucht werden:

Auf der bewusstseinspflichtigen Ziel-Ebene sollen subjektive Theorien koordinierender Personen eruiert werden. Der Gegenstand des Interesses sind die subjektiven Theorien und Heuristiken zur Effektivität ihres Koordinationsverhaltens: Wann verwenden sie insbesondere explizite Mechanismen zur Koordination und wie begründen sie die Verwendung? Welche Bedeutung hat explizite Koordination in ihrem Umgang mit Gruppen? Insofern Teilziele bewusstseinsfähig sind und berichtet werden können, so werden auch diese erfasst (Studie 1 und 2). 
Darüber hinaus wird auf der Handlungsebene untersucht, mit welchen Folgen der Einsatz expliziter Koordinationsmechanismen im Hinblick auf die Gruppenentscheidung verbunden ist. Dazu wird die Effektivität ausgewählter Koordinationsmechanismen experimentell überprüft (Studie 3).

Außerdem kann davon ausgegangen werden, dass die konkrete Ausführung expliziter Koordination nicht unbedingt immer bewusstseinsfähig sein muss, sondern dass das Koordinationsverhalten teilweise auch automatisiert ausgeführt wird. Daher mag sowohl die Erhebung subjektiver Koordinationsperspektiven als auch ihre experimentelle Überprüfung nicht ausreichen, um die situative Effizienz der Koordination zu untersuchen. Es erscheint sinnvoll, die konkrete Umsetzung von Koordinationsmechanismen im Verlauf von Gruppenentscheidungsprozessen $\mathrm{zu}$ untersuchen und erfolgreiche und nicht erfolgreiche Entscheidungsgruppen systematisch diesbezüglich zu untersuchen. Zu diesem Zweck wurden zwei Verhaltensbeobachtungsinstrumente entwickelt (Methodenentwicklung 1 \& 2). Der vorliegenden Arbeit liegt daher eine Kombination aus qualitativen und quantitativen Methoden zugrunde, auf die im Folgenden eingegangen wird.

\subsection{Methodischer Zugang der Arbeit}

Die vorliegende Arbeit soll einen Beitrag zur differenzierten Betrachtung und Präzisierung des Konzepts der expliziter Prozesskoordination leisten. Um dieses Ziel zu erreichen, ist einerseits die Verwendung qualitativer Methoden erforderlich (Interviews und Verhaltensbeobachtung) (Witt, 2001), um neben einer quantitativ-experimentellen Überprüfung Koordination aus verschiedenen Perspektiven betrachten zu können: Subjektive Sichten koordinierender Personen (Studie 1 und Studie 2), Effektivität von Koordinationsmechanismen (Studie 3) und Verbaltensbeobachtung durch Dritte (Methodeentwicklung 1 \& 2).

Die Diskussion um die vermeintliche Überlegenheit der qualitativen oder der quantitativen Methodik besteht unvermindert (z.B. Hackman, 2006), jedoch gibt es bereits etliche 
Bestrebungen, nicht nur eine Koexistenz beider Forschungstraditionen zu tolerieren, sondern sogar integrative Vorgehensweisen zu empfehlen (z.B. Fielding \& Schreier, 2001; Früh, 2004; Kelle, 2001; Kleining \& Witt, 2001).

Bisher ist es nicht gelungen, beide Analysestrategien definitorisch klar voneinander abzugrenzen (Mayring, 2001), dennoch gibt es deutliche Unterschiede. Qualitative und quantitative Analysen unterscheiden sich zunächst in ihrer Begrifflichkeit: Qualitativ legt Qualität und Bedeutung nahe (Witt, 2001), hingegen impliziert der Begriff quantitativ Zahlen und Metrik. Ein weiteres Unterscheidungskriterium betrifft die Datenanalyse (Witt, 2001) und das Skalenniveau der zugrunde liegenden Messung (Mayring, 2000): Qualitative Analysen basieren meist auf nominalskalierten Daten, quantitative Messungen erfordern mindestens ordinalskalierte Daten. Neben verschiedenen Methoden der Datenanalyse und Untersuchungsdurchführung besteht der wesentliche Unterschied im impliziten Wissenschaftsverständnis, das sich auch im Dualismus zwischen Natur- und Geisteswissenschaften widerspiegelt: Verstehen versus Erklären (vgl. Kleining \& Witt, 2001; Mayring, 2000). Die qualitative Forschung habe hauptsächlich das Verstehen von Phänomenen als Ziel, quantitative Forschung diene hingegen der Erklärung und Analyse von Ursache-Wirkungs-Beziehungen. Dieser Dualismus, der weitreichende methodische Konsequenzen hat (z.B. Einzelfallanalysen vs. repräsentative Stichproben bzw. theoretisches versus statistisches Sampling), ist mehrfach kritisiert wurden (Früh, 2004; Mruck, 2000).

Neben dem begrifflichen Dualismus ist der schulenartige und vorurteilsbehaftete Umgang mit dem qualitativen Paradigma problematisch, dem insbesondere in der Psychologie der Charakter des Unwissenschaftlichen und Anekdotischen anhaftet. Dem gegenüber wird das quantitative Paradigma als das einzig wissenschaftliche betrachtet, was sich auch in den Curricula der psychologischen Methodenausbildung widerspiegelt und durch selbige verstärkt wird. Es fällt jedoch auch auf, dass in vielen quantitativen Studien wichtige Konstruktionsprozesse, z.B. das Generieren von Fragebogen-Items oder Beobachtungsskalen, nicht dokumentiert werden. Der/die Leser/-in wird dadurch häufig im Unklaren gelassen, inwieweit diese Verfahren deduktiv 
oder induktiv entwickelt wurden. In allen Fällen bleiben diese Schritte teilweise interpretativ, so dass auch die quantitative Forschung nie gänzlich ohne eigenes Ermessen der Forscher/-innen auskommt. Mittlerweile gibt es Stimmen, die betonen, dass qualitative und quantitative Forschungstraditionen nicht als Gegensätze betrachtet werden sollten (vgl. Früh, 2004; Mayring, 2001). So beinhalten neuere, einschlägige Lehrbücher zur Forschungsmethodik Darstellungen zu qualitativen Methoden (z.B. Howitt \& Cramer, 2005; Langdridge, 2004; Reis \& Judd, 2000). Trotz des Paradigmenkrieges (Kelle, 2001) können qualitative und quantitative Verfahren auf unterschiedlichste Art und Weise kombiniert werden (Mayring, 2001): auf Datenebene durch induktive Kategorienbildung und deduktive Kategorienanwendung, auf Designebene beispielsweise durch ein Vorstudienmodell, auf der Ebene der Forschungslogik durch ein gemeinsames Ablaufmodell (Mayring, 2001). Zur Überwindung des (vermeintlichen) Gegensatzes zwischen qualitativer und quantitativer Forschung ist anzuraten, vor der Bestimmung von Quantitäten eines Forschungsgegenstandes zunächst seine Qualität zu beschreiben. Daher kann am Anfang eines Forschungsprozesses ein qualitativer Schritt stehen (Mayring, 2003), in dem das zu untersuchende Phänomen umfassend beschrieben wird, um die Validität weiterer quantitativer Analysen zu gewährleisten.

In der vorliegenden Arbeit sind die ersten beiden Studien (Studie 1 und 2) insofern qualitativ, als dass die Daten qualitativ erhoben, dann allerdings teilweise quantitativ weiterverarbeitet werden (z.B. Kategorienhäufigkeiten). Das qualitative Vorgehen orientiert sich an der Qualitativen Inhaltsanalyse (Mayring, 2000), die als systematisches und regelgeleitetes Vorgehen eine Art „Zwischenposition“ (Mayring, 2000, S. 7) zwischen quantitativer und qualitativer Forschung einnimmt. Anschließend wird in einem quantitativen Untersuchungsabschnitt die Wirkung von Koordinationsmechanismen experimentell untersucht. Abschließend werden zwei Beobachtungsmethoden vorgestellt, so dass der von Mayring (2003, S. 19) vorgeschlagene Forschungszyklus - „Von der Qualität zur Quantität und wieder zur Qualität" umgesetzt wird. 
Im folgenden Kapitel werden die qualitativen Studien (Studie 1 \& 2) zur Erfassung subjektiver Theorien der Koordination dargestellt. 


\section{Subjektive Theorien expliziter Koordination - Koordination aus Perspektive der Koordinierenden}

Ein Ziel der vorliegenden Arbeit besteht darin, zu untersuchen, inwieweit die explizite Prozesskoordination den Gruppenentscheidungsprozess effektiv unterstützt. Dabei gilt zu beachten, dass es sich bei expliziter Koordination um Verhalten handelt, das nicht geschieht, sondern aktiv von Personen mit der Intention zur Koordination eingesetzt wird. Diese persönlichen Koordinationsintentionen sollen im Folgenden genauer betrachtet werden. Im handlungstheoretischen Sinn handelt es sich dabei um bandlungsleitende Kognitionen, da sie individuelle Koordinationshandlungen beeinflussen. Diese handlungsleitenden Kognitionen werden auch als subjektive Theorien bezeichnet. Ihre Analyse soll im Sinn einer Rekonstruktion der individuellen kognitiven Prozesse der koordinierenden Personen der Untersuchung der beobachtbaren Aspekte der Koordinationshandlungen vorausgehen.

Zunächst wird das Konzept der subjektiven Theorien im allgemeinen erläutert. Anschließend werden subjektive Koordinationstheorien und ihre Untersuchung vorgestellt.

\subsection{Subjektive Theorien}

Es wird davon ausgegangen, dass Menschen sich in ihrem Alltagshandeln auf Wahrnehmungen und Hypothesen stützen, die in Analogie zu wissenschaftlichen Theorien beschrieben werden können (Rheinberg, Bromme, Minsel, Winteler \& Weidemann, 2001 zitiert nach Müller, 2003). Der Terminus Theorie betont die handlungsleitende Funktion dieser Kognitionen, die im Gegensatz zu wissenschaftlichen Theorien nicht aus sozial geteiltem, sondern aus subjektivem Alltagswissen bestehen - daher werden subjektive Theorien auch als Alltags-, Laien- oder implizite Theorien bezeichnet (Iwanowsky \& Beck, 2003). Dem Konstrukt der subjektiven Theorie liegt die Annahme zugrunde, dass menschliches Handeln zielgerichtet und rational ist. Es beschreibt - in Analogie zur wissenschaftlichen Theorie - ein „Aggregat von prinzipiell aktualisierbaren Kognitionen der Selbst -und Weltsicht des Individuums mit 
(zumindest impliziter) Argumentationsstruktur, die sich durch eine Strukturparallelität zu wissenschaftlichen Theorien und ihre Funktion in der Handlungsleitung auszeichnen“ (Müller, 2003, S. 22).

Im Hinblick auf die Unterscheidung subjektiver Theorien von dem in der Psychologie geläufigen Konzept der Kognition konstatiert Groeben (1988), dass unter Kognitionen einfache Phänomene zu verstehen sind, wie beispielsweise Begriffe oder Konzepte. Subjektive Theorien hingegen sind zeitlich stabiler und komplexer, denn sie beinhalten vielschichtige Aggregate von Konzepten, „deren Struktur und Funktion in Parallelität zu wissenschaftlichen Theorien konzipiert bzw. postuliert werden“" (Groeben, 1988, S. 18). Auch vielschichtige und vernetzte Kognitionen (z.B. hierarchisch aufgebaute Begriffsnetze) stellen noch keine subjektive Theorie dar, denn durch den Terminus Theorie wird impliziert, dass die Kognitionen systematisch organisiert bzw. zueinander relational sind, wodurch Schlussfolgern ermöglicht wird (Groeben, 1988; Iwanowsky \& Beck, 2003). Dann und Humpert (1987) sprechen von einer Wissensorganisation in Form von Herstellungswissen. Dabei handelt es sich um HandlungsErgebnis-Erwartungen, in denen individuelle Annahmen über die Beziehungen zwischen Handlungen und ihren Folgen getroffen werden, wie beispielsweise „befindet man sich in Situation A und will Ziel B erreichen, muss man C tun“ (Dann \& Humpert, 1987).

Insgesamt wird davon ausgegangen, dass subjektive Theorien für das menschliche Handeln eine ähnliche Funktion haben wie objektive Theorien für das wissenschaftliche Handeln (Groeben, 1988): Sie erleichtern das Verstehen, Erklären und Vorhersagen von Verhalten (Schmitt \& Hanke, 2003) und dienen darüber hinaus der Handlungssteuerung (Iwanowsky \& Beck, 2003). Auf den Zusammenhang zwischen subjektiven Theorien und individuellem Verhalten wird im folgenden Abschnitt näher eingegangen. 


\subsubsection{Subjektive Theorien und Verhalten}

Inwieweit besteht ein Zusammenhang zwischen den subjektiven Theorien eines Individuums und seinem tatsächlichen Verhalten? Es wird davon ausgegangen, dass subjektive Theorien eine wichtige Rolle in der Handlungsregulation einnehmen (Groeben, 1986; Iwanowsky \& Beck, 2003; Müller, 2003). Empirisch konnte die Handlungswirksamkeit subjektiver Theorien mehrfach aufgezeigt werden (vgl. Müller, 2003). Beispielsweise zeigten Dann und Humpert (1987), dass subjektive Theorien von Lehrern/-innen zu ihrem Umgang mit aggressionshaltigen Unterrichtssituationen sowohl mit selbstberichtetem als auch mit systematisch beobachtetem Verhalten in regelhaftem Zusammenhang stehen. Dieser Zusammenhang lässt sich handlungstheoretisch erklären, da subjektive Theorien handlungsleitende Kognitionen beinhalten:

Die Handlungstheorie geht davon aus, dass bewusste Kognitionen eine handlungssteuernde Funktion haben (Cranach, 1994; Cranach, Kalbermatten, Indermühle \& Gugler, 1980). Die Kernidee der Handlungstheorie besteht in der hierarchisch-sequentiellen Organisation einer Handlung. Diese Idee wurde in Kapitel 3.1.2 bereits auf Gruppenebene erläutert. Hierbei wird davon ausgegangen, dass Handlungen auf mehreren Ebenen hierarchisch organisiert werden (vgl. Abbildung 6). Auf der obersten Ebene, der Zielbestimmung, werden die Hierarchie sowie die Sequenz der Ziele bestimmt. Unter einem Ziel wird der vorgestellte und angestrebte Zustand am Ende einer Handlung verstanden (Cranach et al., 1980). Auf der strategischen Ebene wird der Handlungsverlauf kognitiv gesteuert, in dem er in Form von Plänen (Handlungsentwürfe) und Strategien (Vorzugsordnung von Plänen) nach Unterzielen strukturiert wird (Cranach et al., 1980). Diese ersten beiden Ebenen sind größtenteils bewusst. Auf der operationalen Ebene hingegen werden einzelne Handlungsschritte selbstregulierend und größtenteils unbewusst organisiert (Cranach et al., 1980). 


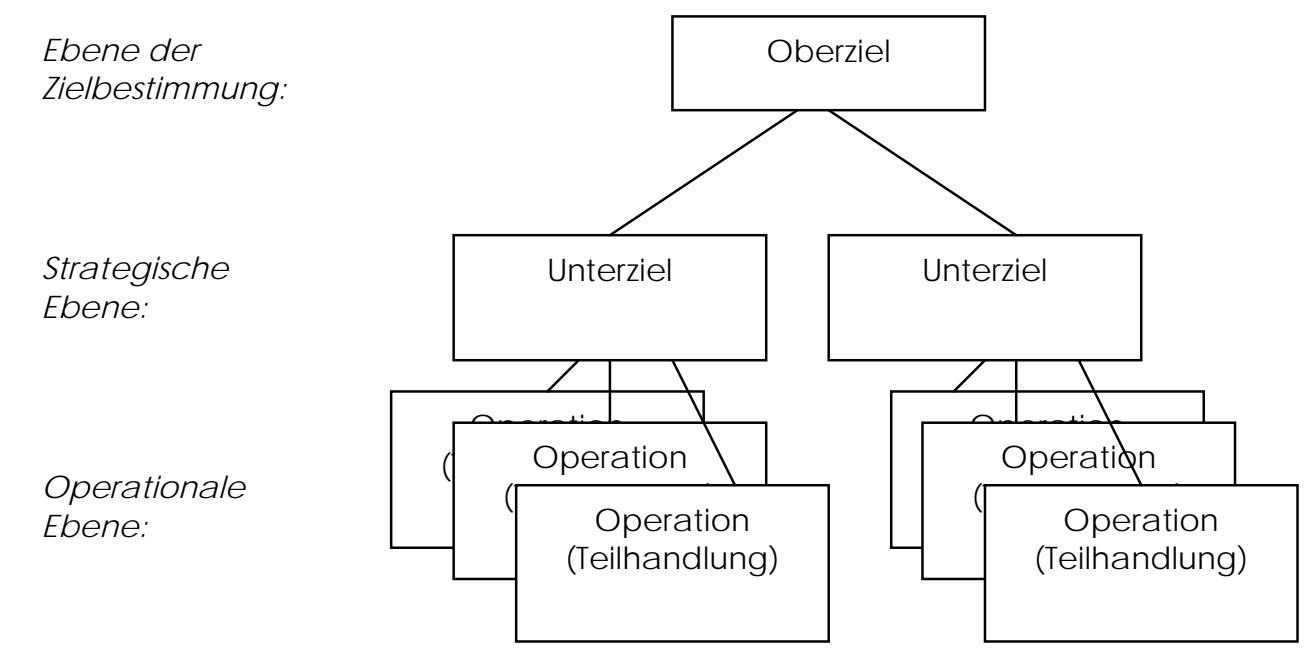

Abbildung 6: Veranschaulichung der hierarchisch-sequentiellen Handlungsorganisation im Rabmen der Handlungstheorie

Vallacher und Wegner (1987) sprechen in diesem Zusammenhang von der Identitätsstruktur einer Handlung, die in einer hierarchischen Anordnung unterschiedlicher Identitäten der Handlung besteht. Handlungsidentitäten stehen in systematischer Beziehung untereinander und bilden eine organisierte, kognitive Repräsentation einer Handlung (Vallacher \& Wegner, 1987). Die Beziehung zwischen einer Handlung und ihrer kognitiven Repräsentation ist nicht unidirektional, sondern zyklisch (Vallacher \& Wegner, 1987). Durch die kognitive Repräsentation wird die Handlung generiert (intent connection), die ausgeführte Handlung kann wiederum zu einer Veränderung der kognitiven Repräsentation führen (reflective connection) (Vallacher \& Wegner, 1987).

Durch die Handlungstheorie wird erklärt, dass die Ausführung einer Handlung eine kognitive Repräsentation der Handlung benötigt. Den bewussten, handlungsbezogenen Kognitionen kommt dabei eine entscheidende Rolle zu, da sie das Handeln steuern und regeln (Cranach et al., 1980). Subjektive Theorien beinhalten nicht nur individuelles Wissen, sondern ebenso bewusste handlungsleitende Kognitionen. Deshalb kann davon ausgegangen werden, dass sie das tatsächliche Verhalten beeinflussen. 


\subsubsection{Subjektive Theorien als Forschungsobjekt}

Aufgrund der Strukturparallelität wissenschaftlicher und subjektiver Theorien werden letztere am Beginn eines Forschungsprozesses häufig zur Ideengenerierung und Theorieentwicklung untersucht. Dabei wird davon ausgegangen, dass subjektive Theorien einen Beitrag zur Entwicklung objektiver Theorien leisten können, weil sie u.a. das Verständnis und die Prognose von Handlungen erleichtern (Schmitt \& Hanke, 2003). Insbesondere in der Lehr- und Lernforschung erhofft man sich von der Untersuchung subjektiver Theorien Ergebnisse, die das Alltagshandeln von Lehrern /-innen beschreiben und erklären sowie seine Veränderung ermöglichen sollen. Beispielsweise werden subjektive Theorien von Mathematikaufgaben im Rahmen der diagnostischen Kompetenz von Lehrern/-innen erfasst (Bruder, Lengnink \& Prediger, 2003). Darüber hinaus sollen entsprechende Unterschiede zwischen erfolgreichen und weniger erfolgreichen Lehrern/-innen sichtbar gemacht werden (Müller, 2003). In der Sportwissenschaft werden subjektive Feedbacktheorien von erfahrenen Trainern/-innen im Hinblick auf ihre Übereinstimmung mit denen ihrer Athleten/-innen untersucht (Schmitt \& Hanke, 2003). In der Organisationswissenschaft werden subjektive Organisationstheorien von Entscheidungsträgern/-innen näher untersucht (Iwanowsky \& Beck, 2003).

Entsprechend des breiten Interesses und Anwendungsbereiches subjektiver Theorien, weisen die Methoden ihrer Erfassung eine große Vielfalt auf. Es existiert keine einheitliche methodische Herangehensweise. Wesentliche Bestimmungsfaktoren des Methodeneinsatzes sind, neben dem Inhalt der Theorie, die zu befragende Zielgruppe und der Rahmen, in dem die Theorie für Untersuchungszwecke verfügbar ist. Neben Interviews oder strukturierten Dialogen (Schmitt \& Hanke, 2003) werden Selbstberichte, Beobachtungen (Dann \& Humpert, 1987), Repertory Grids (Bruder et al., 2003), Stimulated Recalls (Cranach et al., 1980), Strukturlegetechniken etc. (Iwanowsky \& Beck, 2003) verwendet. 


\subsubsection{Subjektive Theorien expliziter Koordination}

Im Fokus der vorliegenden Arbeit steht das Konstrukt der expliziten Prozesskoordination. In dieser Arbeit soll, neben der Überprüfung der Effektivität expliziter Prozesskoordination, die vorhandene Beschreibung dieses Konstruktes im Rahmen von Gruppenentscheidungen erweitert werden. Dazu interessieren auf der Bedeutungsebene zunächst die subjektiven Koordinationstheorien der Personen, die explizite Prozesskoordination zur Koordination von Entscheidungsfindungsgruppen einsetzen. Ihre subjektiven Koordinationstheorien geben Auskunft über die Intentionalität und die Reflexivität ihrer Koordinationshandlungen. Es soll untersucht werden, welche Koordinationshandlungen koordinierenden Personen bekannt sind, mit welchem Ziel diese Handlungen eingesetzt werden und welcher Effekt als daraus resultierend wahrgenommen wird. Durch die Beantwortung dieser Fragen soll zunächst das Konstrukt expliziter Prozesskoordination differenziert werden. Dadurch soll es möglich werden, sowohl seine Wirksamkeit zur Koordination von Gruppenentscheidungsfindungen zu überprüfen (vgl. Studie 3), als auch entsprechende Beobachtungsverfahren zu entwickeln (vgl. Methodenentwicklungen 1 \& 2).

Um der Forderung nach maximaler struktureller Variation der Perspektiven auf den Forschungsgegenstand nachzukommen und die subjektiven Koordinationstheorien möglichst facettenreich beschreiben zu können (vgl. Kleining \& Witt, 2001), sollen sie von möglichst unterschiedlichen Personen erfasst werden. Dafür eignet sich insbesondere die Untersuchung von Experten/-innen und Nicht-Experten/-innen (vgl. Zempel, 2002). Die Kontrastierung der subjektiven Koordinationstheorien beider Gruppen ermöglicht sowohl Aussagen über allgemeines handlungssteuerndes Wissen über Gruppenkoordination als auch über sperielle, erfahrungsbedingte handlungssteuernde Kognitionen. 
Es wurden in Studie 1 zunächst subjektive Koordinationstheorien von Nicht-Experten/innen der Gruppenkoordination erhoben, um das allgemeine, nicht-erfahrungsgebundene, individuelle Wissen über Koordination sowie eigene Koordinationshandlungen zu erfassen.

Müller (2003) geht in Anlehnung an Dann (1994, zitiert nach Müller, 2003) davon aus, dass subjektive Theorien - im Gegensatz zu bewussten momentanen Kognitionen - als relativ stabile kognitive Strukturen gelten, die allerdings durch Erfahrung veränderbar sind. Daher werden in Studie 2 subjektive Koordinationstheorien von in der Koordination von Gruppenentscheidungsprozessen erfahrenen Personen wie Führungskräften und Moderatoren/innen erhoben, um die erfahrungsbedingten handlungsleitenden Vorstellungen zu erfassen. Hierbei interessiert, inwieweit sich die erfahrene Perspektive der Koordination von der naiven unterscheidet und ob sie einen erfahrungsbedingt anderen Fokus der Koordination oder konkreteres Herstellungswissen im Sinne spezifischer Wenn-Dann-Beziehungen beinhaltet.

Die beiden Studien werden im Folgenden dargestellt. 


\subsection{Studie 1: Subjektive Theorien der Koordination: Die naive Perspektive der Koordinierenden}

Das Ziel der ersten Studie besteht in der Erfassung individueller subjektiver Koordinationstheorien von Personen, die in der Gruppenkoordination bisher wenig persönliche Erfahrung gesammelt haben. Es wird davon ausgegangen, dass diese Personen über naive subjektive Koordinationstheorien verfügen. Durch die Erfassung dieser naiven subjektiven Koordinationstheorien soll ein Beitrag zur detaillierten Beschreibung des Konstrukts der expliziten Prozesskoordination geleistet werden. Zur Erhebung der subjektiven Koordinationstheorien wurde eine Szenariostudie durchgeführt, die im Folgenden näher beschrieben wird.

\subsubsection{Methode}

Es wurde bereits darauf hingewiesen, dass die Methoden zur Erfassung subjektiver Theorien vielfältig sind. Im Hinblick auf die Verfügbarkeit potentieller Versuchsteilnehmer/innen konnten in der ersten Studie keine aufwändigen Verfahren wie Interviews oder Strukturlegetechniken verwendet werden. Statt dessen wurde ein Szenarium entwickelt, auf dessen Grundlage die Probanden/-innen zur ihren subjektiven Theorien mittels Fragebogen befragt werden konnten.

Bevor das Szenarium dargestellt wird, werden zunächst die Stichprobe und der Untersuchungsrahmen beschrieben.

\section{Stichprobe}

An der Untersuchung nahmen 56 (81,2\%) Schülerinnen und $13(18,8 \%)$ Schüler $(N=69)$ teil. Ihr Alter reichte von 16 bis 23 Jahren, das durchschnittliche Alter lag bei 18,4 Jahren. 


\section{Untersuchungsdurchführung und Erhebungsinstrument}

Die Studie wurde während der Hochschulinformationstage der Universität Göttingen vom 14. bis 15. März 2005 durchgeführt. Während dieser zwei Tage konnten sich Abiturienten/innen über verschiedene Studienmöglichkeiten an der Universität Göttingen informieren. Die an der Studie teilnehmenden Abiturient/-innen informierten sich über das Studienfach Psychologie.

Als Erhebungsinstrument wurde ein Fragebogen entwickelt, der ein Szenarium mit zugehörigen Fragen enthält. Durch das beschriebene Szenarium soll gewährleistet werden, dass sich die Schüler/-innen bei ihren Überlegungen zu dem interessierenden expliziten Koordinationsverhalten eine konkrete Situation vorstellen können, die darüber hinaus für alle Probanden/-innen gleich ist und tatsächlich expliziter Koordination bedarf. Der gesamte Fragebogen befindet sich im Anhang A.

Das verwendete Szenarium besteht in einer komplexen Problemlöse- und Entscheidungssituation aus dem Schulkontext: Der Fortbestand einer bisher erfolgreichen Schüler/-innen-Arbeitsgemeinschaft (AG) ist ungesichert, wobei sowohl Befürworter/-innen als auch Gegner/-innen der AG davon ausgehen, triftige Gründe für ihre jeweilige Sicht vorzuweisen. In einer gemeinsamen Sitzung soll der Schülersprecher Stefan zwischen Vertretern/-innen gegensätzlicher Interessen vermitteln. Obwohl ihm persönlich auch am Fortbestand der AG liegt, soll der Schülersprecher die Diskussion ausschließlich moderieren und nicht inhaltlich Position beziehen. Er hat in der Vergangenheit mehrfach in ähnlichen Diskussionen zwischen Parteien vermitteln können und durch seine Art zu moderieren meist für einen Konsens gesorgt. Aus diesem Grund wurde er als Diskussionsleiter für die Sitzung berufen.

Den Schülern/-innen wurde erläutert, dass mittels des vorliegenden Fragebogens etwas über ihr allgemeines Wissen zur Gestaltung von Gruppendiskussionen in Erfahrung gebracht werden soll. Sie wurden gebeten, sich das Szenarium durchzulesen und die nachfolgenden Fragen 
zu beantworten. Dabei gäbe es weder richtige noch falsche Antworten, es interessierten ausschließlich ihre persönlichen Erfahrungen und Meinungen. Da im Anschluss an eine erste offene Frage explizite Koordinationshandlungen vorgegeben wurden, wurden die Teilnehmer/innen gebeten, im Vorfeld noch nicht im Fragebogen umher zu blättern. Für den Zweck der Untersuchung sei es wichtig, dass sie die einzelnen Fragen in der Reihenfolge beantworteten, in der sie im Fragebogen aufgelistet sind.

Die Schüler/-innen wurden gebeten, sich die im Szenario dargestellte Situation vorzustellen. Um sicher zu gehen, dass die Teilnehmer/-innen das beschriebene Problem hinreichend verstanden hatten, wurden sie zunächst gefragt, vor welchen Schwierigkeiten der Schülersprecher Stefan während der Diskussion stehe. Anschließend wurde die eigentliche Untersuchungsfrage danach gestellt, was Stefan während der Diskussion machen könne, damit alle Interessen berücksichtigt würden, alle Vertreter/-innen zu Wort kämen und kein Streit, sondern ein Ergebnis entstünde, das von beiden Parteien getragen werden könne. Die Teilnehmer/-innen wurden zusätzlich darauf hingewiesen, dass sie nicht Stefans persönliche Charaktereigenschaften berichten sollten (z. B. „muss freundlich, aufgeschlossen und unvoreingenommen sein"), sondern dass nach seinem konkreten Verhalten in dieser Diskussion gefragt wird (z. B. „er äußert seine Meinung“; ,,weist bei Bedarf wiederholt darauf hin, dass die Gesprächspartner/-innen sich gegenseitig ausreden lassen“).

Im Anschluss an die explorative Frage wurden den Teilnehmer/-innen Verhaltensweisen expliziter Prozesskoordination von Diskussionen vorgegeben, deren Nützlichkeit für die Leitung der vorliegenden Diskussion beurteilt werden sollte. Die Verhaltensweisen (siehe Tabelle 3) $\begin{array}{llll}\text { repräsentieren } & \text { Möglichkeiten } & \text { expliziter } & \text { Prozesskoordination }\end{array}$ Entscheidungsfindungssitzungen durch Kommunikation (vgl. Kapitel 3.4). Sie wurden unter Berücksichtigung praktischer Literatur zu Gesprächsleitung und Moderation (Edmüller \& Wilhelm, 2005; Hartmann, Funk \& Wittkuhn, 2000; Kanitz, 2004) sowie Gruppenentscheidungsfindung (Hirokawa, 1982) konzipiert. Die Probanden/-innen sollten auf 
einer fünfstufigen Skala (1... überhaupt nicht sinnvoll; 5 ... sehr sinnvoll) einschätzen, für wie sinnvoll sie es halten, wenn der Schülersprecher Stefan in der Diskussion diese Verhaltensweisen zeigen würde. Da nicht ausgeschlossen werden kann, dass den Schülern/-innen vereinzelte Verhaltensweisen unbekannt sind, wurde die Skala um die Kategorie „weiß nicht“ erweitert. Somit wird einer möglichen Verzerrung des Antwortverhaltens entgegengewirkt, wonach die Schüler/-innen ihnen unbekannte Verhaltensweisen möglicherweise allein aufgrund ihre Unkenntnis als nicht nützlich beurteilen könnten.

Tabelle 3: ein₹uschätzende Verhaltensweisen und ibre inhaltliche Zuordnung ₹u Oberkategorien

\begin{tabular}{|c|c|c|}
\hline Oberkategorie & Nr. & Verhaltensweisen \\
\hline \multirow[t]{5}{*}{ Fragen } & 1 & Nach einer Information fragen \\
\hline & 2 & Eine Person nach einer Information fragen \\
\hline & 3 & Nach einer Meinung fragen \\
\hline & 4 & Eine Person nach Ihrer Meinung fragen \\
\hline & 5 & Nach Konsens fragen \\
\hline Vorschläge machen & 6 & Ein weiteres Vorgehen vorschlagen \\
\hline \multirow{2}{*}{$\begin{array}{l}\text { Zusammenfassungen } \\
\text { und } \\
\text { Zielformulierungen }\end{array}$} & 7 & Bisher Gesagtes zusammenfassen \\
\hline & 8 & Ein Ziel formulieren \\
\hline \multirow[t]{3}{*}{ Meinungsäußerungen } & 9 & Seine eigene Meinung sagen \\
\hline & 10 & Einem unterbreiteten Vorschlag zustimmen oder diesen ablehnen \\
\hline & 11 & Etwas Gesagtes bewerten \\
\hline \multirow{2}{*}{$\begin{array}{l}\text { Nonverbale } \\
\text { Verhaltensweisen }\end{array}$} & 12 & Nicken \\
\hline & 13 & Den Kopf schütteln \\
\hline Aufforderungen & 14 & Eine Person zum Sprechen auffordern \\
\hline \multirow[t]{2}{*}{ Erklärungen } & 15 & Etwas Gesagtes begründen oder erklären \\
\hline & 16 & Interpretation des Gesagten \\
\hline \multirow{3}{*}{$\begin{array}{l}\text { Andere } \\
\text { Verhaltensweisen }\end{array}$} & 17 & „Metakommunikation“ (über das Gespräch sprechen) \\
\hline & 18 & „Aktives“ Zuhören (z. B. durch wiederholtes „hm“) \\
\hline & 19 & Das Gespräch kurz unterbrechen \\
\hline
\end{tabular}

Anmerkung. Die Oberkategorien waren den Probanden/-innen nicht bekannt. Ihre Bildung wird in Kapitel 4.2.2 dargestellt.

Abschließend wurden die Teilnehmer/-innen gebeten, einige persönliche Angaben zu Alter, Geschlecht, Klassenstufe, Studienwunsch, Leistungsfächer bzw.- kurse und Nationalität zu 
machen. Sie wurden außerdem gefragt, ob sie etwas an dem Fragebogen gestört habe und wenn ja, dies kurz zu erläutern.

Im Folgenden werden die Ergebnisse der Studie dargestellt.

\subsubsection{Ergebnisse}

Zunächst werden die Ergebnisse des Manipulation Checks berichtet. Anschließend werden die Befunde des explorativen Studienteils und der standardisierten Befragung dargestellt.

\section{Manipulation Check - Überprüfung des Verständnisses der Aufgabe}

Bevor die eigentlichen Ergebnisse der Studie betrachtet werden konnten, wurde untersucht, ob das Szenarium die beabsichtigte Situation induziert hat bzw. inwieweit die Teilnehmer/-innen das Szenarium sowie das darin beschriebene Problem verstanden hatten. Dazu wurden die Antworten auf die erste offene Frage „Vor welchen Schwierigkeiten steht Stefan?" analysiert.

\section{Wie sollte die Situation verstanden werden?}

Die Situation sollte als ein komplexes Problemlöse- und Entscheidungsfindungsproblem verstanden werden. Die moderierende Person des Schülersprechers soll sich inhaltlich nicht beteiligen, sondern ausschließlich als Vermittlerin zwischen Repräsentanten/-innen gegnerischer Interessen handeln. Sie soll sich neutral verhalten, ihre eigene Meinung zurückhalten und den Diskussionsprozess durch sachliche, rein vorgehensbezogene Äußerungen strukturieren. Als Schülersprecher hat Stefan keine formale Autorität, er kann deshalb das Gespräch nur durch gelungene Moderationsbeiträge leiten. 


\section{Wie wurde die Situation verstanden?}

Die von den Schülern/-innen genannten Schwierigkeiten konnten von der Autorin in acht Kategorien systematisiert werden. Diese sind der Abbildung 7 zu entnehmen sind. Nahezu zwei Drittel $(62,9 \%)$ benannten die Neutralität des Schülersprechers als wichtig. Ein Viertel $(25,7 \%)$ der Schüler/-innen bezeichneten weiterhin die Zurückhaltung der eigenen Meinung als wichtige Herausforderung. Als weitere Schwierigkeiten wurden Fairness, für Rube und Sachlichkeit sorgen, Diskussion könnte unangenehm und emotional werden und sich nicht beeinflussen lassen genannt. Diese genannten Aspekte entsprechen dem oben geschilderten Soll-Verständnis der Situation.

Nur ein/e Teilnehmer/-in benannte die Vertretung der Meinung der Scbüler/-innen als Probleminhalt. Dies ist eine falsche Interpretation der Situation, da der Schülersprecher die Aufgabe hat, sich eigentlich unparteiisch zu verhalten und die Interessen beider Parteien gleichermaßen zu berücksichtigen. Da jedoch nur eine Person diesen Aspekt erwähnt hat und keine weiteren falschen Interpretationen berichtet wurden, kann davon ausgegangen werden, dass das im Szenario dargestellte Problem angemessen verstanden wurde.

Wie aus Abbildung 7 ersichtlich ist, wurde von über einem Drittel (40\%) der Teilnehmer/-innen auch der innere Konflikt des Schülersprechers erwähnt, der einerseits als Schüler ein klares eigenes Interesse hat (Fortbestand der Arbeitsgemeinschaft), anderseits er sein Interesse nicht zeigen und sich als Moderator nur (neutral) verhalten darf. Diese Interpretation geht ebenfalls aus dem Szenario hervor, die Offensichtlichkeit dieses Problems wurde allerdings nicht in diesem Ausmaß erwartet. Es ist daher zu erwarten, dass sich diese Problemsicht auch in den Antworten auf die Frage nach den konkreten Verhaltensweisen des Schülersprechers niederschlagen wird. 


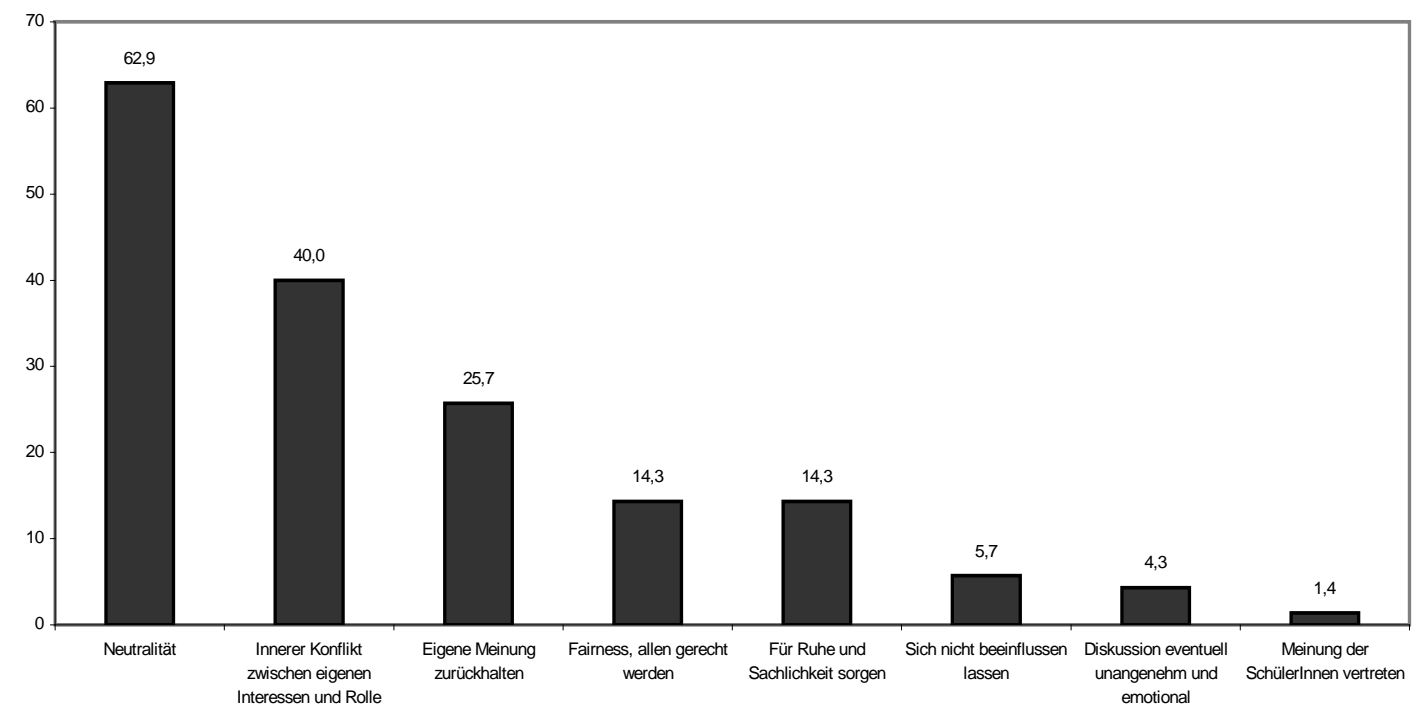

Abbildung 7: Relative Häufigkeiten der kategorisierten Antworten auf die Frage: Vor welchen Schwierigkeiten steht Stefan? Mehrfachnennungen möglich.

Insgesamt haben die Schüler/-innen das im Szenario beschriebene Problem in hohem Maße verstanden. Sie haben erkannt, dass sich der Schülersprecher neutral und unparteiisch verhalten muss und seine eigene Meinung zurückstellen sollte. Außerdem haben die Teilnehmer/-innen zusätzlich Stefans inneren Konflikt erkannt. Die fehlende Autorität und die damit verbundene einzige Möglichkeit des Schülersprechers, die Diskussion durch gelungene vorgehensbezogene Beiträge zu leiten, wurde größtenteils nicht erkannt.

Im folgenden Abschnitt werden die Ergebnisse der explorativen Analyse der berichteten Verhaltensweisen beschrieben.

\section{Ergebnisse des explorativen Untersuchungsteils}

Nachdem die Schüler/-innen sich das Szenario durchgelesen und die Manipulation Check-Frage beantwortet hatten, sollten sie in der Beantwortung der eigentlichen Untersuchungsfrage beschreiben, welche Verhaltensweisen der Schülersprecher während der Diskussion zeigen solle, damit alle Interessen der beteiligten Personen berücksichtigt würden, alle Vertreter/-innen zu Wort kämen und kein Streit, sondern ein Ergebnis entstünde, welches von 
beiden Parteien getragen werden könne. Sie sollten dabei nicht Stefans persönliche Charaktereigenschaften, sondern sein konkretes Verhalten während der Diskussion berichten.

Die Antworten der Probanden/-innen wurden von einer in inhaltsanalytischen Auswertungsmethoden erfahrenen Mitarbeiterin kategorisiert. Eine studentische Hilfskraft, die blind gegenüber dem Ziel der Untersuchung war, überprüfte die entstandenen Kategorien auf Plausibilität und unternahm einen ersten Kodierdurchgang. Anschließend wurden die Kategorien von der Untersuchungsleiterin überarbeitet. Alle Antworten wurden von ihr und einer weiteren Kodiererin danach erneut kodiert (Kappa von .71 bis .99). Insgesamt wurden 201 kodierbare Verhaltensweisen berichtet.

Die berichteten Verhaltensweisen ließen sich drei Hauptkategorien zuordnen: Handlungsanleitungen, Strukturierung des Problemlöseprozesses und Konfliktvermeidung. Diese Hauptkategorien werden im Folgenden näher beschrieben.

\section{$\underline{\text { Handlungsanleitungen }}$}

Die Handlungsanleitungen beinhalten Verhaltensweisen, mit denen die Diskussionsteilnehmer/-innen zu konkreten Handlungen aufgefordert werden. Dazu zählen: Argumente zu Beginn oder im Wechsel vortragen lassen, Rednerliste erstellen bəw. Rederechte vergeben, ausreden lassen bzw. nicht unterbrechen, Regeln festlegen sowie Zeitlimit setzen. Abbildung 8 zeigt die Handlungsanleitungen und ihre relativen Nennungshäufigkeiten. 


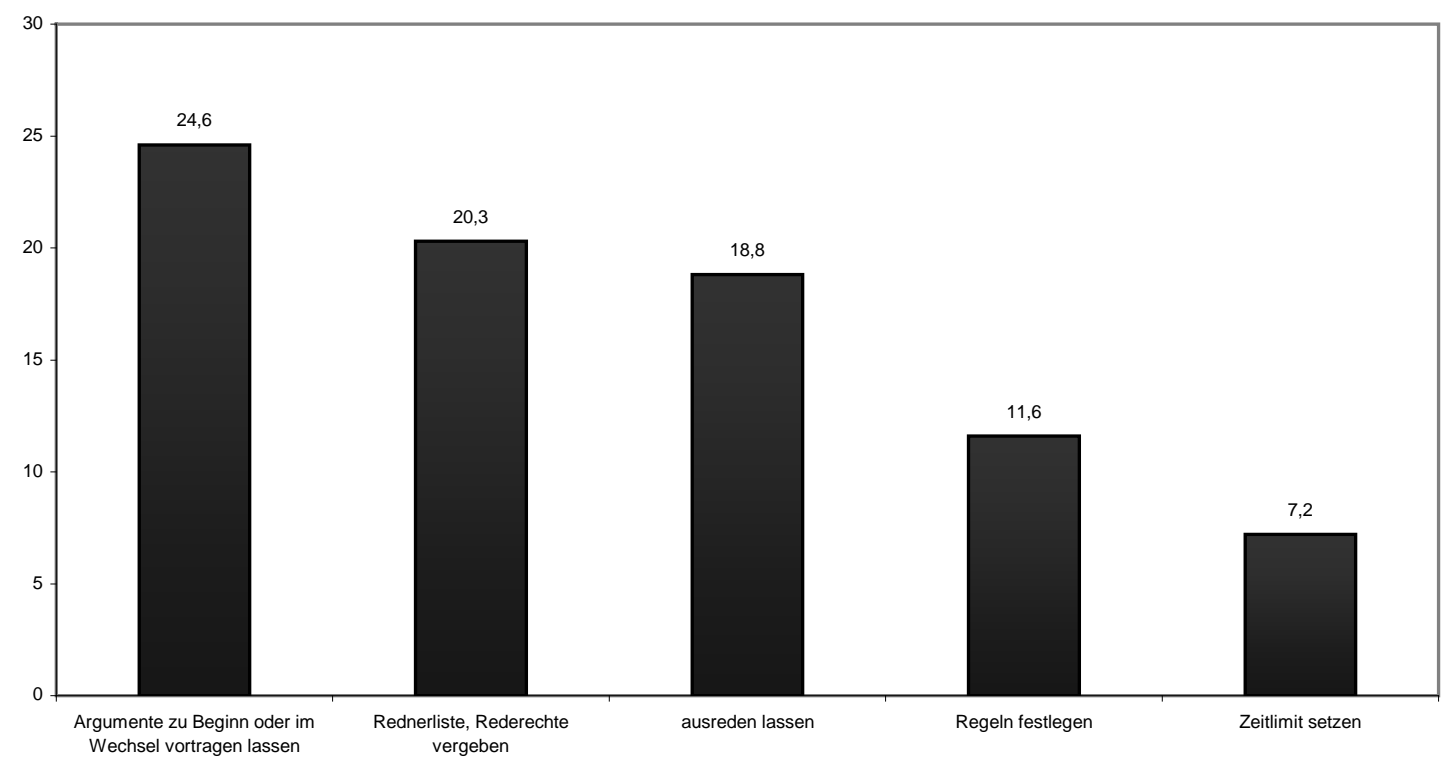

Abbildung 8: Häufigkeiten der Handlungsanleitungen. Mehrfachnennungen möglich.

\section{Strukturierung des Problemlöseprozesses}

Zur Strukturierung des Problemlöseprozesses gehören Verhaltensweisen, mit denen der Informationsaustausch strukturiert wird. Darunter fallen Handlungen wie Zusammenfassen und Wiederholen, Fragen stellen, thematisch strukturieren, Punkte sammeln b₹w. Pro- und Kontra-Liste erstellen und Lösungen sammeln. Abbildung 9 zeigt die genauen Häufigkeiten dieser Kategorien.

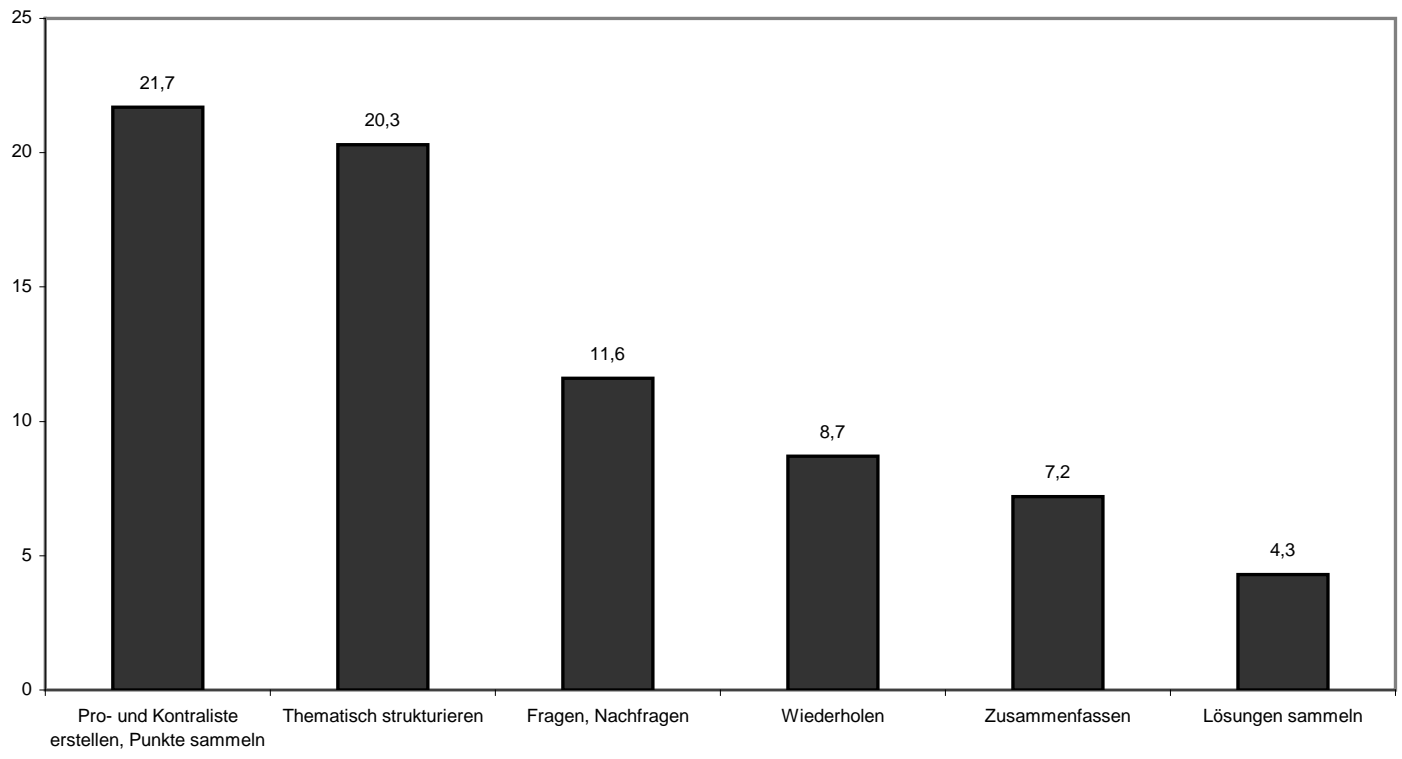




\section{$\underline{\text { Konfliktvermeidung }}$}

Die Oberkategorie Konfliktvermeidung beinhaltet solche Handlungen, durch die Konflikte während der Diskussionen vermieden werden sollen. Wie aus

Abbildung $10 \mathrm{zu}$ entnehmen ist, gehören dazu die Kategorien Gleichberechtigung bzw. jeden $₹ u$ Wort kommen lassen, für Rube und Ordnung sorgen, Einbinden rubiger Diskussionsteilnehmer/-innen, Streit vermeiden sowie auf Konsens bəw. Kompromiss hinarbeiten.

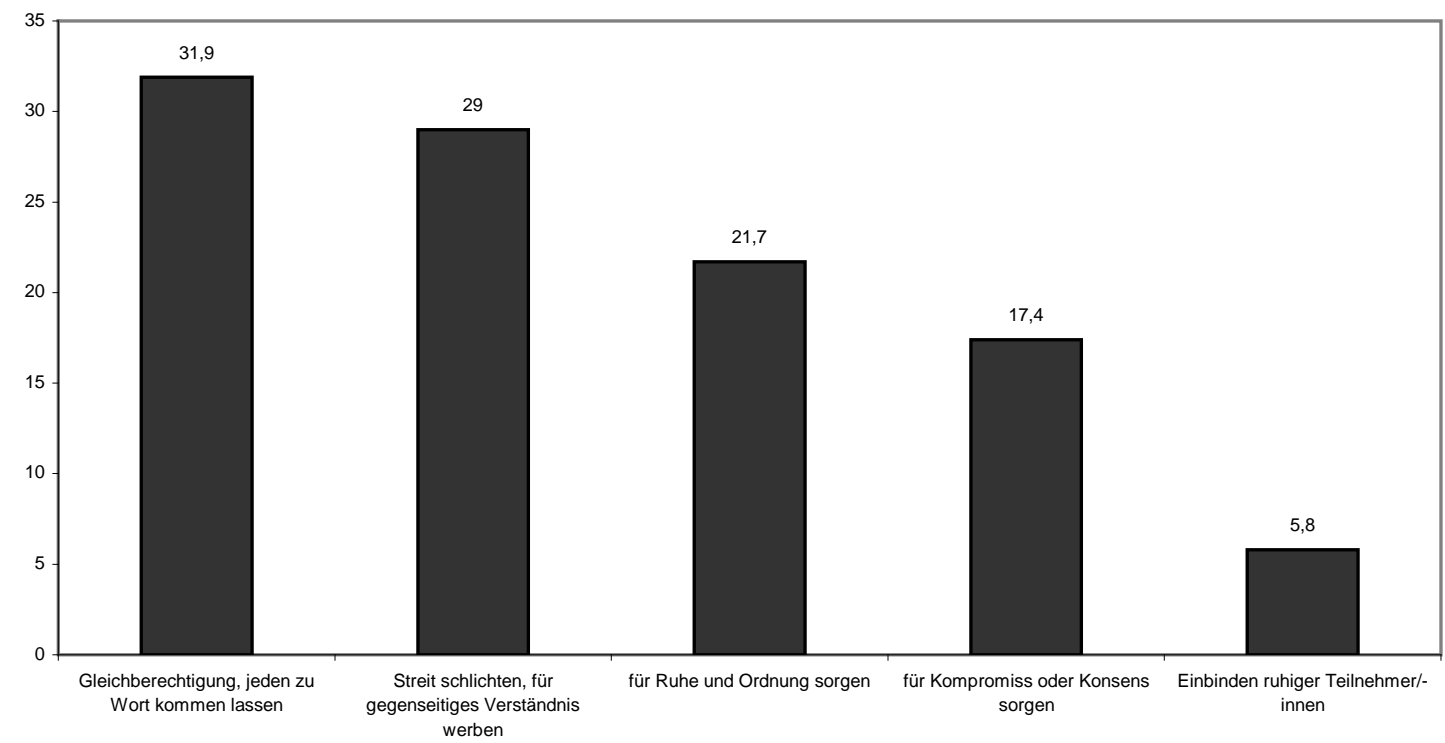

Abbildung 10:Häufigkeiten der Kategorien zur Konfliktvermeidung. Mehrfachnennungen möglich.

Darüber hinaus schlugen wenige Probanden/-innen vor, Vorschläge zur Einigung zu machen (4,3\%) oder die eigene Meinung zu vertreten (1,4\%). 21,7\% der Schüler/-innen berichteten zudem Verhaltensweisen, die der Schülersprecher nicht zeigen sollte, z B. nicht die eigene Meinung sagen, nicht selber Stellung beziehen, eigene Kommentare - außer zum organisatorischen Ablauf vermeiden.

Nachfolgend werden die Ergebnisse zur Einschätzung der Nützlichkeit vorgegebener Verhaltensweisen berichtet. 


\section{Ergebnisse der standardisierten Befragung}

Im Anschluss an den explorativen Part der Studie wurden den Teilnehmer/-innen konkrete Verhaltensweisen expliziter Prozesskoordination (vgl. Tabelle 3) vorgegeben. Sie sollten auf einer fünfstufigen Skala (1 ... überhaupt nicht sinnvoll, 5 ... sehr sinnvoll) einschätzen, für wie sinnvoll sie es halten, wenn der Schülersprecher diese Handlungen während der Diskussion zeigen würde.

\section{Zusammenfassung der Items}

Mit Blick auf eine übersichtliche Auswertung wurden die Items: nach einer Information fragen, eine Person nach einer Information fragen, nach einer Meinung fragen, eine Person nach Ihrer Meinung fragen und nach Konsens fragen entsprechend der Oberkategorie (vgl. Tabelle 3) zusammengefasst zu Fragen stellen.

Bisher Gesagtes zusammenfassen und ein Ziel formulieren wurde zusammengefasst zu Zusammenfassung und Zielformulierung.

Die eigene Meinung sagen, einem unterbreiteten Vorschlag zustimmen oder diesen ablebnen und etwas Gesagtes bewerten wurde zusammengefasst zu eigene Meinung sagen.

Nicken und den Kopf schütteln wurden zu Nonverbalen Verbaltensweisen und etwas Gesagtes begründen oder erklären und Interpretation des Gesagten zu Erklärungen zusammengefasst.

Schließlich wurden Metakommunikation, Aktives Zubören und das Gespräch kurz unterbrechen $\mathrm{zu}$ andere Verbaltensweisen zusammengefasst.

\section{Ergebnisse}

Abbildung 11 zeigt die Nützlichkeitseinschätzungen dieser Verhaltenskategorien. Als sinnvollste Verhaltensweisen werden Zusammenfassen und Ziele formulieren $(M=4,45, S D=0,61)$ eingeschätzt, gefolgt von Vorgehensvorschlägen $(M=4,17, S D=0,96), t(65)=2,55, p<0.05$, 
zweiseitig), Fragen stellen ( $M=3,88, S D=0,61), t(51)=2,72, p<0,01$, zweiseitig), sowie Aufforderungen ( $M=3,56, S D=1,04), t(52)=1,9, p=, 06$, zweiseitig). Als am wenigsten sinnvoll werden nonverbale Äußerungen wie Kopfschütteln und Nicken $(M=2,09, S D=0,77)$ und die eigene Meinung äußern $(M=1,86, S D=0,86)$ angesehen.

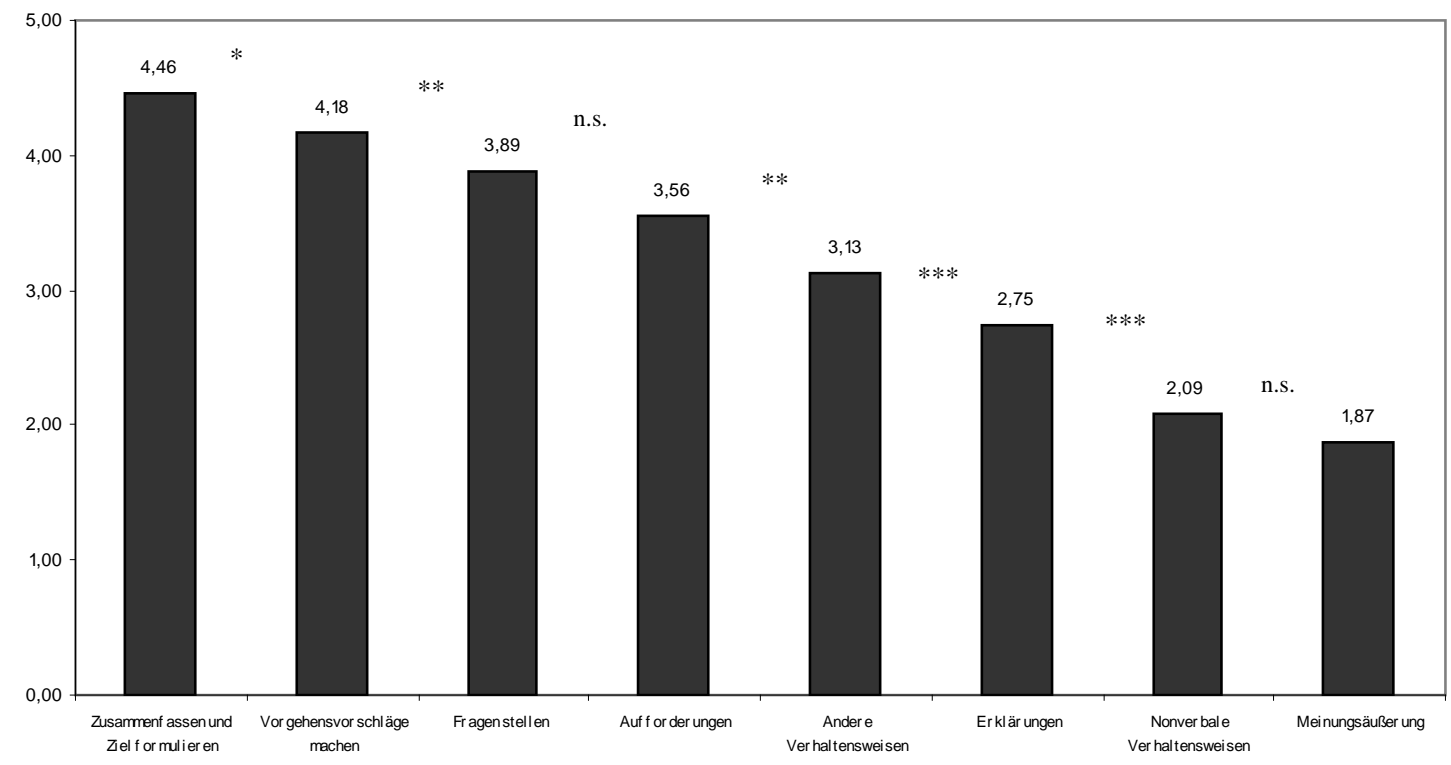

Abbildung 11: Mittelwerte der eingeschätžten Nützlichkeit der vorgegebenen Verbaltensweisen. Anmerkung: Die Skala reichte von $1 . .$. überbaupt nicht sinnvoll bis $5 . .$. sehr sinnvoll; *** bedeutet einen signifikeanten Unterschied auf dem 0,001-Niveau, ** auf dem 0,01-Niveau und * auf dem 0,05-Niveau.

Insgesamt hat sich gezeigt, dass die Verhaltensweisen, die der expliziten Prozesskoordination dienen, von den Probanden/-innen als sinnvolle bis sehr sinnvolle Handlungen während der Problemdiskussion angesehen werden. Plausibel ist auch der Befund, dass die weniger expliziten, nonverbalen Verhaltensweisen als deutlich weniger sinnvoll betrachtet werden. Als am wenigsten sinnvolle Koordinationshandlung in der Moderation einer Problemdiskussion wird das Bekunden der eigenen Meinung angesehen.

Auf die Bedeutung und die Implikationen der Ergebnisse der explorativen und der standardisierten Befragung wird im Folgenden eingegangen. 


\subsubsection{Diskussion}

Zunächst wird auf die Bedeutung der Ergebnisse im Hinblick auf subjektive Koordinationstheorien sowie das Konzept expliziter Prozesskoordination eingegangen. Anschließend wird die Wahl des Szenariums als Methode und dessen Inhalt kritisch reflektiert.

\section{Bedeutung der Ergebnisse}

Das Ziel der Szenariostudie bestand in der Erfassung individueller subjektiver Koordinationstheorien von Personen, die in der Gruppenkoordination bisher wenig persönliche Erfahrungen gesammelt haben. Hierdurch soll ein Beitrag zur differenzierten Beschreibung des Konstrukts der expliziten Prozesskoordination geleistet werden.

Die Ergebnisse der Studie 1 zeigen, dass die Schüler/-innen, als in der Gruppenkoordination unerfahrene Personen, genaue Vorstellungen von Verhaltensweisen haben, die in der Koordination einer Entscheidungsfindungsdiskussion zum Einsatz kommen sollen. Ihre subjektiven Koordinationstheorien beinhalten sowohl Verhaltensweisen, die einen Aufforderungscharakter (Handlungsanleitungen) haben, als auch Handlungen, die den Diskussionsprozess zielführend strukturieren (Strukturierung des Problemlöseprozesses). Nonverbale Verbaltensweisen wie Nicken und Kopfschütteln sowie das Verkünden der eigenen Meinung werden kaum empfohlen bzw. als wenig sinnvoll eingeschätzt. Daraus kann geschlossen werden, dass die subjektiven Koordinationstheorien der Schüler/-innen Inhalte expliziter Prozesskoordination enthalten: Die genannten Verhaltensweisen sind explizit, da sie den Interaktionsprozess durch deutliche verbale Instruktionen (z.B. Rederechte vergeben, Regeln festlegen) oder Strukturierungen (z.B. Fragen stellen, Zusammenfassen, Wiederholen oder Pro- und KontraListen erstellen) koordinieren, mit Koordinationsabsicht ausgeführt werden (vgl. Espinosa et al., 2004) und verbal kommuniziert werden (vgl. Wittenbaum et al., 1998). Damit wird deutlich, dass 
Kommunikation als ein wesentliches Mittel der Koordination von Entscheidungsfindungsgruppen betrachtet wird.

Wittenbaum et al. (1998) betrachten explizite Prozesskoordination von Gruppenarbeit hauptsächlich als Planung und Besprechung des Vorgehens der Aufgabenerledigung durch verbale Abstimmungen, Rollenverteilung und Zuordnung von Teilaufgaben (vgl. Kapitel 3.3.3). Im Hinblick auf Entscheidungsfindung ist diese Form der Koordination auch ein Bestandteil der subjektiven Koordinationstheorien der Schüler/-innen. Diese beinhalten Verhaltensweisen, mit denen die Diskussionsteilnehmer/-innen verbal zu konkreten Handlungen aufgefordert werden, beispielsweise durch die Vergabe von Rederechten. Im Hinblick auf die konkrete Gruppenaufgabe der Entscheidungsfindung beinhalten die subjektiven Koordinationstheorien auch Handlungen zum Management des Informationsaustauschs durch Strukturierungen (z.B. Fragen stellen, Zusammenfassen, Wiederholen oder Pro- und Kontra-Listen erstellen) sowie zum Umgang mit Interessenskonflikten durch Konfliktvermeidungsverhaltensweisen.

Es kann festgehalten werden, dass die subjektiven Theorien zur Koordination einer Gruppenentscheidungsfindung von in der Gruppenkoordination unerfahrenen Personen im wesentlichen Handlungen expliziter Prozesskoordination (Handlungsanleitungen, Strukturierungen des Problemlöseprozesses und Konfliktvermeidung) beinhalten. Dieses Ergebnis steht im Einklang mit den Postulaten Wittenbaums et al. (1998), wonach unsichere Gruppenaufgaben mit herausfordernden Zielen und notwendiger interdependenter Zusammenarbeit der Gruppenmitglieder vermehrt expliziter Koordination bedürfen.

Neben den spezifisch beschriebenen Koordinationshandlungen der moderierenden Person zeigt sich allerdings auch, dass a) als subjektiven Koordinationstheorien der Schüler/innen hauptsächlich Funktionen der diskussionsleitenden Person genannt werden (z.B. für Gleichberechtigung sorgen, Streit vermeiden, für Konsens und Kompromiss sorgen), obwohl diese laut Instruktion explizit nicht aufgeführt werden sollten, sondern konkrete Verhaltensweisen erfragt 
wurden. Daneben gaben b) viele Schüler/-innen Verhaltensweisen an, die Stefan als moderierende Person nicht zeigen sollte (z.B. nicht selber Stellung beziehen). Offenbar fällt es vielen in der Koordination unerfahrenen Schüler/-innen schwer, konkrete Verhaltensweisen zu benennen, was einerseits auf mangelnde Kenntnis, andererseits auf mangelnde Fähigkeit zu Verbalisierung dieses Verhaltens zurückzuführen sein kann. Vielmehr fällt es den Schüler/-innen leichter, allgemeine Verhaltensweisen wie thematisch strukturieren, Streit vermeiden und für Rube und Ordnung sorgen zu benennen und Handlungen aufzuführen, die nicht gezeigt werden sollen. Eventuell sind die konkreten Koordinationsverhaltensweisen kognitiv nicht zugänglich, so dass sie auch nicht ohne Weiteres genannt werden können. Diese Beobachtung lässt sich handlungstheoretisch erklären: Beispielsweise kann Streit vermeiden als ein Unterziel des Oberziels der Koordination einer Gruppenentscheidungsdiskussion betrachtet werden, da es sich um einen vorgestellten und angestrebten Zustand am Ende einer (Teil-)Handlung handelt (vgl. Cranach et al., 1980). Damit ist das Teilziel Streit vermeiden Bestandteil des Plans zur Erreichung des Oberziels und dient der kognitiven Steuerung der zur Erreichung des Oberziels notwenigen Handlungen. Die Ebene der Zielbestimmung (Koordination einer Gruppenentscheidungsdiskussion) und die strategische Ebene (Unterziele wie Streit vermeiden, thematische Strukturierung) können von den Schüler/-innen berichtet werden. Die operationale Ebene, im Sinne einzelner Handlungsschritte, wird jedoch wesentlich seltener berichtet (z.B. Fragen und Nachfragen), obwohl in der Instruktion danach gefragt wurde. Diese Beobachtung stimmt mit den Annahmen der Handlungstheorie überein, wonach die einzelnen Handlungsschritte der operationalen Ebene teilweise unterbewusst ablaufen (Cranach et al., 1980) und daher kognitiv nicht zugänglich sein müssen.

Darüber hinaus fällt auf, dass die konkreten Verhaltensweisen Wiederbolen, Zusammenfassen und Fragen stellen in den Antworten auf die explorative Frage nach konkreten Handlungen nur selten genannt wurden, in der anschließenden standardisierten Nützlichkeitseinschätzung jedoch als sehr sinnvoll eingeschätzt wurden. Dies konnte durch entsprechende experimentelle Befunde gezeigt werden konnte (Larson et al., 1998b). Offenbar werden diese Handlungen als sinnvoll zur 
Koordination von Entscheidungsfindungsdiskussionen anerkannt, jedoch scheinen sie nicht selbst generiert zu werden, da sie aufgrund ihre fehlenden kognitiven Repräsentanz kaum assoziiert werden - möglicherweise fehlt den Schüler/-innen hier das abstrakte und erfahrungsbedingte Wissen zur Koordination von Gruppendiskussionen.

\section{Das Szenarium als Methode der Wabl}

Obwohl die vorgestellte Studie bedeutsame Ergebnisse im Hinblick auf das Konstrukt expliziter Prozesskoordination zeigte, muss das Szenarium als eingesetzte Methode zur Erhebung der subjektiven Theorien kritisch betrachtet werden.

Das Einsetzen eines Szenariums birgt einerseits den Vorteil, dass allen Teilnehmern/innen die gleiche Situation vorliegt. Andererseits kann nur teilweise nachvollzogen werden, inwieweit sich dadurch tatsächlich allen Teilnehmern/-innen das gleiche Problem auftat. Diesbezüglich wurden die Schüler/-innen konkret gefragt, vor welcher Schwierigkeit der Schülersprecher während der Entscheidungsfindungsdiskussion stehe. Die Antworten zeigen, dass das Problem bis auf die fehlende Autorität des Moderators größtenteils richtig erkannt wurde. Außerdem zeigte sich, dass sich die Antworten auf die anschließende, eigentliche Untersuchungsfrage den vorher berichteten Schwierigkeiten sehr ähneln. So wurden beispielsweise für Ruhe sorgen und Fairness bzw. Gleichberechtigung sowohl in der Frage nach den Schwierigkeiten, als auch in der Untersuchungsfrage nach möglichen Koordinationshandlungen berichtet. Offenbar fällt es den Schülern/-innen nicht schwer, das beschriebene Problem zu erkennen und die entsprechenden Handlungsziele zu beschreiben. Eine entsprechende Umsetzung auf der operativen Handlungsebene erfolgte jedoch nicht immer. Das kann einerseits auf die oben erwähnten Verbalisierungsschwierigkeiten zurückzuführen sein. Andererseits kann dafür auch das verwendete Szenario verantwortlich sein. Möglicherweise konnten sich die Teilnehmer/-innen nicht tief genug in die vorgegebene Situation hineinversetzen, die es ermöglicht, entsprechende Verhaltensweisen abzurufen. Dies kann auf ihre fehlende Erfahrung 
oder auf die Unangemessenheit des im Szenario dargestellten Problems hinweisen. Entsprechend der Stichprobe enthielt das Szenario zwar ein Problem aus dem Schulkontext, es ist dennoch möglich, dass die Schüler/-innen das Problem „absurd“ fanden und sich deshalb nicht auf eine genauere Auseinandersetzung damit eingelassen haben. Diese Vermutung wird zum Teil durch die Antworten auf die am Ende des Fragebogens gestellte Kritikfrage bestätigt, in denen neben der Länge des Szenariums und die geringe Zeit, die ihnen zum Ausfüllen des Fragebogens zur Verfügung stand, ebenfalls der im Szenarium beschriebene Konflikt als wenig realistisch kritisiert wurde. Zwar wurde das Szenario im Vorfeld von verschiedenen Personen (Psychologen/-innen und Student/-innen) auf Plausibilität geprüft. Allerdings fehlte eine entsprechende Validierung durch Schüler/-innen. Denkbar ist, im Hinblick auf zukünftige Untersuchungen, den Schülern/innen kein Szenarium vorzulegen, sondern eine entsprechende Situation erinnern zu lassen, die sie individuell erlebt haben. Möglicherweise würde es ihnen dann leichter fallen, aufgrund ihrer eigenen Erfahrung konkretes, selbst ausgeführtes Verhalten zu berichten.

Außerdem wurde in der vorliegenden Studie implizit davon ausgegangen, dass die Schüler/-innen wenig persönliche Erfahrung in der Gruppenkoordination besitzen, ohne dass konkret danach gefragt wurde. Es ist daher denkbar, dass einige Schüler/-innen durch ehrenamtliche Positionen oder Trainings doch über Erfahrungen in der Gruppenleitung verfügen. Für den Großteil der Befragten wird jedoch aufgrund ihres Alters und Lebenskontextes, der hauptsächlich durch die Schule geprägt ist, davon ausgegangen, dass sie keine Koordinationserfahrung besitzen.

\section{Ausblick}

In dieser Studie wurden subjektive Koordinationstheorien von Personen erfasst, die in der Gruppenkoordination bisher wenig persönliche Erfahrungen gesammelt haben. Dadurch konnte das Konstrukt der expliziten Prozesskoordination differenzierter beschrieben werden: Die Annahmen von Wittenbaum et al. (1998) bezüglich des Einsatzes expliziter Koordination lassen 
sich auch in den subjektiven Theorien zur Koordination von Entscheidungsfindungsprozessen finden: Sie beinhalten Verhaltensweisen, mit denen die Diskussionsteilnehmer/-innen verbal zu konkreten Handlungen aufgefordert werden. Darüber hinaus besteht die explizite Prozesskoordination der kollektiven Entscheidungsfindung auch aus Handlungen einerseits zum Management des Informationsaustauschs durch Strukturierungen und andererseits zum Umgang mit Interessenskonflikten durch Konfliktvermeidungsverhaltensweisen.

Die Ergebnisse zeigen die subjektiven Koordinationstheorien von Schülern/-innen, die in der Gruppenkoordination keine langjährige Erfahrung haben und spiegeln daher im Vergleich zu Experten/-innen naive subjektive Theorien wider, die wesentlich durch abstraktes Wissen, weniger durch erlernte und entwickelte Kompetenzen entstanden sind. Nun ist von Interesse, inwieweit Experten/-innen, die sich beruflich mit Gruppenkoordination beschäftigen, ähnliche subjektive Koordinationstheorien besitzen und inwieweit diese zur Präzisierung oder Erweiterung des Konstrukts der expliziten Prozesskoordination beitragen können. Dieser Frage wird in der zweiten Studie nachgegangen. 


\subsection{Studie 2: Subjektive Theorien der Koordination: Die erfahrene Perspektive der Koordinierenden}

Nachdem in der ersten Studie subjektive Theorien expliziter Prozesskoordination von Personen erhoben worden, die in der Gruppenkoordination bisher wenig praktische Erfahrung hatten, sollen die subjektiven Koordinationstheorien nun um die Betrachtung der erfahrenen Perspektive erweitert werden. Durch die Ergänzung der naiven um die erfahrene Perspektive kann der Forderung von Kleining und Witt (2001) nach maximaler struktureller Variation der Perspektiven nachgekommen werden. Hiernach sollen möglichst unterschiedliche Betrachtungspunkte, Methoden, Untersuchungsteilnehmer/-innen und Situationen zur Erforschung eines Konzepts eingesetzt werden. Es interessiert, inwieweit sich die erfahrene Perspektive von der naiven Perspektive unterscheidet, ob es einen gemeinsamen inhaltlichen Kern und einen erfahrungsbedingt anderen Fokus der Koordination gibt, beispielsweise andere Koordinationsziele oder im Vergleich zu den Schülern/-innen konkreteres Herstellungswissen im Sinne spezifischer Wenn-Dann-Beziehungen.

\section{Experte/-in und Expertise}

Expertise kann sowohl herausragende Leistung als auch langjährige Erfahrung in einem spezifischen Kompetenzbereich bedeuten (Zempel, 2002). Der Status eines/r Experten/-in ist relativ und wird der zu befragenden Person begrenzt auf den Forschungsgegenstand zugeschrieben (Meuser \& Nagel, 2005).

In Anlehnung an Bromme (1992) liegt der Studie eine Definition des Experten/der Expertin zugrunde, die Personen beinhaltet, „die berufliche Aufgaben zu bewältigen haben, für die man eine lange Ausbildung und praktische Erfahrung benötigt und die diese Aufgaben erfolgreich lösen“ (S. 7-8). Das Erfolgkriterium ist individuell zu bestimmen und beinhaltet in der vorliegenden Untersuchung das Innehaben einer verantwortlichen Berufsrolle, in der Arbeitsgruppen entweder angeleitet oder durch Moderation unterstützt werden. 
Im Unterschied zu den Teilnehmern/-innen der ersten Studie - in Führung und Gruppenleitung nicht ausgebildete Abiturienten/-innen - verfügen die Koordinationsexperten/innen über besondere Kompetenz im Umgang mit Arbeitsgruppen. Diese haben sie sich durch Training und langjährige Erfahrung erworben. Ihr professionelles Expertenwissen „umfasst also theoretische Elemente, und es besteht aus Faustregeln und praktischen Erfahrungen“ (Bromme, 1992, S. 9). Es enthält Wertvorstellungen und ist in Abhängigkeit der zu bewältigenden praktischen Probleme organisiert (Bromme, 1992).

Es wird davon ausgegangen, dass koordinationserfahrene Personen über umfangreiches strukturiertes Koordinationswissen verfügen und sich in ihren Koordinationshandlungen von weniger erfahrenen oder kompetenten Personen unterscheiden. In Studie 1 konnte gezeigt werden, dass die subjektiven Koordinationstheorien koordinationsunerfahrener Personen neben Handlungen expliziter Prozesskoordination hauptsächlich Funktionen (z.B. für Gleichberechtigung sorgen, Streit vermeiden, für Konsens und Kompromiss sorgen) und nicht zu zeigende Verhaltensweisen (z.B. nicht selber Stellung beziehen) beinhalten. Es wurde diskutiert, dass die Benennung konkreter Verhaltensweisen den Nicht-Experten/-innen eventuell aufgrund mangelnder Kenntnis oder Verbalisierungsschwierigkeiten schwer fällt und sie deshalb eher allgemeine Verhaltensweisen wie thematisch strukturieren, Streit vermeiden und für Rube und Ordnung sorgen, benennen. In Studie 2 interessiert nun, ob die subjektiven Koordinationstheorien der Experten/-innen ähnlich aufgebaut sind (Kleining \& Witt, 2001). Speziell wird den Fragen nachgegangen, inwieweit eher Funktionen oder konkrete Handlungen berichtet werden, wann bzw. in welchen Situationen die Experten/-innen Koordinationsbedarf sehen, wie und mit welchem Grund sie auf diesen Bedarf reagieren und welche Folgen sie als aus ihrem Handeln resultierend wahrnehmen. Zur Beantwortung dieser Fragen wurden fokussierte Interviews mit Koordinationsexperten/-innen durchgeführt. Diese sollen im Folgenden vorgestellt werden. 


\subsubsection{Methode}

Ein übliches Verfahren zur Befragung und Erhebung subjektiver Theorien von Experten/-innen ist das Interview (z.B. Bogner \& Menz, 2005; Schmitt \& Hanke, 2003). Im Gegensatz zu anderen Techniken (z.B. Strukturlegetechnik) kann bei dem Einsatz von Interviews davon ausgegangen werden, dass sie den Experten/-innen prinzipiell vertraut sind und von ihnen aus formaler, inhaltlicher und zeitlicher Sicht akzeptiert werden. Das forschungslogische Ziel der Experteninterviews besteht darin, „Strukturen und Strukturzusammenhänge des ExpertInnenwissens/handelns zu analysieren“ (Meuser \& Nagel, 2005, S. 76). Inwieweit Experteninterviews als eigenständige Methode zu betrachten sind, die sich von anderen Interviewformen unterscheidet (Meuser \& Nagel, 2005), ist umstritten (Kassner \& Wassermann, 2005). Experteninterviews werden auch als Sammelbegriff für offene oder teilstandardisierte Befragungen von Experten/-innen zu einem bestimmten Thema angesehen (Bortz \& Döring, 2002). Diese Sichtweise wird in der vorliegenden Studie geteilt. Formal wurden die Interviews als fokussierte Interviews durchgeführt. Hierauf wird im Folgenden näher eingegangen.

\section{Fokussierte Experteninterviews}

Die Methode des fokussierten Interviews geht auf Merton und Kendall (1946, zit. nach Merton, 1987) zurück. Ausgangspunkt fokussierter Interviews ist die inhaltliche Konzentration auf einen zuvor definierten Gegenstand oder eine Situation, die von den Befragten erlebt wurde und dem/der Forscher/-in bekannt ist (Bortz \& Döring, 2002; Hopf, 1995; Lamnek, 1989; Merton, 1987). Im Gespräch sollen diesbezügliche subjektive Erfahrungen und Interpretationen der befragten Person erfasst werden (Hopf, 1995; Lamnek, 1989). Ziel ist es, die Reichweite des angesprochenen Themas zu maximieren und dem/der Befragten zu ermöglichen, auch über von dem/der Forscher/-in nicht antizipierte Teilaspekte zu sprechen. Ausgehend von dem inhaltlichen Gegenstand des Interviews wird zunächst ein Gesprächsleitfaden entwickelt, der jedoch im Verlauf des Interviews verlassen werden darf, um an für den/die Befragte/n 
wesentlich erscheinende Aspekte zu gelangen (Lamnek, 1989). Der Vorteil des Einsatzes eines offenen Leitfadens besteht darin, dass sowohl dem inhaltlich begrenzten Interesse des/der Forschers/-in als auch dem Experten/-in-Status des/der Gesprächspartners/-in Rechnung getragen (Meuser \& Nagel, 2005) und gleichzeitig sicher gestellt werden kann, dass alle forschungsrelevanten Themen angesprochen werden (Schnell, Hill \& Esser, 1993). Hopf (1995) sieht in dem fokussierten Interview eine Spezialform teilstandardisierter Interviews, wobei alle Befragten in gleicher Weise befragt werden, innerhalb der gleichen Vorgehensart jedoch eine Variabilität besteht, die es dem/der Interviewer/-in ermöglicht, sich jedem/r Befragten individuell anzupassen und damit einerseits die Fragen stellen zu können, die auf jeden Fall gestellt werden müssen, und andererseits das Gespräch aufrechterhalten zu können (Bartholomew, Henderson \& Marcia, 2000). Trinczek (2005) geht davon aus, dass sich leitfadengestützte Interviews besonders zur Befragung von Führungskräften eignen, da sie deren Erwartung einer strukturierten Gesprächssituation und klarer Rollenverteilung (Fragensteller/-in vs. Befragte/r) gerecht werden.

\section{Leiffaden des fokussierten Interviews}

Im Fokus der Interviews stand die explizite Prozesskoordination. Es wurde ein Gesprächsleitfaden konzipiert, der die Erfragung der subjektiven Koordinationstheorien der Experten/-innen ausgehend von einer selbst erlebten Gruppensituation gestattet. Im Gegensatz zur Studie 1 wurde kein für alle Probanden/-innen gleiches Szenario verwendet. Statt dessen sollten die Befragten sich an eine selbst erlebte Situation und ihr entsprechendes Verhalten in dieser Situation erinnern. Damit sollte gewährleistet werden, dass die Befragten ihre konkreten Erfahrungen sowie damit verbundenen subjektiven Koordinationstheorien und nicht nur abstraktes Wissen anwenden.

Zum Interviewbeginn wurden zunächst das Ziel und der Ablauf des Interviews erläutert, wobei das Interesse an Koordination, insbesondere der expliziten Koordination, betont wurde 
(vgl. Anhang B). Anschließend wurden die Befragten gebeten, sich eine problematische Diskussion vorzustellen bzw. eine solche zu erinnern, zu deren Moderation man sie als Experten/-in gebeten hat. Es sollte sich um eine Situation handeln, bei der sie das Gefühl hatten, ihr ganzes Moderationsgeschick aufbieten zu müssen. Die Befragten sollten diese Situation zunächst beschreiben. Sie wurden dann gefragt, vor welchen Schwierigkeiten und Herausforderungen sie ihrer Meinung nach standen und was sie in dieser Situation gedacht und möglicherweise empfunden haben. Die Befragten sollten anschließend berichten, was genau sie in dieser Situation getan haben, um den geschilderten Herausforderungen gerecht werden. Zur genaueren Analyse wurden sie weiter gefragt, ob es unter den geschilderten Verwaltensweisen solche gab, die sie besonders gern oder besonders ungern ausführen und aus welchem Grund. Um spezifisches Herstellungswissen (vgl. Kapitel 4.1) zu erfassen, wurden die Befragten nochmals gebeten, an die von ihnen zuvor beschriebenen Interventionen zu denken und zu überlegen, wann sie diese verwenden und ob sie deren Einsatz von bestimmten vorherigen Situationen oder Verhaltensweisen der Gruppe oder einzelner Gruppenmitglieder abhängig machen. Abschließend wurden die Befragten gebeten, Auskunft darüber zu geben, wo sie ihr Wissen und die Fertigkeiten, die sie während des Interviews berichtet haben, erworben haben. Der gesamte Interviewleitfaden ist im Anhang B einzusehen.

\section{Stichprobe}

Da das Ziel dieser Studie in der facettenreichen Beschreibung subjektiver Expertentheorien zur expliziten Prozesskoordination von Gruppen besteht, muss die Stichprobe insofern repräsentativ sein, als dass sie möglichst unterschiedliche Experten/-innen beinhaltet, so dass nicht nur der typischste Fall vertreten ist (Maas \& Wunderlich, 1972; Witt, 2001). Drei Expertinnen und fünf Experten aus verschiedenen Berufsfeldern und mit unterschiedlicher Erfahrung im Umgang mit Arbeitsgruppen wurden befragt. Ihr Beruf und Arbeitskontext sind in Tabelle 4 dargestellt. Die Experten/-innen wurden aus dem persönlichen und beruflichen 
Arbeitsumfeld der Autorin geworben. Ihnen ist gemeinsam, dass sie langjährige Erfahrung in der Leitung oder Unterstützung von Arbeitsgruppen besitzen.

Tabelle 4: Beruf, Alter, Geschlecht und Erfahrungshintergrund der Experten/-innen

\begin{tabular}{|c|c|c|c|c|}
\hline & Person & $\begin{array}{l}\text { Alter in } \\
\text { Jahren }\end{array}$ & Geschlecht & Arbeitskontext und Erfahrungshintergrund \\
\hline $\mathbf{A}$ & Sozialwirt & 28 & männlich & $\begin{array}{l}\text { seit mehr als } 5 \text { Jahren als Moderator tätig, } \\
\text { Ausbildung in Gestalttherapie }\end{array}$ \\
\hline B & Organisationsberater & 65 & männlich & selbstständiger Trainer, Berater und Moderator \\
\hline C & Organisationsberaterin & 42 & weiblich & $\begin{array}{l}\text { selbstständige Trainerin, Beraterin und } \\
\text { Moderatorin }\end{array}$ \\
\hline $\mathbf{D}$ & Dezernent & 63 & männlich & $\begin{array}{l}\text { Verwaltungsbeamter a. D., Ressort Umwelt, } \\
\text { verantwortlich für Genehmigungsverfahren }\end{array}$ \\
\hline $\mathbf{E}$ & Führungskraft & 48 & weiblich & $\begin{array}{l}\text { Arbeitsgruppenleiterin einer } \\
\text { Forschungseinrichtung (Biologie) }\end{array}$ \\
\hline $\mathbf{F}$ & Führungskraft & 63 & weiblich & $\begin{array}{l}\text { Leiterin des Patientenbüros eines } \\
\text { Unfallkrankenhauses }\end{array}$ \\
\hline G & Führungskraft & 62 & männlich & $\begin{array}{l}\text { Leiter Abteilung Grundstücksmanagement eines } \\
\text { Flughafens }\end{array}$ \\
\hline $\mathbf{H}$ & Führungskraft & 47 & männlich & $\begin{array}{l}\text { Leitender Mitarbeiter Deutsche Bahn (Ressort } \\
\text { Energie) }\end{array}$ \\
\hline
\end{tabular}

\section{Ablauf der Interviews}

Alle Interviews wurden von der Autorin durchgeführt und fanden entweder im Büro oder der Wohnung der Befragten statt. Nach der Begrüßung fand häufig ein kurzes, zwangloses Vorgespräch statt, in dem entweder über persönliche Dinge oder den Anfahrtsweg gesprochen wurde. Zu Beginn des eigentlichen Interviews erläuterte die Interviewerin das Ziel und den Ablauf des Interviews (vgl. Anhang B) und bat um das Einverständnis, das Gespräch auf Tonband aufnehmen zu dürfen, was in keinem Fall abgelehnt wurde. Neben der Tonaufnahme machte sich die Interviewerin Notizen, um wesentliche Äußerungen und Ausgangspunkte für Nachfragen festzuhalten. Am Ende des Interviews bedankte sich die Interviewerin bei den 
Gesprächspartner/-innen und bot bei Interesse an, die Ergebnisse der Interviewstudie zuzuschicken.

Darüber hinaus wurde im Anschluss an das jeweilige Interview ein Nach-InterviewProtokoll angefertigt, in welchem wesentliche Eindrücke festgehalten und Angaben zur Gesprächsatmosphäre gemacht wurden.

Die Dauer der Interviews schwankte zwischen 25 und 90 Minuten.

\subsubsection{Ergebnisse}

Vorgehen bei der Datenauswertung

Das Ziel der fokussierten Interviews bestand in der Erfassung subjektiver Expertentheorien expliziter Prozesskoordination. Mögliche Auswertungsmethoden leitfadenorientierter Interviews sind sehr vielfältig. Für die vorliegende Fragestellung bieten sich inhaltsanalytische Methoden an, da nicht das Interview bzw. Gespräch an sich im Sinne einer Konversations- oder Diskursanalyse (vgl. Howitt \& Cramer, 2005), sondern der Inhalt des Interviews den Interessensgegenstand bildet. Ausgehend von dem erhaltenen Datenmaterial sollen subjektive Theorien expliziter Prozesskoordination induktiv extrahiert werden. Früh (2004) empfiehlt, im Rahmen der Inhaltsanalyse das Selektions- und Abstraktionsinteresse festzulegen. Das Selektionsinteresse bestimmt die Auswahl der Daten und besteht in der vorliegenden Studie in der Erfassung subjektiver Koordinationstheorien von Experten/-innen. Die explizite Prozesskoordination stellt das Abstraktionsinteresse dar, danach werden die Interviewdaten untersucht.

Als inhaltsanalytisches Auswertungsmodell eignet sich Mayrings qualitative Technik der Zusammenfassung bzw. induktiven Kategorienbildung (Mayring, 2003). Er schlägt vor, das vorliegende Material mit Hilfe von vier Interpretationsregeln $\mathrm{zu}$ reduzieren: 1. Paraphrasierung, 2. Generalisierung auf das Abstraktionsniveau, 3. Erste Reduktion, 4. Zweite Reduktion (Mayring, 2003). Auch Früh (2004) schlägt zur empiriegeleiteten Kategorienbildung vier vergleichbare Schritte vor: 
1. Selektion/Reduktion, 2. Bündelung, 3. Generalisierung/Abstraktion/Bezeichnung und 4. Rückbezug auf Theorie. Meuser und Nagel (2005) entwickelten speziell für leitfadenorientierte Experteninterviews eine interpretative Auswertungsstrategie, die aus sechs Schritten besteht: 1 . Transkription, 2. Paraphrase, 3. Überschriften, 4. thematischer Vergleich, 5. soziologische Konzeptualisierung und 6. theoretische Generalisierung. Da dieses Vorgehen mit den einschlägigen inhaltsanalytischen Auswertungsstrategien in wesentlichen Punkten vergleichbar ist (Bartholomew et al., 2000; Früh, 2004; Langdridge, 2004; Mayring, 2003), jedoch speziell für die Anwendung an leitfadenorientierten Experteninterviews konzipiert ist, wurden die vorliegenden Interviews in Anlehnung an Meuser und Nagel (2005) ausgewertet. Die Auswertung wird im Folgenden vorgestellt.

\section{Schritt 1: Transkription}

In Anlehnung an Meuser und Nagel (2005) wurden die auf Tonband aufgenommenen Interviews transkribiert, wobei auf die Erfassung von Pausen, Stimmlagen und anderen nonverbalen Ausdrücken verzichtet wurde. Der Grund für diese sparsame Transkribierung besteht darin, dass für die oben genannte Fragestellung nicht der Diskursverlauf des Interviews Gegenstand der Analyse ist, sondern lediglich die Inhalte der Antworten auf die Interviewfragen. Da der Interviewleitfaden den Gesprächsablauf zwar strukturiert, aber inhaltliche Abschweifungen aus oben genannten Gründen zulässt, gibt es häufig Gesprächsabschnitte, in denen nicht über die eigentliche Thematik gesprochen wurde. Diese Abschnitte wurden ebenfalls nicht transkribiert.

\section{Schritt 2: Paraphrase}

In diesem Schritt wurden die Äußerungen der Experten/-innen mit eigenen Worten wiedergegeben. Dazu „verfolgt [man] den Text in der Absicht, die Gesprächsinhalte der Reihe nach wiederzugeben und den propositionalen Gehalt der Äußerungen zu einem Thema explizit zu machen“ (Meuser \& Nagel, 2005, S. 84). 


\section{Schritt 3: Überschriften}

Im dritten Schritt werden für die paraphrasierten Abschnitte Überschriften gebildet, wobei die Terminologie der Interviewten beibehalten werden sollte (Meuser \& Nagel, 2005). In jedem der acht paraphrasierten Interviews wurden Abschnitte mit gleichen oder ähnlichen Themen unter einer Hauptüberschrift subsummiert. Anhang C enthält pro Interview eine Abbildung, in der die Überschriften und Themen der dazugehörigen Paraphrasen des Interviews systematisch dargestellt sind. Es wurde versucht, die Überschriften so zu systematisieren, dass sie der in der Handlungstheorie angenommenen hierarchischen Handlungsstruktur entsprechen. Allerdings besteht das Ziel der vorliegenden Studie nicht in der Überprüfung einer handlungstheoretischen Struktur subjektiver Koordinationstheorien. Die Handlungstheorie dient lediglich als Darstellungsraster und soll darauf hinweisen, dass auch explizite Koordinationshandlungen hierarchisch organisiert sein können.

Zur Gewährleistung der Übersichtlichkeit werden hier vorerst nur die wesentlichen Charakteristika der acht Experten/-innen zusammengefasst. Der Ergebnisschwerpunkt liegt jedoch in der Präsentation der über alle acht Interviews generalisierten Ergebnisse. Dieses Vorgehen fällt in Anlehnung an Meuser und Nagel (2005) unter den thematischen Vergleich und wird in Schritt 4 dargestellt.

Person A. Der 28jährige Sozialwirt (Ausbildung in Gestalttherapie) berät und moderiert Gruppen im Rahmen der Prozessberatung. Wesentlich sei das Rollenverständnis des Moderierens, der/die Moderator/-in sei nicht für das Gruppenergebnis, sondern für den Arbeitsprozess verantwortlich. Die Moderation sei eher Einstellung als Methodik, baue jedoch auf einem Methodenrepertoire auf. Er betonte die Situationsangemessenheit jeglicher Interventionen in den Gruppenprozess. Entscheidend für die Wahl der Gruppenunterstützungsmittel sei die Art der Gruppenaufgabe.

Person B. Der 65jährige Organisationsberater legt den Fokus auf seine Einstellung als Moderator, die geprägt ist vom personzentrierten Beratungsansatz (z.B. Rogers, 1999). Danach haben die Kontrolle der eigenen Emotionen, die Wertschätzung und Akzeptanz der 
anderen Personen Priorität gegenüber dem Einsatz von Methoden. Die häufigste von ihm genannte Technik besteht in der Verbalisierung (von Gefühlen, Problemen, Absichten).

Person $\boldsymbol{C}$. Die 42jährige Organisationsberaterin (Ausbildung in systematischer Beratung) berichtete sehr differenziert von ihrer prozesssteuernden Rolle als Moderatorin und dem Einsatz von entsprechenden Methoden. Ihr Rollenverständnis beinhaltet die Übernahme der Verantwortung für den Gruppenarbeitsprozess. Daher ist sie in ihrer Moderation sehr klar und direktiv. Neben vielen allgemeinen Techniken der Gruppensteuerung stellte sie einige Beispiele ihres situationsangemessenen Methodeneinsatzes dar, wonach sie auf eine bestimmte Situation oder Äußerung eines Gruppenmitgliedes eine bestimmte Technik einsetzt, die zu einer bestimmten Reaktion der Gruppe führt. Damit gehen auch ihre Annabmen über das Verbalten der Gruppenmitglieder einher.

Person D. Der 63jährige Verwaltungsbeamte berichtete, dass es im Rahmen von Genehmigungsverfahren zu Dienstberatungen mit den Antragstellern/-innen komme, die er als zuständiger Dezernent zu leiten habe. Er berichtete sehr strukturiert von den Zielen dieser Gespräche und den damit verbundenen Anforderungen an ihn als Gesprächsleiter. Er betonte die Vorbereitung dieser Beratungen, insbesondere die Erstellung schriftlicher Unterlagen, die im Verlauf der Beratung ergänzt würden. Neben allgemeinen Techniken der Gesprächsleitung zeigten sich auch Heuristiken im Umgang mit schwierigen Situationen oder zum typischen Sitzungsablauf.

Person E. Die 48jährige Führungskraft hat Erfahrung im Umgang mit Arbeitgruppen durch ihre Funktion als Arbeitsgruppenleiterin in einer Forschungseinrichtung. Sie leitet die regelmäßigen Teamsitzungen mit $\mathrm{dem}$ Ziel des Informationsaustauschs und des Präsentationstrainings für ihre Mitarbeiter/-innen. Trotz ihrer Funktion als Arbeitgruppenleiterin ist ihr Rollenverständnis nicht durch eine klare Übernahme der Führungsrolle charakterisiert. Sie habe das Gefühl, eher gut mit Menschen umgehen zu können, als über besondere und mitteilbare Methoden als Gruppenleiterin zu verfügen.

Person $\boldsymbol{F}$. Die 63jährige Führungskraft arbeitete als Leiterin des Patientenbüros in einem städtischen Krankenhaus. Sie berichtete hauptsächlich von Anlässen und Situationen, in denen Führung notwendig ist und den damit verbundenen Anforderungen an sie. Ähnlich Person D zeigten sich auch hier Heuristiken zum typischen Sitzungsablauf.

Person G. Der 62jährige Leiter der Abteilung für Grundstückmanagement arbeitete als Führungskraft in einem Flughafen. Sein Rollenverständnis ist durch Personalverantwortung und eine deutliche Übernahme der Führungsrolle charakterisiert. Da er für die 
Gruppenentscheidungen verantwortlich sei, treffe er sie im Anschluss an die Dienstberatung. Neben Anforderungen und Methoden der Führung berichtet er Einstellungen gegenüber seinen Mitarbeiter/-innen und der Führungsverantwortung.

Person $\boldsymbol{H}$. Der 47jährige leitende Mitarbeiter ist als Führungskraft bei der Deutschen Bahn tätig. Bezüglich der Rollenklärung unterschied er sehr differenziert zwischen inhaltlicher Beteilung als Führungskraft oder Moderation einer Beratung. Er berichtete neben Einflussfaktoren sowohl Methoden zum grundsätzlichen Vorgehen der Gesprächsleitung als auch spezifische Techniken.

Zusammenfassend lässt sich festhalten, dass die Experten/-innen alle über ein spezifisches Verständnis ihrer Rolle verfügen und sich entweder als moderierende Prozessbegleiter/-innen oder ergebnisverantwortliche Führungskräfte verstehen. Danach richtet sich ihr Entscheidungsverhalten: Die Moderatoren/-innen lassen die Gruppe entscheiden, die Führungskräfte entscheiden selbst.

\section{Schritt 4: Thematischer Vergleich}

Nach der Zuordnung von Überschriften innerhalb der einzelnen Interviews werden die Ergebnisse nun zwischen den Interviews verglichen. Dabei wird, ähnlich dem dritten Schritt, nach thematisch vergleichbaren Abschnitten zwischen den Interviews gesucht und die jeweiligen Überschriften angeglichen (Meuser \& Nagel, 2005). Abbildung 12 zeigt die wesentlichen Themen, die sich in allen Interviews - allerdings mit jeweils unterschiedlichen Schwerpunkten - zeigten: 1. Anlass, Kontext und Bedingungen der Moderation oder Führung, 2. Anforderungen und Kompetenzen des/der Moderators/-in oder Gruppenleiters/-in, 3. Einstellungen des/der Moderators/-in oder Gruppenleiters/-in, 4. Methoden und Techniken der Gruppenunterstützung und 5. Erlernen der Gruppenunterstützung. Diese fünf Themen werden im Folgenden vorgestellt. 


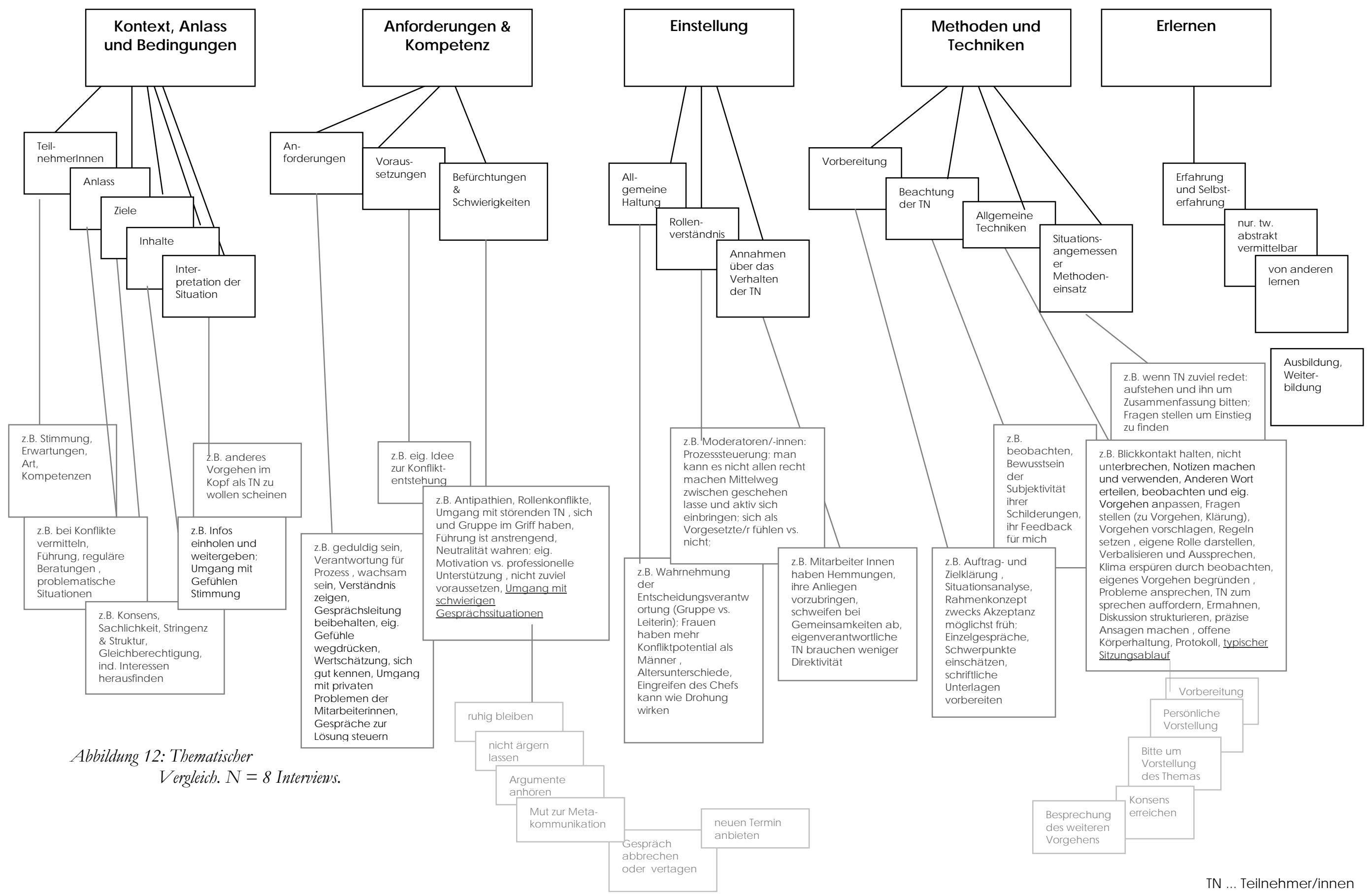




\section{Anlass, Kontext und Bedingungen der Moderation oder Führung}

Alle Experten/-innen berichteten zunächst, in welchen Situationen sie Arbeitsgruppen unterstützen oder leiten. Neben regulären Teamsitzungen wurden Gruppenkonflikte, plötzlich auftretende Probleme oder Dienstberatungen mit externen Personen (z.B. Antragstellern/-innen, Verhandlungspartnern/-innen) genannt. Je nach Rolle des/der Experten/-in leiteten sie die Gruppe entweder als zuständige Person oder reguläre Führungskraft oder sie wurden als externe/n Moderator/-in zur Konfliktlösung oder Moderation von Gruppenentwicklungsseminaren eingesetzt. Es wurden auch ganz spezifische Anlässe zum Einsatz von Koordinationshandlungen berichtet, wie z.B. die Wahrnehmung, dass die Gruppenmitglieder momentan dem vorgeschlagenen Vorgehen nicht folgen oder bei einem Thema länger als vorgesehen beharren. Die Ziele der Unterstützung bestehen hauptsächlich in der Ermöglichung von Gleichberechtigung und Sachlichkeit, der Strukturierung der Diskussion, dem Herausfinden individueller Interessen der Gruppenmitglieder und der Erzielung eines Konsenses. Wesentliche Inhalte der Diskussionen sind das Einholen und Weitergeben von Informationen, das Lösen von Problemen, Treffen von Entscheidungen und (in der Konfliktmoderation) der Umgang mit Gefühlen. Die Experten/-innen antizipieren vor ihrem Einsatz häufig die Erwartungen, Kompetenzen und Stimmungen der Teilnebmer/-innen der Gruppendiskussionen.

2. Anforderungen und Kompetenzen des/der Moderators/-in oder Gruppenleiters/-in

Die Experten/-innen berichten von Anforderungen, denen sie gerecht werden müssen. Dazu zählen Geduld, Wachsamkeit, Verantwortung, Wertschätzung, Umgang mit privaten Problemen der Mitarbeiter/-innen, Selbstkenntnis, Wegdrücken eigener Gefühle und Beibehalten der Gesprächsleitung. Voraussetæungen dafür sind beispielsweise eigene Ideen zur Entstehung von Konflikten. Darüber hinaus berichten die Koordinationsexperten/-innen von Befürchtungen und Schwierigkeiten der Gruppenleitung, z.B. persönliche Antipathien, Rollenkonflikte, Wahrung der Neutralität, Umgang mit störenden Gruppenmitgliedern oder Gesprächssituationen. 
3. Einstellungen des/der Moderators/-in oder Gruppenleiters/-in

Es zeigte sich, dass die Experten/-innen über spezifische Einstellungen gegenüber ihrer Tätigkeit verfügen. Ihre allgemeine Haltung beinhaltet die Wahrnehmung der Entscheidungsverantwortung: Die Führungskräfte fühlen sich für das Entscheidungsergebnis verantwortlich und treffen die Entscheidung, die Moderatoren/-innen fühlen sich ausschließlich für den Entscheidungsprozess verantwortlich, nicht aber für das Ergebnis. Darüber hinaus haben einige Experten/-innen Annahmen gegenüber geschlechtsspezifischem Konfliktpotential, der Wirkung ihrer Interventionen und dem Verbalten der Gruppenmitglieder oder Diskussionsteilnehmer/innen (z.B. Hemmungen, Bedürfnis nach Direktivität, Abschweifen vom Thema). Außerdem verfügen die Experten/-innen über ein spezifisches Rollenverständnis. Es beinhaltet beispielsweise die Unterstützung und Steuerung des Prozesses und ihre Selbstwahrnehmung als Vorgesetzte/r.

\section{Methoden und Techniken der Gruppenunterstützung}

Auf konkretes Nachfragen hin berichteten die Experten/-innen über ihr methodisches Vorgehen während der Moderation oder Gesprächsleitung. Die geschilderten Handlungen lassen sich klassifizieren in Vorbereitung (z.B. Auftrags- und Zielklärung, Rahmenkonzept entwickeln, Einzelgespräche, schriftliche Unterlagen erstellen), Beacbtung der Teilnehmer/-innen (z.B. durch Beobachten, Wahrnehmen ihrer Rückmeldungen), allgemeine Techniken (z.B. Notizen machen, Fragen stellen, Regeln festsetzen, prototypischer Ablauf der Sitzung; vgl. Abbildung 12) und den situationsangemessenen Methodeneinsatz (z.B. wenn ein/e Teilnehmer/-in zuviel redet, wird er/sie aufgefordert, seine/ihre Aussage prägnant zusammenzufassen). Die geschilderten Techniken sind für das Konzept der expliziten Prozesskoordination von besonderem Interesse, da sie konkrete Koordinationshandlungen beinhalten. In Schritt 5 und 6 der Interviewauswertung wird näher auf sie eingegangen. 


\section{Erlernen der Gruppenunterstützung}

Befragt nach dem Erwerb ihrer Kompetenzen in der Moderation oder Führung von Gruppen berichteten die Experten/-innen, dass sie diese hauptsächlich durch Erfahrung oder/und Selbsterfabrung oder Aus- und Weiterbildung (Beratungsausbildung, Führungstrainings) erworben hätten. Es wurde auf das Lernen von anderen Personen, z.B. Vorgesetzen, hingewiesen und betont, dass nicht alle Kompetenzen abstrakt vermittelbar seien und Erfahrung oder Einstellung wesentlicher zum Kompetenzerwerb beitrügen.

Im Folgenden wird überlegt, inwieweit im Rahmen der theoretischen Generalisierung die fünf geschilderten Punkte auf das Koordinationskonzept übertagen werden können.

\section{Schritt 5 und 6: Soziologische Konzeptualisierung und theoretische Generalisierung}

Im fünften und sechsten Auswertungsschritt darf die Terminologie der Interviewten verlassen werden. Im Hinblick auf theoretisches Wissen wird systematisch nach Gemeinsamkeiten im Sinne von Kategorien gesucht und empirisch generalisiert (Meuser \& Nagel, 2005). Die in Schritt 4 gefundenen Kategorien werden auf das Konzept der expliziten Prozesskoordination übertragen. Dieser Schritt ist interpretativ und insofern schwierig, als dass während der Interviews die Terminologie der befragten Experten/-innen beibehalten werden musste (z.B. „Führung der Gruppe“, „Moderation“, „Prozesssteuerung“), nun aber auf das Konstrukt der expliziten Prozesskoordination abstrahiert werden muss. Die fünf gefundenen Themen der Gesprächsleitung von Gruppen (vgl. Abbildung 12) wurden von den Befragten im Rahmen der Führung oder Moderation berichtet. In Kapitel 3.2 wurde Koordination als der aufgabenabhängige Umgang mit Interdependenz zur effektiven Erreichung eines gemeinsamen Ziels im Sinne einer Abstimmung von Teilhandlungen und einer hierarchisch und sequentiell logischen Verarbeitung von Informationen definiert. Sowohl Führung als auch Moderation können nicht mit Koordination gleichgesetzt werden, auch wenn eine definitorische Abgrenzung schwer fällt: 
Unter Führung wird häufig die zielgerichtete Beeinflussung von Mitarbeitern/-innen, aber auch die Unterstützung von Arbeitsprozessen verstanden:

"Leadership is the process of influencing others to understand and agree about what needs to be done and how it can be done effectively, and the process of facilitating individual and collective efforts to accomplish the shared objectives" (Yukl, 2002, S. 7).

Die konzeptuelle Differenzierung von Führung und Koordination wurde bisher nicht wissenschaftlich vorgenommen. Denkbar ist es, den Begriff der Führung als übergeordnetes Konzept und die Koordination, neben Motivation, als wesentliche Aufgabe der Führung zu betrachten. Beispielsweise zählt Yukl (2002) die Koordination einer Arbeitseinheit zu den Führungsaufgaben (S. 66). Anderseits kann argumentiert werden, dass Koordination das übergeordnete Konzept darstellt, da sich die Gruppenmitglieder auch selbstorganisiert zielgerichtet koordinieren können, ohne dass eine Führung übernommen wurde (Schattenhofer, 1995). Diese basale Form der Koordination ist auch im Tierreich zu finden (Kappeler, 2006). In diesem Sinne könnte Führung einen Koordinationsmechanismus darstellen. Diese Sichtweise scheint auch von Grote und Kollegen/-innen (2003) geteilt zu werden. Sie unterscheiden neben expliziter und impliziter Koordination auch Führung und Heedful Interrelating ${ }^{8}$ als Mechanismus im Umgang mit Interdependenzen und hoher Arbeitsbelastung, wobei explizite Koordination und Führung zum Teil mit gleichen Verhaltensindikatoren gemessen werden. Es bleibt dennoch festzuhalten, dass die begriffliche Abgrenzung der Koordination von Führung ein bisher ungelöstes theoretisches Problem darstellt.

Ein vergleichbares Problem besteht in der fehlenden definitorischen Abgrenzung von Koordination und Moderation. Im Gegensatz zur Führung beinhaltet Moderation nicht eine zielgerichtete Einflussnahme von Personen, sondern die Unterstützung der Interaktion und Kommunikation der Gruppenmitglieder.

\footnotetext{
8 Auch wenn es keine eindeutige Definition dieses Konstrukts gibt, so ist darunter eine Art achtsame gegenseitige Bezugnahme während der Zusammenarbeit zu verstehen, die sich z.B. durch Feedback geben oder Hilfe anbieten zeigt (vgl. Grote et al., 2003).
} 
„Bei der Moderation handelt es sich also um eine Methode, mit der Arbeitsgruppen unterstützt werden können, ein Thema, ein Problem oder eine Aufgabe auf die Inhalte konzentriert, eigenverantwortlich, im Umgang miteinander zufrieden stellend und möglichst störungsfrei sowie an der Umsetzung in die alltägliche Praxis orientiert zu bearbeiten“" (Hartmann et al., 2000, S. 13).

Allerdings wird der Begriff der Moderation nicht einheitlich, sondern eher unspezifisch für verschiedene Arten der Steuerung oder Leitung verwendet. (Hartmann et al., 2000). Ein/e Moderator/-in ist im Unterschied zur Führungskraft inhaltlich unparteilich und nur für die methodische Unterstützung des Arbeitsprozesses zuständig. Dadurch ist er/sie in seiner/ihrer Arbeitsweise systematisch, strukturiert und offen (Edmüller \& Wilhelm, 2005). Durch die Moderation wird kein manipulativer Einfluss auf das Gruppenergebnis ausgeübt, sondern der Interaktionsprozess koordiniert. In der vorliegenden Arbeit wird davon ausgegangen, dass die Moderation im Vergleich zur Fübrung eine größere begriffliche Nähe zu Koordination aufweist. Im Folgenden werden die Terminologien der Führung und Moderation verlassen. Die von den Experten/-innen berichteten Methoden und Techniken der Leitung von Gruppendiskussionen werden auf das Koordinationskonzept übertragen. Es wird davon ausgegangen, dass Koordination sowohl während der Führung als auch während der Moderation von Arbeitsgruppen eingesetzt wird.

In Abbildung 13 wird die theoretische Generalisierung der subjektiven Koordinationstheorien dargestellt. Es zeigt sich, dass die Experten/-innen präzise Auskünfte über die Notwendigkeit von Koordinationshandlungen geben können. Sie sehen den Koordinationsbedarf bedingt durch die Gruppenaufgabe (z.B. Austausch von Informationen') und verstehen Koordination als bedeutsam für die Realisierung eines guten Interaktionsprozesses. Die Notwendigkeit effektiver Koordination ergibt sich aus bestimmten Anforderungen, die insbesondere durch die Antizipation des Verhaltens der unterschiedlichen Gruppenmitglieder wahrgenommen werden. Diese Annahmen finden sich auch in den Einstellungen gegenüber

\footnotetext{
${ }^{9} \mathrm{Zu}$ bedenken ist jedoch, dass der Informationsaustausch immer als Aufgabe, nicht jedoch als Prozess oder notwendige Bedingung einer Gruppenentscheidung, betrachtet wurde.
} 
Mitarbeiter/-innen oder Gruppenmitgliedern wieder. Deutlich wird, dass die Experten/-innen (teilweise) zu erreichende Ziele (z.B. Strukturierung der Diskussion, Ermöglichung gleichberechtigten Einbringens aller Teilnehmenden) und dazu notwendige Schritte (z.B. Fragen, Handlungsanleitungen) präzise angeben können. Es kann daher angenommen werden, dass die subjektiven Koordinationstheorien der Experten/-innen handlungstheoretisch strukturiert sind: Den zu erreichenden Zielen (z.B. Gleichberechtigung) werden Operationen auf verschiedenen Ebenen (Rederechte erteilen, bei Nicht-Einhaltung Ermahnung) zugeordnet (bierarchische Organisation) und diese werden teilweise auch in einen zeitlichen Ablauf gebracht (z.B. Kennenlernen der Teilnehmer/-innen, Vorbereitung auf die Diskussion, Beginn der Diskussion, etc.) (sequentielle Organisation). 


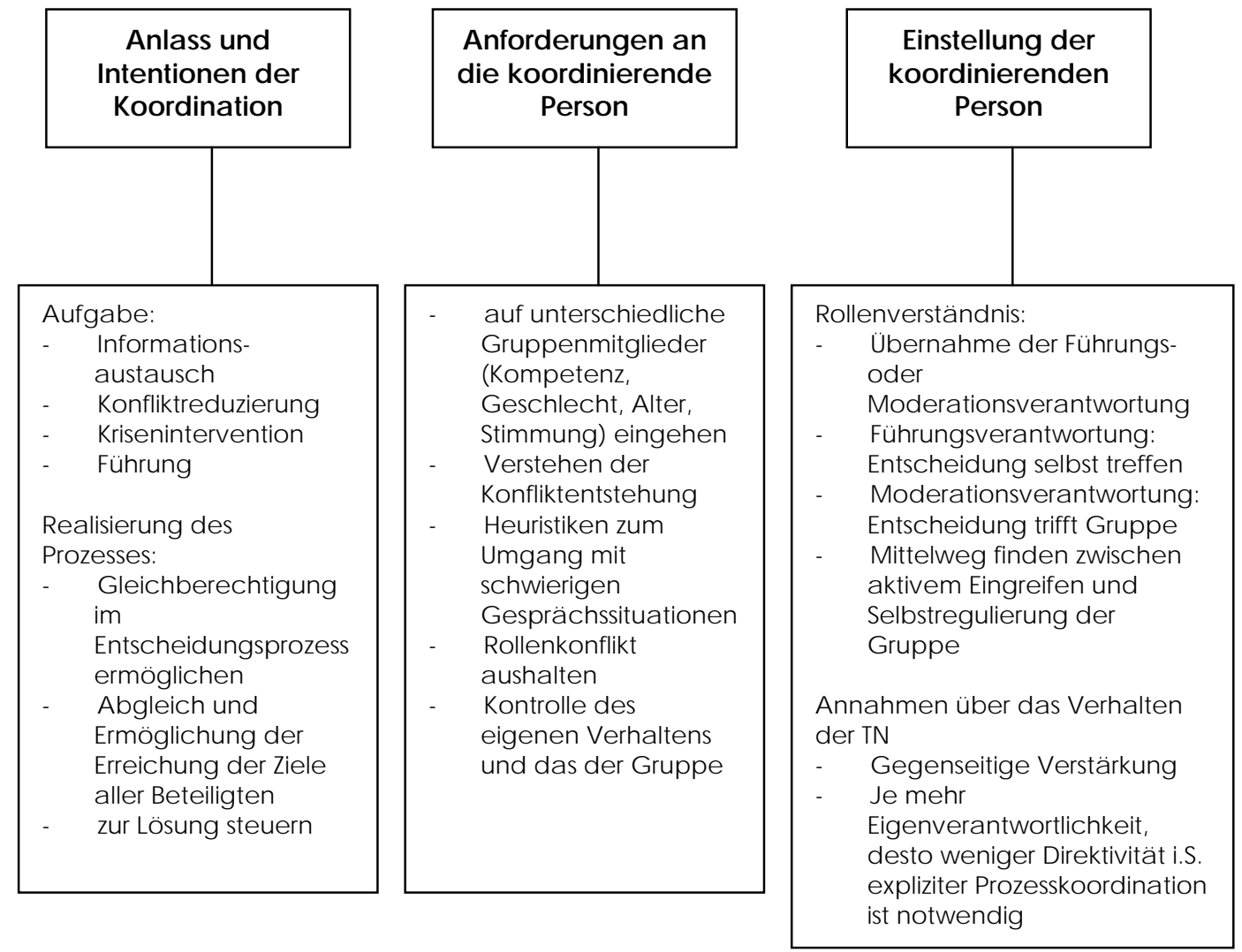

Abbildung 13: Theoretische Generalisierung der subjektiven Koordinationstheorien

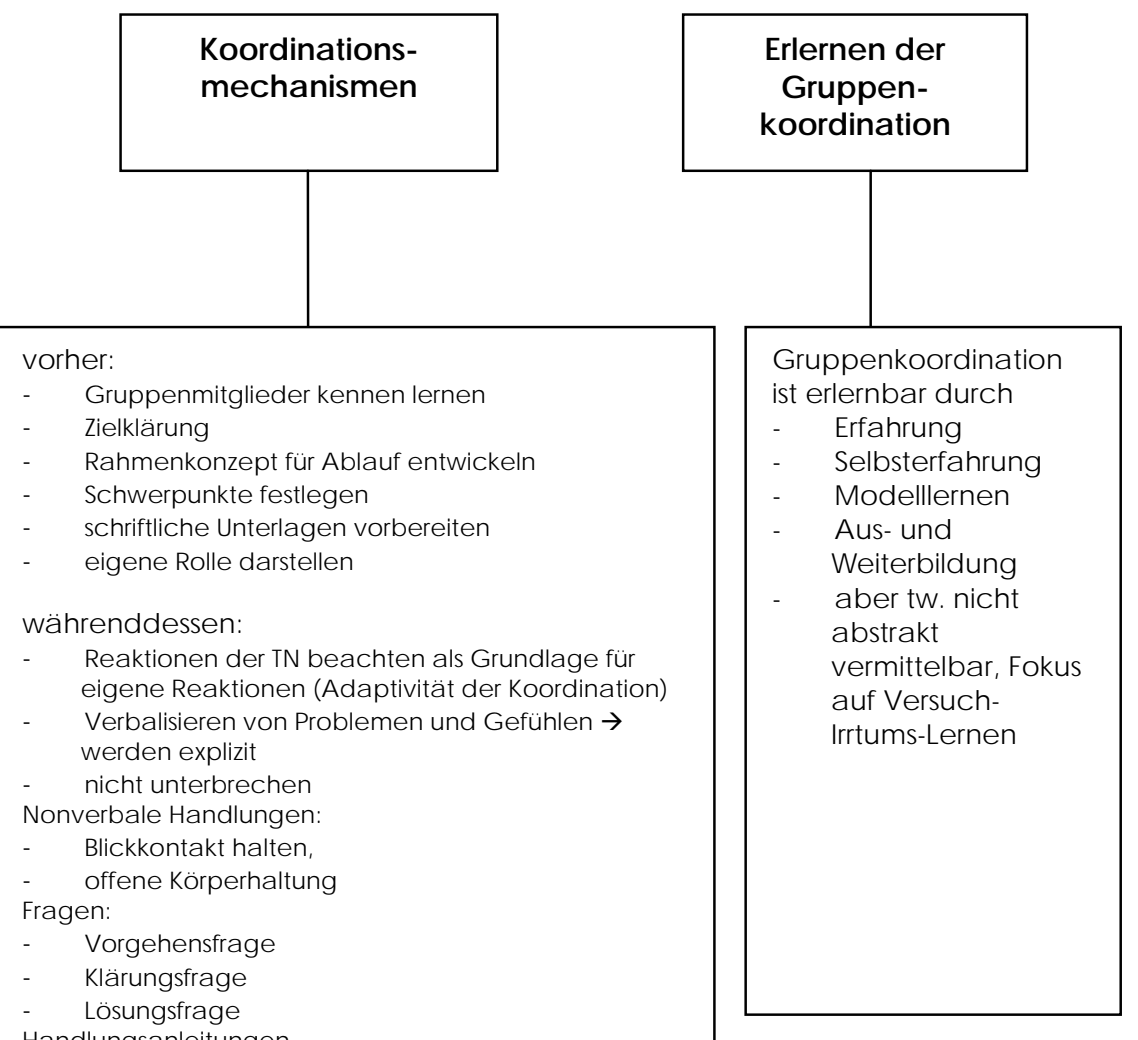

Handlungsanleitungen

anderen Wort erteilen, $\mathbb{T N}$ zum Sprechen

auffordem

Vorgehen vorschlagen, präzise Ansagen machen

Emahnen

Regeln setzen

Strukturienungen

Zel formulieren

eigenes Vorgehen begründen

Notizen machen und verwenden, Protokoll

Ablauf nach Phasen strukturieren: vgl. funktionales

Modell des Gruppenentscheidens

entsc heiden

Situationsangemessener Methodeneinsatz

/Kontingenzen

je nach Situation (z.B. TN redet viel zu lange) Koordinationsmechanismus einsetzen (aufstehen und inn um Zusammenfassung bitten) 
Im Rahmen der subjektiven Theorien interessiert insbesondere das Herstellungswissen der Experten/-innen, welches durch Wenn-Dann-Beziehungen geprägt ist (vgl. Kapitel 4.1). Folgendes konnte diesbezüglich aufgezeigt werden: Ausgehend von einem Problem (z.B. ein/e Teilnehmer/in hört nicht auf zu reden) wird eine koordinierende Intervention eingesetzt (z.B. diese/n Teilnehmer/-in um eine prägnante Zusammenfassung bitten), die mit einem bestimmten Erfolg verbunden ist (z.B. der/die Teilnehmer/-in ist nach der kurzen Zusammenfassung ruhig und die Gruppe kann weiterarbeiten). Auch speziell im Hinblick auf die explizite Prozesskoordination konnte Herstellungswissen gefunden werden: Je eigenverantwortlicher die Gruppenmitglieder wahrgenommen werden, desto weniger würden sie des direktiven Eingreifens durch den/die Gesprächsleiter/-in bedürfen.

Im Hinblick auf die Entscheidungsfindung interessiert besonders, mit welchen Mechanismen die Experten/-innen den Informationsaustausch koordinieren. Die berichteten Koordinationsmechanismen lassen sich klassifizieren (siehe Abbildung 13) in

- vorbereitende Maßnahmen, z.B. Gruppenmitglieder kennen lernen, Schwerpunkte festlegen, schriftliche Unterlagen vorbereiten,

- Mechanismen während der Diskussion, z.B.

Fragen,

Handlungsanleitungen,

Strukturierungen,

nonverbale Verhaltensweisen.

Diese Mechanismen werden je nach Situation angewendet (Herstellungswissen): Ausgehend von einem Koordinationsanlass wird ein spezifischer Koordinationsmechanismus eingesetzt, der mit einer bestimmten Konsequenz verbunden ist. Diese sequentielle Struktur wird in Abbildung 14 veranschaulicht. 


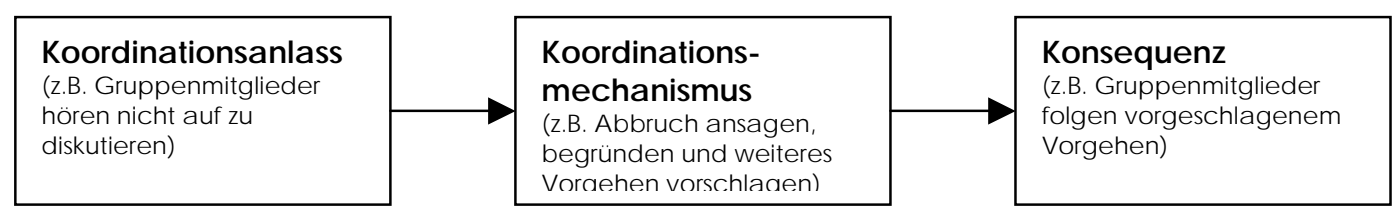

Abbildung 14: Veranschaulichung des Herstellungswissens der Experten/-innen zum situationsangemessenen Einsatz von Koordinationsmechanismen

Nach einer kurzen Zusammenfassung der Ergebnisse der Inhaltsanalyse der Interviews werden die theoretische Einordnung und Bedeutung der Ergebnisse diskutiert.

\section{Zusammenfassung der Ergebnisse der inhaltsanalytischen Auswertung der Interviews}

Es kann festgehalten werden, dass die Analyse der subjektiven Koordinationstheorien der Experten/-innen drei wesentliche Aussagen zu lässt:

1. Die Experten/-innen treffen vor und während der Koordination Annahmen über das Verhalten der zu koordinierenden Gruppenmitglieder. Diese Annahmen beziehen sie in die Wahl der geeigneten Koordinationsmechanismen ein.

2. Die subjektiven Koordinationstheorien beinhalten Intentionen, Anlässe, Einstellungen, Mechanismen und Überlegungen zur Erlernbarkeit. Es wird eine Vielzahl von Koordinationsmechanismen genannt, die als Koordinationshandlungen teilweise sowohl hierarchisch als auch sequentiell organisiert sind.

3. Die Aussagen zum Einsatz der Koordinationsmechanismen lassen sich in einer formalen Sequenz beschreiben, in der ausgehend von einem Koordinationsanlass eine Koordinationshandlung ausgeführt wird, woran sich die Wahrnehmung einer Konsequenz der Koordinationshandlung anschließt (Reaktion der Gruppe).

Die Bedeutung der Ergebnisse der Interviewstudie für das Konzept der expliziten Prozesskoordination wird im folgenden Abschnitt diskutiert. 


\subsubsection{Diskussion}

\section{Bedeutung der Ergebnisse}

In Studie 2 interessierte, inwieweit die subjektiven Koordinationstheorien der Experten/innen eher Funktionen oder konkrete Handlungen beinhalten, wann bzw. in welchen Situationen die Experten/-innen Koordinationsbedarf sehen, wie und mit welchem Grund sie auf diesen Bedarf reagieren und welche Folgen sie als aus ihrem Handeln resultierend wahrnehmen. Im Folgenden wird diskutiert, welche Erkenntnis für das Konzept der expliziten Koordination durch die zweite Studie hinzugewonnen wurden.

Die Ergebnisse der Studie 2 liefern Aussagen über die Intentionen des Einsatzes von Koordinationsmechanismen von koordinationserfahrenen Personen. Sie zeigen, dass die Experten/-innen präzise Aussagen über den Einsatz von Koordinationsmechanismen treffen. Ihre subjektiven Koordinationstheorien beinhalten den Anlass und damit verbundene Intentionen der Koordination, die zu entsprechenden Anforderungen an den/die Koordinatorin führen. Ihre Theorien beinhalten auch spezifische Einstellungen und Annahmen über die Erlernbarkeit der Gruppenkoordination. Abbildung 15 zeigt die Integration dieser Faktoren in das IPO-Modell. Es wird deutlich, dass die Experten/-innen einen bestimmten Anlass als Koordinationsauslöser betrachten, der vor allem durch die Wahrnehmung der Gruppenaufgabe bestimmt wird. Dieser Befund ähnelt den Postulaten von Espinosa et al. (2004) und Wittenbaum et al. (1998), die der Gruppenaufgabe eine moderierende Funktion bezüglich des Einsatzes von Koordinationsmechanismen zusprechen. Die subjektiven Koordinationstheorien lassen aber darauf schließen, dass die Einstellungen, Kompetenzen und Intentionen der Koordinierenden die Interpretation des Koordinationsanlasses beeinflussen und die Beziehung zwischen dem Anlass und dem Einsatz von Koordinationsmechanismen möglicherweise moderieren. 


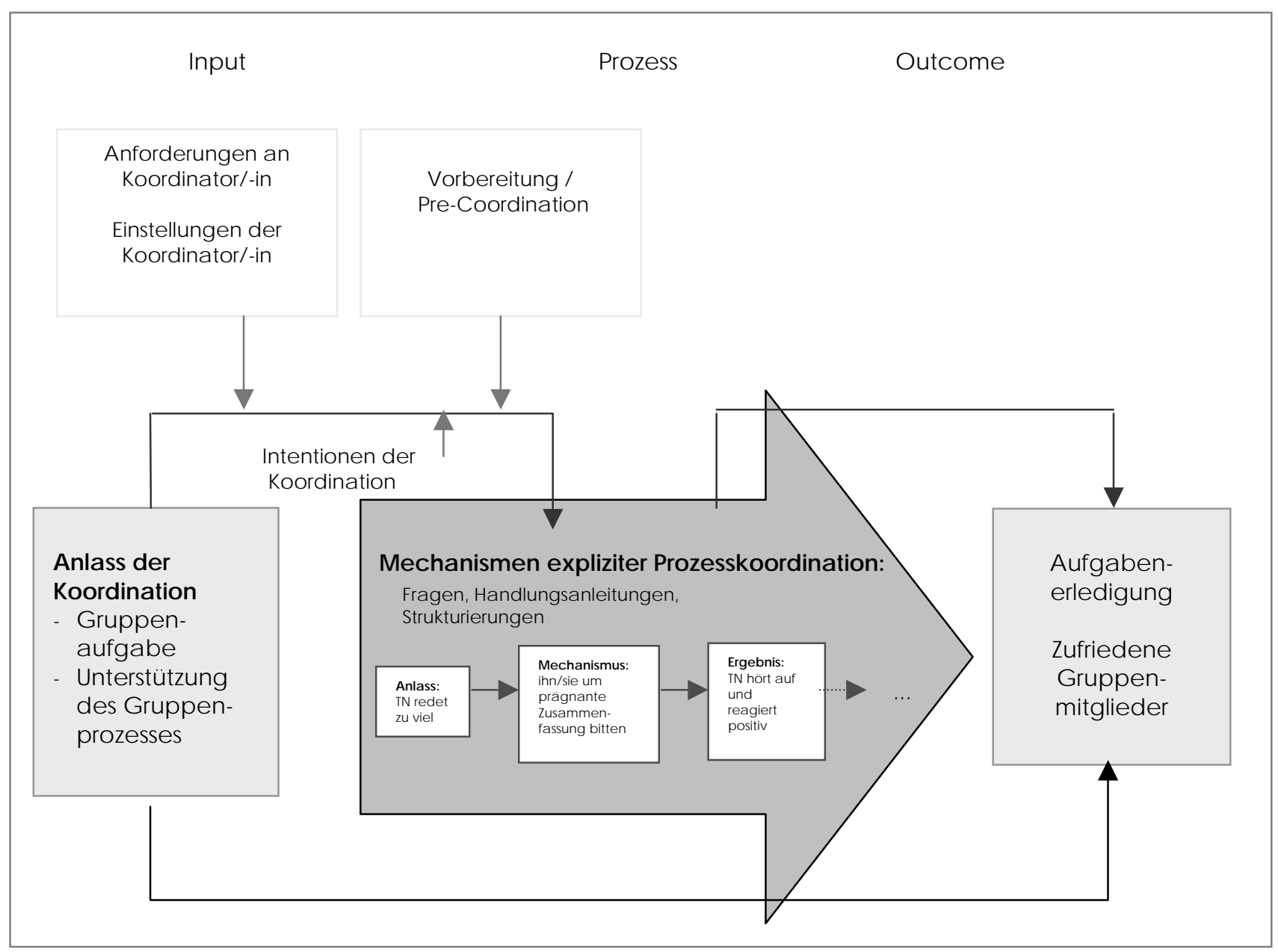

Abbildung 15: Integration der Inbalte der subjektiven Koordinationstheorien in das IPO-Modell

Neben den Einflussfaktoren der Koordination berichten die Experten/-innen verschiedene Koordinationsmechanismen. Diese können in die von Wittenbaum und Kollegen (1998) vorgeschlagene Taxonomie (Wittenbaum et al., 1998) der Koordinationsmodi eingeordnet werden: Danach koordinieren die Experten/-innen vor der Gruppendiskussion

- explizit, indem sie z.B. ein Rahmenkonzept der Diskussion und schriftliche Unterlagen zur Vorbereitung erstellen,

- implizit durch die Antizipation des Verhaltens der Gruppenmitglieder.

Während der Gruppendiskussion koordinieren die Experten/-innen

- explizit durch Fragen, Handlungsanleitungen oder Strukturierungen,

- implizit durch nonverbale Verhaltensweisen wie Blickkontakt, Körperhaltung (siehe Abbildung 16). 


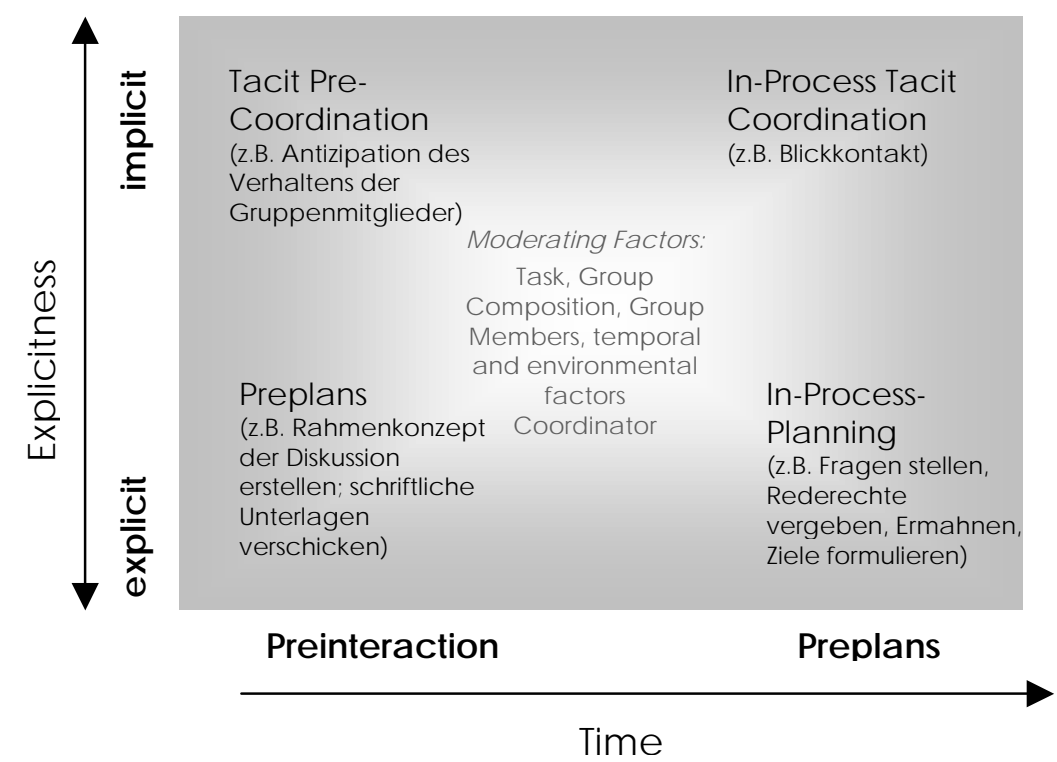

Abbildung 16: Integration von Beispielen der von den Experten/-innen berichteten Koordinationsmechanismen in die Taxonomie der Koordinationsmodi (Wittenbaum et al., 1998).

Abbildung 16 zeigt die Einordnung der von den Experten/-innen berichteten Koordinationsmechanismen in die Taxonomie von Wittenbaums und Kollegen (1998). Dabei wird deutlich, dass sich die Dimensionen Explizitheit und Zeitpunkt zur theoretischen Anordnung der Koordinationsmechanismen eignen.

Die Ergebnisse der zweiten Studie geben allerdings Anlass zu prüfen, ob Explizitheit und Zeitpunkt zur Klassifikation von Koordinationsmechanismen ausreichen. Beispielsweise berichteten die Experten/-innen, dass sie Blickkontakt bewusst zur Koordination einsetzen. Durch seinen bewussten Einsatz ist der Blickkontakt kein impliziter, aber aufgrund der fehlenden Verbalisierung auch kein expliziter Koordinationsmechanismus. Es sollte daher überlegt werden, inwieweit eine zusätzliche Dimension des bewussten Einsatzes impliziter Koordinationsmechanismen in das Modell von Wittenbaum et al. (1998) integriert werden könnte. Um die situative Effizienz der Koordinationsmechanismen bestimmen zu können, sollten ebenfalls die Intentionen der koordinierenden Person betrachtet werden. Diese Betrachtung wäre durch den Einbezug einer 
Dimension der Koordinationsintentionen möglich. Diese könnte als globale Dimension die oben vorgeschlagene Dimension des bewussten Einsatzes impliziter Koordinationsmechanismen ersetzen, da sie sowohl Intentionen über den Einsatz expliziter als auch impliziter Mechanismen beinhaltet. Da Koordination dem Umgang mit Interdependenzen während der Interaktion dient, sollte zusätzlich zur Perspektive der Koordinierenden auch die der Koordinierten im Sinne der Sozialperspektivität in ein Koordinationsmodell integriert werden. Der Grund dafür besteht in dem Einfluss gegenseitiger Koordinationserwartungen (z.B. Annahmen über die Reaktionen der Gruppenmitglieder auf den Koordinationsmechanismus) auf die Koordinationshandlungen und reaktionen.

Die Ergebnisse der zweiten Studie zeigen den Ergänzungsbedarf der von Wittenbaum et al. (1998) vorgeschlagenen Taxonomie. Sie zeigen auch, dass die Dynamik der Verwendung der Koordinationsmechanismen während der Interaktion eine bedeutende Rolle spielt, die in Wittenbaums Taxonomie nicht berücksichtigt ist. Abbildung 14 (S. 104) und Abbildung 15 (S. 106) veranschaulichen, dass die Experten/-innen die Koordinationsmechanismen ausgehend von einer bestimmten Situation bzw. einem Koordinationsanlass einsetzen und der Einsatz mit einer bestimmten Konsequenz verbunden ist, die ihrerseits wieder zur Verwendung eines weiteren Koordinationsmechanismus führt. Solche Mikro-Koordinationszyklen finden während des gesamten Koordinationsprozesses wiederholt statt. Diese Iteration während des Koordinationsprozesses sowie die situationale Abhängigkeit und die Folgen der Koordinationsmechanismen sollten ebenfalls in ein Koordinationsmodell integriert werden, um die Effektivität und letztlich auch die Effizienz der Koordinationsmechanismen überprüfen zu können.

Insgesamt konnte in Studie 2 gezeigt werden, dass die Experten/-innen neben ihren Funktionen in der Koordination im Gegensatz zu den Schüler/-innen in Studie 1 vor und während der Koordination Annahmen über das Verbalten der zu koordinierenden Gruppenmitglieder treffen. Diese Annahmen beziehen sie in die Wahl der geeigneten Koordinationsmechanismen ein. Darüber 
hinaus berichten sie eine Vielzahl von Koordinationsmechanismen, die als Koordinationshandlungen teilweise sowohl hierarchisch als auch sequentiell organisiert und in Form eines Herstellungswissens strukturiert sind: Ausgehend von einem Koordinationsanlass wird eine Koordinationshandlung ausgeführt, woran sich die Wahrnehmung einer Konsequenz der Handlung anschließt.

Im Hinblick auf die explizite Prozesskoordination des Informationsaustauschs werden Verbalisierungen, Fragen, Handlungsanleitungen und Strukturierungen berichtet. Dabei wurde auf die Gefahr des Verlustes von Informationen während der Diskussion in keinem Fall hingewiesen. Lediglich in einem Fall wurde die Bedeutung schriftlicher Unterlagen und ihrer Vervollständigung betont. Im Folgenden wird überlegt, inwieweit durch eine veränderte Methodik noch mehr und spezifischeres Herstellungswissen zur Koordination des Informationsaustauschs hätte eruiert werden können.

\section{Das fokussierte Interview als Methode der Wabl}

„Eine methodische Schwierigkeit der Expertenforschung besteht darin, dass Experten ,gekonnt' und ,intelligent' handeln, aber das Wissen, das sie dazu benötigen, häufig nicht vollständig angeben können" (Bromme, 1992, S. 121).

Die in Studie 2 gewonnenen Ergebnisse lassen umfangreiche Schlüsse über subjektive Koordinationstheorien von Experten/-innen zu. Dennoch kann nicht davon ausgegangen werden, dass die jeweiligen Koordinationsintentionen und -heuristiken vollständig erfasst werden konnten. Subjektive Theorien müssen nicht prinzipiell bewusstseinspflichtig und verbalisierbar sein (Groeben, 1986). Daher stößt das Interview als Erhebungsmethode an seine Grenzen, denn die Handlungsziele sind zwar stets bewusstseinspflichtig, die konkreten Handlungen zur Zielerreichung müssen jedoch nicht unbedingt bewusst und verbalisierbar sein (Cranach et al., 1980). Dieses Phänomen gilt insbesondere für die subjektiven Expertentheorien: Es muss davon ausgegangen werden, dass die Handlungen mit zunehmender Erfahrung automatisiert und die 
automatisierten Handlungskomponenten in größere Einheiten integriert werden, die der bewussten Kontrolle wieder zugänglich sind (Vallacher \& Wegner, 1987).

"In rendering actions progressively more familiar, more automatic, and otherwise easier to do, experience enables action to be understood in terms that transcend the action's mechanistic underpinnings and highlight instead its potential meanings, effects, and implications" (Vallacher \& Wegner, 1987, S. 8).

Daher sind die konkreten Koordinationshandlungen den Experten/-innen auf der operationalen Ebene weniger zugänglich. Dieses Phänomen zeigte sich in einigen Interviews. Die konkreten Verhaltensweisen, die die Experten/-innen zur Koordination einsetzen, konnten größtenteils nur durch wiederholtes Nachfragen der Interviewerin eruiert werden. Dennoch wurden häufig eher Ziele als Verhaltensweisen genannt. Es zeigte sich auch, dass die Experten/innen, die ein spezielles Gruppenmoderationstraining absolviert hatten, über mehr Herstellungswissen als andere berichteten. Dieser Befund weist auf die Erlernbarkeit des Einsatzes von Gruppenkoordinationsmechanismen hin.

Im Hinblick auf die eingeschränkte Anzahl berichteter Mechanismen, mit denen speziell der Informationsaustausch während der Entscheidungsfindung koordiniert wird, wäre der Einsatz einer anderen Methode eventuell angemessener gewesen: In einem Stimulated Recall hätte das Verhalten der Experten/-innen während ihrer Gruppenkoordination gefilmt und sie anschließend anhand des Films zu den Gründen ihres Verhaltens befragt werden können. Dadurch hätten die Experten/-innen möglicherweise die Situation der Entscheidungsfindung stärker fokussiert und mehr über die eventuell unbewusste Koordination des notwendigen Informationsaustauschs berichtet.

Bezüglich der Inhalte der subjektiven Koordinationstheorien fällt auf, dass diese sich nicht nur auf Entscheidungsaufgaben beziehen. Sie beinhalten auch den Umgang mit Konflikten. Angesichts dieses Befundes sollte zukünftig überlegt werden, inwieweit der Umgang mit Interessenskonflikten stärkeres Gewicht in der Forschung zum Informationsaustausch und zur Entscheidungsfindung bekommen sollte. 
Trotz der zu bedenkenden Alternative des Stimulated Recall, stellte das fokussierte Interview im Rahmen der vorliegenden Studie eine geeignete Methode zur Erfassung subjektiver Theorien von Experten/-innen dar. Die Vorteile der Methode bestanden einerseits in der großen Akzeptanz auf Seiten der Befragten, andererseits konnte die Interviewerin durch gezieltes Nachfragen zu den interessierenden Inhalten gelangen.

Nachdem die ersten beiden Studien präsentiert wurden, werden ihre wesentlichen Ergebnisse und deren Bedeutung für das weitere Vorgehen im folgenden Abschnitt beschrieben.

\subsection{Studie 1 und Studie 2 - Implikationen der Studien zur Erfassung subjektiver Koordinationstheorien}

Die Erhebung subjektiver Theorien wird als Verfahren der Phänomenbeschreibung und Theorieentwicklung empfohlen (Bartholomew et al., 2000; Froschauer \& Lueger, 1998). Die subjektiven Theorien zur expliziten Prozesskoordination von Entscheidungsfindungsgruppen bildeten den Fokus der Studien 1 und 2. Zunächst wird auf die Bedeutung der wesentlichen Ergebnisse der Studien eingegangen. Anschließend werden ihre Implikationen für das weitere Vorgehen im Rahmen der vorliegenden Arbeit diskutiert.

\subsubsection{Bedeutung der Ergebnisse}

Ausgangspunkt der ersten beiden Studien war die Überlegung, dass explizite Prozesskoordination nicht geschiebt, sondern aktiv von Personen mit der Intention zur Koordination eingesetzt wird. Diese persönlichen Koordinationsintentionen wurden als bandlungsleitende Kognitionen bzw. subjektive Theorien bezeichnet. Das Ziel der ersten beiden Studien bestand in der Rekonstruktion der subjektiven Koordinationstheorien und geht der Untersuchung der beobachtbaren Aspekte der Koordinationshandlungen (Studie 3) voraus. Die subjektiven Koordinationstheorien geben Auskunft über die Intentionalität und die Reflexivität der Koordination. Es wurde überprüft, welche Koordinationshandlungen koordinierenden 
Personen bekannt sind, mit welchem Ziel die Handlungen eingesetzt werden und welche Wirkung als daraus resultierend wahrgenommen wird. Durch die Beantwortung dieser Fragen sollte sowohl das Konstrukt expliziter Prozesskoordination im Hinblick auf folgende Wirksamkeitsuntersuchungen differenziert als auch die Entwicklung von entsprechenden Beobachtungsverfahren ermöglicht werden. Dabei wurde der Forderung nach maximaler struktureller Variation der Perspektiven (vgl. Kleining \& Witt, 2001) nachgekommen, indem unterschiedliche Personengruppen befragt wurden: Experten/-innen und Nicht-Experten/-innen der Koordination (vgl. Zempel, 2002). Durch die Kontrastierung ihrer subjektiven Koordinationstheorien werden sowohl Aussagen über allgemeines handlungssteuerndes Wissen über Gruppenkoordination als auch über spezielle, erfahrungsbedingte handlungssteuernde Kognitionen möglich.

In Studie 1 wurden subjektive Koordinationstheorien von Nicht-Experten/-innen der Gruppenkoordination erhoben. Es konnte gezeigt werden, dass ihr allgemeines, nichterfahrungsgebundenes, handlungssteuerndes Wissen zur Koordination einer Gruppenentscheidungsfindung im wesentlichen Handlungen expliziter Prozesskoordination (Handlungsanleitungen, Strukturierungen des Problemlöseprozesses und Konfliktvermeidung) beinhaltet. Diese Verhaltensweisen sind explizit, da sie den Interaktionsprozess durch deutliche verbale Instruktionen (z.B. Rederechte vergeben, Regeln festlegen) oder Strukturierungen (z.B. Fragen stellen, Zusammenfassen, Wiederholen oder Pro- und Kontra-Listen erstellen) koordinieren, mit Koordinationsabsicht ausgeführt werden (vgl. Espinosa et al., 2004) und verbal kommuniziert werden (vgl. Wittenbaum et al., 1998). Dieses Ergebnis steht im Einklang mit den Postulaten Wittenbaums et al. (1998), wonach unsichere Gruppenaufgaben mit herausfordernden Zielen und notwendiger interdependenter Zusammenarbeit vermehrt expliziter Koordination bedürfen. Allerdings zeigte sich auch, dass die subjektiven Theorien der Nicht-Experten/-innen, neben einigen spezifischen Koordinationshandlungen, hauptsächlich Funktionen der diskussionsleitenden Person (z.B. für Gleichberechtigung sorgen, Streit vermeiden, für Konsens und Kompromiss sorgen) und 
Verhaltensweisen, die nicht ausgeführt werden sollten (z.B. nicht selbst Stellung beriehen) beinhalten.

Darüber hinaus fällt auf, dass die Koordinationsmechanismen Wiederbolen, Zusammenfassen und Fragen stellen in den Antworten auf die explorative Frage nach konkreten Koordinationshandlungen nur selten genannt wurden.

Anschließend wurden in Studie 2 die erfahrungsbedingten subjektiven Koordinationstheorien von in der Gruppenkoordination erfahrenen Führungskräften und Moderatoren/-innen erhoben. Es zeigte sich, dass die Experten/-innen, im Gegensatz zu den Schüler/-innen in Studie 1, neben ihren Funktionen in der Koordination vor und während der Koordination Annahmen über das Verbalten der zu koordinierenden Gruppenmitglieder treffen, die sie in die Wahl der geeigneten Koordinationsmechanismen einbeziehen. Die Experten/-innen berichteten im Vergleich zu den Nicht-Experten/-innen mehr explizite Koordinationsmechanismen (Handlungsanleitungen, Fragen, Strukturierungen), die als Koordinationshandlungen teilweise sowohl hierarchisch als auch sequentiell bzw. in Form eines Herstellungswissens organisiert sind: Ausgehend von der Wahrnehmung eines Koordinationsanlasses wird eine Koordinationshandlung ausgeführt, woran sich die Wahrnehmung einer Konsequenz der Koordinationshandlung anschließt. Anhand des Vergleiches der Ergebnisse der beiden Studien kann in Anlehnung an Müller (2003) davon ausgegangen werden, dass subjektive Theorien durch Erfahrung veränderbar sind.

Den subjektiven Theorien der Nicht-Experten/-innen und Experten/-innen ist gemein, dass der Umgang mit Interessenskonflikten während des Gruppen(entscheidungs)prozesses einen wesentlichen Bestandteil der Koordination darstellt. Da Interessenskonflikte ein Merkmal von Gruppenentscheidungen sind, sollte nicht nur die Koordination des Informationsaustausches, sondern auch des Konfliktausgleichs, Gegenstand der Koordinationsforschung werden. Die Ergebnisse beider Studien weisen darauf hin, dass die Koordination des Konfliktausgleichs insbesondere durch explizite Prozesskoordination (z.B. Verbalisierung von Emotionen und Gefühlen, Vergabe von Rederechten, Ermahnen) möglich ist. 
Neben dem Einbezug des Umgangs mit Konflikten fällt in beiden Studien auf, dass im Hinblick auf die Koordination des Informationsaustauschs plausibel erscheinende Mechanismen (Fragen, Zusammenfassen, Wiederholen, Themenliste erstellen) nur teilweise genannt werden. Die NichtExperten/-innen berichten Mechanismen wie Pro- und Kontraliste erstellen, Argumente im Wechsel vortragen lassen oder thematisch strukturieren. Die Experten/-innen betonten die Bedeutung des Stellens von Fragen und der Formulierung von Zielen. Der in der Moderation jedoch häufig empfohlene Mechanismus des Zusammenfassens bisher genannter Äußerungen wurde nicht genannt. Ebenso selten wurden Methoden zur Sicherung des Besprochenen durch Wiederholung oder Verschriftlichung genannt.

Bezug nehmend auf das Ziel der Präzisierung des Konzepts der expliziten Prozesskoordination werden in Tabelle 5 alle bisher eruierten Mechanismen zur expliziten Koordination des Entscheidungsprozesses zusammengefasst.

Tabelle 5: Mechanismen expliziter Prozesskoordination von Gruppenentscheidungsprozessen

\begin{tabular}{|c|c|c|c|c|}
\hline \multirow{2}{*}{$\begin{array}{l}\text { Vor der } \\
\text { Interaktion }\end{array}$} & \multicolumn{4}{|c|}{ Während der Interaktion } \\
\hline & $\begin{array}{l}\text { Handlungs- } \\
\text { anleitungen }\end{array}$ & Strukturierungen & Fragen & Andere \\
\hline $\begin{array}{l}\text { Ziel klären } \\
\text { Ablaufplan } \\
\text { entwickeln } \\
\text { Schwerpunkte } \\
\text { festlegen } \\
\text { Schriftliche } \\
\text { Unterlagen } \\
\text { vorbereiten } \\
\text { Rollenklärung }\end{array}$ & $\begin{array}{l}\text { Argumente in einer } \\
\text { Reihenfolge } \\
\text { vortragen lassen } \\
\text { Jemanden zum } \\
\text { Sprechen } \\
\text { auffordern } \\
\text { Rederechte } \\
\text { vergeben } \\
\text { Regeln festlegen } \\
\text { Zeitlimits festlegen } \\
\text { Ermahnen }\end{array}$ & $\begin{array}{l}\text { Ziel formulieren } \\
\text { Eigenes Vorgehen } \\
\text { begründen } \\
\text { Pro- und Kontra- } \\
\text { Liste erstellen } \\
\text { Wiederholen } \\
\text { Zusammenfassen } \\
\text { Sammeln von } \\
\text { Lösungen } \\
\text { Ablauf nach Phasen } \\
\text { strukturieren } \\
\text { Notizen machen / } \\
\text { schriftliche } \\
\text { Unterlagen ergänzen } \\
\text { Entscheiden } \\
\text { Protokoll erstellen } \\
\text { und unterschreiben } \\
\text { lassen }\end{array}$ & $\begin{array}{l}\text { Vorgehensfrage } \\
\text { Klärungsfrage } \\
\text { Lösungsfrage }\end{array}$ & $\begin{array}{l}\text { Nicht } \\
\text { unterbrechen } \\
\text { Verbalisieren } \\
\text { Reaktionen der } \\
\text { Gruppenmitglieder } \\
\text { beobachten }\end{array}$ \\
\hline
\end{tabular}


Im Folgenden werden die Implikationen der Studien 1 und 2 für das weitere Vorgehen im Rahmen der vorliegenden Arbeit beschrieben.

\subsubsection{Implikationen für das weitere Vorgehen}

Die Ergebnisse der Studien 1 und 2 zeigen, welche Mechanismen zur expliziten Koordination von Gruppenentscheidungsprozessen eingesetzt werden können. Offen bleibt allerdings, zu welchen Konsequenzen der Einsatz dieser Mechanismen führt. Daher muss im Folgenden überprüft werden, ob der Informationsaustausch und die Entscheidungsfindung durch die Verwendung expliziter Koordinationsmechanismen tatsächlich verbessert werden. Dazu wird in Studie 3 ein Experiment dargestellt, in dem zwei Mechanismen expliziter Prozesskoordination auf ihre Wirksamkeit überprüft wurden.

Angesichts der Vielzahl berichteter expliziter Koordinationsmechanismen liegt es außerdem nahe, deren tatsächlichen Einsatz im Verlauf von Gruppenentscheidungsprozessen zu verfolgen. Daher wird in Kapitel 6 die Entwicklung eines Klassifikationssystems beschrieben, welches die Erfassung expliziter und impliziter Koordinationsmechanismen in Gruppendiskussionen erlaubt. Dadurch wird die systematische Untersuchung des Auftretens und Wechselspiels von expliziten und impliziten Koordinationsmechanismen möglich. Somit können einerseits Bedingungen und Konsequenzen des Mechanismeneinsatzes geprüft werden. Andererseits können erfolgreiche und nicht erfolgreiche Entscheidungsgruppen bezüglich ihrer Koordination verglichen werden. 


\section{$5 \quad$ Studie 3: Der Einfluss von Koordinationsmechanismen auf die Entscheidungsfindung - eine experimentelle Überprüfung}

Die Studien 1 und 2 haben gezeigt, dass Moderatoren/-innen und Führungskräfte eine ganze Reihe von Mechanismen zur expliziten Koordination von Gruppenentscheidungsprozessen einsetzen. Bisher ist allerdings offen, ob der Entscheidungsprozess durch den Einsatz solcher Koordinationsmechanismen tatsächlich verbessert wird. Daher geht es in der folgenden Studie nicht mehr um die individuelle Bedeutung von Koordination. Der Betrachtungsfokus liegt statt dessen auf den Folgen der Koordinationshandlung für die Gruppenentscheidung. Es wird der Frage nachgegangen, ob die Mechanismen expliziter Prozesskoordination den Informationsaustausch tatsächlich unterstützen und die Gruppenentscheidung verbessern. Zur Beantwortung dieser Frage wurde ein Experiment durchgeführt, in dem zwei Mechanismen expliziter Prozesskoordination auf ihre Wirksamkeit überprüft wurden.

Nach einigen theoretischen Überlegungen (Kapitel 5.1) werden die Hypothesen abgeleitet (Kapitel 5.2). Anschließend werden die experimentelle Methodik (Kapitel 5.3) beschrieben, die Ergebnisse erläutert (Kapitel 5.4) und diskutiert (Kapitel 5.5).

\subsection{Theoretische Überlegungen}

In Kapitel 2 und 3 wurde ausführlich erläutert, dass Gruppenentscheidungen sehr komplexe Aufgaben sind, welche mit hohen Koordinationsanforderungen an die Gruppe verbunden sind. Wesentlich für eine gute Gruppenentscheidung ist ein umfassender Austausch von entscheidungsrelevanten Informationen der Gruppenmitglieder. Empirische Befunde zeigen, dass sowohl der Informationsaustausch als auch die Gruppenentscheidungen häufig nicht optimal sind (zur aktuellen Übersicht der Befunde vgl. Kerschreiter et al., 2003; Mojzisch et al., 2004; Mojzisch \& Schulz-Hardt, 2006; Stasser \& Titus, 2006). Es wird daher nach Interventionsmöglichkeiten gesucht, die den Entscheidungsprozess verbessern sollen. 
Beispielsweise untersuchten Schweiger, Sandberg und Ragan (1986) die Wirksamkeit von drei verschiedenen Interventionsmethoden: Dialektisches Fragen, die Methode des Teufelsanwalts und des Konsensus (zum Inhalt der Methoden siehe Schweiger et al., 1986). Sie konnten zeigen, dass sowohl das Dialektisches Fragen als auch die Teufelsanwaltsmethode zu höherer Entscheidungsqualität führten, die Konsensusmethode führte hingegen zu einer höheren Zufriedenheit der Gruppenmitglieder und zu einer größeren Akzeptanz der Gruppenentscheidung. Ein „programmed conflict“ kann die Entscheidungsqualität also erhöhen (Schweiger et al., 1986, S. 66). Eine konsensorientierte Intervention verbessert nicht die Entscheidung, sondern die Zufriedenheit der Gruppenmitglieder. Ähnliche Studien bestätigen diese Befunde (Schweiger \& Sandberg, 1989; Schweiger, Sandberg \& Rechner, 1988). Inwieweit durch die Teufelsanwaltsmethode neben der Entscheidungsqualität auch der Informationsaustausch verbessert wird, haben Greitemeyer, Brodbeck, Schulz-Hardt und Frey untersucht (2006). Sie zeigten, dass die Teufelsanwaltsmethode im Gegensatz zur freien Diskussion den Informationsaustausch verbessert (höherer Austausch geteilter und ungeteilter Informationen). Der Nennungsvorteil der geteilten Informationen verschwand jedoch auch unter der Intervention nicht, wurde allerdings mit zunehmender Erfahrung der Gruppe in der Teufelsanwaltsmethode schwächer (Greitemeyer et al., 2006). Die Entscheidungsqualität konnte durch den Einsatz der Intervention allerdings nicht verbessert werden, auch nicht bei zunehmender Erfahrung der Gruppe in der Methode (Greitemeyer et al., 2006).

Neben Interventionstechniken wurde auch das Verhalten der Führungskraft als unabhängige Variable im Hinblick auf die Gruppenentscheidung untersucht. Larson, FosterFishman und Franz (1998b) untersuchten, welche Auswirkungen zwei unterschiedliche Führungsstile (partizipativ vs. direktiv) auf den Informationsaustausch und die Gruppenentscheidung ausüben. Sie konnten zeigen, dass Gruppen, die von einer partizipativen Führungskraft geleitet wurden, im Vergleich zu direktiv geleiteten Gruppen sowohl mehr geteilte als auch ungeteilte Informationen diskutierten (Larson et al., 1998b). Partizipative Führungskräfte 
binden die Gruppenmitglieder in den Entscheidungsprozess mit ein und teilen dadurch ihre Macht. Direktive Führungskräfte ermutigen die Gruppenmitglieder hingegen nicht, sich in die Diskussion einzubringen. Statt dessen nutzen sie die Diskussion, um ihre eigene Entscheidungspräferenz durchzusetzen. In den Ergebnissen zeigte sich, dass Gruppen mit einer direktiven Führungskraft, deren Informationen die richtige Entscheidungsalternative implizierten, bessere Entscheidungen trafen. Besaß die direktive Führungskraft allerdings die falschen Informationen, so traf auch die Gruppe die falsche Entscheidung. Bei partizipativ geleiteten Gruppen zeigte sich kein Einfluss der initialen Präferenz der Führungskraft (Larson et al., 1998b). Die Ergebnisse dieser Studie weisen auf zwei wesentliche Punkte hin:

1. Weder partizipativer noch direktiver Führungsstil garantieren einen guten Informationsaustausch oder eine gute Gruppenentscheidung.

2. Es besteht eine Interaktion zwischen dem Führungsstil und dem Wissen einer Führungskraft: Besitzt eine direktive Führungskraft die richtigen Informationen, so ist auch die Wahrscheinlichkeit einer richtigen Gruppenentscheidung hoch. Verfügt sie allerdings über falsche Informationen, so sinkt die Wahrscheinlichkeit einer richtigen Gruppenentscheidung (Cruz, Henningsen \& Smith, 1999). Im Hinblick auf den Führungsalltag ist zu fragen, inwieweit Führungspersonen tatsächlich über die entscheidungsrelevanten und wichtigen Informationen verfügen. Schließlich besteht ihre Rolle per Definition nicht in der Expertise sondern in der Führung.

Die Funktion der Steuerung einer Diskussion wird häufig von Personen übernommen, die in der Gruppe eine besondere Rolle innehaben, weil sie entweder die Führungskraft sind oder als externe Moderatoren/-innen zur Unterstützung und Leistung der Gruppendiskussion hinzugezogen werden (Boos, 1996b). Auf die notwendige Trennung von Führung und Diskussionsleitung hat bereits Maier (1967) hingewiesen. Er betont, dass Führungskräfte während der Gruppenleitung Gefahr laufen, ihre Position auszunutzen und ihre eigenen Ansichten durchzusetzen. Auch Interaktionsprozessanalysen des Problemlösens und 
Entscheidens in Gruppen zeigten, dass die Führungsform (z.B. Vorgesetzte/r entscheidet nach Diskussion allein) einen Einfluss auf die Entscheidungsqualität ausübt (Boos, Scharpf \& Fisch, 1991). Diskussionsleiter/-innen verfügen durch ihre für die Gruppe besondere Position über hohe Positionsmacht und damit über Möglichkeiten der hierarchischen Steuerung der Gruppe (Boos, 1994). Während des Entscheidungsprozesses sollte die Gruppe daher von einer inhaltlich neutralen Person, einem/r Moderator/-in, übernommen werden (Maier, 1967). Im Gegensatz zur Führungskraft trägt ein/e Moderator/-in nicht selbst inhaltlich zur Problemlösung oder Entscheidungsfindung bei, sondern konzentriert sich auf den Prozess der Diskussion (Boos, 1996a). Durch seine/ihre Unparteilichkeit kann der/die Moderator/-in seine/ihre Aufmerksamkeit auf die Koordination des Entscheidungsprozesses legen (vgl. Ergebnisse Studie 2 in Kapitel 4.3.2). Es wird davon ausgegangen, dass Moderationstechniken eine Möglichkeit darstellen, Gruppenentscheidungen zu verbessern (Kerschreiter et al., 2003). In diesem Zusammenhang ist die Bedeutung von Koordination bisher vernachlässigt wurden.

Koordination wird als der aufgabenabhängige Umgang mit Interdependenz zur effektiven Erreichung eines gemeinsamen Ziels definiert. Sie dient speziell der Abstimmung von Teilhandlungen und einer hierarchisch und sequentiell logischen Verarbeitung von Informationen (vgl. Kapitel 3.2). Die Koordination stellt daher eine Aufgabe der Moderation dar. In der vorliegenden Studie werden zwei Mechanismen von einem/r Moderator/-in zur Koordination des Informationsaustauschs eingesetzt. Welche spezifischen Erwartungen damit verbunden sind, wird im Folgenden erläutert. 


\subsection{Fragestellung und Ableitung der Hypothesen}

Ausgehend von dem empirisch vielfach bestätigten Fund, dass Gruppen das Potential des Informationsaustausches nicht nutzen und die Gruppenentscheidung dadurch suboptimal ist, ist es notwendig, Faktoren zu identifizieren, welche die Koordination des Entscheidungsfindungsprozesses ermöglichen. Da die Entscheidungsfindung eine komplexe und interdependente Aufgabe ist, sind die Koordinationsanforderungen vergleichsweise hoch. Insbesondere der Austausch von Informationen und deren Bedeutungen muss koordiniert werden, wofür sich explizite Koordinationsmechanismen anbieten (Wittenbaum et al., 1998). Der Einsatz expliziter Koordinationsmechanismen soll dazu dienen, dass für die Entscheidung wesentliche Informationen in die Diskussion eingebracht werden und während der Diskussion nicht verloren gehen. Das Einbringen und Festhalten von Informationen ist zur Erfüllung aller kritischen Funktionen im Rahmen des Entscheidungsprozesses (Problemanalyse, Festlegung der Bewertungskriterien, Generierung von Entscheidungsalternativen und Evaluation ihrer positiven und negativen Konsequenzen, vgl. Hirokawa, 1985; Orlitzky \& Hirokawa, 2001) erforderlich. In dem folgenden Experiment soll untersucht werden, inwieweit explizite Koordinationsmechanismen (Espinosa et al., 2004; Wittenbaum et al., 1998) den Entscheidungsfindungsprozess und die Entscheidungsgüte verbessern. Es soll die Frage beantwortet werden, welchen Einfluss explizite Koordinationsmechanismen auf die Ergebnisse der Gruppenarbeit bei einer Entscheidungsaufgabe ausüben.

Um dem Nennungsvorteil der geteilten Informationen (Larson et al., 1994; Mojzisch \& Schulz-Hardt, 2006; Stasser \& Titus, 1985) vorzubeugen und das Einbringen von ungeteilten Informationen in den Diskussionsprozess zu ermöglichen, bietet es sich an, während der Diskussion systematisch Informationen zu erfragen (Kerschreiter et al., 2003 ). So kann dazu beigetragen werden, dass der Informationsaustausch sowohl quantitativ als auch qualitativ (ungeteilt bzw. präferenzinkonsistente) verbessert wird. Da Gruppenmitglieder häufig 
präferenzkonsistente Informationen erfragen, die insbesondere bei Hidden-Profile-Aufgaben die falsche Entscheidung implizieren, sollte systematisch sowohl nach geteilten wie ungeteilten als auch präferenzkonsistenten wie -inkonsistenten Informationen gefragt werden, wodurch auch der Gefahr eines vorschnellen Konsenses auf eine falsche Alternative (Gigone \& Hastie, 1993) vorgebeugt wird. Dies ist einer am Entscheidungsprozess beteiligten Person nicht möglich, da sie ihre eigenen Ziele und Präferenzen verfolgen wird (Larson et al., 1998b). Daher sollte das Erfragen von Informationen durch eine/n inhaltlich neutrale/n und nicht in die Entscheidungsaufgabe involvierte/n Moderator/-in geschehen. Dafür spricht auch der Befund, dass die Interessen und das Verhalten einer Führungsperson während des Gruppenprozesses ein besonderes Gewicht haben (Boos, 1996b; Cruz et al., 1999; Henningsen, Henningsen, Jakobsen \& Borton, 2004), das nicht immer positiv auf die effektive Entscheidungsfindung wirkt (Larson et al., 1998b). Damit entspräche das Vorgehen auch dem zunehmenden Trend in Organisationen, zur Unterstützung von Gruppenprozessen eine/e inhaltlich neutral/e, aber in der Diskussionsleitung erfahrene/n Moderator/-in zu engagieren, der/die selbstverständlich auch nicht einschätzen kann, inwieweit eine Information geteilt oder ungeteilt ist.

Das regelmäßige Erfragen von Informationen durch den/die Moderator/-in bildet die erste unabhängige Variable der Untersuchung.

$\mathrm{PH}_{1}$ : Gruppen, in denen ein/-e Moderator/-in zusätəlich zu ausgewählten allgemeinen Gesprächsleitungsregeln regelmäßig Informationsfragen stellt, zeigen ein erböhtes Outcome in verschiedenen Bereichen.

Neben dem Phänomen des späteren bzw. kaum vorhandenen Eintritts ungeteilter Information in die Diskussion können bereits genannte Informationen während des Prozesses wieder verloren gehen, weil sie nicht festgehalten oder zusammengefasst werden (Koordinationsverlust). Um dem Verlust von genannten Informationen während der Diskussion vorzubeugen, bietet es sich an, den Informationsaustausch durch regelmäßiges Wiederholen genannter Information zu koordinieren. Dadurch werden die kognitiven Ressourcen der Gruppenmitglieder geschont und die Aufmerksamkeit der Gruppe wird wieder auf die Information gelenkt (Larson 
et al., 1998b). Darüber hinaus konnte gezeigt werden, dass das Wiederholen informativen Einfluss auf die Gruppenmitglieder ausübt (Henningsen, Henningsen, Borton \& Braz, 2006), unklar ist dennoch, inwieweit die Entscheidung durch das Wiederholen verbessert wird.

Der/die Moderator/-in/ sollte bei der Wiederholung nicht zwischen geteilten, ungeteilten, präferenzkonsistenten oder -inkonsistenten Informationen unterscheiden, damit alle Informationen größere Chancen haben, in die Gruppenentscheidung einzufließen. Das regelmäßige Wiederholen bereits genannter Informationen durch den/die Moderator/-in wird als zweite unabhängige Variable in das Design der Untersuchung aufgenommen.

Für die Untersuchung wird folgendes angenommen:

$\mathrm{PH}_{2}:$ Gruppen, in denen ein/-e Moderator/-in zusätzlich zu ausgewählten allgemeinen Gesprächsleitungsregeln regelmäßig bereits genannte Informationen wiederholt, zeigen ein erböhtes Outcome in verschiedenen Bereichen.

Ob oder inwieweit Gruppen mit einer Moderation, in der beide unabhängigen Variablen umgesetzt werden, ein noch höheres Outcome zeigen, als durch die beiden Haupteffekte von $\mathrm{PH}_{1}$ und $\mathrm{PH}_{2}$ zu erwarten ist, kann nicht bestimmt werden. Die Vorhersage von Interaktionseffekten fällt vor dem aktuellen Wissenshintergrund schwer. Daher hat die folgende Hypothese einen explorativen Charakter.

PH3: In Gruppen, in denen ein/-e Moderator/-in zusätzlich zu ausgewählten allgemeinen Gesprächsleitungsregeln Informationsfragen stellt und Informationen wiederholt, lassen sich Interaktionseffekte in Berug auf das Outcome finden.

Bevor die Hypothesen bezüglich der Outcome- bzw. abhängigen Variablen im folgenden Abschnitt spezifiziert werden, verdeutlicht Abbildung 17 die Forschungsfrage der vorliegenden Untersuchung im Rahmen des IPO-Modells. 
Input

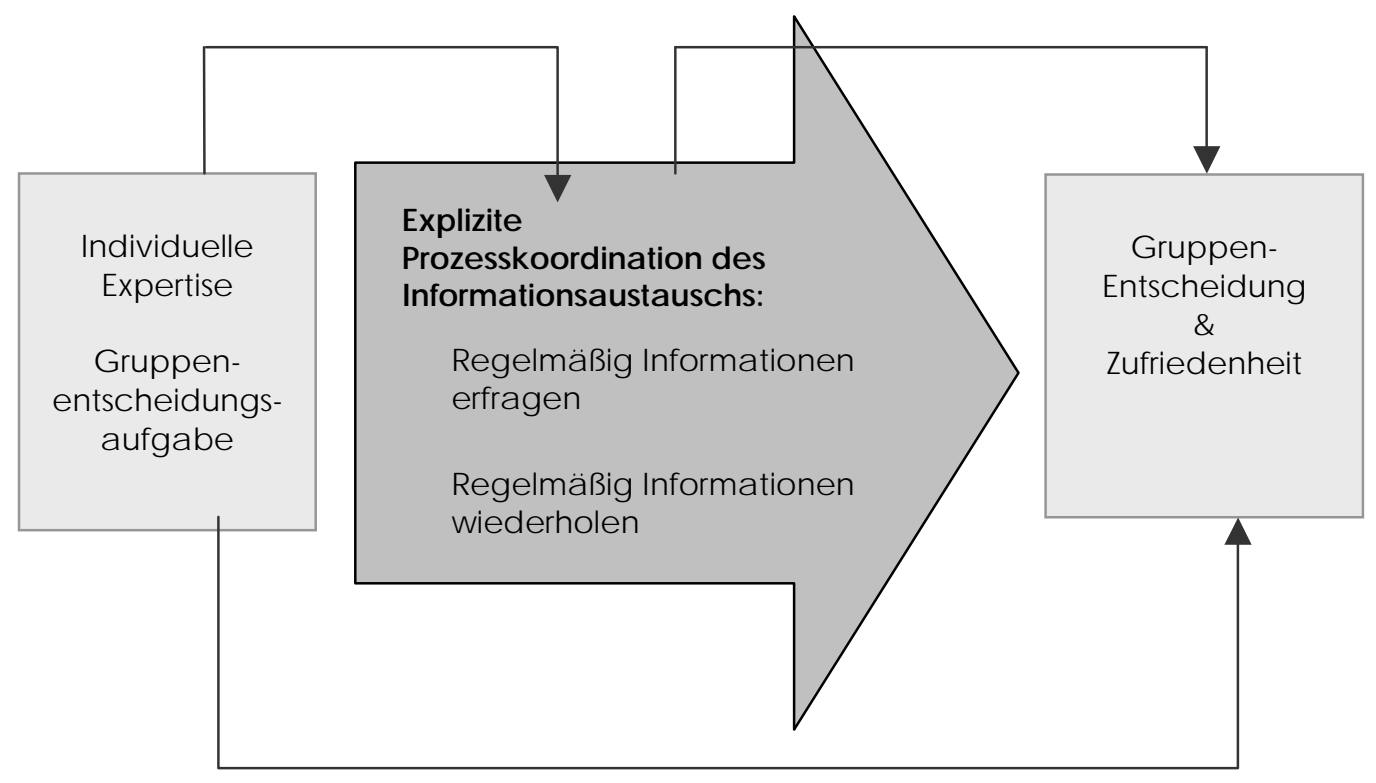

Abbildung 17: Darstellung der Forschungsfrage des Experiments im Rabmen des IPO-Modells

\subsection{Methode}

Zur Darstellung der Methodik des Experiments werden nach einer kurzen allgemeinen Beschreibung der Untersuchung zunächst die unter Abschnitt 5.2 aufgestellten Hypothesen präzisiert. Anschließend werden das experimentelle Design und die Stichprobe beschrieben. Danach wird erläutert, wie die zu untersuchenden Variablen operationalisiert und der Versuch durchgeführt wurde.

\subsubsection{Allgemeine Beschreibung der Untersuchung}

Die Frage nach dem Einfluss der expliziten Koordinationsmechanismen systematisches Erfragen und/oder systematisches Wiederbolen von Informationen auf die Gruppenentscheidungsfindung wurde experimentell geprüft. Das Experiment wurde gemeinsam mit Luisa Becker durchgeführt 
(vgl. Becker, 2006). Als Gruppenentscheidungsaufgabe wurde das Hidden-Profile-Paradigma verwendet. Im Rahmen einer entsprechenden Personalwahlaufgabe müssen drei Personen über die Auswahl eines von vier Kandidaten entscheiden. Dabei implizieren ihre vor der Diskussionen erhaltenen individuellen Informationen eine falsche Entscheidung, die Gesamtinformationen legen allerdings den richtigen Kandidaten nahe und können durch den Informationsaustausch von der Gruppe eruiert werden. Die Umsetzung der unabhängigen Variablen (systematisches Erfragen oder/und Wiederholen von Informationen) erfolgt durch eine vierte Person, die als inhaltlich neutrale/r Moderator/-in den Diskussionsprozess unterstützt.

\subsubsection{Ziele und Design}

Ziel dieses Experiments ist es, zu untersuchen, ob der Einsatz expliziter Koordinationsmechanismen durch eine/n inhaltlich neutrale/n Moderator/-in während des Entscheidungsfindungsprozesses Einfluss auf verschiedene Ergebnisvariablen hat. Im Folgenden werden die relevanten Output-Variablen sowie die entsprechenden Hypothesen spezifiziert.

\section{Entscheidungsgüte $\left(A V_{1}\right)$}

Sechs Variablen, die in der Terminologie des IPO-Modells als Output bezeichnet werden, sollen näher untersucht werden. Hackman und Morris (1975) unterscheiden zwischen performanzbezogenen und anderen Ergebnissen des Gruppenprozesses (vgl. 2.1.2). Zu ersteren zählt vor allem die Entscheidungsgüte, die im Experiment als $A V_{1}$ erfasst wird. Eine Verbesserung der Entscheidungsqualität ist neben dem Wissenszuwachs die Art des Qualitätsgewinns, den man sich durch den Einsatz einer Gruppe erhofft (Kerschreiter et al., 2003). In Bezug auf die $A V_{1}$ werden folgende Hypothesen getestet:
$H_{1-1}$ :
Das Stellen von Informationsfragen verbessert die Entscheidungsgüte.
$H_{1-2}: \quad$ Das Wiederholen von Informationen verbessert die Entscheidungsgüte. 
$\begin{array}{ll}H_{1-3}: & \text { Es lassen sich Interaktionseffekte des Informationserfragens mit dem Informationswiederholen in } \\ & \text { Bezug auf die Entscheidungsgüte finden. }\end{array}$

Bewertung des optimalen Kandidaten $\left(A V_{2}\right)$

Nach dem funktionalen Modell der Gruppenentscheidungen müssen die verschiedenen Entscheidungsalternativen kritisch bewertet werden (Hirokawa, 1985; Orlitzky \& Hirokawa, 2001). Die letztlich beste Entscheidung wird von der Gruppe schließlich ausgewählt. Im organisationalen Alltag ist der Entscheidungsfindungsprozess nach der Diskussion nicht unbedingt endgültig abgeschlossen. Beispielsweise können die Entscheidungsalternativen für die weitere Begutachtung in eine Rangreihe gebracht werden. Denkbar ist auch, dass sich während der Umsetzung der getroffenen Entscheidung unvorhergesehene Schwierigkeiten ergeben. Dann kommen eventuell zuvor verworfene oder weniger positiv bewertete Entscheidungsalternativen wieder ins Spiel. In diesen Fällen spielt die Bewertung derselben eine wichtige Rolle für den weiteren Entscheidungsprozess. Es scheint daher angebracht, die Einschätzung der Gruppenmitglieder bezüglich der Eignung der optimalen Alternative zu erfassen, unabhängig davon, ob sie letztendlich gewählt wurde oder nicht. Besonders im letzten Fall kann eine verbesserte Beurteilung nach der Diskussion entscheidende Konsequenzen für den weiteren Verlauf eventuell folgender Entscheidungsprozesse haben. Als $\mathrm{AV}_{2}$ wird ein Vergleich der Bewertung der optimalen Entscheidungsalternative vor und nach der Gruppendiskussion vorgenommen und überprüft, inwieweit der Einsatz expliziter Koordinationsmechanismen zu einer positiven Einschätzung der korrekten Entscheidungsalternative führt.

Die entsprechenden Hypothesen lauten:

$H_{2-1}$ : Das Stellen von Informationsfragen verbessert die Bewertung der Eignung des optimalen Kandidaten von vor zu nach der Diskussion. 


\section{$H_{2-2}$ : Das Wiederbolen von Informationen verbessert die Bewertung der Eignung des optimalen Kandidaten von vor zu nach der Diskussion. \\ $H_{2-3}: \quad$ Es lassen sich Interaktionseffekte des Informationserfragens mit dem Informationswiederholen in Bezug auf die Bewertung der Eignung des optimalen Kandidaten von vor zu nach der Diskussion finden.}

\section{Informationsaustausch $\left(\mathrm{AV}_{3}\right)$}

Als ein wesentlicher Grund für den häufigen Befund, dass Gruppenentscheidungen qualitativ nicht hochwertig sind, wird der schlechte Informationsaustausch während des Entscheidungsfindungsprozesses angesehen So werden insbesondere ungeteilte Informationen, die den Erkenntnisgewinn und das Synergiepotential der Gruppe ermöglichen würden, im Gegensatz zu geteilten Informationen, weniger häufig in den Diskussionsprozess eingebracht und wiederholt (Kerschreiter et al., 2003; Larson et al., 1994; Stasser \& Titus, 1985).

Der Einsatz expliziter Koordinationsmechanismen soll dazu dienen, dass für die Entscheidung wesentliche Informationen in die Diskussion eingebracht und während der Diskussion nicht verloren gehen. Es wird angenommen, dass durch das systematische Erfragen und Wiederholen das Einbringen und Diskutieren der ungeteilten Informationen verbessert wird. Dazu werden folgenden Hypothesen getestet:

$H_{3-1}$ : Das Stellen von Informationsfragen verbessert den Informationsaustausch während der Diskussion.

$H_{3-2}$ : Das Wiederbolen von Informationen verbessert den Informationsaustausch während der Diskussion.

$H_{3-3:} \quad$ Es lassen sich Interaktionseffekte des Informationserfragens mit dem Informationswiederbolen in Bezug auf den Informationsaustausch während der Diskussion finden. 


\section{Zufriedenbeit mit dem Ergebnis der Diskussion $\left(A V_{4}\right)$}

Ergebnisse, die über die konkrete Leistung der Gruppe hinausgehen, werden im IPOModell nicht spezifiziert (vgl. 2.1.2). Hackman und Morris (1975) nennen einige mögliche Variablen, fügen aber hinzu, dass es neben diesen auch eine Reihe weiterer relevanter Outcomes geben kann. McGrath hingegen (1991, S. 154) geht davon aus, dass alle Gruppen im Sinne dreier wichtiger Funktionen agieren: das Erfüllen der Projektziele (production), die Gruppenerhaltung (well-being) und die Unterstützung einzelner Gruppenmitglieder (member support).

Individuen arbeiten in Gruppen auch in der Erwartung positiver sozio-emotionaler Effekte. Es ist davon auszugehen, dass die Gruppenmitglieder ihre Gruppenarbeit zufrieden beenden wollen, was sich wiederum positiv auf das zukünftige Funktionieren der Gruppe auswirken kann (Fuller \& Aldag, 2001). Auch Sundstrom, De Meuse und Futrell (1990) postulieren, dass die Effektivität einer Arbeitsgruppe sowohl aus der Leistung (Performance) als auch aus Zufriedenheit der Gruppenmitglieder und deren Bereitwilligkeit, die Arbeit mit der Gruppe fortzusetzen (Viability) besteht. Wesentlich ist dabei die Zufriedenheit der Gruppenmitglieder mit dem Entscheidungsprozess, da ihre Unzufriedenheit ihr Verhalten in zukünftigen Diskussion verändern kann (Poole \& Hirokawa, 1996). Die Zufriedenheit der Gruppenmitglieder wird daher als weitere abhängige Variable aufgenommen. In Anlehnung an Fuller und Aldag (2001) wird in die Zufriedenheit mit dem Ergebnis $\left(\mathrm{AV}_{4}\right)$, mit dem Prozess $\left(\mathrm{AV}_{5}\right)$ und dem/der Moderator/-in $\left(\mathrm{AV}_{6}\right)$ erfasst. Die Hypothesen bezüglich der Zufriedenheit mit dem Ergebnis lauten:

$H_{4-1}$ : Das Stellen von Informationsfragen verbessert die Zufriedenheit mit dem Ergebnis der Diskussion.

$H_{4-2}$ : Das Wiederholen von Informationen verbessert die Zufriedenheit mit dem Ergebnis der Diskussion. 
$H_{4-3}$ : Es lassen sich Interaktionseffekte des Informationserfragens mit dem Informationswiederbolen in Bezug auf die Zufriedenheit mit dem Ergebnis finden.

$\mathrm{AV}_{5}$ stellt die Zufriedenheit der Gruppenmitglieder mit dem Verlauf der Diskussion dar. Folgende Vorhersagen werden getestet:

$H_{5-1}$ : Das Stellen von Informationsfragen verbessert die Zufriedenheit mit dem Verlauf der Diskussion.

$H_{5-2}$ : Das Wiederbolen von Informationen verbessert die Zufriedenheit mit dem Verlauf der Diskussion.

$H_{5-3:} \quad$ Es lassen sich Interaktionseffekte des Informationserfragens mit dem Informationswiederholen in Bezug auf die Zufriedenbeit mit dem Verlauf finden.

Zusätzlich wurden über $\mathrm{AV}_{6}$ die Zufriedenheit mit dem/der Moderator/-in erfasst und folgende Hypothesen getestet:

$H_{6-1}$ : Das Stellen von Informationsfragen verbessert die Zufriedenheit mit dem/der Moderator/-in.

$H_{6-2}: \quad$ Das Wiederbolen von Informationen verbessert die Zufriedenheit mit dem/der Moderator/-in.

$H_{6-3}$ : Es lassen sich Interaktionseffekte des Informationserfragens mit dem Informationswiederbolen in Bezug auf die Zufriedenheit mit dem/der Moderator/-in finden.

Alle aufgestellten Hypothesen werden in Tabelle 6 zusammengefasst. 
Tabelle 6: Überblick über die Hypothesen

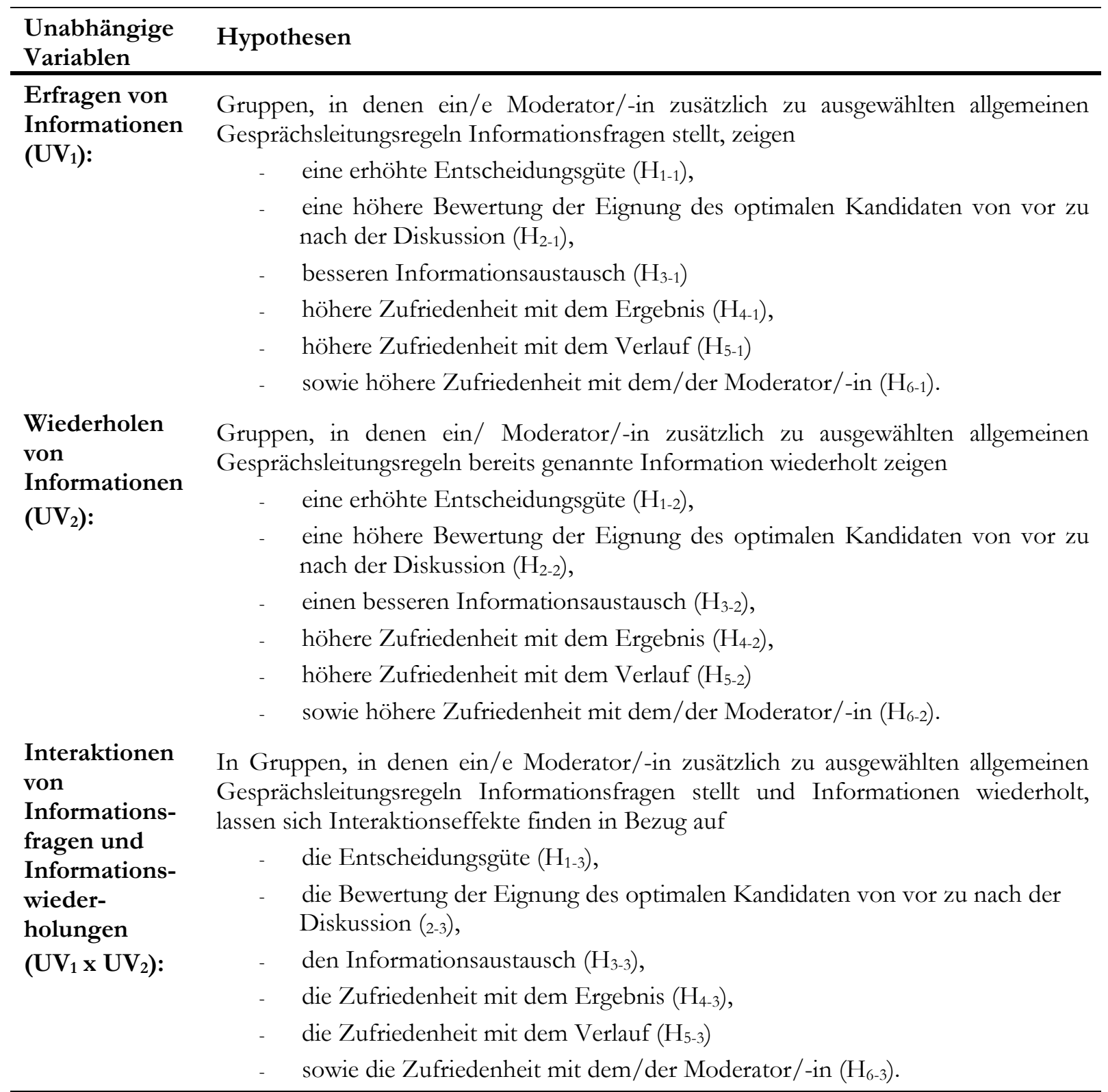

Zur Untersuchung der entwickelten Hypothesen wurde ein Laborexperiment durchgeführt, bei dem Drei-Personen-Gruppen mit Unterstützung eines/er Moderator/-in eine Hidden-Profile-Aufgabe bearbeiten mussten. In einer individuellen Vorbereitungszeit machten sich die Gruppenmitglieder mit der Aufgabe vertraut. Währenddessen wurde der/die Moderator/-in durch ein spezielles Training auf seine/ihre Rolle vorbereitet. Anschließend kamen alle Teilnehmer/-innen für die Gruppendiskussion zusammen, die auf Video aufgezeichnet wurde. Nach Beendigung der Diskussion bearbeiteten die Teilnehmer/-innen verschiedene Fragebögen. 
Die Hypothesen wurden in einem 2x2faktoriellen Between-Groups-Design geprüft. Die erste experimentelle unabhängige Variable (UV) stellte Informationen erfragen $\left(\mathrm{UV}_{1}\right.$ : keine Fragen stellen vs. Fragen stellen) und die zweite Variable Informationen wiederbolen ( $\mathrm{UV}_{2}$ : keine Informationen wiederholen vs. Informationen wiederholen) dar, mit jeweils dichotomer Ausprägung. Beide unabhängigen Variablen werden im Abschnitt 5.3 .4 genauer beschrieben. Tabelle 7 ist der Versuchsplan zu entnehmen.

Tabelle 7: Versuchsplan des Experiments

\begin{tabular}{llll}
\hline & & $\mathrm{UV}_{1}$ : Informationsfragen stellen & \\
& $\mathrm{Ja}$ & Nein \\
\hline $\mathrm{UV}_{2}$ : & $\mathrm{Ja}$ & Experimentalgruppe 3 & Experimentalgruppe 2 \\
Informationen wiederbolen & $\mathrm{Nein}$ & Experimentalgruppe 1 & Kontrollgruppe 0 \\
& & & \\
\hline
\end{tabular}

Insgesamt wurden 66 Gruppen zu je vier Personen untersucht. Davon entfallen sieben Gruppen auf den Pretest. Neun Gruppen mussten aufgrund Problemen in der Videoaufzeichnung (fehlender Ton) ausgeschlossen werden und bei zwei weiteren Gruppen wurde im Anschluss an den Versuch festgestellt, dass die einige Gruppenmitglieder instruktionswidriges Verhalten gezeigt hatten (Erinnerungsfragebogen während der Diskussion benutzt bzw. während der Diskussion mitgeschrieben), diese beiden Gruppen wurden ebenfalls ausgeschlossen. Daher wurden 48 Gruppen in die Auswertung einbezogen, 12 pro Bedingung. Die Gleichverteilung auf die vier Experimentalbedingungen konnte gewährleistet werden, weil die oben geschilderten Probleme graduell auftraten und für jede auszuschließende Gruppe eine weitere erhoben wurde. Die Zuteilung der Gruppen zu den Bedingungen erfolgte randomisiert (vgl. Rack \& Christophersen, 2006).

\subsubsection{Beschreibung der Stichprobe}

Die Datenerhebung erstreckte sich über den Zeitraum von Dezember 2005 bis Juli 2006. An der vorliegenden Studie nahmen insgesamt 264 Personen in 66 Gruppen teil. Sieben Gruppen 
waren Bestandteil der Voruntersuchung, mit deren Hilfe der Versuchsablauf und vor allem die Manipulation der unabhängigen Variablen auf ihre Einsetzbarkeit überprüft wurden sollten. Nach einer Analyse der Videos sowie der Auswertung der schriftlichen und mündlichen Rückmeldungen der Versuchsteilnehmer/-innen zeigte sich, dass eine Überarbeitung der Trainingsunterlagen für die Rolle des/der Moderators/-in notwendig war. Die Instruktionen wurden weiter spezifiziert und die kritischen Koordinationsverhaltensweisen ausführlicher beschrieben. Wichtig schien es, die Versuchspersonen in der Rolle des/der Moderators /-in darauf hinzuweisen, die von ihnen erwünschten Verhaltensweisen (Fragen stellen bzw. Informationen wiederholen) häufig einzusetzen und die Bedeutung der Moderationsrolle für die Gruppenentscheidung zu betonen.

An der Hauptuntersuchung nahmen insgesamt 192 Probanden/-innen teil. Sie wurden per Internetanzeige auf der Homepage der Abteilung für Sozial- und Kommunikationspsychologie, per Aushang im Institut für Psychologie und an anderen Standorten der Universität sowie über persönliche Ansprache auf die Untersuchung aufmerksam gemacht. Als Entschädigung für ihre Teilnahme erhielten die Probanden/-innen entweder zwei Versuchspersonenstunden ${ }^{10}$ oder 16 Euro oder ihnen wurde die Teilnahme an einer Verlosung von Fluggutscheinen ermöglicht. Das Alter der Probanden/-innen reichte von 19 bis zu 45 Jahren, mit einem Durchschnitt von 23,3 Jahren. Es nahmen wesentlich mehr Frauen als Männer an der Untersuchung teil (131 Teilnehmerinnen vs. 61 Teilnehmer, 68,2\% vs. 31,8\%). Der Großteil der Teilnehmenden waren Studierende der Fächer Psychologie bzw. Sozialwissenschaften mit Nebenfach Wirtschafts- und Sozialpsychologie (84 Teilnehmer/-innen). Darüber hinaus nahmen Studierende anderer Fachrichtungen teil, wenige Versuchspersonen hatten ein bereits abgeschlossenes Studium. Nahezu drei Viertel der Studierenden befanden sich zwischen erstem und viertem Semester (132 Teilnehmer, 71,7\%), was insbesondere im Falle der

\footnotetext{
${ }^{10}$ Innerhalb des Grundstudiums müssen Studierende der Psychologie aus Haupt- und Nebenfach eine gewisse Anzahl von Stunden ableisten, während derer sie sich als Versuchspersonen für institutsinterne Untersuchungen zur Verfügung stellen.
} 
Teilnehmer/-innen des Faches Psychologie intendiert war, da diese nach dem vierten Semester spezifisches Wissen zum Hidden-Profile-Paradigma vermittelt bekommen. Aufgrund einer möglichen Verfälschung der Ergebnisse durch Vorwissen bezüglich des Paradigmas wurden Psychologie-Studierende im Hauptstudium und Studierende, die bereits an ähnlichen Versuchen teilgenommen hatten, von der Untersuchung ausgeschlossen.

Diskussionsteilnehmer/-innen und Moderator/-innen unterschieden sich kaum im Hinblick auf die oben genannten Kriterien, ein Überblick findet sich in Tabelle 8.

Tabelle 8: Verteilung von Geschlecht, Alter und Semesterzabl der Probanden/-innen

\begin{tabular}{|c|c|c|c|c|}
\hline & & $\begin{array}{l}\text { Alle Teilnehmer/- } \\
\text { innen }(\mathrm{N}=192)\end{array}$ & $\mathrm{XYZ}(\mathrm{N}=144)$ & $\mathrm{M}(\mathrm{N}=48)$ \\
\hline \multirow[t]{2}{*}{ Geschlecht } & $\hat{0}$ & $61(31.8 \%)$ & $47(32.6 \%)$ & $14(29.2 \%)$ \\
\hline & $q$ & $131(68.2 \%)$ & $97(67.4 \%)$ & $34(70.8 \%)$ \\
\hline \multirow{3}{*}{\multicolumn{2}{|c|}{ Alter }} & $23.32(4.61)$ & $23.67(4.34)$ & $22.25(3.00)$ \\
\hline & & 19-45 Jahre & 19-45 Jahre & 19-30 Jahre \\
\hline & & $56.8 \%-19-22$ & $53.5 \%-19-22$ & $66.7 \%-19-22$ \\
\hline \multirow{3}{*}{\multicolumn{2}{|c|}{ Semester }} & 1.-17. Semester & 1.-15. Semester & 1.- 17. Semester \\
\hline & & 1.-4. Semester: & 1.-4. Semester: & 1.-4. Semester: \\
\hline & & $132(71.7 \%)$ & $96(69.1 \%)$ & $36(80.0 \%)$ \\
\hline
\end{tabular}

Anmerkung. Bei Geschlecht: Anzahl, Prozentwert in Klammern; bei Alter: Mittelwerte,

Standardabweichungen in Klammern, Spannweite; bei Semester: Spannweite, davon Anzahl

Grundstudium, Prozentwert in Klammern. XYZ ... Inhaber/-innen der Rolle der

Diskutant/-innen; M ... Inhaber/-innen der Moderation.

\subsubsection{Operationalisierung der Konstrukte}

\section{Operationalisierung der Unabhängigen Variablen}

Beide unabhängigen Variablen wurden von einem/er inhaltlich neutralen Moderator/-in während der Diskussion ausgeübt. Sie wurden dafür mittels schriftlicher Instruktionen und einem speziellen Training vorbereitet (siehe Abschnitt Moderator/-innen-Training). In der Instruktion wurde zunächst darauf hingewiesen, dass Informationen zu einem Entscheidungsproblem oftmals unterschiedlich auf die Gruppenmitglieder verteilt sind und Gruppen häufig dazu neigen, mehr über Informationen zu sprechen, die allen Mitgliedern bereits vorab bekannt sind, hingegen aber wenig neue Informationen in die Diskussion einbringen. Gründe dafür könnten darin 
bestehen, dass einzelne Gruppenmitglieder sich nicht trauen, neue Informationen zu nennen bzw. bereits genannte Informationen wieder verloren gehen. Um das zu verhindern, solle der/die Moderator/-in die entsprechende Regel beachten und folgende Intervention umsetzen:

Zur Umsetzung der $\mathrm{UV}_{1}$ Fragen stellen in den Bedingungen 1 und 3 sollte der/die Moderator/-in fragen, ob es zu den Kandidaten noch weitere Informationen gäbe. Dabei sollte der/die Moderator/-in regelmäßig und häufig Fragen stellen, sich gleich oft nach allen Kandidaten erkundigen und sowohl nach positiven als auch nach negativen Informationen der Kandidaten fragen.

Zur Umsetzung der $\mathrm{UV}_{2}$ Informationen wiederbolen in den Bedingungen 2 und 3 sollte der/die Moderator/-in regelmäßig genannte Informationen zu den Kandidaten wiederholen. Zu diesem Zweck durfte sich der/die Moderator/-in Notizen machen, diese aber den Gruppenmitgliedern nicht zeigen. Er/sie sollte regelmäßig und häufig Informationen wiederholen, auch ohne darauf zu warten, dass zuvor neue Informationen eingebracht wurden.

$\mathrm{Zu}$ beiden Regeln gab es stichpunktartige Ausführungen, die das Verhalten konkretisierten und Hilfen für Umsetzung gaben (vgl. Anhang D).

\section{Operationalisierung der Abbängigen Variablen}

Sechs abhängige Variablen (AVs) wurden betrachtet. Außer der $A V_{1}$ und der $A V_{2}$ wurden alle weiteren abhängigen Variablen mit einem für die Fragestellung entwickelten Fragebogen erfasst. Die Operationalisierung erfolgte über die Messung der Zustimmung der Probanden/innen zu Fragebogen-Items auf 8-stufigen Skalen mit den Polen von 0 bis 7 (0 ... überhaupt nicht, 7 ... sehr $)^{11}$. Soweit möglich wurde dabei auf Items ähnlicher Untersuchungen zurückgegriffen. Grundlage von drei Fragen war die Untersuchung von Schulz-Hardt et al. (in press), deren Hidden-Profile-Aufgabe auch dieser Studie zugrunde liegt. Daneben wurden zwei Items der

\footnotetext{
${ }^{11}$ Ausnahmen finden sich bei zwei Items: „Wie war die Atmosphäre während der Gruppendiskussion?“ $(0$... konkurrierend, 7 ... kooperativ) und „Wie viel Einfluss hatten Sie auf die Gruppenentscheidung?“ (0 ... überhaupt keinen, 7 ... sehr großen)
} 
Arbeit von Schauenburg (2004) entnommen. Eine Übersicht der Items jeder abhängigen Variable und ihrer Herkunft findet sich in Tabelle 9.

Zur Bestimmung der Zuverlässigkeit der zu Skalen zusammengefassten Items wurde das Maß der internen Konsistenz herangezogen (Cronbachs Alpha), dabei können Werte ab $\alpha=.60$ als ausreichend, Werte $\mathrm{ab} \alpha=.90 \mathrm{als}$ sehr gut angesehen werden (Aron, Aron \& Coups, 2006).

\section{$\mathrm{AV}_{1}$ : Entscheidungsgüte}

Das Hidden-Profile erlaubt eine einfache Bestimmung der Güte der Gruppenentscheidung. Es wird erfasst, ob das Hidden-Profile gelöst und der richtige Kandidat gewählt wurde oder nicht. Diese Variable ist dichotom (Wahl des geeigneten Kandidaten vs. Wahl eines der drei suboptimalen Kandidaten) und konnte dem ausgefüllten Fragebogen zur Gruppenentscheidung direkt entnommen werden.

$\mathrm{AV}_{2}$ : Bewertung der Eignung des optimalen Kandidaten

Jeder der zur Auswahl stehenden vier Kandidaten musste sowohl vor als auch nach der Diskussion individuell von jedem/jeder Teilnehmer/-in hinsichtlich seiner Eignung beurteilt werden (,Für wie geeignet halten Sie Bewerber A/ B/ C/ D? “).

\section{$\mathrm{AV}_{3}$ : Informationsaustausch}

Der Informationsaustausch wurde über die Nennungen geteilter und ungeteilter Informationen erfasst. Er wurde dazu von drei Kodierer/-innen kodiert. Anhand der Kodierungen sind sowohl das Einbringen der Informationen in die Diskussionen (Erstnennung) als auch die Anzahl der geteilten und ungeteilten Informationen (Nennungshäufigkeit) ersichtlich. 
$\mathrm{AV}_{4}:$ Zufriedenheit mit dem Ergebnis der Diskussion

Die Zufriedenheit mit dem Ergebnis wurde anhand einer Skala bestehend aus vier Items erfasst. Zwei Items stellten explizit die Frage nach der Zufriedenheit mit dem Gruppenergebnis (,Wie zufrieden sind Sie mit dem Ergebnis der Diskussion?" und „Wie zufrieden sind Sie mit dem Ergebnis der Zusammenarbeit in der Gruppe?") Über ein Item sollten die Teilnehmer/-innen die Qualität der Entscheidung beurteilen (,Wie gut ist die Entscheidung, die die Gruppe getroffen hat?'). Ein letztes Item erfasste, inwieweit die Teilnehmer/-innen hinter der Entscheidung standen (,Wie sehr stehen Sie qu der Entscheidung, die in der Gruppe getroffen wurde?'). Die aus diesen vier Items gebildete Skala wies eine hohe Reliabilität von $\alpha=.92$ auf.

$\mathrm{AV}_{5}:$ Zufriedenheit mit dem Verlauf der Diskussion

Die Zufriedenheit mit dem Verlauf der Diskussion wurde zunächst über sieben Items operationalisiert. Es schien angebracht, eine Faktorenanalyse durchzuführen, um zu prüfen, inwieweit die Items eine oder mehrere übergeordnete Variablen repräsentieren. Die rotierte Faktorenlösung ergab zwei Komponenten, wobei dem ersten Faktor vier Items zugeordnet wurden, dem zweiten Faktor drei Items. Erstere konnten unter dem Stichwort „Arbeitsklima“ subsumiert werden; hierzu zählten Fragen nach dem Verlauf (,Wie zufrieden sind Sie mit dem Verlauf der Diskussion?"), der Atmosphäre (,Wie war die Atmosphäre während der Gruppendiskussion?" und „Haben Sie sich in der Gruppe wohl gefüblt?`), und der Einschätzung des eigenen Einflusses (,Wie viel Einfluss batten Sie auf die Gruppenentscheidung?" "). Die anderen Items erfassten die „Beitragsmöglichkeiten“ der Teilnehmer/-innen: wie gut sie sich einbringen konnten (,Wie gut konnten Sie das sagen, was Sie sagen wollten?" und „Wie gut konnten Sie sich in die Diskussion einbringen?") und ob auch konträre Meinungen geäußert werden konnten („Ist man auf die Meinung Andersdenkender eingegangen?" "). 
Die internen Konsistenzen liegen für der Skala Arbeitsklima bei $\alpha=.61$ und für die Skala Beitragsmöglichkeiten bei $\alpha=.55$. Bei einer Zusammenfassung von fünf der sieben Items, die augenscheinlich ebenfalls in einen sinnvollen Zusammenhang gebracht werden können, war die Zuverlässigkeit der Skala höher $(\alpha=.73)$. Aus inhaltlichen Gründen wurde aber die Zweifaktorenlösung trotz der gerade ausreichenden Reliabilitäten bevorzugt.

$\mathrm{AV}_{6}$ : Zufriedenheit mit dem/der Moderator/-in

Die Versuchsteilnehmer/-innen schätzten die Zufriedenheit mit dem/der Moderator/-in ein. Sie beurteilten zum einen allgemein seine/ihre Fähigkeiten (Skala Kompetenø). Zum anderen wurde erfragt, inwieweit der/die Moderator/-in der Gruppe helfen konnte (Skala Hilfe). Die Kompetenz wurde durch drei Fragen erhoben, wobei nach den Qualitäten in Bezug auf die Leitung (,Wie zufrieden sind Sie mit der Art und Weise, wie diese Person die Diskussion geleitet hat?'), die Strukturierung (,Wie sehr hat diese Person für eine Strukturierung der Diskussion gesorgt? “) und nach seiner/ihrer Kompetenz („Wie kompetent wirkte diese Person in ihrer Rolle? ') gefragt wurden. Die Skala erreichte eine interne Konsistenz von $\alpha=.87$. Die Hilfe beim Informationsaustausch und bei der Entscheidungsfindung wurde durch zwei Fragen gemessen (,,Wie sehr hat diese Person der Gruppe geholfen, ibre Informationen auszutauschen?") und (,Wie sehr hat diese Person der Gruppe geholfen, eine Entscheidung ₹и treffen? ‘). Die Reliabilität dieser Skala liegt bei $\alpha=.73$. 
Tabelle 9: Übersicht über die Herkunft und Reliabilität der Items der $A V_{2}$ sowie $A V_{4}$ bis $A V_{6}$

Skala/ Item Herkunft

Cronbachs

Alpha

\section{$\mathrm{AV}_{2}$ : Bewertung des optimalen Kandidaten}

Für wie geeignet halten Sie Bewerber A/ B/ C/ D?

\section{$\mathrm{AV}_{4}$ : Zufriedenheit mit dem Ergebnis}

Wie zufrieden sind Sie mit dem Ergebnis der Diskussion?

Wie zufrieden sind Sie mit dem Ergebnis der Zusammenarbeit in der Gruppe?

Wie gut ist die Entscheidung, die die Gruppe getroffen hat?

Wie sehr stehen Sie zu der Entscheidung, die in der Gruppe getroffen wurde?

\section{$\mathrm{AV}_{5}:$ Zufriedenheit mit dem Verlauf}

Wie zufrieden sind Sie mit dem Verlauf der Diskussion?

$\mathrm{BK}$

Wie war die Atmosphäre während der Gruppendiskussion?

BS

Haben Sie sich in der Gruppe wohl gefühlt?

$\mathrm{BK}$

Wie viel Einfluss hatten Sie auf die Gruppenentscheidung?

- AV $V_{5 b}$ : Beitragsmöglichkeiten

$\mathrm{SH}$

Wie gut konnten Sie das sagen, was Sie sagen wollten?

BK

BS

BK

$\mathrm{SH}$

Wie gut konnten Sie sich in die Diskussion einbringen?

Ist man auf die Meinung Andersdenkender eingegangen?

\section{$\mathrm{AV}_{6}:$ Zufriedenheit mit dem Moderator/-in}

\section{- $A V_{6 a}$ : Kompetenz.}

Wie zufrieden sind Sie mit der Art und Weise, wie diese Person die

Diskussion geleitet hat?

Wie sehr hat diese Person für eine Strukturierung der Diskussion gesorgt?

Wie kompetent wirkte diese Person in ihrer Rolle?

- $A V_{6 b}:$ Hilfe

Wie sehr hat diese Person der Gruppe geholfen, ihre Informationen auszutauschen?

Wie sehr hat diese Person der Gruppe geholfen, eine Entscheidung zu treffen?
Anmerkung: BK...selbst konzipierte Items durch Becker \& Kolbe, BS .. Schauenburg (2004), SH ... Schulz-Hardt, Brodbeck, Mojzisch, Kerschreiter \& Frey (in press) 


\subsubsection{Versuchsmaterial}

Die Frage, wie man die Qualität einer Gruppenentscheidung messen kann, beschäftigt die Gruppenforschung seit geraumer Zeit (Boos, 1996a). Da in der vorliegenden Untersuchung neben der Gruppenentscheidung auch der Informationsaustausch im Vordergrund steht, bietet sich der Einsatz des Hidden-Profile-Paradigmas als Untersuchungsinstrument an. Das HiddenProfile kann als Prototyp für Entscheidungssituationen angesehen werden, in denen sich das Synergiepotential der Gruppe durch einen gelungenen Informationsaustausch in einer richtigen Gruppenscheidung zeigen kann.

Das Hidden-Profile eignet sich, trotz später zu diskutierende Schwächen, für die Analyse von Gruppenentscheidungen, da es eine detaillierte Manipulation und Untersuchung der Gruppenentscheidungsaufgabe ermöglicht. Damit kommt es in Ansätzen der Forderung nach Aufgabenanalysen nach (Tschan, 2000): Das Hidden-Profile erlaubt normative Aussagen über den erforderlichen Informationsaustausch, jedoch nicht über seinen hierarchisch-sequentiell Ablauf.

Die hier verwendete Entscheidungsaufgabe wurde von Schulz-Hardt, Brodbeck, Mojzisch, Kerschreiter und Frey (in press) entwickelt und freundlicherweise von Andreas Mojzisch für die vorliegende Untersuchung zur Verfügung gestellt. Es handelt sich um eine Personalauswahlaufgabe, bei der eine Fluglinie einen neuen Piloten für Langstreckenflüge sucht. Die Versuchsteilnehmer/-innen spielen die Rolle von Mitgliedern des Personalauswahlgremiums und müssen eine Entscheidung zwischen den vier zur Verfügung stehenden Piloten A, B, C und D treffen. Insgesamt ist jeder der vier Kandidaten durch jeweils 10 Eigenschaften charakterisiert, wobei die 40 Items gleichermaßen überzeugend ist bzw. die gleiche Wertigkeit aufweisen. Ein Beispiel für ein negatives Attribut wäre „leicht aufbrausend“, ein Beispiel für ein positives Attribut „,kann komplexe Zusammenbänge gut überblicken“. Unter Berücksichtigung der insgesamt vorhandenen Informationen ist Kandidat C die beste Alternative, da er ein Verhältnis von 7 positiven und nur 
3 negativen Eigenschaften aufweist, wohingegen A, B und D jeweils 4 positive und 6 negative Eigenschaften besitzen (vgl. Tabelle 10).

Im Experiment erhält jede/r Teilnehmer/-in eine bestimmte Unterauswahl dieser Eigenschaften, wobei ein Teil der Information allen Teilnehmer/-innen zur Verfügung steht (geteilte Informationen), ein anderer Teil jedoch den drei Diskutanten/-innen individuell zugeordnet wird (ungeteilte Information). Auf dem Kandidatenblatt wird jeder der vier Kandidaten mit jeweils 6 Attributen beschrieben. Es gibt drei Versionen des Informationsblattes, jeweils eines für Diskutant/-in $\mathrm{X}, \mathrm{Y}$ und $\mathrm{Z}$. Zusammengenommen enthalten die drei Informationsblätter 40 Informationen zu den Kandidaten. Für Kandidat A, B und D sind alle positiven Eigenschaften geteilt und alle negativen Eigenschaften ungeteilt. Eine Gesamtübersicht aller Informationen sowie ein exemplarisches Kandidatenblatt für Teilnehmer Y befinden sich in Anhang D.

Tabelle 10: Informationsverteilung der Hidden-Profile-Aufgabe

\begin{tabular}{|c|c|c|c|c|c|}
\hline \multirow[b]{2}{*}{ Informationsart } & \multirow[b]{2}{*}{ Wertigkeit } & \multicolumn{4}{|c|}{ Kandidat } \\
\hline & & $\mathrm{A}$ & $\mathrm{B}$ & $\mathrm{C}$ & $\mathrm{D}$ \\
\hline \multirow[t]{2}{*}{ Geteilte Informationen } & + & 4 & 4 & 1 & 4 \\
\hline & - & 0 & 0 & 3 & 0 \\
\hline \multirow[t]{2}{*}{ Ungeteilte Informationen } & + & 0 & 0 & 6 & 0 \\
\hline & - & 6 & 6 & 0 & 6 \\
\hline \multirow{2}{*}{$\begin{array}{l}\text { Gesamtinformationen auf Gruppenebene } \\
(\mathrm{X}+\mathrm{Y}+\mathrm{Z})\end{array}$} & + & 4 & 4 & 7 & 4 \\
\hline & - & 6 & 6 & 3 & 6 \\
\hline \multirow{2}{*}{$\begin{array}{l}\text { Informationsverteilung auf } \\
\text { Individualebene } \\
\text { (je X, Y, Z) }\end{array}$} & + & 4 & 4 & 3 & 4 \\
\hline & - & 2 & 2 & 3 & 2 \\
\hline
\end{tabular}

Jedes Gruppenmitglied besitzt vor der Diskussion jeweils 4 positive und nur 2 negative Attribute. Für Kandidat C hingegen sind alle negativen Eigenschaften sowie eine positive geteilt, die weiteren positiven Eigenschaften sind ungeteilt. Somit ist $C$ vor der Diskussion für jedes 
Gruppenmitglied durch 3 positive sowie 3 negative Eigenschaften charakterisiert. Entsprechend der ihnen verfügbaren Informationen sollten die meisten Gruppenmitglieder vorab einen der Kandidaten A, B oder D bevorzugen. Da die Differenz im Verhältnis zwischen positiven und negativen Eigenschaften aber nicht enorm ist und stets eine gewisse Variabilität in der Beurteilung der Wichtigkeit der Eigenschaften zu erwarten ist, ist es möglich, dass einige Teilnehmer/-innen auch Kandidat C auswählen (vgl. Schulz-Hardt et al., in press).

\subsubsection{Versuchsdurchführung}

\section{Allgemeine Durchführung}

Das Experiment fand im Kommunikationslabor des Georg-Elias-Müller-Institutes für Psychologie in Göttingen statt. Die Durchführung lehnt sich von ihrer Vorgehensweise teilweise an die Studie von Schulz-Hardt et al. (in press) an. Ad-hoc- gebildete Drei-Personen-Gruppen bearbeiteten im Labor face-to-face eine Entscheidungsfindungsaufgabe, eine weitere Person unterstützte die Gruppe als Moderator/-in. Die Zuteilung zu den Versuchsbedingungen erfolgte randomisiert durch Ziehung eines Loses jeweils kurz vor einem Versuchsdurchgang. Nach der Begrüßung der Teilnehmer/-innen durch Versuchsleiter/-in A und B folgten einige einleitende Worte über Ziele und Ablauf des Experiments. Es wurde darauf hingewiesen, dass in dem Experiment der Prozess und die Qualität von Gruppenentscheidungen untersucht würden. Den Teilnehmenden wurde erklärt, dass drei von ihnen in einer individuellen Vorbereitungsphase Material zu einer Bewerberauswahlaufgabe einer Fluglinie erhielten und bearbeiten müssten. Im Anschluss daran fände eine Gruppendiskussion statt, in der die Gruppe eine Personalentscheidung darüber treffen müsse, welchen der vier zur Auswahl stehenden Piloten die Fluglinie einstellen solle. Der/die vierte Teilnehmer/-in würde nicht direkt inhaltlich an der Diskussion beteiligt sein, sondern die Gruppe während der Entscheidungsfindung unterstützen. Zu diesem Zweck bekäme er/sie ebenfalls eine individuelle Vorbereitung. Außerdem wurde das Einverständnis der Teilnehmer/-innen eingeholt, die Gruppendiskussion für spätere 
Auswertungszwecke auf Video aufzuzeichnen ${ }^{12}$. Anschließend wurde per Los über die Rollenverteilung der Probanden/-innen entschieden (Diskutant/-in X, Y, Z oder Moderator/-in M), um eine randomisierte Zuteilung zu gewährleisten. Moderator/-in und Versuchsleiterin A verließen danach den Raum, so dass sie das Moderator/-in-Training durchführen konnte, dessen Ablauf im folgenden Abschnitt näher beschrieben wird. Versuchsleiter/-in B händigte den anderen drei Personen zunächst ein Deckblatt aus, auf dem einige grundlegende Informationen zur Entscheidungsaufgabe, der Fluggesellschaft und dem weiteren Ablauf vermerkt waren. Außerdem wies er/sie darauf hin, dass Gruppen, die den eindeutig besten Piloten auswählen, an der Verlosung von CD-Gutscheinen im Wert von je $25 €$ pro Person teilnehmen würden. Dies sollte einen zusätzlichen Anreiz schaffen, sich als Gruppe um eine gute Gruppenentscheidung zu bemühen. Die Teilnehmer/-innen notierten auf diesem Deckblatt ihr Geschlecht, Alter, Studienfach und Semesterzahl. Zusätzlich bekam jede/r von ihnen einen Code zugeteilt, bestehend aus dem Buchstaben X, Y oder $\mathrm{Z}$ (entsprechend ihrer Rolle in der Gruppendiskussion) sowie einer sechsstelligen Zahl. Dann wurden sie auf drei unterschiedliche Räume aufgeteilt, in denen sie alle weiteren Unterlagen bearbeiteten, sowohl vor als auch nach der Diskussion.

Zunächst erhielt jede/r Diskutant/-in die Kandidateninformation und den dazugehörigen Bewertungsbogen. Die Teilnehmer/-innen wurden gebeten, die Informationen einzeln auf den Bewertungsbogen zu notieren und im Hinblick auf die Eignung für die Stelle als Pilot auf einer Skala von -3 bis +3 zu bewerten. Für diese Aufgabe standen 15 Minuten zur Verfügung. Außerdem wurden sie gebeten, die Informationen zu allen Kandidaten auswendig zu lernen, da ihnen in der daran anschließenden Diskussion die Informationsblätter nicht mehr zur Verfügung stünden. Wie in der überwiegenden Anzahl von Untersuchungen, die das Hidden-ProfileParadigma verwenden, wurde darauf verzichtet, den Teilnehmern/-innen die Unterlagen während

\footnotetext{
12 Obwohl Videoaufzeichnungen von den Teilnehmer/-innen gelegentlich zunächst als unangenehm empfunden werden, gewöhnen sie sich bereits an kurzer Zeit daran, sodass das keine langfristige Verhaltensänderung zu erwarten ist (vgl. Lamnek, 2005).
} 
der Diskussion zur Verfügung zu stellen (vgl. Schulz-Hardt et al., in press). In einer Untersuchung von Mojzisch, Schulz-Hardt, Vogelgesang und Kerschreiter (2005) wurde der Informationsaustausch verglichen zwischen Gruppen, die während der Diskussion entweder Zugang zu den vorhandenen Informationen hatten oder nicht. Es zeigte sich ein Deckeneffekt in den Gruppen mit Informationszugang: Nahezu 100\% der geteilten sowie 90\% der ungeteilten Informationen wurden ausgetauscht. Ein solcher Effekt würde in dem vorliegenden Experiment die Überprüfung der Wirksamkeit der Interventionen nicht erlauben. Daher wurde darauf verzichtet, die Informationsblätter mit in die Diskussion zu geben.

Nach Ablauf der 15 Minuten zur Bewertung der Kandidateninformationen erfolgte ein kurzer Hinweis des/der Versuchsleiters/-in auf die verstrichene Zeit, dann standen weitere zehn Minuten zum Memorieren und zur Festigung des Wissens zur Verfügung. Insgesamt standen den Probanden/-innen damit 25 Minuten zum Lernen der Einzelinformationen zur Verfügung. Die individuelle Bearbeitungszeit der Teilnehmer/-innen endete mit einer ersten Einzelentscheidung über den geeigneten Kandidaten und dem Ausfüllen des ersten Erinnerungsfragebogens. Hier erhielten die Probanden/-innen die Instruktion, innerhalb von fünf bis sieben Minuten alle Informationen kandidatenspezifisch zu notieren, die sie erinnern konnten. Bei der Ausgabe neuer Versuchsunterlagen wurden die bereits bearbeiteten eingesammelt und standen den Teilnehmern/-innen somit nicht mehr zur Verfügung. Anschließend fanden sich die Teilnehmer/-innen wieder im Gruppenraum ein und nahmen entsprechend eines vorgegebenen Sitzplans im Halbkreis an einem Tisch Platz (vgl. Schulz-Hardt et al., in press).

Der/die Moderator/-in kam nach Abschluss seines/ihres Trainings in den Diskussionsraum zurück. Versuchsleiter/-in B teilte die Gruppeninstruktion aus und las diese laut vor, während Versuchsleiterin A die Videokamera ausrichtete und einschaltete. In Anlehnung an frühere Hidden-Profile-Forschung wies die letzte Instruktion darauf hin, dass nicht allen Gruppenmitgliedern alle Informationen bekannt seien, sondern sie teilweise über unterschiedliche, aber nicht widersprüchliche, Informationen verfügten. Bei Betrachtung aller 
Informationen gäbe es jedoch einen eindeutig besten Kandidaten und es sei Aufgabe der Gruppe, diesen zu finden. Es wurde außerdem auf die unterstützende Rolle des/der Moderators/-in ${ }^{13}$ hingewiesen und angemerkt, dass diese/r gleich die Gruppendiskussion eröffnen würde. Danach verließen beide Versuchsleiter/-innen den Raum und die Gruppendiskussion begann. Zunächst gab es keine zeitliche Vorgabe. Nach 45 Minuten erfolgte jedoch ein Hinweis der/des Versuchsleiters/-in, die Gruppendiskussion innerhalb der nun folgenden 10 Minuten abzuschließen (die maximale Dauer betrug somit 55 Minuten). Die Versuchsleiter/-innen waren während der Diskussion nicht anwesend, hielten sich aber im Nachbarraum auf, um für eventuelle Nachfragen zur Verfügung zu stehen. Nach dem Abschluss der Diskussion informierte der/die Moderator/-in den/die Versuchsleiter/-in, und alle Teilnehmer/-innen kehrten in ihre individuellen Räume zurück. In der Nachbereitungsphase wurde zunächst erneut ein Erinnerungsfragebogen ausgegeben, im dem alle Informationen, die noch erinnert werden konnten - sowohl vor der Diskussion bekannte als auch neu hinzugewonnene Informationen aufgeschrieben werden sollten. Dafür wurden den Teilnehmern/-innen etwa sieben Minuten eingeräumt. Abschließend folgten weitere Fragebögen, die eine Einschätzung der Gruppendiskussion und des/der Moderators/-in erforderten. Die vollständigen Teilnehmerunterlagen befinden sich exemplarisch für Person X im Anhang D. Alle Teilnehmer/innen wurden zum Abschluss von dem/der Versuchsleiter/-in kurz über den Inhalt und Hintergrund der Untersuchung aufgeklärt und mit Dank und Aufwandsentschädigung entlassen. Nach Beendigung der gesamten Versuchsreihe erhielten alle Versuchsteilnehmer/-innen via Email Informationen zur richtigen Lösung, die Namen der Gewinner von CD- und Fluggutscheinen und einige weitere Anmerkungen zum Experiment. Im Durchschnitt dauert ein gesamter Versuch 120 Minuten.

\footnotetext{
${ }^{13}$ Der Terminus „Moderator/-in“ wurde während der Versuchsdurchführung nicht benutzt, da hier der/die Moderator/-in instruktionsbedingt die Diskussion nicht im eigentlichen Sinne moderiert, sondern lediglich die unabhängigen Variablen umsetzt. Dadurch sollte eine Erwartungshaltung der Diskutanten/-innen an den/die Moderator/-in und eine damit verbundene Unzufriedenheit vermieden werden. Stattdessen wurde der Begriff „Unterstützende Person“ verwendet.
} 


\section{Ablauf des Moderator/-in-Trainings}

Das Training des/der Moderators/-in fand in einem separaten Raum durch Versuchsleiterin A statt. Um Effekten, die auf den/die Versuchsleiter/-in zurückgehen, vorzubeugen, führte in allen Fällen dieselbe Versuchsleiterin das Training durch. Das Training lehnt sich in seiner prinzipiellen Vorgehensweise an das Training der Führungspersonen bei Larson et al. (1998b) an. Zunächst erhielt der/die Teilnehmer/-in ein Deckblatt, das nahezu identisch mit der Eingangsinstruktion für die diskutierenden Teilnehmer/-innen war. Jedoch erhielt der/die Moderator/-in keinerlei spezifische Informationen zur Entscheidungsaufgabe, korrekten Lösung und Informationsverteilung.

Zunächst machte der/die Moderator/-in ebenfalls verschiedene demographische Angaben. Sein/ihr Versuchscode bestand aus einem M und der entsprechenden sechsstelligen Zahl. Dann folgten ein Bogen mit einigen einführenden Worten zur Rolle des/der Moderators/in sowie Instruktionen zu den Grundregeln der Moderation (als Überblick in Tabelle 11), die jeweils in Unterpunkten nochmals ausgeführt wurden. Diese Regeln sahen vor, dass der/die Moderator/-in die Diskussion eröffnet und seine/ihre Aufgaben vorstellt, darauf achtet, dass alle Gruppenmitglieder zu Wort kommen und sich nicht unterbrechen, und den Teilnehmern/-innen abschließend den Gruppenentscheidungsbogen überreicht. Außerdem betonten die Regeln die Wichtigkeit, als Moderator/-in inhaltliche Neutralität zu bewahren. Die vollständigen Instruktionen der Moderation befinden sich in Anhang D.

Durch die Grundregeln der Moderation (Baseline-Moderation), die in allen Bedingungen ausgeführt wurden, sollte sichergestellt werden, dass die Rolle des/der Moderators/-in keinen zu eindimensionalen Charakter bekam. Alleiniges Agieren im Sinne der unabhängigen Variablen (Fragen stellen oder/und Informationen wiederholen) hätte eine künstliche Gesprächssituation geschaffen. Die Grundregeln erlaubten es dem/der Moderator/-in, in kontrollierter Weise auch andere Aufgaben wahrzunehmen, als nur nach Informationen zu fragen bzw. Informationen zu wiederholen. In der Kontrollbedingung erfüllte die Baseline-Moderation zusätzlich den Zweck, 
dem/der als unterstützende Person angekündigten Moderator/-in ebenfalls eine Aufgabe zu geben. Die Anwesenheit einer Person, die während der Diskussion keine ersichtliche Funktion erfüllt, wäre für die Diskutanten/-innen sonst irritierend gewesen.

\section{Tabelle 11: Grundregeln der Moderation für Teilnehmer/-in M}

1. Eröffnen Sie die Diskussion.

2. Erklären Sie, dass Sie sich eventuell während der Diskussion Notizen machen werden, die aber nur für Sie bestimmt sind und auf die die Teilnehmer nicht zurückgreifen dürfen.

3. Fragen Sie in die Gruppe, wie zunächst vorgegangen werden soll. Stimmen Sie dem Vorschlag, der gemacht wird, zu.

4. Seien Sie inhaltlich neutral!

5. Sprechen Sie klar und deutlich!

6. Sorgen Sie dafür, dass sich alle ausreden lassen!

7. Schließen Sie die Diskussion ab!

Nachdem der/die Moderator/-in zunächst selbstständig die Instruktionen durchgelesen hatte, besprachen Versuchsleiterin und Moderator/-in alle Instruktionspunkte im Detail. Eventuelle Fragen des/der Moderators/-in wurden beantwortet. Zu den Grundregeln der Moderation wurde ein Video mit vier Sequenzen gezeigt, in denen die wichtigsten Verhaltensweisen veranschaulicht wurden: Diskussionseröffnung, Einbeziehen stiller Teilnehmer/-innen, Ausreden lassen und Diskussionsabschluss. In den Bedingungen mit Einsatz von Koordinationsverhalten (Bedingungen 1, 2 und 3) wurden dann die Zusatzinstruktionen ausgegeben, die entweder im Detail die Aufgabe des Fragenstellens, des Wiederholens von Informationen oder beides erklärten. Zu jeder Bedingung wurde ein zusätzliches Video gezeigt, in dem die anzuwendenden Verhaltensweisen beispielhaft dargestellt wurden. Anschließend hatte der/die Moderator/-in etwa zehn Minuten Zeit, sich Notizen zu den Regeln zu machen, die er/sie mit in die Gruppendiskussion nehmen konnte. Diese wurden nicht ausgewertet. In den Bedingungen mit Informationsfragen und/oder -wiederholungen bearbeitete der/die Moderator/-in außerdem entsprechend der jeweiligen Instruktion vor Abschluss des Trainings einen Übungszettel bestehend aus einem A4-seiten langen Auszug einer Gruppendiskussion. 
$\mathrm{Er} /$ sie musste beispielsweise vermerken, an welchen Stellen er/sie eine Wiederholung bzw. Frage hätte einbringen können.

Die Instruktionen hoben hervor, wie wichtig eine genaue Beachtung aller Regeln sei und gaben an, in der Diskussion nichts über Inhalt und Ablauf des Trainings zu berichten. Um die Motivation des/der Moderators/-in zu erhöhen, ihre Rolle entsprechend auszuüben, konnten sie sich für die Teilnahme an einer Verlosung eines CD-Gutscheins im Wert von $50 €$ qualifizieren. Voraussetzung war, alle geforderten Verhaltensweisen - und nur diese, korrekt einzuhalten. Das komplette Training des/der Moderators/-in dauerte je nach Bedingung zwischen 25 und 40 Minuten.

Nach der Gruppendiskussion füllte der/die Moderator/-in ebenfalls Fragebögen zur Einschätzung der Gruppendiskussion und seiner/ihrer eigenen Rolle aus (siehe Anhang D).

Festzuhalten im Sinne der Umsetzung der unabhängigen Variablen ist, dass der/die Moderator/-in weder nach bestimmten (₹.B. geteilten) Information fragte, noch bestimmte Informationen wiederholte. Da er/sie weder über Wissen über die individuelle Informationsverteilung noch über die korrekte Gruppenentscheidung verfügt, kann er/sie beispielsweise nicht gezielt nach ungeteilten oder der initialen Präferenz widersprechenden Informationen fragen. Diese Manipulation der Intervention wurde bewusst gewählt, um der Validität der Untersuchung Rechnung zu tragen. Der Zweck jeder Moderation besteht in der inhaltlich neutralen Unterstützung des Gruppenprozesses, kein/e Moderator/-in verfügt zu Beginn der Moderation über detaillierte inhaltliche Informationen der Gruppenmitglieder noch kennt er/sie die richtige Lösung des Gruppenproblems.

Eine Übersicht über den gesamten Versuchsablauf für Diskutanten/-innen und Moderator/-in gibt Tabelle 12. 
Tabelle 12: Übersicht über den Versuchsablauf für Diskutant/-innen und Moderator/-in

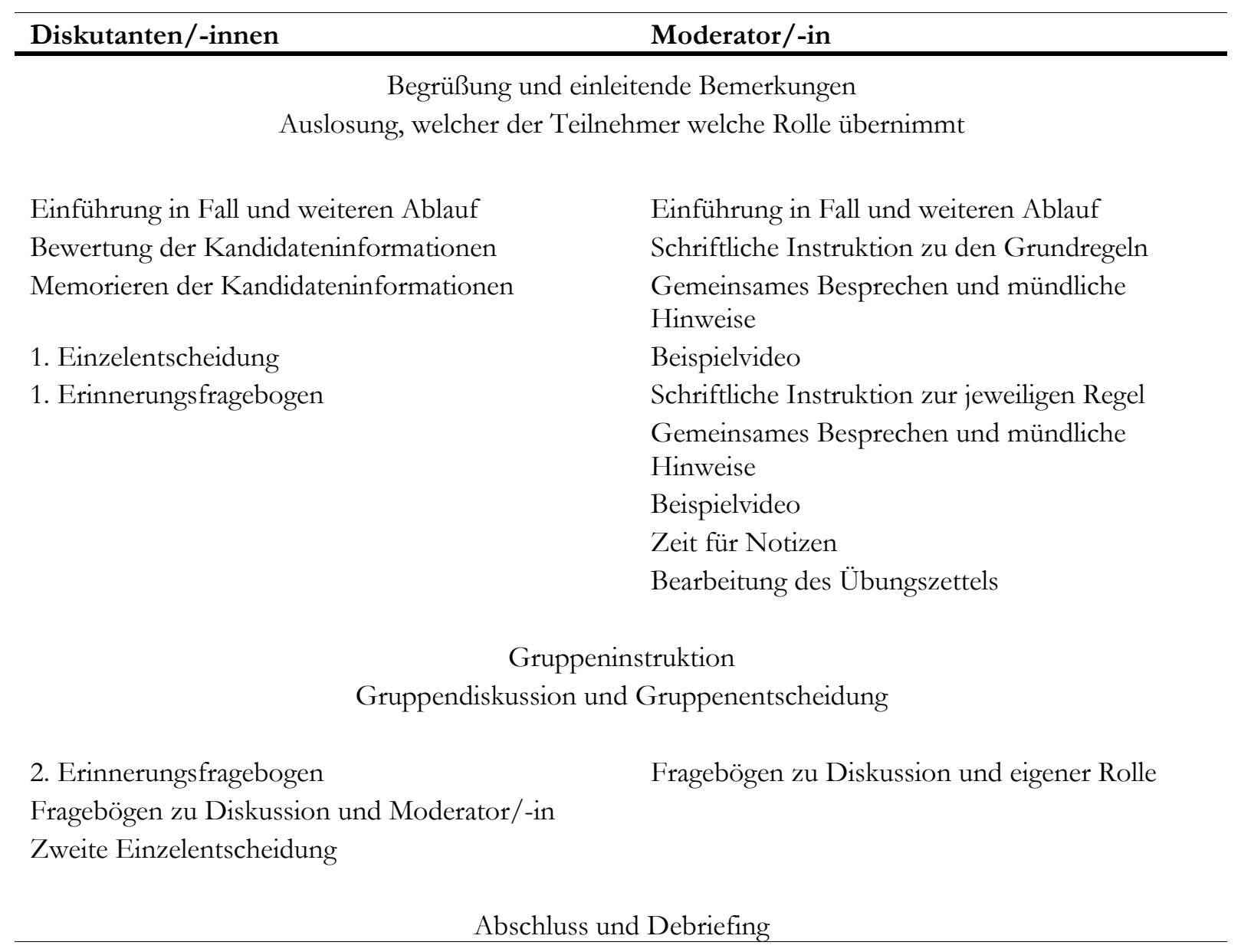

Anmerkung: bei für Diskutanten/-innen und Moderator/-in separat aufgeführten Punkten ist keine zeitliche Kontingenz impliziert

Die Ergebnisse des Experiments werden im folgenden Kapitel dargestellt. 


\subsection{Ergebnisse}

Bevor die Ergebnisse der Hypothesentestungen dargestellt werden, wird zunächst auf allgemeine Ergebnisse eingegangen.

\subsubsection{Allgemeine Ergebnisse}

\section{Manipulation Checks}

Zunächst wurde überprüft, ob die Manipulation der unabhängigen Variablen erfolgreich war. Die Teilnehmer/-innen wurden im Anschluss an die Gruppendiskussion befragt, ob und inwieweit der/die Moderator/-in Fragen gestellt bzw. Informationen wiederholt hat: „Hat diese Person während der Diskussion nach Informationen zu einzelnen Kandidaten gefragt?" bzw. „Hat diese Person während der Diskussion Informationen zu einzelnen Kandidaten wiederholt?" (0 ... überhaupt nicht, 7 ... sehr).

Die Ergebnisse zeigen, dass die Manipulation für beide Bedingungen erfolgreich war. In den Frage-Bedingungen stellt der/die Moderator/-in signifikant mehr Informationsfragen $(M=5.42, S D=1.72)$ als in den Nicht-Frage-Bedingungen $(M=2.52 S D=2.40), t(142)=-8.34, p$ $<.001$, zweiseitig). In den Wiederholungs-Bedingungen wiederholt der/die Moderator/-in signifikant mehr Informationen $(M=6.35, S D=.98)$ als in den Nicht-Wiederholungs-Bedingungen $(M=1.23 S D=1.66), t(142)=-22.44, p<.001$, zweiseitig).

Darüber hinaus wurde die inhaltliche Neutralität des/der Moderators/-in überprüft. Dazu beantworteten die Gruppenmitglieder nach der Diskussion die Frage: „Hat diese Person die Gruppe während der Diskussion in eine bestimmte Richtung gedrängt?" ( 0 ... überhaupt nicht, 7 ... sehr). Die Datenanalyse zeigt, dass der/die Moderator/-in die inhaltliche Neutralität gewahrt hat $(M=.77$ $S D=1.37)$, die sich auch zwischen den vier Versuchsbedingungen nicht unterscheidet $F(3$, $143)=1.72, p=.167)$. 


\section{Weitere allgemeine Befunde}

Neben der Kontrolle der Manipulation wurde überprüft, inwieweit die Gruppen ihre Aufgabe, den richtigen Kandidaten auszuwählen, ernst nahmen. Dazu wurden sie im Anschluss an die Diskussion befragt: „Wie wichtig war es Ihnen bei der Aufgabenstellung, die richtige Entscheidung zu treffen?" (0 ... überhaupt nicht, 7 ... sehr). Insgesamt waren die Gruppenmitglieder sehr motiviert, die richtige Entscheidung zu treffen $(M=6.12 S D=1.06)$, es lassen sich diesbezüglich keine Unterschiede zwischen den Versuchsbedingungen finden $F(3,143)=.37, p=.776)$.

Die Analyse der individuellen initialen Entscheidungspräferenzen vor der Gruppendiskussionen zeigte, dass 37 Gruppenmitglieder Kandidat A wählen, 42 Kandidat B, 22 Kandidat C und 43 Kandidat D (vgl. Abbildung 18). Diese Verteilung zeigt, dass das HiddenProfile erfolgreich umgesetzt werden konnte.

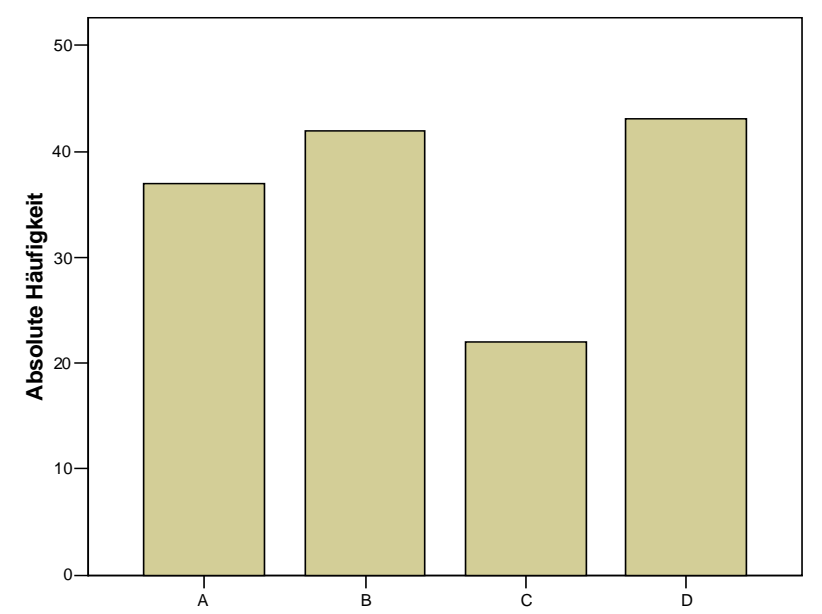

Abbildung 18: Verteilung der individuellen initialen Entscheidungspräferenzen der Kandidaten A, B, C und D

Gefragt nach der Sicherheit, mit die Teilnehmer/-innen ihre Einzelentscheidung vor der Gruppenentscheidung für richtig halten (,Wie sicher sind Sie, dass Ibre Entscheidung richtig ist?“ (0 ... überhaupt nicht, 7 ... sehr), so liegen die Sicherheitseinschätzungen im mittleren Bereich $(M=4.76$ 
$S D=1.30$ ), es lassen sich diesbezüglich keine Unterschiede zwischen den Versuchsbedingungen finden $F(3,142)=.15, p=.931)$.

Im Folgenden werden die Ergebnisse der Hypothesentestung vorgestellt. Die Hypothesen 1 und 2 wurden auf Gruppenebene getestet, die Hypothesen 3 bis 6 auf Individualebene.

\subsubsection{Ergebnisse zu den Hypothesen:}

\section{Ergebnisse zur $A V_{1}$ Entscheidungsgüte}

Von den 48 untersuchten Gruppen entschieden sich 4 für Kandidat A, 18 für Kandidat B, 12 für Kandidat C und 14 für Kandidat D. Die meisten Gruppen (40) trafen ihre Entscheidung einstimmig (vgl. Tabelle 13).

Tabelle 13: Häufigkeiten der Einstimmigkeiten der Gruppenentscheidungen

\begin{tabular}{|c|c|c|c|c|c|}
\hline & & \multicolumn{4}{|c|}{ Gruppenentscheidung } \\
\hline & & $\begin{array}{c}\text { Kandidat } \\
\mathrm{A}\end{array}$ & $\begin{array}{c}\text { Kandidat } \\
\text { B }\end{array}$ & $\begin{array}{c}\text { Kandidat } \\
\mathrm{C}\end{array}$ & $\begin{array}{c}\text { Kandidat } \\
\mathrm{D} \\
\end{array}$ \\
\hline \multirow{3}{*}{$\begin{array}{l}\text { Gruppen- } \\
\text { entscheidung } \\
\text { einstimmig } \\
\text { getroffen }\end{array}$} & ja & 2 & 15 & 11 & 12 \\
\hline & nein & 2 & 3 & 1 & 2 \\
\hline & Gesamt & 4 & 18 & 12 & 14 \\
\hline
\end{tabular}

Zwölf Gruppen (25\%) trafen die richtige Entscheidung. Tabelle 14 enthält die Häufigkeitsverteilung der Entscheidungen in den vier Versuchsbedingungen. Zwei Gruppen der Kontrollbedingung entschieden sich für die richtige Lösung. In Bedingung 1, in der nur nach Informationen gefragt wurde, fand keine Gruppe die richtige Lösung. In Bedingung 2 wurden die Informationen wiederholt, hier entschieden sich 5 Gruppen für die richtige Lösung. In der dritten Bedingung wurden sowohl Informationen erfragt als auch wiederholt, auch hier entschieden sich 5 Gruppen für die richtige Lösung. 
Tabelle 14: Verteilung der Häufigkeiten der Gruppenentscheidungen in den einzelnen Bedingungen

\begin{tabular}{llccc}
\hline & & Keine Fragen & Fragen & Gesamt \\
\hline $\begin{array}{l}\text { Gruppen- } \\
\text { entscheidung } \\
\text { richtig }\end{array}$ & Keine Wiederholungen & 2 & 0 & 2 \\
& Wiederholungen & 5 & 5 & 10 \\
& Keine Wiederholungen & 7 & 5 & 12 \\
$\begin{array}{l}\text { Gruppen- } \\
\text { entscheidung } \\
\text { falsch }\end{array}$ & Wiederholungen & 10 & 12 & 22 \\
& Gesamt & 7 & 7 & 14 \\
\hline
\end{tabular}

Nach den Hypothese $\mathrm{H}_{1-1}$, bis $\mathrm{H}_{1-3}$ wird erwartet, dass durch das Stellen von Informationsfragen und durch das Wiederholen genannter Informationen die Entscheidungsgüte verbessert wird. Zur Überprüfung dieser Hypothese wurde eine loglineare Analyse der Gruppenentscheidung (richtig vs. falsch) mit den Faktoren Fragen (ja vs. nein) und Wiederholen (ja vs. nein) durchgeführt. Das nach Rückwärtseliminierung resultierende Modell beinhaltet ausschließlich den Faktor Wiederholen, der Faktor Fragen und beider Faktoren Interaktion konnten aus dem Modell ausgeschlossen werden, ohne dass es sich signifikant verschlechterte. Der Faktor Wiederholen übt einen signifikanten Haupteffekt auf die Entscheidungsgüte aus $\left(\chi^{2}(1)=7.61, p<.01\right.$; odds ratio $\left.=7.89\right)($ vgl. Abbildung 19). Der Likelihood Ratio des finalen Modells beträgt $\chi^{2}(4)=2.95, p=.565$, demnach gibt es keine signifikanten Unterschiede zwischen dem durch das Modell vorhergesagten und tatsächlichen Daten und es kann davon ausgegangen werden, dass das resultierende Modell eine gute Abbildung der Daten darstellt. 


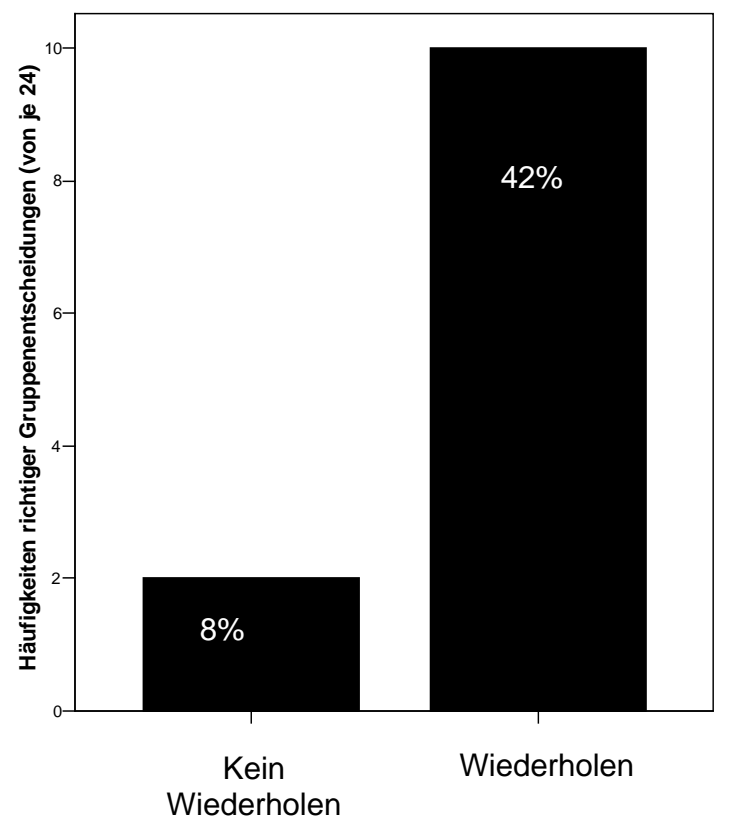

Abbildung 19: Einfluss des regelmäßigen Wiederholens genannter Informationen auf die Entscheidungsgüte

Aus Abbildung 18 geht hervor, dass 15\% der Gruppenmitglieder bereits vor der Diskussion die richtige Entscheidung favorisierten. Daher muss kontrolliert werden, inwieweit lediglich die initiale Entscheidungspräferenz der Mitglieder zur richtigen Gruppenentscheidung führte und welche Rolle das Wiederholen von Informationen unter Kenntnis der initialen Entscheidungspräferenzen spielt. Dazu wurde zunächst die auf Individualebene vorliegende initiale Entscheidungspräferenz in eine Gruppenvariable transformiert $(p=0 . .$. keine $/ \mathrm{r}$ der drei Diskutanten/-innen präferierte die richtige Entscheidung [Kandidat C], $p=.33 \ldots$ ein Gruppenmitglied präferierte C, $p=.67 \ldots$ zwei Gruppenmitglieder präferierten $\mathrm{C}$ und $p=1 \ldots$ alle drei Gruppenmitglieder präferierten C). Die Verteilung dieser initialen Präferenzen in den Bedingungen mit vs. ohne Wiederholen ist Tabelle 15 zu entnehmen. 
Tabelle 15: Absolute Häufigkeiten der korrekten initialen Entscheidungspräferenzen auf Gruppenebene in den Bedingungen mit vs. obne Wiederholung getrennt nach unabbängigen V ariablen

Kein Wiederholen Wiederholen

\begin{tabular}{lccc}
\hline Korrekte initiale &, 00 & 17 & 11 \\
Entscheidungs- &, 33 & 7 & 11 \\
präferenz $(\boldsymbol{p})$ &, 67 & - & 2 \\
& Gesamt & 24 & 24 \\
& & & \\
&
\end{tabular}

Anmerkung: $p=0 . .$. keine/r der drei Diskutanten/-innen präferierte die richtige

Entscheidung [Kandidat C], $p=.33 \ldots$ ein Gruppenmitglied präferierte $C, p=.67$... zwei

Gruppenmitglieder präferierten C.

Zur Kontrolle, inwieweit der gefundene Effekt des Wiederholens von Informationen auf die konfundierte initiale Entscheidungspräferenz zurückgeht, wurde eine logistische Regression berechnet, wobei beide unabhängige Variablen sowie die als intervallskaliert gemessene initiale Entscheidungspräferenz in die Gleichung aufgenommen werden. Tabelle 16 zeigt, dass die initiale Entscheidungspräferenz den erwartbaren signifikanten Einfluss auf die Gruppenentscheidung hat. Allerdings bleibt das Wiederholen von Informationen mit einem Odds Ratio von 5.72 ebenfalls ein sehr guter Prädiktor der Gruppenentscheidung, auch wenn das Signifikanzniveau nun die 5\%-Grenze knapp verfehlt.

Tabelle 16: Logistische Regression zur Überprüfung der Wirkung der unabhängigen Variablen unter Kontrolle der initialen Entscheidungspräferenz.

\begin{tabular}{|c|c|c|c|c|c|c|c|c|}
\hline & $\begin{array}{l}\text { Regressions- } \\
\text { koeffizient B }\end{array}$ & $\begin{array}{c}\text { Standard- } \\
\text { fehler }\end{array}$ & Wald & df & $p$ & \multicolumn{3}{|c|}{$95 \%$ Konfidenzintervall für $\operatorname{Exp}(b)$} \\
\hline & & & & & & $\begin{array}{c}\text { Unterer } \\
\text { Wert }\end{array}$ & $\operatorname{Exp}(b)$ & $\begin{array}{c}\text { Oberer } \\
\text { Wert }\end{array}$ \\
\hline $\begin{array}{l}\text { Initiale } \\
\text { Entscheidungs- } \\
\text { präferenz }\end{array}$ & $-7,60$ & 2,75 & 7,63 & 1 & ,006 & ,000 & ,00 & ,11 \\
\hline Wiederholen & 1,74 & ,95 & 3,36 & 1 & ,067 & 88 & 5,72 & 36,89 \\
\hline Fragen & $-1,00$ &, 89 & 1,23 & 1 & ,266 & ,06 & ,36 & 2,14 \\
\hline Konstante & 2,51 & 1,01 & 6,200 & 1 & ,013 & & 12,36 & \\
\hline
\end{tabular}


Trotz der höheren Zufallswahrscheinlichkeit von $p=.067$ kann die $\mathrm{H}_{1-2}$ angenommen und eine Verbesserung der Gruppenentscheidung durch das Wiederholen von Informationen konstatiert werden.

Insgesamt ergibt sich für die Überprüfung der ersten Hypothese kein einheitliches Bild. Die Hypothese $\mathrm{H}_{1-2}$ konnte angenommen werden, das regelmäßige Wiederholen von Informationen verbessert die Entscheidungsgüte. Die Hypothesen $\mathrm{H}_{1-1}$ und $\mathrm{H}_{1-3}$ konnten nicht angenommen werden. Das Erfragen von Informationen verbessert die Entscheidungsgüte nicht, auch konnte keine Interaktion des Erfragens und Wiederholens von Informationen gefunden werden.

\section{Ergebnisse zur $\mathrm{AV}_{2}$ Bewertung der Eignung des optimalen Kandidaten}

Die Hypothesen $\mathrm{H}_{2-1}$ und $\mathrm{H}_{2-2}$ postulieren, dass sich durch das Erfragen und Wiederholen von Informationen die Bewertung des optimalen Kandidaten C während der Diskussion erhöht. Diese Hypothesen wurden durch eine 2 (Zeit: vor- und nach der Diskussion) x 2 (Bewertung des Kandidaten: ABD-gemittelt vs. C) x 2 (Fragen: keine Fragen vs. Fragen bzw. Wiederholen: kein Wiederholen vs. Wiederholen) Varianzanalyse mit Messwiederholung auf dem ersten Faktor Zeit geprüft. Dabei wurde die von den Teilnehmern/-innen vor der Diskussion gewählte Alternative in die Berechnung einbezogen, da eine Veränderung der Eignungsbewertung von vor zu nach der Diskussion auch davon abhing, ob der bewertete Kandidat bereits vorab die präferierte Alternative des/der Teilnehmers/-in war. Daher wurde die Eignungseinschätzung von $\mathrm{C}_{\text {prä-post }}$ unter Berücksichtigung der initialen Präferenz im Verhältnis zu A, B und D gemittelt dargestellt.

Für den Einfluss des Faktors Fragen $\left(\mathrm{H}_{2-1}\right)$ zeigte sich kein signifikantes Ergebnis ( $F(1$, 139) $=1.34 p=.249)$. Der Faktor Wiederholen hingegen beeinflusst die Bewertung des optimalen Kandidaten: Während in den Bedingungen ohne Informationswiederholung die Bewertung des richtigen Kandidaten nach der Diskussion schlechter ist und die suboptimalen Kandidaten besser bewertet werden (vgl. Abbildung 20), so verbessert sich die Bewertung des richtigen Kandidaten 
in den Bedingungen mit Informationswiederholung und die suboptimalen Kandidaten werden als schlechter bewertet (vgl. Abbildung 21) $(F(1,139)=16.43 p<.001)$.
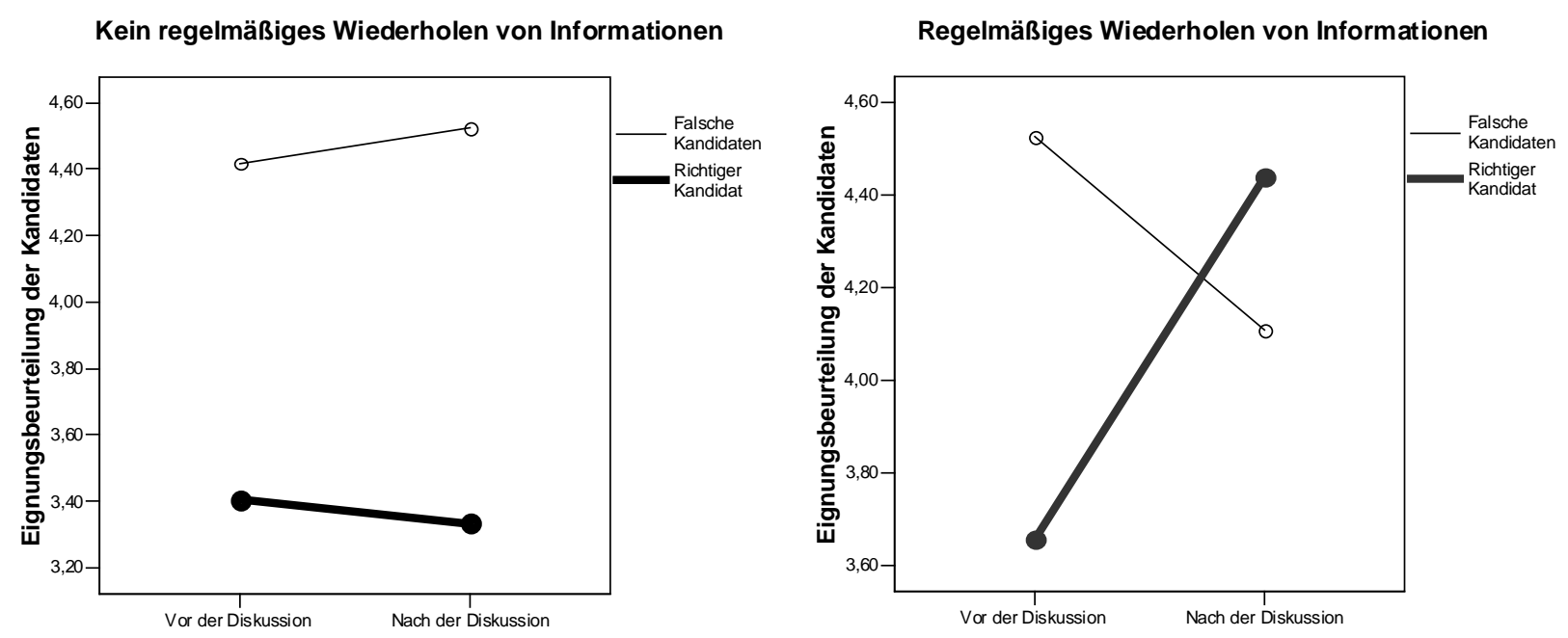

Abbildung 20: Eignungsbeurteilungen der Kandidaten vor im Vergleich zu nach der Diskussion in den Bedingungen obne Wiederbolung von Informationen. Die Ordinate beginnt nicht bei Null.
Abbildung 21: Eignungsbeurteilungen der Kandidaten vor im Vergleich zu nach der Diskussion in den Bedingungen mit Wiederbolung von Informationen. Die Ordinate beginnt nicht bei Null.

Die Effektstärke von $\eta^{2}=.11$ zeigt, dass es sich dabei um einen mittleren bis großen Effekt handelt (vgl. Cohen, 1980, zit. n. Hussy \& Jain, 2002).

Der in der $\mathrm{H}_{2-3}$ postulierte Interaktionseffekt des Erfragens und Wiederholens auf die Eignungseinschätzung zeigt sich nicht $(F(1,139)=.85 p=.360)$. Insgesamt ergibt sich für die Überprüfung der zweiten Hypothese kein einheitliches Bild. Die Hypothese $\mathrm{H}_{2-2}$ konnte angenommen werden, das regelmäßige Wiederholen von Informationen verbessert die Eignungsbeurteilung des richtigen Kandidaten. Die Hypothesen $\mathrm{H}_{2-1}$ und $\mathrm{H}_{2-3}$ konnten nicht angenommen werden.

Neben der Überprüfung der Wirkung der unabhängigen Variablen auf die Bewertung des optimalen Kandidaten interessiert, welche Rolle diese Bewertung in der Vorhersage der korrekten Gruppenentscheidung einnimmt. Dazu konnte in einem Pfadmodell auf Gruppenebene gezeigt 
werden, dass der Anstieg der Bewertung des richtigen Kandidaten tatsächlich verantwortlich für die richtige Gruppenentscheidung ist (vgl. Abbildung 22). Demnach wird die nach der $\mathrm{H}_{1-2}$ angenommene Wirkung des Wiederholens von Informationen auf die Entscheidungsgüte mediiert durch den Bewertungsanstieg der richtigen Entscheidungsalternative während der Diskussion.

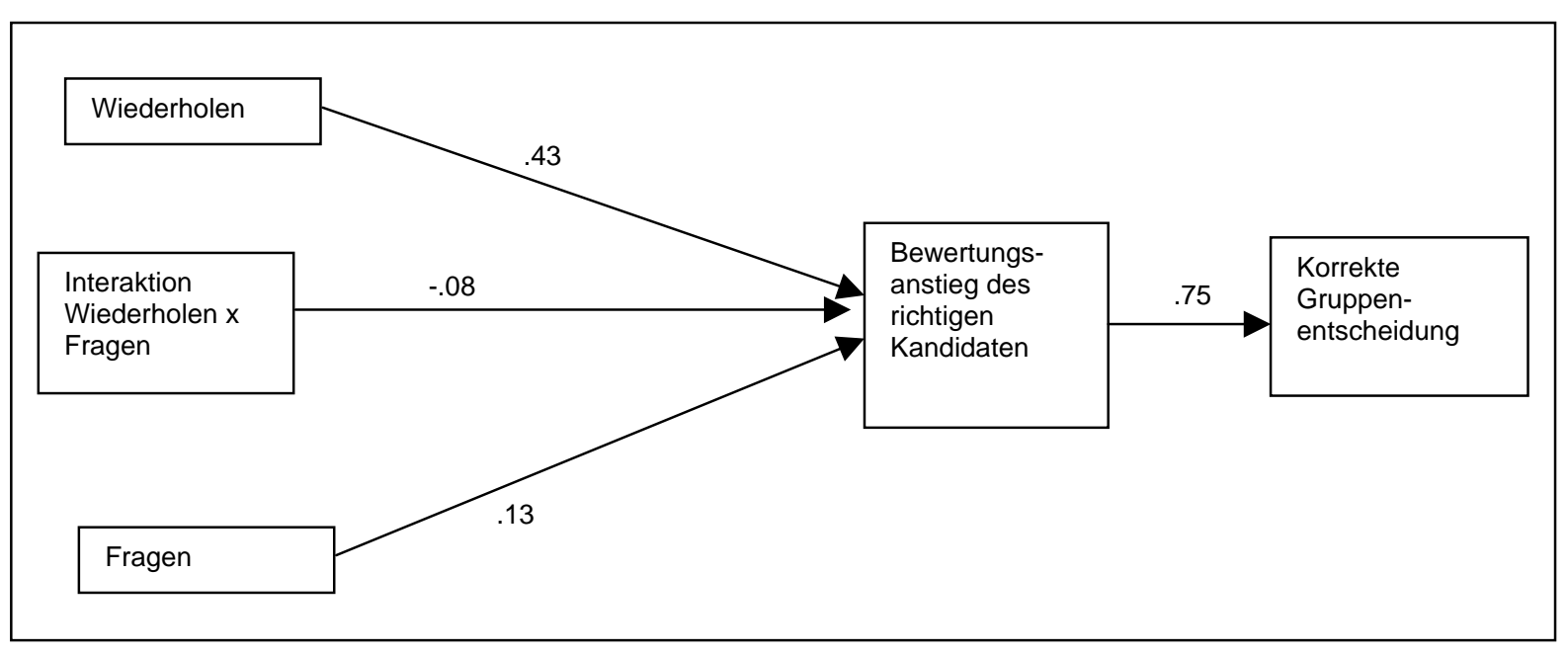

Abbildung 22: Pfadmodell zur Vorhersage der Entscheidungsgüte (Einschluss beider unabhängigen Variablen und deren Interaktion). $N=48$ Gruppen, $D f=3 ; G F I=.94 ; A G F I=.70 ; P G F I=.18 ; \mathrm{RMR}=.06$.

Darüber hinaus konnte gezeigt werden, dass der Mediator (Bewertungsanstieg der richtigen Entscheidungsalternative) nur auf die Wirkung des Wiederholens von Informationen zurückgeht. Werden die anderen Variablen (Erfragen von Informationen; Interaktion) aus der Gleichung eliminiert, so verbessert sich das Modell sogar leicht (vgl. Abbildung 23). 


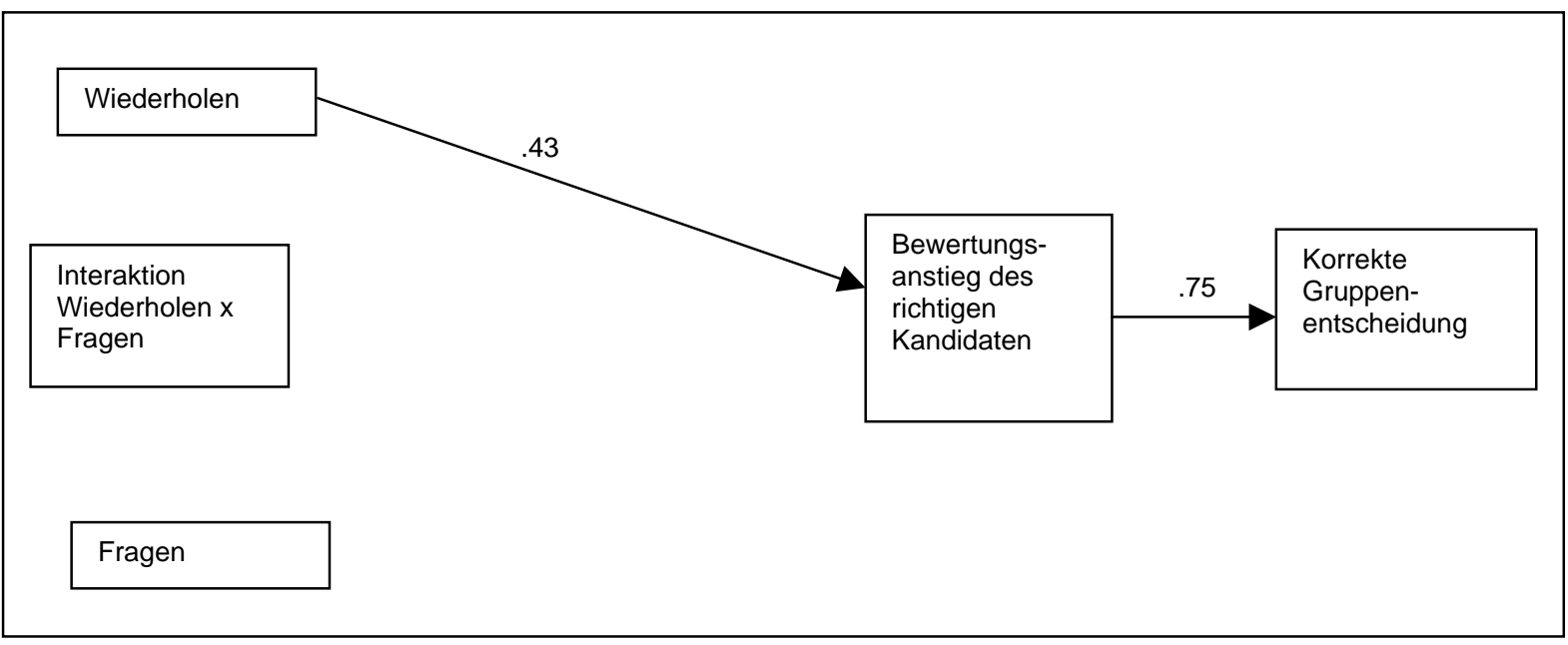

Abbildung 23: Finales Pfadmodell zur Vorbersage der Entscheidungsgüte (Nach Ausschluss nicht beitragender Variablen). $N=48$ Gruppen, $D f=5 ; G F I=.92 ; A G F I=.78 ; P G F I=.31 ; R M R=.05$.

Festzuhalten bleibt, dass das die positive Wirkung des Wiederholens von Informationen auf die Gruppenentscheidung durch den Bewertungsanstieg der richtigen Entscheidungsalternative mediiert wird.

\section{Ergebnisse zur $\mathrm{AV}_{3}$ Informationsaustausch}

Die Hypothesen $\mathrm{H}_{3-1}$ und $\mathrm{H}_{3-2}$ postulieren, dass das Erfragen und Wiederholen von Informationen den Informationsaustausch verbessern.

Von den insgesamt 48 Gruppen wurden die ersten 28 (7 pro Gruppe) in die Untersuchung des Informationsaustauschs einbezogen ${ }^{14}$. Fragt der/die Moderator/-in während der Diskussion regelmäßig nach Informationen, so hat dies weder einen Einfluss auf die Gesamtanzahl der Nennungen geteilter Informationen $(F(1,24)=.25, p=.625)$, noch auf die Erstnennung der geteilten Information $(F(1,24)=.50, p=.485)$. Der gleiche Befund zeigt sich für die ungeteilten Informationen: Fragt der/die Moderator/-in während der Diskussion regelmäßig

\footnotetext{
${ }^{14}$ Die Kodierung des Informationsaustauschs ist sehr zeitaufwendig, so dass sie bis dato nur für eine Teilstichprobe von $n=28$ Gruppen gewährleistet werden konnte.
} 
nach Informationen, so hat dies weder einen Einfluss auf die Gesamtzahl der Nennungen ungeteilter Informationen $(F(1,24)=.08, p=.777)$, noch auf die Erstnennung der ungeteilten Informationen $(F(1,24)=.27, p=.61)$.

Wiederholt der/die Moderatorin während der Diskussion regelmäßig bereits genannte Informationen, so hat dies weder einen Einfluss auf die Gesamtanzahl der Nennungen geteilter Informationen $(F(1,24)=2.94, p=.099)$, noch auf die Erstnennung der Information $(F(1,24)=.06$, $p=.815)$. Allerdings werden insgesamt mehr ungeteilte Informationen genannt $(F(1,24)=4.52$, $\left.p<.05, \eta^{2}=.16\right)(\mathrm{vgl}$. Abbildung 24).

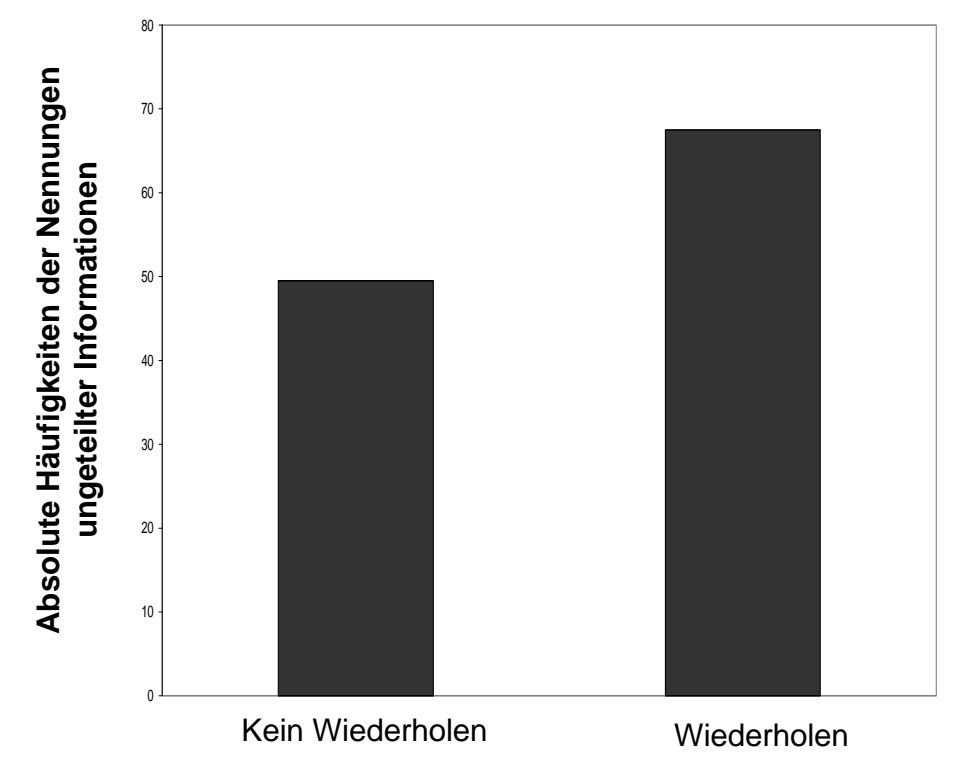

Abbildung 24: Der Einfluss des Wiederholens von Informationen auf die Nennungshäufigkeit ungeteilter Informationen während der Diskussion

Der in $\mathrm{H}_{2-3}$ postulierte Interaktionseffekt des Erfragens und Wiederholens von Informationen auf den Informationsaustausch konnte weder bezüglich der Gesamtanzahl der Nennungen geteilter Informationen $(F(1,24)=1.09, p=.307)$, noch bezüglich der Erstnennung der Information $(F(1,24)=2.75, p=.111)$, noch der Gesamtzahl der Nennungen ungeteilter Informationen $(F(1,24)=.83, p=.371)$, noch bezüglich der Erstnennung der ungeteilten Informationen $(F(1,24)=.03, p=.865)$ gefunden werden. 
Insgesamt zeigt sich, dass das Erfragen von Informationen keinen Einfluss auf den Informationsaustausch ausübte. Das Wiederholen von Informationen bewirkte, dass mehr ungeteilte Informationen genannt wurden.

Darüber hinaus wurde der Informationsaustausch in das in Abbildung 24 dargestellte Pfadmodell integriert, um zu prüfen, welche Rolle er in der Vorhersage der Gruppenentscheidung spielt. Dabei wurde einerseits unterschieden in die Gesamtmenge diskutierter Informationen und der Menge ungeteilter Informationen (vgl. Abbildung 25).

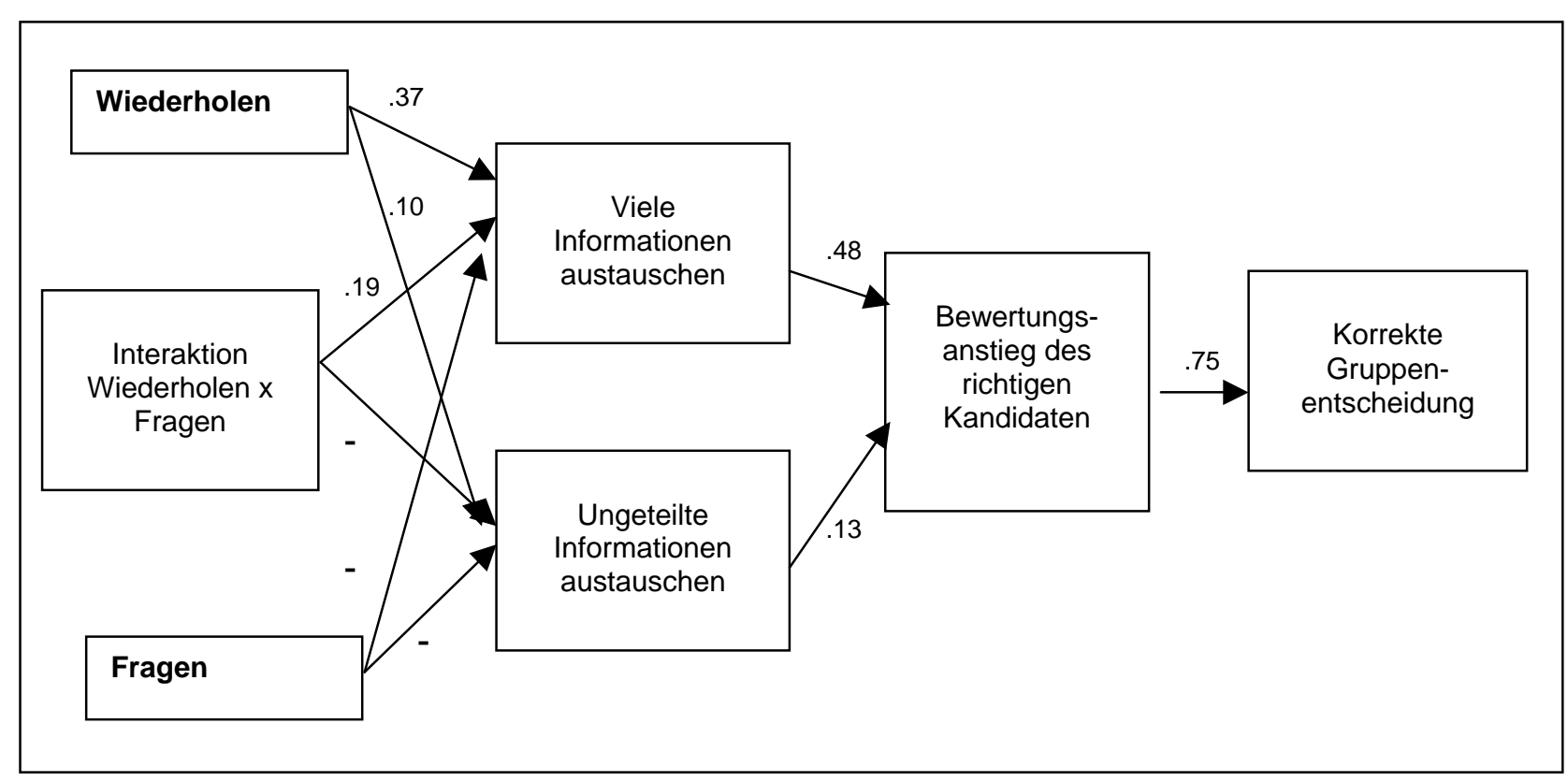

Abbildung 25: Pfadmodell zur Vorhersage der Entscheidungsgüte unter Berücksichtigung des Informationsaustauschs (Einschluss beider unabbängigen Variablen, deren Interaktion als auch des Informationsaustauschs). $N=$ 28 Gruppen $_{s} D f=9 ; G F I=.89 ; A G F I=.65 ; P G F I=.28 ; \mathrm{RMR}=.10$.

Es zeigte sich, dass der Informationsaustausch wesentlich ist für den Bewertungsanstieg des richtigen Kandidaten und damit für die richtige Gruppenentscheidung. Allerdings ist es dabei nicht wichtig, dass hauptsächlich ungeteilte Informationen ausgetauscht werden. Bedeutsamer ist, dass viele Informationen ausgetauscht werden. Wiederholt der/die Moderator/-in regelmäßig Informationen, so führt das dazu, dass insgesamt mehr Informationen ausgetauscht werden, wodurch die Bewertung des richtigen Kandidaten steigt. Die Menge ausgetauschter 
Informationen mediiert also die Wirkung des Wiederholens auf den Bewertungsanstieg der richtigen Entscheidungsalternative und die Gruppenentscheidung (vgl. Abbildung 26).

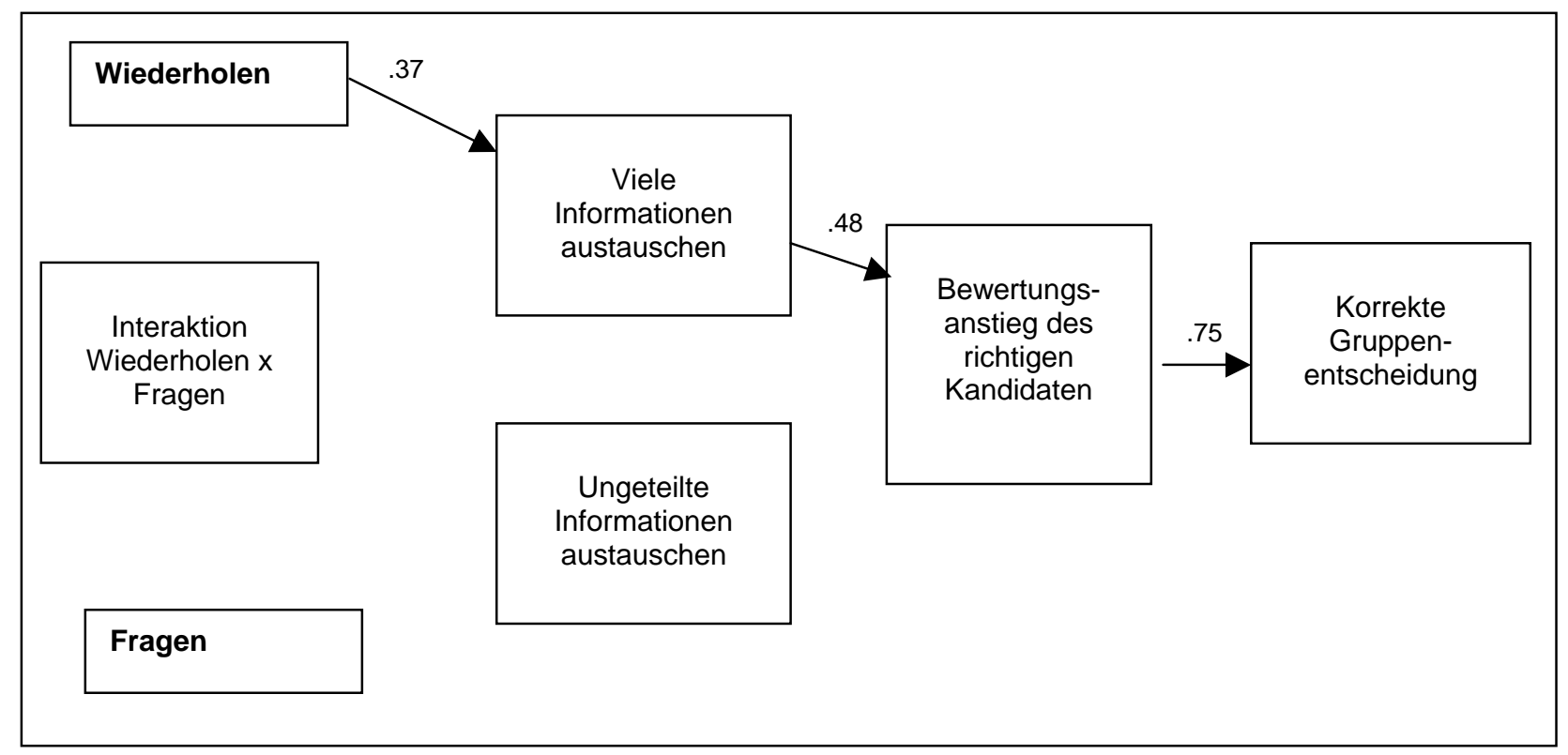

Abbildung 26: Finales Pfadmodell zur Vorhersage der Entscheidungsgüte unter Berücksichtigung des

Informationsaustauschs (nach Ausschluss nicht beitragender Variablen). $N=28$ Gruppen, $_{s} D f=15 ; G F I$ $=.86 ;$ AGFI $=.74 ;$ PGFI $=.46 ; \mathrm{RMR}=.10$.

Nachdem die Ergebnisse im Hinblick auf die Gruppenentscheidungsleistung dargestellt wurden, wird nun die Zufriedenheit der Gruppenmitglieder betrachtet.

\section{Ergebnisse zur $A V_{4}$ Zufriedenheit mit dem Ergebnis der Diskussion}

In den Hypothesen $\mathrm{H}_{4-1}$ und $\mathrm{H}_{4-2}$ wird angenommen, dass das Erfragen bzw. das Wiederholen von Informationen die Zufriedenheit der Gruppenmitglieder mit dem Ergebnis der Diskussion verbessert.

Tabelle 17 zeigt die Mittelwerte und Standardabweichungen der Zufriedenheit mit dem Ergebnis in den verschiedenen Bedingungen.

Tabelle 17: Mittelwerte und Standardabweichungen der Zufriedenheit mit dem Ergebnis in allen Bedingungen

\begin{tabular}{lcccccccccc}
\hline & \multicolumn{3}{c}{$\begin{array}{c}\text { Nach Informationen fragen } \\
\text { nein }\end{array}$} & \multicolumn{3}{c}{ ja } & \multicolumn{3}{c}{ Informationen wiederholen } \\
& $\mathrm{M}$ & $\mathrm{SD}$ & $\mathrm{M}$ & $\mathrm{SD}$ & $\mathrm{M}$ & $\mathrm{SD}$ & $\mathrm{M}$ & $\mathrm{SD}$ \\
& & & & & & & & \\
& $\begin{array}{l}\text { Zufriedenheit mit } \\
\text { dem Ergebnis }\end{array}$ & 5.76 & .90 & 5.85 & 1.00 & 5.61 & 1.04 & 6.00 & .81 \\
\hline
\end{tabular}


Diese Hypothesen wurden mittels einer 2x2 univariaten Varianzanalyse mit den Faktoren Fragen (keine Fragen vs. Fragen) und Wiederholen (kein Wiederholen vs. Wiederholen) überprüft. Die Mittelwerte bezüglich der Ergebniszufriedenheit unterscheiden sich nicht bezüglich des Faktors Fragen $(F(1,140)=.30, p=.586)$. Ein anderes Ergebnis zeigt sich für den Faktor Wiederholen: In den Gruppen, in denen Informationen systematisch wiederholt werden, sind die Gruppenmitglieder zufriedener mit dem Ergebnis der Diskussion als Gruppen, in denen keine Informationen wiederholt werden $F(1,140)=5.59, p<.05, \eta^{2}=.04$. Die in der $\mathrm{H}_{4-3}$ angenommene Interaktionswirkung des Erfragens und Wiederholens von Informationen tritt nicht ein $(F(1,140)=.70 p=.404)$.

\section{Ergebnisse zur $\mathrm{AV}_{5}$ Zufriedenheit mit dem Verlauf der Diskussion}

In den Hypothesen $\mathrm{H}_{5-1}$ und $\mathrm{H}_{5-2}$ wird angenommen, dass das Erfragen bzw. das Wiederholen von Informationen die Zufriedenheit der Gruppenmitglieder mit dem Verlauf der Diskussion verbessert. Die Zufriedenheit mit dem Verlauf wurde über zwei Skalen gemessen: Arbeitsklima und Beitragsmöglichkeiten. Tabelle 18 zeigt die Mittelwerte und Standardabweichungen der Zufriedenheit mit dem Verlauf in den verschiedenen Bedingungen.

Tabelle 18: Mittelwerte und Standardabweichungen der Zufriedenheit mit dem Verlauf in allen Bedingungen

\begin{tabular}{|c|c|c|c|c|c|c|c|c|}
\hline & \multicolumn{4}{|c|}{ Nach Informationen fragen } & \multicolumn{4}{|c|}{ Informationen wiederholen } \\
\hline & \multicolumn{2}{|c|}{ nein } & \multicolumn{2}{|c|}{$\mathrm{ja}$} & \multicolumn{2}{|c|}{ nein } & \multicolumn{2}{|c|}{ ja } \\
\hline & $\mathrm{M}$ & $\overline{\mathrm{SD}}$ & M & $\overline{\mathrm{SD}}$ & $\bar{M}$ & $\overline{\mathrm{SD}}$ & $\bar{M}$ & $\overline{\mathrm{SD}}$ \\
\hline $\begin{array}{l}\text { Zufriedenheit mit } \\
\text { Verlauf } \\
\text { (Arbeitsklima) }\end{array}$ & 5.67 & .77 & 5.62 & .69 & 5.54 & .77 & 5.75 & .68 \\
\hline $\begin{array}{l}\text { Zufriedenheit mit } \\
\text { Verlauf } \\
\text { (Beitragsmöglichkeiten) }\end{array}$ & 5.49 & .94 & 5.39 & .90 & 5.38 & .86 & 5.50 & .98 \\
\hline
\end{tabular}

Die Hypothesen wurden mittels zwei 2x2 univariaten Varianzanalysen mit den Faktoren Fragen (keine Fragen vs. Fragen) und Wiederholen (kein Wiederholen vs. Wiederholen) überprüft. Die Mittelwerte bezüglich des Arbeitsklimas unterscheiden sich weder bezüglich des 
Faktors Fragen $(F(1,140)=.20, p=.658)$ noch bezüglich des Faktors Wiederholen $(F(1,140)=2.80$, $p=.096)$. Auch die Mittelwerte bezüglich der eigenen Beitragsmöglichkeiten unterscheiden sich weder bezüglich des Faktors Fragen $(F(1,140)=.46, p=.499)$ noch bezüglich des Faktors Wiederholen $(F(1,140)=.60, p=.438)$.

Darüber hinaus tritt auch die in der $\mathrm{H}_{5-3}$ angenommene Interaktionswirkung des Erfragens und Wiederholens von Informationen weder für Arbeitsklima $(F(1,140)=.62 p=.433)$ noch für Beitragsmöglichkeiten ein $(F(1,140)=2.64 p=.106)$.

\section{Ergebnisse zur $A V_{6}$ Zufriedenheit mit dem/der Moderator/-in}

In den Hypothesen $\mathrm{H}_{6-1}$ und $\mathrm{H}_{6-2}$ wird angenommen, dass das Erfragen bzw. das Wiederholen von Informationen die Zufriedenheit der Gruppenmitglieder mit dem/der Moderator/-in erhöht. Die Zufriedenheit mit dem/der Moderator/-in wurde über zwei Skalen gemessen: Kompetenz und Hilfe. Tabelle 19 zeigt die entsprechenden Mittelwerte und Standardabweichungen.

Tabelle 19: Mittelwerte und Standardabweichungen der Zufriedenheit mit dem/der Moderator/-in

\begin{tabular}{|c|c|c|c|c|c|c|c|c|}
\hline & \multicolumn{4}{|c|}{ Nach Informationen fragen } & \multicolumn{4}{|c|}{ Informationen wiederholen } \\
\hline & \multicolumn{2}{|c|}{ nein } & \multicolumn{2}{|c|}{ ja } & \multicolumn{2}{|c|}{ nein } & \multicolumn{2}{|c|}{$\mathrm{ja}$} \\
\hline & $\mathrm{M}$ & SD & $\mathrm{M}$ & $\mathrm{SD}$ & $\mathrm{M}$ & $\mathrm{SD}$ & $\mathrm{M}$ & $\mathrm{SD}$ \\
\hline $\begin{array}{l}\text { Zufriedenheit mit } \\
\text { Moderator } \\
\text { (Kompetens) }\end{array}$ & 4.21 & 1.59 & 4.52 & 1.54 & 4.02 & 1.51 & 4.71 & 1.55 \\
\hline $\begin{array}{l}\text { Zufriedenheit mit } \\
\text { Moderator (Hilfe) }\end{array}$ & 3.33 & 1.58 & 3.82 & 1.68 & 2.95 & 1.49 & 4.20 & 1.56 \\
\hline
\end{tabular}

Diese Hypothesen wurden mittels zwei 2x2 univariaten Varianzanalysen mit den Faktoren Fragen (keine Fragen vs. Fragen) und Wiederholen (kein Wiederholen vs. Wiederholen) überprüft. Es zeigte sich, dass das Stellen von Informationsfragen zwar Wahrnehmung der Kompetenz der/des Moderators/-in nicht verbessert $(F(1,140)=1.49 \quad p=.223)$, allerdings 
empfanden die Gruppenmitglieder den/die Moderator/-in tendenziell als hilfreicher, auch wenn die statistische Signifikanz knapp verfehlt wird $\left(F(1,140)=3.85 p=.052 . \eta^{2}=.027\right)$.

Wiederholt der/die Moderator/-in regelmäßig Informationen, wird er/sie sowohl als kompetenter $\left(F(1,140)=7.70 p<.01 . \eta^{2}=.05\right)$ als auch als hilfreicher $(F(1,140)=22.44 p<.001$. $\left.\eta^{2}=.15\right)$ eingeschätzt. Diese Effekte werden in Abbildung 27 und Abbildung 28 dargestellt.

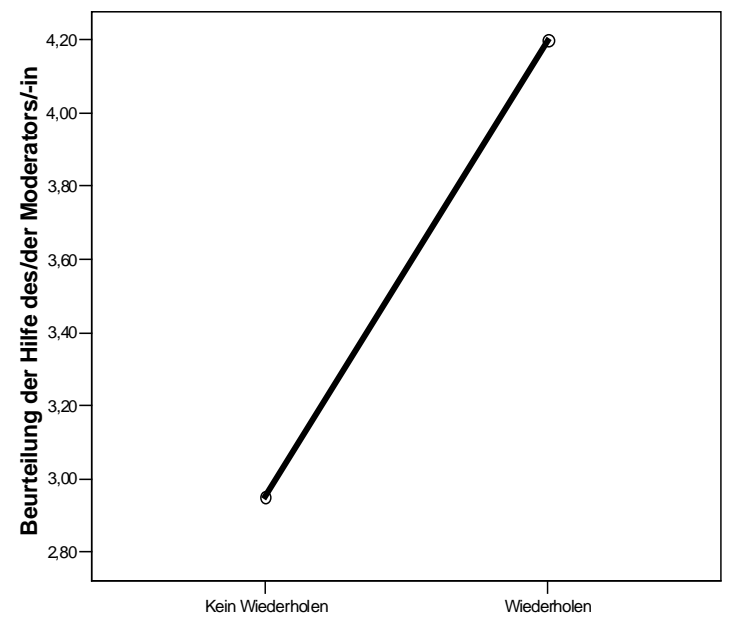

Abbildung 27: Beurteilung der Hilfe der/des Moderators/-in im Vergleich der Bedingungen mit vs. obne Wiederbolen. Die Ordinate beginnt nicht bei Null.

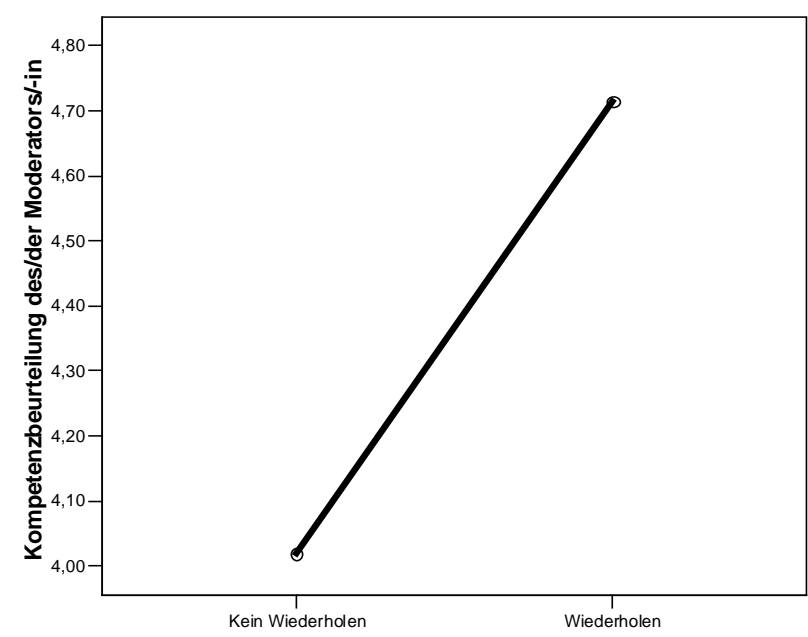

Abbildung 28: Beurteilung der Kompetenz der/ des Moderators/in im Vergleich der Bedingungen mit vs. obne Wiederbolen. Die Ordinate beginnt nicht bei Null.

Die in Hypothese $\mathrm{H}_{6-3}$ postulierte Interaktion des Erfragens und Wiederholens von Informationen wird bezüglich der wahrgenommenen Kompetenz des/der Moderators/-in bestätigt (vgl. Abbildung 29): Fragt der/die Moderator/-in nach Informationen, so muss er/sie ebenfalls Informationen wiederholen, um von den Diskutanten/-innen als kompetent wahrgenommen $\mathrm{zu}$ werden $\left(F(1,140)=5.10, p<.05, \eta^{2}=.04\right)$. Eine Interaktion bezüglich der erlebten Hilfe findet sich nicht $(F(1,140)=.02, p=8.76)$. 


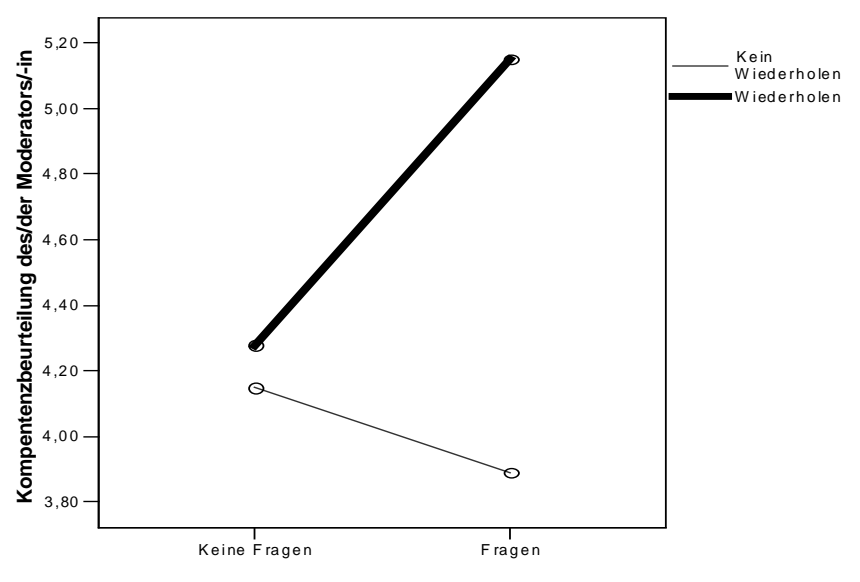

Abbildung 29: Interaktionseffekt des Erfragens und Wiederbolens von Informationen auf die

Kompetenzbeurteilung des/der Moderator/-in. Die Ordinate beginnt nicht bei Null.

Insgesamt ergibt sich für die Überprüfung der sechsten Hypothese kein einheitliches Bild. Die Hypothese $\mathrm{H}_{6-1}$ konnte nur teilweise angenommen werden: Das regelmäßige Erfragen von Informationen seitens des/der Moderators/-in verbessert lediglich die Wahrnehmung seiner/ihrer Unterstützung, nicht aber seiner/ihrer Kompetenz.

Die Hypothese $\mathrm{H}_{6-2}$ konnte angenommen werden, wiederholt der/die Moderator/-in während der Diskussion regelmäßig bereits genannte Informationen, so wird er/sie sowohl als kompetenter als auch als hilfreicher erlebt. Die Hypothese $\mathrm{H}_{6-3}$ zum Interaktionseffekt des Fragens und Wiederholens kann für die wahrgenommene Kompetenz des/der Moderators/-in angenommen werden.

Die Ergebnisse der Hypothesentestung werden im folgenden Kapitel tabellarisch zusammengefasst. 


\subsubsection{Zusammenfassung der Ergebnisse}

Die Tabelle 20 fasst die Ergebnisse aller Hypothesentestungen zusammen.

Tabelle 20: Überblick über die Ergebnisse der Hypothesentestung

\begin{tabular}{|c|c|c|}
\hline $\begin{array}{l}\text { Unabhängige } \\
\text { Variablen }\end{array}$ & Hypothesen & $\begin{array}{l}\text { Annahme } \\
\text { der } \\
\text { Hypothese }\end{array}$ \\
\hline \multirow[t]{7}{*}{$\begin{array}{l}\text { Erfragen von } \\
\text { Informationen } \\
\left(\mathrm{UV}_{1}\right) \text { : }\end{array}$} & $\begin{array}{l}\text { Gruppen, in denen ein/e Moderator/-in zusätzlich zu ausgewählten } \\
\text { allgemeinen Gesprächsleitungsregeln Informationsfragen stellt, } \\
\text { zeigen }\end{array}$ & \\
\hline & - $\quad$ eine erhöhte Entscheidungsgüte $\left(\mathrm{H}_{1-1}\right)$, & Nein \\
\hline & $\begin{array}{l}\text { eine höhere Bewertung der Eignung des optimalen Kandidaten } \\
\text { von vor zu nach der Diskussion }\left(\mathrm{H}_{2-1}\right)\end{array}$ & Nein \\
\hline & - besseren Informationsaustausch $\left(\mathrm{H}_{3-1}\right)$, & Nein \\
\hline & - $\quad$ höhere Zufriedenheit mit dem Ergebnis $\left(\mathrm{H}_{4-1}\right)$, & Nein \\
\hline & - $\quad$ höhere Zufriedenheit mit dem Verlauf $\left(\mathrm{H}_{5-1}\right)$ & Nein \\
\hline & - $\quad$ sowie höhere Zufriedenheit mit dem/der Moderator/-in $\left(\mathrm{H}_{6-1}\right)$. & Zum Teil \\
\hline \multirow{7}{*}{$\begin{array}{l}\text { Wiederholen } \\
\text { von } \\
\text { Informationen } \\
\left(\mathrm{UV}_{2}\right)\end{array}$} & $\begin{array}{l}\text { Gruppen, in denen ein/e Moderator/-in zusätzlich zu ausgewählten } \\
\text { allgemeinen Gesprächsleitungsregeln bereits genannte Information } \\
\text { wiederholt, zeigen }\end{array}$ & \\
\hline & - $\quad$ eine erhöhte Entscheidungsgüte $\left(\mathrm{H}_{1-2}\right)$, & $\mathrm{Ja}$ \\
\hline & $\begin{array}{l}\text { eine höhere Bewertung der Eignung des optimalen Kandidaten } \\
\text { von vor zu nach der Diskussion }\left(\mathrm{H}_{2-2}\right)\end{array}$ & $\mathrm{Ja}$ \\
\hline & - $\quad$ einen besseren Informationsaustausch $\left(\mathrm{H}_{3-2}\right)$ & Zum Teil \\
\hline & - $\quad$ höhere Zufriedenheit mit dem Ergebnis $\left(\mathrm{H}_{4-2}\right)$, & $\mathrm{Ja}$ \\
\hline & - $\quad$ höhere Zufriedenheit mit dem Verlauf $\left(\mathrm{H}_{5-2}\right)$ & Nein \\
\hline & - $\quad$ sowie höhere Zufriedenheit mit dem/der Moderator/-in $\left(\mathrm{H}_{6-2}\right)$. & $\mathrm{Ja}$ \\
\hline
\end{tabular}

Interaktionen
von
Erfragen und
Wiederholen
von
Informationen
$\left(U^{\prime} V_{1} \times V_{2}\right):$

In Gruppen, in denen ein/e Moderator/-in zusätzlich zu ausgewählten allgemeinen Gesprächsleitungsregeln Informationsfragen stellt und Informationen wiederholt, lassen sich Interaktionseffekte finden in Bezug auf

- die Entscheidungsgüte $\left(\mathrm{H}_{1-3}\right)$ Nein

- die Bewertung der Eignung des optimalen Kandidaten von vor Nein zu nach der Diskussion (2-3), den Informationsaustausch $\left(\mathrm{H}_{3-3}\right), \quad$ Nein

die Zufriedenheit mit dem Ergebnis $\left(\mathrm{H}_{4-3}\right), \quad$ Nein

die Zufriedenheit mit dem Verlauf $\left(\mathrm{H}_{5-3}\right) \quad$ Nein

sowie die Zufriedenheit mit dem/der Moderator/-in $\left(\mathrm{H}_{6-3}\right)$. Zum Teil

Es kann festgehalten werden, dass der Koordinationsmechanismus Erfragen von Informationen in dem vorliegenden Experiment fast keinen Einfluss auf die Entscheidungsfindung 
ausübte. Hingegen stellte das Wiederbolen von Informationen einen effektiven Koordinationsmechanismus dar. Diese Ergebnisse werden im folgenden Kapitel diskutiert.

\subsection{Diskussion}

Zunächst werden die Ergebnisse in Bezug auf die eingangs aufgestellten Hypothesen diskutiert (Kapitel 5.5.1). Anschließend wird auf mögliche methodische Einschränkungen des Experiments eingegangen (Kapitel 5.5.2). Schließlich wird die Bedeutung der Ergebnisse im Hinblick auf die praktische Anwendung sowie auf die weitere Forschung diskutiert (Kapitel 5.5.3).

\subsubsection{Interpretation und Diskussion der erhaltenen Ergebnisse}

Das Ziel des Experiments bestand in der Überprüfung der Wirksamkeit expliziter Koordinationsmechanismen. Es wurde überprüft, inwieweit die Koordination des Entscheidungsfindungsprozesses durch systematisches Erfragen oder Wiederholen von Informationen durch eine/n trainierte/n Moderator/-in die Entscheidungsfindung verbessert. Dabei stand nicht nur die Güte der Gruppenentscheidung, sondern auch die Zufriedenheit der Gruppenmitglieder im Fokus.

Insgesamt zeigte sich, dass das regelmäßige Erfragen von Informationen durch eine/n Moderator/-in als Koordinationsmechanismus im Hinblick auf die Entscheidung nahezu wirkungslos ist. Das regelmäßige Wiederholen bereits genannter Informationen ist hingegen sehr effektiv, wobei es hauptsächlich die Qualität der Entscheidung verbessert, jedoch wenig Einfluss auf die Zufriedenheit der Gruppenmitglieder ausübt.

Im Folgenden sollen die Ergebnisse zu den einzelnen Hypothesen diskutiert werden. Dabei wird zunächst auf die performanzbezogenen Kriterien eingegangen. Im Anschluss daran werden die Ergebnisse zur Zufriedenheit der Gruppenmitglieder diskutiert. 
Einfluss der Koordinationsmechanismen auf die Gruppenentscheidung

( $A V_{1}$ bis $\mathrm{AV}_{3}$ : Entscheidungsgüte, Bewertung der richtigen Entscheidungsalternative und

Informationsaustausch)

In den ersten drei Hypothesen steht als performanzbezogenes Kriterium die Gruppenentscheidung im Vordergrund. Es wird erwartet, dass sich die Koordinationsmechanismen Informationen erfragen und Informationen wiederbolen sowohl auf die Gruppenentscheidung, als auch auf die Bewertung der optimalen Entscheidungsalternative und den Informationsaustausch positiv auswirken.

Ausgehend von der Überlegung, dass sich Gruppenentscheidungen qualitativ verbessern, wenn während ihres Entscheidungsprozesses ein intensiver Austausch von entscheidungsrelevantem Wissen stattfindet, wurde angenommen, dass sich das Erfragen von Informationen förderlich auswirkt. Nach Hypothese $\mathrm{H}_{1-1}$ wird erwartet, dass durch das Stellen von Informationsfragen die Entscheidungsgüte verbessert wird. Die Ergebnisse stützen diese Hypothese nicht. Fragt der/die Moderator/-in die Gruppenmitglieder während der Diskussion nach ihrem Wissen über die Entscheidungsalternativen, so hat dies keinen positiven Einfluss auf die Gruppenentscheidung. In den Hypothesen $\mathrm{H}_{2-1}$ und $\mathrm{H}_{3-1}$ wurde erwartet, dass das Erfragen von Informationen die Bewertung der korrekten Entscheidungsalternative sowie den Informationsaustausch verbessert. Auch diese Hypothesen müssen abgelehnt werden. Die genannten Ergebnisse geben Anlass zu Überlegungen, wodurch die fehlende Wirkung des Koordinationsmechanismus Informationsfrage begründet ist. Es erscheint zunächst intuitiv plausibel, zu erwarten, dass bei einer Gruppenaufgabe, die durch den Austausch von Informationen gelöst werden könnte, das Erfragen von Informationen die Gruppenmitglieder dazu ermutigen würde, ihnen bekannte Informationen zu nennen. Diese Vermutung wird durch die Daten nicht gestützt. Es könnte sein, dass das Erfragen wirkungslos blieb, weil sich die Gruppenmitglieder nicht mehr an die erfragten Informationen erinnern konnten. Diese These kann allerdings verworfen werden, da sich die Erinnerungsleistungen der Gruppenmitglieder zwischen den Bedingungen nicht unterschieden. Weiterhin ist denkbar, dass sich die 
Gruppenmitglieder durch das regelmäßige Erfragen von Informationen gestört fühlten und deshalb im Sinne einer Reaktanzreaktion keine Informationen preisgaben. Allerdings kann auch diese Überlegung angesichts des Befundes, dass die Zufriedenheit in den Fragebedingungen nicht schlechter als in der Kontrollgruppe war, verworfen werden.

Die mangelnde Effektivität der Informationsfrage als Koordinationsmechanismus gibt Anlass zu weiterer Forschung. Dabei sollten zwei Punkte im Vordergrund stehen:

1. Der Fragetyp Informationsfrage sollte mit inhaltlich vergleichbaren, aber formal anderen Fragetypen (z.B. Meinungsfrage) verglichen werden. Möglicherweise wird die Frage nach Informationen von den Befragten als zu wenig wertschätzend empfunden. Denkbar ist, dass die Befragten dabei das Gefühl hatten, lediglich als Informationslieferanten/-innen wahrgenommen zu werden, ohne dass ihre persönlichen Meinungen von Interesse waren. Vor dem Hintergrund, dass der Informationsaustausch auch eine motivationale Komponente beinhaltet (Schauenburg, 2004), könnten andere Fragetypen, welche die Persönlichkeit der Befragten stärker betonen, zu einem besseren Outcome führen. Weiterhin ist möglich, dass die Probanden/-innen einige Informationen nicht erinnern konnten und sie die häufigen Nachfragen als Kritik an ihrer schlechten Erinnerungsleistung verstanden haben. Daher ist anzuraten, diesen Fragetyp anhand eines anderen Paradigmas als dem des Hidden-Profiles zu untersuchen.

2. Außerdem sollten die Reaktionen der Gruppenmitglieder auf Informationsfragen im Fokus der Forschung stehen. So kann überprüft werden, mit welchen unmittelbaren Folgen für den Diskussionsprozess das Stellen der Informationsfragen verbunden war. Um dieser Frage nachzugehen, sind Prozessanalysen der Interaktion während der Entscheidungsfindung notwendig. Diese Prozessanalysen sind aufgrund der videographierten und transkribierten Gruppendiskussionen des vorliegenden Experiments möglich. Sie erfordern allerdings ein Kodierverfahren, durch welches die Koordinationsmechanismen und ihre Folgen erfasst werden können. Ein solches Kodierverfahren wurde speziell für diese Fragestellung entwickelt und wird 
in Kapitel 6 vorgestellt. Im Ausblick der Arbeit (Kapitel 8) werden Überlegungen dargestellt, wie die Prozessanalysen methodisch umgesetzt werden können.

Neben der anfänglichen Annahme, dass durch das Erfragen mehr Informationen genannt werden, und sich dadurch die Entscheidung verbessert, wurde davon ausgegangen, dass das Wiederbolen von Informationen verhindert, dass bereits genanntes Wissen während der Diskussion verloren geht, wodurch die Gruppenentscheidung optimiert wird. Nach Hypothese $\mathrm{H}_{1-2}$ wird erwartet, dass durch das regelmäßige Wiederholen von bereits genannten Informationen die Entscheidungsgüte verbessert wird. Die Ergebnisse stützen diese Hypothese: Wiederholt der/die Moderator/-in während der Diskussion regelmäßig bereits genannte Informationen, so verbessert sich die Gruppenentscheidung. Dieser Einfluss wird mediiert durch den Informationsaustausch und den Bewertungsanstieg der richtigen Alternative und bleibt auch unter Berücksichtigung der initialen Entscheidungspräferenz bestehen. In Hypothese $\mathrm{H}_{2-2}$ wurde postuliert, dass sich der Informationsaustausch verbessert, wenn der/die Moderator/-in regelmäßig Informationen wiederholt. Die Ergebnisse zeigen, dass durch das Wiederholen zwar nicht die Gesamtanzahl der Nennungen geteilter Informationen, bzw. die Erstnennung der geteilten und ungeteilten Informationen, steigen. Allerdings werden insgesamt mehr ungeteilte Informationen genannt. Der mangelnde Austausch und die geringe Wiederholungshäufigkeit ungeteilter Informationen werden in der Literatur als wesentliche Ursachen für suboptimale Gruppenentscheidungen genannt (Larson et al., 1998a; Mojzisch \& Schulz-Hardt, 2006; Stasser \& Titus, 2006). Die Ergebnisse des vorliegenden Experiments weisen darauf hin, dass diesem Problem durch einen einfachen Koordinationsmechanismus vorgebeugt werden kann: Das regelmäßige Wiederholen von genannten Informationen durch eine/n Moderator/-in erhöht den Austausch ungeteilter Informationen. Dieser Befund ist auch insofern bedeutsam, als dass der/die Moderator/-in nicht wusste, ob eine Information geteilt oder ungeteilt war. Darüber hinaus ist beachtenswert, dass es in dem vorliegenden Experiment für die richtige Gruppenentscheidung nicht ausschlaggebend 
war, dass die Gruppenmitglieder hauptsächlich ungeteilte Informationen in die Diskussion einbrachten. Vielmehr führte das Wiederholen dazu, dass insgesamt mehr Informationen genannt wurden, wodurch die richtige Entscheidungsalternative besser bewertet und dadurch die Entscheidung optimiert wurde. Daher kann nun konstatiert werden, dass für gute Gruppenentscheidungen neben der Kontroversität (Schulz-Hardt et al., in press) auch die Sicherung der bestehenden Informationen während der Diskussion wesentlich ist.

Es bleibt zu fragen, worin der Wirkmechanismus des Wiederholens besteht. Zu dessen Untersuchung sind die bereits angesprochenen Prozessanalysen unersetzlich. Daneben kann überlegt werden, dass durch das Wiederholen ein sozialer Validierungsprozess angeregt wird. Demnach müsste das Wiederholen der von Person A genannten Information durch eine Person B dazu führen, dass Person A sich in der Richtigkeit dieser Information bestärkt fühlt und diese deshalb während der Diskussion weiter verfolgt. Dieser Zusammenhang konnte bereits im Bereich der Gruppenpolarisierung gefunden werden (Brauer, Judd \& Gliner, 2006). Im Hinblick auf den sozialen Validierungsansatz könnte für die vorliegenden Ergebnisse diskutiert werden, ob das Wiederholen einer Information „stärker“ validierend wirkt als ihre Geteiltheit (vgl. Wittenbaum et al., 1999), denn in den Wiederholungsbedingungen verbesserte sich nicht nur die Bewertung der richtigen Alternative (deren positive Informationen ungeteilt waren), zusätzlich sank die Bewertung der suboptimalen Kandidaten (deren positive Informationen sogar geteilt waren).

Insgesamt kann davon ausgegangen werden, dass durch das regelmäßige Wiederholen von Informationen verhindert wird, dass entscheidungsrelevantes Wissen während der Diskussion vergessen oder nicht in die Entscheidung integriert wird.

In den Hypothesen $\mathrm{H}_{1-3}$ bis $\mathrm{H}_{3-3}$ wurden Interaktionseffekte des Erfragens und Wiederholens in Bezug auf die Entscheidungsfindung postuliert. Diese Hypothesen werden von den Daten nicht gestützt. Dieser Befund verwundert angesichts der fehlenden Wirkung des 
Erfragens von Informationen nicht. Außerdem konnte festgestellt werden, dass zwei der insgesamt 12 Kontrollgruppen die richtige Entscheidung trafen. Dieser Befund zeigt einerseits, dass das Hidden-Profile eine schwierige Gruppenaufgabe ist. Andererseits muss durch weitere Prozessanalysen geklärt werden, wieso es diesen beiden Gruppen obne Koordinationsunterstützung gelang, die richtige Entscheidung zu treffen. Erste Betrachtungen beider Diskussionen weisen darauf hin, dass ihre Moderatoren/-innen instruktionswidrig sehr aktiv waren und die Diskussion tatsächlich moderiert haben. Daraus liesse sich schließen, dass diese Kontrollgruppen aufgrund der koordinierenden Interventionen die richtige Entscheidung trafen.

Einfluss der Koordinationsmechanismen auf die Zufriedenheit der Gruppenmitglieder $\left(A V_{4}\right.$ bis $\left.A V_{6}\right)$

Neben den leistungsbezogenen Kriterien wurde überprüft, welchen Einfluss die Koordinationsmechanismen auf die Zufriedenheit der Gruppenmitglieder ausüben. Dabei wurde sowohl die Zufriedenheit mit dem Ergebnis und dem Prozess der Diskussion als auch mit dem/der Moderator/-in untersucht.

Ausgehend von der Überlegung, dass die Gruppenmitglieder mit einer koordinierten Entscheidungsfindung zufriedener sind und es angenehm finden, persönlich nach Informationen gefragt zu werden, wurde in den Hypothesen $\mathrm{H}_{4-1}$ und $\mathrm{H}_{5-1}$ angenommen, dass das Erfragen von Informationen die Zufriedenheit der Gruppenmitglieder mit dem Ergebnis und dem Prozess der Diskussion verbessert. Beide Hypothesen werden durch die Daten nicht gestützt. Das Erfragen von Informationen erhöht weder die Ergebnis- noch die Prozesszufriedenheit der Gruppenmitglieder. Zur Erklärung dieses Befundes kann die gleiche Überlegung zur „Unpersönlichkeit“ der Informationsfrage wie im vorangegangenen Abschnitt herangezogen werden.

Im Hinblick auf die Wirkung des Wiederholens wurde in den Hypothesen $\mathrm{H}_{4-2}$ und $\mathrm{H}_{5-2}$ angenommen, dass die Gruppenmitglieder der Wiederholungsbedingungen sowohl mit dem 
Ergebnis, als auch mit dem Verlauf der Diskussion, zufriedener sind als Mitglieder solcher Gruppen, in denen keine Informationen wiederholt werden. Diese Annahme wird von den Daten nur teilweise gestützt: Die Verlaufzufriedenheit ändert sich unter den Wiederholungsbedingungen nicht, jedoch steigt die Ergebniszufriedenheit. Dieser Befund erstaunt, denn im Hinblick auf den oben angesprochenen sozialen Validierungsansatz könnte argumentiert werden, dass das Wiederholen eigener Äußerungen durch eine weitere Person als angenehm empfunden wird. Allerdings kann auch vermutet werden, dass das doch sehr „technische“ Wiederholen von Informationen nicht ausreicht, um die Zufriedenheit der Gruppenmitglieder mit dem Entscheidungsprozess zu erhöhen. In diesem Zusammenhang ist es als positiv zu bewerten, dass die Verlaufszufriedenheit nicht gesunken ist, und das Wiederholen die Gruppenmitglieder nicht „genervt“ hat. Durch die gestiegene Ergebniszufriedenheit ist davon auszugehen, dass das Wiederholen bei den Gruppenmitgliedern zu der Ansicht führt, ihre eigenen Informationen hinreichend in die Entscheidung integriert zu haben.

In vergleichbaren Untersuchungen zeigte sich im Hinblick auf die Wirkung von Interventionsmethoden ein ähnliches Bild: Konsensorientierte Interventionen verbessern nicht die Entscheidung, aber die Zufriedenheit. Interventionen zur Förderung des Informationsaustauschs wirken hingegen positiv auf die Entscheidung, nicht aber auf die Zufriedenheit der Mitglieder (Schweiger \& Sandberg, 1989; Schweiger et al., 1986; Schweiger et al., 1988). Daher sollte die in dem vorliegenden Experiment nahezu fehlende individuelle und interaktionale Wirkung der beiden untersuchten Koordinationsmechanismen auf die Verlaufsund Ergebniszufriedenheit nicht überbewertet werden. Es erscheint plausibel, dass die Unterstützung der Gruppendiskussion durch nur eine bzw. zwei einfache koordinierende Verhaltensweise/n nicht unbedingt zu einer erhöhten Zufriedenheit mit dem Diskussionsprozess führen muss.

Im Hinblick auf die Zufriedenheit mit dem/der Moderator/-in wurde in den Hypothesen $\mathrm{H}_{6-1}$ und $\mathrm{H}_{6-2}$ angenommen, dass das Erfragen bzw. das Wiederholen von Informationen die 
Zufriedenheit der Gruppenmitglieder mit dem/der Moderator/-in erhöht. Es zeigte sich, dass der/die Moderator/-in durch das Fragenstellen nicht als kompetenter und nur tendenziell als hilfreicher wahrgenommen wurde. Wiederholt er/sie jedoch regelmäßig Informationen, so wird er/sie sowohl als kompetenter als auch als hilfreicher eingeschätzt. Darüber hinaus zeigte sich ein Interaktionseffekt: Fragt der/die Moderator/-in nach Informationen, so muss er/sie ebenfalls Informationen wiederholen, um von den Diskutanten/-innen als kompetent wahrgenommen zu werden. Diese Interaktion lässt sich durch die fehlende Kompetenzwahrnehmung in der FrageBedingung erklären.

Obwohl die Ergebnisse im Hinblick auf die Zufriedenheit mit dem/der Moderator/-in insgesamt positiv sind, so sollten auch sie nicht überbewertet werden. Angesichts der Tatsache, dass der/die Moderator/-in in der Kontrollbedingung fast überhaupt kein Verhalten zeigen durfte, sollte die höhere Zufriedenheit in den anderen Bedingungen eher als ein erfolgreicher Manipulation Check verstanden werden.

Nach der hypothesenbezogenen Interpretation der Ergebnisse wird nun auf Faktoren eingegangen, welche die Generalisierbarkeit der Ergebnisse des vorliegenden Experiments beeinträchtigen können. Anschließend wird die theoretische und praktische Bedeutung der Ergebnisse diskutiert.

\subsubsection{Mögliche gütereduzierende Faktoren}

Zur Überprüfung der Wirkung von Koordinationsmechanismen wurde ein LaborExperiment durchgeführt. In den folgenden fünf Punkten wird die Angemessenheit dieser Methode im Hinblick auf die Fragestellung bewertet.

1. Im Rahmen des Experiments wurde eine künstliche Gruppenentscheidungssituation geschaffen, die in allen wesentlichen Aspekten kontrolliert werden konnte. Dadurch war es möglich, die Koordinationsmechanismen isoliert und unbeeinflusst zu untersuchen. Allerdings können die gefundenen Ergebnisse nicht ohne weiteres auf reale Gruppenentscheidungsprozesse 
übertragen werden. Obwohl das Wiederholen von Informationen im Rahmen des Experiments zu einer besseren Entscheidung führte, müsste nun im realen Entscheidungsalltag überprüft werden, inwieweit dieser Mechanismus umsetzbar ist. Die unabhängigen Variablen werden in ihrer künstlichen Form nur selten vorkommen. Wahrscheinlich ist, dass die Frage nach Informationen häufig mit der Frage nach Meinungen oder Klärungen kombiniert wird, sowie die Wiederholungen häufig mit Zusammenfassungen verbunden werden.

2. Mit dem ersten Kritikpunkt geht eine weitere methodische Einschränkung einher. Die untersuchten Gruppen wurden mit Studierenden ad hoc gebildet. In der Literatur wird kritisiert, dass diese Gruppen nur methodische Abstraktionen von realen Gruppen seien (McGrath, Arrow \& Berdahl, 2000). Laborgruppen weisen eine andere Dynamik als reale Gruppen auf. Im Hinblick auf die Fragestellung kann allerdings argumentiert werden, dass auch reale Entscheidungsgremien häufig ad hoc zusammengesetzt werden.

3. Als Gruppenentscheidungsaufgabe wurde eine Hidden-Profile-Personalauswahlaufgabe eingesetzt. Dieses methodische Paradigma erlaubt die Manipulation der Informationsverteilung über die Gruppenmitglieder. Dadurch kann einerseits kontrolliert werden, welche Informationen während der Entscheidungsfindung genannt wurden. Anderseits kann auch die Güte der Gruppenentscheidung gemessen werden. Allerdings sind mit diesem Paradigma auch verschiedene Probleme verbunden. So stellt es streng genommen eher eine Problemlöse- und nicht zwingend eine Entscheidungsaufgabe dar, da es eine einzig richtige Entscheidung beinhaltet. Diese ist bei tatsächlichen Entscheidungsaufgaben in der Regel auch post-hoc nicht festzustellen. Außerdem müssen die Lösungsalternativen nicht erst generiert werden (vgl. Gouran \& Hirokawa, 1996), sondern werden bereits vorgegeben. Daher kann nicht von einer uneingeschränkten Operationalisierung einer realen Gruppenentscheidungssituation ausgegangen werden. Ein weiterer Kritikpunkt am Hidden-Profile betrifft die Annahme, dass die Valenz der Informationen über die Entscheidungsalternativen von allen Probanden/-innen in beabsichtigter Weise empfunden wird. Vielmehr werden die Kandidateninformationen von den Probanden/- 
innen als unterschiedlich positiv bzw. negativ erlebt, wodurch sich das Verhältnis von positiven und negativen Eigenschaften für einige Entscheidungsalternativen verschieben, und dadurch die gesamte Informationsmanipulierung verändern kann. Darüber hinaus birgt der Einsatz des Hidden-Profiles eine pragmatische Schwierigkeit: Die Gruppenmitglieder müssen über die entscheidungsrelevanten Informationen verfügen können. Dieses Problem könnte gelöst werden, indem die Probanden/-innen die Informationen mit in die Diskussion nehmen dürften. Es konnte allerdings gezeigt werden, dass die Lösungsrate des Hidden-Profiles bei Informationsverfügbarkeit in face-to-face-Diskussionen derart ansteigt, dass in Bezug auf die experimentelle Manipulation ein Deckeneffekt zu erwarten wäre (Mojzisch et al., 2005). Das zur Verfügung Stellen der Informationen bietet sich daher hauptsächlich für computervermittelte Diskussionen an, in denen die Gruppenmitglieder nicht an einem Tisch sitzen und in Versuchung kommen, die vorhandenen Informationen gemeinsam „durchzugehen“ (z.B. Schauenburg, 2004). Für face-to-face-Diskussionen bedeutet das, dass die Probanden/-innen die Informationen zunächst auswendig lernen müssen. Sie können sich daher während der Diskussion nicht nur auf die Entscheidungsfindung konzentrieren, sondern müssen auch versuchen, die relevanten Informationen $\mathrm{zu}$ erinnern. Dieser doppelte kognitive Aufwand ist bei realen Gruppenentscheidungen nicht zu finden.

4. Zur Generalisierung der Befunde wäre eine höhere Anzahl untersuchter Gruppen pro Bedingung empfehlenswert gewesen, obwohl ein n=12 Gruppen pro Bedingung vergleichbar mit ähnlichen Untersuchungen ist (z.B. Greitemeyer et al., 2006; Henningsen et al., 2004; Larson et al., 1994).

5. Schließlich bleibt anzumerken, dass in dem vorliegenden Experiment der Koordinationsprozess nicht betrachtet wurde. Dadurch sind noch keine vollständigen Aussagen über den Einfluss der Koordinationsmechanismen auf den Interaktionsprozess und auf die Entscheidung möglich (vgl. Hackman \& Morris, 1975). Wie bereits angesprochen, ist zu empfehlen, die erhobenen Daten einer Prozessanalyse zu unterziehen. Dadurch können weitere 
Erkenntnisse im Hinblick auf die Wirkmechanismen des Erfragens und Wiederholens gewonnen werden. Möglicherweise kann dadurch auch erklärt werden, warum das Erfragen von Informationen den Informationsaustausch und die Entscheidungsfindung nicht verbessert hat.

Nachdem im Folgenden die Bedeutung der vorhandenen Ergebnisse diskutiert wird, werden zwei Methoden zur Erfassung von Koordinationsprozessen dargestellt und ihr ergänzender Einsatz im Hinblick auf die vorliegenden Daten diskutiert.

\subsubsection{Bedeutung der Ergebnisse}

Das prägnanteste Ergebnis des vorliegenden Experiments besteht in der unterschiedlichen Koordinationswirkung des Erfragens und Wiederholens von Informationen. Erfragt ein/e Moderator/-in während der Diskussion entscheidungsrelevantes Wissen von den Gruppenmitgliedern, so hat das keinen Einfluss auf die Gruppenentscheidung. Im Gegensatz dazu verbessert das regelmäßige Wiederholen von Informationen die Entscheidung (vgl. Abbildung 30).

Input

Process (UVs)

Outcome (AVs)

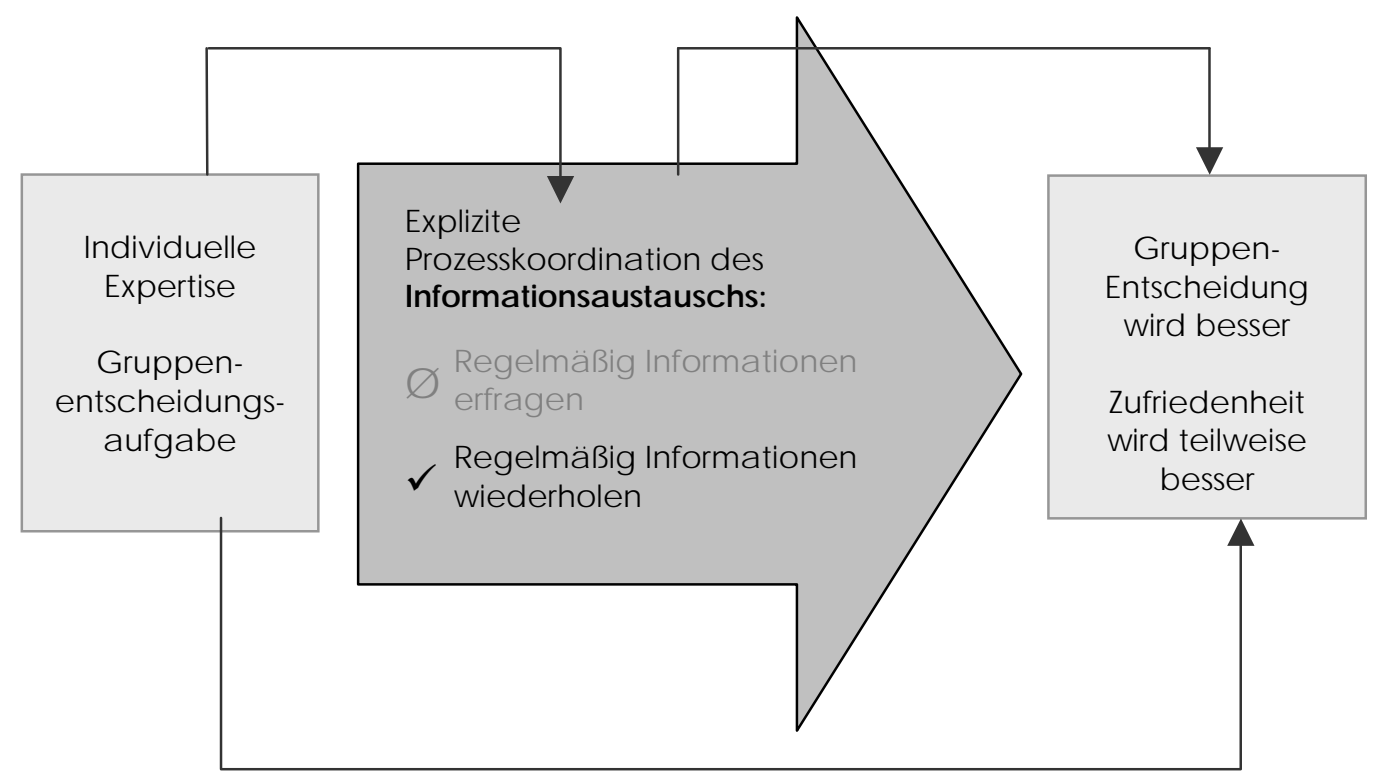

Abbildung 30: Beantwortung der Forschungsfrage des Experiments im Rabmen des IPO-Modells 
Bezugnehmend auf die drei eingangs dargestellten wesentliche Konzepte der vorliegenden Arbeit kann festgehalten werden, dass die Entscheidungsfindung eine komplexe Gruppenaufgabe ist, die durch intensiven Informationstausch bewältigt werden kann, welcher allerdings koordiniert werden muss.

In vergleichbaren Untersuchungen von Interventionen zur Verbesserung von Gruppenentscheidungen konnte hauptsächlich festgestellt werden, dass sich durch Unterstützungsverfahren zwar der Informationsaustausch, nicht aber die Gruppenentscheidung verbessert (z.B. Greitemeyer et al., 2006). Durch die vorliegende Studie konnte gezeigt werden, dass durch Koordination sowohl die Güte des Informationsaustauschs als auch die der Gruppenentscheidung steigt. Darüber hinaus wurde in ähnlichen Studien darauf hingewiesen, dass die Gruppenleitung durch Gruppenmitglieder oder Führungspersonen die Gefahr birgt, dass den Entscheidungspräferenzen dieser Personen ungleich viel Gewicht zukommt (z.B. Larson et al., 1998b). Für das vorliegende Experiment kann festgehalten werden, dass sich die Unterstützung der Gruppe durch eine externe und inhaltlich neutrale Person bewährt hat, da ihre Moderation nicht mit der Durchsetzung ihrer eigenen Interessen konfundiert.

Im Hinblick auf die Wirkung des Wiederholens von Informationen konnten Henningsen et al. (2006) zeigen, dass dieses sowohl normativen als auch informationalen Einfluss ausüben kann. Durch die Ergebnisse der vorliegenden Studie kann nun ergänzt werden, dass das Wiederholen auch eine koordinierende Funktion im Rahmen von Gruppenentscheidungsprozessen ausübt.

In den folgenden Abschnitten wird sowohl die theoretische als auch die praktische Bedeutung der vorliegenden Ergebnisse diskutiert.

\section{Theoretische Bedeutung für das Konstrukt der expliziten Prozesskoordination}

Der Befund der unterschiedlichen Wirkung des Erfragens und Wiederholens verdeutlicht, dass zwischen den einzelnen Koordinationsmechanismen differenziert werden muss. Bereits 
Espinosa et al. (2004) betonten im Hinblick auf die Koordinationsmechanismen: „one size does not fit all“ (S. 107). Diese Äußerung bezog sich allerdings auf die unterschiedliche Effektivität expliziter versus impliziter Mechanismen. Das vorliegende Experiment zeigt, dass es darüber hinaus auch innerhalb der expliziten Mechanismen zu differenzieren gilt. Sowohl das Erfragen als auch das Wiederholen von Informationen sind, im Vergleich $\mathrm{zu}$ Zusammenfassungen oder Zielformulierungen, einfache Koordinationsmechanismen. Trotz ihrer Einfachheit und gleicher Ziele scheinen sie den Informationsaustausch auf unterschiedliche Weise zu beeinflussen. Im übertragenen Sinn kann überlegt werden, dass das Wiederholen den Informationsaustausch aufrechterhält; das Erfragen scheint diese Wirkung offenbar zu verfehlen. Dieser Unterschied wird auch in der Gesprächspsychotherapie bzw. klientenzentrierten Psychotherapie aufgegriffen, die von ihrem Begründer Carl Rogers auch als nicht-direktive Beratung bezeichnet wurde (Rogers, 1999). Danach stellt das Wiederholen im Sinne von Verbalisierungen persönlicher Erlebnisinhalte des/der Klienten/-in ein wesentliches Instrument dar, um Wertschätzung und Verständnis zu kommunizieren, sowie das Erzählen des/der Klienten/-in und seine/ihre Selbstregulationsmechanismen zu verstärken (Biermann-Ratjen, Eckert \& Schwartz, 1995). Hingegen steht das Fragenstellen bei diesem Beratungsansatz nicht im Vordergrund, da angenommen wird, dass es zu direktiv ist und den/die Klienten/-in möglicherweise von seinen/ihren persönlichen Problembewältigungsstrategien ablenkt. Auch im Hinblick auf die Gruppenentscheidungsfindung interessiert nicht nur das Faktenwissen der Gruppenmitglieder, sondern auch ihre Bewertung dieses Wissen. Um die Auseinandersetzung mit den persönlichen Informationsbewertungen während des Entscheidungsprozesses zu ermöglichen, „ist das effektive Fragestellen [...] eine beraterische und therapeutische Kunst" (Bachmair, Faber, Hennig, Kolb \& Willig, 1999, S. 38).

Insgesamt kann festgestellt werden, dass das Konstrukt der expliziten Prozesskoordination der Differenzierung bedarf. Ausgehend von der Taxonomie von Wittenbaum et al. (1998) wurden zwei Mechanismen auf ihre Koordinationswirkung bei 
Gruppenentscheidungen untersucht. Die dargestellten Ergebnisse verdeutlichen die

Notwendigkeit detaillierter Untersuchungen der Wirkungsweisen der Koordinationsmechanismen.

\section{Praktische Bedeutung für die Koordination von Gruppenentscheidungsprozessen}

Im Hinblick auf Ergebnisse der Studien 1 und 2 fällt auf, dass das Erfragen von Informationen häufig als Koordinationsmechanismus genannt wird, insbesondere von den Experten/-innen. Im Vergleich dazu wurde das Wiederholen von Informationen selten erwähnt. Offenbar handelt es sich dabei um einen Koordinationsmechanismus, der im Hinblick auf seine Bedeutung für Gruppenentscheidungsprozesse bisher unterschätzt wurde. Es kann überlegt werden, ob die Effektivität des Erfragens von Informationen bisher möglicherweise überschätzt wurde, was angesichts der Künstlichkeit der experimentellen Umsetzung des Fragens zu bezweifeln ist. Dennoch scheint es empfehlenswert, dass der/die Moderator/-in während der Diskussion die Antworten auf die Informationsfragen sammelt und für alle Gruppenmitglieder sichtbar zur Verfügung stellt. Einerseits könnte die Gruppe dadurch auf einen Antwortpool zurückgreifen, andererseits könnte so der eventuell negative Interviewcharakter der Fragen abgeschwächt werden (vgl. Posthuma, 2002).

Im Hinblick auf die positive Wirkung des Wiederholens kann empfohlen werden, diesen Mechanismus zur Koordination von Gruppenentscheidungen einzusetzen. Dabei sollten allerdings zwei Dinge beachtet werden:

1. Die Informationen sollten von einem/r inhaltlich neutralen Moderator/-in wiederholt werden, nicht von einem involvierten Gruppenmitglied oder einer Führungskraft. Dann bestünde die Gefahr, dass verstärkt präferenzkonsistente Informationen wiederholt werden, was sich negativ auf die Entscheidungsgüte auswirken würde.

2. Der/die Moderator/-in sollte das Wiederholen möglichst auf verschiedene Art und Weise umsetzen. Das schlichte Wiederholen der genannten Informationen, wie es im 
vorliegenden Experiment umgesetzt wurde, mag dauerhaft künstlich wirken und sollte daher durch das Notieren und Visualisieren der Information ergänzt werden. Diese unterschiedlichen Wiederholungsmethoden sollten zukünftig auch bezüglich ihrer Effektivität verglichen werden.

$$
\text { Farit }
$$

Das Ziel des Experiments bestand in der Überprüfung der Wirksamkeit expliziter Koordinationsmechanismen während Gruppenentscheidungsprozessen. Es wurde gezeigt, dass der Mechanismus der Informationsfrage nahezu wirkungslos, das regelmäßige Wiederholen hingegen sehr effektiv ist. Um Aussagen bezüglich der generellen Wirksamkeit expliziter Prozesskoordination bei Gruppenentscheidungen treffen zu können, müssen zukünftig weitere Mechanismen experimentell überprüft werden. Außerdem wurde darauf hingewiesen, dass die experimentellen Befunde um die Betrachtung der Koordination während des Entscheidungsprozesses ergänzt werden müssen. Dazu wurden zwei Methoden entwickelt, durch die der Einsatz und die Folgen von Koordinationsmechanismen im Interaktionsprozess erfasst werden können. Diese Methoden werden in den beiden folgenden Kapiteln dargestellt.

Schließlich gilt es, die durch das vorliegende Experiment noch nicht zu beantwortenden oder neu entstandenen Fragen zu untersuchen. Ideen im Hinblick auf weiterführende Studien werden im Ausblick in Kapitel 8 diskutiert. 


\section{$6 \quad$ Methodenentwicklung 1: \\ Ein Kodiersystem zur mikroanalytischen Erfassung von Koordination in Gruppendiskussionen}

Mit diesem Kodiersystem wird eine Methode vorgestellt, die es ermöglicht, im Rahmen sozialpsychologischer Gruppenforschung Koordinationsmechanismen in Gruppendiskussionen mikroanalytisch zu erfassen. Das Kodiersystem wurde in Zusammenarbeit mit Alexandra Stein (Stein, 2006) entwickelt.

Bevor das Ziel dieser Methode dargestellt wird (Kapitel 6.1.3), werden zunächst der theoretische Hintergrund (Kapitel 6.1.1) und Gegenstand (Kapitel 6.1.2) beschrieben. Anschließend wird die Notwendigkeit der Entwicklung des Kodiersystems begründet (Kapitel 6.1.4). Der eigentliche Kodiervorgang und die Kategorien werden in Kapitel 6.2 bzw. 6.3 erläutert. Abschließend wird in Kapitel 6.4 auf die Güte des Verfahrens eingegangen.

\subsection{Einführung}

Der vorliegenden Arbeit liegt die funktionale Perspektive der Gruppenforschung zugrunde (Wittenbaum et al., 2004b). Diese geht davon aus, dass Inputfaktoren, vermittelt über den Interaktionsprozess der Gruppenmitglieder, die Gruppenleistung beeinflussen. Durch die Analyse des Interaktionsprozesses soll erklärt werden, warum einige Gruppen erfolgreich sind, andere hingegen nicht (Wittenbaum et al., 2004b). Im Hinblick auf Gruppenentscheidungen wird der komplexe Entscheidungsfindungsprozess durch den Einsatz von expliziten und impliziten Koordinationsmechanismen koordiniert. Diese Mechanismen ermöglichen die Koordination des für die gemeinsame Entscheidung notwendigen Austauschs von Informationen. Durch die Erfassung des Einsatzes dieser Mechanismen wird die systematische Untersuchung des Auftretens und des Wechselspiels von expliziten und impliziten Koordinationsmechanismen möglich. Somit können einerseits Bedingungen und Konsequenzen des Mechanismeneinsatzes geprüft werden. Andererseits können erfolgreiche und nicht erfolgreiche Entscheidungsgruppen bezüglich ihrer Koordination verglichen werden. 


\subsubsection{Theoretischer Hintergrund: Mikroanalytische Verhaltensbeobachtung in der Gruppenprozessforschung}

Seit geraumer Zeit steht nicht nur das Ergebnis der Gruppenarbeit, z.B. die Gruppenentscheidung, im Vordergrund der Gruppenforschung. Es interessiert ferner auch der Weg, auf dem die Gruppe dieses Ziel erreicht (Marks et al., 2001; McGrath et al., 2000; Tschan, 2000). Der Gruppenprozess umfasst im Rahmen des funktionalen Ansatzes der Gruppenforschung (Wittenbaum et al., 2004b) die Interaktion der Gruppenmitglieder (vgl. Kapitel 2.1), wobei insbesondere die Kommunikation und die Informationsverarbeitung im Fokus der Betrachtung stehen (Brauner \& Orth, 2002).

Methodisch wird die Kommunikation innerhalb der Gruppe hauptsächlich über Prozessanalysen von Interaktionen erschlossen (Becker-Beck, 1994, 1997; Becker-Beck, Wintermantel \& Borg, 2005; Boos, 1996b; Boos et al., 1990; Brauner, 1998; Kerr, Aronoff \& Messé, 2000; Nägele, 2004).

Die Frage nach dem Einfluss von Interaktion und Kommunikation auf die Entscheidungsfindung konnte bisher nicht eindeutig beantwortet werden, was angesichts der Komplexität sowohl der Aufgabe als auch der Kommunikation nicht verwundert. In älteren Studien, z.B. Lanzetta und Roby (1960) wurde die Beziehung zwischen dem Gruppenprozess und der Gruppenproblemlösung untersucht. Dort konnte gezeigt werden, dass die Gruppeninteraktion den Erfolg der Gruppe besser vorhersagte als aufgabenbezogenes Wissen oder Training der Gruppenmitglieder. Dennoch ist diese Studie heftig kritisiert worden (Hirokawa, 1982; Hirokawa \& Salazar, 1999). Ein Hauptkritikpunkt besteht in der Unangemessenheit der Erfassung des Interaktionsprozesses: Die Interaktion der Gruppe wurde anhand von sieben Kategorien erfasst, deren theoretische Entwicklung jedoch nicht transparent gemacht wurde oder möglicherweise nicht vorhanden ist (Hirokawa, 1982). Hirokawa (1982) fordert, vor der Formulierung von Verhaltensbeobachtungskategorien zunächst ein theoretisches 
Modell zu entwickeln, welches Aussagen über den funktionalen Zusammenhang von

Verhaltensweisen während des Interaktionsprozesses und der Performance postuliert.

„The principle argument advanced in this paper is that previous investigations have generally failed to identify consistent and meaningful relationships because they have typically neglected to operationalize the group interaction process in terms of those communication behaviors which need to occur within the course of the interaction process in order to arrive at a satisfactory decision or solution“ (Hirokawa, 1982, p. 149)

Ausgehend von einem formalen Modell können Kategorien zur Erfassung des relevanten Verhaltens entwickelt werden (Hirokawa, 1982). Damit kann sicher gestellt werden, dass auch tatsächlich nur solches Verhalten kodiert wird, dass kritisch für die Aufgabenerfüllung ist. Ebenso wird kritisiert, dass für viele Fragestellungen zum Zusammenhang des Interaktionsprozesses und der Entscheidungsgüte das Verfahren der Interaktionsprozessanalyse (IPA, Bales, 1950, zit. n. Hirokawa, 1982) zur Erfassung der Interaktion verwendet wird, obwohl die IPA den relevanten Interaktionsmerkmalen gegenüber nicht in allen Bereichen sensitiv ist (Hirokawa \& Salazar, 1999).

Hirokawas (1982) Kritik an der methodischen Umsetzung der Prozessforschung ist angesichts der schwachen Befundlage gerechtfertigt. Allerdings kann seine Implikation der theoriebasierten Entwicklung von Beobachtungsverfahren nicht unumstritten bleiben. Hierauf soll in zwei Punkten eingegangen werden:

1. Einerseits setzt die theoriegebundene Verfahrensentwicklung die Existenz einer geeigneten Theorie voraus. Prozessbeobachtung sollte allerdings auch dann möglich sein, wenn die theoretischen Voraussetzungen schwach sind, da Theorieentwicklung auch durch Induktion möglich ist (Glaser \& Strauss, 2005; Westermann, 2000). Im Hinblick auf die vorliegende Arbeit existiert keine Theorie der Gruppenkoordination. Dennoch wird ausgehend von bestehenden Modellen (Espinosa et al., 2004; Wittenbaum et al., 1998) und eigenen Arbeiten (Studien 1 - 3) in Anlehnung an Boos (2003) ein formales Arbeitsmodell der Koordination postuliert (Abbildung 31). In diesem Modell wird davon ausgegangen, dass dem Einsatz eines Koordinationsmechanismus ein 
bestimmter Koordinationsanlass vorausgeht. Auf den Koordinationsmechanismus folgt wiederum eine Konsequenz, die sich beispielsweise in der Reaktion der Gruppenmitglieder auf den Mechanismeneinsatz manifestiert.

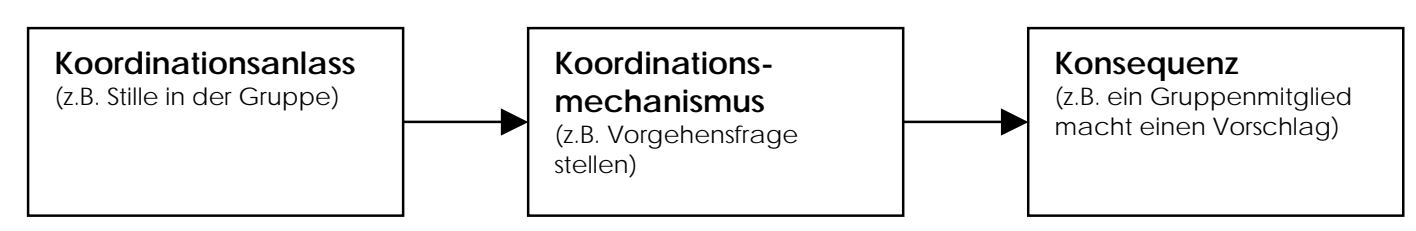

Abbildung 31: Formales Arbeitsmodell der Koordination (in Anlebnung an Boos, 2003)

Es wird davon ausgegangen, dass ein solches Arbeitsmodell nützlich ist, um neben der Phänomenologie die situative Effektivität der Koordinationsmechanismen zunächst im Rahmen von Prozessanalysen zu beobachten und anschließend experimentell zu untersuchen.

2. Bei Betrachtung des postulierten Arbeitsmodells wird deutlich, dass der Auflösungsgrad der zu beobachtenden Interaktion sehr hoch ist. Diese mikroanalytische Erfassung erlaubt sowohl eine detaillierte und sequentielle Beschreibung der Koordinationshandlungen als auch ihrer Anlässe und Konsequenzen. Diese mikroanalytische Struktur der Koordinationshandlungen zeigte sich auch in Form von Herstellungswissen in den subjektiven Koordinationstheorien der Experten/-innen in Studie 2 (vgl. Abbildung 15). Es wird daher argumentiert, dass die Analyseeinheit nicht vorrangig, wie von Hirokawa (1982) vorgeschlagen, auf der Makroebene im Sinne einer Funktion des Gruppenprozesses anzusiedeln ist. Statt dessen ist es empfehlenswert, ausgehend von der Idee des Methodological Fit (Edmondson \& McManus, 2005), in Abhängigkeit des Forschungsgegenstandes auch Untersuchungen auf der Mikroebene durchzuführen. Angesichts einer gewissen Kodier-Erfahrung erscheint es außerdem sehr schwierig, Kodierer/-innen darin zu trainieren, nicht nur deskriptiv, sondern auch funktional zu kodieren. Hirokawas (1982) Forderung nach Transparenz in der Bildung der Kategorien wird durch die detaillierte Darstellung der Kategorienentwicklung in Kapitel 6.3.2 nachgekommen. 
Der im Folgenden vorgestellten Methode zur Erfassung expliziter Prozesskoordination liegt die mikroanalytische Betrachtung des Gruppenprozesses zugrunde.

\subsubsection{Koordinationsmechanismen als Gegenstand des Kodierverfahrens}

Das vorliegende Kategoriensystem dient der Erfassung von Mechanismen, die zur Koordination während Gruppenentscheidungsprozessen eingesetzt werden. Dabei wird unter Koordination der aufgabenabhängige Umgang mit Interdependenz zur effektiven Erreichung eines gemeinsamen Ziels im Sinne einer Abstimmung von Teilhandlungen und einer hierarchisch und sequentiell logischen Verarbeitung von Informationen verstanden (vgl. Kapitel 3.2).

Der Begriff Koordinationsmechanismus beschreibt Verhaltensweisen, durch welche die Koordination während des Interaktionsprozesses umgesetzt wird. Diese Mechanismen werden danach unterschieden, inwieweit ihre Verwendung explizit oder implizit ist. Die konzeptuelle Unterscheidung von expliziten und impliziten Koordinationsmechanismen geht auf die Modelle von Wittenbaum et al. (1998) und Espinosa et al. (2004) sowie auf die in Kapitel 4 und 5 vorgestellten Studien zurück.

Das vorliegende Kategoriensystem wurde mit dem Ziel der Erfassung expliziter Prozesskoordination entwickelt. Die Mechanismen expliziter Prozesskoordination werden als explizit bezeichnet, weil sie mit der Absicht zu koordinieren ausgeführt werden (Espinosa et al., 2004) und in ihrer Botschaft klar und deutlich sind. Diese Mechanismen werden in dem vorliegenden Kategoriensystem operationalisiert durch verbale, an einzelne Gruppenmitglieder oder die gesamte Gruppe gerichtete Handlungsanleitungen, Fragen oder Anreden. Diese können sich zum einen auf zukünftige Aufgabenbereiche jedes einzelnen Teammitglieds beziehen, zum anderen können die Aussagen auch den weiteren Diskussionsverlauf koordinieren und somit nicht den Arbeitsablauf an sich, sondern den Prozess der Zielereichung lenken und für eine Strukturierung der Diskussion sorgen. Explizite Koordination ist in ihrer Absicht deutlich erkennbar. Es wird 
z.B. eindeutig der Aufgabenbereich eines jeden Gruppenmitglieds festgelegt oder einzelne Personen werden in der Diskussion direkt angesprochen.

Trotz der schwerpunktmäßigen Erfassung expliziter Koordinationsmechanismen soll mit dem vorliegenden Verfahren auch der Versuch einer Erhebung impliziter Koordinationsmechanismen unternommen werden. Die Mechanismen impliziter Prozesskoordination sind, im Vergleich zu expliziten Mechanismen, schwerer erkennbar. Implizite Koordination entsteht durch geteilte mentale Modelle und zeigt sich in der Anpassung des eigenen Verhaltens an das erwartete Verhalten der anderen Gruppenmitglieder (Espinosa et al., 2004; Grote et al., 2003; Wittenbaum et al., 1998). Die Erfassung impliziter Mechanismen ist problematisch, da gegenseitige Annahmen nur selten verbalisiert werden (Grote et al., 2003). Für die vorliegende Arbeit wird in Anlehnung an Wittenbaum (1998) davon ausgegangen, dass die Mechanismen impliziter Koordination aufgrund ihres „versteckten“ Charakters nicht sichtbar sind und erst durch die Betrachtung des Verlaufs des Entscheidungsprozesses deutlich werden. Dennoch soll in dem vorliegenden Kategoriensystem versucht werden, Mechanismen mit einem Potential zur impliziten Prozesskoordination zu erfassen. Diese werden operationalisiert durch inhaltliche Beiträge der Gruppenmitglieder, z.B. in Form von Meinungsäußerungen oder Lösungsvorschlägen. Dadurch ist während einer anschließenden Betrachtung des Verlaufs der Interaktion beispielsweise ersichtlich, inwieweit eine Information genannt wurde, ohne dass danach gefragt wurde (vgl. Grote et al., 2003).

Bevor die Notwendigkeit der Entwicklung eines Kategoriensystems zur Erfassung der genannten Mechanismen begründet wird, wird zunächst das Ziel des vorliegenden Verfahrens präzisiert. 


\subsubsection{Ziel des Kategoriensystems}

Das folgende Kategoriensystem dient der Mikroprozessanalyse der Koordination in Gruppendiskussionen, in denen Gruppen ein Problem lösen oder eine Entscheidung treffen. Es soll schwerpunktmäßig der Erfassung von Mechanismen expliziter Prozesskoordination dienen. Diese Form der Koordination soll präzise, transparent und intersubjektiv nachvollziehbar erhoben werden. Darüber hinaus soll das Kategoriensystem auch pragmatisch einsetzbar sein, d. h. eine inhaltlich und zeitlich ökonomische Anwendung erlauben. Der Auflösungsgrad der betrachteten Interaktion ist dabei sehr hoch: Es werden nicht ganze Handlungen oder Redebeiträge einer Person betrachtet. Vielmehr werden individuelle Redebeiträge regelgeleitet in Kodiereinheiten zerlegt, die lediglich eine einzelne Teilhandlung oder -aussage beinhalten (zur Darstellung der Bildung der Kodiereinheiten siehe Kapitel 7). Durch diese mikroanalytische Erhebung der Koordinationsmechanismen können die erhaltenen Diskussionselemente beispielsweise in einer anschließenden Sequenzanalyse auf ihre Konsequenzen im Diskussionsprozess hin untersucht werden.

Neben der schwerpunktmäßigen Erfassung expliziter Prozesskoordination ermöglicht das Kategoriensystem ebenfalls eine Betrachtung impliziter Mechanismen, die über ein Koordinationspotential verfügen können, beispielsweise das ungebetene Nennen von Informationen während der Diskussion. Durch diese inhaltlichen Kategorien können die Reaktionen der Gruppenmitglieder auf den Einsatz eines Koordinationsmechanismus ebenfalls erfasst werden (z.B. auf eine Frage wird eine Information genannt; nach einer Einverständnisfrage erfolgt eine Zustimmungsreaktion).

Außer dem Einsatz in der Forschung bietet das Beobachtungssystem die Möglichkeit, im Rahmen von Schulungen den Teilnehmern/-innen eine beobachtungsbasierte Rückmeldung ihres Koordinationsverhaltens sowohl auf Gruppen- als auch auf Individualebene zu geben. 
Im Folgenden wird erläutert, weshalb die genannten Ziele die Entwicklung eines neuen Kodierverfahrens nahe legen.

\subsubsection{Notwendigkeit des Kategoriensystems}

Das Kategoriensystem dient der Erfassung expliziter Koordination von Gruppenentscheidungsprozessen. Es soll im Hinblick auf Koordination eine vergleichende Untersuchung von Gruppen mit guter vs. schlechter Entscheidungsqualität ermöglichen.

Ein Kategoriensystem, welches dieses Ziel auf den ersten Blick erfüllt, ist die Konferenzkodierung (Konfkod, Beck \& Fisch, 2000; Fisch, 1994, 1998). Konfkod ist ein Beobachtungs- und Kodierverfahren für die teilnehmende Beobachtung des Planens, Beratens, Problemlösens und Entscheidens in Experten/-innen-Gruppen. Es besteht aus 15 Kategorien, welche die inhaltliche und prozedurale Lenkung, inhaltliche Beiträge und sozial-emotionale Aspekte der Diskussion erfassen. Konfkod baut zum einen auf der Interaktionsprozessanalyse (IPA) von Bales (1950, zit. nach Fisch, 1994) und zum anderen auf dem System zur mehrstufigen Beobachtung von Gruppen (SYMLOG) auf (Bales, 1979, zit. nach Fisch, 1994). Da das Ziel des vorliegenden Kategoriensystems in der mikroprozessanalytischen Erfassung der Koordination in Gruppen besteht, wären vor allem die Kategorien der Konferenzkodierung von Bedeutung, welche die inhaltliche und prozedurale Lenkung beschreiben. Diese Kategorien erfassen alle offen erkennbaren und direkten Einflussnahmen, die den Diskussionsprozess regulieren, aufrechterhalten, ihn in eine bestimmte inhaltliche Richtung lenken oder ihm eine bestimmte Form geben (Fisch, 1994). Anhand der Ausführungen von Fisch (1994) lässt sich schlussfolgern, dass die Kategorien zur Lenkung ein sehr umfangreiches und komplexes Gebiet umschreiben. Da verwundert es, dass sowohl die inhaltliche als auch die prozedurale Lenkung aus lediglich zwei Kategorien bestehen. Die Kategorie inhaltliche Lenkung besteht aus den Unterkategorien Frage mit Feststellung und Informationsfrage. Die Kategorie prozedurale Lenkung enthält die beiden Unterkategorien Frage zum Verfahren und Verfahrensgesichtspunkt/Vorschlag zum Verfahren. 
Im Hinblick auf die mikroanalytische Erfassung von Koordinationsmechanismen stellt die Konferenzkodierung kein geeignetes Kategoriensystem dar. In den Studien 1 bis 3 konnte gezeigt werden, dass nicht nur Fragen und Verfahrensvorschläge, sondern auch verschiedene Arten von Handlungsanleitungen und Strukturierungen eine koordinierende Funktion in Entscheidungsprozessen übernehmen. Die Kategorien der Konferenzkodierung sind eher allgemeiner Natur, so dass sie einen guten Ausgangspunkt für die Entwicklung eines erweiterten Kategoriensystems zur Erfassung expliziter Koordination von Entscheidungsprozessen darstellen, diese aber nicht vollständig erfassen können.

Obwohl in der Gruppenforschung zahlreiche und verschiedenartige Kategoriensysteme für die Analyse von Interaktionen in Dyaden oder Gruppen existieren, z.B. das Kasseler Kompetenzraster (Kauffeld, 2003), das Interaktions-Beobachtungssystem SYNPRO (Simon, 1997) oder das Kategoriensystem zur Analyse von komplexem Problemlöseverhalten KATKOMP (Stempfle, 2006), erscheinen diese für die Untersuchung der expliziten Koordination von Entscheidungsfindungsgruppen ebenfalls als nicht geeignet, da sie weniger auf die Koordination von Gruppen als vielmehr auf die Analyse gruppendynamischer Aspekte und des Gruppenklimas fokussieren. Den genannten Methoden ist gemeinsam, dass sie auf Koordinationsmechanismen nur oberflächlich eingehen, eher die verschiedenen Kompetenzarten einzelner Gruppenmitglieder erfassen und teilweise eher für die Makro- und nicht die Mikroebene konstruiert wurden.

Die Literaturrecherche zeigte, dass es kein der Fragestellung angemessenes Erhebungsverfahren gibt, welches die mikroanalytische Erfassung expliziter Prozesskoordination von Gruppenentscheidungsprozessen ermöglicht. Daher kann die Notwendigkeit der Entwicklung eines solchen Verfahrens festgestellt werden. Bevor die Kategorien und ihre Entwicklung erläutert werden, wird auf den Prozess des Kodierens eingegangen. 


\subsection{Kodiervorgang}

Bevor die Kategorien ausführlich beschrieben werden, soll hier zunächst kurz auf die Grundlage der Kodierung und das Kodiervorgehen eingegangen werden.

\subsubsection{Die Kodiereinheiten}

Die Grundlage der Kodierung bilden die Kodiereinheiten. Sie stellen die Bezugsgröße der Kodierung im Text, auf die das Kategoriensystem je einmal anwendbar ist (Früh, 2001) bzw. das spezifische Segment des Inhaltes dar, welches durch die Zuordnung zu einer gegebenen Kategorie charakterisiert wird (Holsti, 1969). Kodiereinheiten sind der kleinste auswertbare Materialbestandteil (Mayring, 2000). Zur Bildung der Kodiereinheiten kann das in Kapitel 7 beschriebene Verfahren zur Einteilung von Kodiereinheiten verwendet werden, da es speziell für die Anwendung in der mikroanalytischen Gruppenprozessforschung entwickelt wurde.

Die Daten sollten möglichst computergestützt kodiert werden, da dies den Kodiervorgang und die weitere Verwendung der Daten, z.B. zur Berechnung der InterraterReliabilitäten, vereinfacht.

\subsubsection{Der Kodierprozess}

Das Kodieren stellt eine komplexe Klassifikationsaufgabe dar (Kriz \& Lisch, 1988, zit. n Wirth \& Lauf, 2001). Die Kodierer/-innen müssen die Grenzen der Kodiereinheiten erkennen, Kategorien identifizieren sowie zwischen den Ober- und Unterkategorien diskriminieren (Holsti, 1969). Wirth und Lauf (2001, S. 161) beschreiben den Kodierprozess wie folgt:

„Kodieren kann als spezifisches Rezeptionshandeln sowie als Problemlöse- und Entscheidungsprozess begriffen werden, bei dem wiederholt nach der Rezeption von systematisch begrenzten Textmengen (Kodiereinheiten) codebuchbasierte Zuordnungsentscheidungen gefällt werden müssen“". 
Das in dieser Arbeit vorgestellte Kodiersystem besteht aus 30 exklusiven Kategorien. Mehrfachkodierungen sind nicht vorgesehen, da diese nachträglich zu einem Auswertungsmehraufwand führen und die sequenzanalytische Auswertung erschweren würden (Scheufele, 2001). Eine Kodiereinheit wird jeweils nur einer Kategorie zugewiesen. Neben der Kategorie werden pro Kodiereinheit die laufende Nummer, die Zeit (Minute) und wer zu wem spricht kodiert.

Das Verfahren wurde zudem so konzipiert, dass auch über mehrere Stunden zuverlässig kodiert werden kann. Die hierarchische Einteilung in Ober- und Unterkategorien erleichtert die Zuordnung der Kategorien. Zuerst wird entschieden, welcher Oberkategorie die vorliegende Kodiereinheit zuzuordnen ist. In einem zweiten Schritt wird die Entscheidung spezifiziert, indem der Kodiereinheit eine Unterkategorie zugewiesen wird. Diese hierarchische Einteilung der Kategorien dient der Reliabilitätssicherung (Holsti, 1969). Dennoch ist ein Training in der Anwendung des Kodiersystems zu empfehlen, um die 30 Kategorien erfolgreich überblicken zu können. 


\subsection{Kategorien}

Zunächst werden die Kategorien und das System ihrer Anordnung überblicksartig vorgestellt (Kapitel 6.3.1). Anschließend wird die Entwicklung der Kategorien beschrieben (Kapitel 6.3.2). Die detaillierten Kodierregeln, Ankerbeispiele und Abgrenzungsregeln der Kategorien können im Anhang E nachgeschlagen werden.

\subsection{1. Überblick über die Kategorien}

Wie Abbildung $32 \mathrm{zu}$ entnehmen ist, besteht das Kodiersystem aus drei Hauptkategorien: Kategorien zur expliziten Prozesskoordination, Kategorien inhaltlicher Beiträge und Zusatzkategorien.

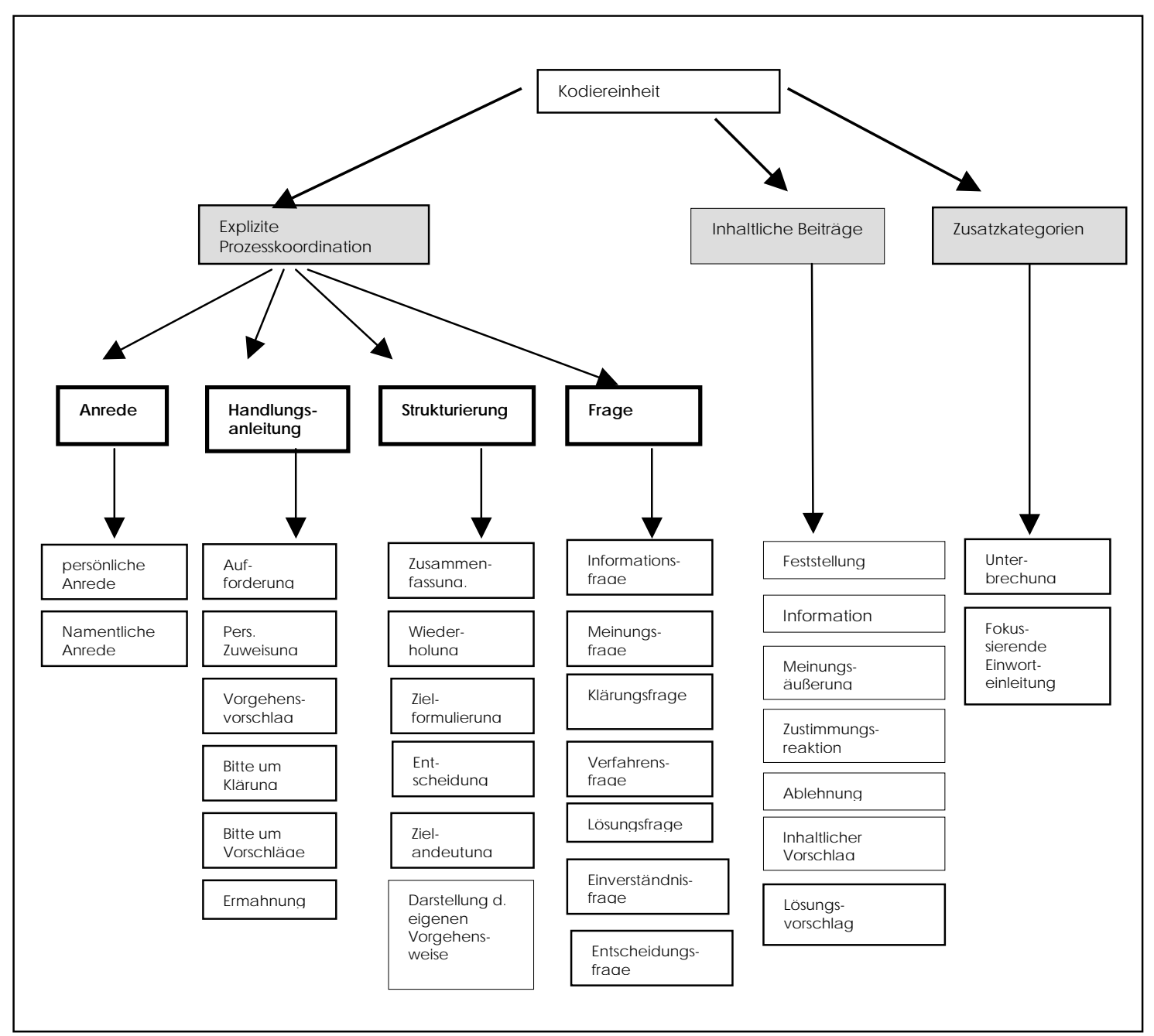

Abbildung 32: Überblick über die Kategorien des Kodiersystems 
Die Kategorien zur expliziten Prozesskoordination beinhalten vier Oberkategorien und entsprechende Unterkategorien: Handlungsanleitungen (Aufforderung, persönliche Zuweisung, Verfahrensvorschlag, erbittet Klärung oder Spezifizierung, erbittet Vorschläge und Ermahnung), Strukturierungen (Zusammenfassung, Wiederholung, Zielformulierung, Entscheidung, Zielandeutung und Darstellung der eigenen Vorgehensweise), Fragen (Informationsfrage, Meinungsfrage, Klärungs- bzw. Spezifizierungsfrage, Verfahrensfrage, Lösungsfrage, Einverständnisfrage und Entscheidungsfrage) sowie Anreden (persönliche Anrede und namentliche Anrede).

Die Kategorien inhalticher Beiträge beinhalten sieben Unterkategorien: Information, Feststellung, Erläuterung, Meinungsäußerung, Zustimmungsreaktion, Ablehnungsreaktion sowie Lösungsvorschlag.

Daneben gibt es die Zusatzkategorien: Unterbrechung und fokussierende Einworteinleitung.

Die detaillierte Darstellung der Kategorien und entsprechender Ankerbeispiele in Form eines Manuals ist im Anhang E enthalten. Im Folgenden wird die Entwicklung der Kategorien beschrieben.

\subsubsection{Entwicklung der Kategorien}

Das Ziel dieses Kategoriensystems besteht schwerpunktmäßig in der Erfassung von Mechanismen expliziter Prozesskoordination. Dazu müssen Kategorien und Verhaltensanker entwickelt werden, durch die die Koordinationsmechanismen abgebildet werden können. Kategorien können nach zwei bekannten Ansätzen entwickelt werden: deduktiv oder induktiv (Mayring, 2000). Bei der deduktiven (theoriegeleiteten) Kategorienentwicklung wird versucht, aufgrund einer bestehenden Theorie und der Forschungsfrage die Kategorien zu operationalisieren (Bilandzic, Koschel \& Scheufele, 2001). Es wird von vorher festgelegten, theoretisch begründeten Konzepten auf Beobachtungsaussagen geschlossen (Früh, 2001, 2004). Sehr wichtig sind dabei 
vor allem die genaue Definition der theoretisch begründeten Kategorien und die Festlegung von Kodierregeln, die angeben, wann ihnen eine Textstelle zugeordnet werden kann (Mayring, 2000).

Bei der induktiven (empiriegeleiteten) Kategorienentwicklung wird aus empirischen Beobachtungen auf theoretische Konzepte geschlossen, die das Definitionskriterium für die zu berücksichtigenden Aspekte im Material festlegen. Die so entwickelten Kategorien werden in einer Rückkopplungsschleife überarbeitet und einer Reliabilitätsprüfung unterzogen (Mayring, 2000). Bei der induktiven Vorgehensweise werden die Kategorien somit datennah gebildet. Dabei kann auch auf bestehende Kategoriensysteme zurückgegriffen werden. Die empiriegeleitete Kategorienbildung kommt unter zwei Voraussetzungen zum Einsatz: Zum einen muss auf das vorliegende Datenmaterial zurückgegriffen werden, wenn noch keine adäquate Theorie zu dem $\mathrm{zu}$ untersuchenden Forschungsgegenstand existiert. Zum anderen wird auch bei einer vorwiegend theoriegeleiteten Kategorienbildung in der Regel ein Teil des Kategoriensystems anhand des empirischen Materials gebildet.

Früh (2004) geht jedoch davon aus, dass eine Trennung von induktiver und deduktiver Kategorienentwicklung, wie sie von Mayring (2000) vorgeschlagen wird, nur begrenzt sinnvoll und umsetzbar ist. In Anlehnung an Poppers These der Theoriebeladenheit jeder Beobachtung (Popper 1974 zit. n. Früh, 2004) postuliert Früh (2004), dass eine reine Induktion nicht möglich sei. Jede Beobachtung sei „theoriegetränkt“, da die Wahrnehmung nicht ohne Interpretation geschehe (Früh, 2004, S. 72). Sollen mit Hilfe von beobachteten Daten schlüssige Aussagen über allgemeine theoretische Annahmen getroffen werden, so müssten die Beobachtungen theorieunabhängig sein, da die gezogenen Schlüsse sonst zirkulär wären. Diese Unabhängigkeit und Offenheit in der Beobachtung, die für eine reine induktive Kategorienentwicklung nötig wäre, sei aber nur schwer, wenn sogar gar nicht, zu realisieren (Früh, 2004).

Aufgrund der mangelnden Realisierbarkeit einer reinen Induktion verbindet Früh (2004) die deduktive mit der induktiven Kategorienentwicklung. Dadurch kann gewährleistet werden, dass ein Gegenstandsbereich vollständig erfasst wird (Brosius \& Koschel, 2001). Daher wurde 
auch während der Entwicklung des vorliegenden Kategoriensystems sowohl theorie- als auch empiriegeleitet vorgegangen. Die einzelnen Entwicklungsschritte werden im Folgenden beschrieben.

\section{Integration der Ergebnisse aus Studie 1 und 2}

Zunächst wurden die in Studie 1 und 2 eruierten Koordinationsmechanismen in einem Kategoriensystem angeordnet. Abbildung 33 zeigt, welche Kategorien auf die Ergebnisse der beiden Studien zurückgehen.

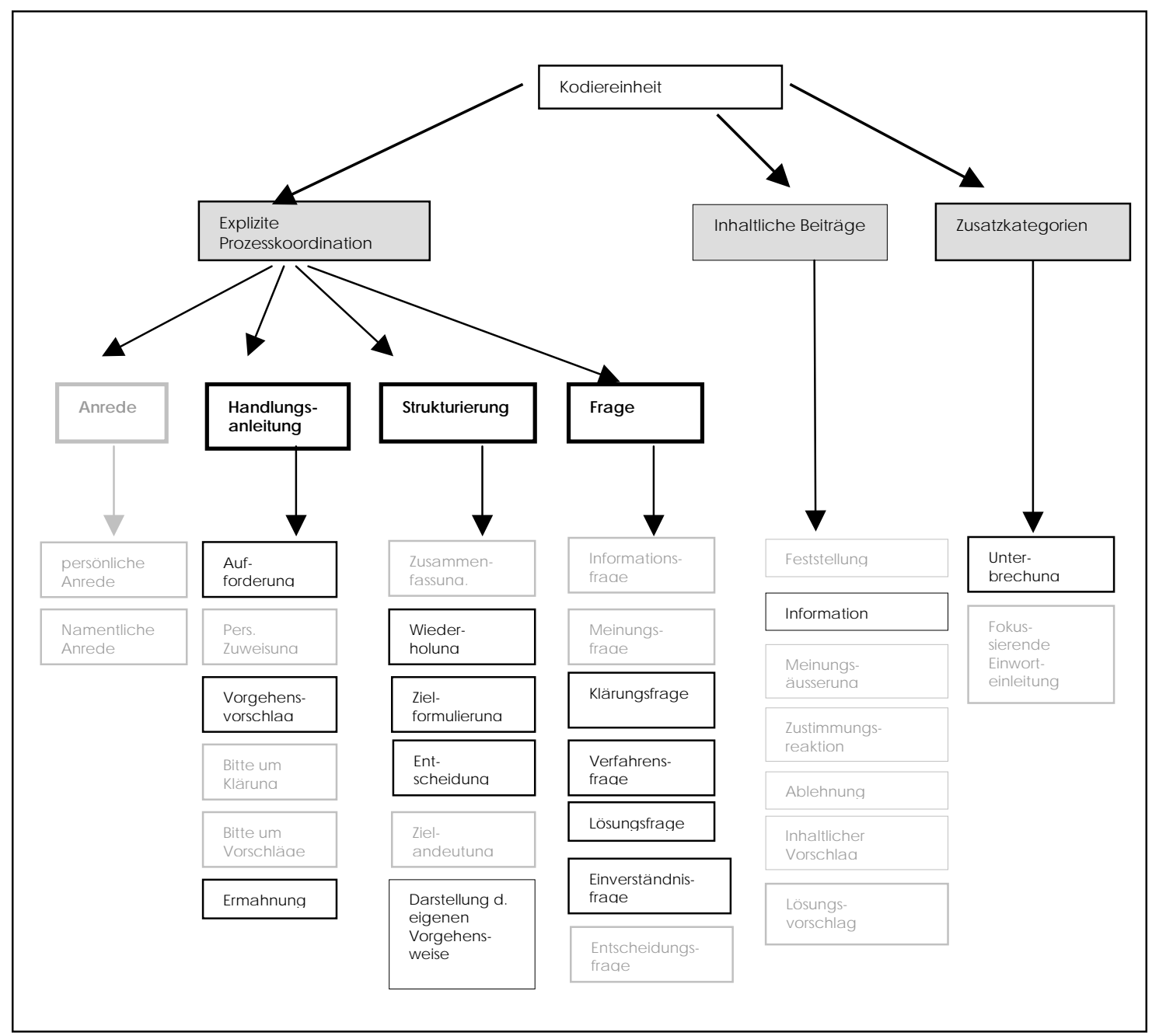

Abbildung 33: Kategorien der Koordinationsmechanismen, die anband der Ergebnisse aus Studie 1 und 2 entwickelt wurden (durch schwarze Rahmen dargestellt). Die grau unterlegten Kategorien wurden nachträglich in das Kodiersystem integriert.

Allerdings wurden nur solche Mechanismen in das Kategoriensystem integriert, die aus konkreten, beobachtbaren Verhaltensweisen bestehen (z.B. Ermahnen). Mechanismen, die sich 
erst im Verlauf des Prozesses zeigen (z.B. ausreden lassen) oder zu unspezifisch und damit nicht genau beobachtbar sind (z.B. thematisch strukturieren, jeden zu Wort kommen lassen) werden nicht als Kategorie aufgenommen, da sie ausschließlich über die Betrachtung des Verlaufs der Diskussion zu erschließen sind.

Anschließend wurden zwei weitere Entwicklungsschritte parallel durchgeführt: Anhand transkribierter Gruppenentscheidungsprozesse wurde nach weiteren Koordinationsmechanismen gesucht, die als Kategorien in das Kodiersystem aufgenommen werden sollten. Außerdem wurde in etablierten Verfahren zur Kodierung von Gruppeninteraktionsprozessen nach relevanten Kategorien recherchiert, die ebenfalls in das Kodiersystem aufgenommen werden konnten oder zur Validierung selbstentwickelter Kategorien dienten. Diese beiden Entwicklungsprozesse werden im Folgenden dargestellt.

\section{Bildung von Kategorien anhand eines Beispieldatensatzes}

Es wurde geprüft, inwieweit sich die bestehenden Kategorien tatsächlich während eines Gruppenentscheidungsprozesses finden lassen. Außerdem wurde davon ausgegangen, dass während des Entscheidungsprozesses noch weitere Mechanismen zur Koordination eingesetzt werden, um die das Kategoriensystem ergänzt werden soll. Da sich Entwicklungs- und Untersuchungsmaterial unterscheiden sollen (Bilandzic et al., 2001), wurde als Grundlage für die induktive Kategorienentwicklung ein separater Datensatz herangezogen, der transkribierte Entscheidungsfindungsdiskussionen von 5-Personen-Gruppen enthält. Eine genaue Beschreibung des Datensatzes findet sich bei Boos (1996b). In sechzigminütigen Gruppendiskussionen sollten im Rahmen eines Planspiels während einer Fortbildung fünf Führungskräfte unterschiedlicher Bereiche der öffentlichen Verwaltung eine Entscheidung erzielen $^{15}$. Da das Kategoriensystem die vorhandenen Daten vollständig erfassen soll, müssen

\footnotetext{
${ }^{15}$ Die Transkripte basieren auf dem Verwaltungsplanspiel „Gipsabbau im Maurerhölzle?“. Es soll über einen Antrag eines Bauunternehmens entschieden werden, Gips in einem Erholungswald am Rande einer Stadt abzubauen. Im Landratsamt kommen verschiedene Personen zusammen, die natürlich unterschiedliche Meinungen vertreten (Landesbeamter, Bau-, Finanz-, Umweltdezernent und Amtsleiter für Natur- und Umweltschutz). Das Ziel ist es,
} 
auch solche Beiträge erfasst werden, die nicht als explizite Koordinationsmechanismen zu kodieren sind. Daher enthält das Kategoriensystem auch Kategorien inhaltlicher Beiträge. Der Schwerpunkt des Systems liegt jedoch auf einer differenzierten Abbildung von Mechanismen expliziter Prozesskoordination. Daher wurde für diese Kategorien im Sinne einer starken Detailtreue ein hoher Auflösungsgrad gewählt. Die inhaltlichen Kategorien wurden hingegen wenig differenziert.

Außerdem kann davon ausgegangen werden, dass durch die inhaltlichen Kategorien Mechanismen erhoben werden, die ein Potential zur Koordination besitzen. Wie bereits geschildert, stellt die Erfassung impliziter Koordinationsmechanismen eine Herausforderung dar, da diese Form der Koordination eher verdeckt und unausgesprochen stattfindet. Es ist allerdings möglich, dass durch eine zeitliche Betrachtung der Folgen von Äußerungen, die einer inhaltlichen Kategorie zugeordnet werden, eine koordinierende Wirkung festgestellt werden kann.

Die eruierten Kategorien wurden in einem ständigen Rückkopplungsprozess an weiteren Ausschnitten des Datensatzes erprobt sowie durch die Recherche ähnlicher Kodierverfahren ergänzt. Darauf wird im Folgenden eingegangen.

\section{Recherche in bestehenden Verfahren zur Kodierung von Gruppeninteraktionsprozessen}

Thematisch relevante, bestehende Kategoriensysteme (CISS, Kasseler Kompetenzraster, Konfkod, SYNPRO und das funktionsorientierte Interaktionsanalysesystem) wurden im Hinblick auf Kategorien expliziter Prozesskoordination gesichtet. Dadurch sollte einerseits ein Überblick über nützliche und erprobte Kategorien gewonnen werden. Andererseits diente dieses Vorgehen der konvergenten Validierung der bereits entwickelten Kategorien. Die genannten Kategoriensysteme wurden ausgewählt, da sie für die Analyse der Interaktion in Dyaden oder Gruppe konstruiert und somit für einen ähnlichen Forschungsinhalt wie das hier beschriebene

eine einheitliche Entscheidung unter Abwägung der ökologischen und wirtschaftlichen Folgen zu treffen, welche von allen Vertretern mitgetragen wird (Boos, 1994, 1996). 
Verfahren gebildet wurden. Die Entwickler/-innen der genannten Methoden haben ihre jeweiligen Kategorien auf unterschiedlichem Wege gewonnen:

Simon (2000) hat die Kategorien des SYNPRO auf der Grundlage von in experimenteller Kleingruppenforschung identifizierten Leistungsdeterminanten im Interaktionsgeschehen von Arbeitsgruppen entwickelt. Dabei hat sie sich am Modell des Problemlösens in Gruppen orientiert (Wetzel, 1995, zit. n. Simon, 2000), welches die Oberkategorien Zielklärung, Prozessklärung, Problemanalyse, Produktionsphase und Prozesskontrolle besitzt.

Gottman (1979) hat die Auswahl der Kategorien des CISS anhand seiner eigenen Forschung und anhand anderer Kategoriensysteme, wie dem „Marital Interaction Coding System“ (MICS) (Hops, Wills, Patterson \& Weiss, 1972, zit. n. Gottman, 1979) und dem „Inventory of Marital Conflict“ (IMC) (Olson \& Ryder, 1970, zit. n. Gottman, 1979) abgeleitet.

Das Kasseler-Kompetenzraster (Kauffeld, 2003) wurde ebenfalls anhand von Forschungsarbeiten entwickelt, allerdings wird die Entwicklung der Kategorien nicht dargestellt.

Fisch (1994) ließ sich während der Entwicklung der Konferen₹kodierung durch den Umgang mit der Interaktionsprozessanalyse (IPA) von Bales (1950, 1970, zit. n. Fisch, 1994) und vom System zur mehrstufigen Beobachtung von Gruppen SYMLOG (Bales 1979/ 1982; Orlik, 1989, zit. n. Fisch, 1994) inspirieren. Die endgültige Entwicklung der Kategorien für die Konferenzkodierung erfolgte datennah mit Hilfe der Analysen von Gruppensitzungen.

Hirokawa (1982) entwickelte das funktionsorientierte Interaktionsanalysesystem deduktiv auf der Grundlage seiner funktionalen Theorie der Gruppenentscheidungsfindung.

Neben Kategoriensystemen wurde außerdem die Literatur zur Gesprächsführung und Moderation von Gruppen im Hinblick auf Koordination untersucht (Edmüller \& Wilhelm, 2005; Hartmann et al., 2000; Kanitz, 2004 ; Seifert, 2005). 
Auf den Ergebnissen der Auseinandersetzung mit den Transkripten und den erwähnten Kategoriensystemen aufbauend fand die deduktive Kategorienbildung statt. Die Daten wurden den zunächst deduktiv entstandenen Kategorien entsprechend kodiert. Gab es bei diesen Kodierdurchgängen noch Kodiereinheiten, die den bereits gebildeten Kategorien nicht zuzuordnen waren, dann wurden induktiv weitere Kategorien gebildet, um sicherzustellen, dass die in den Daten enthaltene Information erschöpfend dargestellt wird.

Um mit der deduktiven Kategorienbildung beginnen zu können, werden zunächst die in Kapitel 6.1.2 beschriebenen Definitionen der explizten und impliziten Koordinationsmechanismen aufgegriffen.

Die Erfassung der impliziten Koordination ist per Definition schwierig, da sie ohne verbale und deutlich erkennbare Mechanismen sowie teilweise automatisch geschieht (Grote et al., 2003). Implizite Koordinationshandlungen lassen sich somit erst erkennen, wenn man den Verlauf der Diskussion betrachtet. Nennt ein Gruppenmitglied beispielsweise ungefragt Informationen, da er/sie aufgrund eines mentalen Bildes weiß, dass ein anderes Teammitglied diese Information benötigt, dann kann man daraus schlussfolgern, dass er/sie mit seiner Aussage die Gruppenarbeit koordiniert. Dieses Beispiel für implizite Koordination ist bei Grote et al. (2003) ebenfalls vorzufinden. Es wird daher vermutet, dass inhaltliche Beiträge, wie beispielsweise die Meinungsäußerung eines Gruppenmitgliedes, ein Potential zur Koordination besitzen können, weil die Gruppe durch solche „Denkanstöße“ in ihrer Entscheidungsfindung vorangebracht wird. Für das Kategoriensystem wurde daher die Oberkategorie Kategorien inhaltlicher Beiträge gebildet. Um zwischen verschiedenen inhaltlichen Beiträgen hinreichend differenzieren zu können, wurde auf bereits existierende Kategoriensysteme zurückgegriffen und überprüft, welche inhaltlichen Kategorien dort vorzufinden sind und in das Kategoriensystem integriert werden können. Zunächst wurde auf die Konferenzkodierung von Fisch (1994) zurückgegriffen. Dort gibt es fünf Kategorien, die den Oberbegriff „aufgabenbezogene Aktivitäten: Inhalte und Sachbeiträge“ tragen. Diese fünf Kategorien: Feststellung, Meinung, Information, Erläuterung und Lösungsvorschlag 
wurden daraufhin für die Oberkategorie Kategorien inhaltlicher Beiträge in das Kategoriensystem aufgenommen. In anderen Kategoriensystemen sind ebenfalls die soeben genannten inhaltlichen Kategorien vorzufinden. So gibt es im Kasseler Kompetenzraster (Kauffeld, 2003) ebenfalls die Erläuterung und den Lösungsvorschlag. Im CISS (Gottman, 1979) wird die Kategorie Lösungsvorschlag ebenso erwähnt. Allen drei zuvor erwähnten Methoden ist zusätzlich gemeinsam, dass sie neben inhaltlichen Kategorien auch Kategorien beinhalten, die emotionale Beiträge kodieren. In Bezug auf das vorliegende Kategoriensystem wurde entschieden, emotionale Beiträge nicht zu erfassen, da es anhand von Transkripten problematisch ist, im Nachhinein festzulegen, inwiefern eine Aussage freundlich oder unfreundlich gemeint war. Das vorliegende Kategoriensystem ist kein teilnehmendes Beobachtungsverfahren, welches simultan zur Diskussion eingesetzt werden kann. Vielmehr soll es erst im Anschluss an die abgeschlossene Diskussion angewendet werden. Die Erfassung von Emotionen scheint daher kaum möglich, da die Kodierer/-innen hierfür die Aussagen nach ihrem emotionalen Gehalt persönlich interpretieren müssten. Diese subjektive Interpretation durch die Kodierer/-innen soll hier vermieden werden. Da allerdings die Kategorien Zustimmung und Ablehnung deutlich erkennbar sind und sowohl in der Konfkod, im Kasseler Kompetenzraster, im CISS als auch bei Hirokawa (1982) vorzufinden sind, werden diese beiden Kategorien in die Kategorien inhaltlicher Beiträge übernommen. Um eine Interpretation der zustimmenden und ablehnenden Aussagen zu vermeiden, werden diese Kategorien in Zustimmungsreaktion und Ablehnungsreaktion umformuliert. Nur eindeutige Zustimmungen und Abweisungen werden kodiert, so dass eine Interpretation durch die Kodierer/-innen vermieden wird.

Zusammenfassend bestehen die Kategorien inhaltlicher Beiträge aus sieben Unterkategorien, deren genauer Bedeutungsinhalt im Anhang E ausführlich erklärt wird:

- Information,

- Feststellung,

- Erläuterung,

- Meinungsäußerung, 
- Zustimmungsreaktion,

- Ablehnungsreaktion,

- Lösungsvorschlag.

Im Folgenden wird die Entwicklung der Kategorien zur expliæiten Koordination beschrieben. Anhand der Ergebnisse aus Studie 1 und 2 sowie des Entwicklungsdatensatzes wurden zunächst vier Oberkategorien gebildet: Handlungsanleitungen, Fragen, Anreden und Strukturierungen. Die Handlungsanleitungen beschreiben Äußerungen, welche zur Koordination durch die Initiierung oder Beendigung von Handlungen eingesetzt werden. Diese Kategorie wurde anhand der Ergebnisse aus Studie 1 und 2 gebildet. Die Oberkategorie Fragen lehnt sich sowohl an die Ergebnisse der Studie 2 als auch an die Konferenzkodierung von Fisch (1994) an, in der es heißt: „Wer fragt, der steuert" (Fisch, 1994, S. 165). Strukturierungen sind ebenfalls ein wichtiges Mittel, um den roten Faden und das Ziel der Diskussion zu verfolgen (Edmüller \& Wilhelm, 2005; Hartmann et al., 2000). Die Kategorie Anreden ist auf induktive Weise entstanden. Durch die mehrmalige Auseinandersetzung mit den Transkripten von Diskussionen wurde deutlich, dass eine einzelne Person oder die ganze Gruppe sehr häufig persönlich oder namentlich angesprochen werden. Es wird vermutet, dass die Aufmerksamkeit damit auf den/die Redner/-in gelenkt werden soll. Denkbar ist auch, dass sich die Gruppenmitglieder durch die konkrete Namensnennung stärker auf den Inhalt einer Aussage konzentrieren sollen. Damit kommt den Anreden eine koordinierende Funktion zu.

Die vier Oberkategorien zur expliziten Koordination (Handlungsanleitungen, Fragen, Anreden und Strukturierungen) werden jeweils in Unterkategorien unterteilt, die im Folgenden beschrieben werden.

Die Handlungsanleitungen dienen der Koordination von Handlungen. Zur ihrer weiteren Unterteilung wurde erneut die Konferenzkodierung (Fisch, 1994) herangezogen. Sie enthält vier Kategorien, welche die Steuerung bzw. Koordination in Gruppendiskussionen erfassen sollen 
(Frage mit Feststellung, Informationsfrage, Frage zum Verfahren und der Vorschlag zum Verfahren). Da eine separate Kategorie für Fragen vorliegt, ist vorerst nur der Vorschlag zum Verfahren als Handlungsanleitung interessant. In weiteren Kategoriensystemen, wie dem Kasseler Kompetenzraster und dem Interaktions-Beobachtungssystem SYNPRO (Simon, 2000), ist der Verfahrensvorschlag ebenfalls als Kategorie vorhanden und wird deshalb für diese Methode übernommen. Das Kasseler Kompetenzraster enthält eine weitere Handlungsanleitung, welche einige Äußerungen der vorliegenden Daten gut umschreibt - die persönliche Zumeisung. Diese Kategorie zählt auch zu den Führungskompetenzen: Yukl (2002) unterscheidet verschiedene Führungskompetenzen, die hilfreiche Hinweise für koordinierende Handlungsanleitungen in Gruppendiskussionen geben. Danach können folgende neue Kategorien gebildet werden: Aufforderung, Erbittet Klärung oder Spezifizierung, Erbittet Vorschläge und Ermahnung. Die Kategorien Erbittet Vorschläge, Verfahrensvorschlag und Persönliche Zuweisung sind zusätzlich auch im InteraktionsBeobachtungssystem SYNPRO (Simon, 1997) enthalten, was für die konvergente Validität dieser Kategorien spricht. Grote et al. (2003) erwähnen die Kategorie Aufforderung ebenfalls als eine Möglichkeit zur expliziten Koordination. Im Überblick bestehen die Handlungsanleitungen aus sechs Unterkategorien, deren genauer Bedeutungsinhalt im Anhang E ausführlich erklärt wird:

- Aufforderung,

- Persönliche Zuweisung,

- Verfahrensvorschlag,

- Erbittet Klärung oder Spezifizierung,

- Erbittet Vorschläge,

- Ermahnung.

Die Oberkategorie Fragen lehnt sich sowohl an die Ergebnisse der Studien 1 und 2 sowie an die Konferenzkodierung von Fisch (1994) an. Diese unterscheidet drei Fragekategorien, die der Koordination in Gruppen dienen. So gehen die Informationsfrage und die Frage zum Verfahren in 
dieses Kategoriensystem ein. Hingegen wird die Konferenzkodierungs-Kategorie Frage mit Feststellung nicht übernommen, da Probekodierungen gezeigt haben, dass sie für die mikroanalytische Verhaltensbeschreibung nicht detailliert genug ist. Die Verfahrensfrage und die Informationsfrage stellen ebenso Kategorien im Kasseler Kompetenzraster dar. Eine andere Fragenart, die bei Kauffeld et al. (2002) zu finden ist und sich in Probekodierungen bewährte, ist die Meinungsfrage. Andere Gruppenmitglieder nach ihrer persönlichen Meinung zu fragen, kann ein Potential zur Gruppenkoordination besitzen, da diese eine Grundlage für neue und wichtige Denkanstöße in der Diskussion liefern können.

Im Interaktions-Beobachtungssystem SYNPRO wiederum sind Frageformen vorzufinden, die sich mit den Handlungsanleitungen decken und die sich bei durchgeführten Probekodierungen als vorteilhaft herausstellten. So gibt es bei SYNPRO das bereits bekannte Kategorienlabel Informationsfrage und zusätzlich noch die Sperifizierungs- und Klärungsfrage (als Pendant zur Kategorie Erbittet Klärung oder Spezifizierung), die Einverständnisfrage sowie die Entscheidungsfrage. Auch Hirokawa (1982) schlägt die Klärungsfrage als Kategorie vor. Grote et al. (2003) geben in ihrem Artikel ebenfalls die Kategorien Informationsfrage und Einverständnisfrage als Beispiele für explizite Koordinationshandlungen an. Aufgrund induktiver Kategorienbildung entstand zusätzlich die Fragekategorie Lösungsfrage. Da die Kategorie Lösungsvorschlag (bei Kategorien inhaltlicher Beiträge) bereits in das Kategoriensystem aufgenommen wurde und sowohl durch die Methoden von Fisch (1994), Kauffeld et al. (2002) als auch Gottman (1979) abgesichert ist, erscheint es logisch, auch die Lösungsfrage zu einer eigenständigen Kategorie zu formulieren (vgl. auch Hirokawa, 1982).

Zusammenfassend bestehen die Fragen aus sieben Unterkategorien, deren genauer Bedeutungsinhalt im Anhang E ausführlich erklärt wird:

- Informationsfrage,

- Meinungsfrage,

- Spezifizierungs- oder Klärungsfrage, 
- Verfahrensfrage,

- Lösungsfrage,

- Einverständnisfrage,

- Entscheidungsfrage.

Die Strukturierungen sind die vorletzte Oberkategorie der Hauptkategorie der expliziten Koordination. Sie sind ein Mittel, um Ordnung in der Diskussion zu ermöglichen, so dass das jeweilige Ziel verfolgt werden kann (Edmüller \& Wilhelm, 2005; Hartmann et al., 2000). Strukturierungen zeigten sich in den Ergebnissen der Studien 1 und 2. In den bisherigen Kategoriensystemen kommen sie allerdings eher selten vor. Bei Hirokawa (1982), Kauffeld et al. (2002) und Grote et al. (2003) gibt es jeweils die Kategorie Zusammenfassung und bei Simon (2000) die Kategorie Entscheidung. Larson et al. (1998b) sowie auch die Studie 3 der vorliegenden Arbeit konnten zeigen, dass die Wiederholung von Äußerungen oder bereits in der Diskussion genannten Informationen einen positiven Einfluss auf die Gruppenentscheidung ausübt. Daher wurden die Kategorien Zusammenfassung, Entscheidung und Wiederholung in dieses Kategoriensystem aufgenommen. Diese Kategorie bewährte sich bei Probekodierungen. Da diese drei Kategorien die Strukturierungen noch nicht vollständig abdecken, wurde induktiv vorgegangen. Bei weiteren Probekodierungen konnte festgestellt werden, dass in den vorliegenden Transkripten sehr häufig Ziel- baw. Aufgabenformulierungen, Zielandeutungen und Darstellungen der eigenen Vorgehensweise aufzufinden waren, so dass diese drei Kategorien ebenfalls in das Kategoriensystem aufgenommen wurden.

Insgesamt bestehen die Strukturierungen aus sechs Unterkategorien, deren genauer Bedeutungsinhalt im Anhang E ausführlich erklärt wird:

- Zusammenfassung,

- Wiederholung,

- Ziel- bzw. Aufgabenformulierung, 
- Entscheidung,

- Zielandeutung,

- Darstellung der eigenen Vorgehensweise.

Darüber hinaus wurde aufgrund induktiver Datenanalyse zu den Kategorien zur expliziten Koordination noch die Oberkategorie Anreden hinzugefügt, die sich wiederum in namentliche und persönliche Anrede aufspaltet.

Um die Vollständigkeit des Kategoriensystems zu gewährleisten, wurden zwei Zusat₹kategorien eingeführt, die sich allerdings den beiden Hauptkategorien Kategorien zur expliziten Koordination und Kategorien inhaltlicher Beiträge nicht zuordnen ließen. Während der induktiven Kategorienentwicklung zeigte sich, dass sowohl Unterbrechungen als auch fokussierende Einworteinleitungen häufig vorkommen. Daher wurden sie als Zusatzkategorien in das Kategoriensystem aufgenommen. Die Kategorie Unterbrechung kann ebenso bei Kauffeld et al. (2002) gefunden werden.

Tabelle 21 fasst die eben erläuterten Kategorien und ihre Herkunft zusammen. 
Tabelle 21: Übersicht über den Ursprung der Kategorien

\begin{tabular}{|c|c|}
\hline Name der Kategorie & Ursprung \\
\hline \multicolumn{2}{|l|}{ Kategorien zur expliziten Koordination } \\
\hline \multicolumn{2}{|l|}{ Handlungsanleitungen: } \\
\hline Aufforderung & Grote et al., 2003, Yukl, 2002, Studien 1 \& 2 \\
\hline persönliche Zuweisung & Kauffeld et al., 2002, Yukl, 2002 \\
\hline Verfahrensvorschlag & Fisch, 1994, Kauffeld et al., 2002, Simon, 2000, Studien $1 \& 2$ \\
\hline Erbittet Klärung oder Spezifizierung & Simon, 2000, Yukl, 2002 \\
\hline Erbittet Vorschläge & Simon, 2000, Yukl, 2002 \\
\hline Ermahnung & Yukl, 2002, Studien $1 \& 2$ \\
\hline \multicolumn{2}{|l|}{ Strukturierungen } \\
\hline Zusammenfassung & Grote et al., 2003, Kauffeld et al., 2002 \\
\hline Wiederholung & Larson et al., 1998, Studien 1 - 3 \\
\hline Zielformulierung & induktiv, Studien $1 \& 2$ \\
\hline Entscheidung & Simon, 2000, Studien $1 \& 2$ \\
\hline Zielandeutung & induktiv \\
\hline Darstellung der eigenen Vorgehensweise & induktiv, Studien $1 \& 2$ \\
\hline \multicolumn{2}{|l|}{ Fragen } \\
\hline Informationsfrage & $\begin{array}{l}\text { Fisch, 1994, Grote et al., 2003, Kauffeld et al., 2002, } \\
\text { Simon, } 2000\end{array}$ \\
\hline Meinungsfrage & Kauffeld et al., 2002 \\
\hline Spezifizierungs- oder Klärungsfrage & Simon, 2000, Yukl, 2002, Studien 1 \& 2 \\
\hline Verfahrensfrage & Fisch, 1994, Kauffeld et al., 2002, Studien 1 \& 2 \\
\hline Lösungsfrage & induktiv, Studien 1 \& 2 \\
\hline Einverständnisfrage & Grote et al., 2003, Simon, 2000, Studien $1 \& 2$ \\
\hline Entscheidungsfrage & Simon, 2000 \\
\hline \multicolumn{2}{|l|}{ Anreden } \\
\hline Persönliche Anrede & induktiv \\
\hline Namentliche Anrede & induktiv \\
\hline
\end{tabular}

\section{Kategorien inhaltlicher Beiträge}

Feststellung

Information

Erläuterung

Meinungsäußerung

Zustimmungsreaktion

Ablehnungsreaktion

Lösungsvorschlag
Fisch, 1994

Fisch, 1994, Studien 1 \& 2

Fisch, 1994,

Kauffeld et al., 2002

Fisch, 1994

Fisch, 1994, Gottman, 1979, Kauffeld et al., 2002

Fisch, 1994, Gottman, 1979, Kauffeld et al., 2002

Fisch, 1994, Gottman, 1979, Kauffeld et al., 2002

\section{Zusatzkategorien}

Unterbrechung

Kauffeld et al., 2002, Studien 1 \& 2

Fokussierende Einworteinleitung induktiv 


\subsection{Die Güte des Kategoriensystems}

Das folgende Kapitel widmet sich der Güte des Kategoriensystems. Zunächst werden in Kapitel 6.4.1 Angaben zur Reliabilität des Verfahrens gemacht. In Kapitel 6.4.2 wird auf die Validität und abschließend auf die Nebengütekriterien eingegangen (Kapitel 6.4.3).

\subsubsection{Reliabilität}

Im Bezug auf das Kodiersystem ist die Reliabilität ein Kriterium, welches die Genauigkeit bzw. Zuverlässigkeit (Bortz, 1999) der Zuteilung der verschiedenen Kategorien jedes/r Kodierers/-in kennzeichnet. Außerdem wird überprüft, ob das Verfahren korrekt angewendet wurde. Eine geringe Reliabilität legt eine Umformulierung der Kategorien nahe. Eine hohe Reliabilität bedeutet wiederum, dass den Kodiereinheiten bei einer wiederholten Kategorienzuteilung dieselben Kategorien zugeordnet werden.

Folglich ist die Reproduzierbarkeit inhaltsanalytischer Ergebnisse ein Kriterium für die Reliabilität des Verfahrens. Werden dieselben Kodiereinheiten in einem gewissen zeitlichen Abstand wiederholt von einem/r Kodierer/-in kodiert (Intrarater-Reliabilität) oder kodieren verschiedene Personen dasselbe Textmaterial und kommen dabei jeweils zum selben Ergebnis (Interrater-Reliabilität), ist die Zuverlässigkeit des Verfahrens gegeben. Bei schlechter InterraterReliabilität kann durch die Berechnung der Intrarater-Reliabilität geprüft werden, ob die mangelnde Zuverlässigkeit eventuell nur auf die mangelnde Sorgfalt eines/r einzigen Kodierer/in zurückgeht. Bei dieser Person müsste die Intrarater-Reliabilität in diesem Fall sehr niedrig sein.

Für das vorliegende Kodiersystem wird nur die Interrater-Reliabilität bestimmt, für die in einigen Publikationen das Synonym Intercoder-Reliabilität verwendet wird (vgl. Mayring, 2003; Merten, 1995). Eine zusätzliche Bestimmung der Intrarater-Reliabilität gäbe lediglich an, inwiefern ein/e Kodierer/-in bei wiederholten Kodierdurchgängen zu konsistenten Kodierungen kommt. Das zentrale Problem bei der Bestimmung dieser Form der Reliabilität liegt allerdings darin, dass 
bei individuell systematisch unterschiedlichem Kategorienverständnis dennoch jede/r für sich allein in seinem/ihrem Urteil konsistent sein kann, ohne dass eine hohe Reliabilität qwischen den Kodierern/-innen besteht. Es könnte somit auch bei wiederholten Kodiervorgängen keine Aussage dazu gemacht werden, ob andere Kodierer/-innen zu ähnlichen oder identischen Kodierungen kommen. Außerdem kann dieses Maß der Stabilität bezüglich einer Kodierung Erinnerungseffekten unterliegen und damit die Intrarater-Reliabilität sehr schnell verfälschen (Wirtz \& Caspar, 2002). Dies ist dann wahrscheinlich, wenn zwischen den Kodierzeitpunkten keine großen Zeitabstände liegen. Daher wird im Folgenden ausschließlich die Interrater- und nicht die Intrarater-Reliabilität bestimmt.

Die Interrater-Reliabilität bestimmt die Korrelation der Urteile zweier beliebiger Kodierer/-innen bzw. den Anteil der Varianz der wahren Merkmalsausprägungen, der entweder durch die Urteile eines/r Kodierers/-in oder durch den Mittelwert mehrerer Kodierer/-innen erklärt werden kann (Wirtz \& Caspar, 2002). In der Literatur werden hauptsächlich drei Typen der Interrater-Reliabilität unterschieden:

Typ 1: Hier wird überprüft, wie stark die paarweise Übereinstimmung von jeweils zwei Kodierern/-innen bei h Kodierern/-innen ist (Schnittmenge von jeweils zwei der h Kodierer/-innen) (Merten, 1995).

Typ 2: Hier wird überprüft, wie stark die gemeinsame Übereinstimmung bei h Kodierern/innen ist (Schnittmenge bei h Kodierern/-innen) (Merten, 1995).

Typ 3: Hier wird überprüft, wie sich eine Mehrheit der Kodierer/-innen verhält. Im einfachsten Fall wird das Kriterium „two out of three“ angewendet, also wie stark zwei von drei Kodierern/-innen (bzw. allgemein m von h Kodierern/-innen) übereinstimmen (Merten, 1995).

Wird die Interrater-Reliabilität ausschließlich zwischen zwei Kodierern/-innen überprüft, wie es in der Forschung üblich ist (vgl. Früh, 2004) und auch bei dem vorliegenden Verfahren vorgenommen wird, so sind alle drei Typen identisch (Merten, 1995). 
Vor der Überprüfung der Interrater-Reliabilität sollten die Kodierer/-innen in der Anwendung des Kategoriensystems geschult werden. Nur so kann gewährleistet werden, dass alle Beteiligten die Kategorien verstanden haben und sie korrekt unterscheiden können. Erst anschließend ist es sinnvoll, die Gütekriterien mit Hilfe empirischer Methoden zu überprüfen (Früh, 2004).

Ein übliches Verfahren zur Berechung der Interrater-Reliabilität eines Kategoriensystems stellt die Berechung des Kappa-Maßes dar. Es beruht auf der Analyse der Urteilshäufigkeiten zweier Kodierer/-innen. Das Kappa-Maß (k) von Cohen erlaubt die Überprüfung der Übereinstimmung zweier Klassifikationen derselben Objekte (Bortz, 1999). Man berechnet k anhand folgender Gleichung:

$$
k=\frac{p_{0}-p_{e}}{1-p_{e}}
$$

$\mathrm{P}_{0}$ bezeichnet hierbei den empirischen Anteil konkordanter Urteile und $\mathrm{p}_{\mathrm{e}}$ steht für den Anteil konkordanter Urteile, die man rein zufällig aufgrund der Randsummen erwarten kann. Diese beiden Urteile werden im Kappa-Maß gegenübergestellt (Bortz, Lienert \& Boehnke, 2000). Mit dem Ausdruck 1- $\mathrm{p}_{\mathrm{e}}$ wird der über den Zufall hinausgehende, theoretisch mögliche Anteil konkordanter Urteile gekennzeichnet. Hingegen bezeichnet $\mathrm{p}_{0}-\mathrm{p}_{\mathrm{e}}$ den über den Zufall hinausgehenden, tatsächlich aufgetretenen, Anteil konkordanter Urteile.

Ein optimales Kappa $k=1$ ist nur dann möglich, wenn unabhängig von den Randverteilungen nur konkordante Urteile auftreten und damit $\mathrm{p}_{0}=1$ ist. In der Literatur existiert die Faustregel, dass ein Wert von $k>0.75$ als Indikator für eine sehr gute und ein $k$ zwischen .60 und .75 als Indikator für eine gute Übereinstimmung angesehen werden können (Fleiss \& Cohen, 1973). Ein k zwischen 0.4 und 0.6 kann als akzeptable Übereinstimmung betrachtet werden (Wirtz \& Caspar, 2002). Jedoch fordern Bakeman und Gottmann (1986) sowie Frick und Semmel (1978) einen strengeren Maßstab und empfehlen, erst bei einem Kappa von k>0.7 bzw. k>0.75 von einem zufrieden stellenden Wert auszugehen. Früh (2004) ist jedoch der Meinung, dass der absolute 
Ausprägungsgrad eines Reliabilitätskoeffizienten nichts über die Qualität der Kodierung aussagt.

Die erreichbare Übereinstimmung sei von verschiedenen Faktoren abhängig:

- Sorgfalt und intellektuelle Fähigkeit der Kodierer/-innen,

- Differenziertheit des Kategoriensystems (Anzahl der Kategorien),

- hierarchische Struktur des Kategoriensystems (Ober- und Unterkategorien),

- Trennschärfe der Kategorien (Früh, 2004).

Zur Berechnung der Interrater-Reliabilität erhielten zwei Kodierer/-innen einen zehnminütigen Auszug einer Diskussion aus der Studie von Boos (1996, vgl. Kapitel 6.3.2). Sie bildeten zunächst Kodiereinheiten anhand des Verfahrens, welches in Kapitel 7 beschrieben wird. Das ermittelte Kappa beträgt $\mathrm{k}=0.69$ und kann in Anlehnung an die zuvor erwähnten Orientierungswerte als gut oder zumindest hinreichend angesehen werden. Die Reliabilität des Kodiersystems ist somit noch verbesserungswürdig. Es ist allerdings anzunehmen, dass bei weiteren Kodierschulungen, einem ausreichenden Training, angemessener Übung und daraus resultierender Kodiererfahrung das Kappa-Maß erhöht werden kann. Die hohe Anzahl von Kategorien stellt eine erhöhte Anforderung an die Kodierer/-innen dar (Früh, 2004). Eine geringere Kategorienanzahl würde die Zuordnung erleichtern, da die Kategorien schneller erlernbar wären. Besonders am Anfang des Kodierprozesses mag es schwierig sein, die hohe Anzahl an Kategorien komplett zu überblicken, so dass mit Sicherheit noch einige Kodierfehler unterlaufen werden, die nach ausreichender Übung verhindert werden können. Inwieweit eine Modifikation der Kategorien notwendig ist, wird sich nach weiteren Anwendungen zeigen.

\subsubsection{Validität}

Unter Validität wird die Gültigkeit einer Methode verstanden (Bortz et al., 2000). Es muss daher überprüft werden, inwieweit die Kategorien tatsächlich Mechanismen expliziter Prozesskoordination erfassen. Im Vergleich zur Reliabilität, die sich nur auf die eigentliche 
Kodierprozedur beschränkt, bezieht sich die Validität auf den gesamten Erhebungsvorgang (Merten, 1995). Die Validität ist damit das weiter gefasste Kriterium, da sie die Reliabilität voraussetzt. Umgekehrt kann das Ergebnis eines Kodierungsprozesses zuverlässig sein, ohne dass es gültig sein muss (Merten, 1995). Nach Krippendorff (1980) und Holsti (1969) lassen sich in der Inhaltsanalyse verschiedene Konzepte der Validität unterscheiden, welche Mayring (2003) zu drei Hauptgruppen zusammengefasst hat - die materialorientierte, die prozessorientierte und die ergebnisorientierte Validität. Diese drei Begriffe werden von Mayring nicht definiert, sondern lediglich durch eine von ihm vorgeschlagene Zuordnung anderer Validitätsarten beschrieben. Die Namen lassen allerdings vermuten, dass sich die materialorientierte Validität auf das während der Studie von dem/der Forscher/-in verwendete Material bezieht. Dazu gehören z.B. die Stichprobenvalidität, welche die üblichen Kriterien exakter Stichproben verlangt (Mayring, 2003) und die semantische Validität, die auf die Bedeutungszuweisung bestimmter Textstellen Bezug nimmt (Bos, 1989).

Die prozessorientierte Validität bezieht sich auf den gesamten Forschungsablauf. Zur prozessorientierten Validität zählt z.B. die Konstruktvalidität. Hier werden aufgrund der theoretischen Vorstellungen des/der Forschers/-in Aussagen über die zu erwartenden Ergebnisse getroffen und anschließend mit den erhaltenen Ergebnissen verglichen. Stimmen beide Resultate größtenteils überein, wird von einer hohen Konstruktvalidität gesprochen. Da diese Form der Kontrolle allerdings vom Wissen des/der Forschers/-in abhängig ist, kann durch sie kein verbindlicher Nachweis über die Fehlerfreiheit der Analyse erbracht werden. Der Fehler kann immer auch in den theoretischen Kenntnissen des/der Forschers/-in liegen (Bos, 1989).

Die ergebnisorientierte Validität stützt sich auf die Gültigkeit der durch die Studie erzielten Resultate. Dazu zählt z.B. die Vorhersagevalidität. Sie verlangt eine Validierung der gewonnenen Ergebnisse in konkreten Verwendungszusammenhängen. Darunter ist zu verstehen, dass aus den Resultaten der Inhaltsanalyse Prognosen ableitbar sein müssen, deren Eintreffen anschließend untersucht wird (Mayring, 2003). 
Allerdings scheint der Nachweis der Validität bei Inhaltsanalysen im Allgemeinen nicht unproblematisch zu sein. In der Praxis beschränkt sich die Validitätsüberprüfung zumeist nur auf die Inhaltsvalidierung, da die Kriteriumsvalidierung oft daran scheitert, dass keine geeigneten Kontrolluntersuchungen vorliegen (Bos, 1989).

So stellt sich auch die Frage, wie sich die Validität bei dem vorliegenden Kodiersystem überprüfen lässt. Prinzipiell kann die Validität nicht verifiziert, sondern nur falsifiziert werden. Lässt sich somit kein Einwand gegen die Gültigkeit des Kodiersystems und gegen deren Anwendung finden, so kann die Validität bis auf weiteres angenommen werden (Merten, 1995). Früh (2004) hat ein Verfahren zur Überprüfung der Validität vorgeschlagen, welches im Folgenden vorgestellt wird.

Früh (2004) postuliert, dass die Forscher/-innen davon ausgehen, dass sie über präzises Wissen über die durch die Kategorien zu repräsentierenden Inhalten verfügen. Daher sollten ihre Kodierungen, abgesehen von einigen Flüchtigkeitsfehlern, korrekt sein. Es wird davon ausgegangen, dass die Entwickler/-innen des Kategoriensystems die vorliegenden Inhalte so kodieren, wie es ihrem eigenen Verständnis nach wiederholten Probekodierungen entspricht. Ihre Kodierung ist somit per normativer Setzung korrekt im Sinne von valide. Hingegen muss sie nicht zwingend korrekt im Sinne der vorliegenden operationalen Definitionen sein, welche in dem Kodiersystem explizit stehen (Früh, 2004).

Die trainierten Kodierer/-innen sollten sich während des Kodierens an die Anweisungen und Regeln des Kodiersystems halten. Kommen sie zum selben Kodierergebnis wie die Entwickler/-innen des Systems, so kann man daraus schließen, dass ihre Kategorienzuordnungen valide sind. Es darf dabei nur zu leichten Abweichungen kommen, die sich zumeist dann ergeben, wenn die Definitionen der Kategorien einem zulässigen, aber kontrollierten, Interpretationsspielraum unterliegen. Es ist schließlich immer möglich, dass zwei Kodierer/innen aufgrund ihres eigenen Sprachverständnisses manche Inhalte unterschiedlich interpretieren. 
Diese möglichen Abweichungen sollten sich allerdings stets in einem nur kleinen Rahmen bewegen (Früh, 2004).

Der Grad der Übereinstimmung zwischen den Kodierern/-innen und den Verfahrensentwickler/-innen lässt sich mit Hilfe der bereits im Kapitel 6.4.1 vorgestellten Methode der Reliabilitätsberechnung feststellen. Mit dieser Methode wird nun die Validität überprüft (Früh, 2004). Die Validität mit Hilfe eines Reliabilitätstests zu kontrollieren, mag auf den ersten Blick etwas irritierend erscheinen, kann aber in Anbetracht der Vorstellungen von Früh (2004) als sinnvoll und verständlich angesehen werden. Treten bei der Gültigkeitsüberprüfung Abweichungen auf, so muss überprüft werden, ob der Interpretationsspielraum der Kodierer/-innen eingeschränkt, die Kategorien umformuliert oder die Kodiererschulung verbessert werden sollten. Insgesamt bietet das Testergebnis einen Anhaltspunkt, inwiefern der Bedeutungsinhalt der Kategorien von Forscher/-in und Kodierer/in übereinstimmend interpretiert wird. Im Zweifelsfall ist dann zu überprüfen, ob es sich bei den Abweichungen im Sinne der Kategoriendefinition um eine zulässige alternative Interpretation der Regeln oder um Kodierfehler handelt. Kann man diese Faktoren ausschließen, dann sagt die Übereinstimmung zwischen Forscher/-in und anderen Kodierern/-innen etwas über die Validität des Kodiersystems aus: Das von dem/der Forscher/-in zu messen beabsichtigte theoretische Konstrukt stimmt mit dem, was die Kodierer/-innen tatsächlich messen, überein ${ }^{16}$ (Früh, 2004).

Zusammengefasst kann festgehalten werden, dass eine Reliabilitätsprüfung zwischen Forscher/-in und Kodierer/-in hinsichtlich der Zuordnung einzelner Kategorien einer Prüfung und Optimierung der Validität dienen kann. Somit werden die Gütekriterien Reliabilität und Validität in dem vorgestellten Verfahren nach Früh (2004) gewissermaßen vereint.

\footnotetext{
${ }^{16}$ Früh (2004) schränkt ein, dass dies mit Sicherheit ein anderer Validitätsbegriff ist, als er in den meisten Lehrbüchern dargestellt wird. Aber die dort vorgestellten Definitionen lassen sich nur schwer bzw. kaum auf Kodiersysteme wie das vorliegende anwenden. Es gilt deshalb, eine geeignete Beschreibung der Validität zu finden, ohne dabei deren ursprüngliche Bedeutung aus den Augen zu verlieren.
} 
Im Hinblick auf die Validität des vorliegenden Kategoriensystems wurden die Kategorien während des Konzeptionsprozesses regelmäßig anhand von Probekodierungen korrigiert und präzisiert. Damit sollte erreicht werden, dass die Kategorienzuordnungen den relevanten Bedeutungsinhalt so messen, wie die Entwickler/-innen ihn aufgrund ihrer Kenntnisse und Kompetenzen auffassen. Es musste geprüft werden, inwieweit es gelungen ist, die Kodierregeln so zu beschreiben, dass die Kodierer/-innen dieselbe Bedeutung mit den Kategorien verbinden, wie die Entwickler/-innen des Kodiersystems. Empirisch überprüfen lässt sich dies, indem kontrolliert wird, ob sich die als valide gesetzte Interpretationsweise der Forscher/-innen den Kodierern/-innen adäquat vermitteln lässt. Ist dies der Fall, so sind auch die Kodierungen in diesem Sinne valide (Früh, 2004).

Ein zehnminütiger Ausschnitt einer Diskussion aus der Studie von Boos (1996) (vgl. Kapitel 6.3.2) wurde von einem trainierten Kodierer und einer Entwicklerin mit dem vorliegenden Kategoriensystem kodiert. Auf Grundlage der erhaltenden Kodierungen wurde die Validität bestimmt. Es zeigte sich, dass das Kappa für das gesamte Kategoriensystem $k=0.37$ beträgt. Dieser Wert muss auf Grundlage der Orientierungswerte, die im Kapitel 6.4.1 beschrieben wurden, als ungenügend angesehen werden. Es ist daher erstrebenswert, die Validität des Kodiersystems zu erhöhen, um eine statistische Auswertung der mit dieser Methode gewonnenen Daten zuzulassen. Bei genauerer Betrachtung der Kodierungen, die in die Validitätsberechnung eingingen, wird deutlich, dass auf Seiten des Kodierers viele unnötige (persönliche Anrede wird mit namentlicher Anrede vertauscht) und unlogische Fehler auftraten (eine eindeutige Zustimmungsreaktion „Ja“ wird mehrfach als fokussierende Einworteinleitung kodiert, die Kategorie „Feststellung““ wird überzufällig häufig verwendet). Dies lässt vermuten, dass die Kodiererschulung nicht intensiv genug verlief, so dass der Kodierer nicht in der Lage war, die 30 Kategorien vollständig zu überblicken und korrekt anzuwenden. Es erscheint daher empfehlenswert, nach einer erneuten Kodierschulung und anschließender sorgfältigerer 
Kodierung die Validität erneut zu überprüfen. Außerdem muss überprüft werden, inwieweit es noch nicht hinreichend gelungen ist, die Kodierregeln so zu beschreiben, dass sie sowohl für die Verfahrensentwickler/-innen als auch für Kodierer/-innen semantisch eindeutig sind. Die Kategorien, Ankerbeispiele und Abgrenzungsregeln müssen weiterhin präzisiert werden. Darüber hinaus mag die der Validitätsberechnung zugrundeliegende Stichprobe zu gering gewesen sein. Sie bestand aus einem zehnminütigen Diskussionsausschnitt, der die zur Reliabilitätsprüfung empfohlene 30malige Mindestvergabe einer Kategorie nicht ermöglichte.

Insgesamt bleibt festzuhalten, dass sich das Kodiersystem noch in der Erprobungsphase befindet. Zukünftig sollten weitere Validitätsuntersuchungen an größeren Stichproben, auch im Hinblick auf die Urteilskonkordanz jeder einzelnen Kategorie, erfolgen.

\subsubsection{Nebengütekriterien}

Neben der Reliabilität und Validität sollte ein Kodiersystem weiteren Gütekriterien genügen: Intersubjektive Nachvollqiebbarkeit, Nützlichkeit, Transparenz und Replizierbarkeit der Kategorienbestimmung sollen gewährleistet werden.

Nach Lienert und Ratz (1998) ist ein Verfahren ökonomisch, wenn es eine kurze Durchführungszeit beansprucht, wenig Material verbraucht, einfach zu handhaben und schnell und bequem auszuwerten ist. Nützlich ist es, wenn es eine Verhaltensweise misst bzw. vorhersagt, für deren Untersuchung ein praktisches Bedürfnis besteht (Lienert \& Raatz, 1998).

Ein weiterer zentraler Punkt für die Entwicklung der Kategorien selbst ist, dass die Kategorien vollständig und trennscharf sein müssen. Vollständig bedeutet, dass die Kategorien das Textmaterial in Richtung der zentralen Fragestellung bestmöglich ausschöpfen (Brosius \& Koschel, 2001). Die Kategorien sind trennscharf, wenn sich die einzelnen Ausprägungen wechselseitig ausschließen und wenn sich alle Ausprägungen auf das gleiche Merkmal beziehen. Demnach wäre die Trennschärfe nicht gegeben, wenn eine Kategorie Antworten unter sich vereinte, die auch in andere Kategorien passen würden (Brosius \& Koschel, 2001). Die letzten 
beiden Anforderungen können vor allem durch eine klare, umfassende und präzise Definition der Kategorien erfüllt werden (Früh, 2001).

Es wird davon ausgegangen, dass dieses Kodiersystem aufgrund der klaren Kategorienbeschreibungen und -unterteilungen für intersubjektive Nachvollziehbarkeit, Transparenz, Replizierbarkeit und Vollständigkeit der Kategorienzuordnung sorgt. Inwieweit die Praktikabilität, Ökonomie und Trennschärfe gewährleistet sind, wird die weitere Anwendung zeigen.

Darüber hinaus kann die Güte eines Kategoriensystems durch eine unsaubere Bildung der Kodiereinheiten - der Grundlage der Kodierung - erheblich eingeschränkt werden. Auf dieses Problem wird im folgenden Kapitel näher eingegangen. Es wird ein regelgeleitetes Vorgehen für die Bildung von Kodiereinheiten vorgeschlagen. 


\section{$7 \quad$ Methodenentwicklung 2: \\ Eine Methode zur Bildung von Kodiereinheiten in Transkripten verbaler Interaktionsprozesse}

"Research on interaction poses methodological problems sufficient to give even the most energetic and skillful researchers pause" (Poole \& Hirokawa, 1996, S. 5).

In den bisherigen Kapiteln wurde die Bedeutung von Prozessanalysen im Rahmen sozialpsychologischer Forschung betont. Darüber hinaus wurde zur Erfassung von Koordinationsmechanismen während des Interaktionsprozesses ein Kodiersystem entwickelt. Dabei wurde darauf hingewiesen, dass die Güte eines Kategoriensystems durch eine unsystematische und uneinheitliche Bildung der Kodiereinheiten - der Grundlage der Kodierung - erheblich reduziert werden kann. Das fehlende regelgeleitete Vorgehen in der Bildung von Kodiereinheiten stellt ein ungelöstes Problem der psychologischen Interaktionsforschung dar. Im Folgenden wird eine Methode vorgestellt, die es ermöglicht, im Rahmen sozialpsychologischer, mikroprozessanalytischer Kommunikationsforschung Redebeiträge in Gruppendiskussionen regelgeleitet in Kodiereinheiten zu zergliedern, so dass diese mit Hilfe eines Kategoriensystems kodiert werden können.

Zunächst werden das Ziel dieser Methode und der theoretische Hintergrund dargestellt, wobei die bisher bestehende Problematik der intransparenten Bildung von Kodiereinheiten diskutiert wird. Im Anschluss werden verschiedene Formen inhaltsanalytischer Einheiten sowie die formale Gestaltung der Zergliederung einzelner Redebeiträge beschrieben. Danach werden die Trennregeln kurz vorgestellt. Ihre ausführliche Erläuterung befindet sich in Form eines Manuals im Anhang F. Abschließend wird auf die Bestimmung der Güte des Verfahrens eingegangen.

\subsection{Ziel der Methode und theoretischer Hintergrund}

Auf die Bedeutung mikroanalytischer Prozessbetrachtungen wurde bereits in Kapitel 6.1.1 hingewiesen. Wesentlich für das vorliegende Verfahren ist die Unterscheidung zwischen 
Prozessanalysen auf der Mikro- und Makroebene. Die Mikroanalyse von Kommunikation untersucht Merkmale der einzelnen Kommunikationshandlungen oder die Entwicklung des Kommunikationsprozesses. Die makroanalytische Perspektive erfasst den gesamten Kommunikationsprozess als Einheit und zergliedert ihn nicht weiter. Für viele mikroprozessanalytische Verfahren (z.B. die Konferenzkodierung nach Fisch, 1994 oder das in Kap. 7 vorgestellte Kodiersystem) müssen die verbalen Daten zunächst transkribiert und für die weitere Kodierung aufbereitet werden. Während es für die Transkribierung bereits einige etablierte Verfahren gibt (z.B. Burger, Günther, Häcki Buhofer, Rüegg \& Schneider, 1998; Dittmar, 2002; Howitt \& Cramer, 2005; Selting et al., 1998), fehlen hinreichend standardisierte und überprüfte Vorgehensweisen für die Bildung von Kodiereinheiten. Eine Ausnahme bildet das von Selting (2000) empfohlene Verfahren, welches klare Regeln für die Konstruktion von Kodiereinheiten vorschlägt. Diese Regeln schließen neben syntaktischen Elementen vor allem die Beachtung von Intonation und Prosodie ein und setzen daher eine entsprechend umfangreiche Transkription voraus (Selting et al., 1998). Dieses aufwendige Verfahren zur Bildung der „smallest interactionally relevant complete linguistic unit“ (Selting, 2000, S. 477) ist für die „,interaktionale Linguistik“ (Selting \& Couper-Kuhlen, 2000) und die klassische Konversationsanalyse eine sehr geeignete und angemessene Methode, um die Struktur und den Verlauf von Gesprächen, insbesondere den Sprecherwechsel, zu untersuchen.

Für die Fragestellungen im Rahmen der sozialpsychologischen Kleingruppenforschung ist Seltings Verfahren aufgrund seiner Aufwendigkeit weniger angemessen, da hier zum einen die Prosodie des Gesprächs weniger interessiert und zum anderen meist Aussagen über andauernde Interaktionen in vielen Gruppen getroffen werden sollen. Psychologische Forschungsparadigmen gestehen gewonnenen Erkenntnissen eine Aussagekraft erst ab einer gewissen statistischen Power zu (Hager, 2004). Ein Verfahren zur Bestimmung von Kodiereinheiten für umfangreiches Gesprächsmaterial muss daher einerseits ein präzises, transparentes und intersubjektiv nachvollziehbares Vorgehen gewährleisten, andererseits auch pragmatisch einsetzbar sein, d. h. 
eine inhaltlich und zeitlich ökonomische Anwendung erlauben. Dies soll durch das hier vorgestellte Verfahren gewährleistet werden.

\section{Wieso ist es notwendig, Redebeiträge in Kodiereinheiten zu zerlegen?}

Die Reliabilität des Kodierens hängt neben der Präzision der Kategorien auch entscheidend von der Genanigkeit der Einheitenbildung ab (Guetzkow, 1950), wobei diese insbesondere bei solchen Daten schwierig sei, die durch kontinuierliche Beobachtung des verbalen und nonverbalen Verhaltens gewonnen wurden - eben die gängige Methode sozialpsychologischer Mikroprozessforschung.

Für die Inhaltsanalyse von Gesprächen mehrerer Personen stellt sich häufig das Problem, dass die Gesprächsteilnehmer/-innen unterschiedlich lange und unterschiedlich inhaltsreiche Redebeiträge äußern. Mitunter sagt Person A in einem Beitrag nur ein oder zwei Worte, Person B allerdings redet anderthalb Minuten lang über verschiedene Dinge. Sollen die Äußerungen im Hinblick auf eine spezifische Fragestellung verschiedenen Kategorien zugeordnet werden, so wären ungleiche Analyseeinheiten die Ausgangsbasis. Die Aussage der Person A wäre wahrscheinlich leicht einer Kategorie zuzuordnen, die Aussage von B müsste aber mehreren Kategorien zugeordnet werden. Letzteres wäre mit verschiedenen Nachteilen verbunden: Die Mehrfachkodierung einer einzelnen Aussage hätte zur Folge, dass in der Auswertung die Reihenfolge der Inhalte verloren ginge. Dadurch wird eine sequenzanalytische Auswertung, insbesondere eine Lag Sequential Analysis (Bakeman \& Gottman, 1986) als häufig eingesetztes Verfahren zur Untersuchung von Prozessverläufen (Van Hooff, 1982), erschwert. Die Daten sollten also möglichst sequentiell kodierbar sein. Daher ist es für solche Fragestellungen, in denen der Verlauf einer Kommunikation über ein Zeitintervall hinweg untersucht werden soll, sinnvoll, Redebeiträge in ihrer inhaltlichen Breite in vergleichbare Einheiten zu zerlegen. Damit ermöglicht man die anschließende Kodierung der gebildeten Einheiten in disjunkte Kategorien (Bakeman, 2000). Bilandzic, Koschel und Scheufele (2001) betonen, dass die Segmentierung des 
Datenmaterials die „intersubjektiv nachvollziehbare Basis für die Kategorienbildung“ (S. 103) schafft. In ihrem Vorschlag der theoretisch-heuristischen Segmentierung soll das Segmentierungskriterium (Kriterium für die Bildung einzelner Kodiereinheiten) im Sinne einer heuristischen Kategorie aus dem Forschungsanliegen abgeleitet werden. Diese sei „empirisch gehaltlos“ (S. 105) und diene der Entwicklung der eigentlichen Kategorien (Bilandzic et al., 2001), indem sie ein „einheitliches Abstraktionsniveau“ (S. 107) sichere. Damit verdeutlichen Bilandzic et al. (2001), dass ausgehend von der Fragestellung (z.B. Mikroprozessanalyse) überlegt werden muss, wie die vorhandenen Daten segmentiert werden sollen (z.B. Mikro- vs. MakroKodiereinheiten). Das entwickelte Kriterium für die Segmentierung wird im Sinne einer heuristischen Kategorie angewendet (z.B. das vorliegende Trennmanual).

Eine vergleichende Untersuchung, wie sich verschiedene Methoden der Kodiereinheitenbildung auf die Kategorisierung auswirken, steht bisher aufgrund eines Mangels an vergleichbaren Verfahren aus. Eine Ausnahme ist die Untersuchung von Geller, Kaplan und Lasswell (1942), in der vier verschieden lange Kodiereinheiten in Zeitungsleitartikeln überprüft wurden (Satz, Abschnitt, drei Sätze \& gesamter Artikel). Die verschiedenen Kodiereinheiten führten teilweise zu unterschiedlichen Kategorienzuweisungen. Dieser Befund verdeutlicht die Bedeutung einer angemessenen Kodiereinheitenbildung für die weiterführenden Analysen. Die Entscheidung darüber, wie die Einheiten zu bilden sind, ist insbesondere bei komplexen Inhalten mit weitreichenden Konsequenzen für die weitere Datenverarbeitung verbunden (Abbott \& Tsay, 2000).

"A discipline that cannot even come to agreement on its basic unit of analysis would seem to be a discipline based on shifting sands at best and, at worst, doomed to theoretical dead ends and contradictions" (Vallacher \& Wegner, 1987, S. 13).

Im Folgenden wird überlegt, welche Methoden zur Definition von Kodiereinheiten herangezogen werden können. 


\section{Die Syntax als Grundlage der Bestimmung von Kodiereinheiten}

Insgesamt liefert die Literatur zur Inhaltsanalyse und zur Beobachtung kaum konkrete Hinweise zur Bildung von Kodiereinheiten (z.B. Bakeman, 2000; Früh, 2004). Die klassische Literatur zur qualitativen Inhaltsanalyse sieht zwar die Festlegung von Analyseeinheiten vor und unterscheidet zwischen Kodier-, Kontext- und Auswertungseinheiten (siehe Kapitel 7.2), trifft aber keine genauen Aussagen zur Bildung dieser verschiedenen Einheiten (Mayring, 2000). Neben der oft unzureichenden Explikation der eigentlichen Kategorienentwicklung (Bilandzic et al., 2001) wird damit ein wesentlicher Schritt in der Auswertung qualitativer Daten der Intuition und damit der Erfahrung des jeweiligen Forschers überlassen.

Die Beschreibung des Segmentierungskriteriums macht die sonst intuitive Vorgehensweise der Kodiereinheitenbildung explizit. Bilandzic et al. (2001) betonen, dass ein einheitliches Segmentierungskriterium für verschiedenste Forschungsanliegen nicht sinnvoll sei, sondern dass es sich vielmehr um eine jeweils anzupassende „Arbeitsweise“ als um eine starres „Rezept“ handele (S. 106). Allerdings, so kritisiert auch Früh (2001), stellen die Autoren anschließend keine Verfahrensweisen zur Segmentierung vor, und ebenso wenig explizieren sie, was genau die segmentierten Stücke enthalten. Es wird kein formales und objektiv zu entwickelndes Kriterium zur Bildung von Kodiereinheiten vorgelegt (Früh, 2001). In der Beschreibung der Kategorienentwicklung empfehlen Bilandzic et al. (2001), dass in einem Segment die Kernaussage erhalten bleiben soll, „wie sie am ehesten mit einer Proposition (grundlegende »kleinste« Wissensstruktur) vergleichbar ist“" (S. 108). Nun ist der Begriff Proposition allerdings mit verschiedenen Bedeutungen behaftet. Einerseits gilt die Proposition im Rahmen der Sprechakttheorie ${ }^{17}$ als eine Sprachfunktion (Krallmann \& Ziemann, 2001). Laut Duden ist eine Proposition u. a. die Ankündigung eines Themas oder der Satz als

\footnotetext{
${ }^{17}$ Im Rahmen der Sprechakttheorie (Searle, 1969) können unter einem Sprechakt Handlungen verstanden werden, die eine Sprecherin ausführt, indem sie etwas äußert. Dieser Terminus ist nicht unbedingt gleichbedeutend mit dem des Redebeitrages, da er vor allem auf die dem Redebeitrag innewohnenden Intentionen und Handlungen hinweist. In der linguistischen Pragmatik steht man aber ebenfalls vor dem Problem, einzelne Sprechakte bzw. Sprachhandlungen in Gesprächen zu identifizieren.
} 
Informationseinheit (Dudenredaktion, 1990). Aufgrund dieser unterschiedlichen Bedeutungen erscheint der Begriff der Proposition wenig geeignet, um als eindeutiges und transparentes Segmentierungskriterium zur Bildung von Kodiereinheiten benutzt zu werden, zumal selbst bei einheitlicher Definition nicht klar ist, wie eine Proposition eindeutig zu erkennen sei bzw. als transparentes Segmentierungskriterium gelten könne.

Auch die Größe der zu bildenden Kodiereinheit muss genau überlegt werden, denn sie bestimmt die Häufigkeit, mit der wiederholte Items als separate Ereignisse klassifiziert werden und muss den Anforderungen des Kategoriensystems entsprechen (Guetzkow, 1950). Ansonsten wäre eine Klassifikation des zu analysierenden Materials schwierig: Ist die gewählte Einheit zu groß, könnte sie mehr als nur einer Kategorie zuzuordnen sein. Ist die gewählte Einheit aber zu klein, steigt vermutlich die Anzahl der Zuordnungen zur „Restkategorie“ (Guetzkow, 1950).

Die bisher übliche Unterteilung von Redebeiträgen in „Sinneinheiten“ durch rein semantische Kriterien nimmt auf diese Größenunterschiede nicht explizit Bezug. Sie ist zudem stark von dem/der jeweiligen Kodierer/-in abhängig, da es keine allgemeinen Regeln dafür gibt, was eine „semantische Einheit“ oder „Sinneinheit“ darstellt. Der/die Kodierer/-in müsste jeweils subjektiv entscheiden, ob es sich bei einem Redebeitrag um eine oder mehrere semantische Einheit(en) handelt. Dieser subjektive Entscheidungsprozess ist später sowohl intrasubjektiv als auch intersubjektiv nicht mehr nachvollziehbar und kann dadurch die Reliabilität eines Verfahrens beeinträchtigen. Früh (2001) weist auf die Notwendigkeit eines „formalen Kriteriums“ (S. 122) zur Bildung einer systematischen Segmentierungsregel hin.

Das im Folgenden vorgestellte Verfahren zur Bestimmung von Kodiereinheiten soll diesem Anspruch gerecht werden. Zugrunde liegt die Idee, dass Kodiereinheiten weder intuitiv abgegrenzt werden sollen, noch dass auf die unzureichend standardisierte Bildung von Sinneinheiten unklaren Inhalts zurückgegriffen werden muss. Damit die Kodiereinheiten regelgeleitet, nachvollziehbar und sowohl intersubjektiv als auch über verschiedene Zeitpunkte hinweg replizierbar definiert werden können, müssen die entsprechenden Regeln einen formalen 
Charakter besitzen und möglichst wenig Interpretationsspielraum zulassen. Die Syntax (Satzlehre) der deutschen Sprache bietet diese Möglichkeit.

Die Syntax beschreibt Regeln, wie sprachliche Elemente angeordnet und zu Sätzen zusammengefügt werden (Bünting \& Bergenholtz, 1979). Es wird ein Mechanismus vorgeschlagen, in dem auf syntaktischen Informationen aufbauende Regeln zur Bildung von Kodiereinheiten herangezogen werden. Syntaktische Regeln haben den Vorteil, dass sie klare Entscheidungsregeln vorgeben, nach denen Redebeiträge zu unterteilen sind. Dadurch sollen intersubjektive Nachvollziehbarkeit und Replizierbarkeit der Kodiereinheitenbestimmung gewährleistet werden. Allerdings ist diese Idee nicht neu. Holzinger ist beispielsweise der Meinung, dass die Syntax Sprechakte „ohnehin nicht vernünftig“ abgrenze (S. 427). Trotz fehlender Begründung ihrer radikalen Ansicht weist Holzinger (2001) damit auf die Problematik der formalen Abgrenzung von Sprechakten hin. Da Sprechakte im Vergleich zu Kodiereinheiten wesentlich inhaltsreicher sind, erscheint es jedoch plausibel, dass allein syntaktische Informationen zu ihrer Abgrenzung nicht ausreichen. Eine alternative Vorgehensweise zur Segmentierung von Sprechakten schlägt Holzinger (2001) nicht vor.

Das in diesem Manual dargestellte Verfahren soll nicht als „Rezept“ für jede Art von Kodiereinheitenbildung betrachtet werden. Es ist allein für solche Fragestellungen gedacht, in denen in der sozialpsychologischen Interaktionsforschung Kommunikationsprozesse mikroanalytisch untersucht und kategorisiert werden sollen. Dazu zählen beispielsweise Kategoriensysteme wie die Konferenzkodierung (Fisch, 1998) oder das in Kapitel 6 vorgestellte Kategoriensystem zur Erfassung von expliziten Koordinationsmechanismen in Gruppendiskussionen, aber auch vergleichbare Prozessanalysen, in denen der Einfluss der Kommunikation auf die Gruppenleistung untersucht wurde (z. B. Gurtner, 2003; Kanki \& Foushee, 1989; Kanki et al., 1989; Nägele, 2004; Tschan, 2000).

In ferner Zukunft mag das vorgestellte Verfahren vielleicht dazu dienen, den Pluralismus und die große Variabilität der Kodiermethoden zu Gunsten einer erhöhten Vergleichbarkeit 
einzuschränken. Schließlich liegt der Vorteil einer syntaktischen Bildung von Kodiereinheiten in der Balance zwischen strikt formaler Abgrenzung durch eine rein zeitliche Abtrennung, bei der z.B. alle 10 Sekunden eine neue Kodiereinheit gebildet wird, welches zwar sehr reliabel ist, aber auf Kosten der inhaltlichen Validität geht und einer rein inhaltlichen Abtrennung, bei der die Vorgehensweise unklar und deshalb nicht reliabel ist und die inhaltliche Validität zudem von der Vorgehensweise abhängig ist (vgl. Faßnacht, 1995). Syntaktische Regeln liefern einerseits formale Entscheidungshilfen, gewährleisten aber im Gegensatz zu rein zeitlicher Abtrennung eine inhaltlich sinnvolle Einheitenbildung, da die deutsche Syntax nicht der deutschen Sprache auferlegt wurde, sondern sich als eine Teilkomponente mit ihr entwickelt hat und sich z.B. „in erheblich geringerem Umfang ändert als der Wortschatz oder die Ausspracheregeln“ (Erben, 1984, S. 87).

Die Trennregeln wurden induktiv anhand von Diskussionstranskripten erstellt und sind das Ergebnis eines langen Entwicklungsprozesses. Die Entscheidungen für die einzelnen Regeln gehen auf kritische Diskussionen und reifliche Überlegungen zurück. Der Schwerpunkt ihrer Darstellung soll daher nicht in ihrer Begründung, sondern in der Art ihrer Anwendung liegen. Die Regeln werden in Kapitel 7.6 sowie im Anhang F dargestellt.

Zur Begriffsklärung werden im Folgenden zunächst die verschiedenen inhaltsanalytischen Einheiten vorgestellt. Anschließend werden das formale Vorgehen der Kodiereinheitenbildung im Transkript, die Voraussetzungen sowie das grammatikalische Vorwissen der Kodierer/-innen erläutert.

\subsection{Inhaltsanalytische Einheiten}

In der Literatur zur Kodierung wird zwischen folgenden inhaltsanalytischen Einheiten unterschieden: 


\section{Analyseeinheit}

Einerseits wird unter einer Analyseeinheit die Größe verstanden, über die im Sinne eines „Falls“ in der Untersuchung eine Aussage getroffen werden kann (Früh, 2001). Andererseits wird der Begriff Analyseeinheit auch als Obergriff für die zu kodierenden Einheiten benutzt (Holsti, 1969).

\section{Kodiereinheit}

Nach Mayring (2000) ist der kleinste Materialbestandteil, der ausgewertet werden darf, die Kodiereinheit. Sie wird auch als die Bezugsgröße der Kodierung im Text bezeichnet, auf die das Kategoriensystem je einmal anwendbar ist (Früh, 2001) bzw. als das spezifische Segment des Inhalts, das durch die Zuordnung zu einer gegebenen Kategorie charakterisiert wird (Holsti, 1969). Durch die vorliegende Methode sollen solche Kodiereinheiten in Transkripten von Diskussionen zu bilden sein.

\section{Kontexteinheit}

Die Kontexteinheit legt die Grenzen fest, in denen weitere Informationen zur Charakterisierung der Kodiereinheit gesucht werden können (Holsti, 1969). Mayring (2000) versteht darunter die Festlegung des größten Textbestandteils, der unter eine Kategorie fallen kann. Im Hinblick auf eine Kodiereinheit kann die Kontexteinheit aus dem jeweils vorausgehenden und folgenden Redebeitrag bestehen.

\section{Messeinheit und Auswertungseinheit}

Früh (2001) unterscheidet weiterhin die Messeinheit, die zumeist ein numerischer Zähler für die Häufigkeit sei, mit der die Kodiereinheiten im untersuchten Material vorkommen. Die Auswertungseinheit legt nach Mayring (2000) diejenigen Textteile fest, die jeweils nacheinander ausgewertet werden. 
Ziel dieses Manuals ist die Bildung solcher Einheiten, denen die Kategorien eines Kodierverfahrens zur Erfassung von Diskussionsprozessen auf Mikroebene zugeordnet werden können. Folglich sollen, um in der vorgestellten Terminologie zu bleiben, Kodiereinheiten gebildet werden.

\subsection{Formale Gestaltung der Zergliederung einzelner Redebeiträge in Kodiereinheiten:}

Im Folgenden wird erläutert, wie einzelne Redebeiträge zerlegt werden. Dabei sind folgende für die maschinelle Auswertung, z.B. mit Excel, wichtige Punkte zu berücksichtigen:

- Das Zergliedern der bestehenden Beiträge in Kodiereinheiten wird deutlich gemacht durch das Einfügen eines Rautezeichens (\#) an der Schnittstelle (vgl. Abbildung 34). Dieses zeigt an, dass es sich um eine künstliche Unterteilung handelt. Das Rautenzeichen wird am Ende der Kodiereinheiten eingefügt, wobei zu beachten ist, dass zwischen dem letzen Wort bzw. Satzzeichen und dem Rautezeichen in der Kodiereinheit ein Leerzeichen einzusetzen ist.

- Erfolgt die Zergliederung aufgrund eines Sprecherwechsels, so wird ein Prozentzeichen (\%) anstatt eines Rautezeichens eingefügt. So können Sprecherwechsel leichter nachvollzogen und bei Bedarf separat betrachtet werden (vgl. Abbildung 34).

- Jede eigenständige Kodiereinheit bekommt eine separate Zeile. Falls mit Excel gearbeitet wird, müssen beispielsweise bei der Unterteilung eines Satzes je nach Anzahl der neu zu bildenden Kodiereinheiten weitere Zeilen in die Excel-Tabelle eingefügt werden.

- Bereits bestehende Interaktnummern werden erst nach abgeschlossener Einheitenbildung aktualisiert.

Ursprungsdialog:

Anna: Wollen wir uns heute Abend einen Film zusammen ansehen? Hast du Lust?

Nicole: Das würde ich sehr gerne machen. Was hältst du von einer Komödie... 
Daraus werden im Transkript folgende Kodiereinheiten:

\begin{tabular}{|l|l|}
\hline Anna: & Wollen wir uns heute Abend einen Film zusammen ansehen? \# \\
\hline & Hast du Lust? \% \\
\hline Nicole: & Das würde ich sehr gerne machen. \# \\
\hline & Was hältst du von einer Komödie... \\
\hline
\end{tabular}

Abbildung 34: Beispiel für die formale Gestaltung der Zergliederung von Redebeiträgen

\subsection{Voraussetzungen des Transkripts}

Nachfolgend wird kurz auf die Informationen eingegangen, die das Transkript zu Bildung der Kodiereinheiten beinhalten soll.

Die Daten sollten so transkribiert sein, dass aus dem Transkript mindestens hervorgeht,

- wer (Abkürzung für die Person)

- was (wörtlicher Inhalt)

- wann (Zeit in Sekunden oder Minuten)

- zu wem (Abkürzung der Person)

gesagt hat und

- wer (Abkürzung für die Person)

- wann (Zeit in Sekunden oder Minuten)

- wen (Abkürzung der Person)

unterbrochen hat.

Demnach muss das oben aufgeführte Beispiel vervollständigt werden (Abbildung 35):

\begin{tabular}{|l|l|l|l|l|}
\hline $\begin{array}{l}\text { Laufende } \\
\text { Nr. }\end{array}$ & Wer & $\begin{array}{l}\text { Zu } \\
\text { Wem }\end{array}$ & $\begin{array}{l}\text { Zeit in } \\
\text { min }\end{array}$ & Inhalt \\
\hline 1 & Anna & Nicole & 1 & $\begin{array}{l}\text { Wollen wir uns heute Abend einen Film zusammen ansehen? } \\
\#\end{array}$ \\
\hline 2 & & & & Hast du Lust? \% \\
\hline 3 & Nicole & Anna & 2 & Das würde ich sehr gerne machen. \# \\
\hline 4 & & & & Was hältst du von einer Komödie... \\
\hline
\end{tabular}

Abbildung 35: Beispiel für die Voraussetzungen des Transkripts 


\subsection{Grammatikalisches Vorwissen}

Die Instruktionen zur Bildung der Kodiereinheiten basieren hauptsächlich auf syntaktischen bzw. grammatikalischen Regeln. Dennoch muss der/die Kodierer/-in über kein spezielles grammatikalisches Vorwissen verfügen. Sämtliche in den Regeln auftauchende grammatikalische Begriffe werden in einer Fußnote präzise erläutert und können im Glossar (Anhang F) nachgeschlagen werden.

\section{6. Übersicht über die Trennregeln}

Zur Gewährung der Übersichtlichkeit werden die Trennregeln an dieser Stelle nur überblicksartig in Tabelle 22 dargestellt. Ihre vollständige Erläuterung ist in Form eines Manuals im Anhang F enthalten.

Das Ziel des vorliegenden Verfahrens besteht darin, dass ein Redebeitrag so in einzelne Kodiereinheiten eingeteilt werden kann, dass alle enthaltenen Inhalte im Sinne des jeweiligen Kodiersystems möglichst einzeln kodierbar sind. Es sollen kodierbare Einheiten gebildet werden, welche soviel wie nötig und so wenig wie möglich Information beinhalten, dass sie eindeutig gegebenen Kategorien zugeordnet werden können. Dieses Ziel soll mit Hilfe von drei Schritten erreicht werden:

1. Schritt: Trennung von Redebeiträgen verschiedener Personen (Regel 1)

2. Schritt: Trennung nach grammatikalischen Gesichtspunkten innerhalb individueller Redebeiträge (Regeln 2-6)

3. Schritt: Regeln für unterbrochene Äußerungen und grammatikalische Phänomene gesprochener Sprache (Regeln 6-10)

Diese drei Schritte müssen nicht nacheinander abgearbeitet werden. Ein paralleles Vorgehen ist möglich. Anhang F enthält die Erläuterung der einzelnen Trennregeln. Dort werden zur besseren Veranschaulichung der Regeln einige Beispiele aufgeführt und es wird begründet, wieso die Trennung in unterschiedliche Kodiereinheiten an dieser Stelle erfolgt. 
Tabelle 22: Übersicht über die Trennregeln des Verfabrens zur Einteilung von Kodiereinheiten

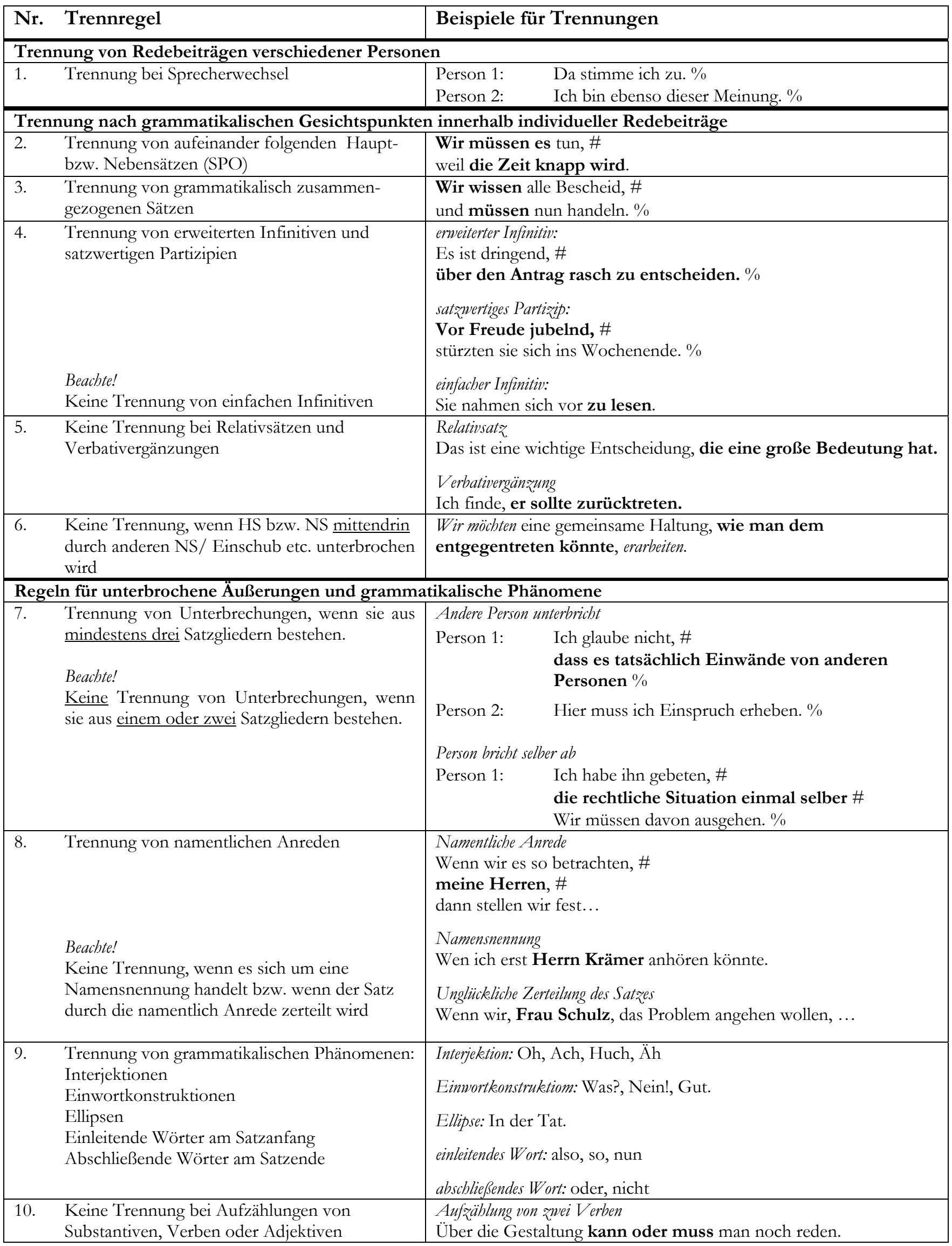


Dennoch ist es weiterhin möglich, dass gewisse Zweifelsfälle vorkommen, bei denen die Trennregeln nicht greifen werden. Wenn dies auftritt, müssen die Kodierer/-innen nach Absprache oder reflektiert selbst entscheiden, an welcher Stelle eine Trennung vorgenommen wird.

Darüber hinaus ist es wichtig, dass man bereits gegebene Einteilungen sowie die bestehende Zeichensetzung im Transkript kritisch betrachtet, da Trennungen, die möglicherweise implizit beim Transkribieren vorgenommen wurden, die Kodierer/-innen bei der Festlegung der eigentlichen Kodiereinheiten stark beeinflussen.

Zusätzlich ist es empfehlenswert, dass die Kodierer/-innen ihre Einteilung mindestens einmal überprüfen. Die Transkripte sollten mindestens zweimal durchgegangen werden, um sie nach den vorliegenden Trennregeln einzuteilen. Oft werden am Anfang einige Dinge übersehen oder Fehler begangen, die bei einer nochmaligen Überarbeitung verbessert werden können.

Darüber hinaus ist es empfehlenswert, nicht mehr als 45 Minuten am Stück Kodiereinheiten zu bilden, da ansonsten die Fehlerwahrscheinlichkeit aufgrund nachlassender Konzentration zunimmt.

\subsection{Gütekriterien}

Im Folgenden soll auf die Güte des vorgestellten Verfahrens zur Bestimmung von Kodiereinheiten eingegangen werden. Hauptsächlich wird auf die Reliabilität des Segmentierungsverfahrens eingegangen (Kapitel 7.7.1). Anschließend werden einige Angaben zur Validität (Kapitel 7.7.2) und zu den Nebengütekriterien (Kapitel 7.7.3) gemacht.

\subsubsection{Reliabilität}

Die Reliabilität des vorliegenden Verfahrens gibt an, wie zuverlässig die Kodiereinheiten gebildet wurden. Eine geringe Reliabilität würde auf die Notwendigkeit der Umformulierung der 
Trennregeln hinweisen. Eine hohe Reliabilität würde bedeuten, dass mehrere Kodierer/-innen während der Abgrenzung der Kodiereinheiten die Diskussionsbeiträge an denselben Stellen segmentieren.

In Anlehnung an Guetzkow (1950) gibt es bei der Bildung von Kodiereinheiten zwei wesentliche Fehlerquellen, welche die Reliabilität des vorliegenden Segmentierungsverfahrens gefährden können und deshalb möglichst klein gehalten werden sollten. So kann es vorkommen, dass zwei Kodierer/-innen zwar die gleiche Anzahl von Einheiten innerhalb eines Redebeitrags definiert haben, diese Einheiten aber inhaltlich voneinander abweichen. Zudem besteht die Gefahr, dass zwei Kodierer/-innen einen Redebeitrag nicht in die gleiche Anzahl von Kodiereinheiten zerlegen.

Um die Interrater-Reliabilität bezüglich der Einheiteneinteilung zu bestimmen, ist es notwendig, ein Maß zu finden, welches die Urteile der Kodierer/-innen miteinander vergleichen kann (Bortz et al., 2000). Cohens Kappa (k) (Bortz et al., 2000; Cohen, 1960) (Darstellung vgl. Kapitel 6.4.1) kann aus folgenden Gründen nicht für die Reliabilitäten-Bestimmung des vorliegenden Verfahrens verwendet werden: Zwar kann $\mathrm{p}_{0}$ berechnet werden, allerdings kann man kein $\mathrm{p}_{\mathrm{e}}$ bestimmen, da es keine konkordanten Urteile gibt, die sich rein zufällig ergeben könnten. Es liegt keine feste Anzahl an Einheiten vor, zu denen Kategorien einfach zugeordnet werden können. Mit Hilfe der Trennregeln müssen die Einheiten erst bestimmt werden, so dass vorher nicht sichergestellt werden kann, wie groß ihre Anzahl am Ende sein wird. Vielmehr entstehen durch die Einteilungen der Einheiten von beiden Kodierern/-innen zwei Zeichenketten, die jeweils aus einer Aneinanderreihung von einzelnen Kodiereinheiten bestehen. Diese müssen miteinander verglichen werden, um den Übereinstimmungsgrad festzustellen. Um ein Vergleichsmaß für die Urteile der Kodierer/-innen zu ermitteln, muss man die Mindestanzahl an Änderungen an den Zeichenketten bestimmen, die notwenig sind, um die Sequenzen der beiden Kodierer/-innen ineinander zu überführen. Man benötigt somit ein Distan₹maß, welches das oben beschriebene Problem lösen kann. Die Levenshtein-Metrik (Levenshtein, 1966) ist ein 
entsprechendes Maß. Sie kann die erhaltenen Zeichenketten miteinander in Beziehung setzen und vergleichen (Abbott \& Tsay, 2000).

Das Prinzip jedes Verfahrens auf Basis der Levenshtein-Metrik ist es, die Unterschiede zwischen Zeichenketten durch Angabe einer Menge von Operationen zu beschreiben, welche die Zeichenketten mit einer minimalen Anzahl an Operationen ineinander überführt (Sörensen, 2003). Die Operationen, die hierfür verwendet werden, sind das Ersetzen eines Zeichens durch ein anderes (Substitution), das Löschen (Deletion) sowie das Einfügen (Insertion) eines Zeichens (Abbott \& Tsay, 2000; Brüderl \& Scherer, 2004; Heeringa, 2004; Kessler, 1995; Nerbonne, Heeringa \& Kleiweg, 1999; Sörensen, 2003; Wagner \& Fischer, 1974). Diese Operationen unterscheiden sich hinsichtlich des Ausmaßes der durch sie veranlassten Änderung. So ist das Löschen eines Zeichens, gefolgt von einem Einfügen eines Zeichens an der gleichen Stelle in einer Zeichenkette, gleichbedeutend mit einer Austauschoperation eines Zeichens an dieser Stelle durch ein anderes. Aus diesem Grund werden Einfüge- und Löschoperationen gemeinsam als Indels bezeichnet. Das Indel steht hierbei für INsertion und DELetion (Levenshtein, 1966). Wenn die Sequenzen identisch sind und man keine Operationen anwenden muss, was für die Reliabilität äußerst positiv zu bewerten wäre, so spricht man von einem Match. Zusammengefasst bezeichnet die Levenshtein-Distanz ein Maß für den Unterschied zwischen zwei Zeichenketten bezüglich der minimalen Anzahl der Operationen Einfügen, Löschen und Ersetzen, die nötig sind, um die eine Zeichenkette in die andere zu überführen

Die verschiedenen Zeichenketten, die entstehen, wenn Kodierer/-innen eine Diskussion anhand des vorliegenden Verfahrens einteilen, können durch Indels aneinander angeglichen werden. Für die Bestimmung der Interrater-Reliabilität des Segmentierungsverfahrens ist es notwendig, die Anzahl der Indels zu bestimmen, die notwendig wären, um die beiden Kodiereinheiten-Sequenzen ineinander zu überführen. Damit erhält man ein Maß für die Levenshtein-Distanz bzw. die Unähnlichkeit zweier Sequenzen. Allerdings liegen die Zeichenketten der beiden Kodierer/-innen nur selten in derselben Sequenzlänge vor, so dass 
dadurch sehr leicht Verzerrungen der Ergebnisse auftreten können (Conroy, 2001). Conroy (2001) hat deshalb vorgeschlagen, eine Normalisierung der Werte vorzunehmen. Dies erreicht man, indem die Levenshtein-Distanz durch die durchschnittliche Länge der beiden vorliegenden Sequenzen teilt. Erst ein auf diese Weise normalisierter Wert ermöglicht es, die entstandenen Werte und somit die Interrater-Reliabilitäten miteinander zu vergleichen. Der ermittelte Wert für die normalisierte Levenshtein-Distan₹ (Levenshtein-Distanz/ durchschnittliche Sequenzlänge der gebildeten Einheiten) gibt dann an, inwiefern sich die Kodierer/-innen hinsichtlich der Einheiteneinteilung unterscheiden.

Die eben geschilderte Problematik soll nun zum besseren Verständnis an einem einfachen Beispiel erläutert werden. Es liegen in Abbildung 36 und Abbildung 37 von zwei Kodierern/innen durchgeführte Segmentierungen der Redebeiträge in Kodiereinheiten vor (Vergleich Kapitel 7.3 zur formalen Gestaltung der Zergliederung einzelner Redebeiträge).

\begin{tabular}{|ll|}
\hline Isabelle: & Anna, wir haben uns lange nicht mehr gesehen. \# \\
& Wie geht es dir? \% \\
Anna: & Mir geht es gut, Isabelle. \# \\
& Ich bin gerade auf der Suche nach einem Geschenk. \% \\
\hline
\end{tabular}

Abbildung 36: Beispieltrennung von Kodierer/-in 1. Das Zeichen \# steht für die Trennung des Redebeitrages durch den Kodierer. Das \%-Zeichen verweist auf einen Sprecherwechsel

\begin{tabular}{|ll|}
\hline Isabelle: & Anna, \# \\
& wir haben uns lange nicht mehr gesehen. \# \\
& Wie geht es dir? \% \\
Anna: & Mir geht es gut, \# \\
& Isabelle. \# \\
& Ich bin gerade auf der Suche nach einem Geschenk. \% \\
\hline
\end{tabular}

Abbildung 37: Beispieltrennung von Kodierer/-in 2. Das Zeichen \# steht für die Trennung des Redebeitrages durch den Kodierer. Das \%-Zeichen verweist auf einen Sprecherwechsel

Wie man erkennen kann, haben beide Kodierer/-innen teilweise an den gleichen und teilweise an unterschiedlichen Stellen eine Trennung der Redebeiträge vorgenommen. Das Ziel ist jetzt, diese beiden Trennungssequenzen einander anzupassen. Kodierer/-in 2 hat an zwei Stellen 
eine Trennung vorgenommen, an denen Kodierer/-in 1 diese nicht vornahm. Um beide Sequenzen einander anzugleichen, ist es also nötig, entweder die beiden entsprechenden Trennungen von Kodierer/-in 2 rückgängig zu machen oder zwei zusätzliche Trennungen bei Kodierer/-in 1 vorzunehmen. Man wendet somit entweder eine Einfüge- oder eine Löschoperation an. Es macht allerdings hinsichtlich der Levenshtein-Distanz keinen Unterschied, welches Indel man verwendet. Der Einfachheit halber werden ausschließlich Einfügeoperationen benutzt, deshalb werden bei Kodierer/-in 1 an den entsprechenden Stellen zwei zusätzliche Trennungen durchgeführt. Die beiden Sequenzen werden dadurch einander angeglichen und sind nun vollkommen identisch (siehe Abbildung 38). Es wurden in diesem Beispiel somit insgesamt zwei Einfügungen vorgenommen (2 Indels gesetzt), so dass die Levenshtein-Distanz $D=2$ beträgt.

\begin{tabular}{|c|c|}
\hline Kodierer/-in 1 & Kodierer/-in 2 \\
\hline $\begin{array}{l}\text { Anna, \# } \\
\text { wir haben uns lange nicht mehr } \\
\text { gesehen. \# } \\
\text { Wie geht es dir? \% } \\
\text { Mir geht es gut, \# } \\
\text { Isabelle. \# } \\
\text { Ich bin gerade auf der Suche nach } \\
\text { einem Geschenk. \% }\end{array}$ & $\begin{array}{l}\text { Anna, \# } \\
\text { wir haben uns lange nicht mehr } \\
\text { gesehen. \# } \\
\text { Wie geht es dir? \% } \\
\text { Mir geht es gut, \# } \\
\text { Isabelle. \# } \\
\text { Ich bin gerade auf der Suche nach } \\
\text { einem Geschenk. \% }\end{array}$ \\
\hline
\end{tabular}

Abbildung 38: Einander angepasste Sequenzen der beiden Kodierer. Die eingefïgten Trennungen werden durch fettgedruckte Rautezeichen gekenn reichnet.

Dieses Verfahren wird nun auf die gesamte Diskussion, die von den Kodierern/-innen eingeteilt wurde, angewendet. Zur Bestimmung der Reliabilität ist es anschließend nötig, die Levenshtein-Distanz und die Anzahl der Sprecherwechsel (=Redebeiträge-1) auszuzählen bzw. zu erfassen. Vorher sollte ebenso vermerkt werden, in wie viele Einheiten die beiden Kodierer /innen die Diskussion eingeteilt haben, da diese Zahlen für die spätere Normalisierung nach Conroy (2001) benötigt werden. 
Wie oben beschrieben, kann die errechnete Levenshtein-Distanz nicht als alleiniges Übereinstimmungsmaß verwendet werden, da sie als absolute Größe nichts darüber aussagt, wie gut oder schlecht die Übereinstimmung zweier Kodierer/-innen ist. Es ist vielmehr notwendig, die beiden Sequenzen in Bezug zueinander zu setzen, um erkennen zu können, wie hoch bzw. wie niedrig die Reliabilität ist. Hierfür muss die Levenshtein-Distanz normalisiert werden (Conroy, 2001), d. h. sie muss durch die durchschnittliche Sequenzlänge aller Kodierer/-innen geteilt werden. Durch diese Normalisierung der Levenshtein-Distanz wird die Länge der Zeichenketten aller Kodierer/-innen in Betracht gezogen (Conroy, 2001). Von der durchschnittlichen Sequenzlänge muss aber noch die Anzahl der Sprecherwechsel abgezogen werden. Dies ist notwendig, da die Sprecherwechselregel (Regel 1) sehr eindeutig ist und theoretisch von jedem/r Kodierer/-in sehr leicht korrekt angewendet werden müsste. Hinzu kommt, dass der Sprecherwechsel in Transkripten oftmals schon implizit enthalten ist. Wenn man diese Trennregel in die Reliabilitätsberechnung einbeziehe, käme es zu einer künstlichen Verbesserung des Reliabilitätswertes. Um dies zu verhindern, wird die Anzahl der Sprecherwechsel im gesamten Transkript von der durchschnittlichen Sequenzlänge abgezogen. Die Formel für die normalisierte Levenshtein-Distanz $n D$ sieht dann wie folgt aus:

$$
\mathrm{nD}=\frac{\text { Levenshtein }- \text { Distanz }}{\text { durchschnittliche Anzahl aller Trennungen im Transkript }- \text { Anzahl aller Sprecherwechsel }} .
$$

Im Endeffekt werden also alle Trennungen, die im Vergleich zweier Kodierer/-innen nicht übereinstimmen, ausgezählt und durch die durchschnittliche Gesamtanzahl aller Trennungen, abzüglich der Anzahl aller Sprecherwechsel, geteilt. Der Quotient, der sich aus diesem Bruch ergibt, schwankt zwischen 0 und 1. Er würde einen Wert genau zwischen 0 und 1.0 annehmen, wenn man die Levenshtein-Distanz lediglich durch die Anzahl aller Trennungen im Transkript teilen würde, die sich nach der vollständigen Angleichung der Zeichenketten ergibt. Da dieses Vorgehen allerdings verworfen und die Anzahl der Trennungen aufgrund des 
Sprecherwechsels von der Anzahl der Gesamttrennungen abgezogen werden sollen, kann sich für $n D$ theoretisch auch ein negativer Wert ergeben, z.B. dann, wenn die Kodierer/-innen einen Fehler bei der Sprecherwechselregel begehen würden (was jedoch nicht anzunehmen ist). Je näher $n D$ die Zahl 0 anstrebt, desto besser wäre die Reliabilität eines Segmentierungsverfahrens einzuschätzen. Eine solche geringe Levenshtein-Distanz würde bedeuten, dass die Kodierer/innen in ihren Segmentierungsurteilen übereinstimmen. Ein Wert von 1.0 würde bedeuten würde, dass die Kodierer/-innen in keinem einzigen Fall der Einheiteneinteilung übereingekommen sind.

Für die Levenshtein-Distanz bzw. für den normalisierten Wert nach Conroy (2001) gibt es keine Orientierungshilfen, die Aussagen darüber treffen, ob die Übereinstimmung zweier Werte (im vorliegendem Fall die Übereinstimmung der Kodierer/-innen) als sehr gut, gut, ausreichend oder ungenügend anzusehen ist. Das Problem besteht auch für ähnliche Maße, die nach einem vergleichbaren Prinzip verfahren und eine Aussage über die Ähnlichkeiten zweier Werte treffen (Bortz, 1999; Bortz \& Döring, 2002; Bortz et al., 2000; Bos \& Tarnai, 1989; Conroy, 2001; Früh, 2004; Holsti, 1969; Krippendorf, 1980; Levenshtein, 1966; Mayring, 2003; Merten, 1995; Sankoff \& Kruskal, 1983; Simon, 2000; Sörensen, 2003; Wagner \& Fischer, 1974; Wirth \& Lauf, 2001; Wirtz \& Caspar, 2002). Es werden lediglich Aussagen darüber getätigt, welcher Vergleichswert besser oder schlechter ist (je nachdem, welche Zahl näher an der 1 bzw. 0 liegt). Es wird aber nicht gesagt, ob der ermittelte Wert allein betrachtet ein gutes oder schlechtes Ergebnis darstellt (Vgl. Richtlinien für Effektstärken nach Cohen in Bortz \& Döring, 2002).

Früh (2004) vertritt die Ansicht, dass der absolute Ausprägungsgrad eines Reliabilitätskoeffizienten nichts über die Qualität der Kodierung aussagt. Die erreichbare Übereinstimmung ist von verschiedenen Faktoren abhängig, auf die bereits in Kapitel 6.4.1 eingegangen wurde.

Die in Kapitel 6.4.1 beschriebenen Angaben zur Interpretation des Kappa-Wertes zur Grundlage nehmend, wird für das vorliegende Verfahren empfohlen, dass die normalisierte Levenshtein-Distanz einen Wert von $n D<0.10$ nicht übersteigen sollte. Dieser vergleichsweise 
strenge Wert scheint angemessen, da die normalisierte Levenshtein-Distanz, im Vergleich zu Cohens Kappa, kein zufallskorrigiertes Maß ist. Zur Bestimmung der Interrater-Reliabilität des vorgestellten Verfahrens haben zwei Kodierer/-innen ein Training in der Anwendung der vorliegenden Methode erhalten und im Anschluss einen sechzigminütigen Ausschnitt einer Diskussion aus der Studie von Boos (1996) in Kodiereinheiten unterteilt. Die ermittelte normalisierte Levenshtein-Distanz betrug hierbei einen Wert von $n D=0.19$ und kann somit als gut oder zumindest hinreichend angesehen werden, da der vorgeschlagene angestrebte Wert von $n D=0.10$ lediglich um 0.09 Punkte überschritten wurde. Die Reliabilität des Manuals ist somit gegeben, aber noch verbesserungswürdig. Es ist anzunehmen, dass bei weiteren Kodierschulungen und einem ausreichenden Training $n D$ minimiert werden kann, da von den Kodierern/-innen vorrangig noch viele Flüchtigkeitsfehler begangen wurden und notwendige Trennungen trotz eindeutiger Regeln übersehen wurden (z.B. Regel 2: Trennung von Haupt- und Nebensatz und Regel 5: Trennung von erweiterten Infinitivsätzen).

Im Folgenden wird auf die Validität des vorliegenden Verfahrens eingegangen.

\subsubsection{Validität}

Unter Validität wird die Gültigkeit eines Verfahrens verstanden (Bortz et al., 2000). Für die vorliegende Methode muss überprüft werden, inwieweit die vorgeschlagenen Regeln eine sinnvolle Verfahrensweise zur Bildung von Kodiereinheiten darstellen.

Während der Konzeption der Methode wurde ihre Validität einer Prozessevaluation unterzogen: Die Trennregeln und Kodiereinheiten wurden regelmäßig anhand von Probekodierungen korrigiert und präzisiert. Darüber hinaus wurden die anhand der vorliegenden Methode gebildeten Einheiten einer Kodierung anhand des in Kapitel 6 beschriebenen Kategoriensystems unterzogen. Dadurch konnte überprüft werden, inwieweit die erhaltenen Einheiten sinnvolle Kodiereinheiten darstellen. 
Zur empirischen Überprüfung der Validität wurde nach Früh (2004) dasselbe Verfahren angewendet, welches bereits in Kapitel 6.4 .2 zur Validitätsprüfung des Kategoriensystems beschrieben wurde. Dabei wird die Validität als Reliabilität zwischen Verfahrensentwicklern/innen und Anwendern/-innen operationalisiert. Ein zehnminütiger Ausschnitt einer Diskussion aus der Studie von Boos (1996) wurde separat von einem Kodierer und einer Verfahrensentwicklerin in Kodiereinheiten unterteilt. Die ermittelte normalisierte LevenshteinDistanz betrug $n D=0.23$ und kann als zumindest hinreichend angesehen werden. Der vorgeschlagene angestrebte Wert von $n D=0.10$ wurde um 0.13 Punkte überschritten. Es zeigte sich, dass es auf Seiten des Kodierers noch einige Flüchtigkeitsfehler gab, die es zu minimieren gilt. Es ist anzunehmen, dass bei zusätzlichen Kodierschulungen und einem ausreichenden Training $n D$ verringert und somit die auch Validität des vorliegenden Verfahrens verbessert werden kann.

In Bezug auf die Validität des vorliegenden Verfahrens scheint Folgendes wesentlich: Das Verfahren muss zukünftig wiederholt zur Aufbereitung unterscbiedlicher transkribierter Gespräche (moderierte und nicht moderierte Gruppendiskussionen, Gespräche in Dyaden, etc.) angewendet werden. Die resultierenden Kodiereinheiten sollten für den Einsatz verschiedener Kodiersysteme verwendet werden. Erst dann kann gezeigt werden, inwieweit das vorliegende Verfahren tatsächlich eine sinnvolle Methode zur Bildung von Kodiereinheiten darstellt.

Abschließend wird auf die Nebengütekriterien eingegangen.

\subsubsection{Nebengütekriterien}

Das Ziel der vorliegenden Methode besteht in der transparenten Bildung von Kodiereinheiten. Aufgrund der zehn Trennregeln kann davon ausgegangen werden, dass die Bildung von Kodiereinheiten nun nicht mehr der Intuition des/der Forscher/-in überlassen wird, sondern sowohl intersubjektiv nachvolliziehbar als auch replizierbar erfolgen kann. 
Bezüglich der Praktikabilität muss überlegt werden, inwieweit die Anzahl der Regeln zukünftig reduziert werden kann, damit der Umgang mit der Methode vereinfacht wird.

Im Hinblick auf die Nützlichkeit des Segmentierungsverfahrens kann davon ausgegangen werden, dass es nicht nur in der sozialpsychologischen Gruppenforschung, sondern auch für Fragestellungen anderer Gebiete interessant ist. Beispielsweise könnte das Verfahren in der Therapieforschung zur Analyse von Gesprächen zwischen Patient/-in und Therapeut/-in Anwendung finden. Denkbar ist auch sein Einsatz in der Entwicklungs-, Kommunikations- und Medienpsychologie.

Im folgenden Kapitel wird überlegt, wie die eben geschilderten Methoden zur vergleichenden Untersuchung von Koordination in Entscheidungsfindungsgruppen angewendet werden können. 


\section{Ausblick}

In Studie 3 konnte gezeigt werden, dass die expliziten Koordinationsmechanismen Erfragen und Wiederholen von Informationen mit unterschiedlichen Konsequenzen für die Gruppenentscheidung verbunden waren. Das Wiederholen führte sowohl zu einem verbesserten Informationsaustausch, als auch zu einer besseren Gruppenentscheidung. Das Erfragen war hingegen erfolglos. Es wurde diskutiert, dass zur Analyse möglicher Ursachen eine Betrachtung der Koordinationsmechanismen im Verlauf der Entscheidungsfindung notwendig ist. Darauf soll im Folgenden eingegangen werden.

In der sozialpsychologischen Gruppenforschung gibt es seit einiger Zeit eine methodologische Diskussion, in der die Forderung deutlich wurde, die während des Gruppenprozesses gewonnenen Daten nicht lediglich zu aggregieren, sondern ihren Verlauf zu untersuchen (McGrath et al., 2000). Diese Forderung wird bereits seit einigen Jahren sehr erfolgreich umgesetzt (Becker-Beck, 1994, 1997; Becker-Beck, Beck \& Eberhardt, 1998; Boos et al., 1990; Nägele, 2004; Tschan, 2000). Der Grundgedanke ist, dass sich interaktive Verlaufsmuster als Prozesseigenschaften von Gruppen zeigen lassen. Diese Prozesseigenschaften können sich auf der Mikroebene in einer charakteristischen Häufung oder Abfolge von Interaktionen manifestieren (Boos, 1994). Durch die Analyse der sequentiellen Struktur, beispielsweise durch die Lag Sequential Analysis (Bakeman, 2000; Bakeman \& Gottman, 1986; Bakeman \& Quera, 1995; Nägele, 2004), können Sequenzähnlichkeiten innerhalb und zwischen Gruppen definiert werden. Beispielsweise konnten Larson et al. (1994) zeigen, dass sich durch die Intervention eines Gruppenentscheidungstrainings (Training zur strategischen Planung und Wachsamkeit bezüglich Informationssuche und -verwertung) sowohl der Austausch geteilter als auch ungeteilter Information erhöhte und sich der sequentielle Ablauf veränderte, in dem die geteilten und ungeteilten Informationen in die Diskussion gebracht wurden: Untrainierte Gruppen sprachen zunächst über geteilte Informationen und gingen dann zur Diskussionen 
ungeteilter Informationen über. Trainierte Gruppen hingegen zeigten diese sequentielle Veränderung nicht (Larson et al., 1994).

Im Hinblick auf die vorliegende Arbeit ist in Studie 3 die Frage offen geblieben, warum der Koordinationsmechanismus Informationsfrage weder den Informationsaustausch noch die Entscheidungsfindung verbessert hat. Zur Beantwortung dieser Frage soll der Einsatz der Koordinationsmechanismen im Verlauf der Diskussion untersucht werden. Ziel ist es, die erfolgreichen und erfolglosen Entscheidungsgruppen unter allen 4 Bedingungen des Experiments systematisch bezüglich ihrer Koordinationsprozesse zu vergleichen. Dazu wurde aufgrund der Studien 1 bis 3 sowie in Anlehnung an Boos (2003) ein formales Arbeitsmodell der Koordination postuliert (vgl. Abbildung 31, Seite 184). In diesem Modell wird davon ausgegangen, dass dem Einsatz eines Koordinationsmechanismus ein bestimmter Koordinationsanlass vorausgeht. Auf den Koordinationsmechanismus folgt wiederum eine Konsequenz, die sich in der Reaktion der Gruppenmitglieder auf den Mechanismeneinsatz manifestiert. Im Hinblick auf die Ergebnisse der Studie 3 kann nun zwischen sieben Bedingungen unterschieden werden (vgl. Tabelle 23): In den ursprünglich drei Experimentalbedingungen und der einen Kontrollbedingung haben die Gruppen entweder die richtige oder die falsche Entscheidung getroffen. Eine Ausnahme stellt die Bedingung dar, in der nur nach Informationen gefragt wurde, hier wurde in keinem Fall die richtige Entscheidung getroffen.

Bezüglich der Befunde aus Studie 3 können folgende Hypothesen aufgestellt werden:

1. Angesichts des Befundes, dass Gruppenmitglieder oder -leiter/-innen zur Koordination aufgefordert werden müssen (Larson et al., 1998a), wird erwartet, dass sich in den Entscheidungsdiskussionen der Gruppen, in denen Informationen erfragt und/oder wiederholt wurden, insgesamt mehr Koordinationsmechanismen finden lassen als in den Kontrollbedingungen (Häufigkeit des Einsatzes von Koordinationsmechanismen).

2. Angesichts des Ergebnisses, dass zwei Gruppen der Kontrollbedingung die richtige Entscheidung trafen, wird erwartet, dass sich in diesen Gruppen insgesamt mehr Koordinationsmechanismen finden lassen als in den Kontrollbedingungen, in denen nicht die richtige Entscheidung getroffen wurde (Häufigkeit des Einsatzes von Koordinationsmechanismen). 
3. Im Hinblick auf das formale Arbeitsmodell der Koordination wird erwartet, dass sich die Gruppen, welche die richtige Entscheidung trafen, unabhängig von der Bedingung, in ihren Koordinationsmustern von den Gruppen unterscheiden, welche nicht die richtige Entscheidung trafen (Koordinationsmuster zwischen den Gruppen).

Das Design zur Untersuchung dieser Hypothesen wird in Tabelle 23 dargestellt. Es wird empfohlen, aus den nun möglichen sieben Bedingungen randomisiert jeweils 5 Gruppen für die vergleichende Prozessanalyse auszuwählen (soweit möglich). In den transkribierten Gruppendiskussionen werden zunächst nach dem in Kapitel 7 vorgestellten Verfahren Kodiereinheiten gebildet. Diese werden anschließend anhand des in Kapitel 6 erläuterten Kategoriensystems kodiert. Die entstandenen Kodierungen des Prozesses können zunächst pro Gruppe mittels der Lag Sequential Analysis (Bakeman, 2000; Bakeman \& Gottman, 1986; Bakeman \& Quera, 1995) nach signifikanten sequentiellen Mustern untersucht werden. Anschließend kann geprüft werden, ob sich die innerbalb einer Gruppe gefundenen Koordinationsmuster auch zur Diskriminierung zwischen den Gruppen eignen.

Tabelle 23: Design zur vergleichenden Analyse der Koordinationsprozesse der Entscheidungsfindungsgruppen aus Studie 3

\begin{tabular}{|c|c|c|c|c|}
\hline & $\begin{array}{l}\text { Information } \\
\text { erfragen }\end{array}$ & $\begin{array}{l}\text { Information } \\
\text { wiederholen }\end{array}$ & $\begin{array}{l}\text { Information } \\
\text { erfragen und } \\
\text { wiederholen }\end{array}$ & $\begin{array}{l}\text { keine Koordinations- } \\
\text { mechanismen }\end{array}$ \\
\hline $\begin{array}{l}\text { Richtige Gruppen- } \\
\text { entscheidung } \\
\text { („Löser-Gruppen“) }\end{array}$ & $0(0)$ & $\begin{array}{c}n=5(5) \\
\text { Bedingung } 2\end{array}$ & $\begin{array}{c}\mathrm{n}=5(5) \\
\text { Bedingung } 4\end{array}$ & $\begin{array}{c}\mathrm{n}=2(2) \\
\text { Bedingung } 6\end{array}$ \\
\hline $\begin{array}{l}\text { Falsche Gruppen- } \\
\text { entscheidung } \\
\text { („Nicht-Löser- } \\
\text { Gruppen“) }\end{array}$ & $\begin{array}{c}\mathrm{n}=5(12) \\
\text { Bedingung } 1\end{array}$ & $\begin{array}{c}\mathrm{n}=5(7) \\
\text { Bedingung } 3\end{array}$ & $\begin{array}{c}\mathrm{n}=5(7) \\
\text { Bedingung } 5\end{array}$ & $\begin{array}{c}\mathrm{n}=5(10) \\
\text { Bedingung } 7\end{array}$ \\
\hline
\end{tabular}

Anmerkung: In den Klammern stehen die ursprünglichen Fallzahlen aus Studie 3.

Mit diesem Vorgehen kann festgestellt werden, welche konkreten Interaktionen dem Einsatz der jeweiligen Mechanismen vorausgegangen sind und mit welchen spezifischen Konsequenzen der Einsatz verbunden war. Diese Ergebnisse können Auskunft über die situative Effektivität und Effizienz der Koordinationsmechanismen geben. 
Neben der eben skizzierten vergleichenden Prozessanalyse gibt die vorliegende Arbeit Anlass zu weiteren Forschungsfragen:

1. Wesentlich für das ganzheitliche Verständnis von Gruppenkoordinationsprozessen ist das Wechselspiel zwischen impliziten und expliziten Koordinationsmechanismen. Wann wechseln Gruppenmitglieder und -leiter/-innen von impliziter zu expliziter Koordination? Wie effektiv sind diese Wechsel?

2. Welche Rolle spielt die Koordination während der sequentiellen Handlungsregulation von Entscheidungsgruppen? Werden die Wechsel zwischen den Handlungsphasen explizit oder implizit koordiniert?

3. Welche Rolle spielt die langfristige Gruppenentwicklung (Gersick, 1988; Wheelan, Davidson \& Tilin, 2003) für den Koordinationsbedarf von Entscheidungsgruppen? Wann bedürfen die Gruppen verstärkt expliziter Prozesskoordination (vgl. Hackman \& Wageman, 2005)? Wie funktioniert in diesem Zusammenhang die Koordination anhand des Transaktiven Gedächtnissystems bei Gruppenentscheidungsprozessen?

4. Um Aussagen über die situative Effektivität von Koordinationsmechanismen treffen zu können, müssen die Kontextfaktoren der Gruppenentscheidung stärker in die Forschung einbezogen werden. Bedeutsam sind solche Untersuchungen insbesondere für die Gruppenentscheidungen in risikoreichen Arbeitsumgebungen, z.B. im OP. Im Rahmen von Simulationsstudien können die Wirkungen von Zeitdruck, Struktur der Gruppe (z.B. Team vs. Crew) und anderen Faktoren systematisch im Hinblick auf ihren Koordinationsbedarf untersucht werden. Das langfristige Ziel derartiger Studien sollte in der Entwicklung einer Kontingenztheorie der Koordination bestehen, welche die Vorhersage des Erfolgs bestimmter Koordinationsmechanismen anhand situativer Gegebenheiten ermöglicht.

5. Weiterhin ist unklar, inwieweit kulturelle Unterschiede den Einsatz expliziter und impliziter Koordinationsmechanismen mitbestimmen. 
6. Im Hinblick auf computergestützte Entscheidungsfindung sollte untersucht werden, inwieweit sich der Koordinationsbedarf je nach Kommunikationsmedium ändert (vgl. Hron, Hesse, Cress \& Giovis, 2000; McGrath \& Hollingshead, 1994).

7. Die Entwicklung einer Theorie der Gruppenkoordination sollte fortgesetzt werden. Diese sollte nicht nur auf Entscheidungsfindungsprozesse anwendbar sein, sondern sich universal auf mehrere Gruppenaufgabentypen beziehen. Ausgehend von den Befunden der vorliegenden Arbeit sollten sowohl die Perspektive der Koordinierenden als auch die der Koordinierten in die Theorie integriert werden.

Schließlich sollte bei aller Forschung zur Koordination nicht vergessen werden, dass Gruppenentscheidungsprozesse auch von der Motivation der Gruppenmitglieder bestimmt werden. Die Möglichkeiten, durch den Einsatz von Koordinationsmechanismen die Gruppenentscheidung zu verbessern, sind begrenzt. Schließlich spielen auch die individuellen Motive der Gruppenmitglieder, bestimmte Informationen zu nennen oder nicht, eine wesentliche Rolle (Steiner, 1972; Wittenbaum et al., 2004a). Trotz expliziter Prozesskoordination könnten Informationen demnach aufgrund fehlender Motivation nicht genannt oder aufgrund spezifischer Motivation zwar genannt, aber verzerrt dargestellt werden, so dass ihre Wirkung präferenzkonsistent verzerrt wird (Schauenburg, 2004).

"When a group appears to be making a decision, members may have other goals or interests in mind, and when they are engaged in other activities, members may be tacitly working on the decision" (Poole \& Hirokawa, 1996, S. 11).

Weitere Arbeiten könnten Aufschluss über das Wechselspiel von motivationalen und koordinierenden Prozessen während der Entscheidungsfindung von Gruppen geben. 


\section{Literaturverzeichnis}

Abbott, A., \& Tsay, A. (2000). Sequence analysis and optimal matching methods in sociology. Sociological Methods and Research, 29, 3-33.

Aron, A., Aron, E. N., \& Coups, E. J. (2006). Statistics for psychology (4th ed.). Upper Saddle River, NJ: Pearson Education.

Arrow, H., McGrath, J. E., \& Berdahl, J. L. (2000). Small groups as complex systems: Formation, coordination, development, and adaption. Thousand Oaks: Sage.

Arrow, H., Poole, M. S., Henry, K. B., Wheelan, S. A., \& Moreland, R. (2004). Time, change, and development. A temporal perspective on groups. Small Group Research, 35, 73-105.

Bachmair, S., Faber, J., Hennig, C., Kolb, R., \& Willig, W. (1999). Beraten will gelernt sein. Weinheim: Beltz.

Bakeman, R. (2000). Behavioral observation and coding. In H. T. Reis \& C. M. Judd (Eds.), Handbook of research methods in social and personality psychology. New York, NY: Cambridge University Press.

Bakeman, R., \& Gottman, J. M. (1986). Observing interaction: An introduction to sequential analysis. Cambridge: Cambridge University Press.

Bakeman, R., \& Quera, V. (1995). Analyzing interaction: Sequential analysis with SDIS \& GSEQ. Cambridge: Camdridge University Press.

Bartholomew, K., Henderson, A. J. Z., \& Marcia, J. E. (2000). Coded semistructured interviews on social psychology research. In H. T. Reis \& C. M. Judd (Eds.), Handbook of research in social and personality psychology (pp. 286-312). New York, NY: Cambridge University Press.

Bechky, B. A. (2003). Coordination as the practice of organizational accountability and common ground. Academy of Management Proceedings, 2003, 11-16.

Beck, D., \& Fisch, R. (2000). Argumentation and emotional processes in group decision-making: Illustration of a multi-level interaction process analysis approach. Group Processes and Intergroup Relations, 3, 183-201.

Becker, L. (2006). Der Einfluss ausgewählter expliziter Koordinationsmechanismen während der Diskussion auf die Entscheidungsfindung in Gruppen. Unpublished Diploma Thesis, University of Goettingen, Goettingen.

Becker-Beck, U. (1994). Strukturanalyse des Interaktionsverhaltens in Diskussionsgruppen. Gruppendynamik, 25, 95-106.

Becker-Beck, U. (1997). Soziale Interaktion in Gruppen: Struktur- und Prozessanalyse. Opladen: Westdeutscher Verlag.

Becker-Beck, U., Beck, D., \& Eberhardt, D. (1998). Muster und Strategien in der sozialen Interaktion zwischen Gruppen - Illustrative Interaktionsanalysen des Verlaufs zweier Erörterungstermine. In E. Ardelt-Gattinger, H. Lechner \& W. Schlögl (Eds.), 
Gruppendynamik. Anspruch und Wirklichkeit der Arbeit in Gruppen (pp. 96-112). Göttingen: Verlag für Angewandte Psychologie.

Becker-Beck, U., Wintermantel, M., \& Borg, A. (2005). Principles of regulating interaction in teams practicing face-to-face communication versus teams practicing computer-mediated communication. Small Group Research, 36, 499-536.

Berteotti, C. R., \& Seibold, D. R. (1994). Coordination and role-definition problems in healthcare team: A hospice case study. In L. R. Frey (Ed.), Group communication in context: Studies of natural groups (pp. 107-131). Hillsdale, NJ: Lawrence Erlbaum Associates.

Biermann-Ratjen, E.-M., Eckert, J., \& Schwartz, H.-J. (1995). Gesprächspsychotherapie. Stuttgart: Kohlhammer.

Bilandzic, H., Koschel, F., \& Scheufele, B. (2001). Theoretisch-heuristische Segmentierung im Prozeß der empiriegeleiteten Kategorienbildung. In W. Wirth \& E. Lauf (Eds.), Inhaltsanalyse (pp. 98-116). Köln: Herbert von Halem Verlag.

Bogner, A., \& Menz, W. (2005). Expertenwissen und Forschungspraxis: Die modernisierungstheoretische und die methodische Debatte um die Experten. In A. Bogner, B. Littig \& W. Menz (Eds.), Das Experteninterview. Theorie, Methode, Anwendung (2nd ed., pp. 7-30). Wiesbaden: VS Verlag für Sozialwissenschaften.

Boos, M. (1994). Entscheidungen in der öffentlichen Verwaltung. Die aufgabenorientierte Dynamik bei drei Modellen der Führung und Zusammenarbeit. Gruppendynamik, 25, 185202.

Boos, M. (1996a). Die Effektivität der Moderation von Projektgruppen. Gruppendynamik, 27(4), 353-369.

Boos, M. (1996b). Entscheidungsfindung in Gruppen: Eine Prozessanalyse. Bern: Huber.

Boos, M. (1997). Gruppenprozesse. In J. Straub, W. Kempf \& H. Werbik (Eds.), Psychologie. Eine Einfübrung. Grundlagen, Methoden, Perspektiven. München: Deutscher Taschenbuch Verlag.

Boos, M. (2003). Prozessteuerung in Gruppendiskussionen. Unpublished manuscript, Universität Göttingen.

Boos, M., Kolbe, M., \& Strack, M. (2006). Gruppenkoordination - Modellierung der Mechanismen. Vortrag auf dem 45. Kongress der Deutschen Gesellschaft für Psychologie, Nürnberg, Germany.

Boos, M., Morguet, M., Meier, F., \& Fisch, R. (1990). Zeitreihenanalysen von Interaktionsprozessen bei der Bearbeitung komplexer Probleme in Expertengruppen. Zeitschrift für Sozialpsychologie, 21, 53-64.

Boos, M., \& Sassenberg, K. (2001). Koordination in verteilten Arbeitsgruppen. In E. H. Witte (Ed.), Leistungsverbesserungen in aufgabenorientierten Kleingruppen: Beiträge des 15. Hamburger Symposiums zur Methodologie der Sozialpsychologie (pp. 198-216.). Lengerich: Papst.

Boos, M., Scharpf, U., \& Fisch, R. (1991). Eine Methode zur Analyse von Interaktionsprozessen beim Problemlösen und Entscheiden in Sitzungen. Zeitscbrift für Arbeits- und Organisationspsychologie, 35, 115-121. 
Bornstein, G., Gneezy, U., \& Nagel, R. (2002). The effect of intergroup competition on group coordination: An experimental study. Games and Economic Behavior, 41, 1-25.

Bortz, J. (1999). Statistik für Sozialwissenschaftler. Berlin: Springer.

Bortz, J., \& Döring, N. (2002). Forschungsmethoden und Evaluation. Berlin: Springer.

Bortz, J., Lienert, G. A., \& Boehnke, K. (2000). Verteilungsfreie Methoden in der Biostatistik. Berlin: Springer.

Bos, W. (1989). Reliabilität und Validität in der Inhaltsanalyse. Ein Beispiel zur Kategorienoptimierung in der Analyse chinesischer Texzbücher für den muttersprachlichen Unterricht von Auslandschinesen. In W. Bos \& C. Tarnai (Eds.), Angewandte Inhaltsanalyse in Empirischer Pädagogik und Psychologie (pp. 61-72). Münster: Waxmann.

Bos, W., \& Tarnai, C. (1989). Angewandte Inhaltsanalyse in Empirischer Pädagogik und Psychologie. Münster: Waxmann.

Brannick, M. T., \& Prince, C. (1997). An overview of team performance measurement. In M. T. Brannick, E. Salas \& C. Prince (Eds.), Team performance assessment and measurement. Theory, methods, and applications (pp. 3-16). Mahwah, NJ: Lawrence Erlbaum.

Brauer, M., Judd, C. M., \& Gliner, M. D. (2006). The effects of repeated expressions on attitude polarization during group discussion. In R. Moreland (Ed.), Small groups (pp. 265-287). New York and Hove: Psychology Press.

Brauner, E. (1998). Die Qual der Wahl am Methodenbuffet oder: Wie der Gegenstand nach der passenden Methode sucht. In E. Ardelt-Gattinger, H. Lechner \& W. Schlögl (Eds.), Gruppendynamik. Anspruch und Wirklichkeit der Arbeit in Gruppen (pp. 176-193). Göttingen: Verlag für Angewandte Psychologie.

Brauner, E. (2001). Wissenstransfer in Projektgruppen: Die Rolle des transaktiven Gedächtnisses. In R. Fisch, D. Beck \& B. Englich (Eds.), Projektgruppen in Organisationen (pp. 237-248). Göttingen: Hogrefe.

Brauner, E., \& Orth, B. (2002). Strukturen von Argumentationssequenzen in Gruppen. Zeitschrift für Sozialpsychologie, 33, 65-81.

Bromme, R. (1992). Der Lehrer als Experte. Zur Psychologie des professionellen Wissens. Bern: Huber.

Brosius, H., \& Koschel, F. (2001). Methoden der empirischen Kommunikationsforschung - Eine Einführung. Wiesbaden: Westdeutscher Verlag.

Bruder, R., Lengnink, K., \& Prediger, S. (2003). Ein Instrumentarium zur Erfassung subjektiver Theorien über Mathematikaufgaben. Retrieved 20.05, 2006, from http://wwwbib.mathematik.tudarmstadt.de/Math-Net/Preprints/Listen/files/2265.pdf

Brüderl, J., \& Scherer, S. (2004). Methoden zur Analyse von Sequenzdaten. Kölner Zeitscbrift für Soziologie und Sozialpsychologie, Sonderheft 44, 330-347.

Bünting, K.-D., \& Bergenholtz, H. (1979). Einführung in die Syntax. Königstein/Ts.: Athenäum. 
Burger, H., Günther, U., Häcki Buhofer, A., Rüegg, R., \& Schneider, H. (1998). Richtlinien zur Transkription von Texten. Zürich: Linguistische Abteilung des Deutschen Seminars der Universität Zürich.

Burleson, B. R. (1984). Decision-making procedure and decision quality. Human Communication Research, 10(4), 557-574.

Campion, E. W. (2003). A death at Duke. New England Journal of Medicine, 348, 1083-1084.

Cannon-Bowers, J. A., \& Salas, E. (2001). Reflection on shared cognition. Journal of Organizational Behavior, 22, 195-202.

Clark, H. H. (1991). Grounding in communication. In L. B. Resnick, J. M. Levine \& S. D. Teasley (Eds.), Perspectives on socially shared cognition. Washinton, DC: American Psychological Association.

Clark, H. H. (1999). On the origins of conversation. Verbum, 21, 147-161.

Clark, H. H. (2004). Coordinating with language. Paper presented at the Postgraduate Workshop on Task Analysis and Collaborative Work Processes, Engelberg, Switzerland.

Clark, H. H., \& Brennan, S. E. (1991). Grounding in communication. In L. B. Resnick, J. M. Levine \& S. D. Teasley (Eds.), Perspectives on socially shared cognition. Washinton, DC: American Psychological Association.

Cohen, J. (1960). A coefficient of agreement for nominal scales. Educational and Psychological Measurement, 20, 37-46.

Conroy, R. (2001). Spatial navigation in immersive virtual environments. University College London, London.

Cranach, M. v. (1994). Die Unterscheidung von Handlungstypen - Ein Vorschlag zur Weiterentwicklung der Handlungspsychologie. In B. R. Bergmann, P. (Ed.), Die Handlungstheorie. Von der Praxis einer Theorie (pp. 69-88). Göttingen: Hogrefe.

Cranach, M. v. (1996). Toward a theory of the acting group. In E. H. Witte \& J. H. Davis (Eds.), Understanding group behavior: Small group processes and interpersonal relations (pp. 147-187). Hillsdale, NJ: Erlbaum.

Cranach, M. v., Kalbermatten, U., Indermühle, K., \& Gugler, B. (1980). Zielgerichtetes Handeln. Bern: Huber.

Cranach, M. v., Ochsenbein, G., \& Valach, L. (1986). The group as self-active system: Outline of a theory of group action. European Journal of Social Psychology, 16, 193-229.

Crowell, L., \& Schneider, T. M. (1961). Categories for analysis of idea development in discussion groups. The Journal of Social Psychology, 54, 155-168.

Cruz, M. G., Henningsen, D. D., \& Smith, B. A. (1999). The impact of directive leadership on group information sampling, decisions, and perceptions of the leader. Communication Research, 26, 349-369.

Daft, R. L., \& Lengel, R. H. (1984). Information richness: A new approach to managerial behavior and organizational design. Research in Organizational Behavior, 6, 191-233. 
Dann, H.-D., \& Humpert, W. (1987). Eine empirische Analyse der Handlungswirksamkeit subjektiver Theorien von Lehrern in aggressionshaltigen Unterrichtssituationen. Zeitschrift für Sozialpsychologie, 18, 40-49.

Dennis, A. R., \& Valacich, J. S. (1999). Rethinking media richness: Towards a theory of media synchronicity. In R. Sprague (Ed.), Proceedings of the 32nd Hawaii International Conference on System Science. Los Alamitos, CA: IEEE Computer Society.

Dickinson, T. L., \& McIntyre, R. M. (1997). A conceptual framework for teamwork measurement. In M. T. Brannick, E. Salas \& C. Prince (Eds.), Team performance assessment and measurement (pp. 19-43). Mahwah, NJ: Lawrence Erlbaum.

Diehl, M., \& Stroebe, W. (1987). Productivity loss in brainstorming groups: Toward the solution of a riddle. Journal of Personality and Social Psychology, 53, 497-509.

Dietrich, R. (2004). Determinants of effective communication. In R. Dietrich \& T. M. Childress (Eds.), Group interaction in high risk environments (pp. 185-207). Aldershot: Ashgate.

Dittmar, N. (2002). Transkription. Ein Leitfaden mit Aufgaben für Studenten, Forscher und Laien. Opladen: Leske + Budrich.

Dudenredaktion (Ed.). (1990). Duden. Band 5: Fremdwörterbuch. Mannheim: Dudenverlag.

Edmondson, A. C., \& McManus, S. E. (2005). Methodological fit in management field research. Retrieved 23.08., 2006, from http://comm.research.arizona.edu/cgi bin/ingroup/list docs.cgi

Edmüller, A., \& Wilhelm, T. (2005). Moderation (3. Auflage ed.). Planegg: Haufe.

Ellis, D. G., \& Fisher, B. A. (1994). Small group decision making. Communication and the group process. Boston: McGraw-Hill.

Ellwein, T. (1990). Koordination ohne Ende. In R. Fisch \& M. Boos (Eds.), Vom Umgang mit Komplexität in Organisationen (pp. 165-179). Konstanz: Konstanzer Beiträge zur sozialwissenschaftlichen Forschung, Band 5.

Engel, U. (1996). Deutsche Grammatik. Heidelberg: Julius Groos.

Erben, J. (1984). Deutsche Syntax. Bern: Peter Lang.

Espinosa, A., Lerch, F. J., \& Kraut, R. E. (2004). Explicit vs. implicit coordination mechanisms and task dependencies: One size does not fit all. In E. Salas \& S. M. Fiore (Eds.), Team cognition: Understanding the factors that drive process and performance (pp. 107-129). Washington, DC: American Psychological Association.

Faßnacht, G. (1995). Systematische Verbaltensbeobachtung (2. ed.). München: Reinhardt.

Fielding, N., \& Schreier, M. (2001). Introduction: On the compability between qualitative and quantitative research methods. Forum Qualitative Sozialforschung. Retrieved 03.01, 2006, from http://qualitative-research.net/fqs/fqs.htm.

Fisch, R. (1994). Eine Methode zur Analyse von Interaktionsprozessen beim Problemlösen. Gruppendynamik, 25(2), 149-168. 
Fisch, R. (1998). Konferenzkodierung: Eine Methode zur Analyse von Problemlöseprozessen in Gruppen. In E. Ardelt-Gattinger, H. Lechner \& W. Schlögl (Eds.), Gruppendynamik: Anspruch und Wirklichkeit der Arbeit in Gruppen (pp. 194-206). Göttingen: Verlag für Angewandte Psychologie.

Fleiss, J. L., \& Cohen, J. (1973). The equivalence of weighted kappa and the intraclass correlation coefficient as measures of reliability. Educational and Psychological Measurement, 33, 613-619.

Foushee, H. C. (1982). The role of communication, socio-psychological, and personality factors in the maintenance of crew coordination. Aviation, Space, and Environmental Medicine, 53, 1062-1066.

Frey, L. R. (2004). The symbolic-interpretive perspective on group dynamics. Small Group Research, 35, 277-306.

Frick, T., \& Semmel, M. I. (1978). Observer agreement and reliabilities of classroom observational measures. Review of Educational Research, 48, 157-184.

Froschauer, U., \& Lueger, M. (1998). Das qualitative Interview zur Analyse sozialer Systeme. Wien: WUV Universitätsverlag.

Früh, W. (2001). Kategorienexplikation bei der Inhaltsanalyse. Basiswissengeleitete offene Kategorienfindung (BoK). In W. Wirth \& E. Lauf (Eds.), Inhaltsanalyse (pp. 117-139). Köln: Herbert von Halem Verlag.

Früh, W. (2004). Inhaltsanalyse. Konstanz: UVK.

Fuller, S. R., \& Aldag, R. J. (2001). The GGPS Model: Broadening the perspective on group problem solving. In M. E. Turner (Ed.), Groups at work: Theory and research (pp. 3-24). Mahwah, NJ: Erlbaum.

Fussell, S. R., Kraut, R. E., Lerch, F. J., Scherlis, W. L., McNally, M. M., \& Cadiz, J. J. (1998). Coordination, overload and team performance: Effects of team communication strategies. In Proceedings of CSCW 1998 (pp. 275-284). New York: Academic Press.

Geller, A., Kaplan, D., \& Lasswell, H. D. (1942). An experimental comparison of four ways of coding editorial content. Journalism Quaterly, 19, 362-370.

Gersick, C. J. G. (1988). Time and transitions in work teams: Toward a new model of group development. The Academy of Management Journal, 31, 9-41.

Gersick, C. J. G. (1989). Marking time: Predictable transitions in task groups. The Academy of Management Journal, 32, 274-309.

Gigone, D., \& Hastie, R. (1993). The Common Knowledge Effect: Information sharing and group judgement. Journal of Personality and Social Psychology, 65, 959-974.

Glaser, B. G., \& Strauss, A. L. (2005). Grounded Theory. Strategien qualitativer Forschung (2nd ed.). Bern: Huber.

Godart, C., Bouthier, C., Canalda, P., Charoy, F., Molli, P., Perrin, O., et al. (2001). Asynchronous coordination of virtual teams in creative applications (co-design or coengineering): Requirements and design criteria. In Proceedings of the workshop on Information Technology for Virtual Enterprises (pp. 135-142). 
Goerges, M. (2005). Steuerung und Koordination von Arbeitsgruppen aus systemtheoretischer Perspektive. Unpublished Diploma Thesis, Georg-August-Universität Göttingen, Göttingen.

Gottman, J. M. (1979). Mariatal interactions. Experimental investigations. New York: Academic Press.

Gouran, D. S., \& Hirokawa, R. Y. (1996). Functional theory and communication in decisionmaking and problem-solving groups. An expanded view. In R. Y. Hirokawa \& M. S. Poole (Eds.), Communication and group decision making (pp. 55-80). Thousand Oaks, CA: Sage.

Greitemeyer, T., Brodbeck, F. C., Schulz-Hardt, S., \& Frey, D. (2006). Information sampling and group decision making: The effects of an advocacy decision procedure and task experience. Journal of Experimental Psychology: Applied, 12, 31-42.

Greitemeyer, T., \& Schulz-Hardt, S. (2003). Preference-consistent evaluation of information in the hidden profile paradigm: beyond group-level explanations for the dominance of shared information in group decisions. Journal of Personality \& Social Psychology, 84(2), 322339.

Greitemeyer, T., Schulz-Hardt, S., \& Frey, D. (2003). Präferenzkonsistenz und Geteiltheit von Informationen als Einflussfaktoren auf Informationsbewertung und intendiertes Diskussionsverhalten bei Gruppenentscheidungen. Zeitschrift für Sozialpsychologie, 34, 9-23.

Grice, H. P. (1998). Studies in the way of words. Cambridge: Harvard University Press.

Groeben, N. (1986). Handeln, Tun, Verhalten als Einheiten einer verstehend-erklärenden Psychologie. Tübingen: Francke.

Groeben, N. (1988). Explikation des Konstrukts 'Subjektive Theorie'. In N. Groeben, D. Wahl, J. Schlee \& B. Scheele (Eds.), Forschungsprogramm Subjektive Theorien (pp. 17-24). Tübingen: Francke.

Grote, G., Zala-Mezö, E., \& Grommes, P. (2003). Effects of standardization on coordination and communication in high workload situations. In R. Dietrich (Ed.), Communication in bigh risk environments. (pp. 127-154). Hamburg: Helmut Buske.

Grote, G., Zala-Mezö, E., \& Grommes, P. (2004). The effects of different forms of co-ordination on coping with workload. In R. Dietrich \& T. M. Childress (Eds.), Group interaction in high risk environments (pp. 39-54). Aldershot: Ashgate.

Guetzkow, H. (1950). Unitizing and categorizing problems in coding qualitative data. Journal of Clinical Psychology, 6, 47-58.

Gurtner, A. (2003). Zweimal musst du es schon sagen. Strategieentwicklung und Kommunikationsmuster in hierarchisch organisierten Teams. Dissertation, Universität Bern:http://www.stub.unibe.ch/download/eldiss/03gurtner a.pdf.

Gurtner, A., Tschan, F., Semmer, N. K., \& Nägele, C. (in press). Getting groups to develop good strategies: Effects of reflexivity interventions on team process, team performance, and shared mental models. Organizational Behavior and Human Decision Processes.

Hackman, J. R. (2006). Conditions, levels, and processes: Three imperatives for new perspectives. Paper presented at the First Annual INGRoup Conference, Pittsburgh, PA. 
Hackman, J. R., Brousseau, K. R., \& Weiss, J. A. (1976). The interaction of task design and group performance strategies in determining group effectiveness. Organizational Behavior and Human Performance, 16, 350-365.

Hackman, J. R., \& Morris, C. G. (1975). Group tasks, group interaction process, and group performance effectiveness: A review and proposed integration. In L. Berkowitz (Ed.), Advances in experimental social psychology (Vol. 8, pp. 45-99). New York: Academic Press.

Hackman, J. R., \& Wageman, R. (2005). A theory of team coaching. The Academy of Management Review, 30, 269-287.

Hager, W. (2004). Testplanung zur statistischen Prüfung psychologischer Hypothesen. Göttingen: Hogrefe.

Hartmann, M., Funk, R., \& Wittkuhn, K. D. (2000). Gekonnt moderieren. Teamsitzung, Besprechung und Meeting: zielgerichtet und ergebnisorientiert. Weinheim: Beltz.

Heeringa, W. (2004). Measuring dialect pronunciation differences using Levenshtein Distance. Groningen dissertations in linguistics 46 (http://irs.ub.rug.nl/ppn/258438452), Groningen:

University of Groningen.

Henningsen, D. D., Henningsen, M. L. M., Jakobsen, L., \& Borton, I. (2004). It's good to be a leader: The influence of randomly and systematically selected leaders on decision-making groups. Group Dynamics: Theory, Resarch, and Practice, 8, 62-76.

Henningsen, M. L. M., Henningsen, D. D., Borton, I. M., \& Braz, M. (2006). The influence of repeated information: Testing perceptions of repetition in an information sampling study. Unpublished manuscript, DeKalb, IL.

Hirokawa, R. Y. (1982). Group communication and problem-solving effectiveness I: A critical review of inconsistent findings. Communication Quarterly, 30(2), 134-141.

Hirokawa, R. Y. (1983). Group communication and problem-solving effectiveness: An investigation of group phases. Human Communication Research, 9, 291-305.

Hirokawa, R. Y. (1985). Discussion procedures and decision-making performance. A test of a functional perspective. Human Communication Research, 12(2), 203-224.

Hirokawa, R. Y. (1990). The role of communication in group decision-making efficacy. A taskcontingency perspective. Small group research, 21(2), 190-204.

Hirokawa, R. Y., \& Salazar, A. J. (1999). Task-group communication and decision-making performance. In L. R. Frey, D. S. Gouran \& M. S. Poole (Eds.), The bandbook of group communication theory and research (pp. 167-191). Thousand Oaks, CA: Sage.

Hogg, M. A., Abrams, D., Otten, S., \& Hinkle, S. (2004). The social identity perspective: Intergroup relations, self-conception, and small groups. Small Group Research, 35, 246-276.

Hollingshead, A. B., \& Poole, M. S. (2004). Interdisciplinary theoretical perspectives of small groups part II. Small Group Research, 35, 243-245.

Holsti, O. R. (1969). Content analysis for the social sciences and bumanities. Reading, MA: AddisonWesley. 
Holzinger, K. (2001). Verhandeln statt Argumentieren oder Verhandeln durch Argumentieren? Eine empirische Analyse auf der Basis der Sprechakttheorie. Politische Vierteljahresschrift, 42, 414-446.

Hopf, C. (1995). Qualitative Interviews in der Sozialforschung. Ein Überblick. In U. Flick, E. von Kardorff, H. Keupp, L. von Rosenstiel \& S. Wolff (Eds.), Handbuch Qualitative Sozialforschung (pp. 177-182). München: PVU.

Howitt, D., \& Cramer, D. (2005). Introduction to research methods in psychology. Harlow: Pearson Education.

Hron, A., Hesse, F. W., Cress, U., \& Giovis, C. (2000). Implicit and explicit dialogue structuring in virtual learning groups. British Journal of Edcuational Psychology, 70.

Huguet, P., Charbonnier, E., \& Monteil, J.-M. (1999). Productivity loss in performance groups: People who see themselves as average do not engage in social loafing. Group Dynamics, 3(2), 118-131.

Hussy, W., \& Jain, A. (2002). Experimentelle Hypothesenprüfung in der Psychologie. Göttingen: Hogrefe.

Hyey, B. M., \& Wickens, C. D. (1993). Team leadership and crew coordination. In B. M. Hyey \& C. D. Wickens (Eds.), Workload transition: Implications for individual and team performance (pp. 229-247). Washington, DC: National Academy Press.

Ilgen, D. R., Hollenbeck, J. R., Johnson, M., \& Jundt, D. (2005). Teams in organizations: From input-process-output models to IMOI models. Annual Review of Psychology, 56, 517-543.

Iwanowsky, S., \& Beck, D. (2003). Modernisierungsprozesse in Organisationen in der öffentlichen Verwaltung zwischen Praxis und Wissenschaft - Wissen und Vorstellungen von Entscheidungsträgern über Organisationswandel und -design. Eine Zwischenbilanz zu Theorien, Methoden und empirischen Befunden der kognitiven Organisationspsychologie. FÖV-Discussion Paper aus dem Forschungsprojekt I/ 22: Fisch/Beck: Aufgaben für die öffentliche Verwaltung im 21. Jahrbundert. Konsequenzen für die Organisationsgestaltung.

Jung, W. (1973). Grammatik der deutschen Sprache. Leipzig: VEB Verlag Enyklopädie.

Kanitz, v. A. (2004). Gesprächstechniken. Planegg: Haufe.

Kanki, B. G., \& Foushee, H. C. (1989). Communication as group process mediator of aircrew performance. Aviation, Space, and Environmental Medicin, 60, 402-410.

Kanki, B. G., Lozito, S., \& Foushee, H. C. (1989). Communication indices of crew coordination. Aviation, Space, and Environmental Medicin, 60, 56-60.

Kappeler, P. (2006). Verbaltensbiologie. Berlin, Heidelberg: Springer.

Karau, S. J. W., Kipling D. (1993). Social loafing: A meta-analytic review and theoretical integration. Journal of Personality \& Social Psychology, 65(4), 681-706.

Kassner, K., \& Wassermann, P. (2005). Nicht überall, wo Methode draufsteht, ist auch Methode drin. Zur Problematik der Fundierung von ExperInneninterviews. In A. Bogner, B. Littig \& W. Menz (Eds.), Das Experteninterview. Theorie, Methode, Anwendung (2nd ed., pp. 95-111). Wiesbaden: VS Verlag für Sozialwissenschaftenq. 
Katz, N., Lazer, D., Arrow, H., \& Contractor, N. (2004). Network theory and small groups. Small Group Research, 35, 307-332.

Kauffeld, S. (2003). Das Kasseler-Kompetenz-Raster (KKR). Retrieved 26.08.03, from www.ifa.earbeit.biz/fg1/downloads/docs/KKR\%20skizze.pdf

Kauffeld, S., Frieling, E., \& Grote, S. (2002). Soziale, personale, methodische oder fachliche: Welche Kompetenzen zählen bei der Bewältigung von Optimierungsaufgaben in betrieblichen Gruppen? Zeitschrift für Psychologie, 210, 197-208.

Kelle, U. (2001). Sociological explanations between micro and macro and the integration of qualitative and quantitative methods. Forum Qualitative Sorialforschung. Retrieved 02.03, 2006, from http://qualitative-research.net/fqs

Kerr, N. L., Aronoff, J., \& Messé, L. A. (2000). Methods of small group research. In H. T. Reis \& C. M. Judd (Eds.), Handbook of research methods in social and personality psychology (pp. 160189). New York, NY: Cambridge University Press.

Kerr, N. L., \& Tindale, R. S. (2004). Group performance and decision-making. Annual Review of Psychology, 55, 623-655.

Kerschreiter, R., Mojzisch, A., Schulz-Hardt, S., Brodbeck, F. C., \& Frey, D. (2003). Informationsaustausch bei Entscheidungsfindungsprozessen in Gruppen: Theorie, Empirie und Implikationen für die Praxis. In S. Stumpf \& A. Thomas (Eds.), Teamarbeit und Teamentwicklung (pp. 85-118). Göttingen: Hogrefe.

Kessler, B. (1995). Computational dialectology in Irish Gaelic. In Proceedings of the European Association for Computational Linguistics (pp. 60-67). Dublin: EACL.

Kleining, G., \& Witt, H. (2001). Discovery as basis methodology of qualitative and quantitative research. Forum Qualitative Sozialforschung. Retrieved 02.03, 2006, from http://qualitativeresearch.net/fqs

Klosa, A. (2001). Duden: Richtiges und gutes Deutsch. Mannheim: Bibliographisches Institut.

Krallmann, D., \& Ziemann, A. (2001). Grundkurs Kommunikationswissenschaft. München: Wilhelm Fink.

Krippendorf, K. (1980). Content analysis. An introduction to its methodology. Beverly Hills, CA: Sage.

Lamnek, S. (1989). Qualitative Sozialforschung. Band 2: Methoden und Techniken. München: PVU.

Lamnek, S. (2005). Gruppendiskussionen (2nd ed.). Weinheim: Beltz.

Langdridge, D. (2004). Introduction to research methods and data analysis in psychology. Edinburgh: Pearson Education.

Lanzetta, J. T., \& Roby, T. (1960). The relationship between certain group process variables and group problem-solving efficiency. Journal of Social Psychology, 52, 135-148.

Larson, J. R., Christensen, C., Franz, T. M., \& Abbott, A. S. (1998a). Diagnosing groups: The pooling, management, and impact of shared and unshared case information in team-based medical decision making. Journal of Personality and Social Psychology, 75, 93-108. 
Larson, J. R., Foster-Fishman, P. G., \& Franz, T. M. (1998b). Leadership style and the discussion of shared and unshared information in decision-making groups. Personality and Social Psychology Bulletin, 24, 482-495.

Larson, J. R., Foster-Fishman, P. G., \& Keys, C. B. (1994). Discussion of shared and unshared information in decision-making groups. Journal of Personality and Social Psychology, 67, 446461.

Larson, J. R., \& Schaumann, L. J. (1993). Group goals, group coordination, and group member motivation. Human Performance, 6, 49-69.

Leedom, D. K., \& Simon, R. (1995). Improving team coordination: A case for behavior-based team training. Military Psychology, 7, 109-122.

Levenshtein, V. I. (1966). Binary codes capable of correcting deletions, insertions and reversals. Soviet Physics-Doklady, 10, 707-710.

Lienert, G. A., \& Raatz, U. (1998). Testaufbau und Testanalyse. Weinheim: Beltz.

Maas, U., \& Wunderlich, D. (1972). Pragmatik und sprachliches Handeln. ?: Althenäum Verlag.

Maier, N. R. F. (1967). Assets and liabilities in group problem solving: The need for an integrative function. Psychological Review, 74, 239-249.

Malone, T. W., \& Crowston, K. (1994). The interdisciplinary study of coordination. ACM Computing Surveys, 26, 87-119.

Marks, M. A., Mathieu, J. E., \& Zaccaro, S. J. (2001). A temporally based framework and taxonomy of team processes. Academy of Management Review, 26, 356-376.

Mathieu, J. E., Goodwin, G. F., Heffner, T. S., Salas, E., \& Cannon-Bowers, J. A. (2000). The influence of shared mental models on team process and performance. Journal of Applied Psychology, 85, 273-283.

Mayring, P. (2000). Qualitative Inbaltsanalyse. Weinheim: Beltz.

Mayring, P. (2001). Kombination und Integration qualitativer und quantitativer Analyse. Forum Qualitative Sozialforschung 2. Retrieved 02.03., 2006, from http://qualitative-research.net/fqs/fqs.htm.

Mayring, P. (2003). Qualitative Inbaltsanalyse. Grundlagen und Techniken (8th ed.). Weinheim und Basel: UTB.

McGrath, J. E. (1970). Social psychology. A brief introduction. London: Holt, Rinehart \& Winston.

McGrath, J. E. (1984). Groups, interaction and performance. Englewood Cliffs, NJ: Prentice-Hall.

McGrath, J. E. (1991). Time, interaction, and performance (TIP). A theory of groups. Small Group Research, 22, 147-174.

McGrath, J. E., Arrow, H., \& Berdahl, J. L. (2000). The study of groups: past, present, and future. Personality and Social Psychology Review, 4(1), 95-105.

McGrath, J. E., \& Hollingshead, A. B. (1994). Groups interacting with technology: Ideas, evidence, issues, and an agenda. Thousand Oaks: Sage. 
McGrath, J. E., \& Tschan, F. (2004). Dynamics in Groups and Teams. In M. S. Poole \& A. H. Van de Ven (Eds.), Handbook of organizational change and innovation (pp. 50-72). Oxford: Oxford University Press.

McLeod, P. L., \& Kettner-Polley, R. B. (2004). Contributions of psychodynamic theories to understanding small groups. Small Group Research, 35, 333-361.

Merten, K. (1995). Inbaltsanalyse. Opladen: Westdeutscher Verlag.

Merton, R. K. (1987). The focussed interview and focus groups: Continuities and discontinuities. The Public Opinion Quaterly, 51, 550-566.

Meuser, M., \& Nagel, U. (2005). ExpertInneninterviews- vielfach erprobt, wenig bedacht. Ein Beitrag zur qualitativen Methodendiskussion. In A. Bogner, B. Littig \& W. Menz (Eds.), Das Experteninterview. Theorie, Methode, Anwendung (2nd ed., pp. 71-93). Wiesbaden: VS Verlag für Sozialwissenschaften.

Mojzisch, A., Kerschreiter, R., Schulz-Hardt, S., \& Frey, D. (2004). Wissensmanagement bei Entscheidungen in Gruppen. In G. Reimann \& H. Mandl (Eds.), Psychologie des Wissensmanagements: Perspektiven, Theorien und Methoden (pp. 178-203). Göttingen: Hogrefe.

Mojzisch, A., \& Schulz-Hardt, S. (2006). Information sampling in group decision making. Sampling Biases and their consequences. In K. Fiedler \& P. Juslin (Eds.), Information sampling and adaptive cognition (pp. 299-325). Cambridge: University Press.

Mojzisch, A., Schulz-Hardt, S., Vogelgesang, F., \& Kerschreiter, R. (2005). The effects of informational access during discussion and prediscussion dissent on group decision quality in bidden profile situations. Paper presented at the SPUDM, Stockholm, Schweden.

Mruck. (2000). Qualitative Sorialforschung in Deutschland. Forum Qualitative Sozialforschung, 1. Retrieved 15.08, 2006, from http://www.qualitative-research.net/fqs-texte/1-00/1-00mruckmeyd.pdf

Müller, C. T. (2003). Subjektive Theorien und handlungsleitende Kognitionen von Lehrern als Determinanten schulischer Lehr-Lern-Prozesse im Physikunterricht. Unveröffentlichte Dissertation. Kiel: Universität Kiel.

Nägele, C. (2004). Dynamic group processes in co-acting virtual teams. Dissertation, Neuchâtel.

Nerbonne, J., Heeringa, W., \& Kleiweg, P. (1999). Comparison and classification of dialects. In Proceedings of the European Association for Computational Linguistics (pp. 281-282). Dublin: EACL.

Orlitzky, M., \& Hirokawa, R. Y. (2001). To err is human, to correct for it divine. A meta-analysis of research testing the functional theory of group decision-making effectiveness. Small Group Research, 32, 313-341.

Poole, M. S., \& Hirokawa, R. Y. (1996). Introduction. Communication and group decision making. In R. Y. Hirokawa \& M. S. Poole (Eds.), Communication and group decision making (pp. 3-18). Thousand Oaks, CA: Sage.

Poole, M. S., Hollingshead, A. B., McGrath, J. E., Moreland, R. L., \& Rohrbaugh, J. (2004). Interdisciplinary perspectives on small groups. Small Group Research, 35, 3-16. 
Posthuma, B. W. (2002). Small groups in counselling and therapy. Process and leadership (4th ed.). Boston: Allyn and Bacon.

Rack, O., \& Christophersen, T. (2006). Experimente. In S. Albers, D. Klapper, U. Konradt, A. Walter \& J. Wolf (Eds.), Methoden der empirischen Forschung (pp. 19-36). Wiesbaden: DVU.

Reis, H. T., \& Judd, C. M. (2000). Handbook of research methods in social and personality psychology. New York, NY: Cambridge University Press.

Rogers, C. R. (1999). Die nicht-direktive Beratung. Frankfurt a. M.: Fischer.

Sankoff, D., \& Kruskal, J. B. (1983). Time warps, string edits, and macromolecules: The theory and practica of sequence comparison. Reading, MA: Addison-Wesley.

Scharpf, U. (1988). Entscheidungsfindung im Gruppenprozess. Konstanz: Hartung-Gorre Verlag.

Schattenhofer, K. (1995). Selbstorganisation in Gruppen. Entwicklungs- und Steuerungsprozesse in Gruppen. Kölner Zeitschrift für Soziologie und Sozialpsychologie, 47, 186-187.

Schauenburg, B. (2004). Motivierter Informationsaustausch in Gruppen: Der Einfluss individueller Ziele und Gruppenziele. Dissertation, Goettingen:http://webdoc.sub.gwdg.de/diss/2004/schauenburg/.

Scherm, M. (1998). Synergie in Gruppen - mehr als eine Metapher? In E. Ardelt-Gattinger, H. Lechner \& W. Schlögl (Eds.), Gruppendynamik. Anspruch und Wirklichkeit der Arbeit in Gruppen (pp. 62-70). Göttingen: Verlag für Angewandte Psychologie.

Scheufele, B. (2001). Notwendigkeit, Nutzen und Aufwand von Mehrfach- und Sondercodierungen. In W. Wirth \& E. Lauf (Eds.), Inhaltsanalyse (pp. 82-97). Köln: Herbert von Halem Verlag.

Schmitt, K., \& Hanke, U. (2003). Subjektive Feedbacktheorien von ExpertentrainerInnen und ihren AthletInnen in der Leichtathletik. Forum Qualitative Sozialforschung / Forum: Qualitative Social Research [Online Journal], 4, http://www.qualitative-research.net/ [27.06.2006].

Schnell, R., Hill, P. B., \& Esser, E. (1993). Methoden der empirischen Sozialforschung. München, Wien: R. Oldenbourg.

Schulz-Hardt, S., Brodbeck, F. C., Mojzisch, A., Kerschreiter, R., \& Frey, D. (in press). Group decision making in hidden profile situations: Dissent as a facilitator for decision quality. Journal of Personality and Social Psychology.

Schurf, B. (2001). Deutschbuch: Orientierungswissen. Berlin: Cornelsen.

Schwabe, G. (2001a). Koordinationswerkzeuge. In G. Schwabe, N. Streitz \& R. Unland (Eds.), CSCW Kompendium - Lehr- und Handbuch zur Computerunterstützung (pp. 174-180). Berlin: Springer.

Schwabe, G. (2001b). "Mediensynchronizität" - Theorie und Anwendung bei Gruppenarbeit und Lernen. In F. Hesse \& H. Friedrich (Eds.), Partizipation und Interaktion in virtuellen Seminaren. Münster: Waxmann. 
Schweiger, D. M., \& Sandberg, W. R. (1989). Experiential effects of dialectical inquiry, devil's advocacy and consensus approaches to strategic decision making. Academy of Management Journal, 32, 745-772.

Schweiger, D. M., Sandberg, W. R., \& Ragan, J. W. (1986). Group approaches for improving strategic decision making: A comparative analysis of dialectical inquiry, devil's advocacy, and consensus. Academy of Management Journal, 29, 51-71.

Schweiger, D. M., Sandberg, W. R., \& Rechner, P. (1988). A longitudinal comparative analysis of dialectical inquiry, devil's advocacy and consensus approaches to strategic decision making. Academy of Management Proceedings, 32-36.

Searle, J. R. (1969). Speech acts: An essay in philosophy of language. Cambridge: Cambridge University Press.

Seifert, J. W. (2005). 30 Minuten für professionelles Moderieren. Offenbach: Gabal.

Selting, M. (2000). The construction of units in conversational talk. Language in Society, 29, 477517.

Selting, M., Auer, P., Barden, B., Bergmann, J., Couper-Kuhlen, E., Günthner, S., et al. (1998). Gesprächsanalytisches Transkriptionssystem (GAT). Linguistische Berichte, 173, 91-122.

Selting, M., \& Couper-Kuhlen, E. (2000). Argumente für die Entwicklung einer 'interaktionalen Linguistik'. Gesprächsforschung, 1, 76-95.

Sexton, J. B. (Ed.). (2004). The better the team, the safer the world. Golden rules of group interaction in high risk environments: Evidence based suggestions for improving performance. Ladenburg and Rüschlikon: Gottlieb Daimler and Karl Benz Foundation and Swiss Center for Global Dialog.

Sexton, J. B., Grommes, P., Zala-Mezö, E., Grote, G., Helmreich, R. L., \& Häusler, R. (2004). Leadership and co-ordination. In R. Dietrich \& T. M. Childress (Eds.), Group interaction in high risk environments (pp. 165-184). Aldershot: Ashgate.

Sexton, J. B., \& Helmreich, R. L. (1999). Analysing cockpit communication: The links between language, performance, error, and workload. In Proceedings of the Tenth International Symposium on Aviation Psychology (pp. 689-695). Columbus: OH: The Ohio State University.

Simon, P. (1997). SYNPRO. Interaktions-Beobachtungssystem. Manual zur Kodierung: Unveröffentlichtes Manuskript, Universität Regensburg.

Simon, P. (2000). Die Entwicklung eines Modells der Gruppeneffektivität und eines Analyseinstruments zur Erfassung des Leistungspotentials von Arbeitsgruppen. Landau: Verlag für Empirische Pädagogik.

Smith, B. N. K., Natalie A. Markus, Michael J. Stasson, Mark F. (2001). Individual differences in social loafing: Need for cognition as a motivator in collective performance. Group Dynamics, 5(2), 150-158.

Sörensen, K. (2003). Distance measures based on the edit distance for permutation type representation. In Proceedings of the workshop on analysis and design of representation and operators (pp. 8-14). 
Stasser, G., \& Titus, W. (1985). Pooling of unshared information in group decision making: Biased information sampling during discussion. Journal of Personality and Social Psychology, 48, 1467-1578.

Stasser, G., \& Titus, W. (1987). Effects of information load and percentage of shared information on the dissemination of unshared information during group discussion. Journal of Personality and Social Psychology, 53, 81-93.

Stasser, G., \& Titus, W. (2006). Pooling of unshared information in group decision making: Biased information sampling during discussion. In J. M. Levine \& R. Moreland (Eds.), Small groups (pp. 227-239). New York and Hove: Psychology Press.

Stein, A. (2006). Einwicklung einer Methode zur mikroanalytischen Verhaltensbeobachtung in Gruppen. Georg-August-Universität Göttingen, Göttingen.

Steiner, I. D. (1972). Group processes and productivity. New York: Academic Press.

Stempfle, J. (2006). Kommunikation und Problemlösen in Gruppen: Unveröffentlichte Dissertation, Universität Bamberg.

Stroebe, W., \& Frey, B. S. (1982). Self-interest and collective action: The economics and psychology of public goods. British Journal of Social Psychology, 21, 121-137.

Sundstrom, E. (1990). Work teams. Applications and effectiveness. American Psychologist, 45(2), 120-133.

Thiele, K., \& Manser, T. (2001). Soziotechnische Systemanalyse im Krankenhaus. Eine arbeitspsychologische Fallstudie in der Anästhesiologie. Harburger Beiträge zur Psychologie und Soziologie der Arbeit, 27.

Thompson, L. L. (2004). Making the team. A guide for managers (2nd ed.). Upper Saddle River, New Jersey: Pearson Education.

Trinczek, R. (2005). Wie befrage ich Manager? Methodische und methodologische Aspekte des Experteninterviews als qualitative Methode empirirscher Sozialforschung. In A. Bogner, B. Littig \& W. Menz (Eds.), Das Experteninterview. Theorie, Methode, Anwendung (pp. 209222). Wiesbaden: VS Verlag für Sozialwissenschaften.

Tschan, F. (1995). Communication enhances small group performance if it conforms to task requirements: The concept of ideal communication cycles. Basic and Applied Social Psychology, 17, 371-393.

Tschan, F. (2000). Produktivität in Kleingruppen. W as machen produktive Gruppen anders und besser? Bern: Huber.

Tschan, F., \& Semmer, N. K. (2001). Wenn alle dasselbe denken: Geteilte Mentale Modelle und Leistung in der Teamarbeit. In R. Fisch, D. Beck \& B. Englich (Eds.), Projektgruppen in Organisationen (pp. 217-235). Göttingen: Verlag für angewandte Psychologie.

Tschan, F., Semmer, N. K., Nägele, C., \& Gurtner, A. (2000). Task adaptive behavior and performance in groups. Group Processes and Intergroup Relations, 3, 367-386.

Vallacher, R. R., \& Wegner, D. M. (1987). What do people think they're doing? Action identification and human behavior. Psychological Review, 94, 3-15. 
Van de Ven, A. H., Delbecq, A. L., \& Koenig, R. J. (1976). Determinants of coordination modes within organizations. American Sociological Review, 41, 322-338.

Van Hooff, J. A. R. A. M. (1982). Categories and sequences of behavior: Methods of description and analysis. In K. R. Scherer \& P. Ekman (Eds.), Handbook of methods in nonverbal behavior research (pp. 362-439). Cambridge, UK: Cambridge University Press.

Wagner, R. A., \& Fischer, M. J. (1974). The string-to-string correction problem. Journal of the Assoziation for Computing Machinery, 21, 168-173.

Weick, K. E. (1995). Der Prozeß des Organisierens. Frankfurt am Main: Suhrkamp.

West, M. A. (2004). Effective teamwork. Practical lessons from organizational research (2nd ed.). Oxford: BPS Blackwell.

Westermann, R. (2000). Wissenschaftstheorie und Experimentalmethodik. Göttingen: Hogrefe.

Wheelan, S. A., Davidson, B., \& Tilin, F. (2003). Group development across time - Realitiy or illusion? Small group research, 34, 223-245.

Wilke, H. (2001). Systemtheorie III: Steuerungstheorie (3rd ed.). Stuttgart: Lucius \& Lucius.

Wilson, G. L. (2005). Groups in context. Leadership and participation in small groups. Boston: McGrawHill.

Winquist, J. R., \& Larson Jr., J. R. (1998). Information pooling: When it impacts group decision making. Journal of Personality and Social Psychology, 74, 371-377.

Wirth, W., \& Lauf, E. (2001). Inhaltsanalyse. Köln: Herbert von Halem Verlag.

Wirtz, W., \& Caspar, E. (2002). Beurteilerübereinstimmung und Beurteilerreliabilität. Göttingen: Hogrefe.

Witt, H. (2001). Forschungsstrategien bei quantitativer und qualitativer Sozialforschung. Forum qualitative Sozialforschung. Retrieved 02.03, 2006, from http://qualitative-research.net/fqs

Witte, E. H. (2001). Entwicklung einer Gruppenmoderationstheorie für Projektgruppen und ihre empirische Überprüfung. In E. H. Witte (Ed.), Leistungsverbesserungen in aufgabenorientierten Kleingruppen (pp. 217-235). Lengerich: Papst.

Wittenbaum, G. M., Hollingshead, A. B., \& Botero, I. C. (2004a). From cooperative to motivated information sharing in groups: Moving beyond the Hidden Profile paradigm.

Communication Monographs, 71, 286-310.

Wittenbaum, G. M., Hollingshead, A. B., Paulus, P. B., Hirokawa, R. Y., Ancona, D. G., Peterson, R. S., et al. (2004b). The functional perspective as a lens for understanding groups. Small Group Research, 35, 17-43.

Wittenbaum, G. M., Hubbell, A. P., \& Zuckerman, C. (1999). Mutual enhancement: Toward an understanding of the collective preference for shared information. Journal of Personality and Social Psychology, 77, 967-978.

Wittenbaum, G. M., Stasser, G., \& Merry, C. J. (1996). Tacit coordination in anticipation of small group task completion. Journal of Experimental Social Psychology, 32, 129-152. 
Wittenbaum, G. M., Vaughan, S. I., \& Stasser, G. (1998). Coordination in task-performing groups. In R. S. Tindale, L. Heath, J. Edwards, E. J. Posavac, F. B. Bryant, Y. SuarezBalcazar, E. Henderson-King \& J. Myers (Eds.), Theory and research on small groups. New York: Plenum Press.

Yukl, G. (2002). Leadership in organizations (5 ed.). Upper Saddle River, NJ: Prentice Hall.

Zempel, J. (2002). Strategien der Handlungsregulation. Dissertation, Giessen:http://psydok.sulb.unisaarland.de/volltexte/2006/841/pdf/ZempelJeannette-2003-02-12.pdf. 


\section{Verzeichnis der Abbildungen}

Abbildung 1: Einordnung der Konzepte Entscheidungsfindung, Informationsaustausch und explizite Prozesskoordination in das funktionale Paradigma .....

Abbildung 2: Traditionelles Paradigma zur Analyse der Gruppeninteraktion als Mediator von Gruppenergebnissen. Nach Hackman und Morris (1975) sowie McGrath (1970).....

Abbildung 3: Exemplarische Darstellung der hierarchisch-sequentiellen Struktur der Gruppenentscheidungsaufgabe

Abbildung 4: Integriertes Rahmenmodell der Teamkoordination und Leistung (Espinosa et al., 2004, S. $110 \& 118)$

Abbildung 5: Modell der Koordinationsmodi in Task-Performing-Gruppen (Wittenbaum et al., $1998, \mathrm{~S}$. 178).

Abbildung 6: Veranschaulichung der hierarchisch-sequentiellen Handlungsorganisation im Rahmen der Handlungstheorie.

Abbildung 7: Relative Häufigkeiten der kategorisierten Antworten auf die Frage: Vor welchen Schwierigkeiten steht Stefan? Mehrfachnennungen möglich.

Abbildung 8: Häufigkeiten der Handlungsanleitungen. Mehrfachnennungen möglich.

Abbildung 9: Häufigkeiten der Strukturierungen des Problemlöseprozesses. Mehrfachnennungen möglich.

Abbildung 10:Häufigkeiten der Kategorien zur Konfliktvermeidung. Mehrfachnennungen möglich. .75

Abbildung 11: Mittelwerte der eingeschätzten Nützlichkeit der vorgegebenen Verhaltensweisen. Anmerkung: Die Skala reichte von $1 . .$. überhaupt nicht sinnvoll bis 5 ...sehr sinnvoll; *** bedeutet einen signifikanten Unterschied auf dem 0,001-Niveau, ** auf dem 0,01-Niveau und * auf dem 0,05-Niveau.

Abbildung 12: Thematischer Vergleich. $\mathrm{N}=8$ Interviews.

Abbildung 13: Theoretische Generalisierung der subjektiven Koordinationstheorien 102

Abbildung 14: Veranschaulichung des Herstellungswissens der Experten/-innen zum situationsangemessenen Einsatz von Koordinationsmechanismen

Abbildung 15: Integration der Inhalte der subjektiven Koordinationstheorien in das IPO-Modell .......... 106

Abbildung 16: Integration von Beispielen der von den Experten/-innen berichteten Koordinationsmechanismen in die Taxonomie der Koordinationsmodi (Wittenbaum et al., 1998).

Abbildung 17: Darstellung der Forschungsfrage des Experiments im Rahmen des IPO-Modells .... 123

Abbildung 18: Verteilung der individuellen initialen Entscheidungspräferenzen der Kandidaten A, B, C und D

Abbildung 19: Einfluss des regelmäßigen Wiederholens genannter Informationen auf die Entscheidungsgüte

Abbildung 20: Eignungsbeurteilungen der Kandidaten vor im Vergleich zu nach der Diskussion in den Bedingungen ohne Wiederholung von Informationen. Die Ordinate beginnt nicht bei Null.... 155

Abbildung 21: Eignungsbeurteilungen der Kandidaten vor im Vergleich zu nach der Diskussion in den Bedingungen mit Wiederholung von Informationen. Die Ordinate beginnt nicht bei Null....... 155

Abbildung 22: Pfadmodell zur Vorhersage der Entscheidungsgüte (Einschluss beider unabhängigen Variablen und deren Interaktion). $\mathrm{N}=48$ Gruppen $_{s} \mathrm{Df}=3$; GFI = .94; $\mathrm{AGFI}=.70 ; \mathrm{PGFI}=.18$; $\mathrm{RMR}=.06$. 
Abbildung 23: Finales Pfadmodell zur Vorhersage der Entscheidungsgüte (Nach Ausschluss nicht beitragender Variablen). $\mathrm{N}=48$ Gruppen, $\mathrm{Df}=5$; GFI $=.92$; AGFI $=.78$; PGFI $=.31$; RMR $=$ .05 .

Abbildung 24: Der Einfluss des Wiederholens von Informationen auf die Nennungshäufigkeit ungeteilter Informationen während der Diskussion...

Abbildung 25: Pfadmodell zur Vorhersage der Entscheidungsgüte unter Berücksichtigung des Informationsaustauschs (Einschluss beider unabhängigen Variablen, deren Interaktion als auch des Informationsaustauschs). $\mathrm{N}=28$ Gruppen, $_{s} \mathrm{Df}=9$; GFI = .89; $\mathrm{AGFI}=.65$; PGFI =.28; $\mathrm{RMR}=.10$.

Abbildung 26: Finales Pfadmodell zur Vorhersage der Entscheidungsgüte unter Berücksichtigung des Informationsaustauschs (nach Ausschluss nicht beitragender Variablen). $\mathrm{N}=28$ Gruppen, $_{s} \mathrm{Df}=$

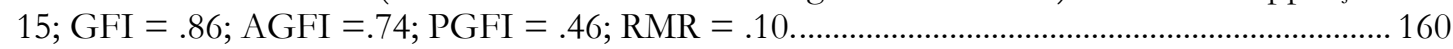

Abbildung 27: Beurteilung der Hilfe der/des Moderators/-in im Vergleich der Bedingungen mit vs. ohne Wiederholen. Die Ordinate beginnt nicht bei Null.

Abbildung 28: Beurteilung der Kompetenz der/des Moderators/-in im Vergleich der Bedingungen mit vs. ohne Wiederholen. Die Ordinate beginnt nicht bei Null.

Abbildung 29: Interaktionseffekt des Erfragens und Wiederholens von Informationen auf die Kompetenzbeurteilung des/der Moderator/-in. Die Ordinate beginnt nicht bei Null. 164

Abbildung 30: Beantwortung der Forschungsfrage des Experiments im Rahmen des IPO-Modells........ 176

Abbildung 31: Formales Arbeitsmodell der Koordination (in Anlehnung an Boos, 2003) ........................ 184

Abbildung 32: Überblick über die Kategorien des Kodiersystems

Abbildung 33: Kategorien der Koordinationsmechanismen, die anhand der Ergebnisse aus Studie 1 und 2 entwickelt wurden (durch schwarze Rahmen dargestellt). Die grau unterlegten Kategorien wurden nachträglich in das Kodiersystem integriert.

Abbildung 34: Beispiel für die formale Gestaltung der Zergliederung von Redebeiträgen

Abbildung 35: Beispiel für die Voraussetzungen des Transkripts

Abbildung 36: Beispieltrennung von Kodierer/-in 1. Das Zeichen \# steht für die Trennung des Redebeitrages durch den Kodierer. Das \%-Zeichen verweist auf einen Sprecherwechsel.

Abbildung 37: Beispieltrennung von Kodierer/-in 2. Das Zeichen \# steht für die Trennung des Redebeitrages durch den Kodierer. Das \%-Zeichen verweist auf einen Sprecherwechsel. 233

Abbildung 38: Einander angepasste Sequenzen der beiden Kodierer. Die eingefügten Trennungen werden durch fettgedruckte Rautezeichen gekennzeichnet. 


\section{Verzeichnis der Tabellen}

Tabelle 1: Übersicht über den Forschungsplan der vorliegenden Arbeit..........................................................5

Tabelle 2: Definitionen von Koordination und ihre Merkmale...........................................................................32

Tabelle 3: einzuschätzende Verhaltensweisen und ihre inhaltliche Zuordnung zu Oberkategorien .............69

Tabelle 4: Beruf, Alter, Geschlecht und Erfahrungshintergrund der Experten/-innen ..................................89

Tabelle 5: Mechanismen expliziter Prozesskoordination von Gruppenentscheidungsprozessen............... 114

Tabelle 6: Überblick über die Hypothesen................................................................................................. 129

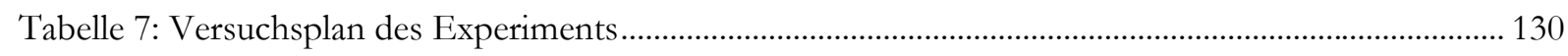

Tabelle 8: Verteilung von Geschlecht, Alter und Semesterzahl der Probanden/-innen .............................. 132

Tabelle 9: Übersicht über die Herkunft und Reliabilität der Items der $\mathrm{AV}_{2}$ sowie $A V_{4}$ bis $\mathrm{AV}_{6} \ldots \ldots \ldots \ldots \ldots . . . .137$

Tabelle 10: Informationsverteilung der Hidden-Profile-Aufgabe ............................................................... 139

Tabelle 11: Grundregeln der Moderation für Teilnehmer/-in M ............................................................. 145

Tabelle 12: Übersicht über den Versuchsablauf für Diskutant/-innen und Moderator/-in........................ 147

Tabelle 13: Häufigkeiten der Einstimmigkeiten der Gruppenentscheidungen ............................................ 150

Tabelle 14: Verteilung der Häufigkeiten der Gruppenentscheidungen in den einzelnen Bedingungen .... 151

Tabelle 15: Absolute Häufigkeiten der korrekten initialen Entscheidungspräferenzen auf Gruppenebene in den Bedingungen mit vs. ohne Wiederholung getrennt nach unabhängigen Variablen ............... 153

Tabelle 16: Logistische Regression zur Überprüfung der Wirkung der unabhängigen Variablen unter Kontrolle der initialen Entscheidungspräferenz ………………................................................... 153

Tabelle 17: Mittelwerte und Standardabweichungen der Zufriedenheit mit dem Ergebnis in allen

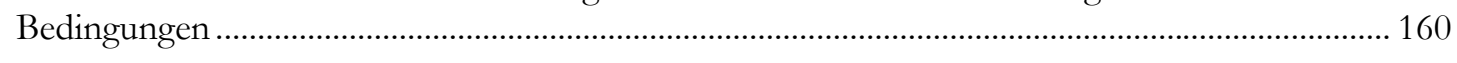

Tabelle 18: Mittelwerte und Standardabweichungen der Zufriedenheit mit dem Verlauf in allen

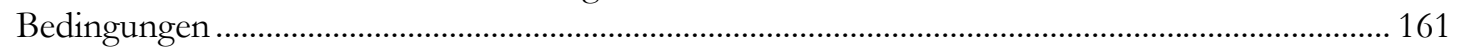

Tabelle 19: Mittelwerte und Standardabweichungen der Zufriedenheit mit dem/der Moderator/-in ...... 162

Tabelle 20: Überblick über die Ergebnisse der Hypothesentestung .............................................................. 165

Tabelle 21: Übersicht über den Ursprung der Kategorien ........................................................................ 206

Tabelle 22: Übersicht über die Trennregeln des Verfahrens zur Einteilung von Kodiereinheiten............. 229

Tabelle 23: Design zur vergleichenden Analyse der Koordinationsprozesse der Entscheidungsfindungsgruppen aus Studie 3 


\section{Anhang}

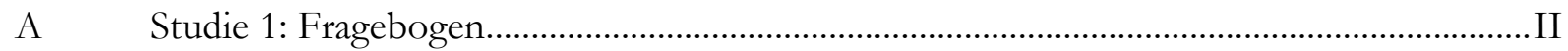

B Studie 2: Leitfaden des fokussierten Interviews............................................................... IX

C Studie 2: Auswertung der Interviews: strukturierte Darstellung der gebildeten Überschriften ...................................................................................................................... XIII

D Studie 3: Untersuchungsmaterial des Experiments ......................................................... XXII

E Kategorien des Kategoriensystems zur mikroanalytischen Erfassung von Koordination in Gruppendiskussionen ........................................................................................................

F Regeln des Manuals zur Einteilung von Kodiereinheiten ........................................ LXVIII 


\section{A Studie 1: Fragebogen}

Liebe Schülerinnen, liebe Schüler,

mit dem vorliegenden Fragebogen möchten wir etwas über Ihre Erfahrungen zur Gestaltung von Gruppendiskussionen erfahren.

Bitte lesen Sie sich das unten beschriebene Szenario durch und beantworten Sie die nachfolgenden Fragen. Dabei gibt es weder richtige noch falsche Antworten. Wir sind ausschließlich an Ihren persönlichen Erfahrung und Meinungen interessiert.

Selbstverständlich werden Ihre Antworten anonym und ausschließlich zum Zweck dieser Untersuchung verwendet.

Bitte blättern Sie trotz Neugier im Vorfeld noch nicht im Fragebogen umher. Für den Zweck der Untersuchung ist es wichtig, dass Sie die einzelnen Fragen in der Reihenfolge beantworten, in der sie im Fragebogen aufgelistet sind. Vielen Dank.

\section{Szenario. Bitte stellen Sie sich folgende Situation vor:}

Im Francke-Gymnasium haben sieben naturwissenschaftlich engagierte Schülerinnen und Schüler gemeinsam mit der Biologie-Lehrerin Frau Weber eine Arbeitsgemeinschaft namens Virtual Science gegründet. Es verbindet sie ihre Begeisterung für die Biologie und das Internet. Am Anfang stand der Wunsch, ein interaktives Computer-Lernprogramm zu gestalten, mit dem biologische Phänomene spielerisch bestaunt und erlernt werden können. Nach einem halben Jahr intensiver Arbeit und Tüftelei ist es der AG gelungen, eine solche Lernumgebung zu entwickeln. Mittlerweile wird sie von vielen SchülerInnen des Francke-Gymnasiums begeistert genutzt. Die entscheidende Innovation dieser Lernumgebung besteht darin, dass sie nicht für den sprachlichen, sondern für den naturwissenschaftlichen Unterricht konzipiert ist. Sie unterscheidet sich daher maßgeblich von anderen Lernprogrammen, die fast ausschließlich zum Erlernen von Fremdsprachen verwendet werden können und stellt somit eine Marktneuheit dar. Das fand auch das Wissenschaftsmagazin NATUR LERNEN und nahm es in die Endausscheidung zum Jugendinnovationspreis 2005 auf.

Leider hat die AG Virtual Science zur Zeit ein anderes Problem: Aufgrund der Einsparungen im Bildungssystem des Landes Niedersachsen soll auch weniger Geld in die Schule fließen. Die jetzige Betreuungslehrerin, Frau Weber, soll eine andere, angeblich wichtigere Aufgabe in der Schule übernehmen. Die Betreuung der AG soll zukünftig wegfallen. Ohne Betreuung durch eine Lehrperson darf die AG allerdings nicht stattfinden.

In einer Diskussion treffen nun die Schulleiterin und drei Vertreterinnen der AG Virtual Science aufeinander. Die Schulleiterin muss die Interessen des Landes Niedersachsen vertreten und sollte eigentlich dafür sorgen, dass Frau Weber die AG nicht mehr betreut sondern ausschließlich für die Oberstufenkoordination zuständig ist. Die Schülerinnen wollen die Schulleiterin überzeugen, dass Frau Weber unbedingt die AG-Betreuung weiter übernehmen müsse, da sie sonst vor dem Aus stünden. Schließlich muss nach einer Lösung gesucht werden, die der Notwendigkeit der finanziellen Einsparung einerseits und dem AG-Fortbestand andererseits gerecht wird.

Moderiert wird diese Diskussion von Stefan. Er ist kein AG-Mitlied, ist allerdings als Schülersprecher ein Vermittler zwischen Schülerlnnen und Schulleitung. Stefan übernimmt in der Diskussion eine neutrale Position. Obwohl ihm persönlich auch am Fortbestand der AG liegt, soll Stefan die Diskussion ausschließlich moderieren und nicht inhaltlich Position beziehen. Er hat in der Vergangenheit mehrfach in ähnlichen Diskussionen zwischen Parteien vermitteln können und durch seine Art zu moderieren meist für einen gemeinsamen Konsens gesorgt. 
Vor welchen Schwierigkeiten steht Stefan?

(Stichpunkte genügen)

Was kann Stefan während der Diskussion machen, damit alle Interessen berücksichtigt werden, alle Vertreterinnen zu Wort kommen und kein Streit, sondern ein Ergebnis entsteht, das von beiden Parteien getragen werden kann? Bitte beachten Sie, dass nicht nach Stefans persönlichen Charaktereigenschaften gefragt wird (z. B. "muss freundlich, aufgeschlossen und unvoreingenommen sein"), sondern dass nach seinem konkreten Verhalten in dieser Diskussion gefragt wird ( $z$. B. „er äußert seine Meinung“; "weist bei Bedarf wiederholt darauf hin, dass die Gesprächsparterinnen sich gegenseitig ausreden lassen“).

(Stichpunkte genügen) 


\section{Bitte blättern Sie erst weiter, wenn Sie die beiden Fragen der vorherigen Seite beantwortet haben.}

Danke! 
Eben sind Sie nach möglichen Verhaltensweisen von Stefan gefragt worden, die er einsetzen könnte, damit in der Diskussion alle Interessen berücksichtig werden, alle Vertreterinnen zu Wort kommen und kein Streit, sondern ein Ergebnis entsteht, was von beiden Parteien getragen werden kann.

Im Folgenden sind einige mögliche Verhaltensweisen aufgelistet. Bitte geben Sie an, für wie sinnvoll Sie es halten, wenn Stefan in der Diskussion dieses Verhalten zeigt.

Bitte kreuzen Sie das Ihrer Meinung nach Zutreffende an.

1. Nach einer Information fragen

überhaupt nicht eher nicht manchmal

eher sinnvoll sehr sinnvoll

weiss nicht sinnvoll sinnvo

$\square$

\section{Nach einer Meinung fragen}

überhaupt nicht eher nicht manchmal sinnvoll sinnvoll sinnvoll

eher sinnvoll sehr sinnvoll

weiss nicht

$\square$

$\square$

$\square$

\section{Ein weiteres Vorgehen vorschlagen}
überhaupt nicht eher nicht manchmal sinnvoll sinnvoll sinnvoll

eher sinnvoll sehr sinnvoll

weiss nicht

4. Bisher Gesagtes zusammenfassen

überhaupt nicht eher nicht manchmal sinnvoll sinnvoll sinnvoll

eher sinnvoll

sehr sinnvoll

weiss nicht

5. Seine eigene Meinung sagen

überhaupt nich sinnvoll

eher nicht

manchmal sinnvoll sinnvoll

eher sinnvoll

sehr sinnvoll

weiss nicht

6. Nicken

überhaupt nich sinnvoll

eher nicht sinnvoll manchmal sinnvoll eher sinnvoll sehr sinnvoll

weiss nicht

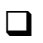




\section{Den Kopf schütteln}

überhaupt nicht eher nicht manchmal sinnvoll sinnvoll

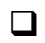

sinnvoll

eher sinnvoll

sehr sinnvoll

weiss nicht

\section{Eine Person zum Sprechen auffordern}

überhaupt nicht eher nicht manchmal sinnvoll sinnvoll eher sinnvoll sehr sinnvoll

weiss nicht

\section{Eine Person nach Ihrer Meinung fragen}
überhaupt nicht sinnvoll
eher nicht sinnvoll
manchmal sinnvoll

eher sinnvoll

sehr sinnvoll

weiss nicht $\square$

\section{Eine Person nach einer Information fragen}

$\begin{array}{cccccc}\text { überhaupt nicht } & \text { eher nicht } & \text { manchmal } & \text { eher sinnvoll } & \text { sehr sinnvoll } & \text { weiss nicht } \\ \text { sinnvoll } & \text { sinnvoll } & \text { sinnvoll } & \square & \square & \end{array}$

\section{Nach Konsens fragen}

überhaupt nicht eher nicht sinnvoll sinnvoll

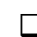

$$
\begin{gathered}
\text { manchmal } \\
\text { sinnvoll }
\end{gathered}
$$

eher sinnvoll

sehr sinnvoll

$\square$

\section{Ein Ziel formulieren}

$\begin{array}{cccccc}\begin{array}{c}\text { überhaupt nicht } \\ \text { sinnvoll }\end{array} & \begin{array}{c}\text { eher nicht } \\ \text { sinnvoll }\end{array} & \begin{array}{c}\text { manchmal } \\ \text { sinnvoll }\end{array} & \text { eher sinnvoll } & \text { sehr sinnvoll } & \text { weiss nicht } \\ \square & \square & \square & \square & \square & \square\end{array}$

\section{Einem unterbreiteten Vorschlag zustimmen oder diesen ablehnen}

überhaupt nicht eher nicht manchmal sinnvoll

$$
\begin{aligned}
& \text { manchmal } \\
& \text { sinnvoll }
\end{aligned}
$$

eher sinnvoll weiss nicht

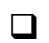


14. Etwas Gesagtes begründen oder erklären

überhaupt nicht
sinnvoll $\begin{gathered}\text { eher nicht } \\ \text { sinnvoll }\end{gathered} \quad \begin{gathered}\text { manchmal } \\ \text { sinnvoll }\end{gathered}$ eher sinnvoll sehr sinnvoll weiss nicht $\begin{array}{llllll}\square & \square & \square & \square & \square & \square\end{array}$

15. „Metakommunikation“ (über das Gespräch sprechen)

überhaupt nicht eher nicht manchmal sinnvoll sinnvoll sinnvoll

eher sinnvoll sehr sinnvoll

weiss nicht

$\square$

$\square$

$\square$

a

\section{Das Gespräch kurz unterbrechen}

überhaupt nicht eher nicht manchma sinnvoll sinnvoll sinnvoll

eher sinnvoll sehr sinnvoll

weiss nicht

口

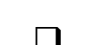

口

a

17. „Aktives“ Zuhören (z. B. durch wiederholtes „hm“)

$\begin{gathered}\text { überhaupt nicht } \\ \text { sinnvoll }\end{gathered}$
$\begin{gathered}\text { eher nicht } \\ \text { sinnvoll }\end{gathered}$

18. Interpretation des Gesagten

$\begin{array}{cccccc}\text { überhaupt nicht } & \text { eher nicht } & \text { manchmal } & \text { eher sinnvoll } & \text { sehr sinnvoll } & \text { weiss nicht } \\ \text { sinnvoll } & \text { sinnvoll } & \text { sinnvoll } & \square & \square & \square \\ \square & \square & \square & \square & \square\end{array}$

19. Etwas Gesagtes bewerten

$\begin{array}{cccccc}\text { überhaupt nicht } & \text { eher nicht } & \text { manchmal } & \text { eher sinnvoll } & \text { sehr sinnvoll } & \text { weiss nicht } \\ \text { sinnvoll } & \text { sinnvoll } & \text { sinnvoll } & \square & \square & \square \\ \square & \square & \square & \square & \square\end{array}$


Noch ein paar persönliche Angaben:

Alter:

Geschlecht: weiblich $\square$

männlich $\square \quad$ (Zutreffendes bitte

Klassenstufe:

Studienwunsch:

Leistungsfächer

bzw. -kurse:

Nationalität:

Hat Sie an diesem Fragebogen irgendetwas gestört? Wenn ja, bitte geben Sie kurz an, was Sie gestört hat.

Herzlichen Dank für Ihre Teilnahme an dieser Untersuchung! 


\section{B Studie 2: Leitfaden des fokussierten Interviews}

\section{Leitfaden für ein fokussiertes ExpertInneninterview}

\section{Ziel des Interviews:}

- Ich beschäftige mich in meiner Promotion mit Kommunikation und Koordination in Kleingruppen. Mich interessiert, wie sich explizite Koordination durch eine Diskussionsleiterin / einen Diskussionsleiter im Verlauf von Problemlöse- und Entscheidungsfindungsdiskussionen zeigt.

- Mit expliziter Koordination meine ich direktes, steuerndes, meist auch verbales Eingreifen der Diskussionsleiterin / des Diskussionsleiters in die Diskussion.

- Es gibt dato empirische Untersuchung zu ausgewählten, spezifischen Interventionen ( $z$. B. zum Wiederholen von Informationen) und dessen Einfluss auf die Entscheidungsgüte. Aber es gibt noch keine umfassende psychologische Theorie expliziter Koordination und Moderation - daher führe ich diese Interviews, um mehr darüber zu erfahren, was erfahrene ModeratorInnen / Führungskräfte darüber denken.

\section{Ablauf des Interviews:}

- Dauer ca. eine halbe bis dreiviertel Stunde

- Ich werde beginnen, indem ich Sie bitte, sich eine bestimmte Situation vorzustellen, anschließend sprechen wir über mögliches Verhalten in dieser Situation.

- Da ich an Ihren Erfahrungen und Ideen interessiert bin, werde ich Sie erzählen lassen und selbst nicht sehr viel sagen.

- Ich bitte Sie, mir alle Gedanken zu der Situation und zum Thema Moderationsverhalten zu sagen, auch wenn diese Ihnen selbst trivial erscheinen.

- Ich würde das Gespräch gern auf Tonband aufnehmen. Ist das für Sie in Ordnung?

Tonband ein. 
Bitte versuchen Sie sich eine problematische Diskussion vorzustellen bzw. zu erinnern, zu der man Sie als Expertin/en und erfahrene/n Moderator/in gebeten hat, um diese prekäre oder spannungsgeladene oder sonst irgendwie schwierige Diskussion zu moderieren, wobei Sie das Gefühl hatten, Ihr ganzes Moderationsgeschick aufbieten zu müssen.

Bitte beschreiben Sie diese Situation.

Vor welchen Schwierigkeiten und Herausforderungen standen Sie Ihrer Meinung nach?

Was haben Sie in dieser Situation gedacht (und möglicherweise empfunden)?

Was genau haben Sie in dieser Situation gemacht, um den geschilderten Herausforderungen gerecht werden? 
Gibt es darunter Verhaltensweisen, die Sie besonders gern oder besonders ungern ausführen? Warum?

Wenn Sie an die eben beschriebenen Interventionen (z. B. ..) denken, können Sie diese von bestimmten vorherigen Situationen oder Verhaltensweisen der Gruppe / einzelner Mitglieder abhängig machen?

Es geht darum, präzise zu überlegen, wann Sie bestimmte Interventionen oder Verhaltensweisen ausführen.

Z. B. Fragen Sie eine Person, die zuvor lange nichts gesagt hat, nach ihrer Meinung. $($ Wenn $\rightarrow$ Dann)

Woher haben Sie Ihr Wissen und die Fertigkeiten, die Sie eben berichtet haben, erworben? (Erfahrung?, Training?, Lektüre einschlägiger Literatur?) 
Abschließend habe ich noch ein paar soziodemografische Fragen:

Wie alt sind Sie:

\section{Geschlecht:}

Wie lange arbeiten

Sie bereits als

Moderatorin?

Hat Sie an den Fragen oder an dem Interview insgesamt irgendetwas gestört? Wenn ja, würden Sie das bitte kurz schildern?

Herzlichen Dank, dass Sie sich für dieses für Interview bereit erklärt haben!

Falls Interesse besteht, kann ich Ihnen gern die Auswertung der Interviewergebnisse zukommen lassen. 
C Studie 2: Auswertung der Interviews: strukturierte Darstellung der gebildeten Überschriften 


\section{Studie 2, Person A}

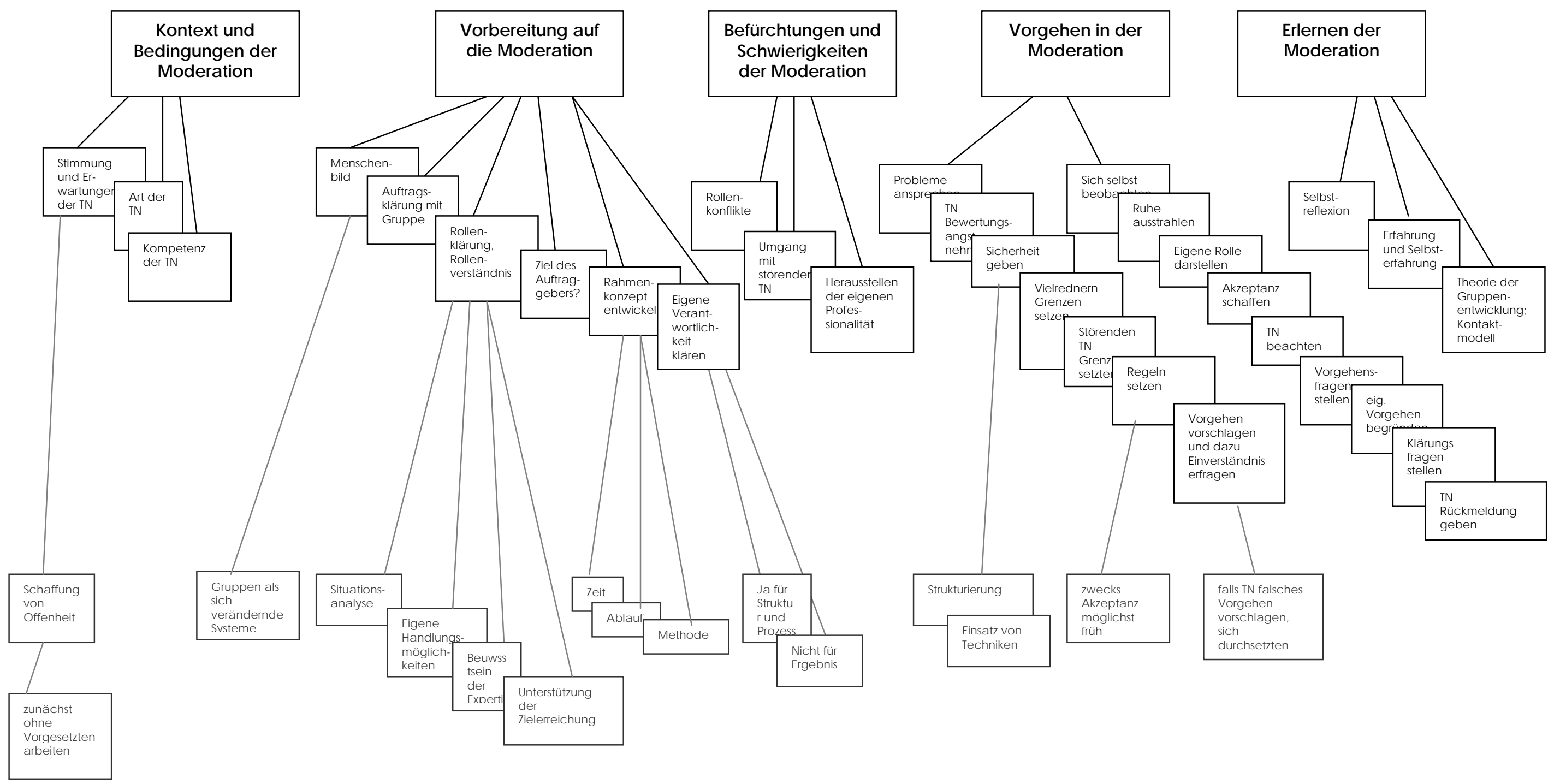




\section{Studie 2, Person B}

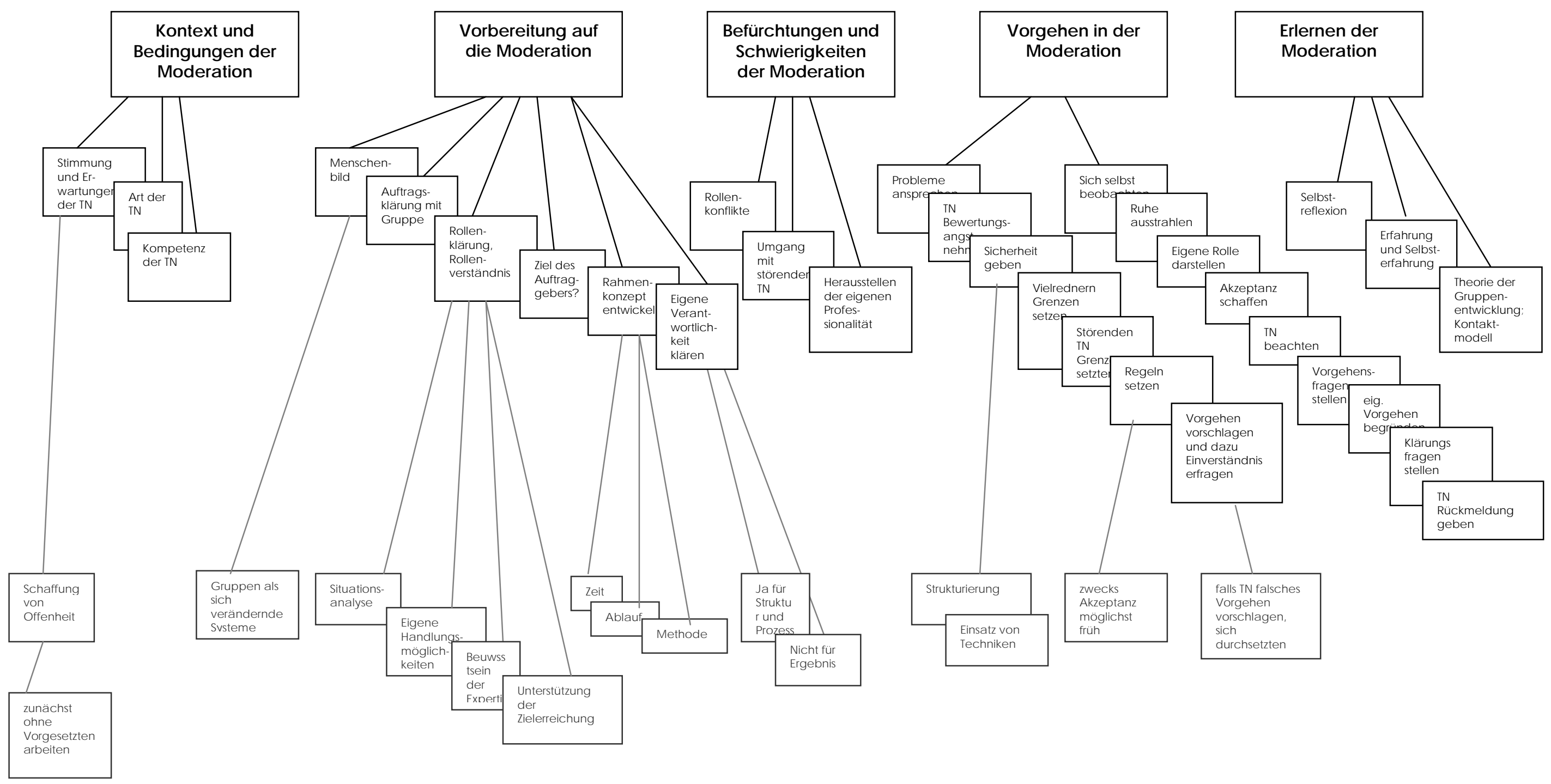


Studie 2, Person C

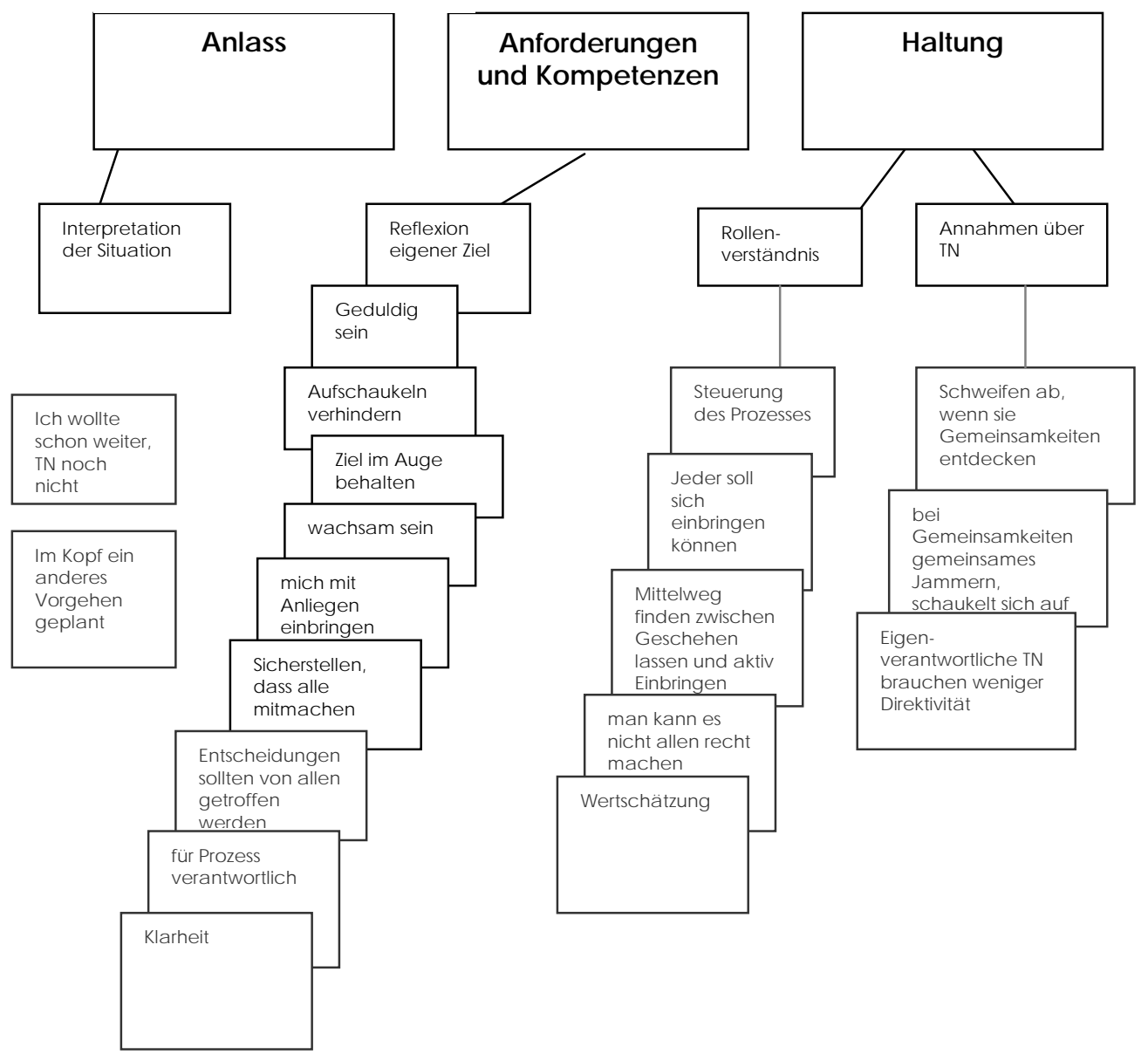

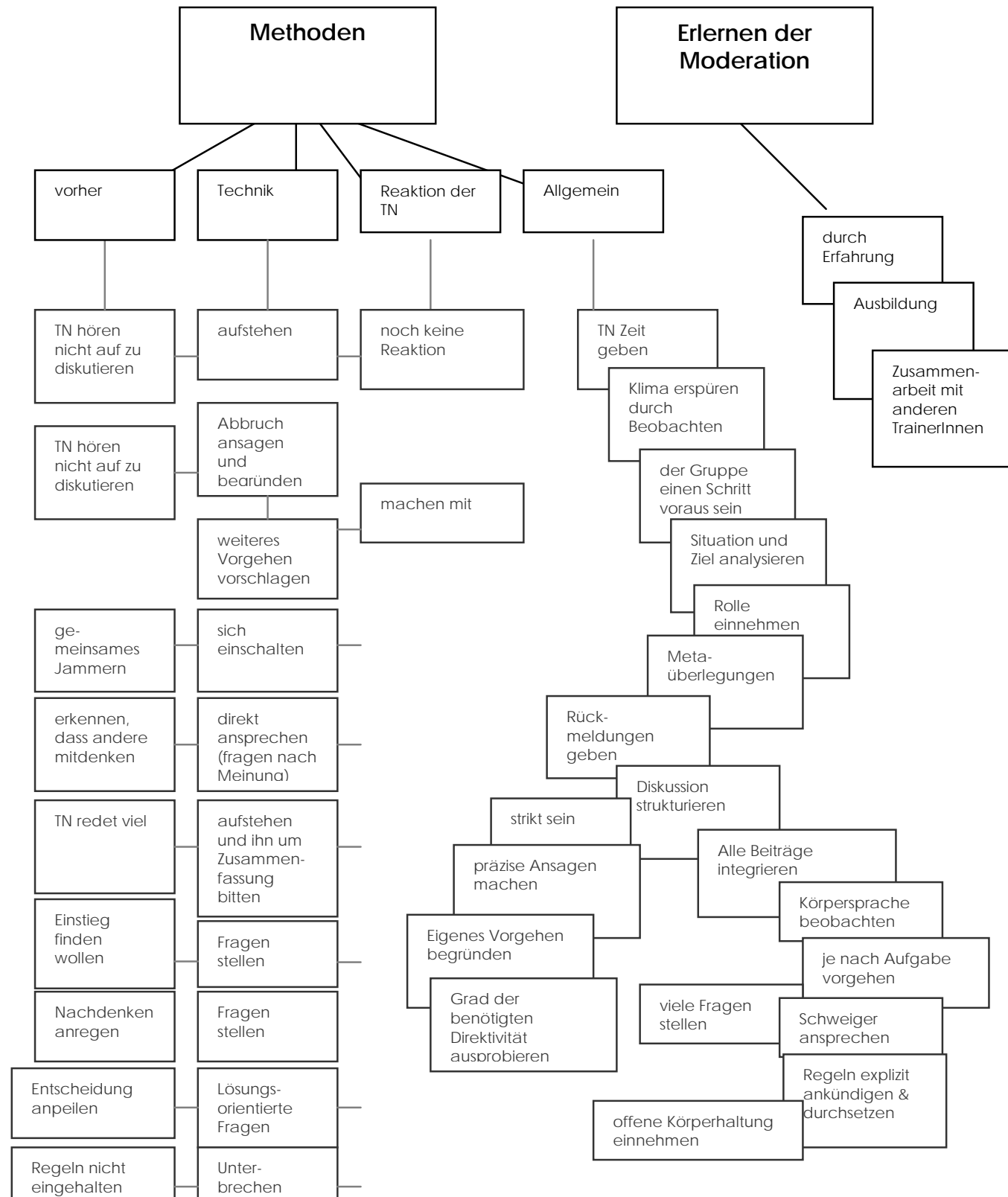

TN ... Teilnehmer/innen 


\section{Studie 2, Person D}

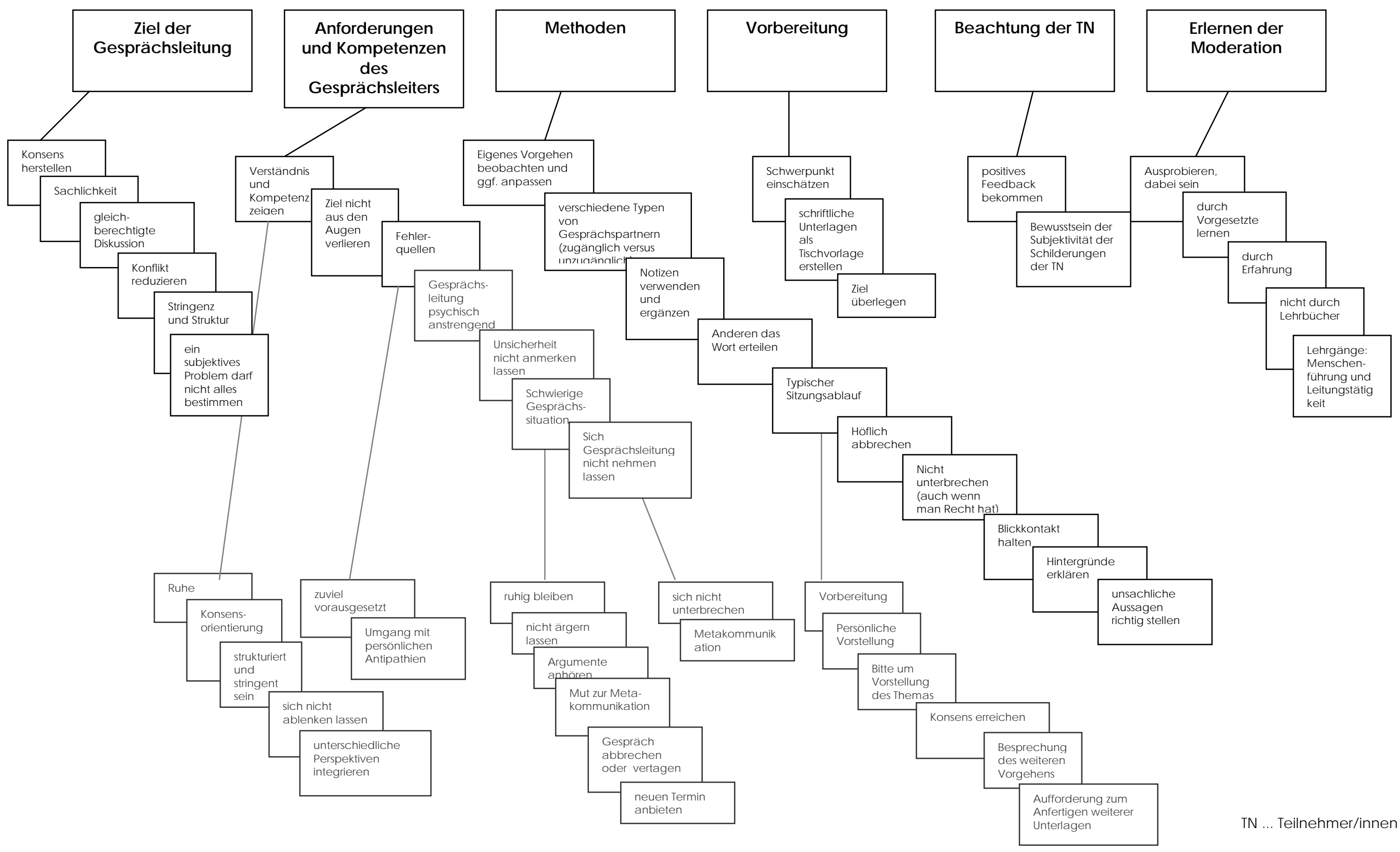




\section{Studie 2, Person E}

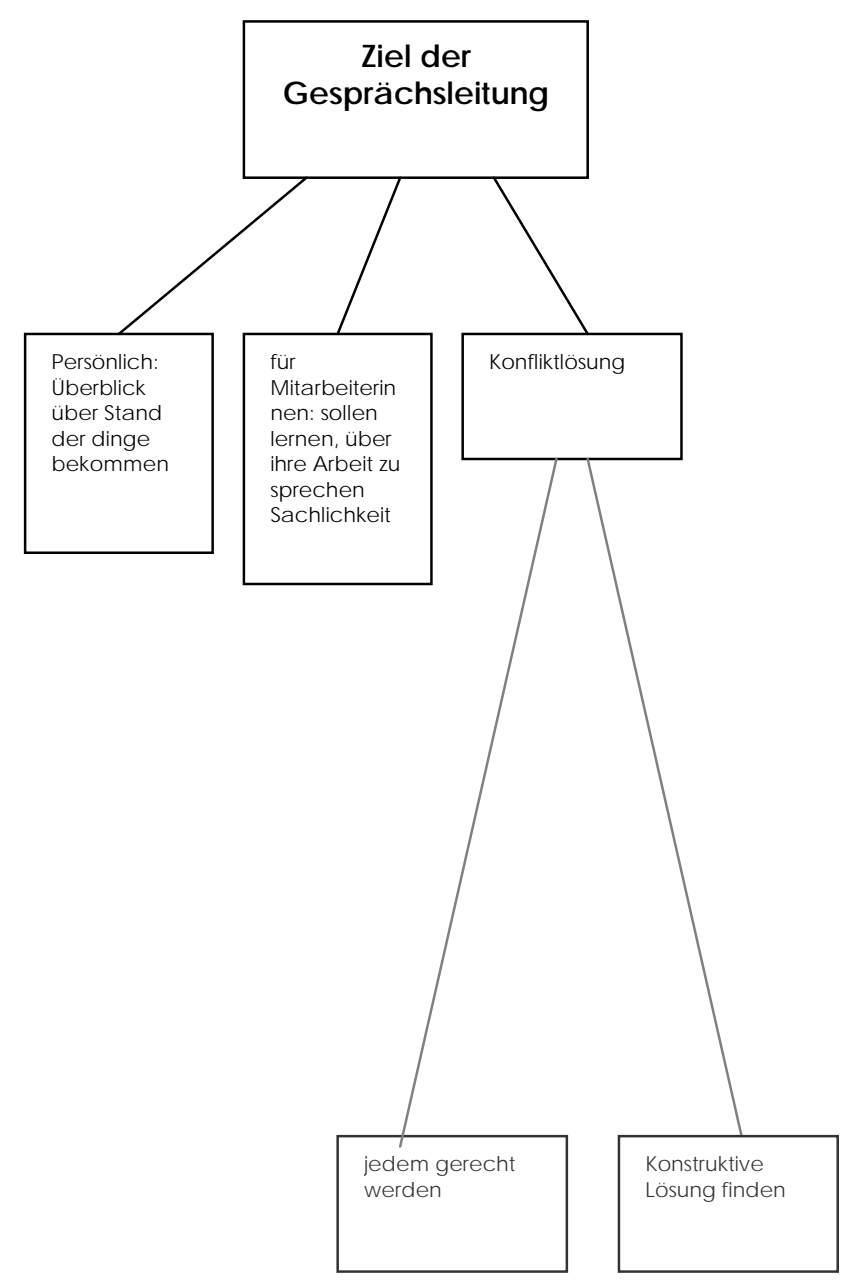

Anfordenungen und Kompetenzen der

Gesprächsleiterin

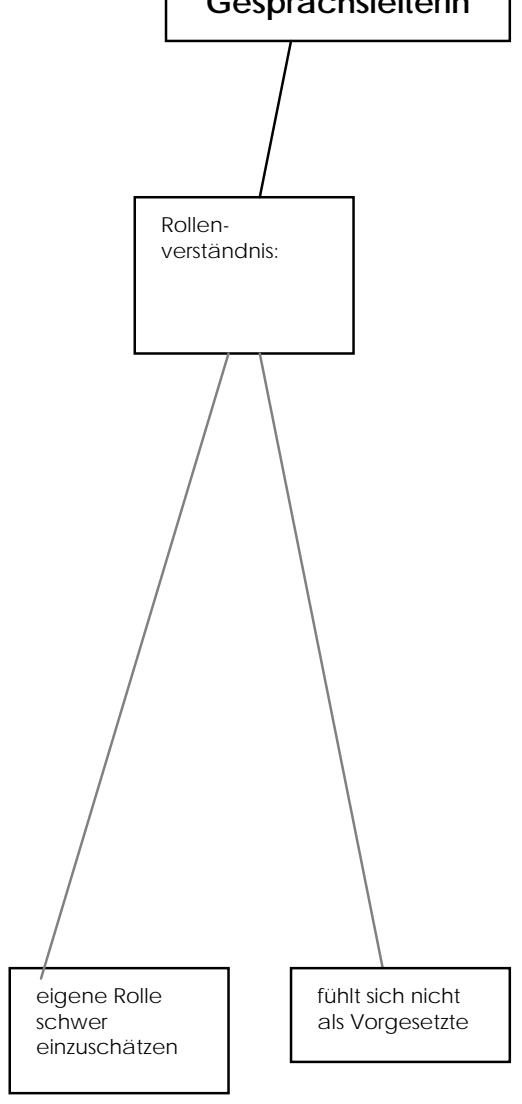

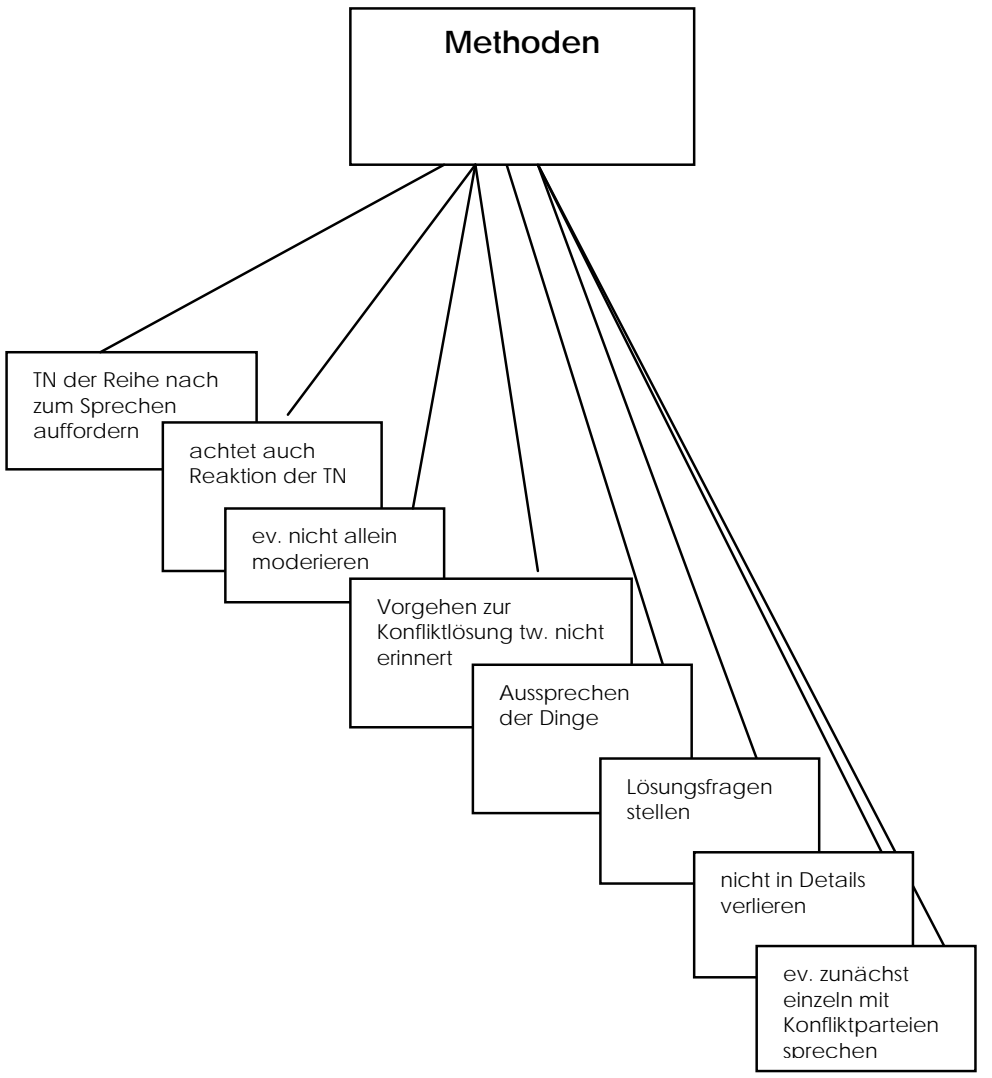




\section{Studie 2, Person F}
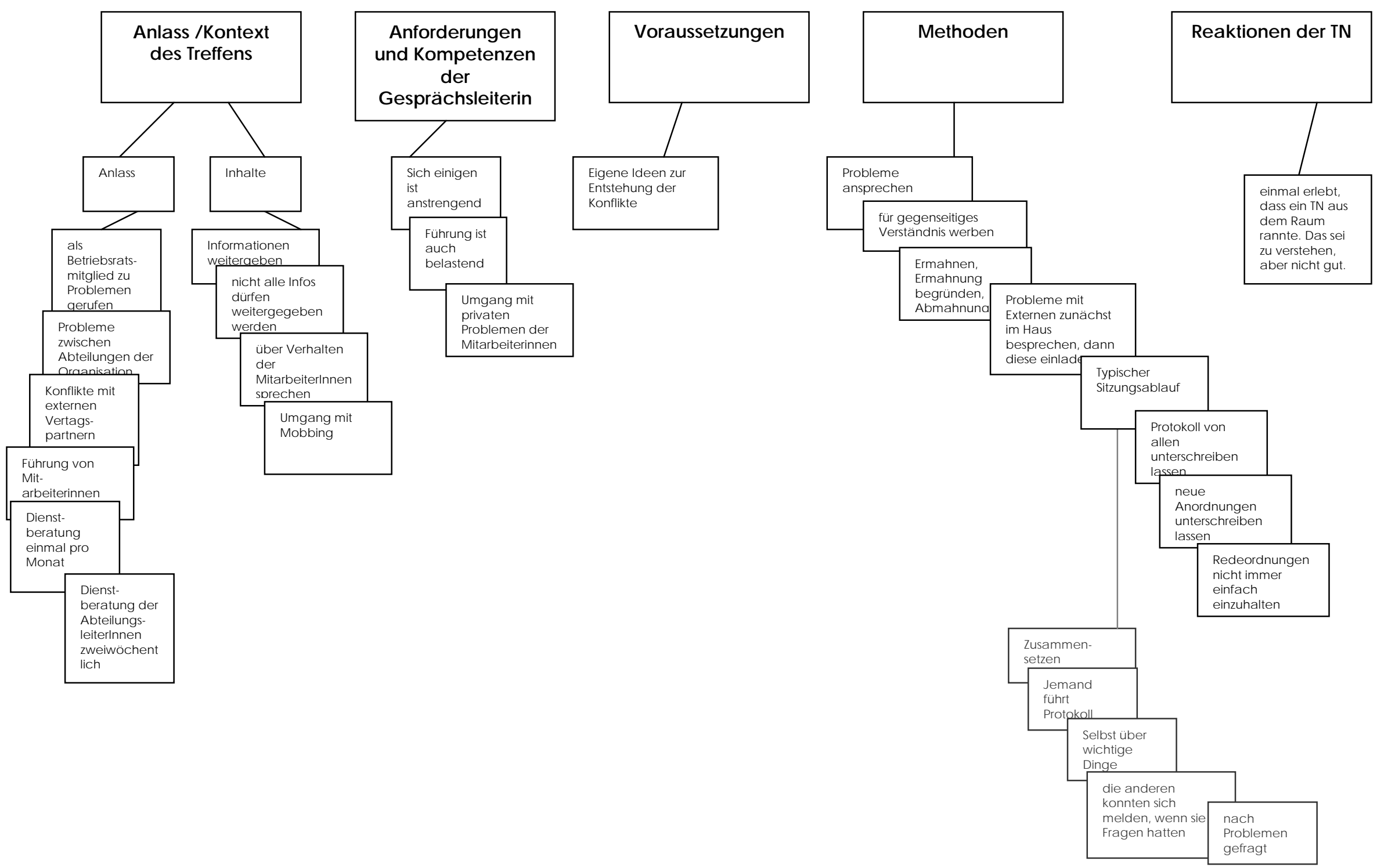
Studie 2, Person G

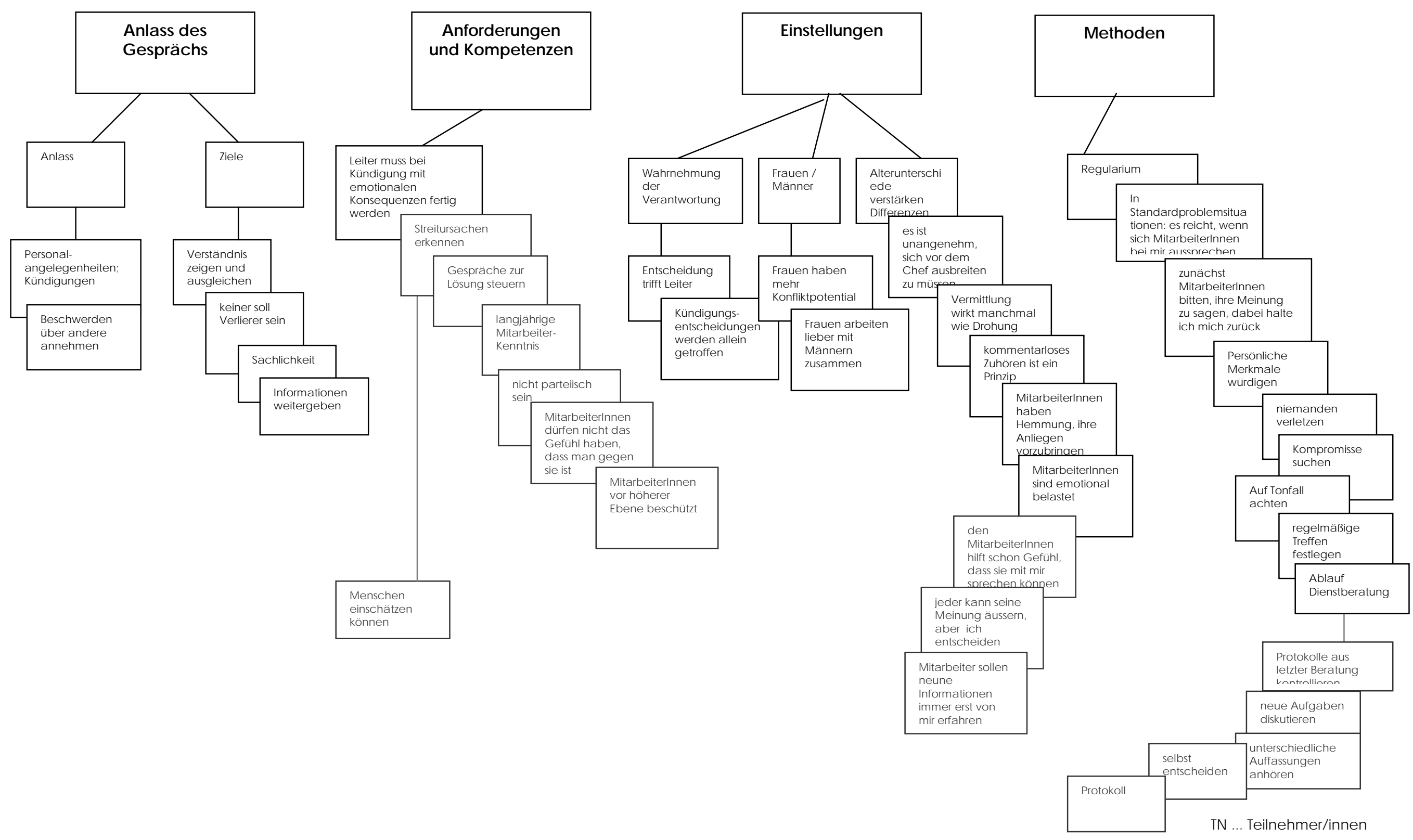


Studie 2, Person H
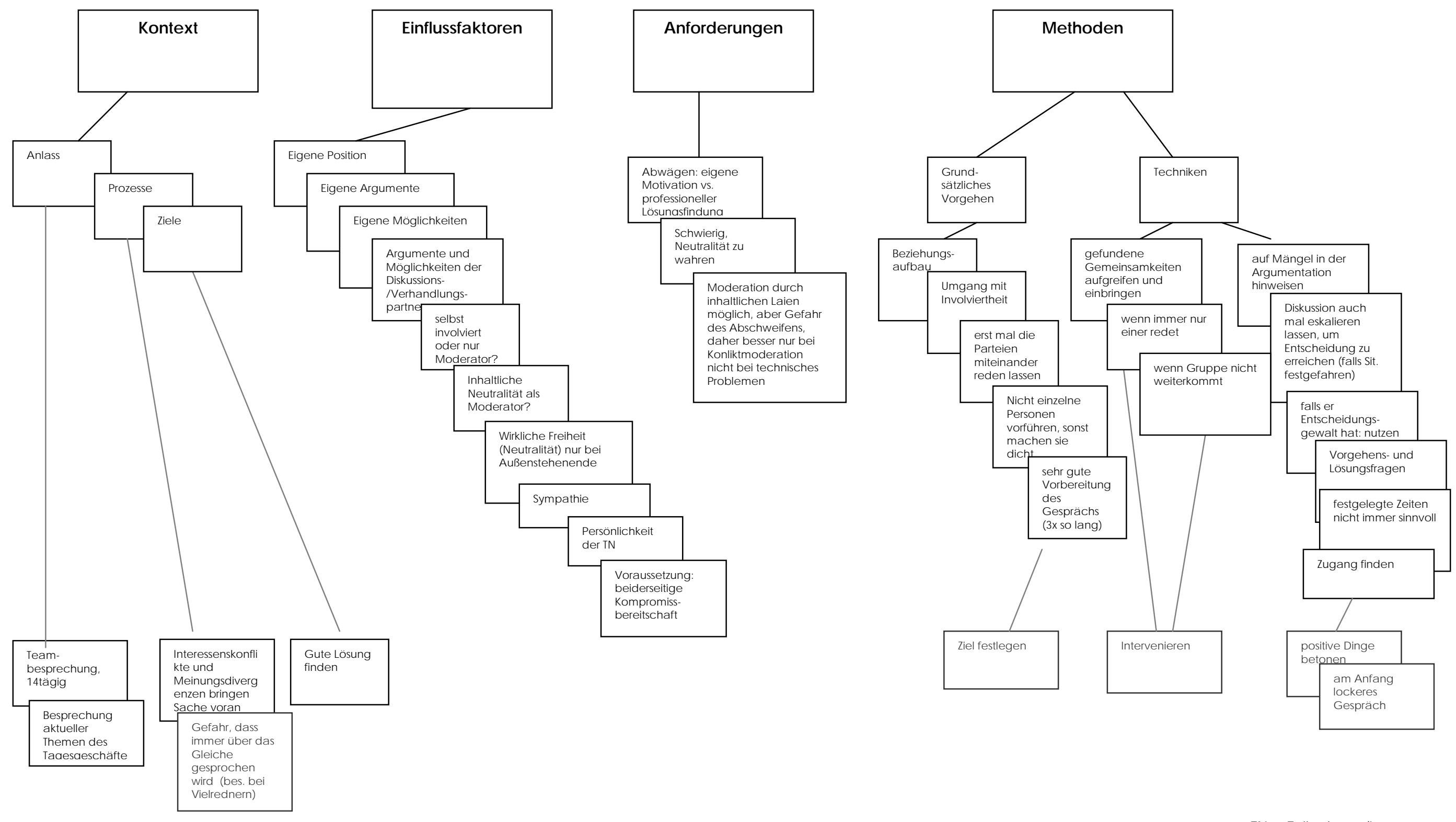

TN ... Teilnehmer/innen 
D Studie 3: Untersuchungsmaterial des Experiments

1. Informationsverteilung im „Pilotenfall“

2. Kandidatenblatt, exemplarisch für Diskutant/-in $Y$

3. Unterlagen für die Diskutanten/-innen, exemplarisch für Diskutant/-in X

4. Instruktionen für die Moderatoren/-innen, Bedingungen 0 bis 3

5. Fragebogen für die Moderatoren/-innen 


\section{Informationsverteilung im „Pilotenfall“}

\section{Kandidat A}

Positive Items:

(1.) verfügt über ein sehr gutes Gespür für Gefahrensituationen

(2.) kann komplexe Zusammenhänge gut überblicken

geteilt

(3.) hat ein ausgezeichnetes räumliches Vorstellungsvermögen

geteilt

(4.) weist sehr gute Führungsqualitäten auf

geteilt

geteilt

Negative Items:
(5.) verträgt bisweilen keine Kritik
(6.) ist manchmal etwas hektisch
(7.) gilt als Angeber
(8.) gilt als nicht offen für Neuerungen
(9.) ist unfreundlich
(10.) ernährt sich sehr ungesund

Gruppenmitglied X Gruppenmitglied $X$ Gruppenmitglied $Y$ Gruppenmitglied $Y$ Gruppenmitglied Z Gruppenmitglied $Z$

\section{Kandidat B}

Positive Items:
(11.) behält in Krisensituationen einen kühlen Kopf
geteilt
(12.) man kann sich hundertprozentig auf ihn verlassen
geteilt
(13.) kann Wetterlagen sehr gut einschätzen
geteilt
(14.) verfügt über sehr gute fachspezifische Computerkenntnisse
geteilt

Negative Items:

(15.) gilt als nörglerisch

(16.) gilt als wenig kooperativ

(17.) verfügt über ein unterdurchschnittliches Zahlengedächtnis

(18.) lästert über seine Mitarbeiter

(19.) gilt als arrogant

(20.) vergreift sich manchmal im Ton

Gruppenmitglied X Gruppenmitglied $X$ Gruppenmitglied $Y$ Gruppenmitglied $Y$ Gruppenmitglied Z Gruppenmitglied $Z$

\section{Kandidat C}

Positive Items:

(21.) kann sehr schnell die richtigen Entscheidungen treffen

geteilt

(22.) ist stressresistent

(23.) fördert ein gutes Klima innerhalb der Crew

(24.) ist sehr gewissenhaft

(25.) ist im Umgang mit komplizierter Technik sehr geschickt

(26.) die Sicherheit inm anvertrauter Personen geht inm über alles

(27.) zeigt sehr gute Leistungen hinsichtlich der Daueraufmerksamkeit

Gruppenmitglied $X$ Gruppenmitglied $X$ Gruppenmitglied $Y$ Gruppenmitglied $Y$ Gruppenmitglied $Z$ Gruppenmitglied Z

Negative Items:
(28.) ist verbal nicht gewandt
(29.) gilt als Egozentriker
geteilt
(30.) nimmt an Fortbildungen nur ungern teil

\section{Kandidat D}

Positive Items:

(31.) ist in der Lage, auf unvorhergesehene Ereignisse adäquat zu reagieren geteilt

(32.) kann sich sehr gut konzentrieren

(33.) verfügt über eine hohe Problemlösefähigkeit

(34.) ist sehr verantwortungsbewusst

geteilt

geteilt

geteilt

Negative Items:

(35.) gilt als überheblich

(36.) ist nicht sehr gut für die Leitung eines Teams geeignet

(37.) gilt als besserwisserisch

(38.) ist leicht aufbrausend

(39.) gilt als launisch

(40.) gilt als Einzelgänger 


\section{Kandidatenblatt, exemplarisch für Diskutant/-in $Y$}

\section{Kandidat A}

- verfügt über ein sehr gutes Gespür für Gefahrensituationen

- gilt als Angeber

- kann komplexe Zusammenhänge gut überblicken

- hat ein ausgezeichnetes räumliches Vorstellungsvermögen

- gilt als nicht offen für Neuerungen

- weist sehr gute Führungsqualitäten auf

\section{Kandidat B}

- behält in Krisensituationen einen kühlen Kopf

- verfügt über ein unterdurchschnittliches Zahlengedächtnis

- man kann sich hundertprozentig auf inn verlassen

- kann Wetterlagen sehr gut einschätzen

- lästert über seine Mitarbeiter

- verfügt über sehr gute fachspezifische Computerkenntnisse

\section{Kandidat C}

- ist verbal nicht gewandt

- kann sehr schnell die richtigen Entscheidungen treffen

- gilt als Egozentriker

- ist sehr gewissenhaft

- nimmt an Fortbildungen nur ungern teil

- ist im Umgang mit komplizierter Technik sehr geschickt

\section{Kandidat D}

- ist in der Lage auf unvorhergesehene Ereignisse adäquat zu reagieren

- gilt als besserwisserisch

- kann sich sehr gut konzentrieren

- verfügt über eine hohe Problemlösefähigkeit

- ist leicht aufbrausend

- ist sehr verantwortungsbewusst 


\section{Unterlagen für die Diskutanten/-innen, exemplarisch für Diskutant/- in $\mathrm{X}$}

Sehr geehrte/r Versuchsteilnehmerlin,

eine deutsche Fluggesellschaft will in den nächsten Jahren mehr Langstreckenflüge anbieten. Da für Langstreckenflüge ausschließlich Flugkapitäne mit langjähriger Berufserfahrung eingesetzt werden und die Fluggesellschaft nicht über genügend derartige Piloten verfügt, wurden über Stellenausschreibungen Flugkapitäne mit langjähriger Berufserfahrung gesucht.

Derartige Entscheidungen werden in der Regel von Expertengruppen getroffen. Wir haben hier einen realen Entscheidungsfall übernommen und für Sie anonymisiert.

Sie werden im Folgenden schriftliche Informationen zu mehreren Piloten erhalten, die sich auf die Stellenausschreibung beworben haben. Alle Piloten verfügen über eine in etwa gleich hohe Anzahl an Flugstunden und langjährige Flugerfahrung. Alle Informationen stammen ausschließlich aus zuverlässigen Quellen, beispielsweise aus etablierten psychologischen und medizinischen Testverfahren sowie aus Beurteilungen von mehreren Kollegen und Vorgesetzten.

Sie sollen die Ihnen individuell vorliegenden Informationen durchgehen und eine individuelle Einschätzung abgeben. Diese individuelle Arbeit wird ca. eine halbe Stunde dauern, in der Ihnen die Versuchsleiterin die Informationen zur Verfügung stellen und verschiedene Fragebögen austeilen wird. Anschließend sollen Sie mit zwei anderen Personen in einer Gruppe Informationen zu diesem Entscheidungsfall austauschen, über die Eignung der vier Bewerber diskutieren und gemeinsam versuchen, den besten Bewerber herauszufinden. In der Diskussion werden Sie durch eine vierte Person unterstützt, die Sie bei der Entscheidungsfindung begleitet. Wenn sich Ihre Gruppe für den eindeutig besten Piloten entscheidet, nimmt sie an einer Verlosung teil, bei der es für jedes Gruppenmitglied einen CD-Gutschein im Wert von $25 €$ zu gewinnen gibt.

Bitte machen Sie zunächst noch folgende allgemeine Angaben zu Ihrer Person.

\begin{tabular}{|rl|}
\hline Geschlecht: & \\
\cline { 2 - 2 } Alter: & \\
Studienfach: & \\
Semesterzahl: & \\
Code: & \\
\cline { 2 - 2 } & (wird von der Versuchsleiterin vergeben) \\
\hline
\end{tabular}


Code:

\section{Bewertung der Informationen zu den vier Bewerbern}

Bitte ordnen Sie für jeden der vier Bewerber die Informationen danach, ob diese Information für den Bewerber spricht, d. h. für seine Eignung als Pilot, oder ob sie gegen den Bewerber spricht.

Geben Sie bitte darüber hinaus an, wie positiv oder negativ diese Informationen ihrer Ansicht nach im Hinblick auf die Eignung des Bewerbers für die Pilotenstelle sind.

\section{Bewerber A}

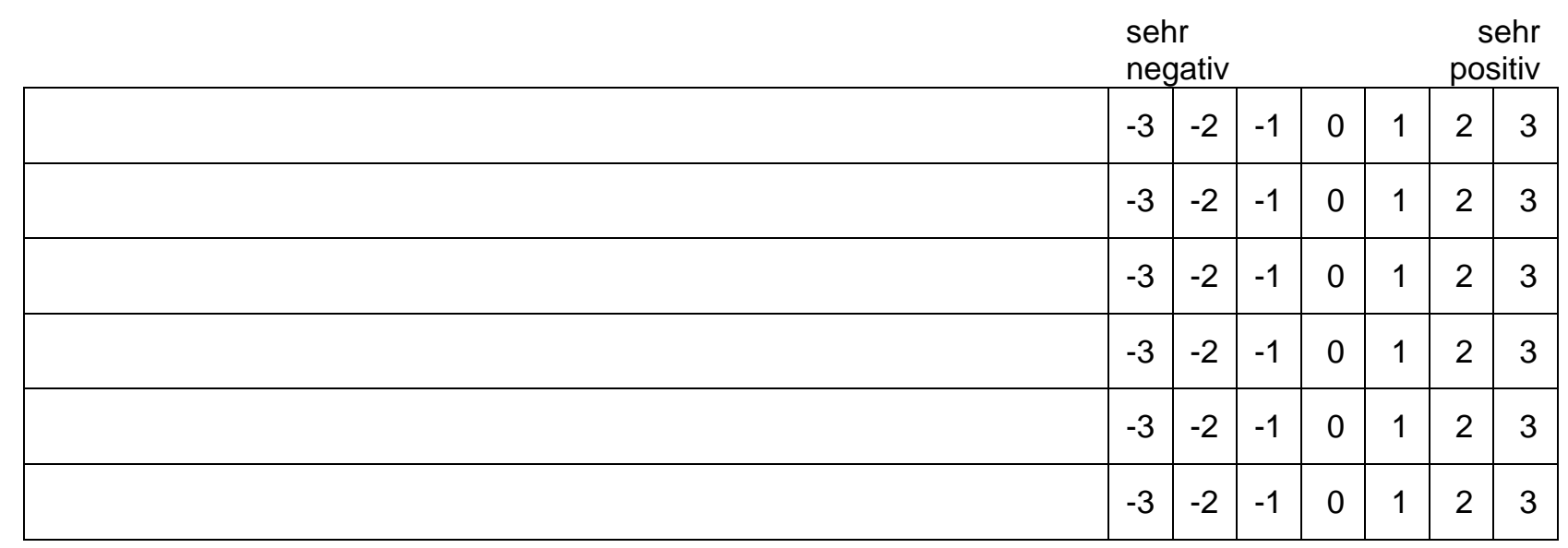

\section{Bewerber B}

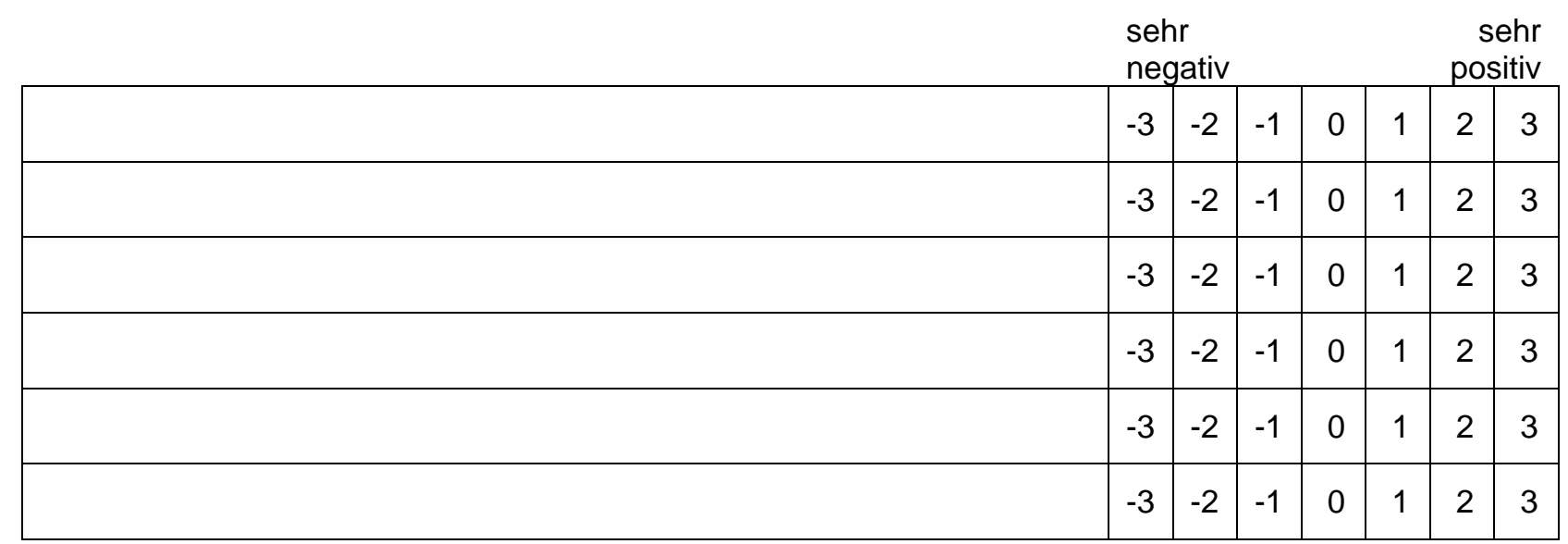


Code:

\section{Bewerber C}

\begin{tabular}{|l|l|l|l|l|l|l|l|}
\multicolumn{4}{c}{ sehr } \\
\hline & \multicolumn{3}{c}{ sehr nesiv } \\
\hline & -3 & -2 & -1 & 0 & 1 & 2 & 3 \\
\hline & -3 & -2 & -1 & 0 & 1 & 2 & 3 \\
\hline & -3 & -2 & -1 & 0 & 1 & 2 & 3 \\
\hline & -3 & -2 & -1 & 0 & 1 & 2 & 3 \\
\hline & -3 & -2 & -1 & 0 & 1 & 2 & 3 \\
\hline & -3 & -2 & -1 & 0 & 1 & 2 & 3 \\
\hline
\end{tabular}

Bewerber D

\begin{tabular}{|l|l|l|l|l|l|l|l|}
\multicolumn{4}{c}{ sehr } \\
\hline & \multicolumn{3}{c}{ sehr nosiv } \\
\hline & -3 & -2 & -1 & 0 & 1 & 2 & 3 \\
\hline & -3 & -2 & -1 & 0 & 1 & 2 & 3 \\
\hline & -3 & -2 & -1 & 0 & 1 & 2 & 3 \\
\hline & -3 & -2 & -1 & 0 & 1 & 2 & 3 \\
\hline & -3 & -2 & -1 & 0 & 1 & 2 & 3 \\
\hline & -3 & -2 & -1 & 0 & 1 & 2 & 3 \\
\hline
\end{tabular}


Code:

\section{Einzelentscheidung}

Treffen Sie nun bitte auf Grundlage der Ihnen vorgelegten Informationen eine vorläufige Entscheidung: Welcher der Bewerber ist Ihrer Meinung nach am besten geeignet?

\begin{tabular}{|ll|}
\hline Pilot A & o \\
Pilot B & 0 \\
Pilot C & 0 \\
Pilot D & 0 \\
\hline
\end{tabular}

\section{Beantworten Sie bitte die folgenden Fragen:}

1. Wie sicher sind Sie, dass Ihre Entscheidung richtig ist?

\begin{tabular}{llllllllll} 
überhaupt nicht & 0 & 1 & 2 & 3 & 4 & 5 & 6 & 7 & sehr \\
\hline
\end{tabular}

2. Für wie geeignet halten Sie Bewerber $A$ ?

\begin{tabular}{llllllllll|} 
überhaupt nicht & 0 & 1 & 2 & 3 & 4 & 5 & 6 & 7 & sehr \\
\hline
\end{tabular}

3. Für wie geeignet halten Sie Bewerber B?

\begin{tabular}{lllllllllll|} 
überhaupt nicht & 0 & 1 & 2 & 3 & 4 & 5 & 6 & 7 & sehr \\
\hline
\end{tabular}

4. Für wie geeignet halten Sie Bewerber C?

\begin{tabular}{lllllllllll|} 
überhaupt nicht & 0 & 1 & 2 & 3 & 4 & 5 & 6 & 7 & sehr \\
\hline
\end{tabular}

5. Für wie geeignet halten Sie Bewerber D?

\begin{tabular}{llllllllll|} 
überhaupt nicht & 0 & 1 & 2 & 3 & 4 & 5 & 6 & 7 & sehr \\
\hline
\end{tabular}

6. Für wie wahrscheinlich halten Sie es, dass Sie Ihre Einzelentscheidung im Laufe der anschließenden Gruppendiskussion revidieren?

\begin{tabular}{|lllllllllll|} 
überhaupt nicht & 0 & 1 & 2 & 3 & 4 & 5 & 6 & 7 & sehr \\
\hline
\end{tabular}

7. Wie wichtig war es Ihnen bei der Aufgabenstellung, die richtige Entscheidung zu treffen?

\begin{tabular}{|llllllllll|} 
überhaupt nicht & 0 & 1 & 2 & 3 & 4 & 5 & 6 & 7 & sehr \\
\hline
\end{tabular}


Code:

\section{Erinnerungsfragebogen}

Um den Prozess der Entscheidungsfindung besser zu verstehen, ist es für uns wichtig zu wissen, über welche Informationen Sie nun verfügen.

Bitte schreiben Sie alle Informationen zu den vier Bewerbern auf, an die Sie sich noch erinnern können.

\section{Bewerber A}

\section{Bewerber B}




\section{Bewerber C}

Bewerber D 


\section{Instruktion zur Gruppendiskussion}

Sie sollen nun als Gruppe versuchen, aufgrund der Informationen, die Sie von uns erhalten haben, den besten Bewerber herauszufinden.

Bitte beachten Sie: Bei derartigen Entscheidungen kommt es nur sehr selten vor, dass alle Personen, die an der Entscheidung beteiligt sind, über die gleichen Informationen verfügen. In der Regel ist es vielmehr eher so, dass die einzelnen Personen über unterschiedliche Informationen verfügen und versuchen müssen, sich auf den besten Bewerber zu einigen.

Um dieser realen Situation möglichst nahe zu kommen, haben auch Sie nicht alle exakt die gleichen Informationen bekommen. Es wurde jedoch sorgfältig darauf geachtet, dass Sie keine widersprüchlichen Informationen erhalten haben.

\section{Wichtig:}

Aufgrund der Gesamtinformation, die sie als Gruppe erhalten haben, gibt es gemäß übereinstimmendem Expertenurteil einen eindeutig besten Piloten.

Während der Diskussion werden Sie von einer weiteren Person unterstützt, die von uns während Ihrer individuellen Vorbereitung mit grundsätzlichen Regeln der Gesprächsleitung vertraut gemacht wurde. Er/Sie ist oberflächlich mit dem Fall vertraut, verfügt allerdings nicht über Ihre jeweiligen individuellen Informationen. Daher ist er/sie inhaltlich unparteilich, dass heißt, er/sie wird keine inhaltlichen Beiträge zur Diskussion beisteuern.

Diese Person wird sich gleich vorstellen und anschließend die Diskussion eröffnen. 


\section{Gruppenentscheidung}

Code

Code

Code

Treffen Sie nun bitte als Gruppe auf Grundlage der Ihnen insgesamt vorliegenden Informationen eine Entscheidung.

\section{Pilot A o}

Pilot Bo

Pilot Co

Pilot Do

\section{Beantworten Sie bitte gemeinsam die folgenden Fragen:}

Handelte es sich bei Ihrer Entscheidung um eine einstimmige Entscheidung?

ja

nein, Gruppenmitglied war für Pilot (ausfüllen, falls keine einstimmige Entscheidung)

1. Wie sicher sind Sie, dass Ihre Entscheidung richtig ist?

\begin{tabular}{|clllllllll|}
\hline überhaupt nicht & 0 & 1 & 2 & 3 & 4 & 5 & 6 & 7 & sehr \\
\hline
\end{tabular}

2. Für wie geeignet halten Sie Bewerber $A$ ?

\begin{tabular}{|llllllllll|}
\hline überhaupt nicht & 0 & 1 & 2 & 3 & 4 & 5 & 6 & 7 & sehr \\
\hline
\end{tabular}

3. Für wie geeignet halten Sie Bewerber B?

\begin{tabular}{|llllllllll|}
\hline überhaupt nicht & 0 & 1 & 2 & 3 & 4 & 5 & 6 & 7 & sehr \\
\hline
\end{tabular}

4. Für wie geeignet halten Sie Bewerber $C$ ?

\begin{tabular}{|llllllllll|}
\hline überhaupt nicht & 0 & 1 & 2 & 3 & 4 & 5 & 6 & 7 & sehr \\
\hline
\end{tabular}

5. Für wie geeignet halten Sie Bewerber D?

\begin{tabular}{|llllllllll|}
\hline überhaupt nicht & 0 & 1 & 2 & 3 & 4 & 5 & 6 & 7 & sehr \\
\hline
\end{tabular}

6. Wie wichtig war es Ihnen bei der Aufgabenstellung, die richtige Entscheidung zu treffen?

\begin{tabular}{|llllllllll|}
\hline überhaupt nicht & 0 & 1 & 2 & 3 & 4 & 5 & 6 & 7 & sehr \\
\hline
\end{tabular}

7. Wie überzeugt sind Sie, dass Sie sich für den richtigen Bewerber entschieden haben?

\begin{tabular}{|llllllllll|} 
überhaupt nicht & 0 & 1 & 2 & 3 & 4 & 5 & 6 & 7 & sehr \\
\hline
\end{tabular}


Code:

\section{Fragen zur Beurteilung der Gruppendiskussion}

\section{Bitte erinnern Sie sich zum Abschluss nochmals an die Gruppendiskussion und an die Gruppenentscheidung. Beantworten Sie hierzu bitte folgende Fragen:}

1. Wie sehr stehen Sie zu der Entscheidung, die in der Gruppe getroffen wurde?

$\begin{array}{llllllllll}\text { überhaupt nicht } & 0 & 1 & 2 & 3 & 4 & 5 & 6 & 7 & \text { sehr }\end{array}$

2. Wie gut ist die Entscheidung, die die Gruppe getroffen hat?

$\begin{array}{lllllllllll}\text { überhaupt nicht } & 0 & 1 & 2 & 3 & 4 & 5 & 6 & 7 & \text { sehr }\end{array}$

3. Haben die anderen Gruppenmitglieder während der Diskussion Informationen genannt, die Sie nicht wussten?

$\begin{array}{llllllllll}\text { überhaupt nicht } & 0 & 1 & 2 & 3 & 4 & 5 & 6 & 7 & \text { sehr }\end{array}$

4. Wie zufrieden sind Sie mit dem Ergebnis der Zusammenarbeit in der Gruppe?

\begin{tabular}{lllllllllll} 
überhaupt nicht & 0 & 1 & 2 & 3 & 4 & 5 & 6 & 7 & sehr \\
\hline
\end{tabular}

5. Wie war die Atmosphäre während der Gruppendiskussion?

$\begin{array}{llllllllll}\text { konkurrierend } & 0 & 1 & 2 & 3 & 4 & 5 & 6 & 7 & \text { kooperativ }\end{array}$

6. Fanden Sie die anderen Gruppenmitglieder nett?

$\begin{array}{llllllllll}\text { überhaupt nicht } & 0 & 1 & 2 & 3 & 4 & 5 & 6 & 7 & \text { sehr }\end{array}$

7. Wie viele Informationen haben Sie in die Gruppendiskussion eingebracht?

\begin{tabular}{|llllllllll|}
\hline keine & 0 & 1 & 2 & 3 & 4 & 5 & 6 & 7 & sehr viele \\
\hline
\end{tabular}

8. Welche Qualität hatten Ihre Argumente?

\begin{tabular}{|llllllllll|}
\hline nicht & 0 & 1 & 2 & 3 & 4 & 5 & 6 & 7 & sehr überzeugend \\
\hline
\end{tabular}

9. Wie sehr haben Sie zum Diskussionserfolg beigetragen?

\begin{tabular}{llllllllll} 
überhaupt keinen & 0 & 1 & 2 & 3 & 4 & 5 & 6 & 7 & sehr großen \\
\hline
\end{tabular}

10. Wie viele Informationen hat Gruppenmitglied $Y$ in die Gruppendiskussion eingebracht?

\begin{tabular}{|llllllllll|}
\hline keine & 0 & 1 & 2 & 3 & 4 & 5 & 6 & 7 & sehr viele \\
\hline
\end{tabular}

11. Welche Qualität hatten die Argumente von Gruppenmitglied Y?

\begin{tabular}{|llllllllll|}
\hline nicht & 0 & 1 & 2 & 3 & 4 & 5 & 6 & 7 & sehr überzeugend \\
\hline
\end{tabular}

12. Wie sehr hat Gruppenmitglied $Y$ zum Diskussionserfolg beigetragen?

\begin{tabular}{|llllllllll|}
\hline überhaupt keinen & 0 & 1 & 2 & 3 & 4 & 5 & 6 & 7 & sehr großen \\
\hline
\end{tabular}

13. Wie viele Informationen hat Gruppenmitglied Z in die Gruppendiskussion eingebracht? 
keine

0

$\begin{array}{llllll}2 & 3 & 4 & 5 & 6 & 7\end{array}$

sehr viele

14. Welche Qualität hatten die Argumente von Gruppenmitglied Z?

\begin{tabular}{llllllllll|} 
nicht & 0 & 1 & 2 & 3 & 4 & 5 & 6 & 7 & sehr überzeugend
\end{tabular}

15. Wie sehr hat Gruppenmitglied Z zum Diskussionserfolg beigetragen?

$\begin{array}{llllllllll}\text { überhaupt keinen } & 0 & 1 & 2 & 3 & 4 & 5 & 6 & 7 & \text { sehr großen }\end{array}$

16. Gab es während der Diskussion Unstimmigkeiten oder/und Konflikte?

$\begin{array}{llllllllll}\text { überhaupt nicht } & 0 & 1 & 2 & 3 & 4 & 5 & 6 & 7 & \text { sehr }\end{array}$

17. Wie kontrovers wurde in der Gruppe diskutiert?

\begin{tabular}{|llllllllll|}
\hline überhaupt nicht & 0 & 1 & 2 & 3 & 4 & 5 & 6 & 7 & sehr \\
\hline
\end{tabular}

18. Sind Sie der Auffassung, dass man in Ihrer Gruppe auf die Meinung

"Andersdenkender"

eingegangen ist?

$\begin{array}{llllllllll}\text { überhaupt nicht } & 0 & 1 & 2 & 3 & 4 & 5 & 6 & 7 & \text { sehr }\end{array}$

19. Wie gut konnten Sie das sagen, was Sie sagen wollten?

\begin{tabular}{llllllllll|} 
überhaupt nicht & 0 & 1 & 2 & 3 & 4 & 5 & 6 & 7 & sehr \\
\hline
\end{tabular}

20. Haben Sie das Gefühl, dass die Diskussion unterschiedlicher Meinungen vermieden wurde?

\begin{tabular}{|llllllllll|} 
überhaupt nicht & 0 & 1 & 2 & 3 & 4 & 5 & 6 & 7 & sehr \\
\hline
\end{tabular}

21. Wie gut konnten Sie sich in die Diskussion einbringen?

\begin{tabular}{|clllllllll|}
\hline überhaupt nicht & 0 & 1 & 2 & 3 & 4 & 5 & 6 & 7 & sehr \\
\hline
\end{tabular}

22. Sind Sie der Meinung, dass Sie schnell zu einem Ergebnis gelangt sind?

\begin{tabular}{|llllllllll|}
\hline überhaupt nicht & 0 & 1 & 2 & 3 & 4 & 5 & 6 & 7 & sehr \\
\hline
\end{tabular}

23. Wie zufrieden sind Sie mit dem Ergebnis der Diskussion?

\begin{tabular}{|llllllllll|}
\hline überhaupt nicht & 0 & 1 & 2 & 3 & 4 & 5 & 6 & 7 & sehr \\
\hline
\end{tabular}

24. Wie zufrieden sind Sie mit dem Verlauf (dem Prozess, nicht dem Ergebnis!) der Diskussion?

\begin{tabular}{|llllllllll|}
\hline überhaupt nicht & 0 & 1 & 2 & 3 & 4 & 5 & 6 & 7 & sehr \\
\hline
\end{tabular}

25. Haben Sie sich in der Gruppe während der Diskussion wohl gefühlt?

\begin{tabular}{|llllllllll|}
\hline überhaupt nicht & 0 & 1 & 2 & 3 & 4 & 5 & 6 & 7 & sehr \\
\hline
\end{tabular}

26. War es Ihnen wichtig, eine gute Entscheidung zu treffen?

\begin{tabular}{|llllllllll|}
\hline überhaupt nicht & 0 & 1 & 2 & 3 & 4 & 5 & 6 & 7 & sehr \\
\hline
\end{tabular}

27. Wie viel Einfluss hatten Sie auf die Gruppenentscheidung? 
\begin{tabular}{|llllllllll}
\hline überhaupt keinen & 0 & 1 & 2 & 3 & 4 & 5 & 6 & 7 & sehr großen
\end{tabular}

28. Wie viel Einfluss hatte Gruppenmitglied $Y$ auf die Gruppenentscheidung?

\begin{tabular}{|llllllllll|}
\hline überhaupt keinen & 0 & 1 & 2 & 3 & 4 & 5 & 6 & 7 & sehr großen \\
\hline
\end{tabular}

29. Wie viel Einfluss hatte Gruppenmitglied Z auf die Gruppenentscheidung?

\begin{tabular}{|llllllllll|}
\hline überhaupt keinen & 0 & 1 & 2 & 3 & 4 & 5 & 6 & 7 & sehr großen \\
\hline
\end{tabular}


Code:

\section{Fragen zur Beurteilung der Sie unterstützenden Person}

\section{Bitte erinnern Sie sich auch an die Rolle der 4. Person in der Gruppe, die Sie unterstützt hat. Beantworten Sie hierzu bitte folgende Fragen:}

1. Wie zufrieden sind Sie mit der Art und Weise, wie diese Person die Diskussion geleitet hat?

\begin{tabular}{llllllllll|} 
überhaupt nicht & 0 & 1 & 2 & 3 & 4 & 5 & 6 & 7 & sehr \\
\hline
\end{tabular}

2. Wie sehr hat diese Person der Gruppe geholfen, eine Entscheidung zu treffen?

$\begin{array}{llllllllll}\text { überhaupt nicht } & 0 & 1 & 2 & 3 & 4 & 5 & 6 & 7 & \text { sehr }\end{array}$

3. Wie sehr hat diese Person der Gruppe geholfen, ihre Informationen untereinander auszutauschen?

\begin{tabular}{|llllllllll|} 
überhaupt nicht & 0 & 1 & 2 & 3 & 4 & 5 & 6 & 7 & sehr \\
\hline
\end{tabular}

4. Wie sehr hat diese Person für eine Strukturierung der Diskussion gesorgt?

\begin{tabular}{|llllllllll|}
\hline überhaupt nicht & 0 & 1 & 2 & 3 & 4 & 5 & 6 & 7 & sehr \\
\hline
\end{tabular}

5. Wie kompetent in ihrer Rolle wirkte diese Person?

\begin{tabular}{|llllllllll|}
\hline überhaupt nicht & 0 & 1 & 2 & 3 & 4 & 5 & 6 & 7 & sehr \\
\hline
\end{tabular}

6. Hat diese Person während der Diskussion Informationen wiederholt?

\begin{tabular}{|llllllllll|}
\hline überhaupt nicht & 0 & 1 & 2 & 3 & 4 & 5 & 6 & 7 & sehr \\
\hline
\end{tabular}

7. Hat diese Person während der Diskussion nach Informationen zu einzelnen Kandidaten gefragt?

\begin{tabular}{|llllllllll|}
\hline überhaupt nicht & 0 & 1 & 2 & 3 & 4 & 5 & 6 & 7 & sehr \\
\hline
\end{tabular}

8. Hat diese Person die Gruppe während der Diskussion in eine bestimmte Richtung gedrängt?

$\begin{array}{lllllllllll}\text { überhaupt nicht } & 0 & 1 & 2 & 3 & 4 & 5 & 6 & 7 & \text { sehr }\end{array}$

Was mir an der Gesprächsleitungsweise dieser Person besonders gut gefallen hat...

Was mir an der Gesprächsleitungsweise dieser Person überhaupt nicht gefallen hat... 
Code:

\section{Zweite Einzelentscheidung}

Treffen Sie nun bitte auf Grundlage aller Ihnen zur Verfügung stehenden Informationen eine Entscheidung, welcher der Bewerber Ihrer Meinung nach am besten geeignet ist. Diese Entscheidung kann, muss aber nicht mit der Gruppenentscheidung übereinstimmen.

\begin{tabular}{|ll|}
\hline Pilot A & o \\
Pilot B & 0 \\
Pilot C & 0 \\
Pilot D & 0 \\
\hline
\end{tabular}

\section{Beantworten Sie bitte die folgenden Fragen:}

1. Wie sicher sind Sie, dass Ihre Entscheidung richtig ist?

\begin{tabular}{|llllllllll|}
\hline überhaupt nicht & 0 & 1 & 2 & 3 & 4 & 5 & 6 & 7 & sehr \\
\hline
\end{tabular}

2. Für wie geeignet halten Sie Bewerber $A$ ?

\begin{tabular}{|llllllllll|}
\hline überhaupt nicht & 0 & 1 & 2 & 3 & 4 & 5 & 6 & 7 & sehr \\
\hline
\end{tabular}

3. Für wie geeignet halten Sie Bewerber B?

\begin{tabular}{|llllllllll|}
\hline überhaupt nicht & 0 & 1 & 2 & 3 & 4 & 5 & 6 & 7 & sehr \\
\hline
\end{tabular}

4. Für wie geeignet halten Sie Bewerber C?

\begin{tabular}{|llllllllll|}
\hline überhaupt nicht & 0 & 1 & 2 & 3 & 4 & 5 & 6 & 7 & sehr \\
\hline
\end{tabular}

5. Für wie geeignet halten Sie Bewerber D?

\begin{tabular}{|llllllllll|}
\hline überhaupt nicht & 0 & 1 & 2 & 3 & 4 & 5 & 6 & 7 & sehr \\
\hline
\end{tabular}

6. Wie wichtig war es Ihnen bei der Aufgabenstellung, die richtige Entscheidung zu treffen?

\begin{tabular}{|llllllllll|} 
überhaupt nicht & 0 & 1 & 2 & 3 & 4 & 5 & 6 & 7 & sehr \\
\hline
\end{tabular}

7. Wie überzeugt sind Sie, dass Sie sich für den richtigen Bewerber entschieden haben?

\begin{tabular}{|llllllllll|}
\hline überhaupt nicht & 0 & 1 & 2 & 3 & 4 & 5 & 6 & 7 & sehr \\
\hline
\end{tabular}

8. Inwieweit ist der vorliegende Fall subjektiv (Geschmacksfrage) oder objektiv (Sachproblem) lösbar?
subjektiv
0
1
34
5
$6 \quad 7$
objektiv 
Code:

\section{Zweiter Erinnerungsfragebogen}

Bitte schreiben Sie nun alle Informationen zu den vier Bewerbern auf, an die Sie sich noch erinnern können, d. h. die Informationen, die Sie bereits zu Beginn hatten, und die Informationen, die Sie während der Gruppendiskussion zusätzlich erfahren haben.

\section{Bewerber A}

Bewerber B 


\section{Bewerber C}

Bewerber D 
Vielen Dank für Ihre Teilnahme! 


\section{Instruktionen für die Moderatoren/-innen - Bedingung k}

Sehr geehrte/r Versuchsteilnehmer/in,

die anderen Teilnehmer/innen sollen im Anschluss an ihre individuelle Vorbereitung eine Personalentscheidung treffen. Sie sollen eine Stelle eines Piloten besetzen und sich hierfür zwischen vier Bewerbern entscheiden. Dazu haben sie folgende Instruktion bekommen (siehe Kasten) und jetzt etwa eine halbe Stunde Zeit, sich mit den Informationen zu den einzelnen Kandidaten auseinanderzusetzen. Nach der individuellen Vorbereitung wird eine Gruppendiskussion stattfinden. Im Anschluss an diese Gruppendiskussion sollen die Gruppenmitglieder die Personalentscheidung treffen.

Sehr geehrte/r Versuchsteilnehmer/in,

eine deutsche Fluggesellschaft will in den nächsten Jahren mehr Langstreckenflüge anbieten. Da für Langstreckenflüge ausschließlich Flugkapitäne mit langjähriger Berufserfahrung eingesetzt werden und die Fluggesellschaft nicht über genügend derartige Piloten verfügt, wurden über Stellenausschreibungen Flugkapitäne mit langjähriger Berufserfahrung gesucht.

Derartige Entscheidungen werden in der Regel von Expertengruppen getroffen. Wir haben hier einen realen Entscheidungsfall übernommen und für Sie anonymisiert.

Sie werden im Folgenden schriftliche Informationen zu mehreren Piloten erhalten, die sich auf die Stellenausschreibung beworben haben. Alle Piloten verfügen über eine in etwa gleich hohe Anzahl an Flugstunden und langjährige Flugerfahrung. Alle Informationen stammen ausschließlich aus zuverlässigen Quellen, beispielsweise aus etablierten psychologischen und medizinischen Testverfahren sowie aus Beurteilungen von mehreren Kollegen und Vorgesetzten.

Sie sollen die Ihnen individuell vorliegenden Informationen durchgehen und eine individuelle Einschätzung abgeben. Diese individuelle Arbeit wird ca. eine halbe Stunde dauern, in der Ihnen die Versuchsleiterin die Informationen zur Verfügung stellen und verschiedene Fragebögen austeilen wird. Anschließend sollen Sie mit zwei anderen Personen in einer Gruppe Informationen zu diesem Entscheidungsfall austauschen, über die Eignung der vier Bewerber diskutieren und gemeinsam versuchen, den besten Bewerber herauszufinden. In der Diskussion werden Sie durch eine vierte Person unterstützt, die Sie bei der Entscheidungsfindung begleitet. Wenn sich Ihre Gruppe für den eindeutig besten Piloten entscheidet, nimmt sie an einer Verlosung teil, bei der es für jedes Gruppenmitglied einen CD-Gutschein im Wert von $25 €$ zu gewinnen gibt.

Bitte machen Sie nun zunächst noch folgende allgemeine Angaben zu Ihrer Person.

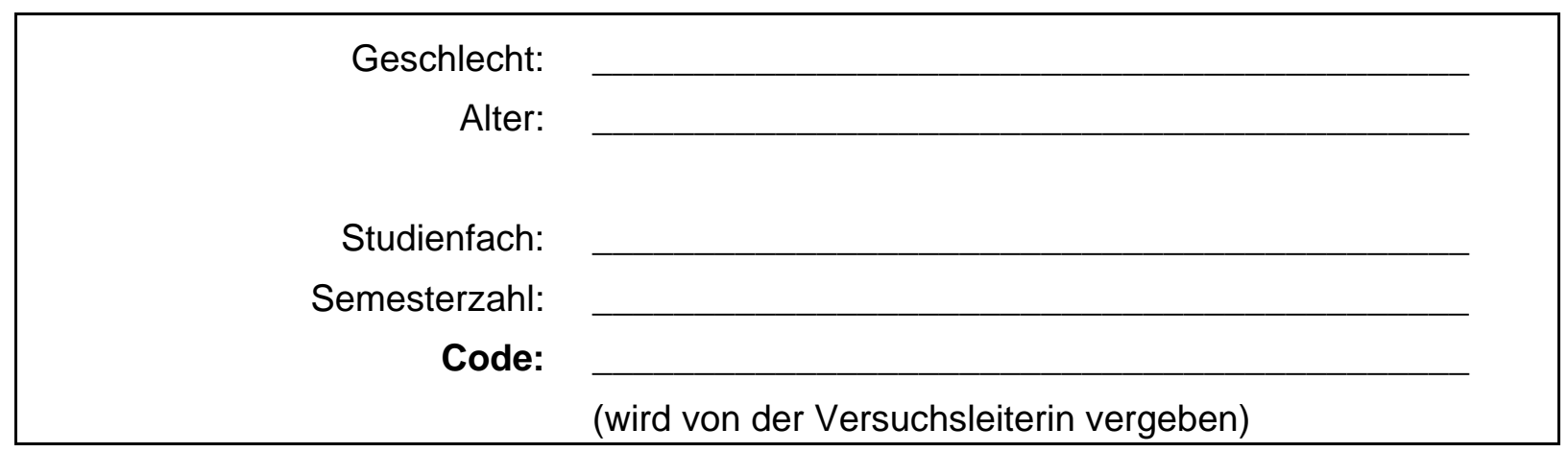


Ihre Aufgabe besteht darin, die drei Personen während der Diskussion zu unterstützen. Ihnen kommt die wichtige Rolle der Leitung des Gruppengesprächs zu. Sie helfen der Gruppe, zu einer Entscheidung zu kommen, indem Sie den Austausch und die Diskussion zwischen den Gruppenteilnehmern unterstützen. Nur durch eine angemessene Unterstützung von Ihnen ist es der Gruppe möglich, zu einer richtigen Entscheidung zu kommen.

Ihre Aufgabe besteht darin, zuzuhören und einzugreifen, wenn es die unten genannten Regeln erfordern. Dennoch sollen Sie keine allzu dominierende Rolle in der Gruppe einnehmen. Sie diskutieren nicht inhaltlich mit.

Damit Sie die Diskussion angemessen leiten können, beachten Sie bitte die nun folgenden Regeln. Es ist wichtig, dass Sie diese Regeln in standardisierter Weise einsetzen. Dafür soll Ihnen ein kurzes Training genau zeigen, welche spezifischen Verhaltensweisen Sie während der Diskussion ausführen und welche Sie unterlassen müssen. Erwähnen Sie bitte in der Gruppendiskussion nichts zum Inhalt und Ablauf des Trainings.

Sie können diese Unterlagen nicht mit in die Gruppendiskussion nehmen, aber sich gern selbst einige Notizen machen, die sie mitführen dürfen. 


\section{Eröffnen Sie die Diskussion.}

a. Kurze Begrüßung und Vorstellung

b. Kurze Vorstellung der Teilnehmer/innen vorschlagen

c. Kurze Vorstellung Ihrer Aufgaben

i. „Ihr sollt aufgrund der vorliegenden Informationen eine Entscheidung treffen."

ii. „Ich werde Euch während der Entscheidungsfindung unterstützen und die Diskussion leiten."

iii. „Ich werde beispielsweise darauf achten, dass sich alle gleichberechtigt in die Diskussion einbringen können."

iv. "Ich werde mich inhaltlich nicht einmischen."

v. „Die Entscheidung treffe nicht ich, sondern Ihr."

d. Weisen Sie die Gruppe darauf hin, dass die Teilnehmer/innen dennoch selbstständig diskutieren sollen und Sie nur bei Bedarf eingreifen.

e. Weisen Sie die Gruppe darauf hin, dass es zunächst keine Zeitvorgabe gibt. Die Gruppenmitglieder haben so viel Zeit für die Entscheidung, wie sie brauchen.

\section{Erklären Sie, dass Sie sich eventuell während der Diskussion Notizen machen} werden, die aber nur für Sie bestimmt sind und auf die die Teilnehmer nicht zurückgreifen dürfen und können.

3. Fragen Sie in die Gruppe, wie zunächst vorgegangen werden soll. Stimmen Sie dem Vorschlag, der gemacht wird, zu.

\section{Seien Sie inhaltlich neutral!}
a. Ergreifen Sie nicht Partei.
b. Alle Nennungen und Personen sind gleich wichtig.
c. Die Beiträge der Diskussionsteilnehmer/innen sollen Sie nicht bewerten oder kommentieren.
d. Geben Sie keinem Recht oder Unrecht.
e. Deklarieren Sie keine Nennung als richtig/falsch.
f. Bitte nicken Sie möglichst nicht und schütteln auch möglichst nicht den Kopf.

\section{Sprechen Sie klar und deutlich!}
a. Nicht zu leise.
b. Nicht zu laut. 


\section{Sorgen Sie dafür, dass sich alle ausreden lassen!}

a. Es spricht nur eine/r zur selben Zeit.

b. Bremsen Sie Vielredner/innen, ermuntern Sie stille Teilnehmer/innen.

c. Alle Teilnehmer/innen sollen sich möglichst gleichberechtigt beteiligen, niemand soll die Gruppe unfair dominieren.

\section{Schließen Sie die Diskussion ab!}

a. Nachdem sich die Teilnehmer/innen einig sind, übergeben Sie der Gruppe das Formular, in dem die Gruppenentscheidung eingetragen werden soll.

b. Anschließend beenden Sie die Diskussion.

Sie haben die Möglichkeit, an einer Verlosung teilzunehmen, bei der es einen CD-Gutschein im Wert von $50 €$ zu gewinnen gibt. Allerdings qualifizieren Sie sich nur dafür, wenn Sie während der Diskussion alle Verhaltensweisen korrekt, regelmäßig und in entsprechender Häufigkeit anwenden.

Wir wissen, dass es für Sie möglicherweise ungewohnt ist, eine Diskussion ausschließlich mit den oben genannten Regeln zu leiten. Dennoch ist es für den Zweck der Studie notwendig, dass Sie sich bemühen, nach den aufgeführten Regeln vorzugehen. Sie können dabei nichts falsch machen. 


\section{Instruktionen für die Moderatoren/-innen der Bedingung 1 (zusätzlich zu den Instruktionen der Bedingung k)}

\section{Wesentlich ist auch, dass Sie die folgenden Informationen beachten:}

Die Informationen zu einem Entscheidungsproblem können vor der Gruppendiskussion unterschiedlich auf die Gruppenmitglieder verteilt sein. Einige Informationen sind sicher vorab allen Mitgliedern bekannt, andere Informationen liegen nur jeweils einem Gruppenmitglied vor. Verschiedene Untersuchungen zum Thema haben gezeigt, dass Gruppen mehr über den erstgenannten Typ, die geteilten Informationen sprechen als über die ungeteilten. Dies kann unter anderem daran liegen, dass sich einzelne Gruppenmitglieder beispielsweise nicht trauen, solche Informationen zu nennen, die noch nicht im Verlauf der Diskussion aufgetaucht sind.

Daher ist es für Sie in ihrer unterstützenden Rolle wichtig, während der Gruppendiskussion darauf zu achten, dass möglichst viele Informationen über die Kandidaten in die Diskussion eingebracht werden. Um das zu erreichen, beachten Sie bitte zusätzlich zu den allgemeinen Regeln folgende Regel:

Fragen Sie, ob es zu den Kandidaten noch weitere Informationen gibt.

- Bitte fragen Sie ausschließlich nach Informationen, nicht nach Meinungen!

- Formulieren Sie Ihre Fragen klar, fragen Sie dabei kurz und verständlich, z. B.

"Gibt es noch andere Informationen, die für Kandidaten B sprechen?“ „Welche Informationen sprechen gegen Kandidaten B?"

- Achten Sie bitte darauf, dass über alle Kandidaten etwa gleich viel gesprochen wird.

- Achten Sie beispielsweise darauf, zu jedem der Kandidaten zu fragen, was für negative bzw. positive Informationen die Gruppe noch nennen kann.

- Fragen Sie regelmäßig (mindestens alle 2 Minuten).

- Fragen Sie gleich oft nach Informationen zu allen vier Kandidaten.

- Passen Sie sich dabei an das, worüber die Gruppe gerade spricht an (Beispiel: Gruppe redet über gute Eigenschaften von A, scheint auf keine noch nicht genannten negativen Informationen zu kommen, also könnten Sie fragen: Was gibt es für Informationen, die gegen A sprechen?)

- Gelegentlich kommt es vor, dass die Gesprächsteilnehmer/innen sehr schnell von einem zum anderen Kandidaten springen. Hier bietet sich folgendes an:

„Bevor Ihr jetzt zu Kandidat A übergeht - welche Informationen gibt es noch zu Kandidat D?"

- Achten Sie darauf, dass Ihre Fragen Gehör finden und beantwortet werden.

- Eine Strichliste hilft Ihnen dabei festzuhalten, wie oft Sie sich schon zu einem Kandidaten erkundigt haben, damit ein ausgeglichenes Fragen sichergestellt ist. 


\section{Instruktionen für die Moderatoren/-innen der Bedingung 2 (zusätzlich zu den Instruktionen der Bedingung k)}

\section{Wesentlich ist, dass Sie die folgenden Informationen beachten:}

Die Informationen zu einem Entscheidungsproblem können vor der Gruppendiskussion unterschiedlich auf die Gruppenmitglieder verteilt sein. Einige Informationen sind sicher vorab allen Mitgliedern bekannt, andere Informationen liegen nur jeweils einem Gruppenmitglied vor. Verschiedene Untersuchungen zum Thema haben gezeigt, dass Gruppen mehr über den erstgenannten Typ, die geteilten Informationen sprechen als über die ungeteilten. Dies kann unter anderem daran liegen, dass die ungeteilten Informationen im Verlauf des Gesprächs verloren gehen, da sie ja nur einer Person bekannt waren.

Daher ist es für Sie in ihrer leitenden Rolle wichtig, während der Gruppendiskussion darauf zu achten, dass möglichst viele Informationen über die Kandidaten in der Diskussion erhalten bleiben. Um das zu erreichen, beachten Sie bitte zusätzlich zu den allgemeinen Regeln folgende Regel:

\section{Wiederholen Sie regelmäßig genannte Informationen zu den Kandidaten.}

Zum Beispiel: Jedes Mal, wenn drei neue Informationen genannt wurden:

„Also, ihr habt jetzt über Kandidat A gesagt, dass er ...“

"Also, ich wiederhole mal, was Ihr eben gesagt habt:...."

- Wiederholen Sie häufig Informationen, lassen Sie die Gruppe also nicht zu lange sammeln, bevor Sie etwas sagen.

- Es kann auch vorkommen, dass Sie wiederholt dieselben Informationen wiederholen (bspw. wenn die Gruppe länger über zwei oder 3 Informationen diskutiert), warten Sie also nicht darauf, dass neue Informationen ins Gespräch eingebracht werden, bevor Sie wiederholen. Ihr regelmäßiges, häufiges Wiederholen ist entscheidend.

- Wiederholen Sie regelmäßig (mindestens nach jeweils 5 Redebeiträgen).

- Fassen Sie die Informationen nicht zusammen, sondern wiederholen Sie lediglich, was gesagt wurde.

- Fragen Sie nicht nach Informationen.

- Lassen Sie sich nicht unterbrechen. 


\title{
Instruktionen für die Moderatoren/-innen der Bedingung 3 (zusätzlich zu den Instruktionen der Bedingung k)
}

\author{
Wesentlich ist, dass Sie die folgenden Informationen beachten:
}

Die Informationen zu einem Entscheidungsproblem können vor der Gruppendiskussion unterschiedlich auf die Gruppenmitglieder verteilt sein. Einige Informationen sind sicher vorab allen Mitgliedern bekannt, andere Informationen liegen nur jeweils einem Gruppenmitglied vor. Verschiedene Untersuchungen zum Thema haben gezeigt, dass Gruppen mehr über den erstgenannten Typ, die geteilten Informationen sprechen als über die ungeteilten. Dies kann unter anderem daran liegen, dass sich einzelne Gruppenmitglieder beispielsweise nicht trauen, solche Informationen zu nennen, die noch nicht im Verlauf der Diskussion aufgetaucht sind und dass die ungeteilten Informationen im Verlauf des Gesprächs verloren gehen, da sie ja nur einer Person bekannt waren.

Daher ist es für Sie in ihrer leitenden Rolle wichtig, während der Gruppendiskussion darauf zu achten, dass möglichst viele Information über die Kandidaten in die Diskussion eingebracht und erhalten werden. Um das zu erreichen, beachten Sie bitte zusätzlich zu den allgemeinen Regeln die folgenden beiden Regeln:

\section{Fragen Sie, ob es zu den Kandidaten noch weitere Informationen gibt.}

- Bitte fragen Sie ausschließlich nach Informationen, nicht nach Meinungen!

- Formulieren Sie Ihre Fragen klar, fragen Sie dabei kurz und verständlich, z. B.

"Gibt es noch andere Informationen, die für Kandidaten B sprechen?"

„Welche Informationen sprechen gegen Kandidaten B?"

- Achten Sie bitte darauf, dass über alle Kandidaten etwa gleich viel gesprochen wird.

- Achten Sie beispielsweise darauf, zu jedem der Kandidaten zu fragen, was für negative bzw. positive Informationen die Gruppe noch nennen kann.

- Fragen Sie regelmäßig (mindestens alle 2 Minuten).

- Fragen Sie gleich oft nach Informationen zu allen vier Kandidaten.

- Passen Sie sich dabei an das, worüber die Gruppe gerade spricht an (Beispiel: Gruppe redet über gute Eigenschaften von A, scheint auf keine noch nicht genannten negativen Informationen zu kommen, also könnten Sie fragen: Was gibt es für Informationen, die gegen A sprechen?)

- Gelegentlich kommt es vor, dass die Gesprächsteilnehmer/innen sehr schnell von einem zum anderen Kandidaten springen. Hier bietet sich folgendes an:

Kandidat $D$ ?

„Bevor Ihr jetzt zu Kandidat A übergeht - welche Informationen gibt es noch zu

- Achten Sie darauf, dass Ihre Fragen Gehör finden und beantwortet werden.

- Eine Strichliste hilft Ihnen dabei festzuhalten, wie oft Sie sich schon zu einem Kandidaten erkundigt haben, damit ein ausgeglichenes Fragen sichergestellt ist. 


\section{Wiederholen Sie regelmäßig genannte Informationen zu den Kandidaten.}

Zum Beispiel: Jedes Mal, wenn drei neue Informationen genannt wurden:

„Also, ihr habt jetzt über Kandidat A gesagt, dass er ...“

„Also, ich wiederhole mal, was Ihr eben gesagt habt:...."

- Wiederholen Sie häufig Informationen, lassen Sie die Gruppe also nicht zu lange sammeln, bevor Sie etwas sagen.

- Es kann auch vorkommen, dass Sie wiederholt dieselben Informationen wiederholen (bspw. wenn die Gruppe länger über zwei oder 3 Informationen diskutiert), warten Sie also nicht darauf, dass neue Informationen ins Gespräch eingebracht werden, bevor Sie wiederholen. Ihr regelmäßiges, häufiges Wiederholen ist entscheidend.

- Wiederholen Sie regelmäßig (mindestens nach jeweils 5 Redebeiträgen).

- Fassen Sie die Informationen nicht zusammen, sondern wiederholen Sie lediglich, was gesagt wurde.

- Lassen Sie sich nicht unterbrechen. 


\section{Fragebogen für die Moderatoren/-innen}

\section{Fragen zur Beurteilung der Gruppendiskussion}

Bitte erinnern Sie sich zum Abschluss nochmals an die Gruppendiskussion und an die Gruppenentscheidung. Beantworten Sie hierzu bitte folgende Fragen:

Wie gut ist Ihrer Meinung nach die Entscheidung, die die Gruppe getroffen hat?

\begin{tabular}{|llllllllll|} 
überhaupt nicht & 0 & 1 & 2 & 3 & 4 & 5 & 6 & 7 & sehr \\
\hline
\end{tabular}

Wie zufrieden sind Sie mit dem Ergebnis der Zusammenarbeit in der Gruppe?

\begin{tabular}{|llllllllll|}
\hline überhaupt nicht & 0 & 1 & 2 & 3 & 4 & 5 & 6 & 7 & sehr \\
\hline
\end{tabular}

Wie war die Atmosphäre während der Gruppendiskussion?

\begin{tabular}{|llllllllll|}
\hline konkurrierend & 0 & 1 & 2 & 3 & 4 & 5 & 6 & 7 & kooperativ \\
\hline
\end{tabular}

Fanden Sie die anderen Gruppenmitglieder nett?

\begin{tabular}{llllllllll|} 
überhaupt nicht & 0 & 1 & 2 & 3 & 4 & 5 & 6 & 7 & sehr \\
\hline
\end{tabular}

Gab es während der Diskussion Unstimmigkeiten oder/und Konflikte?

\begin{tabular}{llllllllll|} 
überhaupt nicht & 0 & 1 & 2 & 3 & 4 & 5 & 6 & 7 & sehr \\
\hline
\end{tabular}

Wie kontrovers wurde in der Gruppe diskutiert?

\begin{tabular}{llllllllll|} 
überhaupt nicht & 0 & 1 & 2 & 3 & 4 & 5 & 6 & 7 & sehr \\
\hline
\end{tabular}

Sind Sie der Auffassung, dass man in der Gruppe auf die Meinung "Andersdenkender" eingegangen ist?

\begin{tabular}{|llllllllll|}
\hline überhaupt nicht & 0 & 1 & 2 & 3 & 4 & 5 & 6 & 7 & sehr \\
\hline
\end{tabular}

Haben Sie das Gefühl, dass die Diskussion unterschiedlicher Meinungen vermieden wurde?

\begin{tabular}{|clllllllll|}
\hline überhaupt nicht & 0 & 1 & 2 & 3 & 4 & 5 & 6 & 7 & sehr \\
\hline
\end{tabular}

Sind Sie der Meinung, dass die Gruppe schnell zu einem Ergebnis gelangt ist?

$\begin{array}{llllllllll}\text { überhaupt nicht } & 0 & 1 & 2 & 3 & 4 & 5 & 6 & 7 & \text { sehr }\end{array}$

Wie zufrieden sind Sie mit dem Verlauf (dem Prozess, nicht dem Ergebnis!) der Diskussion?

\begin{tabular}{llllllllll|} 
überhaupt nicht & 0 & 1 & 2 & 3 & 4 & 5 & 6 & 7 & sehr \\
\hline
\end{tabular}

Für welchen Piloten hätten Sie sich, nachdem Sie die Diskussion über die Kandidaten verfolgt haben, entschieden?

\begin{tabular}{|ll}
\hline Pilot A & o \\
Pilot B & o \\
Pilot C & o \\
Pilot D & o
\end{tabular}


Code:

\section{Fragen zur Beurteilung meiner Rolle}

\section{Bitte erinnern Sie sich auch an Ihre Rolle während der Gruppendiskussion. Beantworten Sie hierzu bitte folgende Fragen:}

Empfanden Sie die Vorbereitung auf ihre Aufgabe während der Gruppendiskussion als ausreichend?

\begin{tabular}{|llllllllll|} 
überhaupt nicht & 0 & 1 & 2 & 3 & 4 & 5 & 6 & 7 & sehr \\
\hline
\end{tabular}

Als wie leicht bzw. schwer empfanden Sie die Umsetzung der Regeln währen der Gruppendiskussion?

\begin{tabular}{|llllllllll|}
\hline sehr leicht & 0 & 1 & 2 & 3 & 4 & 5 & 6 & 7 & sehr schwer \\
\hline
\end{tabular}

Wie zufrieden sind Sie mit der Art und Weise, wie Sie die Diskussion geleitet haben?

\begin{tabular}{|llllllllll|}
\hline überhaupt nicht & 0 & 1 & 2 & 3 & 4 & 5 & 6 & 7 & sehr \\
\hline
\end{tabular}

Wie sehr haben Sie ihrer Meinung nach der Gruppe geholfen, eine Entscheidung zu treffen?

\begin{tabular}{|llllllllll|} 
überhaupt nicht & 0 & 1 & 2 & 3 & 4 & 5 & 6 & 7 & sehr \\
\hline
\end{tabular}

War es Ihnen wichtig, dass die Gruppe eine gute Entscheidung trifft?

\begin{tabular}{|llllllllll|}
\hline überhaupt nicht & 0 & 1 & 2 & 3 & 4 & 5 & 6 & 7 & sehr \\
\hline
\end{tabular}

Wie sehr haben Sie der Gruppe geholfen, ihre Informationen untereinander auszutauschen?

\begin{tabular}{llllllllll|} 
überhaupt nicht & 0 & 1 & 2 & 3 & 4 & 5 & 6 & 7 & sehr \\
\hline
\end{tabular}

Wie sehr haben Sie für eine Strukturierung der Diskussion gesorgt?

\begin{tabular}{|llllllllll|}
\hline überhaupt nicht & 0 & 1 & 2 & 3 & 4 & 5 & 6 & 7 & sehr \\
\hline
\end{tabular}

Haben Sie während der Diskussion Informationen wiederholt?

\begin{tabular}{llllllllll|} 
überhaupt nicht & 0 & 1 & 2 & 3 & 4 & 5 & 6 & 7 & sehr \\
\hline
\end{tabular}

Haben Sie während der Diskussion nach Informationen zu einzelnen Kandidaten gefragt?

\begin{tabular}{|llllllllll|}
\hline überhaupt nicht & 0 & 1 & 2 & 3 & 4 & 5 & 6 & 7 & sehr \\
\hline
\end{tabular}

Haben Sie sich wohl gefühlt in Ihrer Rolle während der Diskussion?

\begin{tabular}{|llllllllll|}
\hline überhaupt nicht & 0 & 1 & 2 & 3 & 4 & 5 & 6 & 7 & sehr \\
\hline
\end{tabular}

Weitere Anmerkungen: 
E Kategorien des Kategoriensystems zur mikroanalytischen Erfassung von Koordination in Gruppendiskussionen

\section{Kategorien zur expliziten Koordination}

\subsection{Handlungsanleitungen}

Die Handlungsanleitungen sind dadurch gekennzeichnet, dass sie keinen inhaltlichen Beitrag zur Diskussion beinhalten, sondern inhaltliche Beiträge fordern bzw. auslösen. Es werden sechs Handlungsanleitungen unterschieden, die im folgenden erläutert werden:

- $\quad$ Aufforderung,

- persönliche Zuweisung,

- Verfahrensvorschlag,

- $\quad$ erbittet Klärung oder Spezifizierung,

- $\quad$ erbittet Vorschläge,

- $\quad$ Ermahnung.

\section{Aufforderung (AF)}

In diese Kategorie fallen alle Äußerungen, mit deren Hilfe anderen Personen in der Diskussion eine direkte Anweisung, Bestimmung, Instruktion, Order oder ein direkter Auftrag oder Befehl gegeben wird (Grote et al., 2003). Aufforderungen dienen dazu, einem/einer Gesprächspartner/-in ein Anliegen, ein Nachsuchen oder einen Wunsch unmittelbar deutlich zu machen. Mit Hilfe der Aufforderung soll erreicht werden, dass andere Personen etwas Bestimmtes ausführen bzw. unterlassen.

Typische Beispiele für die Kategorie Aufforderung sind folgende Kodiereinheiten:

Ich möchte Sie bitten, das genauer zu recherchieren!

Würden Sie sich dazu äußern!

Schlagen Sie die Frage nach! 


\section{Persönliche Zuweisung (PZ)}

Bei einer persönlichen Zuweisung weist der/die Sprecher/-in darauf hin, dass ein/e andere/r Diskussionsteilnehmer/-in für ein bestimmtes Gebiet zuständig ist bzw. die gestellte Frage am besten beantworten oder die exaktesten Informationen liefern kann. Die Person verweist z.B. auf eine/n Spezialisten/-in, der/die genaue Argumente und Informationen zu einem Thema vortragen kann (Kauffeld et al., 2002).

Typische Beispiele für die Kategorie Persönliche Zuweisung sind folgende Kodiereinheiten:

Das ist Ihr Metier.

Dafür ist Herr Marx zuständig.

Dazu kann Frau Schultz besser Auskunft erteilen.

\section{Verfahrensvorschlag (VV)}

In einem Verfahrensvorschlag wird das weitere Vorgehen in der Diskussion angesprochen (Kauffeld et al., 2002). Durch einen Verfahrensvorschlag wird der Diskussionsverlauf koordiniert. Er bezieht sich direkt auf die Vorgehensweise, nicht auf die inhaltliche Problemlösung (Fisch, 1994). Verfahrensvorschläge werden oft bei Unstimmigkeiten, Konfliktregelungen, Abstimmungen zu Meinungsbildungen und Entscheidungen verwendet (Fisch, 1994). Sie dienen ebenfalls dazu, die Reihenfolge der nächsten Arbeitsschritte festzulegen. Dementsprechend wird mit Verfahrensvorschlägen auch die Bearbeitungsabfolge der einzelnen Problemaspekte festgelegt (Simon, 1997).

Abgrenzung zum Lösungsvorschlag: In einem Verfahrensvorschlag wird das Verfahren und das Vorgehen in der Diskussion angesprochen. Ein Verfahrensvorschlag enthält keine inhaltlichen Beiträge, wohingegen Lösungsvorschläge einen inhaltlichen Beitrag zur Problemlösung liefern. Falls bei einer Aussage Unsicherheiten auftreten sollen oder es nicht eindeutig ist, ob es sich um einen Verfahrens- oder eine Lösungsvorschlag handelt, dann ist immer der Lösungsvorschlag zu kodieren.

Typische Beispiele für die Kategorie Verfahrensvorschlag sind folgende Kodiereinheiten:

Ich schlage vor, jeder stellt der Reihe nach seinen Standpunkt dar.

Hier sollten wir so verfahren, dass...

Machen wir doch Folgendes... 
Als Lösungsvorschlag hingegen wird kodiert:

Ich schätze, wir sollten das, bevor wir eine Entscheidung fällen, erst rechtlich abklären.

\section{Erbittet Klärung oder Spezifizierung (EKS)}

Der/die Fragesteller/-in erbittet eine genauere Erklärung oder Erläuterung eines Sachverhaltes, der kurz zuvor diskutiert oder angesprochen wurde. Dies erfolgt meistens dann, wenn ein/e Teilnehmer/-in die Ausführungen eines/e anderen nicht genau verstanden hat und dadurch Unsicherheiten beim Verstehen der zuvor genannten Äußerungen verhindern möchte. Er/sie möchte somit sichergehen, den Bedeutungsinhalt der Aussage des/der Vorgängers/-in richtig verstanden zu haben (Simon, 1997).

Typische Beispiele für die Kategorien Erbittet Klärung oder Spezifizierung sind folgende Kodiereinheiten:

Bitte erklären Sie uns das!

Schildern Sie es genauer!

Stellen die ihren angesprochenen Punkt deutlicher dar.

\section{Erbittet Vorschläge (EV)}

Es werden Vorschläge angefordert, die dabei helfen sollen, ein Problem zu lösen. Im Gegensatz zur Aufforderung wird hier ein/e Gesprächsteilnehmer/-in explizit um einen inhaltlichen Vorschlag gebeten.

Typische Beispiele für die Kategorien Erbittet Vorschläge sind folgende Kodiereinheiten:

Machen Sie bitte weitere Vorschläge!

Schlagen Sie doch bitte weiteres vor!

\section{Ermahnung (EM)}

Alle Appelle, Mahnungen, Ordnungsrufe, Mahnrufe oder Mahnworte, die von einem/r Diskussionsteilnehmer/-in an eine andere Person gerichtet werden, zählen zu dieser 
Kategorie. Zudem werden Aussagen als Ermahnung gesehen, die den anderen Personen die Bedeutung ihrer derzeitigen Situation, die Verantwortung, die sie dabei tragen und die Konsequenzen ihres Handelns deutlich vor Augen führen.

Typische Beispiele für die Kategorie Ermahnung sind folgende Kodiereinheiten:

Ich bitte Sie!

So geht das nicht!

Äußern Sie sich konstruktiv.

Lassen Sie ihn ausreden!

Sie wissen, welche Verantwortung Sie bei dieser Entscheidung tragen!

\subsection{Strukturierungen}

Die Oberkategorie Strukturierungen beschreibt alle Äußerungen mit dem Ziel, Ordnung in die Diskussion zu bringen, indem beispielsweise das Ziel der Diskussion genannt wird oder bisherige Lösungen zusammengefasst werden.

Die Oberkategorie Strukturierungen beinhaltet sechs Kategorien:

- Zusammenfassung,

- Wiederholung,

- Zielformulierung,

- Entscheidung,

- Zielandeutung,

- Darstellung der eigenen Vorgehensweise.

\section{Zusammenfassung (ZF)}

Der/die Sprecher/-in fasst bisher Gesagtes zusammen (Grote et al., 2003) oder formuliert es prägnant mit eigenen Worten. Die bisher erzielten Resultate der Diskussion werden somit nochmals wiedergegeben (Kauffeld et al., 2002). Darunter können auch Resümees fallen. Es wird eine Kurzfassung über das bisher Gesagte gegeben und der/die Sprecher/-in gibt seine/ihre eigenen vorangegangenen Aussagen oder auch die eines/r anderen wieder. Zusammenfassungen werden zumeist kurz und knapp gehalten.

Typische Beispiele für die Kategorie Zusammenfassung sind folgende Kodiereinheiten: 
Um es kurz in eigenen Worten zu sagen,...

Was ich/ er/ sie damit sagen wollte, ...

Wir haben somit festgestellt,...

\section{Wiederholung (WD)}

Der Sprecher wiederholt bisher genannte Informationen, Feststellungen, Meinungen oder Vorschläge. Im Gegensatz zur Zusammenfassung sind Wiederholungen eher am ursprünglichen Wortlaut der zu wiederholenden Inhalte orientiert.

Die Aussagen müssen nicht unbedingt kurz und knapp sein.

Typische Beispiele für die Kategorie Wiederholung sind folgende Kodiereinheiten:

Um es nochmals zu sagen,...

Ich wiederhole meine Aussagen, ...

Du hast ja vorhin gesagt, dass

Neben diesen typischen Beispielen sind auch Informationen, die bereits mehrmals genannt werden, nicht unter Information, sondern unter Wiederholung zu kodieren. Der/die Kodierer/in muss während des Kodiervorgangs das Gespräch inhaltlich mitverfolgen, um beurteilen zu können, ob eine Informationen zum ersten Mal (und damit als Information kodiert wird) oder schon zum zweiten Mal genannt wird (ab hier als Wiederholung kodiert wird).

\section{Zielformulierung (ZF)}

Hiermit sind Aussagen gemeint, in denen das Ziel der gesamten Sitzung oder das Ziel eines abgesteckten Zeitrahmens definiert, erwähnt bzw. betont wird. Sie können sich sowohl auf Oberziele als auch auf Unterziele beziehen. Die Absicht, das Bestreben, die Intention, der Plan, der Sinn, das Vorhaben, der Vorsatz bzw. die Zielsetzung, die Zielvorstellung oder der Zweck, den die Diskussion besitzt, wird hierbei explizit genannt.

Typische Beispiele für die Kategorie Zielformulierung sind folgende Kodiereinheiten:

Wir müssen eine Einigung betreffs des Problems treffen erreichen,...

Unser Ziel/Unsere Aufgabe/Unser Anliegen ist... 


\section{Entscheidung (EN)}

Der/die Sprecher/-in trifft explizit eine Entscheidung zu einem Problem und nimmt z.B. einen Vorschlag direkt an (Simon, 1997).

Typische Beispiele für die Kategorie Entscheidung sind folgende Kodiereinheiten:

Dann machen wir das so.

Dann ist die Entscheidung gefallen.

Dann investieren wir das Geld.

\section{Zielandeutung (ZA)}

Bei Zielandeutungen handelt es sich um Aussagen, die indirekt auf das zu erreichende Ziel eingehen. In Abgrenzung zur Kategorie Zielformulierung wird das Ziel jedoch nicht explizit angesprochen. Stattdessen liefert der/die Sprecher versteckte bzw. implizite Hinweise, von denen er/sie sich dann erhofft, dass der/die Zuhörer/-in die eigentliche Botschaft schlußfolgert.

Typische Beispiele für die Kategorie Zielandeutung sind folgende Kodiereinheiten:

Damit könnten wir uns möglicherweise einer Lösung nähern.

Wir sollten den Bogen nicht überspannen.

\section{Darstellung der eigenen Vorgehensweise (DAV)}

Hiermit sind alle Aussagen gemeint, mit denen der/die Sprecher/-in seine/ihre eigene Vorgehensweise darlegt. Er/sie beschreibt kurz, wie und warum er/sie vorgegangen ist oder vorgehen wird.

Typische Beispiele für die Kategorie Darstellung der eigenen Vorgehensweise sind folgende Kodiereinheiten

Aus rechtlicher Sicht muss ich dazu Stellung nehmen.

Ich werde mich zu dem eben angesprochenen Punk jetzt äußern müssen. 


\subsection{Fragen}

Die Oberkategorie Fragen beschreibt alle Äußerungen, die in der Frageform gestellt werden. Die Fragen sind dadurch gekennzeichnet, dass sie keinen inhaltichen Beitrag zur Diskussion liefern, sondern inhaltliche Beiträge fördern bzw. auslösen.

Die Oberkategorie unterscheidet sieben Kategorien:

- Informationsfrage,

- Meinungsfrage,

- Klärungs- bzw. Spezifizierungsfrage,

- Verfahrensfrage,

- Lösungsfrage,

- Einverständnisfrage,

- $\quad$ Entscheidungsfrage.

\section{Informationsfrage (IF)}

In dieser Kategorie wünscht der/die Fragensteller/-in Informationen (Grote et al., 2003), er/sie möchte damit etwas Bestimmtes wissen und fragt so, dass informiert werden kann (Fisch, 1994). Es geht hierbei um verifizierbare Tatbestände, Wissen oder Fakten (Fisch, 1994), aber nicht um die eigene Meinung oder den persönlichen Standpunkt zu diesem Wissen. Die Informationen werden hierbei durch einfaches Nachfragen in der Gruppe gewonnen (Kauffeld et al., 2002). Informationsfragen dienen auch dazu, Informationen über die Arbeit eines einzelnen Gruppenmitgliedes einzuholen (Simon, 1997).

Typische Beispiele für die Kategorie Informationsfrage sind folgende Kodiereinheiten.

Welche Arbeit ist damit verbunden?

Wo kommt das fehlende Material her?

Was stand in dem Brief?

Wie viel wird uns das kosten?

\section{Meinungsfrage (MF)}

Der/die Fragesteller/-in wünscht, dass andere ihre persönliche Meinung zum Ausdruck bringen und spricht deshalb alle oder einzelne Teilnehmer/-innen direkt auf ihre Meinung an. 
Die Meinungen der anderen Teilnehmer/-innen werden hierbei durch einfaches Nachfragen in der Gruppe eingeholt (Kauffeld et al., 2002).

Typische Beispiele für die Kategorie Meinungsfrage sind folgende Kodiereinheiten:

Was denken Sie?

Was halten Sie davon?

Was ist Ihre Meinung?

\section{Spezifizierungs- oder Klärungsfrage (SKF)}

Der/die Fragesteller/-in wünscht eine genauere Erklärung oder Erläuterung eines Sachverhaltes, der kurz zuvor von einem/r einzelnen oder den anderen Teilnehmer/n/-innen diskutiert oder angesprochen wurde. Dies erfolgt meistens dann, wenn ein/e Teilnehmer/-in die Ausführungen eines/r anderen nicht genau verstanden hat und dadurch Unsicherheiten beim Verstehen der zuvor genannten Äußerungen verhindern möchte. Es handelt sich hierbei um Rück- und Verständnisfragen nach dem Bedeutungsinhalt der Aussage eines Vorgängers (Simon, 1997).

Typische Beispiele für die Kategorie Spezifizierungs- oder Klärungsfrage sind folgende Kodiereinheiten:

Können Sie mir das erklären?

Können Sie das genauer ausführen?

Könnten Sie das eventuell genauer beschreiben?

Ist diese Entscheidung wirklich richtig?

Wie meinst Du das mit der Aufgabenteilung?

\section{Verfahrensfrage (VF)}

Der/die Fragesteller/-in wünscht Vorschläge zum weiteren Vorgehen (Kauffeld et al., 2002), die nicht themenrelevant sind, da sich die Frage ausschließlich auf das methodische Vorgehen bezieht. Die Frage zielt somit auf die Vorgehensweise in der Diskussion ab (Fisch, 1994). Der/die Fragesteller/-in hat die Intention, sich mit den anderen Beteiligten abzustimmen und sie nach ihrer Meinung zu einem Verfahrensvorschlag zu fragen bzw. selbst alternative Vorgehensweisen zur Auswahl anzubieten (Fisch, 1994). 
Typische Beispiele für die Kategorie Verfahrensfrage sind folgende Kodiereinheiten:

Wie sollen wir hier verfahren/ vorgehen/ fortfahren?

Was halten Sie davon, wenn wir zunächst alle Themen sammeln?

Wollen wir das dann Schritt für Schritt abarbeiten?

Sollen wir jetzt darüber diskutieren?

Wollen wir diesen Punkt abschließen?

\section{Lösungsfrage (LF)}

Der/die Fragesteller/-in erfragt oder erbittet inhaltliche Vorschläge oder Lösungen, welche sich auf das Thema der Diskussion beziehen sollen. Die Frage zielt also daraufhin ab, eine Lösung für das zur Diskussion stehende Problem zu finden. Im Gegensatz zur Meinungsfrage werden hier konkrete inhaltliche Lösungsvorschläge erfragt.

Typische Beispiele für die Kategorie Lösungsfrage sind folgende Kodiereinheiten:

Wie können wir das Problem lösen/ angehen?

Hat jemand einen guten Lösungsvorschlag parat?

Wie können wir die Unstimmigkeiten beseitigen?

\section{Einverständnisfrage (EF)}

Der/die Fragesteller/-in erfragt hierbei die Zustimmung, das Einverständnis oder die Bestätigung zu einem von ihm/ihr oder von einem/r anderen Teilnehmer/-innen unterbreiteten Vorschlag oder einer Aussage. Dadurch wird ein Feedback bzw. eine Absicherung eines Vorschlages durch andere Gruppemitglieder erwartet (Grote et al., 2003). In diese Kategorie fallen ebenfalls rückversichernde Fragen nach der Richtigkeit des zum Ausdruck gebrachten Inhaltes (Simon, 1997).

Abgrenzung zur Klärungsfrage: Einverständnisfragen beziehen sich immer auf die Aussagen, die man selber getätigt hat. Man möchte sicherstellen, dass alle Gruppenmitglieder die eigenen Ausführungen verstanden haben und damit einverstanden sind bzw. man fragt nach, ob die eigenen Aussagen der Richtigkeit entsprechen. Bei der Klärungsfrage fragt im Gegensatz dazu eine Person nach, ob sie den Redebeitrag eines 
Vorredners richtig verstanden hat. Die Klärungsfrage bezieht sich somit immer auf Beiträge von anderen Personen, während sich die Einverständnisfrage auf die zuvor getätigten eigenen Aussagen bezieht.

Typische Beispiele für die Kategorie Einverständnisfrage sind folgende Kodiereinheiten:

Sind damit alle einverstanden?

Ist das jetzt so angenommen?

Ist das so in Ordnung?

Sind wir darin uns einig?

Oder?

Nicht wahr?

Habe ich das richtig dargelegt? (rückversichernde Frage)

\section{Entscheidungsfrage (ENF)}

Der/die Diskussionsteilnehmer/-in erfragt explizit eine Entscheidung. Er/sie möchte, dass die Teilnehmer/-innen bezüglich eines Diskussionspunktes eine Einigung erzielen und damit einen Abschluss der Diskussion erwirken. Die Gruppenmitglieder sollen eine Entscheidung zu einem Problemaspekt herbeiführen, der Prozess der Entscheidungsfindung soll mit dieser Frage vorangetrieben werden (Simon, 1997).

Typische Beispiele für die Kategorie Entscheidungsfrage sind folgende Kodiereinheiten:

Wie sieht unsere Entscheidung nun aus?

Wie entscheiden wir nun?

\subsection{Anreden}

Die Oberkategorie Anreden beschreibt alle Äußerungen, in der einzelne Personen oder die gesamte Gruppe direkt angesprochen werden. Es werden zwei Anreden unterschieden: 
- persönliche Anrede,

- $\quad$ namentliche Anrede.

\section{Persönliche Anrede (PA)}

Bei einer persönlichen Anrede werden die Diskussionsteilnehmer/-innen direkt angesprochen. Der Name einer Person wird allerdings nicht direkt genannt. Wird der Name der Person genannt, so ist die namentliche Anrede zu kodieren.

Typische Beispiele für die Kategorie Persönliche Anrede sind folgende Kodiereinheiten:

Meine Herren, ...

Stellen Sie sich vor...

Sie kennen ja...

Kollegen,

\section{Namentliche Anrede (NA)}

Bei einer namentlichen Anrede wird ein/e andere/r bzw. werden die anderen Diskussionsteilnehmer/-innen direkt mit seinem/ ihren Namen angesprochen. Es reicht allerdings nicht, dass innerhalb eines Redebeitrages der Name einer Person genannt wird (z. B. Frau Schultz ist heute nicht da), sondern die andere Person muss direkt namentlich

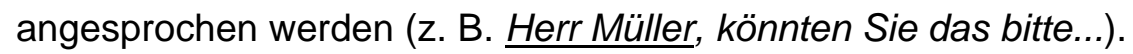

Typische Beispiele für die Kategorie Namentliche Anrede sind folgende Kodiereinheiten:

Frau Schultz, ...

\section{Kategorien inhaltlicher Beiträge}

Die Kategorien inhaltlicher Beiträge beschreiben Äußerungen, die einen inhaltlichen Beitrag zur Diskussion liefern und somit ebenfalls der Lösungsfindung dienen. Diese Beiträge können über Potential zur impliziten Koordination des Gruppenprozesses verfügen. Ob die 
jeweilige Kategorie allerdings vergeben wird, hängt ausschließlich von ihrem inhaltlichen Gehalt, nicht von ihrer eventuellen Koordinationswirkung ab.

Diese Oberkategorie beinhaltet sieben Kategorien inhaltlicher Beiträge:

- Information,

- Feststellung,

- $\quad$ Erläuterung,

- Meinungsäußerung,

- Zustimmungsreaktion,

- Ablehnungsreaktion,

- Lösungsvorschlag.

\section{Information (I)}

Als Informationen werden Äußerungen, die der Aufklärung dienen bzw. Antworten, Hinweise, Auskünfte auf offene Fragen, kodiert. Entscheidend ist der informierende Charakter der Aussage (Fisch, 1994) und die Möglichkeit, die Richtigkeit der Aussage zu überprüfen. Die Informationen dienen dazu, um das Problem, worüber diskutiert wird, zu lösen. Sie sind für die Lösungsfindung somit äußerst relevant (Yukl, 2002).

Abgrenzung zur Feststellung, Meinungsäußerung und Erläuterung: Im Unterschied zur Feststellung kann hier die Richtigkeit der Information nachgewiesen werden, da z.B. ein Gesetz zitiert oder ein Brief vorgelesen wird, anhand derer man die Authentizität der Aussage überprüfen kann. Im Unterschied zur Meinungsäußerung wird bei der Information die Darstellung der eigenen Sichtweise weder explizit betont noch beinhaltet die Aussage eine reflexive Komponente (Fisch, 1994). Die Erläuterung unterscheidet sich von der Information, da der illustrierende, erklärende und erweiternde Charakter im Vordergrund steht (Fisch, 1994).

Typische Beispiele für die Kategorie Information sind folgende Kodiereinheiten:

Der Paragraph 6 besagt....

Der Flächennutzungsplan sieht vor, dass... 


\section{Feststellung (F)}

Hierunter fällt eine Äußerung, die einer Person dazu dient, eine Anmerkung zu machen, ihre persönliche Auffassung darzulegen, eine einfache Aussage, Äußerung, Behauptung, Bemerkung, Erwähnung zu tätigen (Fisch, 1994) oder ihre eigene Meinung hervorzuheben.

Abgrenzung zur Information, Meinungsäußerung und Erläuterung: Im Gegensatz zur Information kann die Richtigkeit der Aussage nicht nachgewiesen werden, da z.B. kein Gesetz zitiert oder ein Brief vorgelesen wird, anhand derer man die Authentizität der Aussage überprüfen kann. Im Unterschied zur Meinungsäußerung wird bei der Feststellung die Darstellung der eigenen Sichtweise nicht explizit betont. Entscheidend ist immer die reflexive Komponente in der Aussage (Fisch, 1994). Die Erläuterung unterscheidet sich von der Feststellung, indem bei ihr der illustrierende, erklärende und erweiternde Charakter im Vordergrund steht (Fisch, 1994).

Typische Beispiele für die Kategorie Feststellung sind folgende Kodiereinheiten:

Das ist eine wichtige Frage.

Das lässt sich im Moment noch nicht abschließend behandeln.

\section{Erläuterung (E)}

Die Erläuterung dient der näheren Beschreibung zuvor genannter Äußerungen (Kauffeld et al., 2002). Eine Erläuterung kann als Anmerkung, Ausführung, Bericht oder Beschreibung angesehen werden, die der Konkretisierung, Veranschaulichung bzw. Verdeutlichung einer Äußerung dient. Sie ist meist dadurch gekennzeichnet, dass sie den zuvor mitgeteilten Sachverhalt illustriert, erklärt oder erweitert (Fisch, 1994). Erläuterungen kommen häufig in der Form von Nebensätzen vor, die mit Konjunktionen eingeleitet werden.

Abgrenzung zur Feststellung, Information und Meinungsäußerung: Im Unterschied zur Feststellung, Information und Meinungsäußerung steht der illustrierende, erklärende und erweiternde Charakter im Vordergrund (Fisch, 1994).

Typische Beispiele für die Kategorie Erläuterung sind folgende Kodiereinheiten: 
(Das ist sicherlich erst einmal die erste Prämisse, die man hier treffen muss,) wenn man überhaupt zu einem Abbau positiv kommen wird.

(Das lässt sich im Moment noch nicht abschließend behandeln,) weil die

Stellungnahmen des Wasserwirtschaftsamtes einfach noch nicht präzise genug sind.

\section{Meinungsäußerung (M)}

Mit einer Meinungsäußerung gibt die Person ihre eigenen Annahmen, Anschauungen, Auffassungen, Behauptungen, Betrachtungsweisen, Einstellungen, Haltungen, Meinungen, Positionen, Sichtweisen, Überzeugungen, Werte bzw. den eigenen Blickwinkel oder Standpunkt wieder.

Meinungen sind immer eindeutig auf die eigene Person bezogen und spiegeln nicht die Haltungen der gesamten Gruppe wider. Es wird somit explizit und eine oft persönlich eingefärbte Meinung zum Ausdruck gebracht (Fisch, 1994).

Abgrenzung zur Feststellung, Information und Erläuterung: Im Gegensatz zur Feststellung, Information und Erläuterung kündigt die Person ihre Meinungsäußerung explizit an durch Satzanfänge, wie „ich denke“, „ich meine“, „ich bin der Meinung“, „ich möchte sagen“... an. Es wird explizit die Darstellung der eigenen Sichtweise betont.

Typische Beispiele für die Kategorie Meinungsäußerung sind folgende Kodiereinheiten:

Wir werden uns um dieses Thema, glaube ich, nicht drücken können.

Das scheint mir also ganz klar zu sein.

Beachte!

Meinungsäußerungen sind vom Vorgehensvorschlag abzugrenzen: Eine Äußerung wie: „Ich finde, wir sollten die Dinge der Reihe nach besprechen." beinhaltet einen Vorgehensvorschlag und wird daher als solcher kodiert.

\section{Zustimmungsreaktion (Z)}

Zustimmungen sind Äußerungen, die explizit positive Einstellungen, Meinungen, Bewertungen etc. zu einer Information ausdrücken (Kauffeld et al., 2002). Sie dienen dazu, 
eine eigene Bejahung oder das Einverständnis zu einem Vorschlag, einer Idee, einem Gedanken oder einer anderen Aussage auszudrücken. Man bringt durch seine Zustimmung zum Ausdruck, dass man z.B. die Ansicht oder die Meinung eines anderen Diskussionsteilnehmers teilt (Gottman, 1979). Eine inhaltliche Begründung ist dafür nicht notwendig (Fisch, 1994).

Entschuldigungen und Aussagen darüber, dass man eine persönliche Kritik akzeptiert und annimmt oder dass man seine Meinung doch überdacht hat oder kleine verbale Reaktionen (z.B. „mmhmm“) fallen auch in diese Kategorie (Gottman, 1979).

Typische Beispiele für die Kategorie Zustimmung sind folgende Kodiereinheiten:

Ja.

Das ist gut.

Gut.

Super.

Du hast Recht.

So habe ich es noch nie gesehen.

Meine übertriebene Reaktion tut mir jetzt Leid.

Mmhmm.

\section{Ablehnungsreaktion (A)}

Eine Ablehnung ist eine Äußerung, mit der man seine ablehnende Haltung zu einem Vorschlag oder einer Aussage kund tut. Sie enthält immer eine explizit negative Bewertung bzw. Ablehnung einer Information, Meinung etc. Man stellt sich gegen einen Vorschlag, ein Ergebnis oder sogar eine Person (Fisch, 1994) oder man teilt die Meinung mit einer anderen Person nicht (Gottman, 1979).

Typische Beispiele für die Kategorie Ablehnung sind folgende Kodiereinheiten:

Nein.

Das ist hier nicht wichtig.

Das ist keine gute Lösung.

Dem stimme ich nicht zu.

Das sehe ich nicht so. 


\section{Lösungsvorschlag (LV)}

Bei einem Lösungsvorschlag wird ein Plan oder eine Methode unterbreitet, der/die dazu dienen soll, das zu lösende Problem zu bewältigen (Gottman, 1979). Es wird ein Verhalten vorgeschlagen, welches inhaltlich zur Problemlösung oder Entscheidungsfindung beitragen soll. Dabei kann eine Lösung bzw. eine Teillösung genannt werden (Kauffeld et al., 2002).

Diese Vorschläge sind immer im Sinne der Aufgabenbewältigung. Hierunter fallen auch Beiträge, die als Lösungsvorschlag oder Anmerkung bezüglich der Abwicklung oder Umsetzung der Lösung gelten.

Abgrenzung zum Verfahrensvorschlag: Bei einem Lösungsvorschlag ist der inhaltliche Charakter der Aussage entscheidend, dass heißt, es wird explizit und eindeutig gesagt, was inhaltlich gemacht werden soll, um das Ziel der Diskussion zu erreichen bzw. das Problem zu lösen. Ein Verfahrensvorschlag enthält im Gegensatz keine inhaltlichen Beiträge. Falls bei einer Aussage Unsicherheiten auftreten sollen oder es nicht eindeutig ist, ob es sich um einen Verfahrens- oder Lösungsvorschlag handelt, dann ist immer der Lösungsvorschlag zu kodieren.

Typische Beispiele für die Kategorie Lösungsvorschlag sind folgende Kodiereinheiten:

Wir sollten erst den Bescheid der Baubehörde abwarten.

Wir müssen das Gesetz noch in Betracht ziehen.

\section{Zusatzkategorien}

Ergänzend werden noch zwei Kategorien erfasst: Unterbrechungen und fokussierende Einwort-Einleitungen.

\section{Unterbrechung (U)}

Eine Unterbrechung wird für eine Person B kodiert, wenn sie von einer anderen Person A unterbrochen wird und ihren Beitrag nicht zu Ende führen kann. Eine Person A schneidet 
einer anderen Person B somit das Wort mitten im Satz ab (Kauffeld et al., 2002). Außerdem kann eine Person selbst ihre Äußerung oder ihren Gedankengang abbrechen. Die Aussagen sind dann dadurch gekennzeichnet, dass sie in nicht grammatikalisch vollständigen Sätzen vorliegen.

Typische Beispiele für die Kategorie Unterbrechung sind folgende Kodiereinheiten:

Dann muss $[\ldots]$

Die Abteilung [...]

Vielleicht musst das [...]

\section{Fokussierende Einworteinleitungen (FEE)}

Fokussierende Einworteinleitungen dienen augenscheinlich dazu, besondere Aufmerksamkeit auf den nachfolgenden eigenen Redebeitrag zu lenken. Sie werden allerdings nur zu Beginn eines Redebeitrages kodiert, da sie nur dort ihre koordinierende Einleitungsfunktion besitzen. Das bedeutet, das der/die Kodierer/-in bei der Betrachtung über die Kodiereinheit hinaus gehen und überprüfen muss, ob die Äußerung lediglich am Anfang einer Kodierheit, aber inmitten eines gesamten Redebeitrages, vorkommt (dann wird die Kategorie nicht vergeben, z.B. „....., aber, ich finde, ...") oder ob die Äußerung tatsächlich am Anfang eines Redebeitrages steht (dann wird Kategorie vergeben, z.B. „Okay, dann machen wir folgendes...").

Typische Beispiele sind für diese Kategorie sind folgende Kodiereinheiten:

aber

nur 


\section{F Regeln des Manuals zur Einteilung von Kodiereinheiten}

Es empfiehlt sich, zunächst alle zehn Regeln der Reihe nach durchzulesen. Auf diese Weise lassen sich mögliche Unklarheiten beseitigen, die eventuell bei der erstmaligen Auseinandersetzung mit jeder einzelnen Regel auftreten könnten. Einige Regeln mögen einzeln betrachtet zunächst verwirren, werden jedoch in Anwendung des gesamten Regelsystems schlüssig.

Die Regeln zur Bildung von Kodiereinheiten beruhen auf der deutschen Syntax und enthalten teilweise grammatikalische ${ }^{18}$ Begriffe. Diese werden im laufenden Text anhand von Fußnoten erklärt und können im Glossar (am Ende der Regeln) nachgeschlagen werden.

Während der detaillierten Beschreibung der Regeln wird zur besseren Veranschaulichung ein fiktiver Dialog (siehe Abbildung 39) verwendet, an dem die Einteilung der Kodiereinheiten Regel für Regel dargestellt wird. So kann der Leser Schritt für Schritt die Regelanwendung nachvollziehen. Zum Ende des Leitfadens liegt dann eine vollständige Segmentierung des Gespräches vor.

\footnotetext{
In diesem Dialog treffen sich zwei Freundinnen zufällig in der Stadt:

Isabelle: Anna, wir haben uns schon lange nicht mehr gesehen. Wie geht es dir denn?

Anna: Mir geht es ganz gut, Isabelle. Ich bin gerade auf der Suche nach einem Geburtstagsgeschenk für eine Freundin. Was treibt dich denn in die Stadt?

Isabelle: Ach, ich will einfach nur ein bisschen herumschlendern und in ein paar Läden nach neuen Klamotten Ausschau halten. Ich habe mir eben sogar schon eine Jeans gekauft. Warte, ich zeige sie dir mal. Die ist doch toll, oder?

Anna: Ja, in der Tat. Wo hast du die Hose denn gekauft? Die wäre wirklich super als Geschenk!

Isabelle: Also, die kannst du im neuen Geschäft, du weißt schon, an der nächsten Ecke erstehen. In dem Laden scheinen ganz...

Anna: Oh, das ist doch der Laden, der letzte Woche eröffnet und gerade lauter Sonderangebote hat.

Isabelle: Ja, ganz genau!

Anna: $\quad$ Dann werde ich da gleich vorbeischauen, um nach Schnäppchen Ausschau zu halten. Ich hoffe ganz stark, dass wir uns bald mal wieder sehen.

Isabelle: Viel Spaß noch und bis bald.
}

\section{Abbildung 39: Fiktiver Beispieldialog}

Im folgenden Kapitel werden die zehn Trennregeln an diesem fiktiven Dialog erläutert.

\footnotetext{
18 In diesem Manual wird nicht auf die Diskussion über die unterschiedliche oder synonyme Verwendung der Begriffe „grammatisch“ versus „grammatikalisch“ eingegangen. Nach Aussage des Dudens bedeutet grammatikalisch „,..., ,a) die Grammatik betreffend; vgl. grammatisch (a); b) sprachkundlich“ (S. 288) und grammatisch ..., „a) die Grammatik betreffend; vgl. grammatikalisch; b) der Grammatik gemäß; sprachrichtig; nicht ungrammatisch“ (Dudenredaktion, 1990). Da hier die bestehende Grammatik als sprachkundliches Phänomen herangezogen wird, wird der Begriff grammatikalisch verwendet.
} 


\section{Trennung von Redebeiträgen verschiedener Personen}

\section{Regel 1: Sprecherwechsel}

Zuerst wird innerhalb des Transkripts eine grobe Trennung gesetzt, wenn eine Person zu sprechen aufhört und eine andere Person zu sprechen beginnt. Diese Regel lehnt sich an gängige sprachwissenschaftliches Vorgehen an (Crowell \& Schneider, 1961; Fisch, 1994; Kauffeld et al., 2002; Simon, 2000) und ist unproblematisch, da sie häufig schon im Transkript enthalten ist, indem jeder individuelle Redebeitrag in eine andere Zeile aufgenommen wurde.

Diese Regel wird nun auf den fiktiven Dialog angewendet (siehe Abbildung 40).

In dem Beispieldialog ist diese Trennung im Transkript ebenfalls schon enthalten. Es wird zur Kennzeichnung des Sprecherwechsels das Prozentzeichen (\%) als Trennzeichen gesetzt.

Isabelle: Anna, wir haben uns schon lange nicht mehr gesehen. Wie geht es dir denn? \%

Anna: Mir geht es ganz gut, Isabelle. Ich bin gerade auf der Suche nach einem Geburtstagsgeschenk für eine Freundin. Was treibt dich denn in die Stadt? \%

Isabelle: Ach, ich will einfach nur ein bisschen herumschlendern und in ein paar Läden nach neuen Klamotten Ausschau halten. Ich habe mir eben sogar schon eine Jeans gekauft. Warte, ich zeige sie dir mal. Die ist doch toll, oder? \%

Anna: Ja, in der Tat. Wo hast du die Hose denn gekauft? Die wäre wirklich super als Geschenk! \%

Isabelle: Also, die kannst du im neuen Geschäft, du weißt schon, an der nächsten Ecke erstehen. In dem Laden scheinen ganz... \%

Anna: Oh, das ist doch der Laden, der letzte Woche eröffnet und gerade lauter Sonderangebote hat. \%

Isabelle: Ja, ganz genau! \%

Anna: $\quad$ Dann werde ich da gleich vorbeischauen, um nach Schnäppchen Ausschau zu halten. Ich hoffe ganz stark, dass wir uns bald mal wieder sehen. \%

Isabelle: $\quad$ Viel Spaß noch und bis bald. \%

Abbildung 40: Beispiel für Regel 1: Trennung bei Sprecherwechsel

\section{Zusammenfassend kann festgehalten werden:}

- Es wird zunächst jedes Mal eine eigenständige Kodiereinheit gebildet, wenn eine andere Person beginnt, eine Aussage zu tätigen.

- $\quad$ Trennungen ergeben sich immer genau zwischen den Redebeiträgen der Personen.

\section{Trennung nach grammatikalischen Gesichtspunkten innerhalb individueller Redebeiträge}

Zunächst wird zwischen Haupt- und Nebensätzen getrennt (Regel 2). Da sich allerdings in der gesprochenen Sprache nicht immer Haupt- und Nebensätze aneinander anreihen, sondern diese Teilsätze umgangssprachlich miteinander verbunden werden, wird anschließend auf „grammatikalisch zusammengezogene“ Sätze eingegangen (Regel 3). Im 
Weiteren wird der Umgang mit speziellen Satzformen (Infinitivgruppen und satzwertige Partizipien: Regel 4; Relativsätze und Verbativergänzungen: Regel 5) und Einschüben erläutert (Regel 6).

Für die Regeln 2 und 3 gilt, dass die Sätze nur dann abgetrennt werden, wenn sie aus mindestens drei Satzgliedern $\underline{19}$ bestehen. Ein aussagekräftiger Satz setzt sich immer aus drei verschiedenen Satzgliedern zusammen, zum Beispiel aus einem Subjekt, einem Prädikat und einem Objekt (SPO) (Schurf, 2001). Wir empfehlen deshalb, nur solche Äußerungen als eigenständige Kodiereinheit zu betrachten, die diese Mindestanforderungen an einen gehaltvollen Satz erfüllen. Nur so erscheint es inhaltlich sinnvoll, den Satz als eigenständige Kodiereinheit aufzufassen. Würde man lediglich ein bzw. zwei Satzglieder als ausreichende Bestandteile einer trennungswürdigen Einheit ansehen, so erhielte man jede Menge einzelner Satzfragmente, die im Rahmen der eingangs genannten Fragestellung für die weitere Kodierung nicht verwendbar wären.

\section{Regel 2: Trennung zwischen Haupt- und Nebensätze}

Zur Bildung der Kodiereinheiten werden aufeinander folgende abgeschlossene und aussagekräftige Haupt- bzw. Nebensätze voneinander abgetrennt. Als aussagekräftig gilt ein Teilsatz, der aus mindestens drei Satzgliedern besteht. Dadurch enthält jede entstehende Kodiereinheit mindestens ein Subjekt und ein Prädikat sowie ein zusätzliches drittes Satzglied, z. B. ein Objekt.

Für den Beispieldialog ergeben sich somit die ersten Trennungen von Redebeiträgen (siehe Abbildung 41).

In unserem Beispiel werden nun alle abgeschlossenen Haupt- und Nebensätze, die ein Subjekt und ein vollständiges Prädikat und nicht nur eine finite Verbform besitzen und die noch aus mindestens einem dritten Satzglied bestehen, in eine neue Zeile eingefügt. An die Trennstelle wird, wie in Kapitel 1.3 erwähnt, das Trennzeichen „\#“ mit vorhergehendem Leerzeichen gesetzt. Es entsteht folgendes neues Bild:

Isabelle: Anna, wir haben uns schon lange nicht mehr gesehen. \# Wie geht es dir denn? \%

Anna: $\quad$ Mir geht es ganz gut, Isabelle. \# Ich bin gerade auf der Suche nach einem Geburtstagsgeschenk für eine Freundin. \# Was treibt dich denn in die Stadt? \%

Isabelle: Ach, ich will einfach nur ein bisschen herumschlendern und in ein paar Läden nach neuen Klamotten Ausschau halten. \# Ich habe mir eben sogar schon eine Jeans gekauft. \# Warte, ich zeige sie dir mal. \#

\footnotetext{
${ }^{19}$ Die Bausteine, aus welchen Sätze zusammengesetzt werden, bezeichnet man als Satzglieder. Ein Satzglied kann sowohl aus einzelnen Wörtern als auch aus Wortgruppen bestehen (Engel, 1996). Zur Ermittlung der Satzglieder wurde eine Methode entwickelt: die Umstellprobe. Sie zeigt, dass bestimmte Wörter nur zusammen im Satz verschoben werden können (Schurf, 2001). Ein Beispiel für die Umstellprobe: Der berühmte Schriftsteller/schreibt/ ein neues Buch/ in diesem Jahr. In diesem Jahr/ schreibt/ der berühmte Schriftsteller/ ein neues Buch. Ein neues Buch/schreibt/ der berühmte Schriftsteller/ in diesem Jahr.
} 


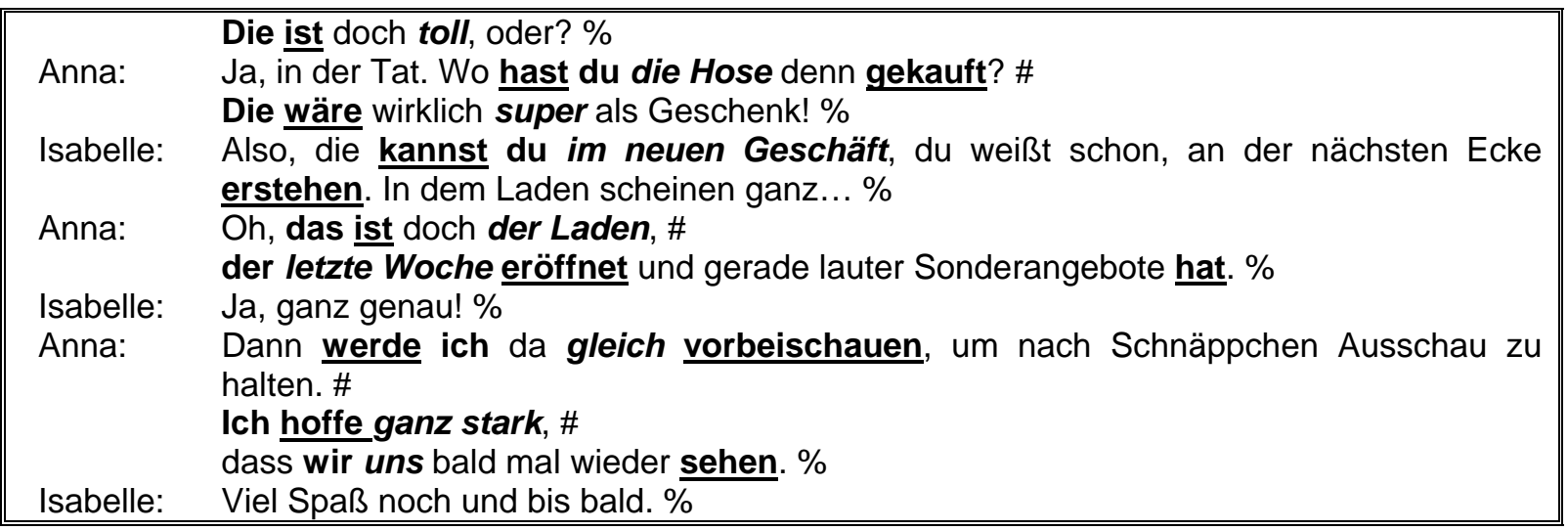

Abbildung 41: Beispiel für Regel 2: Trennung der Haupt- und Nebensätze

Hauptsätze, die ein Subjekt und ein vollständiges Prädikat und nicht nur eine finite Verbform besitzen, werden voneinander abgetrennt, da sie sprachwissenschaftlich gesehen autonome Sätze darstellen (Engel, 1996).

Nebensätze, die ein Subjekt und ein vollständiges Prädikat und nicht nur eine finite Verbform besitzen, werden ebenso abgetrennt, obwohl sie unselbstständige Sätze sind, die nur in Abhängigkeit von einem Hauptsatz vorkommen (Engel, 1996). Dennoch stellen sie eine eigenständige Einheit sprachlicher Kommunikation dar, denn jeder Satz - egal ob Hauptoder Nebensatz - ist „eine Sinneinheit innerhalb eines größeren Gedankenzusammenhanges“ (Jung, 1973, S. 1). Nebensätze drücken beispielsweise Überlegungen, Begründungen, Folgen und andere gedankliche zu dem im Hauptsatz Gesagtem Inhalte aus (Schurf, 2001).

\section{Zu beachten ist insbesondere:}

Für die Fälle, in denen spezielle Nebensätze (z.B. Relativsätze) lediglich ein Subjekt, Verb o.ä. näher bestimmen oder in denen Satzeinschübe vorliegen, gibt es Ausnahmeregeln (siehe Regeln 5 und 6).

\section{Zusammenfassend kann festgehalten werden:}

Trennungen werden vorgenommen zwischen:

- $\quad$ zwei aufeinander folgenden Hauptsätzen (HS)

- $\quad$ einem HS und einem Nebensatz (NS) oder

- $\quad$ zwei aufeinander folgenden Nebensätzen.

Bedingung: Die Sätze bestehen aus mindestens drei Satzgliedern.

Die Stelle der jeweiligen Trennung ist:

- $\quad$ vor Beginn eines neuen Satzes oder 
vor einer Konjunktion ${ }^{20}$.

\section{Regel 3: Grammatikalisch zusammengezogene Sätze}

Die gesprochene Sprache unterscheidet sich in vielerlei Hinsicht von der Schriftsprache. So werden beispielsweise aus Gründen der Ökonomie, d. h. um in kurzer Zeit möglichst viel Informationen auszudrücken, kurze und einfache Sätze gesprochen. Dasselbe Subjekt wird darin nicht wiederholt, da ein/e Sprecher/-in davon ausgehen kann, dass die Empfänger/innen wissen, dass er/sie selbiges meint (Jung, 1973). Zum Beispiel wird im zweiten Hauptsatz des folgenden Satzes das Subjekt, da es in beiden Teilsätzen identisch ist, aus vermutlich ökonomischen Gründen weggelassen (siehe Abbildung 42).

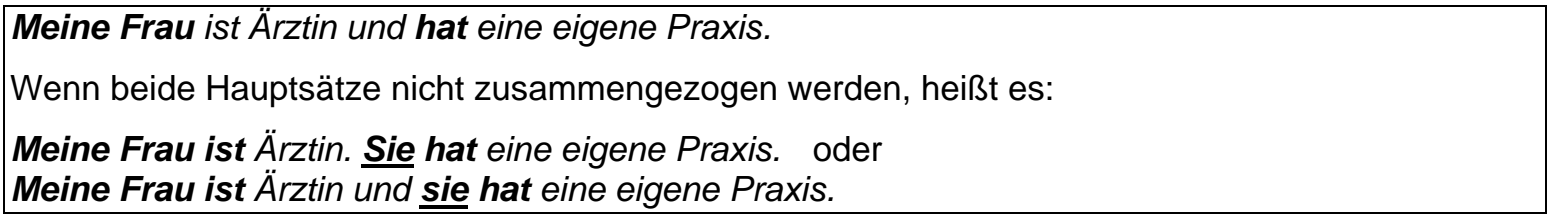

Abbildung 42: Beispiel 1 für ökonomisch gesprochene Sprache

Der zusammengezogene Satz gilt als ein aus mehreren Teilsätzen zusammengesetztes Satzganzes (Jung, 1973). Das heißt, man könnte den zusammengezogenen Satz in mehrere eigenständige Sätze umformulieren, die jeweils mit einer Interpunktion (Punkt, Fragezeichen oder Ausrufezeichen) enden. Zur Verdeutlichung sei noch ein anderes Beispiel angeführt (siehe Abbildung 43):

Sie haben das Bild in der Wohnung hängen und den Sessel in der Ecke stehen sowie das Buch auf dem Tisch liegen.

Dieses ist genau ein zusammengezogener Satz, aus dem man drei eigenständige Sätze bilden kann.

Sie haben das Bild in der Wohnung hängen. Sie haben den Sessel in der Ecke stehen. Sie haben das Buch auf dem Tisch liegen.

Abbildung 43: Beispiel 2 für ökonomisch gesprochene Sprache

Dieses Phänomen der gesprochenen Sprache muss in der Analyse und Aufbereitung von Gesprächstranskripten berücksichtigt werden. Daher schlagen wir vor, Haupt- bzw. Nebensätze voneinander abzutrennen, die in der gesprochenen Sprache grammatikalisch zusammengezogen wurden und aus mindestens drei Satzgliedern bestehen.

\footnotetext{
${ }^{20}$ Konjunktionen sind Wörter, die andere Wörter, Wortgruppen und Sätze miteinander verbinden. Sie werden deshalb auch Bindewörter genannt. Als lediglich verbindende Glieder gehören sie nicht zum Satz und haben somit keinen Satzgliedwert. Der Satz ergibt auch ohne die Konjunktion Sinn. Beispiele sind: aber, denn, oder, und, sondern, wo, wenn, nachdem, während, weil, dass, obwohl, da, ob (Jung, 1973).
} 
In Abbildung 44 ist die Anwendung der dritten Regel dargestellt.

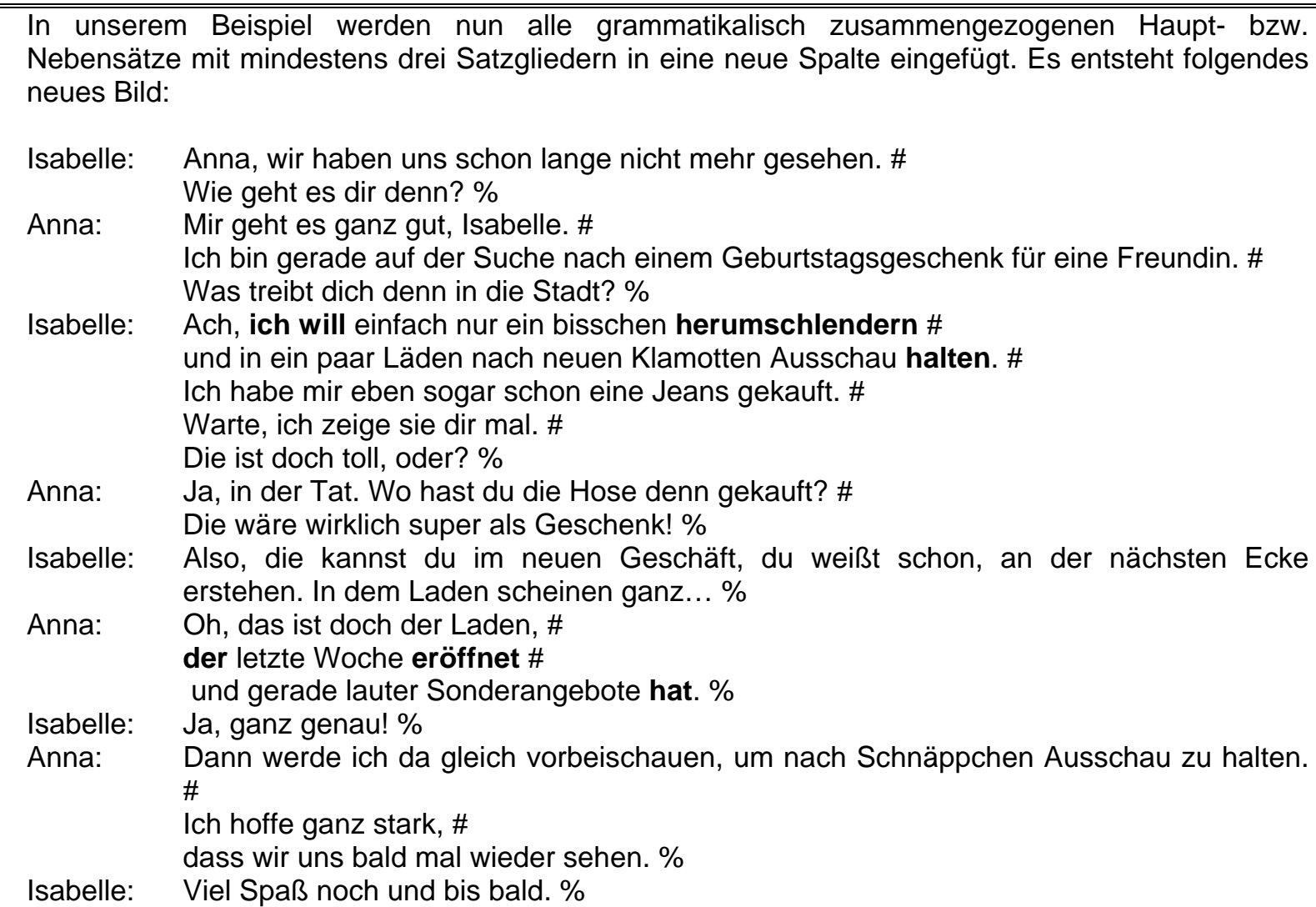

Abbildung 44: Beispiel für Regel 3: Trennung grammatikalisch zusammengezogener Sätze

Es kommt also in der gesprochenen Sprache durch das Zusammenziehen von Sätzen zur Einsparung von Satzgliedern, die im gleichen oder in einem benachbarten Satz mehr als einmal vorkommen. Gesprächspartner/-innen versuchen, eine Verständigung untereinander mit möglichst wenig Aufwand zu realisieren (Clark \& Brennan, 1991; Grice, 1998). Im Rahmen des Grounding spricht man auch von dem „principle of least collaborative effort“ (Clark, 1999). Da es sich um ein Zusammenziehen von Sätzen aus Gründen der Sparsamkeit handelt (vgl. Clark \& Brennan, 1991), aber dennoch unterschiedliche Aussagen kommuniziert werden, erfolgt eine Trennung. Somit wird gewährleistet, dass unterschiedliche Aussagen, die nur vermeintlich zusammengehören, auch unterschiedlich kodierbar sind.

\section{Zu beachten ist insbesondere:}

Es darf nicht vergessen werden, dass im grammatikalisch zusammengezogenen Satz mindestens ein Satzglied nicht erwähnt wird und somit fehlt, da es, wie oben beschrieben, aus ökonomischen Gründen weggelassen wird. Trotzdem werden die fehlenden Wörter oder Wortgruppen im entsprechenden Satz als Satzglieder gewertet (siehe Abbildung 45). 
Ich will noch einkaufen und das Auto waschen.

Hierbei handelt es sich um zwei grammatikalisch zusammengezogene Sätze, wobei im 2. Satz das Subjekt und das finite Verb weggelassen wurden. Der Teilsatz besteht somit auf den ersten Blick nur aus zwei Satzgliedern („das Auto“ und „waschen“) und wird demnach nicht getrennt. Da die Sätze allerdings eigenständig ausgesprochen aus jeweils mindestens drei Satzgliedern bestehen, werden beide Sätze voneinander getrennt.

Ich will noch einkaufen und ich will das Auto waschen.

Abbildung 45: Beispiel für besondere Zählung der Satzglieder im grammatikalisch zusammengezogenen Satz

\section{Zusammenfassend kann festgehalten werden:}

Trennungen werden vorgenommen zwischen:

- $\quad$ zwei zusammengezogenen Hauptsätzen (HS)

- $\quad$ oder zwischen zwei zusammengezogenen Nebensätzen (NS).

Bedingung: Die Sätze bestehen aus mindestens drei Satzgliedern.

Die Stelle der Trennungen erfolgt:

- $\quad$ vor einer Konjunktion. 


\section{Regel 4: Infinitivgruppen und satzwertige Partizipien}

Wenn ein Satz einen erweiterten Infinitiv ${ }^{21}$ (siehe Abbildung 46) oder mehrere erweiterte Infinitivgruppen bzw. satzwertige Partizipien ${ }^{22}$ (siehe Abbildung 47) am Anfang oder am Ende des Satzes enthält, so wird jede dieser Satzergänzungen einzeln vom restlichen Satz abgetrennt.

Ein erweiterter Infinitiv, der abgetrennt wird, wäre:

Wir müssen uns heute treffen, um eine wichtige Entscheidung zu treffen.

In diesem Fall würde man den erweiterten Infinitiv „um eine wichtige Entscheidung zu treffen“ abtrennen (die unterstrichenen Worte stellen die Erweiterung des Infinitivs dar).

Abbildung 46: Beispiel für einen erweiterten Infinitiv

Ein satzwertiges Partizip wurde im folgenden Satz verwendet:

Konzentriert lesend, überhörte er das Klopfen an der Tür.

Das satzwertige Partizip „konzentriert lesend“ würde vom restlichen Satz abgetrennt werden.

Abbildung 47: Beispiel für ein satzwertiges Partizip

Erweiterte Infinitive und satzwertige Partizipien werden abgetrennt, weil sie dem Charakter eines Nebensatzes nahekommen (Klosa, 2001). Dies gilt nicht für einfache Infinitive, die lediglich aus einem „Infinitiv mit zu“ bestehen. Sie werden daher nicht abgetrennt. Diese Unterscheidung wird in Abbildung 48 veranschaulicht.

Ein einfacher Infinitiv, der nicht getrennt wird, wäre:

Sie trafen sich, um zu reden.

In diesem Beispiel besteht der „Infinitiv mit zu“ nur aus den Wörtern „um zu reden“. Würde der „Infinitiv mit zu“ erweitert werden, dann würde wiederum ein Trennung erfolgen:

Sie trafen sich, um über ihr Referat zu reden.

Hier wurde der "Infinitiv mit zu“ um das Satzglied „über ihr Referat" erweitert.

Abbildung 48: Beispiel für die Unterscheidung von einfachen und erweiterten Infinitiven

In Abbildung 49 wird die Anwendung der vierten Regel auf den eingangs eingeführten Beispieldialog gezeigt.

\footnotetext{
${ }^{21}$ Der einfache oder erweiterte Infinitiv ist eine Wortgruppe aus einem Infinitiv mit qu und meist einem oder mehreren davon abhängenden Gliedern (Klosa, 2001).

22 Man nennt sie auch Partizipgruppen (Schurf, 2001). Das satzwertige Partizip ist ein Partizip, das mit einer näheren Bestimmung größeren Umfangs verbunden ist. Im Unterschied zum einfachen Partizip, das fast immer einfaches Satzglied oder Attribut ist, ist das satzwertige Partizip aus dem übergeordneten Satz herausgelöst, d. h., es stellt neben dem Verb dieses Satzes einen eigenen Verbalbereich und somit auch inhaltlichen Bereich dar (Klosa, 2001).
} 
In unserem Beispieldialog gibt es einen erweiterten Infinitiv, der natürlich abgetrennt wird. Es ergibt sich eine neue Kodiereinheit:

Isabelle: Anna, wir haben uns schon lange nicht mehr gesehen. \#

$\begin{array}{ll} & \text { Wie geht es dir denn? \% } \% \\ \text { Anna: } & \text { Mir geht es ganz gut, Isabelle. \# }\end{array}$

Ich bin gerade auf der Suche nach einem Geburtstagsgeschenk für eine Freundin. \#

Was treibt dich denn in die Stadt? \%

Isabelle: Ach, ich will einfach nur ein bisschen herumschlendern \#

und in ein paar Läden nach neuen Klamotten Ausschau halten. \#

Ich habe mir eben sogar schon eine Jeans gekauft. \#

Warte, ich zeige sie dir mal. \#

Die ist doch toll, oder? \%

Anna: $\quad$ Ja, in der Tat. Wo hast du die Hose denn gekauft? \#

Die wäre wirklich super als Geschenk! \%

Isabelle: Also, die kannst du im neuen Geschäft, du weißt schon, an der nächsten Ecke erstehen. In dem Laden scheinen ganz... \%

Anna: $\quad$ Oh, das ist doch der Laden, \#

der letzte Woche eröffnet \#

und gerade lauter Sonderangebote hat. \%

Isabelle: Ja, ganz genau! \%

Anna: $\quad$ Dann werde ich da gleich vorbeischauen, \# um nach Schnäppchen Ausschau zu halten. \#

Ich hoffe ganz stark, \#

dass wir uns bald mal wieder sehen. \%

Isabelle: Viel Spaß noch und bis bald. \%

Abbildung 49: Beispiel für Regel 4: Trennung von erweiterten Infinitiven und satzwertigen

Partizipien.

Der erweiterte Infinitiv gibt als satzwertiger Infinitiv einen eigenständigen Inhalt wieder. Im Unterschied zum reinen Infinitiv ist er aus dem übergeordneten Satz herausgelöst, d.h. er stellt neben dem Verb dieses Satzes einen eigenen inhaltlichen Bereich dar (Klosa, 2001) und sollte somit separat kodiert werden.

Satzwertige Partizipien werden abgetrennt, obwohl sie kein finites Verb in der Personalform enthalten, da sie im Satz die Funktion von Nebensätzen übernehmen können (Schurf, 2001) und somit eine eigene Kodiereinheit darstellen.

\section{Zu beachten ist insbesondere:}

Wenn ein Infinitiv oder ein satzwertiges Partizip inmitten eines Satzes vorzufinden ist, bei dem der eigentliche Haupt- oder Nebensatz vor der Satzergänzung noch nicht beendet ist, also im Anschluss weitergeführt wird, so wird der erweiterte Infinitiv bzw. das satzwertige Partizip nicht abgetrennt (siehe Regel 6: Einschübe), da sonst der Haupt- oder Nebensatz in anschließend nicht mehr kodierbare Segmente zerfällt. 
Zusätzlich ist zu berücksichtigen, dass im Hinblick auf Regel 5 „Relativsätze und Verbativergänzungen“ ein erweiterter Infinitiv nicht abzutrennen ist, wenn es sich bei dem Satzteil vor dem „Infinitiv mit zu“, um eine Verbativergänzung handelt, da Regel 5 hierarchisch höher als Regel 4 zu betrachten ist. Somit werden Sätze, wie „sie nahmen sich vor, das Buch zu lesen.“ nicht getrennt, obwohl ein erweiterter Infinitiv („das Buch zu lesen“) vorliegt, da die Wortgruppe "sie nahmen sich vor" eine Verbativergänzung ist (siehe Regel 5).

\section{Zusammenfassend kann festgehalten werden:}

Trennungen werden vorgenommen bei:

- $\quad$ erweiterten Infinitiven oder

- $\quad$ bei satzwertigen Partizipien.

Die Stelle der Trennungen ist:

- $\quad$ vor dem Beginn der Satzergänzung oder

- $\quad$ vor einer Konjunktion (bei Infinitivgruppen oft „um“).

\section{Regel 5: Relativsätze und Verbativergänzungen (keine Trennung)}

Wenn ein Haupt- oder Nebensatz durch einen oder mehrere Relativsätze ${ }^{23}$ (siehe Abbildung 12), welche ein Nomen oder Pronomen näher beschreiben (z.B. die Frau, die die Entscheidung trifft), erläutert wird bzw. wenn ein Verb durch eine Verbativergänzung ${ }^{24}$ in Form eines Satzes komplettiert wird (siehe Abbildung 13), so ergibt sich keine Trennung. Aufzählungen von Relativsätzen (siehe Abbildung 50) bzw. von Verbativergänzungen werden ebenso nicht getrennt (siehe Abbildung 51).

\section{Einfacher Relativsatz}

Das ist eine wichtige Entscheidung, die eine große Bedeutung hat.

Aufzählung von mehreren Relativsätzen

\footnotetext{
23 Der Relativsatz ist ein Nebensatz, der durch ein Relativpronomen mit einem Haupt- oder Nebensatz verknüpft wird. Relativpronomen sind: der, die, das, welcher, welche, welches. Diese dürfen nicht verwechselt werden mit der, die, das in der Funktion des Artikels oder Demonstrativpronomens (hinweisendes Fürwort). Nur das Relativpronomen kann durch welcher, welche, welches ersetzt werden (Schurf, 2001).

${ }^{24}$ Eine Verbativergänzung ist immer seine Ergänzung zu einem Verb, die nur als satzartiges Konstrukt realisiert werden kann. Sie enthält immer ein Verb und ist nie gegen eine nicht satzförmige Ergänzung austauschbar. Ein typisches Beispiel für eine Verbativergänzung ist: Ich finde, dass Hannah Recht hat (Engel, 1996).
} 
Der 100m-Lauf ist eine Disziplin, die sehr kraftaufwendig ist und die der Leichtathletik zuzuordnen ist.

Abbildung 50: Beispiele für Relativsätze

Verbativergänzungen gehen oft Verben wie sein, werden, bleiben, scheinen (Jung, 1973) oder Nebensatzverben (z.B. bedeuten, finden, sich fragen, es heißt) zuvor (Engel, 1996). Typische Beispiele für Satzanfänge, denen eine Verbativergänzung folgt, sind:

- Ich bin der Meinung/ Ansicht/ Auffassung, ...

- Ich denke/ meine/ finde/ sage, ...

- Ich möchte vorschlagen, ...

- Es wird sich die Frage stellen, ...

- Das heißt/ bedeutet/ scheint, ...

- Ich frage mich, ...

- Ich nehme mir vor,...
Verbativergänzung
Ich finde, er sollte zurücktreten.
Aufzählung von mehreren Verbativergänzungen
Ich finde, er sollte zurücktreten und in den Ruhestand gehen.

\section{Abbildung 51: Beispiel für Verbativergänzungen}

Einfache Relativsätze und Aufzählungen von Relativsätzen werden nicht abgetrennt, da der Relativsatz als Attribut ${ }^{25}$ kein eigenständiges Satzglied ist, sondern lediglich eine genauere Erläuterung eines Satzbestandteiles darstellt (Schurf, 2001). Wichtiger sind aber vielmehr die Konsequenzen, die eine Abtrennung von Relativsätzen mit sich zögen: Der den Relativsatz einschließende Haupt- oder Nebensatz würde so „zerrissen“, dass die verbleibenden Fragmente für eine weitere Kodierung keinen Sinn machten. Demnach würde eine zusätzliche Erfassung der Relativsätze den Umgang mit den verbleibenden Satzbestandteilen stark erschweren (vgl. Regel 6). Wenn man den Relativsatz weglassen würde, ergäbe der verbleibende Haupt- oder Nebensatz eine in sich geschlossene Aussage, die eben nicht zu trennen ist.

\footnotetext{
${ }^{25}$ Attribute sind immer Teil eines Satzglieds, also keine selbstständigen Satzglieder. Diese Satzteile stehen vor oder hinter ihren Bezugswörtern (z.B. Nomen) und bestimmen sie näher (Schurf, 2001).
} 
Verbativergänzungen werden ebenfalls nicht abgetrennt, da sie benötigt werden, um dem vorangegangen Satz einen Sinn zu geben. Ohne diese Ergänzung wäre der Satz unvollständig und unlogisch (Engel, 1996). Wenn ich z.B. nur sagen würde „ich finde.“, dann stellt sich automatisch die Frage „was finde ich?“. Der Satz ist nur komplett, wenn ich inn weiterführe, z.B. in der Form „ich finde, dass du Recht hast“. Da der vorangegangene Hauptsatz in unserem Beispiel „ich finde“ nur durch den Nebensatz bzw. genauer gesagt der Verbativergänzung „dass du Recht hast“ komplett ist, werden beide Teile nicht voneinander getrennt. Verbativergänzungen ergeben somit nie eine eigenständige Sinneinheit, sondern bilden immer mit dem voran- oder nachgestellten Satz eine Einheit.

\section{Zu beachten ist insbesondere}

Regel 5 über den Umgang mit Verbativergänzungen ist hierarchisch höher gestellt als Regel 4 über erweiterte Infinitive! Erweiterte Infinitive werden somit nicht abgetrennt, wenn ihnen nur eine Verbativergänzung vorausgeht (siehe Abbildung 52).

Ich nahm mir vor, das Buch zu lesen.

In diesem Beispiel besteht der „Infinitiv mit zu“ aus den Wörtern „das Buch zu lesen“. Die Verbativergänzung ist „ich nahm mir vor". Es erfolgt keine Trennung, da ausschließlich eine Verbativergänzung und ein erweiterter Infinitiv vorliegen.

Ich finde, wir müssen uns treffen, um eine Entscheidung zu fällen.

In diesem Beispiel erfolgt eine Trennung, da die Verbativergänzung (,ich finde“) dem erweiterten Infinitiv („um eine Entscheidung zu fällen“) nicht genau vorangestellt ist. Die Trennung sieht wie folgt aus.

Ich finde, wir müssen uns treffen, \#

um eine Entscheidung zu fällen.

Abbildung 52: Beispiel für Verbativergänzung und erweiterte Infinitiv

Die neue Trennregel wird in Abbildung 53 am Beispieldialog verdeutlicht.

Für unser Beispiel bedeutet dies, dass wir Trennungen, die wir aufgrund von Regel 1 (Trennung von Haupt- und Nebensätzen) getätigt haben, wieder rückgängig machen müssen, wenn es sich hierbei um einen Relativsatz oder eine Verbativergänzung handelt.

Isabelle: Anna, wir haben uns schon lange nicht mehr gesehen. \# Wie geht es dir denn? \%

Anna: $\quad$ Mir geht es ganz gut, Isabelle. \# Ich bin gerade auf der Suche nach einem Geburtstagsgeschenk für eine Freundin. \# Was treibt dich denn in die Stadt? \%

Isabelle: Ach, ich will einfach nur ein bisschen herumschlendern \# und in ein paar Läden nach neuen Klamotten Ausschau halten. \# Ich habe mir eben sogar schon eine Jeans gekauft. \# 


\begin{tabular}{|c|c|}
\hline \multirow{3}{*}{ Anna: } & Warte, ich zeige sie dir mal. \# \\
\hline & $\begin{array}{l}\text { Die ist doch toll, oder? \% } \\
\text { Ja, in der Tat. Wo hast du die Hose denn qekauft? \# }\end{array}$ \\
\hline & Die wäre wirklich super als Geschenk! \% \\
\hline Isabelle: & $\begin{array}{l}\text { Also, die kannst du im neuen Geschäft, du weißt schon, an der nächsten Ecke } \\
\text { erstehen. In dem Laden scheinen ganz...\% }\end{array}$ \\
\hline Anna: & $\begin{array}{l}\text { Oh, das ist doch der Laden, der letzte Woche eröffnet und gerade lauter } \\
\text { Sonderangebote hat. } \%\end{array}$ \\
\hline Isabelle: & Ja, ganz genau! \% \\
\hline Anna: & $\begin{array}{l}\text { Dann werde ich da gleich vorbeischauen, \# } \\
\text { um nach Schnäppchen Ausschau zu halten. \# } \\
\text { Ich hoffe, dass wir uns bald mal wieder sehen.\% }\end{array}$ \\
\hline Isabelle: & Viel Spaß noch und bis bald. \% \\
\hline
\end{tabular}

Abbildung 53: Beispiel für Regel 5: Keine Abtrennung von Relativsätzen und Verbativergänzungen

Zusammenfassend kann festgehalten werden:

Keine Trennungen werden vorgenommen bei:

- $\quad$ einfachen Relativsätzen

- Verbativergänzungen und

- $\quad$ Aufzählungen von mehreren Relativsätzen und Verbativergänzungen.

\section{Regel 6: Einschübe (keine Trennung)}

Die folgende Regel ähnelt dem Umgang mit Relativsätzen (vgl. Regel 5). Wenn ein Hauptoder Nebensatz mittendrin durch einen oder mehrere Hauptsätze, Nebensätze, erweiterte Infinitive oder anderweitige Einschübe (z.B. satzwertige Partizipien oder Apposition ${ }^{26}$ ) näher erläutert bzw. unterbrochen wird, so ergibt sich keine Trennung (siehe Abbildung 55). Würde man den Einschub bzw. die Einschübe entfernen, so ergäbe der verbleibende Haupt- oder Nebensatz weiterhin eine korrekte und fließende Aussage.

Unter Einschüben werden grammatische Satzformen verstanden, bei denen der eigentliche Haupt- oder Nebensatz vor dem Einschub bzw. vor den Einschüben noch nicht beendet ist und im Anschluss nach dem Einschub noch weitergeht. Dies trifft z.B. bei folgender grammatikalischer Form zu:

\section{Anfang des Hauptsatzes - Einschub - Fortsetzung des Hauptsatzes}

Der Einschub wird nicht abgetrennt, da er nicht den Hauptsinn des Satzes widerspiegelt, den der/die Sprecher/-in mit seiner Aussage tätigen will. Wenn man den Einschub in Gedanken

\footnotetext{
${ }^{26}$ Appositionen sind nachgestellte nähere Bestimmungen, die im gleichen Kasus stehen wie das Bezugswort, meist ein Nomen (Schurf, 2001). Attribute beziehen sich nie auf das Prädikat und sind somit kein selbständiges Satzglied (Jung, 1973).
} 
weglässt, hat man trotzdem eine in sich geschlossene Aussage vorliegen, welche die Hauptintention des/der Sprechers/-in abbildet.

Ein weiterer Grund gegen die Abtrennung von Einschüben besteht darin, dass es durch die Zerteilung des eigentlichen Hauptsatzes bzw. Nebensatzes in zwei eigenständige, jedoch „zerrissene“ Einheiten später zu Problemen bei der Kategorienbestimmung kommen könnte (siehe Abbildung 54). In diesem Fall beschränkt man sich auf die vollständige Erhaltung des sprachwissenschaftlich gesehen übergeordneten Satzes (entweder ein Hauptsatz oder ein übergeordneter Nebensatz).

Würde man den Satz „Herr Müller, der Chef des Unternehmens, hat Einspruch erhoben“ wie folgt trennen, käme es zu inhaltlichen Problemen.

Herr Müller, \# der Chef des Unternehmens, \# hat Einspruch erhoben.

Herr Müller würde wahrscheinlich, da dieses Satzglied jetzt einzeln steht, mit einer namentlichen Anrede (siehe Regel 8) verwechselt werden. Der Satz heißt aber vollständig:

Herr Müller hat Einspruch erhoben.

Hier erkennt man, dass es sich keineswegs um eine namentliche Anrede von Herrn Müller handelt, sondern dass „Herr Müller" eine Namensnennung darstellt, die das Subjekt des Satzes ist. Eine Zerteilung dieses Satzes aufgrund des Einschubes „der Chef des Unternehmens“ könnte also inhaltliche Probleme nach sich ziehen, weswegen Einschübe inmitten des Satzes nicht abgetrennt und somit auch nicht separat kodiert werden.

Abbildung 54: Veranschaulichung der Einschub-Problematik

\section{Beispiele für Einschübe, bei denen keine Trennung erfolgt:}

Eingeschobener Hauptsatz

Das ist aber, glaube ich, verbesserbar.

Der Hauptsatz "Das ist aber verbesserbar." wird durch einen anderen Hauptsatz "glaube ich“ unterbrochen. Es findet keine Trennung statt.

ODER

Eingeschobener erweiterter Infinitiv

Wir müssen, um eine Entscheidung zu treffen, schneller arbeiten.

Der Satz „Wir müssen schneller arbeiten." wird durch den eingeschobenen erweiterten Infinitiv „um eine Entscheidung zu treffen“ unterbrochen. Es findet keine Trennung statt.

ODER

Eingeschobenes satzwertiges Partizip

Ihre Freundin, das gerade eingegangene Fax lesend, freute sich über die Einladung.

Der Satz "Ihre Freundin freute sich über die Einladung." wird durch das eingeschobene satzwertige Partizip "das gerade eingegangene Fax lesend“ unterbrochen. Es findet keine Trennung statt.

\section{Abbildung 55: Beispiele für Einschübe}


Abbildung 56 zeigt die Anwendung der sechsten Regel auf den eingangs eingeführten Beispieldialog.

Für unser Beispiel bedeutet dieses, dass „du weißt schon“ ein Einschub ist und den Satz „Also, die kannst du im neuen Geschäft an der nächsten Ecke erstehen.“ unglücklich zerteilen würde. „Du weißt schon" wird deshalb nicht in eine einzelne Zeile eingefügt, um eine eigenständige Kodiereinheit zu bilden:

Isabelle: Anna, wir haben uns schon lange nicht mehr gesehen. \# Wie geht es dir denn? \%

Anna: $\quad$ Mir geht es ganz gut; Isabelle. \# Ich bin gerade auf der Suche nach einem Geburtstagsgeschenk für eine Freundin. \# Was treibt dich denn in die Stadt? \%

Isabelle: $\quad$ Ach, ich will einfach nur ein bisschen herumschlendern \# und in ein paar Läden nach neuen Klamotten Ausschau halten. \# Ich habe mir eben sogar schon eine Jeans gekauft. \# Warte, ich zeige sie dir mal. \# Die ist doch toll, oder? \%

Anna: $\quad$ Ja, in der Tat. Wo hast du die Hose denn gekauft? \# Die wäre wirklich super als Geschenk! \%

Isabelle: Also, die kannst du im neuen Geschäft, du weißt schon, an der nächsten Ecke erstehen. In dem Laden scheinen ganz... \%

Anna: Oh, das ist doch der Laden, der letzte Woche eröffnet und gerade lauter Sonderangebote hat. \%

Isabelle: Ja, ganz genau! \%

Anna: $\quad$ Dann werde ich da gleich vorbeischauen, \# um nach Schnäppchen Ausschau zu halten. \# Ich hoffe, dass wir uns bald mal wieder sehen. \%

Isabelle: Viel Spaß noch und bis bald. \%

Abbildung 56: Beispiel für Regel 6: Umgang mit Einschüben

\section{Zusammenfassend kann festgehalten werden:}

Keine Trennungen bei Einschüben wie:

- $\quad$ eingeschobenen Hauptsätzen

- $\quad$ eingeschobenen Nebensätzen

- $\quad$ eingeschobenen erweiterten Infinitiven

- $\quad$ eingeschobenen satzwertigen Partizipien oder

- Appositionen.

\section{Regeln für unterbrochene Äußerungen und grammatikalische Phänomene}

\section{Regel 7: Umgang mit Unterbrechungen}

Es gehört zu den Phänomenen gesprochener Sprache, dass sich die Gesprächspartner /innen mitunter nicht ausreden lassen. Daher müssen solche Sätze, die mittendrin 
abgebrochen und somit nicht beendet werden oder plötzlich von einer anderen Person weitergeführt, erweitert bzw. vervollständigt werden, gesondert betrachtet werden.

Wir schlagen vor, dass unterbrochene Äußerungen erst dann als eigenständige Kodiereinheit bestimmt werden, wenn der unterbrochene Teil ein gewisses Maß an Information bzw. Inhalten enthält.

Ein aussagekräftiger Satz setzt sich zumeist aus mindestens drei verschiedenen Satzgliedern zusammen: Subjekt, Prädikat und Objekt (SPO) (Schurf, 2001). Wir empfehlen, nur solche unterbrochenen Äußerungen als eigenständige Kodiereinheit zu betrachten, die aus mindestens drei Satzgliedern ${ }^{27}$, bestehen (vgl. Abbildung 57 und Abbildung 58). Nur so erscheint es inhaltlich sinnvoll, dass man die Unterbrechung als eigenständige Kodiereinheit auffasst. Würde man lediglich ein bzw. zwei Satzglied(er) als ausreichende Bestandteile einer trennungswürdigen Unterbrechung ansehen, so erhielte man jede Menge Satzfragmente, die im Rahmen der eingangs genannten Fragestellung für die weitere Kodierung nicht verwendbar wären.

Die unterbrochenen Äußerungen, die diese Bedingung der drei Satzglieder erfüllen, werden als Kodiereinheit erfasst, da sie eine koordinierende Rolle in der Kommunikation einnehmen (Clark \& Brennan, 1991).

Diese Regel ist unabhängig davon, ob die Person ihren Gedanken selber abbricht und etwas Neues aufwirft oder ob sie von einer anderen Person unterbrochen wird.

Person 1: In finde, wir sollten uns noch einmal treffen. Es ist doch möglich, \# dass es tatsächlich Einwände von anderen Personen \%

Person 2: Nein, genau das glaube ich nicht! \%

Person 1 wird von Person 2 unterbrochen. Der unterbrochene Satzbestandteil „dass es tatsächlich Einwände von anderen Personen" wird als eigenständige Kodiereinheit aufgefasst, da er aus mehr als zwei Satzgliedern besteht. Die Satzglieder sind: „es“, „tatsächlich Einwände“ und „Von anderen Personen“. Die Trennung erfolgt vor der Konjunktion „dass“.

Abbildung 57: Beispiel für eine abzutrennende Unterbrechung

Person 1: Sind Sie sich da sicher? Sonst \%

Person 2: Ich nehme das so an. \%

Person 1 wird von Person 2 unterbrochen. Der Satzbestandteil „sonst" wird nicht als eigenständige Kodiereinheit aufgefasst, da er aus nicht mehr als zwei Satzgliedern besteht.

\section{Abbildung 58: Beispiel für eine nicht abzutrennende Unterbrechung}

\footnotetext{
$27 \mathrm{Zu}$ beachten ist, dass Konjunktionen, wie z.B. „denn“, „oder“, „und“, „dann“, „weil“, „deshalb“, „wo“, „während“. „nachdem“ nicht als eigenständige Satzglieder betrachtet werden, da sie Bindewörter sind. Sie verbinden Sätze, Nebensätze, Teile von Sätzen oder Wörter und haben somit keinen Satzgliedstatus (Jung, 1973). Relativsätze (Regel 5) und Einschübe (Regel 6) werden ebenfalls als genau ein Satzglied angesehen.
} 
Abbildung 59 zeigt die Anwendung der siebenten Regel auf den eingangs eingeführten Beispieldialog.

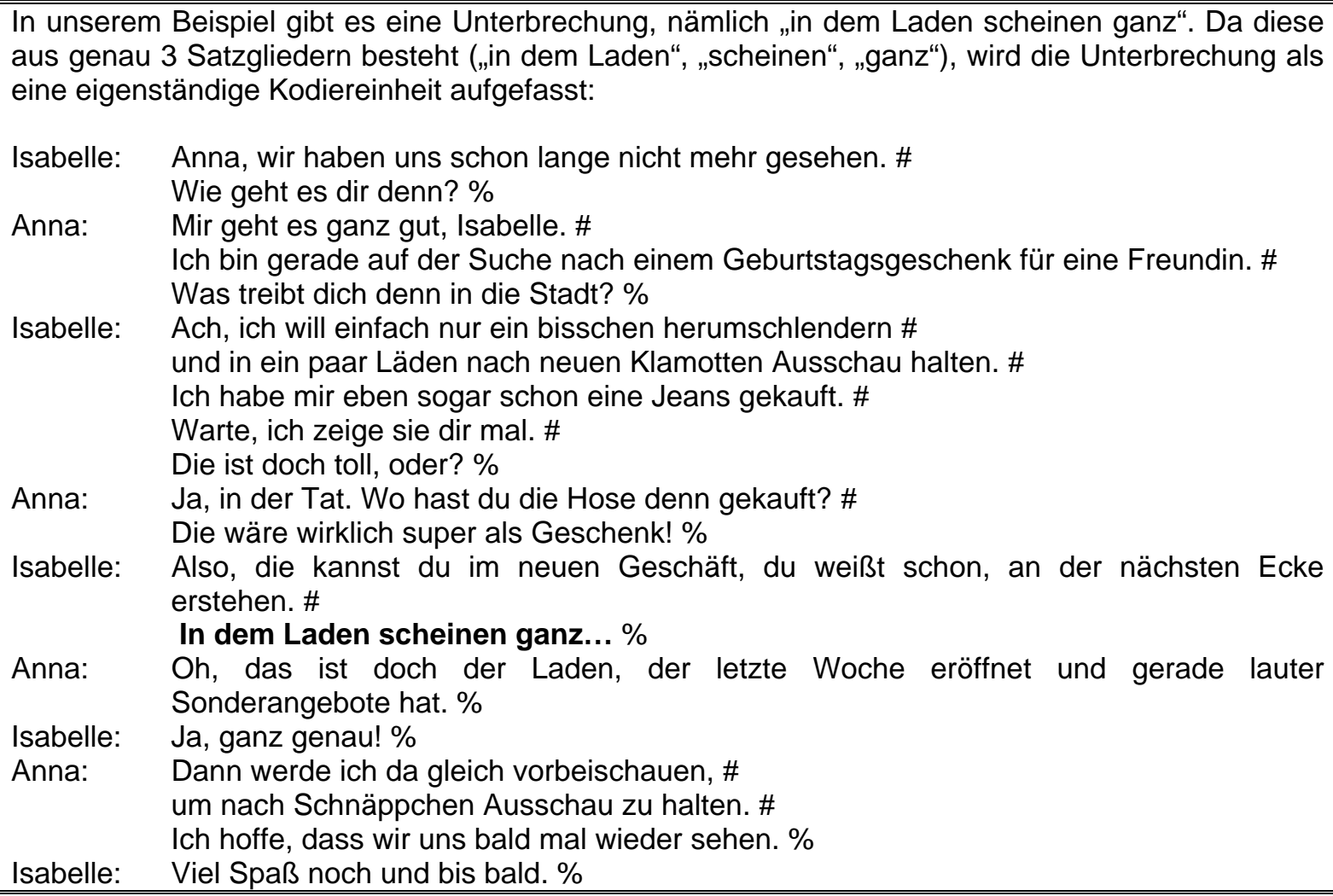

Anna: Ja, in der Tat. Wo hast du die Hose denn gekauft? \# Die wäre wirklich super als Geschenk! \%

Isabelle: Also, die kannst du im neuen Geschäft, du weißt schon, an der nächsten Ecke erstehen. \#

In dem Laden scheinen ganz... \%

Anna: Oh, das ist doch der Laden, der letzte Woche eröffnet und gerade lauter Sonderangebote hat. \%

Isabelle: Ja, ganz genau! \%

Anna: $\quad$ Dann werde ich da gleich vorbeischauen, \# um nach Schnäppchen Ausschau zu halten. \#

Ich hoffe, dass wir uns bald mal wieder sehen. \%

Isabelle: Viel Spaß noch und bis bald. \%

\section{Abbildung 59: Beispiel für die Anwendung von Regel 7: Umgang mit Unterbrechungen}

\section{Zusammengefasst kann festgehalten werden: Unterbrechungen sind:}

- $\quad$ nicht als eigenständige Kodiereinheit aufzufassen, soweit sie aus weniger als drei Satzgliedern bestehen

- $\quad$ immer als eigenständige Kodiereinheit aufzufassen, soweit sie aus mindestens drei Satzgliedern bestehen.

Wenn eine Unterbrechung abgetrennt wird, dann:

- $\quad$ an der Stelle, wo der direkt vorangehende Satzbestandteil beendet wurde

- $\quad$ vor einer Konjunktion.

Wenn eine Unterbrechung nicht abgetrennt wird, dann wird sie:

- $\quad$ der vorangehenden Kodiereinheit zugeordnet, wenn die Unterbrechung inmitten oder am Ende eines Satzes stattfindet,

- der nachfolgenden Kodiereinheit zugeordnet, wenn sie am Anfang eines Satzes vorliegt. 
Die bisherigen Kodiereinheiten werden nun noch einmal betrachtet, um namentliche Ansprachen, die man ebenso gut hätte weglassen können, sowie grammatikalische Phänomene gesprochener Sprache in Kodiereinheiten zu segmentieren.

\section{Regel 8: Namentliche Anrede}

Namentliche Anreden werden dann abgetrennt, wenn der/die Sprecher/-in sie auch hätte weglassen können, ohne dabei den Sinn des Satzes zu entstellen. Hierbei ist es gleichgültig, ob die persönliche Anrede zu Beginn oder am Ende eines Haupt- bzw. Nebensatzes erfolgt. Trennungen erfolgen immer so, dass die namentliche Anrede allein eine Kodiereinheit darstellt (siehe Abbildung 60).

Texteröffnende namentliche Anreden werden abgetrennt und als eigenständige Kodiereinheit betrachtet:

\section{Herr Schütz, \#}

Sie können ganz kurz über dieses Thema berichten.

Namentliche Anreden, die im Textinneren - aber am Ende des Teilsatzes - vorkommen, werden ebenfalls abgetrennt und als eigenständige Kodiereinheit betrachtet:

Wenn wir das ganze von einer anderen Seite betrachten, \#

meine Herren, \#

dann stellen wir fest,...

Ebenso werden textschließende namentliche Anreden werden abgetrennt und als eigenständige Kodiereinheit betrachtet:

Was denken Sie darüber, \#

Herr Meier?

Abbildung 60: Beispiele für namentliche Anreden, die abgetrennt werden

Abbildung 61 zeigt die Anwendung der achten Regel auf den eingangs eingeführten Beispieldialog.

Für unser Beispiel ergibt sich folgendes:

Isabelle: Anna, \#

wir haben uns schon lange nicht mehr gesehen. \#

Wie geht es dir denn? \%

Anna: $\quad$ Mir geht es ganz gut, \#

Isabelle. \#

Ich bin gerade auf der Suche nach einem Geburtstagsgeschenk für eine Freundin. \# Was treibt dich denn in die Stadt? \% 


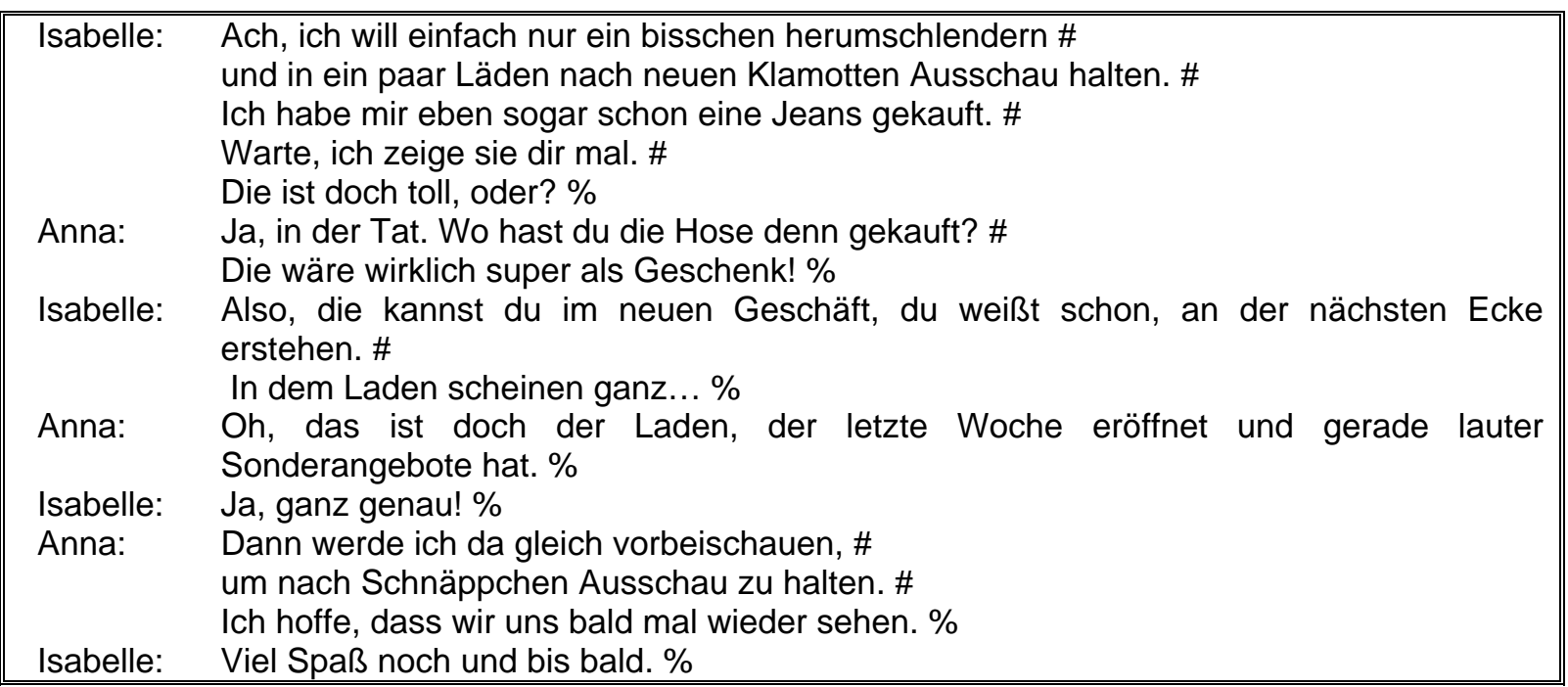

Abbildung 61: Beispiel für die Anwendung der Regel 8: Namentliche Anreden

Der Grund für die Abtrennung der namentlichen Anreden besteht in ihrer Funktion: Sie dienen der Kontakteröffnung, der Kontaktsicherung oder der Kontaktverstärkung (Engel, 1996).

$\mathrm{Zu}$ beachten ist allerdings, dass namentliche Anreden, die einen Satz zerteilen, nicht abgetrennt werden (siehe Abbildung 62). Hier greift Regel 6 „Einschübe“.

Namentliche Anreden werden nicht abgetrennt, wenn sie den Text zerteilen.

Wenn wir, Frau Schulz, das Problem angehen wollen, \#

dann müssen wir...

Abbildung 62: Beispiel für Namensansprachen, die aufgrund der Einschubregel (Regel 6) nicht abgetrennt werden

Weiterhin ist zu beachten, dass der Name einer Person nicht in eine separate Zeile gefügt wird, wenn es sich nicht um eine direkte namentliche Anrede handelt (siehe Abbildung 63). Eine Namensnennung kann auch die Rolle eines Subjektes oder Objektes einnehmen. Es handelt sich somit um eine in sich geschlossene Aussage. Die Namensnennung ist nötig, um dem Satz einen eindeutigen Sinn zu geben. Der Name stellt also keine eigenständige Kodiereinheit dar, wenn er einen Satzgliedstatus besitzt und Teil eines Subjekts oder Objekts ist.

\section{Namensnennung als Objekt}

Wenn ich erst Herrn Krämer anhören könnte.

Es handelt sich hierbei um keine namentliche Anrede! Lässt man das Objekt „Herrn Krämer“ weg, so ergibt sich keine geschlossene Aussage: Wenn ich erst anhören könnte. 
Namensnennung als Subjekt

Herr Müller müsste dafür zuständig sein.

Auch hier liegt keine namentliche Anrede vor. Der Name „Herr Müller“ ist in diesem Fall das Subjekt und kann natürlich nicht abgetrennt werde.

Abbildung 63: Beispiele für Namensnennungen, die keine namentlichen Anreden sind

\section{Zusammenfassend kann festgehalten werden:}

Namentliche Anreden werden immer in eine separate Zeile eingefügt, sofern sie den Text nicht unzumutbar zerteilen.

\section{Regel 9: Grammatikalische Phänomene gesprochener Sprache $\underline{28}$}

Grammatikalische Phänomene gesprochener Sprache werden als eigenständige Kodiereinheiten angesehen, wenn sie als eigenständiger Satz bzw. am Anfang oder am Ende eines Satzes verwendet werden.

Zu den abzutrennenden grammatikalischen Phänomenen gesprochener Sprache zählen:

- Interjektionen ${ }^{29}$

o Beispiele: oh, ach, huch, äh, hm

- Einwortkonstruktionen ${ }^{30}$

o Beispiele: Was?, Nein!, Ja!, Bitte., Gut., Klar., Okay.

- Ellipsen ${ }^{31}$

o Beispiele:

In der Tat.

Das allein dazu.

Im Gegenteil.

Sozusagen.

- Einleitende Wörter am Satzanfang

o Beispiele: so, also, nun

- Abschließende Wörter am Satzende

\footnotetext{
${ }^{28}$ Grammatikalische Phänomene gesprochener Sprache findet man meistens im alltäglichen mündlichen Sprachgebrauch. Typisch für die Umgangssprache sind kurze und einfache, aber auch unvollständige Sätze (Schurf, 2001).

${ }^{29}$ Die Interjektionen (Empfindungswörter) sind keine Wortart im eigentlichen Sinne. Sie geben Gemüts- und Willensregungen und sinnliche Eindrücke wieder. Typische Beispiele sind au, ach, oh, hm, aha, brr (Jung, 1973).

${ }^{30}$ Einwortkonstruktionen bestehen, wie der Name schon sagt, aus genau einem Wort. Sie unterscheiden sich insofern von anderen Aussagen, die ebenfalls nur aus einem Wort bestehen, dass sie in sich geschlossen und klar verständlich sind.

${ }^{31}$ Ellipsen sind unvollständige Sätze, bei denen Satzglieder oder Worte, die aus dem Kontext heraus leicht ergänzbar sind, ausgelassen werden (Schurf, 2001). Die Bedeutung und der Sinn des Satzes sind klar verständlich, da sich der Inhalt aus der Situation ergibt (Jung, 1973).
} 
o Beispiele: oder?, nicht?

Grammatikalische Phänomene gesprochener Sprache werden immer abgetrennt, so dass sie alleine eine Kodiereinheit darstellen. Wenn mehrere grammatikalische Phänomene direkt hintereinander auftreten, werden auch diese von einander abgetrennt und somit als eigenständige Sinneinheiten kodiert (siehe Abbildung 65).

Abbildung 64 zeigt die Anwendung der neunten Regel auf den eingangs eingeführten Beispieldialog.

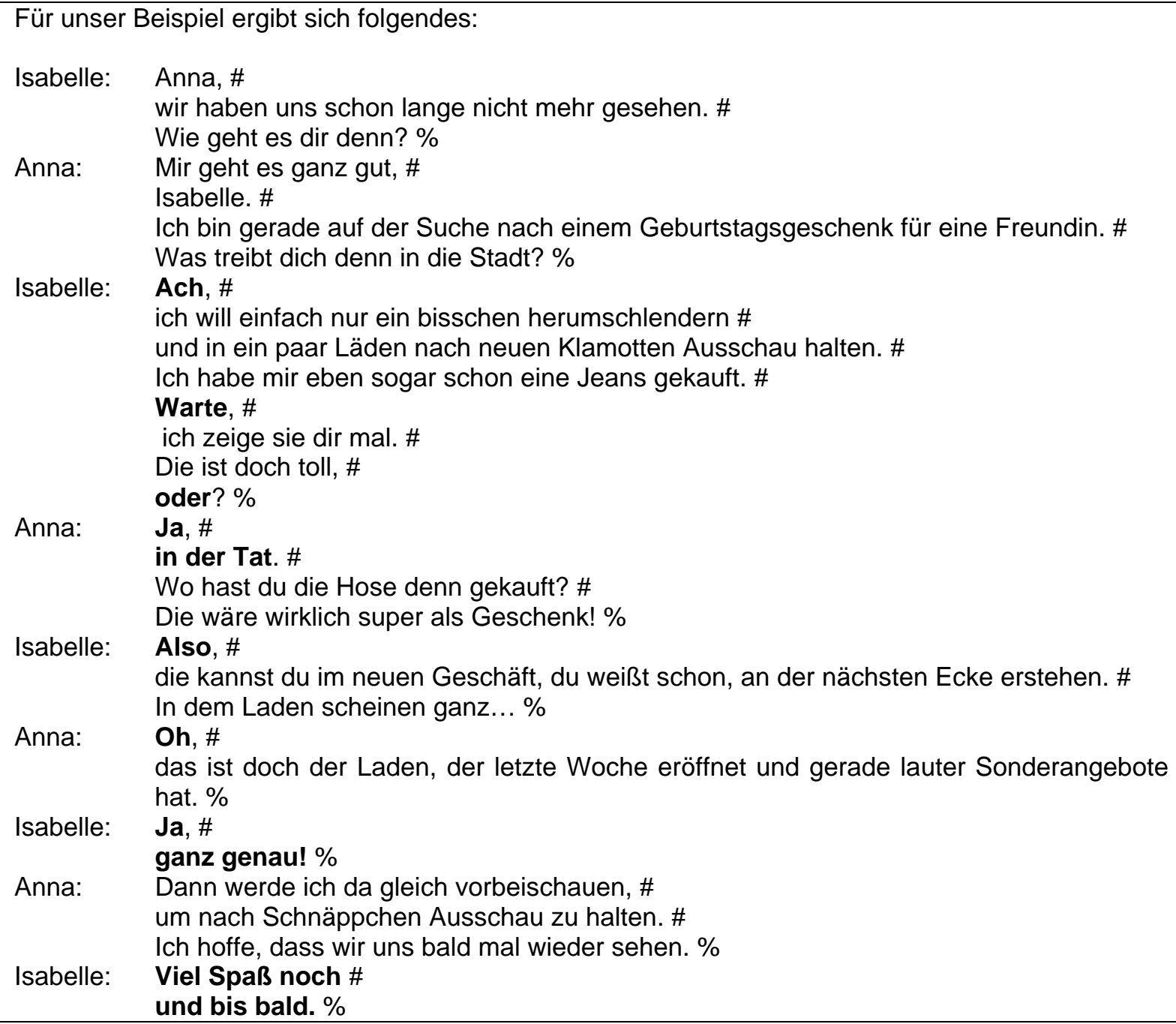

Anna: $\quad$ Oh, \#

das ist doch der Laden, der letzte Woche eröffnet und gerade lauter Sonderangebote hat. $\%$

Isabelle: $\mathrm{Ja}, \#$

ganz genau! \%

Anna: $\quad$ Dann werde ich da gleich vorbeischauen, \# um nach Schnäppchen Ausschau zu halten. \#

Abbildung 64: Anwendung Regel 9: Abtrennung grammatikalischer Phänomene gesprochener Sprache

Interjektionen, Einwortkonstruktionen und Ellipsen werden abgetrennt, da sie zwar keine Wortarten im eigentlichen Sinne sind, aber trotzdem einen Satzwert, wenn auch ohne Satzform, besitzen (Jung, 1973). Sie sind in der gesprochenen Sprache im gesprochenen Dialog ein wichtiges Ausdrucks- und Kommunikationsmittel (Engel, 1991). 
Wörter wie „okay“, „ja“, „oder“, „also“ sind typische Beispiele für Ausdrücke, die Personen im Rahmen des least collaborative effort verwenden. Die Gründe hierfür sind vielfältig, aber vor allem die Zeitersparnis scheint hierbei eine wichtige Rolle einzunehmen (Clark \& Brennan, 1991).

Einleitende und abschließende Wörter werden abgetrennt, wenn der Satz seinen inhaltlichen Sinn auch ohne die Verwendung dieser Einleite- bzw. Schlusswörter behält. Man hätte diese Wörter also ohne Sinnentstellung des Satzes weglassen können.

Die Trennung von zwei aufeinander folgenden Einwortkonstruktionen erfolgt so, dass jedes grammatikalische Phänomen eine Kodiereinheit darstellt.

Ja, \#

gut.

Die Trennung von einer Einwortkonstruktionen und Ellipse:

Ja, \#

in der Tat.

Person 2 beginnt ihren Redebeitrag mit einem einleitenden Wort. Es erfolgt eine Trennung, so dass das Einleitewort eine eigenständige Kodiereinheit ergibt.

Person 1: Was denkst du denn? \%

Person 2: Also, \#

wir müssen eine Entscheidung treffen. \%

ODER

Die Person beendet ihren Redebeitrag mit dem abschließenden Wort „oder“. Die Trennung erfolgt so, dass das abschließende Wort „oder" als eine eigenständige Kodiereinheit klassifiziert wird.

Person 1: $\quad$ Das sehen sie doch auch so, \# oder? \%

Person 2: Ich bin ganz ihrer Meinung. \%

Abbildung 65: Beispiele für die Anwendung der 9. Regel: Abtrennung von grammatikalischen Phänomenen gesprochener Sprache

\section{Zusammengefasst kann festgehalten werden:}

Grammatikalische Phänomene gesprochener Sprache werden immer in eine separate Zeile eingefügt, sofern sie den Text nicht unzumutbar zerteilen. Die Trennung erfolgt so, dass jedes einzelne grammatikalische Phänomen eine Kodiereinheit darstellt.

\section{Regel 10: Aufzählungen (keine Trennung)}

Hierzu zählen Aufzählungen von Substantiven, Verben und Adjektiven (siehe Abbildung 66).

\section{Substantiven}

Wir müssen zunächst mal die Zukunftsperspektive des Betriebes, die finanzielle Solvenz des Betriebes exakt ermitteln. 
Über die Ausgestaltung kann oder muss man noch reden.

Adjektive

Gibt es spezifische, immissionsschutzrechtliche Gesichtspunkte?

Abbildung 66: Beispiele für Aufzählungen

Aufzählungen werden nicht abgetrennt, da sie lediglich eine Häufung bzw. Aneinanderreihung gleichartiger ${ }^{32}$ und gleichrangiger ${ }^{33}$ Elemente darstellen (Engel, 1996; Klosa, 2001). Aufzählungen von Substantiven, Verben bzw. Adjektiven haben keinen Satzwert, sondern sie besitzen lediglich einen Satzgliedstatus (Engel, 1996), es besteht somit kein Anlass zur Trennung.

Abbildung 67 zeigt die Anwendung der zehnten Regel auf den eingangs eingeführten Beispieldialog.

Für unser Beispiel ergibt sich keine Änderung und die Kodiereinheiteneinteilung der Unterhaltung liegt in der endgültigen Form vor.

Isabelle: Anna, \#

wir haben uns schon lange nicht mehr gesehen. \#

Wie geht es dir denn? \%

Anna: $\quad$ Mir geht es ganz gut, \#

Isabelle. \#

Ich bin gerade auf der Suche nach einem Geburtstagsgeschenk für eine Freundin. \# Was treibt dich denn in die Stadt? \%

Isabelle: Ach, \#

ich will einfach nur ein bisschen herumschlendern \#

und in ein paar Läden nach neuen Klamotten Ausschau halten. \#

Ich habe mir eben sogar schon eine Jeans gekauft. \#

Warte, \#

ich zeige sie dir mal. \#

Die ist doch toll, \#

oder? \%

Anna: Ja, \#

in der Tat. \#

Wo hast du die Hose denn gekauft? \#

Die wäre wirklich super als Geschenk! \%

Isabelle: Also, \#

die kannst du im neuen Geschäft, du weißt schon, an der nächsten Ecke erstehen. \# In dem Laden scheinen ganz... \%

Anna: $\quad$ Oh, \#

das ist doch der Laden, der letzte Woche eröffnet und gerade lauter Sonderangebote hat. \%

Isabelle: Ja, \#

ganz genau! \%

Anna: $\quad$ Dann werde ich da gleich vorbeischauen, \#

um nach Schnäppchen Ausschau zu halten. \#

Ich hoffe, dass wir uns bald mal wieder sehen. \%

\footnotetext{
32 Gleichrangig sind Elemente beliebigen Umfangs, die von einem und demselben Wort unmittelbar abhängen oder in gleicher Weise unabhängig sind (Engel, 1996), so zum Beispiel: alte und neue Bücher.

${ }^{33}$ Gleichartig sind Elemente, die der gleichen Klasse syntaktischer Glieder angehören (Engel, 1996), so zum Beispiel: weil er Angst hatte und eine Lösung nicht in Sicht war (Nebensatz).
} 
Isabelle: Viel Spaß noch \#

und bis bald. $\%$

Abbildung 67: Beispiele für die Anwendung der 10. Regel: Keine Abtrennung von

Aufzählungen

Zusammengefasst kann festgehalten werden:

Aufzählungen von Substantiven, Verben und Adjektiven werden nicht abgetrennt.

\section{Glossar der grammatikalischen Begriffe}

Apposition

Attribut

Einwort-

konstruktion

Ellipse

Infinitivgruppe

Interjektion

Konjunktion

Relativsatz

Satzglied
Appositionen sind nachgestellte nähere Bestimmungen, die im gleichen Kasus stehen wie das Bezugswort, meist ein Nomen (Schurf, 2001). Attribute beziehen sich nie auf das Prädikat und sind somit kein selbständiges Satzglied (Jung, 1973).

Attribute sind immer Teil eines Satzglieds, also keine selbstständigen Satzglieder. Diese Satzteile stehen vor oder hinter ihren Bezugswörtern (z.B. Nomen) und bestimmen sie näher (Schurf, 2001).

Einwortkonstruktionen bestehen, wie der Name schon sagt, aus genau einem Wort. Sie unterscheiden sich insofern von anderen Aussagen, die ebenfalls nur aus einem Wort bestehen, dass sie in sich geschlossen und klar verständlich sind.

Ellipsen sind unvollständige Sätze, bei denen Satzglieder oder Worte, die aus dem Kontext heraus leicht ergänzbar sind, ausgelassen werden (Schurf, 2001). Die Bedeutung und der Sinn des Satzes sind trotzdem klar verständlich, da sich der Inhalt aus der Situation ergibt (Engel, 1996).

Der einfache oder erweiterte Infinitiv ist eine Wortgruppe aus einem Infinitiv mit zu und meist einem oder mehreren davon abhängenden Gliedern (Klosa, 2001).

Die Interjektionen (Empfindungswörter) sind keine Wortart im eigentlichen Sinne. Sie geben Gemüts- und Willensregungen und sinnliche Eindrücke wieder. Typische Beispiele sind au, ach, oh, hm, aha, brr (Jung, 1973).

Konjunktionen sind Wörter, die andere Wörter, Wortgruppen und Sätze miteinander verbinden. Sie werden deshalb auch Bindewörter genannt. Als lediglich verbindende Glieder gehören sie nicht zum Satz und haben somit keinen Satzgliedwert. Der Satz ergibt auch ohne die Konjunktion Sinn. Konjunktionen sind zum Beispiel aber, denn, oder, und, sondern. wo, wenn, nachdem, während, weil, dass, obwohl, da, ob (Jung, 1973).

Der Relativsatz ist ein Nebensatz, der durch ein Relativpronomen mit einem Haupt- oder Nebensatz verknüpft wird. Relativpronomen sind: der, die, das/ welcher, welche, welches. Diese dürfen nicht verwechselt werden mit der, die, das in der Funktion des Artikels oder Demonstrativpronomens (hinweisendes Fürwort). Nur das Relativpronomen kann durch welcher, welche, welches ersetzt werden (Schurf, 2001).

Die Bausteine, aus welchen Sätze zusammengesetzt werden, bezeichnet man als Satzglieder. Ein Satzglied kann sowohl aus einzelnen Wörtern als auch aus Wortgruppen bestehen (Engel, 1996). Zur Ermittlung der Satzglieder wurde eine Methode entwickelt: die Umstellprobe. Sie zeigt, dass bestimmte Wörter nur zusammen im Satz verschoben werden können (Schurf, 2001).

Ein Beispiel für die Umstellprobe:

Der berühmte Schriftsteller/ schreibt/ ein neues Buch/ in diesem Jahr. In diesem Jahr/ schreibt/ der berühmte Schriftsteller/ ein neues Buch. Ein neues Buch/ schreibt/ der berühmte Schriftsteller/ in diesem Jahr. 
Satzwertiges Partizip

Verbativergänzung
Man nennt sie auch Partizipgruppen (Schurf, 2001). Das satzwertige Partizip ist ein Partizip, das mit einer näheren Bestimmung größeren Umfangs verbunden ist. Im Unterschied zum einfachen Partizip, das fast immer einfaches Satzglied oder Attribut ist, ist das satzwertige Partizip aus dem übergeordneten Satz herausgelöst, d. h., es stellt neben dem Verb dieses Satzes einen eigenen Verbalbereich und somit auch inhaltlichen Bereich dar (Klosa, 2001).

Eine Verbativergänzung ist immer seine Ergänzung zu einem Verb, die nur als satzartiges Konstrukt realisiert werden kann. Sie enthält immer ein Verb und ist nie gegen eine nicht satzförmige Ergänzung austauschbar. Ein typisches Beispiel für eine Verbativergänzung ist: Ich finde, dass Hannah Recht hat (Engel, 1996). 


$\begin{aligned} \text { Name } & \text { Michaela Kolbe } \\ \text { Geburtsdatum } & \text { 07.06.1977 } \\ \text { Geburtsort } & \text { Halle/Sa ale } \\ \text { Staatsangehörigkeit } & \text { Deutsch }\end{aligned}$

\section{AUSBILDUNG}

Georg-August-Universität Göttingen:

Diplom in Psychologie

Georg-August-Universität Götingen und Martin-Luther-Universität Halle-

Wittenberg

Psychologiestudium

Abitur

Landesgymna sium

Latina August Hemann Francke in Ha lle

Polytechnisc he Oberschule „Arthur Becker"

in Halle

\section{BERUFUC HER WERDEG ANG}

Forsc hung sa ufentha It an der Université de Neuchâtel, Groupe de Psychologie Appliquée, Sc hweiz (DAAD-Stipendium)

Wissenschaftlic he Mitarbeiterin in der Abteilung für Sozial- und Kommunikationspsychologie an der GeorgAugust-Universität Göttingen

Benufspra ktikum im Institut für Trainings- und Untemehmensberatung (ift) in Köln

Betriebspsychologisches Pra ktikum bei der Psycholog isc hen Personalberatung der

\section{Deutsc hen Luthansa}

Studentische Hilfskraft in der Abteilung für Sozial- und Kommunikationspsychologie an der Georg-August-Universität Göttingen

Studentisc he Hilfskraft in der Beratungsstelle für Studierende der Georg-August-Universität

\section{Göttingen}

Interviewerin im J ugendstra fvollzug in Ha lle im Rahmen eines Forschungsp rojekts des

Kriminologischen Forsc hungsinstitutes
J a nuar 2003

O kto ber 1996 - J a nuar 2003

Sommer 1996

August 1991 - Sommer 1996

September 1984 bis J uli 1991

J uli - September 2004

Seit Februar 2003

Oktober - November 2001

J uni - J uli 2001

A pril 2001 - J uni 2002

Mai 2000 - April 2001

Februar 1998 - Dezember 1999 